GÖTTINGER SCHRIFTEN ZUR INTERNETFORSCHUNG

Hg.: S. Hagenhoff, D. Hogrefe, E. Mittler,
M. Schumann, G. Spindler, V. Wittke

\title{
Rechtliche Probleme der elektronischen \\ Langzeitarchivierung wissenschaftlicher Primärdaten
}

\section{Tobias Hillegeist}



Tobias Hillegeist

Rechtliche Probleme der elektronischen Langzeitarchivierung wissenschaftlicher Primärdaten

This work is licensed under the

Creative Commons License 3.0 "by-nd", allowing you to download, distribute and print the document in a few copies for private or educational use, given that the document stays unchanged and the creator is mentioned. You are not allowed to sell copies of the free version. 
erschienen als Band 8 in der Reihe „Göttinger Schriften zur Internetforschung“ im Universitätsverlag Göttingen 2012 
Tobias Hillegeist

Rechtliche Probleme der elektronischen

Langzeitarchivierung

wissenschaftlicher

Primärdaten

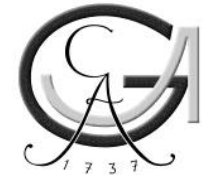

Universitätsverlag Göttingen 2012 


\title{
Bibliographische Information der Deutschen Nationalbibliothek
}

Die Deutsche Nationalbibliothek verzeichnet diese Publikation in der Deutschen Nationalbibliographie; detaillierte bibliographische Daten sind im Internet über $<$ http://dnb.ddb.de $>$ abrufbar.

\section{Reihe}

Band 8 der Reihe „Göttinger Schriften zur Internetforschung“ in der qualitätsgeprüften Sparte des Universitätsverlags Göttingen.

Herausgeber der Reihe: Svenja Hagenhoff, Dieter Hogrefe, Elmar Mittler, Matthias Schumann, Gerald Spindler und Volker Wittke.

\author{
Anschrift des Autors \\ Tobias Hillegeist \\ e-mail: hillegeist@hillegeist-partner.de
}

Dieses Buch ist auch als freie Onlineversion über die Homepage des Verlags sowie über den OPAC der Niedersächsischen Staats- und Universitätsbibliothek (http://www.sub.uni-goettingen.de) erreichbar und darf gelesen, heruntergeladen sowie als Privatkopie ausgedruckt werden. Es gelten die Lizenzbestimmungen der Onlineversion. Es ist nicht gestattet, Kopien oder gedruckte Fassungen der freien Onlineversion zu veräußern.

Satz und Layout: Tobias Hillegeist

Umschlaggestaltung: Martin Kaspar und Margo Bargheer

(C) 2012 Universitätsverlag Göttingen http://univerlag.uni-goettingen.de ISBN: 978-3-86395-066-8

ISSN: 1863-0944 


\section{Vorwort}

Die vorliegende Arbeit wurde im Wintersemester 2009/2010 von der Juristischen Fakultät der Georg-August-Universität Göttingen als Dissertation angenommen. Das Manuskript wurde im Januar 2010 fertiggestellt. Soweit dies möglich und inhaltlich sinnvoll war, wurden auch Publikationen bis zum Zeitpunkt des Rigorosums im Frühjahr 2011 berücksichtigt.

Mein herzlicher Dank gilt meinem Doktorvater, Herrn Prof. Dr. Gerald Spindler, der das Thema der vorliegenden Arbeit anregte und mir während der Erstellung stets beratend zur Seite stand. Nicht zuletzt wegen der Eindrücke und Erfahrungen, die ich während der äußerst schönen und lehrreichen Zeit in seinem Lehrstuhlteam verbringen durfte, reifte in mir schließlich der Entschluss, zu promovieren. Herrn Prof. Dr. Andreas Wiebe danke ich für die Erstellung des Zweitgutachtens.

Besonderer und großer Dank gilt meinen Eltern für Ihre Förderung während meines bisherigen Lebensweges und ihre Unterstützung während meines Studiums. Ihnen widme ich diese Arbeit.

Für Ihre jahrelange treue Freundschaft und einer damit einhergehenden großartigen Studienzeit in Göttingen danke ich Herrn Jens Bogner, Herrn Björn Bogner, Herrn Robert Aue, Herrn Niklas Stahnke, Herrn Ramin Wais und Dr. Jörn Heckmann. Letzterem danke ich außerdem für die zahlreichen fachlichen Diskussionen während der Entstehung der Dissertation sowie die gemeinsame Zeit in 
den Büros der Wilhelm-Weber-Straße. Für die Mithilfe im Rahmen der Erstellung der Druckfahnen danke ich meiner Mutter, meinen Schwestern Catharina und Johanna sowie Herrn Christoph Schulze.

Schließlich danke ich von ganzem Herzen meiner Lebensgefährtin Frau Karina Kaspareit für ihre grenzenlose und liebevolle Unterstützung und Hilfsbereitschaft während der gesamten Promotionszeit. 


\section{Inhaltsverzeichnis}

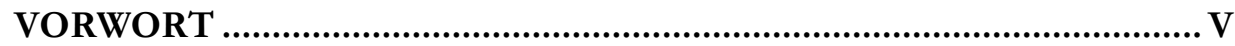

INHALTSVERZEICHNIS ........................................................... VII

LITERATURVERZEICHNIS .............................................................

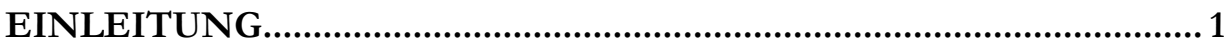

KAPITEL 1 - URHEBERRECHTLICHE PROBLEMATIK..................... 5

A. Urheberrechtliche Schutzfähigkeit wissenschaftlicher Primärdaten...............5

I. Urheberrechtlicher Schutz von Daten und wissenschaftlichen

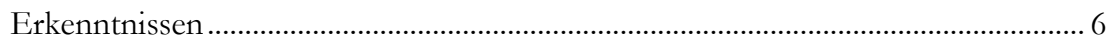

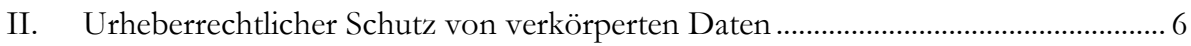

1. Voraussetzungen eines urheberrechtlichen Schutzes ………………….................. 6

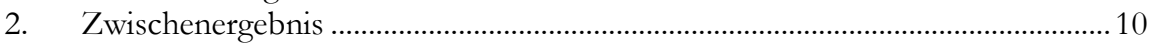

3. Urheber- und leistungsrechtlicher Schutz aufgrund der Zusammenstellung und Sammlung von wissenschaftlichen Primärdaten........................................... 10

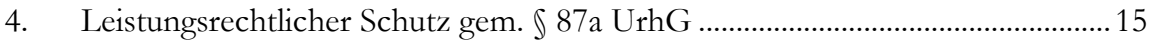

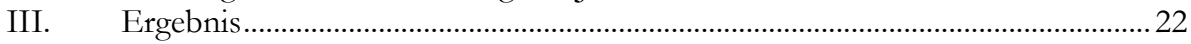

B. Rechtliche Probleme bei der Speicherung und Bearbeitung

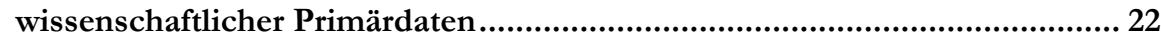

I. Vorüberlegungen.................................................................................................. 22

II. Urheberrechtliche und leistungsrechtliche Zulässigkeit der Kopie und

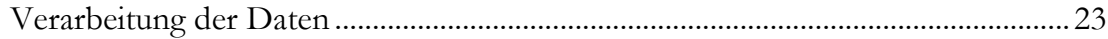

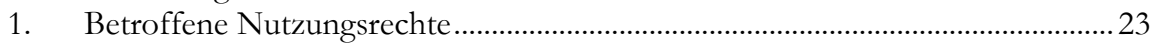

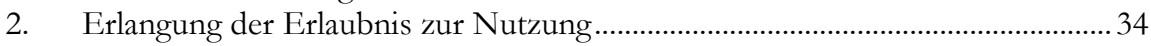

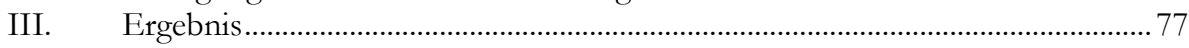

C. Rechtliches Verhältnis zwischen archivierender Einrichtung und

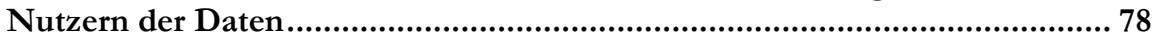

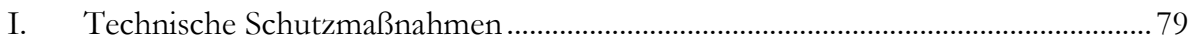




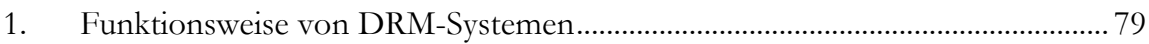

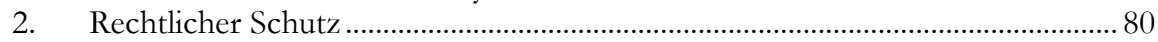

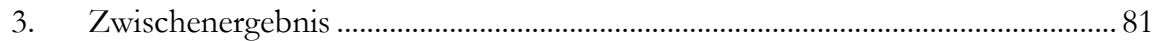

II. Rechtlicher Schutz vor der Weitergabe durch Fremdforscher ................................. 81

1. Weitergabeverbot aufgrund vertraglicher Vereinbarung ….................................. 82

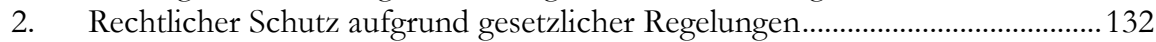

3. Rechtsfolgen der unerlaubten Verwertung des Werkes .......................................134

4. Urheber- oder leistungsrechtlicher Schutz an dem

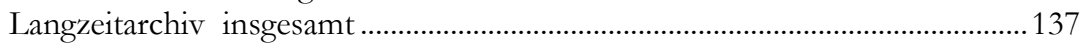

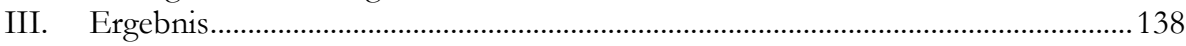

IV. Anlage Entwurf einer Verschwiegenheitsklausel .................................................... 139

\section{KAPITEL 2 - DATENSCHUTZRECHTLICHE PROBLEMATIKEN .141}

A. Datenschutzrechtliche Zulässigkeit der Langzeitarchivierung wissenschaftlicher Daten mit Personenbezug .............................................141

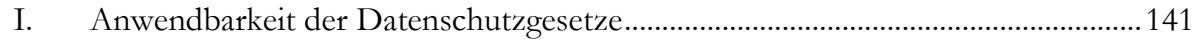

1. Anwendbarkeit der Landesdatenschutzgesetze ..................................................... 142

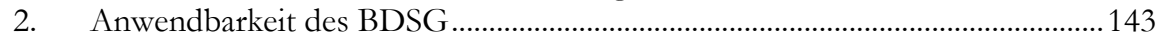

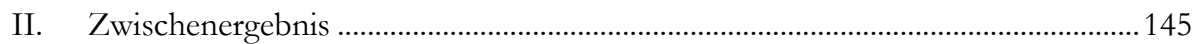

B. Rechtliche Einschränkungen im Rahmen der Archivierung wissenschaftlicher Primärdaten mit Personenbezug .................................. 146

I. Zulässigkeit der Archivierung medizinischer personenbezogener

Forschungsdaten nach den Vorschriften des BDSG und der

Landesdatenschutzgesetze.

1. Rechtliche Anforderungen an eine Einverständniserklärung des

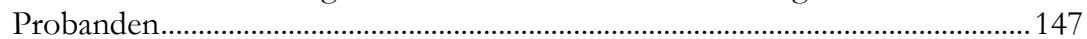

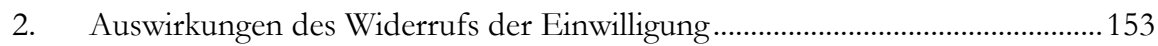

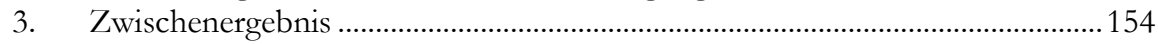

4. Erlaubnistatbestände des BDSG und der Landesdatenschutzgesetze ...............155

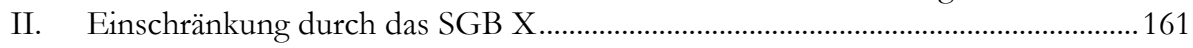

1. Anwendbarkeit des $\int 67 b$ SGB X auf die vorliegenden Fälle.............................. 161

2. Zulässigkeit der Archivierung aufgrund der Erlaubnisnorm

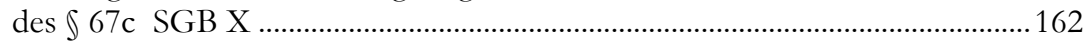

III. Entbindung des Arztes von seiner Schweigepflicht ...........................................164

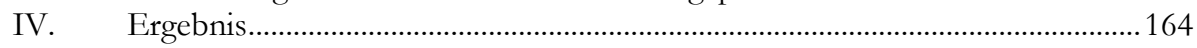

C. Technische und organisatorische Zugangssicherungsmaßnahmen

bei der Langezeitarchivierung personenbezogener Daten ........................ 165

I. Gesetzliche Vorgaben zur Anonymisierung und Pseudonymisierung .................... 165

1. Pflicht zur gesonderten Speicherung von Merkmalen, anhand derer ein Personenbezug hergestellt werden kann .............................................................. 165

2. Pflicht zur separaten Speicherung aufgrund teleologischer Auslegung ............ 166

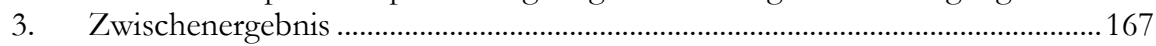

II. Organisationspflichten der archivierenden Einrichtung .......................................... 167

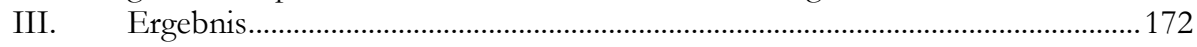


D. Rechtsfolgen des Verstoßes gegen einzelne Bestimmungen des BDSG, des SGB X oder der Landesdatenschutzgesetze.........................................173

I. Strafrechtliche Konsequenzen und Ordnungswidrigkeiten ...................................174

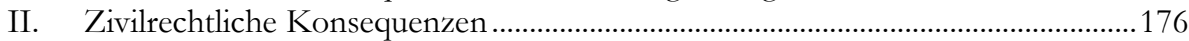

1. Zivilrechtliche Konsequenzen im Anwendungsbereich des BDSG ..................176

2. Zivilrechtliche Konsequenzen im Anwendungsbereich der Landesdatenschutzgesetze

E. Rechtliche Anforderungen an Verträge über die Archivierung

personenbezogener Forschungsdaten ...................................................182

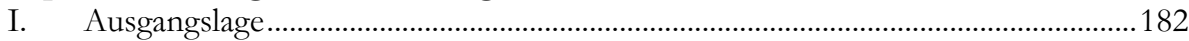

II. Gesetzliche Vorschriften hinsichtlich des Vertragsinhalts ......................................183

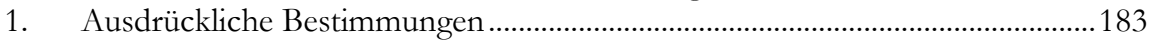

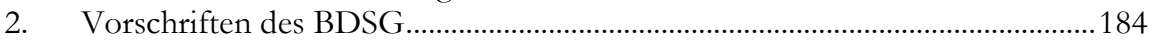

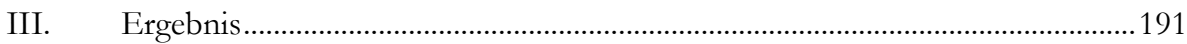

KAPITEL 3 - HAFTUNGSRECHTLICHE PROBLEME ......................193

A. Rechtliche Pflicht zur Langzeitarchivierung und Verantwortlichkeit...........194

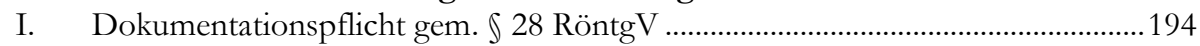

II. Dokumentationspflicht gem. $\int 42$ Strahlenschutzverordnung (StrlSchV)............195

III. Dokumentationspflicht gem. $\int 1$ der Verordnung über Aufzeichnungen bei gentechnischen Arbeiten oder über Freisetzungen (GenTAufzV) .................196

IV. Dokumentationspflicht gem. \10 Berufsordnung der Ärztekammer Niedersachsen......................................................................................197

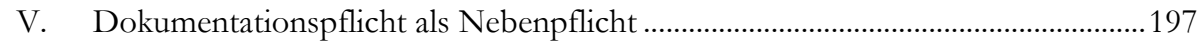

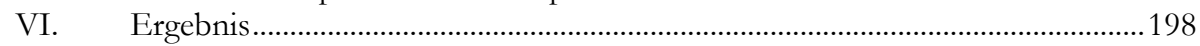

B. Sicherstellung der Authentizität und Integrität der archivierten Daten ........199

I. Systembezogene und organisatorische Sicherungsmittel ........................................200

II. Datenträgerbezogene Sicherungsmittel.......................................................................20

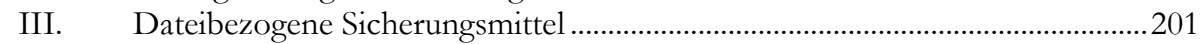

1. Voraussetzungen einer qualifizierten elektronischen Signatur ...........................201

2. Lebensdauer der Signatur vs. Archivierungsdauer...............................................202

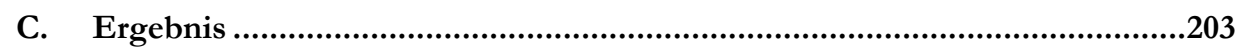

D. Haftung für Datenfehler, die im Rahmen der Archivierung verursacht werden..................................................................................204

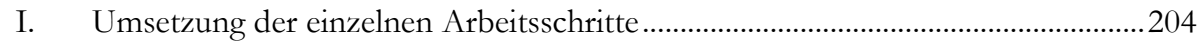

1. Archivierung durch Dritte .....................................................................................204

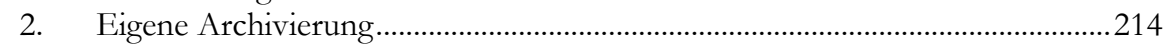

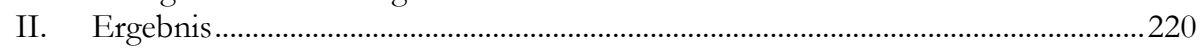

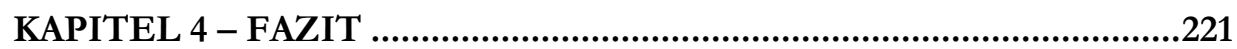





\section{Literaturverzeichnis}

Arlt, Cristian, $\quad$ Digital Rights Management Systeme: Der Einsatz technischer Maßnahmen zum Schutz digitaler Inhalte, 2006.

Bamberger, Heinz G./Roth, Kommentar zum Bürgerlichen Gesetzbuch, Herbert (Hrsg.), Bd. 1, 2. Aufl. 2007.

Bamberger, Heinz G./Roth, Kommentar zum Bürgerlichen Gesetzbuch, Herbert (Hrsg.), Bd. 2, 2. Aufl. 2008.

Bechtold, Stefan, Vom Urheber- zum Informationsrecht: Implikationen des Digital Rights Management, 2002. 
Bensinger, Viola,

Berger, Christian,

Berger, Christan,

Bernuth, Wolfgang von.,

Beushausen, Till,

Beutelspacher, Alb-

recht/Schwenk,

Jörg/Wolfenstetter, Klaus-

Dieter,

Bundesamt für Sicherheit in der Informationstechnik BSI

(Hrsg.),

Dannecker, Gerhard,
Sui-generis Schutz für Datenbanken - Di EG-Datenbankrichtlinie vor dem Hintergrund des nordischen Rechts, 1999.

„Der Schutz elektronischer Datenbanken nach der EG-Richtlinie vom 11. 3. 1996“,GRUR 1997, 169.

Die Neuregelung der Privatkopie in $\ 53$ Abs. 1 UrhG im Spannungsverhältnis von geistigem Eigentum, technischen Schutzmaßnahmen und Informationsfreiheit", ZUM 2004, 257-266.

„Streitpunkt - der Regelungsgehalt des \$ 52a UrhG“, ZUM 2003, 438.

„Modernes Wissensmanagement trotz ,amtlicher Werke“? - Zur Urheberrechtlichen Schutzfähigkeit kommunaler Datenbanken“, MMR 2007, Heft 1 VIII-X.

Moderne Verfahren der Kryptographie : von RSA zu Zero-Knowledge, 2006.

Handbuch für die sichere Anwendung der Informationstechnik, 1992, abrufbar unter: http://www.bsi.bund.de/literat/kriterie.ht m.

„Rechtsschutz nach der DatenbankRichtlinie: Einführung ,geeigneter Sanktionen“،, K\&R 1999, 529-541.

Kommentar zum NDSG; abrufbar unter: http:/ /www.lfd.niedersachsen.de/master/ C52351301_N38588372_L20_D0_I560.ht ml, (Stand: 09.01.2009).
Der Landesbeauftragte für den Datenschutz Niedersachsen, 
Derclaye, Estelle,

Deutsch, Erwin,

Diemar, Undine von,

Dittmann, Jana,

Dreier, Thomas,

Dreier, Thomas/Schulze, Gernot,

Dreier, Thomas in Lehmann (Hrsg.),

Dreyer, Gunda/Kotthoff, Jost/Meckel, Astrid (Hrsg.),

Dusollier, Séverine,
„Database Sui Generis Right: What is a Substantial Investment? A Tentative Definition“, IIC 2005, 2-30.

„Das Persönlichkeitsrecht des Patienten“, AcP 192 (1992), 162.

„Kein Recht auf Privatkopien - Zur Rechtsnatur der gesetzlichen Lizenz zu Gunsten der Privatvervielfältigung", GRUR 2002, 587.

Digitale Wasserzeichen: Grundlagen, Verfahren, Anwendungsgebiete, 2000.

„Die Harmonisierung des Rechtsschutzes von Datenbanken in der EG“ GRUR Int. 1992, 739.

Urheberrechtsgesetz: Kommentar, 3. Aufl., München 2008.

Internet- und Multimediarecht (Cyberlaw), Stuttgart 1997.

Heidelberger Kommentar zum Urheberrecht, 2. Aufl., Heidelberg 2009.

„Exceptions and Technological Measures in the European Copyright Directive of 2001 - An Empty Promise“, IIC 2003, 62. 
Ehrmann, Timo/Fischer, Oliver,

Erdmann, Willi,

Euler, Ellen,

Flechsig, Norbert P.,
"Zweitverwertung rechtswissenschaftlicher Texte im Internet", GRUR Int. 2008, 284293.

„Schutz der Kunst im Urheberrechtsgesetz" in Erdmann, W/Mees, H.-K./Piper, H/Otto, T. (Hrsg.), Festschrift für O.-F. Frhr. v. Gamm, 1990, S. 389-404.

„Zur Langzeitarchivierung digital aufgezeichneter Werke und ihrer urheberrechtlichen Einordnung und Beurteilung", AfP 2008, 474.

„Der rechtliche Rahmen der europäischen Richtlinie zum Schutz von Datenbanken“, ZUM 1997, 577.

Frey, Dieter/Rudolph, Matt- „Verfügungen über unbekannte Nutzungshias, arten: Anmerkungen zum Regierungsentwurf des Zweiten Korbs“, ZUM 2007, 1323.

Fromm, Friedrich K. / Nordemann, W. (Hrsg.),

Gaster, Jens-Lienhard, Der Rechtsschutz von Datenbanken, Köln 1999.

Urheberrecht: Kommentar zum Urheberrechtsgesetz, 10. Aufl., Stuttgart 2009.

Gaster, Jens-Lienhard, „Zur anstehenden Umsetzung der EGDatenbankrichtlinie (II)“, CR 1997, 717.

Goebel, Jürgen W./

„Zum Begriff des Erscheinens beim Elektronischen Publizieren“, GRUR 1986, 355361. 
Götting, Horst-P./Schertz, Handbuch des Persönlichkeitsrechts, MünChristian/Seitz, Walter (Hrsg.), chen 2008.

Gola, Peter/Schomerus, Rudolph,

Gottschalk, Eckart,

Gounalakis, Georgios

Guibault, Lucie,

Haberstumpf, Helmut,

Heckmann, Jörn/Weber, Marc,

Hoebbel, Christoph,

Hoeren, Thomas
BDSG: Kommentar, 10. Aufl., München 2010.

„Digitale Musik und Urheberrecht aus USamerikanischer Sicht“, GRUR Int. 2002, 95.

Elektronische Kopien für Unterricht und Forschung ( $\int 52 \mathrm{a}$ UrhG) im Lichte der Verfassung, Tübingen 2003.

„Contracts and Copyright Exemptions“ in Hugenholtz (Hrsg.), Copyright and Electronic Commerce, Den Haag 2000, S. 125 $-163$.

„Der Schutz elektronischer Datenbanken nach dem Urheberrechtsgesetz", GRUR 2003, 14-31.

„Elektronische Netzpublikationen im Lichte des Gesetzes über die Deutsche Nationalbibliothek (DNBG)“, AfP 2008, 269276.

„EG-Richtlinienentwurf über den Rechtsschutz von Datenbanken“, CR 1993, 12-18.

Rechtsfragen zu Langzeitarchivierung (LZA) und zum Anbieten von digitalen Dokumenten durch Archivbibliotheken unter besonderer Berücksichtigung von Online-Hochschulschriften, 2005, abrufbar unter:

http://www.langzeitarchivierung.de/downl oads/mat/hoeren.pdf 
Hoeren, Thomas,

Höftberger, Christian,

Hohagen, Gisbert,

Holznagel, Bernd,

Hubmann, Heinrich,

Jarass, Hans D./

Pieroth, Bodo (Hrsg.),

Katzenberger, Paul,

Katzenberger, Paul,

Kern, Bernd-Rüdiger,
„Der Zweite Korb - Eine Übersicht zu den geplanten Änderungen im Urheberrechtsgesetz“, MMR 2007, 615-620.

Österreichische Rechtsprechung zur Arzthaftung, MedR 2000, 505-511.

„Überlegungen zur Rechtsnatur der Kopierfreiheit" in Ohly/Bodewig/Dreier/ Götting/Haedicke/Lehmann (Hrsg.), Perspektiven des Geistigen Eigentums und Wettbewerbsrechts, Festschrift für Gerhard Schricker, München 2005, S. 353 - 368. Recht der IT-Sicherheit, München 2003.

„Zum Rechtsbegriff des Erscheinens", ZUM 1980, 537-541.

Grundgesetz für die Bundesrepublik Deutschland, 11. Aufl., München 2011.

„Neue Urheberrechtsprobleme der Photographie - Reproduktionsphotographie, Luftbild- und Satellitenaufnahmen", GRUR-Int. 1989, 116.

„Urheberrecht und Datenbanken“, GRUR 1990, 94-100.

„Organisationsverschulden - Ausdruck institutioneller Sorgfaltspflichtverletzungen“, MedR 2000, 347-351. 
Klein, Andreas,

Klickermann, Paul H.,

Klöhn, Lars,

Köhler, Helmut,

Kolle, Gert,

Kotthoff, Jost,

Kroll, Joachim,

Die Landesbeauftragte für den Datenschutz und für das Recht auf Akteneinsicht Brandenburg (Hrsg.),

Laufs, Adolf/Kern, Bernd-R. (Hrsg.),
„Die Zweitverwertung von Stellenanzeigen“, GRUR 2005, 377-383.

„Urheberrechtsschutz bei zentralen Datenspeichern“, MMR 2007, 7-11.

„Unbekannte Nutzungsarten nach dem „Zweiten Korb“ der Urheberrechtsreform“", K\&R 2008, 77-83

„Die Haftung des Betriebsinhabers für Wettbewerbsverstöße seiner Angestellten und Beauftragten (\13IV UWG)“, GRUR 1991, 344-355.

„Der angestellte Programmierer - Zur rechtlichen Zuordnung von in Arbeitsverhältnissen geschaffenen, insbesondere urheberrechtlich geschützten Softwareprodukten“, GRUR 1985, 1016-1024.

„Zum Schutz von Datenbanken beim Einsatz von CD-ROMs in Netzwerken", GRUR 1997, 597-603.

Datenschutz im Arbeitsverhältnis, Königstein 1981.

Brandenburgisches Informationsgesetzbuch; Teil 1: Datenschutzgesetze; Heft 1 Brandenburgisches Datenschutzgesetz, 4. Auflage, Kleinmachnow 2008.

Handbuch des Arztrechts, 4. Aufl., München 2010 . 
Lehmann, Michael,

Leistner Matthias,

Leistner Matthias,

Leistner Matthias,

Leistner Matthias,

Lippert, Hans-D.,

Loewenheim, Ulrich (Hrsg.),
„The Theory of Property Rights and the Protection of Intellectual and Industrial Property - „Property rights develop to internalize externalities when the gains of internalization become larger than the cost of internalization“"،, IIC 1985, 525-540.

„Verwandte Schutzrechte im europäischen Urheberrecht: Eine Untersuchung anhand des Datenbankherstellerschutzes" in Urheberrecht Gestern - Heute - Morgen: Festschrift für A. Dietz zum 65. Geburtstag, 2001, S. 493-516.

„Der neue Rechtsschutz des Datenbankherstellers - Überlegungen zu Anwendungsbereich, Schutzvoraussetzungen, Schutzumfang sowie zur zeitlichen Dauer des Datenbankherstellerrechts gemäß \$S 87a ff. UrhG“, GRUR Int.1999, 819-839.

Der Rechtsschutz von Datenbanken im deutschen und europäischen Recht, München 2000 .

"Last exit" withdrawal?", K\&R 2007, 457465.

„Wem gehören Daten, die im Rahmen von Forschungsprojekten gewonnen werden?““ in Ahrens, H.-J./Bar, C. v./Fischer, G./Spickhoff, A./Taupitz, J. (Hrsg.), Medizin und Haftung - Festschrift für Erwin Deutsch zum 80. Geburtstag, Berlin Heidelberg 2009, S. 359-369.

Handbuch des Urheberrechts, 2. Aufl., München 2010. 
Lorenz, Stefan,

Maaßen, Wolfgang

Mangoldt, Hermann v./

Klein, Friedrich/Starck,

Christian (Hrsg.),

Mathis, Hans-P.,

Mehrings, Josef,

Möhring, Philipp/Niccolini, Urheberrechtsgesetz, 2.Aufl., München Käte (Hrsg.),

Musielak, Hans-J. (Hrsg.),

Nack, Ralph,

„Nacherfüllungskosten und Schadensersatz nach neuem Schuldrecht - was bleibt vom Dachziegel-Fall?“, ZGS 2004, 408-411.

„Urheberrechtliche Probleme der elektronischen Bildverarbeitung“, ZUM 1992, 338352.

Kommentar zum Grundgesetz, Band 1, 6 . Aufl., München 2010.

Der Arbeitnehmer als Urheber, Frankfurt a.M. 1988.

Der Rechtsschutz computergestützter Fachinformationen - unter besonderer Berücksichtigung von Datenbanken, Baden-Baden 1990.

Schütze, Rolf A./Weipert, Lutz Münchener Vertragshandbuch, Bd. 3 Wirt(Hrsg.), schaftsrecht II, 6. Aufl., München 2009. 2000.

Kommentar zur Zivilprozessordnung, 8. Aufl., München 2011.

„Nationaler und internationaler Rechtsschutz von Datenbanken“, GRUR Int. 2004, 227-234. 
Neuroth, Heike/ Oßwald, Achim/Scheffel, Regine/Jehn, Mathias/Strathmann, Stefan (Hrsg.),

Nolte, Georg,

Ortner, Franz-J./Geis, Ivo,

Palandt, Otto (Hrsg.),
Nestor, Eine kleine Enzyklopädie der digitalen Langzeitarchivierung, Version 2.0, Boizenburg 2009.

„Paperboy oder die Kunst den Informationsfluss zu regulieren“, ZUM 2003, 540549.

„Die elektronische Patientenakte“, MedR 1997, 337-341.

Bürgerliches Gesetzbuch, 71. Aufl., München 2012.

Podlech, Adalbert/Pfeifer, „Die informationelle Selbstbestimmung im Michael, Spannungsverhältnis zu modernen Werbestrategien“, RDV 1998, 139-154.

Prütting, Hanns/Wegen, Ge- BGB: Kommentar, 6. Aufl., Köln 2011. rhard/Weinreich, Gerd (Hrsg.),

Raue, Peter/Bensinger, Viola, „Umsetzung des sui-generis-Rechts an Datenbanken in den $\iint 87 \mathrm{a}$ ff. UrhG“, MMR 1998, 507-512.

Redeker, H. (Hrsg.),

Handbuch der IT-Verträge, Bd. I, Stand: Okt. 2007.

Rehbinder, M.,

,Zu den Nutzungsrechten an Werken von Hochschulangehörigen" in Forkel, H/Kraft, A. (Hrsg.), Beiträge zum Schutz der Persönlichkeit und ihrer besonderen schöpferischen Leistung, Festschrift für H. Hubmann zum 70. Geburtstag, 1985, S. 359-372. 
Ders.,

Reinhard, T./Pohl, Capellaro, H.-C. (Hrsg.),

Roßnagel A./Schmücker, P.,

Roßnagel, A. (Hrsg.),

Ders.,

Ders.,

Roßnagel, A./Fischer-Dieskau, S./Jandt, S./Knopp, M.,

Roßnagel, A./Garstka, H., A./Pfitzmann, Modernisierung des Datenschutzrechts:

Roßnagel, A./Scholz, P., Gutachten im Auftrag des Bundesministeriums des Inneren, 2001.

Urheberrecht: Ein Studienbuch, 15. Aufl. 2008.

L./ IT-Sicherheit und Recht - Rechtliche und technisch-organisatorische Aspekte für Unternehmen, Berlin 2007.

Beweiskräftige elektronische Archivierung: Bieten elektronische Signaturen Rechtssicherheit?, 2006.

Recht der Multimediadienste: Kommentar zum IuKDG und zum MDStV, Losebl.Ausg., 7. Aufl. 2005, Stand: April 2005.

Handbuch Datenschutzrecht: Die neuen Grundlagen für Wirtschaft und Verwaltung, 2003.

„Datenschutz in globalen Netzen - Das TDSG - Ein wichtiger Schritt“, DuD 1999, 253.

Langfristige Aufbewahrung elektronischer Dokumente, 2007.

„Datenschutz durch Anonymität und Pseudonymität - Rechtsfolgen der Verwendung anonymer und pseudonymer Daten “, MMR 2000, 721. 
Säcker, F.S./Rixecker, R. Münchener Kommentar zum Bürgerlichen (Hrsg.),

Gesetzbuch, Bd. 2, Schuldrecht Allgemeiner Teil, 5. Aufl. 2007.

Dies.,

Münchener Kommentar zum Bürgerlichen Gesetzbuch, Bd. 3, Schuldrecht Besonderer Teil I, 6. Aufl. 2012.

Schack, H.,

„Rechtsprobleme der Onlineübermittlung“, GRUR 2007, 639.

Ders., „Schutz digitaler Werke vor privater Vervielfältigung - zu den Auswirkungen der Digitalisierung auf $₫ 53$ UrhG“, ZUM 2002, 497.

Schmechel-Gaumé,

K\&R 2001. 74, 76

Schmidt-Bleibtreu,

Kommentar zum Grundgesetz, 11. Aufl. B./Hofmann, H./Hopfauf, A. 2008.

(Hrsg.),

Schneider, J., Handbuch des EDV-Rechts, 3. Aufl. 2003.

Scholz in Mestmäcker/Schulze Urheberrechtskommentar, Stand: 47. Ak(Hrsg.), tualisierungslieferung, August 2008, Bd. 1.2, München.

Schricker, G. (Hrsg.),

Urheberrecht: Kommentar, 3. Aufl. 2006. 
Schütze, R.A./Weipert, L. Münchener Vertragshandbuch, Bd.3 Wirt(Hrsg.), schaftsrecht II, 2009.

Sendrowski, H., „Zum Schutzrecht „sui generis“ an Datenbanken“, GRUR 2005, 369-377.

Simitis, S. (Hrsg.),

Bundesdatenschutzgesetz, 7. Aufl. 2011.

Sonntag, Matthias,

IT-Sicherheit kritischer Infrastrukturen, München 2005.

Spindler, G.,

„Europäisches Urheberrecht in der Informationsgesellschaft“, GRUR 2002, 105120.

Spindler G. (Hrsg.),

Vertragsrecht der Internet-Provider, 2. Aufl. 2004

ders. (Hrsg.),

„Rechtliche Rahmenbedingungen von Open Access - Publikationen", Göttingen 2006.

ders.,

„Die Entwicklung des EDV-Rechts 2007/2008“, K\&R 2008, 565.

ders.,

„Reform des Urheberrechts im „Zweiten Korb“"،, NJW 2008, 9. 
Spindler, G./Heckmann, J., „Retrodigitalisierung verwaister Printpublikationen - Die Nutzungsmöglichkeiten von „orphan works" de lege lata und de lege ferenda“, GRUR Int. 2008, 271-284.

Sprang, C./Ackermann, A., „Der „Zweite Korb“ aus Sicht der (Wissenschafts-)Verlage“, K\&R 2008, 7-11.

Stoffels, M.,

AGB-Recht, 2. Aufl. 2009.

Stumpf, H./Groß, M.,

Der Lizenzvertrag, 8. Aufl., Frankfurt/a.M. 2005.

Süßenberger, C./Czychowski, „Das „Erscheinen“ von Werken ausschließC., lich über das Internet und ihr urheberrechtlicher Schutz in Deutschland - Einige Argumente Pro und Contra“, GRUR 2003, 489.

Tinnefeld, M.-T./Ehmann, Einführung in das Datenschutzrecht, 4. E./Gerling, R.W. (Hrsg.), Aufl., München Wien 2005.

Trayer, M.,

Technische Schutzmaßnahmen und elektronische Rechtewahrnehmungssysteme: Die Umsetzung von Art. 6 und 7 der EUUrheberrechtsrichtlinie in deutsches Recht und der Schutz des Nutzers urheberrechtlicher Werke, 2003.

Thüringer Innenministerium

Hinweise des Thüringer Innenministerium zum Thüringer Datenschutzgesetz ThürDSG - vom 07. Februar 2003, abrufbar unter: http://www.thueringen.de/de/tim/rechtsg rundlagen/datenschutz/ 
Ulmer/Brandner/Hensen (Hrsg.),

Vinje, T.C.,

Vogel, M.,

Wandtke, A.,

Wandtke, A.,

Wandtke, A.,

Weber, R.H./Willi, A.,

Wiebe, A., (Hrsg.),
AGB-Recht: Kommentar, 11. Aufl. 2011.

„Copyright Imperilled“, E.I.P.R. 1999, 21 (4), 192.

„Die Umsetzung der Richtlinie 96/9/EG über den rechtlichen Schutz von Datenbanken in Art. 7 des Regierungsentwurfs eines Informations- und Kommunikationsdienstegesetz“, ZUM 1997, 592.

Die Rechte der Urheber und ausübenden Künstler im Arbeits- und Dienstverhältnis, Berlin 1993.

„Reform des Arbeitnehmerurheberrechts?", GRUR 1999, 390.

„Copyright und virtueller Markt in der Informationsgesellschaft - oder das Verschwinden des Urhebers im Nebel der Postmoderne?", GRUR 2002, 1, 10.

Wandtke, A./Bullinger, W. Praxiskommentar zum Urheberrecht, 3. Aufl. 2009.

IT-Sicherheit und Recht - Grundlagen eines integrativen Gestaltungskonzepts, Zürich 2006.

„Rechtsschutz von Datenbanken und europäische Harmonisierung“, CR 1996, 198. 
Wohlgemut, Hans. H/Gerloff, Datenschutzrecht: Eine Einführung mit J., $\quad$ praktischen Fällen, 3. Aufl. 2005, Rn. 182.

Wolter, H.T., „Rechtliche Betrachtung von Digital Rights Management in Peer-to-Peer-Netzwerken“, JurPC Web-Dok. 160/2008.

Wolters, K., Datenschutz und medizinische Forschungsfreiheit, 1988.

Zöllner, W., „Die Reichweite des Urheberrechts im Arbeitsverhältnis untypischer Urheber" in Forkel, H/Kraft, A. (Hrsg.), Beiträge zum Schutz der Persönlichkeit und ihrer besonderen schöpferischen Leistung, Festschrift für H. Hubmann zum 70. Geburtstag, 1985, S. 523-539. 


\section{Einleitung}

Kernaufgabe der Wissenschaft ist das ernsthafte Streben nach neuen Erkenntnissen, wozu auch die Bewahrung und Erhaltung bereits vorhandenen Wissens zählt, aus dem neue Erkenntnisse gewonnen werden können. Im Rahmen von Publikationen wird diese Aufgabe hauptsächlich von Bibliotheken, wie etwa den Universitätsbibliotheken und der Deutschen Nationalbibliothek, sowie einer Vielzahl von Archiven wahrgenommen. Neben wissenschaftlichen Publikationen werden auch die in wissenschaftlichen Forschungsprojekten gewonnenen Primärdaten archiviert.

War dies früher gerade bei umfangreichen Datensätzen sehr aufwendig, können im heutigen Zeitalter der elektronischen Datenverarbeitung auch umfangreiche Datenmengen problemlos in digitaler Form gespeichert werden. Eine elektronische Archivierung hat darüber hinaus den Vorteil, dass die Daten mittels eines Netzwerkes anderen Forschern zur Verfügung gestellt werden können, da diesen lediglich der Zugang zu den betreffenden Dateien eingeräumt werden muss. Bedeutung hat dies zum einen bei großen Forschungseinrichtungen, da diese ihren angestellten Wissenschaftlern den Zugang zu bereits erhobenen Daten ermöglichen können. Zum anderen besteht die Möglichkeit, Daten mit anderen Forschungseinrichtungen im Rahmen von Kooperationen auszutauschen.

Die elektronische Archivierung und die damit einhergehende Übermittlung wissenschaftlicher Primärdaten begegnet jedoch einer Reihe von rechtlichen Prob- 
lemen. So entbrennt nicht selten ein Streit darüber, wer „Inhaber“ der betreffenden Daten ist. Dabei stehen sich regelmäßig die Forschungseinrichtung, die das jeweilige Forschungsprojekt finanziert hat und der beziehungsweise die Wissenschaftler gegenüber, die das Projekt geleitet und durch ihre fachliche Kompetenz dazu beigetragen haben, dass die Daten überhaupt gewonnen werden konnten. Von den Vertretern der Forschungseinrichtungen wird in diesem Zusammenhang oftmals geltend gemacht, dass diese aufgrund der getätigten Investitionen Inhaber der Daten seien. Die Wissenschaftler stützen sich hingegen regelmäßig darauf, dass ihnen angeblich ein Urheberrecht an den betreffenden Daten zustehe. Ob an wissenschaftlichen Primärdaten ein urheberrechtlicher Schutz entstehen kann, soll daher im Rahmen dieser Arbeit geprüft werden.

Sofern diese Frage zu bejahen ist, kommt es ferner darauf an, wer Inhaber der Nutzungsrechte an diesen Daten ist und ob diese Rechte durch eine elektronische Archivierung berührt werden. Sofern die letzte Frage zu bejahen ist, kommt es für archivierende Einrichtungen darauf an, ob sie die Daten trotzdem archivieren dürfen, wobei diesbezüglich vor allem zu untersuchen ist, inwieweit urheberrechtliche Schranken im Rahmen der elektronischen Archivierung Anwendung finden. Darüber hinaus stellt sich für Forschungseinrichtungen die Frage, wie sie die zur Archivierung erforderlichen Nutzungsrechte erlangen können, sofern keine urheberrechtliche Schranke greifen sollte. In diesem Zusammenhang sind insbesondere diejenigen Fallkonstellationen zu betrachten, in denen der Rechteinhaber zum Zeitpunkt der Rechteerlangung in einem Angestelltenverhältnis zur archivierenden Einrichtung stand. Des Weiteren ist zu prüfen, inwieweit die archivierende Einrichtung die benötigten Nutzungsrechte aufgrund der vom Gesetzgeber zum 01.01.2008 in das Urheberrechtsgesetz eingefügten Rechteübertragungsfiktion des \1371 UrhG erlangen kann.

Für den Fall, dass urheberrechtliche Nutzungsrechte bei der archivierenden Einrichtung selbst entstehen sollten, ist ferner bedeutend, wie diese sich vor einem Missbrauch dieser Daten durch Dritte schützen kann. Relevant ist dies vor allem in den bereits oben angesprochenen Fällen, in denen die Daten nicht lediglich gespeichert, sondern auch für Fremdforscher und andere Forschungseinrichtungen zur Verfügung gestellt werden. In diesem Zusammenhang ist außerdem von Bedeutung, inwieweit die Verträge mit den Nutzern der Daten ausgestaltet sein müssen. Insbesondere soll im Folgenden geprüft werden, ob durch eine derartige Vereinbarung urheberrechtliche Schranken der Nutzer abbedungen werden und ob eine solche Abbedingung urheberrechtlicher Schranken zulässig ist.

Neben diesen urheberrechtlichen Fragen können im Rahmen der digitalen Langzeitarchivierung weitere rechtliche Probleme auftreten, sofern personenbezogene Daten archiviert werden sollen, was vor allem im medizinischen Bereich denkbar ist. Diesbezüglich soll im Folgenden dargelegt werden, ob und unter welchen Voraussetzungen in den vorliegend im Fokus stehenden Fällen datenschutz- 
rechtliche Vorschriften Anwendung finden und welche rechtlichen Einschränkungen sich daraus für die Archivierung ergeben.

Daneben ist für die archivierende Einrichtung von Bedeutung, welche rechtlichen Konsequenzen eine unrechtmäßige Archivierung personenbezogener Daten nach sich ziehen würde. In diesem Zusammenhang ist außerdem zu beachten, dass gerade kleine Forschungseinrichtungen oftmals nicht über die notwendigen technischen und personellen Ressourcen verfügen, um die Archivierung selbst durchzuführen, weshalb nicht selten ein Fremdunternehmen mit diesen Aufgaben beauftragt wird. Insofern stellt sich die Frage, was die archivierende Einrichtung bei der Auftragserteilung aus datenschutzrechtlicher Sicht beachten muss.

Schließlich ist zu bedenken, dass bei der Durchführung von Archivierungsmaßnahmen Dateien gelöscht oder beschädigt werden können. Die hieraus entstehenden haftungsrechtlichen Probleme sollen in dieser Arbeit ebenfalls untersucht werden. 



\section{Kapitel 1 - Urheberrechtliche Problematik}

\section{A. Urheberrechtliche Schutzfähigkeit wissenschaftlicher Primärdaten}

Entscheidend für die Beantwortung der folgenden Fragen ist zunächst, ob an wissenschaftlichen Primärdaten ein urheberrechtlicher Schutz entsteht und, sofern diese Frage zu bejahen ist, wem die aus dem Urheberrecht folgenden Nutzungsrechte daran zustehen. Dies hängt insbesondere davon $a b$, ob wissenschaftliche Primärdaten überhaupt urheberrechtsfähig sind. Dabei ist danach zu differenzieren, ob, und wenn ja in welcher Form, die Daten verkörpert sind. Aus diesem Grund wird im Folgenden danach differenziert, ob an einem Datum an sich, also dem Ergebnis einer Messung oder eines Versuchs, und der daraus resultierenden Erkenntnis ein urheberrechtlicher Schutz entstehen kann (I.) oder ob die Daten auf Papier oder einem digitalen Datenträger verkörpert sein müssen bzw. ob diese Verkörperung bestimmten Anforderungen entsprechen muss, damit ein urheberrechtlicher Schutz entsteht(II.1.). Des Weiteren stellt sich die Frage, welche Auswirkungen es auf die urheberrechtliche Schutzfähigkeit hat, wenn mehrere Daten miteinander kombiniert und beispielsweise in einer Tabelle zusammengefasst werden(II.3.). 
I. Urheberrechtlicher Schutz von Daten und wissenschaftlichen Erkenntnissen

Zunächst stellt sich die Frage, ob an den Daten an sich, die als Ergebnis einer Studie oder einer statistischen Untersuchung an der Universität erhoben werden, unabhängig davon, ob und in welcher Form sie gespeichert worden sind, ein rechtlicher Schutz nach dem Urheberrechtsgesetz (UrhG) entstehen kann. Das UrhG schützt gem. $\int 1$ und 2 individuelle Geisteswerke. ${ }^{1}$ Dabei folgt nach der heute überwiegenden Ansicht in Rechtsprechung und Literatur, dass der Werkbegriff eine persönliche Schöpfung des Urhebers mit einem geistigen Gehalt voraussetzt, die eine wahrnehmbare Formgestaltung gefunden hat und in der die Individualität des Urhebers zum Ausdruck kommt. ${ }^{2}$ Unabhängig von der Frage der persönlichen geistigen Schöpfung und dem geistigen Gehalt der Daten, muss ein urheberrechtlicher Schutz der Daten an sich, genauer gesagt, der Erkenntnis, die aus einer Messung oder einer Untersuchung folgt, bereits an der fehlenden wahrnehmbaren Formgestaltung ausscheiden. Zwar muss die Formgestaltung nicht schriftlich oder körperlich festgehalten sein, sie muss jedoch wahrgenommen werden können. ${ }^{3}$ Demzufolge scheidet ein urheberrechtlicher Schutz an nicht verkörperten Daten, also an der bloßen Erkenntnis an sich, schon an der fehlenden Wahrnehmbarkeit aus. ${ }^{4}$

\section{Urheberrechtlicher Schutz von verkörperten Daten}

Ein urheber- oder leistungsrechtlicher Schutz kommt indes in Betracht, sofern die zu archivierenden Daten bereits auf einem Datenträger festgehalten worden sind, was wohl in nahezu allen Fällen gegeben sein wird. Dabei ist zunächst fraglich, ob die Voraussetzungen für einen urheberrechtlichen Schutz bei wissenschaftlichen Primärdaten vorliegen, die auf einem Datenträger gespeichert sind.

\section{Voraussetzungen eines urbeberrechtlichen Schutzes}

Der urheberrechtliche Schutz eines Werkes ergibt sich aus $\int 1$ und $\int 2$ des Urheberrechtsgesetzes. Gem. $\int 1$ UrhG genießen die Urheber von Werken der Literatur, Wissenschaft und Kunst für Ihre Werke Schutz nach Maßgabe des UrhG. In diesem Zusammenhang zählt \ 2 UrhG enumerativ auf, welche Werke der Literatur, Wissenschaft und Kunst insbesondere geschützt sind. Entscheidend ist also,

${ }^{1}$ Rehbinder, Urheberrecht, 15. Aufl. 2008, Rn. 145 f.

2 Loewenheim in Schricker/Loewenheim (Hrsg.), Urheberrecht, 4. Aufl. 2010, § 2 Rn. 9; Dreyer in Dreyer/Kotthoff/Meckel, Urheberrecht, 2. Aufl. 2009, \ 2 Rn. 12; Dreier in Dreier/Schulze, Urheberrechtsgesetz, 3. Aufl. 2009, \2 Rn. 8 ff.; A. Nordemann in Fromm/Nordemann, Urheberrecht, 10. Aufl. 2009, \2 Rn. 20; Erdmann in FS Gamm, 1990, S. 389, 395 ff.

${ }^{3}$ Loewenheim in Schricker/Loewenheim, $\sqrt{ } 2$ Rn. 20; Schulze in Dreier/Schulze, $\int 2$ Rn. 13; A. Nordemann in Fromm/Nordemann, $\int 2$ Rn. 23.

4 So auch A. Nordemann in Fromm/Nordemann, \ 2 Rn. 23; Schulze in Dreier/Schulze, \ 2 Rn. 41; Loewenheim in Schricker/Loewenheim, \ 2 Rn. 61, 65. 
ob wissenschaftliche Primärdaten unter den Werkbegriff des $₫ 2$ UrhG fallen und damit einen urheberrechtlichen Schutz gem. \1 UrhG genießen.

a. Werkeigenschaft gem. \2 UrhG

Zunächst könnte es sich bei den verkörperten Daten um Sprachwerke i.S.d. \ 2 I Nr. 1 UrhG handeln. Unter einem Sprachwerk versteht man dabei solche Werke, bei denen der gedankliche Gehalt mit Mitteln der Sprache ausgedrückt wird und die damit der Informationsvermittlung dienen. ${ }^{5}$ Bei wissenschaftlichen Primärdaten handelt es sich um Daten, die bei einer Datenerhebung unmittelbar gewonnen werden. Es kann sich dabei also beispielsweise um Werte einer medizinischen Untersuchung, Messergebnisse, allgemeine Ergebnisse oder auch statistische Werte handeln, die im Rahmen einer Befragung gewonnen werden. Unabhängig davon, auf welche Weise oder für welchen Zweck die Daten gewonnen werden, dienen sie doch der Informationsvermittlung, da hier ein gedanklicher Inhalt mit Mitteln der Sprache ausgedrückt wird. Dem steht auch nicht entgegen, dass es sich bei den Daten oftmals um mathematische Zeichen (wie beispielsweise bei dem Ausdruck eines EKG oder den Ergebnissen physikalischer oder chemischer Untersuchungen) oder Zahlen handelt (wie zum Beispiel bei statistischen Werten oder bei den Ergebnissen von medizinischen Messungen), da auch diese ein Sprachwerk i.S.d. \ 2 I Nr.1 UrhG darstellen können. ${ }^{6}$ Damit könnten wissenschaftliche Primärdaten grundsätzlich ein Sprachwerk i.S.d. \2 I Nr. 1 UrhG darstellen.

Die verkörperten Daten genießen allerdings nur dann urheberrechtlichen Schutz, wenn sie gem. \2 II UrhG eine persönliche geistige Schöpfung darstellen. ${ }^{7}$ Darunter versteht man solche Leistungen, die auf einer menschlichgestalterischen Tätigkeit des Urhebers beruhen. ${ }^{8}$ Die Daten müssten also durch ihre Form oder ihren Inhalt den individuellen Geist des Urhebers ausdrücken. ${ }^{9}$

$\mathrm{Ob}$ eine solche gestalterische Tätigkeit bei wissenschaftlichen Primärdaten vorliegt, erscheint indes fraglich. Eine gestalterische Leistung läge dann vor, wenn die einzelnen Daten ihre Gestalt jeweils dem Tun desjenigen, der sie erhebt, verdan-

\footnotetext{
${ }^{5}$ BGH GRUR 1959, 251- Einheitsfahrschein; Ablberg in Möhring/Niccolini (Hrsg.), Urheberrechtsgesetz, 2.Aufl. 2000, \2 Rn.3; Loewenheim in Schricker/Loewenheim, \$2 Rn. 79; A. Nordemann in Fromm/Nordemann, $\mathbb{} 2$ Rn. 54; Schulze in Dreier/Schulze, \ 2 Rn. 81.

${ }^{6}$ RGZ 121, 357, 358 - Rechentabellen; BGH GRUR 1959, 251; OLG Karlsruhe GRUR 1983, 300, 306 - Inkasso-Programm; A. Nordemann in Loewenheim (Hrsg.), Handbuch des Urheberrechts, 2. Aufl. 2010, \9 Rn. 6; Loewenheim in Schricker/Loewenheim, \ 7 Rn. 80; Ablberg in Möhring/Niccolini, \2 Rn. 3; Schulze in Dreier/Schulze, $\$ 2$ Rn. 81.

7 Ablberg in Möhring/Niccolini, $\ 2$ Rn. 43; Dreyer in Dreyer/Kotthoff/Meckel, $\ 2$ Rn. 12; Schulre in Dreier/Schulze; $\$ 2$ Rn. 16.

${ }^{8}$ Dreyer in Dreyer/Kotthoff/Meckel, $\ 2$ Rn. 17; A. Nordemann in Fromm/Nordemann, $\ 2$ Rn. 21; Loewenheim in Schricker/Loewenheim, $\$ 2$ Rn. 11.

9 BGHZ 9, 262, 268; Ablberg in Möhring/Niccolini, \2 Rn. 53; Loewenheim in Schricker/Loewenheim, \2 Rn. 18; A. Nordemann in Fromm/Nordemann, \2 Rn. 24; Schulze in Dreier/Schulze, $\$ 2$ Rn. 23.
} 
Kapitel 1 - Urheberrechtliche Problematik

ken würden. ${ }^{10}$ Die Tatsache, dass zur Erlangung der Daten oftmals Apparate, wie beispielsweise Messinstrumente zu Hilfe genommen werden, steht dabei einer persönlichen geistigen Schöpfung allein noch nicht entgegen. Viele dieser Instrumente und Maschinen werden in der Regel von einem Menschen eingestellt und bedient. ${ }^{11}$ Jedoch verdanken die einzelnen Daten ihre Gestalt nicht dem Tun desjenigen, der sie erhebt. Vielmehr würden diese Daten bei jeder Person entstehen, die die entsprechende Messung oder den entsprechenden Versuch vornimmt, aus dem die Daten als Ergebnis hervorgehen, und sie auf einem Datenträger oder in analoger Form festhält.

Demzufolge drücken die verkörperten Daten gerade nicht den individuellen Geist desjenigen aus, der sie durch Versuche oder Experimente erlangt hat. Vielmehr sind die Daten von vornherein vorgegeben und müssen lediglich festgestellt und dokumentiert werden. Dies gilt auch dann, wenn zur Erlangung der Daten eine neuartige Methode eingesetzt wird, die bis dahin unbekannt war. Dann würden die Daten zwar mit Hilfe dieser Methode gewonnen werden. Ihre jeweilige Gestalt würde jedoch auch davon nicht beeinflusst. Vielmehr würde das neuartige Verfahren lediglich die Gewinnung der Daten ermöglichen. Damit ist eine persönliche geistige Schöpfung bei wissenschaftlichen Primärdaten grundsätzlich nicht gegeben, so dass das einzelne Datum an sich keinen urheberrechtlichen Schutz nach $\iint 1,2$ I Nr. 1 UrhG genießt.

\section{b. Werkeigenschaft gem. \2 I Nr. 5 UrhG}

Gem. \ 2 Abs. 1 Nr.5 gehören zu den urheberrechtlich geschützten Werken Lichtbildwerke einschließlich der Werke, die ähnlich wie Lichtbildwerke geschaffen werden. Unter die zu archivierenden Daten würden im Bereich der medizinischen Fakultät auch Röntgenbilder fallen. ${ }^{12}$ Diese könnten gem. \ 2 I Nr. 5 UrhG urheberrechtlich geschützt sein. Danach sind Lichtbildwerke einschließlich der Werke, die ähnlich wie Lichtbildwerke geschaffen werden, urheberrechtlich geschützt. Unter einem Lichtbildwerk versteht man dabei jedes Bild für dessen Herstellung strahlungsempfindliche Schichten chemisch oder physikalisch durch Strahlung in einer Weise verändert wurden, dass eine Abbildung entsteht und das Bild eine persönliche geistige Schöpfung i.S.d. \ 2 II UrhG darstellt. ${ }^{13}$ Selbst wenn man jedoch Röntgenbilder unter ,ähnliche“ Werke i.S.d. \ 2 I Nr. 5 UrhG fassen würde, so ist auch hier wieder fraglich, ob die Röntgenbilder eine persönliche

\footnotetext{
${ }^{10}$ Dreyer in Dreyer/Kotthoff/Meckel, $\ 2$ Rn. 19; Bullinger in Wandtke/Bullinger (Hrsg.), Praxiskommentar zum Urheberrecht, 3. Auflage 2009, \$2 Rn. 15; Loewenheim in Schricker/Loewenheim, $\ 2$ Rn. 12.

${ }^{11}$ Bullinger in Wandtke/Bullinger, \2 Rn. 16; Dreier in Dreier/Schulze, \ 2 Rn. 8; A. Nordemann in Fromm/Nordemann, \ 2 Rn. 21; Loewenheim in Schricker/Loewenheim, \ 2 Rn. 13; Ablberg in Möhring/Niccolini, \2 Rn. 51.

12 Vgl. dazu auch Lippert in FS Deutsch, S. 359, 368.

13 BGHZ 37, 1, 6; Dreyer in Dreyer/Kotthoff/Meckel, \2 Rn. 233; Loewenheim in Schricker/Loewenheim, \ 2 Rn. 179; Scbulze in Dreier/Schulze, \ 2 Rn. 199.
} 
geistige Schöpfung i.S.d. \ 2 II UrhG darstellen. Ein menschliches Schaffen kann dabei grundsätzlich bejaht werden, da der Radiologe bzw. die Person, die die Aufnahme erstellt, über die Position des Patienten und den Winkel entscheidet, in dem das zu röntgende Körperteil bestrahlt wird. ${ }^{14}$ Allerdings stellt sich auch hier wieder das Problem, dass es an der Individualität des Röntgenbildes fehlen wird. Die Röntgenaufnahme wäre nur dann individuell, wenn sie eine Aussage des Fotografen bzw. desjenigen enthielte, der die Röntgenaufnahme erstellt hat und die auf einer Gestaltung beruht, wie etwa der Auswahl des Motivs oder der Belichtungsdauer. ${ }^{15}$ Daran fehlt es bei einer Röntgenaufnahme jedoch regelmäßig. Zwar muss der Radiologe stets entscheiden, aus welcher Perspektive er den betroffenen Körperteil bestrahlt, um eine effektive Diagnose zu ermöglichen. Jedoch steckt hinter dieser Auswahl keine Aussage. Vielmehr ergibt sich die Auswahl aus der Natur der Sache, nämlich der für die Diagnose günstigsten Aufnahmeperspektive. Demzufolge fehlt es Röntgenbildern an der für einen Schutz nach \ 2 I Nr. 5 UrhG erforderlichen, persönlichen geistigen Schöpfung.

\section{c. Leistungsrechtlicher Schutz gem. \ 72 UrhG}

Ein urheberrechtlicher Schutz von Röntgenbildern könnte sich indes aus \ 72 UrhG ergeben. Danach werden Lichtbilder und Erzeugnisse, die ähnlich wie Lichtbilder hergestellt werden, in entsprechender Anwendung der für Lichtbildwerke geltenden Vorschriften des Teils 1 des UrhG geschützt. \ 72 UrhG schützt dabei einerseits auf herkömmlicher Technologie hergestellte Fotografien. Aufgrund der Formulierung „Erzeugnisse, die ähnlich wie Lichtbilder hergestellt werden“, sind andererseits jedoch auch Techniken der Bilderzeugung erfasst, die unter Einsatz strahlender Energie zu fotografieähnlichen Bildern führen, wie dies auch bei Röntgenaufnahmen der Fall ist. ${ }^{16}$

Röntgenaufnahmen werden also gem. $\int 72$ I UrhG in entsprechender Anwendung der für Lichtbildwerke geltenden Vorschriften des Ersten Teils des UrhG urheberrechtlich geschützt. Dieser Schutz erlischt gem. \ 72 III UrhG allerdings fünfzig Jahre nach dem Erscheinen des Lichtbildes bzw. fünfzig Jahre nach der Herstellung, wenn es innerhalb dieses Zeitraums nicht erschienen ist.

\footnotetext{
${ }^{14}$ Loewenheim in Schricker/Loewenheim, \ 2 Rn. 183 f.; A. Nordemann in Loewenheim, \9 Rn. 131; ders. in Fromm/Nordemann, \2 Rn. 197; Katzenberger, GRUR-Int. 1989, 116, 118 f.

15 OLG Köln GRUR 2000, 43, 44 - Klammerpose; OLG Hamburg GRUR 1999, 717 - WagnerFamilienfotos; Loewenheim in Schricker/Loewenheim, \2 Rn. 182, 184; A. Nordemann in Loewenheim, $₫ 9$ Rn. 134; ders. in Fromm/Nordemann, \2 Rn. 196; Dreyer in Dreyer/Kotthoff/Meckel, \2 Rn. 239.

${ }_{16}$ BGHZ 37, 1, 6; Vogel in Loewenheim, $\int 37$ Rn. 9; Loewenheim in Schricker/Loewenheim, $\int 72$ Rn.

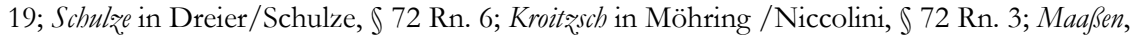
ZUM 1992, 338, $339 \mathrm{f}$.
} 
d. Werkeigenschaft gem. \2 I Nr.7 UrhG

Damit stellt sich weiterhin die Frage, ob auch sonstige Daten unter das UrhG fallen und damit geschützt sind. In Betracht käme ein Schutz nach \ 2 I Nr. 7 UrhG, wonach zu den geschützten Werken insbesondere Darstellungen wissenschaftlicher oder technischer Art, wie Zeichnungen, Pläne, Karten, Skizzen, Tabellen und plastische Darstellungen gehören. Dabei schützt \2 I Nr. 7 UrhG allerdings nicht den wissenschaftlichen Inhalt der Darstellung, sondern nur deren Formgestaltung. Das wissenschaftliche Gedankengut eines Werkes wird nämlich gerade nicht durch das UrhG geschützt, da die wissenschaftliche Lehre und das wissenschaftliche Gedankengut frei sind. ${ }^{17}$ Allerdings setzt auch der Schutz nach \2 I Nr. 7 UrhG voraus, dass das Werk gem. \2 II UrhG eine persönliche geistige Schöpfung darstellt. Da es daran aber, wie oben bereits festgestellt, regelmäßig fehlen wird, genießen wissenschaftliche Primärdaten grundsätzlich auch keinen urheberrechtlichen Schutz nach $₫ 2$ I Nr. 7 UrhG.

\section{Zwischenergebnis}

Aufgrund der fehlenden persönlichen geistigen Schöpfung, scheidet ein urheberrechtlicher Schutz von einzelnen wissenschaftlichen Primärdaten damit grundsätzlich aus. In Betracht kommt lediglich ein leistungsrechtlicher Schutz von Röntgenaufnahmen nach $₫ 72 \mathrm{I}$ UrhG. Dabei ist zu beachten, dass dieser Schutz gem. \ 72 III UrhG zeitlich begrenzt ist, was für die vorliegenden Fälle insbesondere dann relevant ist, wenn alte Röntgenaufnahmen elektronisch archiviert werden sollen.

3. Urheber- und leistungsrechtlicher Schutz aufgrund der Zusammenstellung und Sammlung von wissenschaftlichen Primärdaten

Sofern Daten erhoben oder gewonnen werden, werden sie in der Regel nicht isoliert gespeichert, sondern im Zusammenhang mit anderen Daten in Tabellen dokumentiert oder angeordnet. Es stellt sich deshalb die Frage, ob ein urheberrechtlicher Schutz, wenn auch nicht an den einzelnen Daten, zumindest an einer solchen Datensammlung entsteht, wenn die Daten miteinander kombiniert dargestellt werden.

a. Urheberrechtlicher Schutz gem. \4 UrhG

Ein urheberrechtlicher Schutz könnte zunächst gem. \4 Abs. 1 bzw. Abs. 2 UrhG bestehen. Nach dem Wortlaut von Absatz 1 der Vorschrift werden Sammlungen von Werken, Daten oder anderen unabhängigen Elementen, die aufgrund der Auswahl oder Anordnung der Elemente eine persönliche geistige Schöpfung dar-

17 BGH GRUR 1985; 1041, 1047 - Inkasso-Programm; GRUR 1991, 130, 132 - Themenkatalog; GRUR 1981, 352, 353 - Staatsexamensarbeit; Ablberg in Möhring/Niccolini, \ 2 Rn. 36; Scbulze in Dreier/Schulze, $\ 2$ Rn. 223; Loewenheim in Schricker/Loewenheim, \ 2 Rn. 62. 
stellen, unbeschadet eines an den einzelnen Elementen gegebenenfalls bestehenden (oder nicht bestehenden) Urheberrechts oder verwandten Schutzrechts, wie selbständige Werke geschützt. Dabei stellen die in Abs. 2 der Vorschrift genannten Datenbankwerke einen Unterfall der Sammelwerke dar. ${ }^{18}$ Insofern käme damit ein urheberrechtlicher Schutz, wenn auch nicht an den einzelnen Daten, zumindest an einer Sammlung mehrerer Daten in Betracht, wenn diese beispielsweise in einer Tabelle angeordnet oder gesammelt werden. Des Weiteren könnte die Zusammenstellung von Primärdaten die Voraussetzungen einer Datenbank erfüllen und damit dem Leistungsschutzrecht nach $\int 87$ a UrhG unterfallen. Dies würde zwar expressis verbis nichts daran ändern, dass die in der Tabelle gesammelten Daten für sich immer noch nicht urheberrechtlich geschützt wären, da nur die in der Anordnung oder Auswahl der Daten erbrachte Leistung durch das Urheberrecht geschützt würde; ${ }^{19}$ allerdings würde im Rahmen einer Archivierung der an der Universität erhobenen Daten die komplette Tabelle und nicht lediglich die darin enthaltenen Daten archiviert werden, so dass die Frage des urheber- bzw. leistungsschutzrechtlichen Schutzes an diesen Datensammlungen für die Frage der Langzeitarchivierung durchaus relevant ist. Aus diesem Grund soll im Folgenden festgestellt werden, ob und unter welchen Voraussetzungen eine Sammlung von wissenschaftlichen Primärdaten unter den urheberrechtlichen Schutz des \4 UrhG bzw. den leistungsschutzrechtlichen Schutz des $\int 87$ a UrhG fällt.

\section{i. Voraussetzungen des $\int 4 \mathrm{I}$ UrhG}

Damit eine Zusammenfassung von Daten unter den urheberrechtlichen Schutz als Sammelwerk i.S.d. \4 UrhG fällt, müsste es sich dabei zunächst um eine Sammlung im Sinne von Absatz 1 handeln. Darüber hinaus müsste diese Sammlung, wie auch beim Werkbegriff nach \2 UrhG, eine persönliche geistige Schöpfung darstellen. ${ }^{20}$

\section{(1) Sammlung von Werken, Daten oder anderen unabhängigen Elementen}

Zunächst müsste es sich bei der Zusammenstellung der Daten um eine Sammlung nach $\int 4$ Abs. 1 UrhG handeln. Dabei erfasst der Schutzbereich der Norm seinem Wortlaut nach ausdrücklich auch Daten. ${ }^{21}$ Unter einer Sammlung i.S.d. \ 4 Abs. 1 UrhG wird in diesem Zusammenhang jede Zusammenstellung mehrerer Werke, Daten oder anderer unabhängiger Elemente verstanden. Im Rahmen der Erfas-

${ }^{18}$ Loewenheim in Schricker/Loewenheim, \4 Rn. 32; Kotthoff in Dreyer/Kotthoff/Meckel, $\ 4$ Rn. 2; Dreier in Dreier/Schulze, \4 Rn.16; Vogel, ZUM 1997, 592, 599.

19 BGH GRUR 1992, 382, 384 - Leitsätze; KG GRUR 1973, 602, 603; Dreier in Dreier/Schulze, \ 4

Rn. 4; Loewenheim in Schricker/Loewenheim, \4 Rn. 6, 27; Cyychowski in Fromm/Nordemann, \ 4 Rn. 1.

${ }^{20}$ Kotthoff in Dreyer/Kotthoff/Meckel, $₫ 4$ Rn. 8; Dreier in Dreier/Schulze, $\ 4$ Rn. 1; Loewenheim in Schricker/Loewenheim, \4 Rn. 37; Caychowski in Fromm/Nordemann, \4 Rn.12.

${ }^{21}$ Dreier in Dreier/Schulze, $\ 4$ Rn. 1; Loewenheim in Schricker/Loewenheim, $\$ 4$ Rn. 5; Ablberg in Möhring/Niccolini, $\ 4$ Rn. 8. 
Kapitel 1 - Urheberrechtliche Problematik

sung von wissenschaftlichen Primärdaten ist es dabei durchaus denkbar, dass mehrere dieser Daten beispielsweise in Tabellen oder Diagrammen zusammengefasst werden. Meist wird eine solche Zusammenstellung sogar der Regelfall sein, da sich gerade im wissenschaftlichen Bereich der Nutzen dieser Daten vornehmlich aus ihrer Zusammenstellung ergibt. Das Merkmal der Sammlung von Daten wäre damit bei zusammengestellten wissenschaftlichen Primärdaten in der Regel erfüllt.

\section{(2) Persönliche geistige Schöpfung}

Die bloße Zusammenstellung der Daten allein genügt indes nicht, verlangt doch auch $\int 4$ Abs. 1 UrhG, dass die Auswahl oder Anordnung der einzelnen Elemente beziehungsweise Daten eine persönliche geistige Schöpfung darstellt. ${ }^{22}$ Dabei ist an das Tatbestandsmerkmal der ,persönlichen geistigen Schöpfung“ der Maßstab des $\int 2$ Abs. 2 UrhG anzulegen. ${ }^{23}$ Eine persönliche geistige Schöpfung ist danach dann gegeben, wenn die Kombination der im Sammelwerk enthaltenen Elemente besondere Strukturen in deren Auswahl oder Anordnung und das Gewebe der persönlichen geistigen Schöpfung des Sammelwerks erkennen lässt. ${ }^{24}$ Es muss sich also ein neuer geistiger Gehalt ergeben, der über die bloße Summe der in ihr enthaltenen Daten hinausgeht. ${ }^{25}$

Dabei dürfen jedoch aufgrund einer richtlinienkonformen Auslegung von $\int 4$ Abs. 1 im Lichte von Art. 3 Abs. 1 S. 2 der Richtlinie 96/9/EG (im Folgenden „Datenbankrichtlinie“) andere Kriterien als die Auswahl oder Anordnung der Elemente nicht herangezogen werden, so dass es nicht auf die Qualität oder den ästhetischen Wert der Sammlung ankommt. ${ }^{26}$ Die Zusammenstellung mehrerer Messdaten oder statistischer Werte in einer Tabelle würde demnach nicht automatisch ein Sammelwerk i.S.d. \ 4 Abs. 1 UrhG darstellen. Vielmehr müsste die Kombination der enthaltenen Daten eine besondere Struktur in der Auswahl oder Anordnung erkennen lassen. In diesem Zusammenhang setzt das Tatbestandsmerkmal der „Auswahl“ ein Sammeln, Sichten, Bewerten und Zusammenstellen

22 BT-Drucks. 13/7934 S. 43; Dreier in Dreier/Schulze, \ 4 Rn. 11, 19; Loewenheim in Schricker/Loewenheim, \& 4 Rn. 9; Ablberg in Möhring/Niccolini, $\int 4$ Rn. 8; Caychowski in Fromm/Nordemann, \ 4 Rn. 12; Kotthoff in Dreyer/Kotthoff/Meckel, \& 4 Rn. 8; Leistner, MMR 1999, 636, 637.

${ }^{23}$ Caychowski in Fromm/Nordemann, $\int 4$ Rn. 21; Dreier in Dreier/Schulze, $\int 4$ Rn. 19; Loewenheim in Loewenheim, \& 9 Rn. 229.

${ }^{24}$ BGH GRUR 1992, 382, 384 - Leitsätze; 1990, 669, 673 - Bibelreproduktion; OLG Hamburg GRUR 2000, 319, 320 - Börsendaten; Dreier in Dreier/Schulze, \ 4 Rn. 11, 19; Kotthoff in Dreyer/Kotthoff/Meckel, \4 Rn. 8; Loewenheim in Loewenheim, \9 Rn. 229; ders. in Schricker/Loewenheim, \4 Rn. 9; Czychowski in Fromm/Nordemann, \4 Rn. 13.

25 OLG Nürnberg GRUR 2002, 607; Schulze in Dreier/Schulze, $\int 4$ Rn. 11; Loewenheim in Schricker/Loewenheim \ 4 Rn. 9.

${ }^{26}$ Kotthoff in Dreyer/Kotthoff/Meckel, \4 Rn. 8; Loewenheim in Schricker/Loewenheim, \4 Rn. 37 ; Ablberg in Möhring/Niccolini, \ 4 Rn. 21; Czychowski in Fromm/Nordemann, \& 4 Rn. 14; siehe auch 16. Erwägungsgrund der Richtlinie 96/9/EG. 
von Daten zu einem bestimmten Thema unter Beachtung besonderer Auswahlkriterien voraus. ${ }^{27}$ So würde es an der erforderlichen Struktur fehlen, wenn die Messwerte eines Versuchs lediglich in der zeitlichen Reihenfolge ihrer Erfassung zusammengestellt würden, ihre Anordnung also logisch vorgegeben wäre. ${ }^{28}$ In diesem Fall würden die Daten zwar gesammelt, jedoch nicht bewertet und anhand dieser Bewertung zusammengestellt. So hat die Rechtsprechung bei einer umfangreichen Sammlung bibliographischer Daten ohne erkennbare konzeptionelle Leistungen $^{29}$ und bei gesammelten Börsendaten, die auf Berechnungen von Wirtschaftsfaktoren durch Börsenanalysten beruhten ${ }^{30}$ sowie einem Telefonverzeichnis auf CD-ROM ${ }^{31}$ eine Sammelwerkseigenschaft verneint.

Bejaht wurde dagegen die Sammelwerkeigenschaft unter anderem bei Gesetzessammlungen ${ }^{32}$ oder bei einem aus wissenschaftlichen Beiträgen einzelner Verfasser zusammengestellten Archiv ${ }^{33}$. Bei einer Zusammenfassung wissenschaftlicher Primärdaten kommt es jedoch grundsätzlich gerade auf eine vollständige Erfassung der gewonnenen Daten an, sei es bei der Erfassung der Versuchsergebnisse im Rahmen eines wissenschaftlichen Experimentes oder bei der Erfassung von Umfrageergebnissen. In den meisten Fällen werden sich nämlich verlässliche wissenschaftliche Erkenntnisse nur aus der Gesamtheit der ermittelten Daten ergeben. So ist es kaum vorstellbar, dass bei der Auswahl dieser Daten ein Spielraum besteht und bestimmte Daten bei einer Sichtung aussortiert werden. Auch wird die Anordnung der Daten im Regelfall kraft Natur der Sache vorgegeben sein, wie etwa der zeitlichen Entstehung der Ergebnisse oder der Einordnung von Umfragewerten in bestimmte Gruppen. Aus diesem Grund wird bei einer Zusammenfassung von wissenschaftlichen Primärdaten mangels persönlicher geistiger Schöpfung im Regelfall auch kein urheberrechtlicher Schutz gem. \4 UrhG an der Sammlung der Daten entstehen.

\section{ii. Voraussetzungen des $₫ 4$ II UrhG}

Des Weiteren wäre ein urheberrechtlicher Schutz an der Datensammlung nach $\int 4$ Abs. 2 UrhG denkbar, sofern die gesammelten Daten ein Datenbankwerk darstellen. Nach der Legaldefinition des $\int 4$ Abs. 2 UrhG ist eine Datenbank ein Sammelwerk, dessen Elemente systematisch oder methodisch angeordnet und einzeln

\footnotetext{
27 Ablberg in Möhring/Niccolini, \4 Rn. 15; Caychowski in Fromm/Nordemann, \4 Rn. 12; Loewenbeim in Schricker/Loewenheim, \4 Rn. 9; Berger, GRUR 1997, 169, 173.

${ }^{28}$ BGH GRUR 2005, 857, 858 - HIT BILANZ; OLG Nürnberg GRUR 2000, 607; Dreier in Dreier/Schulze, \4 Rn. 12; Cyychowski in Fromm/Nordemann, \4 Rn. 12; Loewenheim in Loewenheim, \9 Rn. 229; ders. in Schricker/Loewenheim, \4 Rn. 10.

29 OLG Hamburg ZUM 1997, 145.

30 OLG Hamburg GRUR 2000, 319 - Börsendaten.

31 BGH GRUR 1999, 923 - Tele-Info-CD.

32 OLG Frankfurt GRUR 1986, 242 - Gesetzessammlung.

33 OLG Frankfurt GRUR 1967, 151 - Archiv; weitere Beispiele siehe bei Loewenheim in Loewenheim, \9 Rn. 230; ders. in Schricker/Loewenheim, \4 Rn. 16.
} 
Kapitel 1 - Urheberrechtliche Problematik

mit Hilfe elektronischer Mittel oder auf andere Weise zugänglich sind. Das Datenbankwerk stellt somit einen Unterfall des bereits oben erörterten Sammelwerks nach $\int 4$ Abs. 1 UrhG dar. ${ }^{34}$

\section{(1) Systematische oder methodische Anordnung der Daten}

Dabei folgt aus der Voraussetzung einer systematischen oder methodischen Anordnung, dass die einzelnen Elemente nach bestimmten Ordnungsgesichtspunkten angeordnet sein müssen und nicht lediglich willkürlich angehäuft sein dürfen. ${ }^{35}$ Systematisch ist eine Datensammlung in diesem Zusammenhang dann, wenn sie sich an einer Klassifizierung oder einem Ordnungsschema orientiert. ${ }^{36}$ Methodisch ist die Anordnung hingegen, wenn sie einem bestimmten Plan oder einer bestimmten ordnenden Handlungsanweisung folgt. ${ }^{37}$ Sofern wissenschaftliche Primärdaten gesammelt werden, wird eine solche systematische oder methodische Anordnung wohl in den meisten Fällen vorliegen. Ferner werden die einzelnen Daten der Sammlung mit Hilfe elektronischer Mittel oder auf andere Weise zugänglich sein, d.h. der Zugriff wird in der Regel, anders als zum Beispiel bei digitalen Musikstücken, auf ein einzelnes Element erfolgen. ${ }^{38}$

\section{(2) Persönliche geistige Schöpfung}

Aus dem Verweis in $\int 4$ Abs. 2 auf Abs. 1 folgt jedoch, dass das Datenbankwerk als Unterfall eines Sammelwerkes, ebenfalls eine persönliche geistige Schöpfung darstellen muss. Die bloße Ansammlung der Daten allein reicht demzufolge also nicht für das Vorliegen eines Datenbankwerkes nach \ 4 Abs. 2 UrhG aus. ${ }^{39}$ Diese persönliche geistige Schöpfung kann dabei in der Anordnung oder der Auswahl der Daten zum Ausdruck kommen. ${ }^{40}$ Die Auswahl der Daten kann jedoch nur dann zur Schöpfungshöhe beitragen, wenn ein entsprechender Entscheidungsspielraum besteht. ${ }^{41}$ An einem solchen Entscheidungsspielraum fehlt es allerdings

34 Ablberg in Möhring/Niccolini \4 Rn. 10; Kotthoff in Dreyer/Kotthoff/Meckel, \4 Rn. 11; Marquardt in Wandtke/Bullinger, \4 Rn. 8; Loewenheim in Loewenheim, \9 Rn. 243; siehe auch BTDrucks. 13/7934, S. 34.

35 OLG München GRUR-RR 2001, 228, 229; KG GRUR-RR 2001, 102; Dreier in Dreier/Schulze, \4 Rn. 17; Loewenheim in Loewenheim, \4 Rn. 244; ders. in Schricker/Loewenheim, \4 Rn. 41; Cyychowski in A. Nordemann, \4 Rn. 35; Flechsig, ZUM 1997, 577, 580.

36 A. Nordemann in Fromm/Nordemann, \4 Rn. 34; Dreier in Dreier/Schulze, \4 Rn. 17; Loewenheim in Schricker/Loewenheim, \4 Rn. 42.

${ }^{37}$ Dreier in Dreier/Schulze, $₫ 4$ Rn. 17; Loewenheim in Schricker/Loewenheim, $\ 4$ Rn. 41; Bullinger in Wandtke/Bullinger, \4 Rn. 10.

38 Vgl. Marquardt in Wandtke/Bullinger, \ 4 Rn. 10.

${ }^{39}$ Caychowskei in Fromm/Nordemann, \4 Rn. 10, 12; Dreier in Dreier/Schulze, $\int 4$ Rn. 19, 11; Loewenheim in Loewenheim, \9 Rn. 245; Kotthoff in Dreyer/Kotthoff/Meckel, \4 Rn. 11;

${ }^{40}$ Dreier in Dreier/Schulze, $\ 4$ Rn. 19, 11; Kotthoff in Dreyer/Kotthoff/Meckel, $\ 4$ Rn. 8; Loewenheim in Loewenheim \9 Rn. 245; ders. in Schricker/Loewenheim, \4 Rn. 38.

${ }^{41}$ Loewenheim in Schricker/Loewenheim, \4 Rn. 38; Dreier in Dreier/Schulze, $\ 4$ Rn. 19, 12;

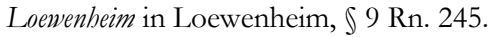


zumeist in den Fällen von Fachdatenbanken, die auf Vollständigkeit angelegt sind..$^{42}$ Wie bereits erläutert wurde, ${ }^{43}$ ist dies bei der Sammlung wissenschaftlicher Primärdaten gerade der Fall, da es zur Erlangung der wissenschaftlichen Erkenntnisse im Allgemeinen auf die Vollständigkeit der erfassten Daten ankommt, ein Entscheidungsspielraum für die Auswahl also gerade nicht besteht. Insofern wird bei der Sammlung von wissenschaftlichen Primärdaten eine schöpferische Höhe durch die Auswahl der Daten grundsätzlich nicht erreicht werden. Eine Schöpfungsqualität könnte somit nur durch die Anordnung der Daten erreicht werden. Die Anordnung wissenschaftlicher Primärdaten folgt zumeist gängigen Ordnungskriterien, ${ }^{44}$ wie beispielsweise chronologischen oder numerischen Prinzipien, so dass eine schöpferische Gestaltungshöhe grundsätzlich auch nicht durch die Anordnung der Daten in Betracht kommt. Hinzu kommt, dass bei elektronischen Datenbanken die Anordnung im Speichermedium technisch vorgegeben ist oder auf Computerprogrammen beruht, welche gem. $\int 4$ Abs. 2 S. 2 UrhG nicht Bestandteile des Datenbankwerks sind. ${ }^{45}$ Damit scheidet ein urheberrechtlicher Schutz von Zusammenstellungen wissenschaftlicher Primärdaten als Datenbankwerk nach $\int 4$ Abs. 2 UrhG mangels einer persönlichen geistigen Schöpfung grundsätzlich ebenfalls aus.

b. Ergebnis zu 3.

Ein urheberrechtlicher Schutz an Tabellen und Sammlungen, in denen wissenschaftliche Primärdaten enthalten sind, entsteht aufgrund der fehlenden persönlichen geistigen Schöpfung grundsätzlich nicht, da derartige Datensammlungen gerade im wissenschaftlichen Bereich meistens auf Vollständigkeit angelegt sind und ihre Anordnung in der Regel gängigen Ordnungskriterien unterliegt, weshalb es bei der Auswahl und Kombination der Daten an einem Entscheidungsspielraum und somit der erforderlichen geistigen Schöpfungshöhe fehlt.

\section{Leistungsrechtlicher Schutz gem. \87aUrbG}

Die Sammlung wissenschaftlicher Primärdaten könnte indes einem leistungsrechtlichen Schutz nach \87b UrhG unterfallen. Nach \87b Abs. 1 S. 1 UrhG hat der Datenbankhersteller das ausschließliche Recht, die Datenbank insgesamt oder einen nach Art oder Umfang wesentlichen Teil der Datenbank zu vervielfältigen, $\mathrm{zu}$ verbreiten und öffentlich wiederzugeben. Aus diesem Grund könnte in dem

42 OLG Düsseldorf MMR 1999, 729, 731; Loewenheim in Loewenheim, \9 Rn. 245; Csychowski in Fromm/Nordemann, \4 Rn. 32; Loewenheim in Schricker/Loewenheim, \4 Rn. 38; Berger, GRUR 1997, 169, 174; a.A. Dreier in Dreier/Schulze, \4 Rn. 12.

${ }^{43}$ Siehe dazu die Ausführungen auf S. 12

${ }^{44}$ Siehe dazu ebenfalls die Ausführungen auf S. 12

${ }^{45}$ Loewenheim in Loewenheim, $\$ 9$ Rn. 246; Caychowski in Fromm/Nordemann, $\$ 4$ Rn. 32; Berger, GRUR 1997, 169, 174; Dreier, GRUR Int. 1992, 739, 740 f.; Hoebbel, CR 1993, 12, 15; Wiebe, CR 1996, 198, 201; Mehrings, Der Rechtsschutz computergestützter Fachinformationen, 1990, S. 143. 
Fall, dass eine Sammlung wissenschaftlicher Primärdaten die Anforderungen an eine Datenbank i.S.d. \87 b Abs. 1 S.1 UrhG erfüllt, das Leistungsschutzrecht des Datenbankherstellers betroffen sein.

a. Vorliegen einer Datenbank i.S.d. \87a UrhG

Zunächst müsste es sich bei der Sammlung von Daten um eine Datenbank handeln. Nach $\int 87$ a UrhG wäre dies der Fall, wenn die gesammelten Daten systematisch oder methodisch angeordnet und einzeln mit Hilfe elektronischer Mittel oder auf andere Weise zugänglich sind und die Beschaffung, Überprüfung oder Darstellung eine nach Art und Umfang wesentliche Investition erfordert. Die Definition stimmt demzufolge mit der in $\int 4$ Abs. 2 überein, wobei die Datenbank i.S.d. $\int 87 \mathrm{a}$ UrhG im Gegensatz zum Datenbankwerk nach \4 Abs. 2 UrhG jedoch keine persönliche geistige Schöpfung erfordert. ${ }^{46}$

i. Systematische oder methodische Anordnung der Daten

Wie oben bereits unter II.3.a.ii dargelegt, ist es der Regelfall, dass wissenschaftliche Daten, sofern sie gesammelt werden, systematisch oder methodisch angeordnet sind, da sich ja erst aufgrund dieser Anordnung eine Vergleichbarkeit der Daten ergibt und sich auf diese Weise erst fundierte wissenschaftliche Erkenntnisse ziehen lassen. Als Beispiel sei nur eine Sammlung von medizinischen Daten genannt, die während einer Studie erhoben wurden und nach chronologischen Kriterien in einer Tabelle aufgelistet werden, um so etwa Entwicklungen der Blutwerte des Patienten kenntlich zu machen. Demzufolge wird bei Sammlungen von wissenschaftlichen Primärdaten das Merkmal der systematischen oder methodischen Anordnung regelmäßig erfüllt sein. ${ }^{47}$

\section{ii. Zugang zu einzelnen Elementen}

Darüber hinaus setzt $\int 87$ a UrhG voraus, dass die Daten „einzeln mit Hilfe elektronischer Mittel oder auf andere Weise zugänglich sind“. Einzeln zugänglich wären die Daten dann, wenn der Zugriff durch den Benutzer der Datenbank bestimmungsgemäß von Fall zu Fall auf ein einzelnes Element erfolgt, wie dies beispielsweise der Fall ist, wenn das einzelne Datum durch das Aufschlagen eines Buches, das Blättern in einer Kartei, in der die einzelnen Daten angeordnet sind, durch das Ansteuern auf einer CD-ROM oder durch den Abruf aus einer OnlineDatenbank zur Kenntnis genommen werden kann. ${ }^{48}$ Dabei stellt $\int 87$ a UrhG so-

${ }^{46}$ Kotthoff in Dreyer/Kotthoff/Meckel, \4 Rn. 11; Cayychowski in Fromm/Nordemann, \87a Rn. 11; Dreier in Dreier/Schulze, $\ 4$ Rn. 20; Loewenheim in Schricker/Loewenheim, $\ 4$ Rn. 32; Vogel in Schricker/Loewenheim, \87a Rn. 5, 26.

47 Siehe dazu die Ausführungen auf S. 14.

48 Vogel in Schricker/Loewenheim, \87a Rn. 25; Kotthoff in Dreyer/Kotthoff/Meckel, \87a Rn. 21; Dreier in Dreier/Schulze, \87a Rn. 8; Decker in Möhring/Niccolini, $\int 87 a$ Rn. 10; Tbum in Wandtke/Bullinger, \87a Rn. 13; Leistner, MMR 1999, 636, 639. 
wohl elektronische und elektrooptische als auch herkömmliche, auf analoger Technik basierende Datenbanken unter Schutz, was an der Formulierung „mit elektronischen oder anderen Mitteln zugänglich macht", deutlich wird. ${ }^{49}$ Wie bereits oben festgestellt sind bei Sammlungen von wissenschaftlichen Primärdaten die enthaltenen Elemente einzeln zugänglich, da man, anders als zum Beispiel bei den einzelnen Bits auf einer Musik-CD, gerade ein Interesse an den einzelnen Daten hat und damit auch einzeln auf diese zugreifen können muss.

\section{iii. Wesentliche Investition}

Der leistungsrechtliche Schutz einer Sammlung von wissenschaftlichen Primärdaten als Datenbank i.S.d. $\int 87$ a UrhG setz ferner voraus, dass die Beschaffung, Überprüfung oder Darstellung der Daten eine nach Art oder Umfang wesentliche Investition erfordert. Insofern gilt es im Folgenden festzustellen, ob bzw. wann eine solche wesentliche Investition bei Datenbanken mit wissenschaftlichen Primärdaten vorliegt. Eine Investition i.S.d. \ 87a UrhG kann dabei in der Bereitstellung von finanziellen Mitteln sowie im Einsatz von Zeit, Arbeit, Energie, ${ }^{50}$ aber auch in der eingehenden Überprüfung des Inhalts der Datenbank bestehen. ${ }^{51} \mathrm{Da}$ bei muss die Investition jedoch expressis verbis nach Art oder Umfang ,wesentlich“ sein. Der Begriff der Wesentlichkeit ist allerdings weder im nationalen Gesetz, noch in der Datenbankrichtlinie 96/9/EG legal definiert wird. Vielmehr hat der deutsche Gesetzgeber die Konkretisierung dieses unbestimmten Rechtsbegriffs der Rechtsprechung überlassen. ${ }^{52}$

Die Rechtsprechung zum Begriff der wesentlichen Investition ist jedoch nicht einheitlich. Wurde teilweise ein erheblicher finanzieller Personal- oder Sachaufwand verlangt, ${ }^{53}$ lag in anderen Fällen die Schwelle der Wesentlichkeit sehr niedrig. ${ }^{54}$ Teilweise ließen es die Gerichte sogar ausreichen, dass die Datenbank regelmäßig aktualisiert wurde. ${ }^{55}$ Einigkeit besteht jedoch dahingehend, dass bei der Bestimmung der Wesentlichkeit objektive Kriterien anzulegen sind und es demzu-

\footnotetext{
${ }^{49}$ EuGH GRUR Int. 2005, 239, 240 - Fixtures-Fußballpläne I; BGH GRUR 1999, 923, 925 - TeleInfo-CD; Dreier in Dreier/Schulze, $\ 87$ a Rn. 8; Thum in Wandtke/Bullinger, $\ 87$ a Rn. 14; Vogel in Schricker/Loewenheim, $\int 87$ a Rn. 25; Kotthoff in Dreyer/Kotthoff/Meckel, $\int 87$ a Rn. 22; Loewenheim in Loewenheim, $\int 43$ Rn. 6; Cayychowski in Fromm/Nordemann, $\int 87$ a Rn. 13; siehe auch 14. Erwägungsgrund der Datenbankrichtlinie.

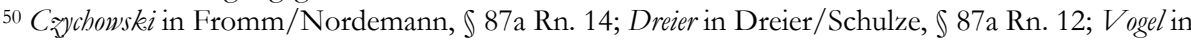
Schricker/Loewenheim, \87a Rn. 48, 56; Loewenheim in Loewenheim, \43 Rn. 8; Beushausen, MMR 2007, Heft 1 IX; siehe auch 40. Erwägungsgrund der Datenbankrichtlinie.

${ }^{51}$ Kotthoff in Dreyer/Kotthoff/Meckel, \87a 27; Loewenheim in Loewenheim, \43 Rn. 8; Vogel in Schricker/Loewenheim, \87a Rn. 50; siehe auch 55. Erwägungsgrund der Datenbankrichtlinie

52 Dreier in Dreier/Schulze, \87a Rn. 15; Vogel in Schricker/Loewenheim, \87a Rn. 41.

53 LG Köln CR 2000, 400, 401; CR 1999, 593; siehe auch Cayychowski in Fromm/Nordemann, \87a Rn. 16.

54 AG Rostock CR 2001, 786.

${ }^{55}$ LG Köln CR 1999, 593, 594; CR 2000, 400, 401; LG Berlin CR 1999, 388.
} 
folge auf die Zweckrichtung der Investition gerade nicht ankommt. ${ }^{56}$ Insofern kommt es im Rahmen von an der Universität gewonnenen Primärdaten, die gesammelt worden sind und damit eine Datenbank i.S.d. \87a UrhG darstellen könnten, darauf an, ob bei der Beschaffung, Überprüfung oder Darstellung der Daten eine nach Art oder Umfang nach objektiven Kriterien wesentliche Investition erforderlich gewesen ist.

\section{(1) Investitionen bei der Datengewinnung}

In Betracht kämen in dieser Hinsicht dabei wohl vor allem Investitionen bei der Datengewinnung, wie beispielsweise der Finanzierung einer medizinischen Studie, durch die neue Daten gewonnen werden. Da die Finanzierung dieser Studien zumeist sehr kostenaufwändig ist, wäre das Merkmal der Wesentlichkeit wohl in den meisten Fällen nach allen genannten Ansichten unproblematisch gegeben. Aber auch bei der Gewinnung von Umfragewerten wäre die Wesentlichkeit wohl nicht von vornherein abzulehnen, da zur Durchführung dieser Umfragen in den meisten Fällen ein Zeitaufwand erforderlich ist, der nach objektiven Kriterien auch bei einer stringenten Auslegung der Norm als wesentlich anzusehen ist.

In diesem Zusammenhang stellt sich allerdings die Frage, ob die Kosten bzw. der Zeitaufwand zur Gewinnung dieser Daten überhaupt als Investition i.S.d. $\int 87 \mathrm{a}$ UrhG anzusehen sind. Dies wird von Teilen der Literatur und Rechtsprechung bejaht, da die Kosten für die Erstellung der Datenbank von solchen, die für die Gewinnung der Daten anfallen, oftmals nur schwer abzugrenzen seien, gehe doch in vielen Fällen mit der Informationsgewinnung auch eine ordnende Tätigkeit einher. ${ }^{57}$

Dagegen spricht allerdings zum einen der Schutzzweck der $\iint 87$ a ff. UrhG sowie der Richtlinie 96/9/EG sei, der darin besteht, einen Anreiz für die Erstellung von Informationssystemen zu bieten, nicht jedoch die Generierung der Informationen als solche zu schützen. ${ }^{58}$ Dies folgt unter anderem aus den Erwägungsgründen 9, 10 und 12 der Datenbankrichtlinie, wonach diese die erforderlichen „Investitionen in moderne Datenspeicher- und Datenverarbeitungs-

${ }^{56}$ EuGH GRUR Int. 2005, 247 Tz. 42 - BHB v. William Hill; AG Rostock MMR 2001,631, 632; LG Köln CR 2000, 400, 401; Kotthoff in Dreyer/Kotthoff/Meckel, \87a Rn. 30; Vogel in Schricker/Loewenheim, \87a Rn. 43.

${ }^{57}$ OLG Dresden ZUM 2001, 595 f.; Leistner in FS Dietz, 2001, S. 493; Haberstumpf, GRUR 2003, 14, 26; a.A. hingegen BGH GRUR 1999, 926.

${ }^{58}$ EuGH GRUR 2005, 254, 256 Tz. 40 ff. - Fixtures-Fußballspielpläne II; EuGH C-46/02 Tz. 44 ff.; EuGH GRUR 2005, 252, 253 - Fixtures-Fußballspielpläne I; siehe auch Erwägungsgrund 9, 10 und 12 der RL96/9/EG; Vogel in Schricker/Loewenheim, \87a Rn. 52; Leistner, K\&R 2007, 457, 460 (nach dem die Kosten für die „Erzeugung“ jedoch wie Beschaffungsinvestitionen behandelt werden sollen, sofern die Informationen mit einem vergleichbaren Aufwand hätten beschafft werden können); ders., GRUR Int. 1999, 819, 832; a.A. Cyychowski in Fromm/Nordemann, \87a Rn. 19; so auch Spindler, JZ 2004, 150, 152 (Anmerkung zu BGH Urt. v. 17.7.2003 - Paperboy). 
Systeme“ schützen soll, die „für die Entwicklung des Informationsmarktes in der Gemeinschaft von großer Bedeutung [sind]“. Die Investitionen für die Erzeugung der Informationen werden dabei gerade nicht genannt, woraus im Umkehrschluss folgt, dass diese auch nicht von der Richtlinie geschützt werden sollen. ${ }^{59}$

Des Weiteren lässt auch Erwägungsgrund 19 der Datenbankrichtlinie darauf schließen, dass Erhebungskosten nicht unter die wesentlichen Investitionen fallen. Dieser besagt, dass die Zusammenstellung mehrerer Musikaufzeichnungen auf einer CD normalerweise keine ausreichende Investitionsleistung darstellt, die unter den Schutz des Rechts sui generis fällt. Da diese Kosten für die Aufzeichnung der Musikstücke, die in der Regel nicht unerheblich sein werden, jedoch gerade Investitionen im Rahmen der Gewinnung darstellen, folgt aus Erwägungsgrund 19 der Richtlinie, dass die Kosten für die reine Datengewinnung nach dem Willen des Richtliniengebers nicht unter den Schutz sui generis fallen sollen. ${ }^{60}$

Aus diesen Gründen sind Kosten zur Neugewinnung von Informationen durch wissenschaftliche Untersuchungen oder Messungen neuer Daten, bei der Beurteilung der Wesentlichkeit der Investition gerade nicht zu berücksichtigen. ${ }^{61}$ Insofern kann bei einer Datensammlung ein leistungsrechtlicher Schutz gem. I 87a UrhG nicht schon deshalb angenommen werden, weil die wissenschaftlichen Untersuchungen oder Umfragen, aus denen die in der Datenbank enthaltenen Daten hervorgegangen sind, mit wesentlichen Kosten verbunden waren. Vielmehr entsteht dieser Schutz erst dann, wenn der Datenbank ihre Funktion der Informationsverarbeitung verliehen wird. ${ }^{62}$ Es muss demzufolge bei den in Frage stehenden Datenbanken stets geprüft werden, ob Investitionen lediglich im Stadium der Datenerhebung erbracht wurden. Ist dies der Fall, scheidet ein leistungsrechtlicher Schutz nach der hier vertretenen Ansicht des EuGH und weiten Teilen der Literatur grundsätzlich aus, da andernfalls eine Monopolisierung von Daten drohen würde. ${ }^{63}$

Etwas anderes gilt hingegen, wenn die „erzeugten“ Daten mit einem vergleichbaren Aufwand „,beschafft“" werden könnten, da in derartigen Konstellationen die Gefahr der gerade genannten derartigen Fälle auch anderweitig verfügbar sind. ${ }^{64}$ Es kommt demnach darauf an, welche weiteren Tätigkeiten bei der Erstellung einer Datenbank als wesentliche Investition in Betracht kommen. Da bei der

\footnotetext{
${ }^{59}$ EuGH MMR 2005, 29; BGH GRUR 2005, 857, 858; Leistner, GRUR Int. 1999, 819, 826.

${ }^{60}$ Ebenso EuGH MMR 2005, 29, 30; Leistner, GRUR Int. 1999, 819, 827; siehe auch Derclaye, IIC 2005, 2, $6 \mathrm{f}$.

${ }^{61}$ EuGH MMR 2005, 29, 30-BHB; EuGH GRUR 2005, 254- Fixtures-Fußballspielpläne II; EuGH GRUR 2005, 252 - Fixtures-Fußballspielpläne I; BGH GRUR 2005, 857, 858 f.; BGH GRUR 2005, 940, 942; Thum in Wandtke/Bullinger, \87a Rn. 36; Leistner, K\&R 2007, 457, 460; Sendrowski, GRUR 2005, 369, 371; a.A.: Haberstumpf, GRUR 2003, 24, 26.

${ }^{62}$ Vogel in Schricker/Loewenheim, \87a Rn. 52.

${ }^{63}$ EuGH GRUR 2005, 254, 256 Tz. 40; Leistner, K\&R 2007, 457, 460; a.A. Haberstumpf, GRUR 2003, 14, 26.

${ }^{64}$ Leistner, K\&R 2007, 457, 460.
} 
Kapitel 1 - Urheberrechtliche Problematik

Sammlung wissenschaftlicher Primärdaten in den meisten Fällen ausnahmslos alle gewonnenen Daten in die Datenbank eingepflegt werden, scheidet eine wesentliche Investition zur Beschaffung, also einer sichtenden Tätigkeit, in den meisten Fällen wohl aus. Darüber hinaus werden diese Daten in den meisten Fällen auch nicht anderweitig verfügbar sein, da die Durchführung wissenschaftlicher Experimente und Untersuchungen dazu dient, neue Erkenntnisse zu erlangen.

\section{(2) Investition im Rabmen der Darstellung}

Allerdings könnte eine wesentliche Investition für die Darstellung der Daten bereits darin liegen, dass Tabellen oder Abfragesysteme erstellt werden, durch die der Einzelzugriff auf die Primärdaten erst möglich wird. Die Erstellung dieser Tabellen erfordert zum einen Zeit, zum anderen werden derartige Tabellen heutzutage meist mit Computerprogrammen erstellt, deren Anschaffungskosten ebenfalls als wesentliche Investition i.S.d. $\int 87$ a UrhG anerkannt werden. ${ }^{65}$ Darüber hinaus gelten in diesem Zusammenhang auch die Kosten für die Datenträger bzw. das Papier, auf dem die Daten verkörpert sind, als Investition im Rahmen der Datendarstellung. ${ }^{66}$

\section{iv. Zwischenergebnis zu II.4.a.}

Somit bleibt festzuhalten, dass in den Fällen, in denen wissenschaftliche Primärdaten in Tabellen, Karteien oder auch Online-Datenbanken eingepflegt wurden, ein leistungsrechtlicher Schutz dieser Datensammlungen nach \87a UrhG in Betracht kommt. Bei der Frage ob eine wesentliche Investition i.S.d. \ 87a UrhG vorliegt, dürfen die Kosten, die bei der Gewinnung der Daten entstanden sind, wie etwa die Finanzierung einer klinischen Studie oder einer statistischen Umfrage, nicht berücksichtigt werden. Etwas anderes gilt in den Fällen, in denen die erzeugten Daten jedoch anderweitig beschafft werden können, da in diesen Fällen ausnahmsweise keine Informationsmonopolisierung droht.

In den vorliegend relevanten Fällen, wird dies jedoch äußerst selten zutreffen, da die durchgeführten wissenschaftlichen Experimente gerade der Gewinnung neuer Daten gedient haben werden. Es kommt somit darauf an, dass bei der tatsächlichen Beschaffung, Überprüfung oder Darstellung der Daten wesentliche Investitionen nach Art oder Umfang getätigt worden sind. Dies ist zumindest dann der Fall, wenn für die Erstellung der Datenbank kostspielige Computerprogramme erworben wurden oder die Darstellung der Datenbank erhebliche personelle oder zeitliche Investitionen erfordert hat. Letztlich kommt es auf den jeweiligen Einzelfall an. Eine bloße Exceltabelle, in die lediglich einige Daten eingegeben

\footnotetext{
65 KG CR 2000, 812, 813; Thum in Wandtke/Bullinger, \ 87a Rn. 39; Czychowski in Fromm/Nordemann, \87a Rn. 20; Vogel in Schricker/Loewenheim, \ 87a Rn. 48, 51. ${ }^{66}$ KG ZUM 2001, 70, 71 f.; OLG Dresden, 2001, 595 f.; Vogel in Schricker/Loewenheim, $\int 87 a$ Rn.

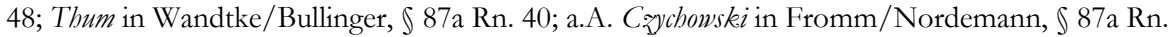
19.
} 
wurden, wird daher den Voraussetzungen von $\int 87$ a UrhG regelmäßig nicht genügen. Anders kann es sich hingegen mit umfangreichen und aufwendig gestalteten Datenbanken verhalten.

\section{b. Datenbankhersteller i.S.d. \87a UrhG}

Des Weiteren muss in den Fällen, in denen eine Datenbank unter den leistungsrechtlichen Schutz der $\iint 87$ a ff. UrhG fällt, vor der Archivierung geklärt werden, wer der Datenbankhersteller ist, da dieser gem. \87b Abs. 1 UrhG Inhaber der Nutzungsrechte ist, die durch eine elektronische Archivierung tangiert werden könnten ${ }^{67}$. Als Datenbankhersteller gilt in diesem Zusammenhang derjenige, der die wesentliche Investition bei der Beschaffung, Darstellung oder Überprüfung des Datenbankinhalts vorgenommen hat. ${ }^{68}$ Dabei kommt es jedoch nicht zwangsläufig darauf an, wer die Finanzierung übernommen hat oder wer die Tabelle erstellt hat, in welche die Daten eingepflegt wurden; ${ }^{69}$ vielmehr gilt derjenige als Hersteller der Datenbank, der die Initiative ergreift und das Investitionsrisiko trägt. ${ }^{70}$

Im Rahmen von Datenbanken, die an der archivierenden Einrichtung erhobene Daten beinhalten, werden die notwendigen Arbeiten zur Beschaffung, Überprüfung oder Darstellung der Daten von Angestellten der Einrichtung erfolgen, so dass die Investitionen in Form der anfallenden Lohnkosten von dieser getragen werden. Darüber hinaus wird die Einrichtung auch die Erwerbskosten für die zur Erstellung der Datenbanken erforderlichen Computerprogramme tragen. Diese Kosten stellen, sofern sie nicht lediglich zur Datengewinnung notwendig sind, Investitionen i.S.d. $\int 87$ a UrhG dar.

Demzufolge wird in den meisten Fällen, in denen Investitionen i.S.d. \ 87a UrhG getätigt worden sind, die archivierende Einrichtung Datenbankherstellerin und damit Inhaberin der genannten Leistungsschutzrechte sein. Die Tatsache, dass es sich dabei regelmäßig um eine juristische Person handeln wird, steht dem auch nicht entgegen. Im Gegensatz zum Urheberrecht, welches nur natürliche Personen innehaben können, ${ }^{71}$ können Datenbankhersteller auch juristische Personen sein. ${ }^{72}$

${ }^{67}$ Zur Frage, welche Nutzungsrechte durch die Archivierung im Einzelnen betroffen sein können, siehe S. $23 \mathrm{ff}$.

${ }^{68}$ Dreier in Dreier/Schulze, $\int 87 a$ Rn. 19; C Cyychowski in Fromm/Nordemann, $\int 87 a$ Rn. 25; Vogel in Schricker/Loewenheim, \87a Rn. 70.

${ }^{69}$ Decker in Möhring/Niccolini, $\int 87$ a Rn. 16; Dreier in Dreier/Schulze, $\int 87$ a Rn. 19; Vogel in Schricker/Loewenheim, \87a Rn. 70; Berger, GRUR 1997, 169, 172.

${ }^{70}$ Caychowski in Fromm/Nordemann, \87a Rn. 25; Decker in Möhring/Niccolini, \87a Rn. 16; Dreier in Dreier/Schulze, \87a Rn. 19; siehe auch 41. Erwägungsgrund Satz 2 der RL 96/9/EG.

${ }^{71}$ Loewenheim in Schricker/Loewenheim, $\mathbb{} 7$ Rn. 2; W. Nordemann in Fromm/Nordemann, $\ 7$ Rn. 9; Schulze in Dreier/Schulze, $\$ 7$ Rn.2.

72 Kotthoff in Dreyer/Kotthoff/Meckel, $\int 87 a$ Rn. 40; Csychowskei in Fromm/Nordemann, $\int 87 a$ Rn. 25, 27; Dreier in Dreier/Schulze, \87a Rn. 20. 
Kapitel 1 - Urheberrechtliche Problematik

c. Zwischenergebnis zu 4.

Werden die an der Universität gewonnenen Primärdaten gesammelt, so können sie aufgrund der vorangegangenen Überlegungen grundsätzlich dem leistungsrechtlichen Schutz der $\iint 87$ a ff. UrhG unterfallen. Allerdings muss jeweils im Einzelfall geprüft werden, ob eine wesentliche Investition i.S.d \ 87a Abs.1 UrhG vorliegt, wobei die Kosten, die bei der Gewinnung der Daten entstanden sind, aufgrund der hier vertretenen Ansicht nicht zu berücksichtigen sind. Sofern im Einzelfall doch wesentliche Investitionen getätigt worden sind, wird die archivierende Einrichtung regelmäßig Trägerin des Investitionsrisikos und damit Datenbankherstel-

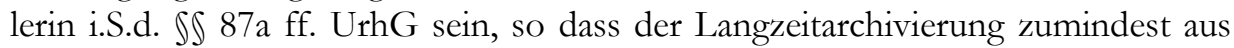
urheberrechtlicher Sicht nichts entgegenstünde. Sofern ausnahmsweise jedoch ein außenstehender Dritter Datenbankhersteller wäre, muss geprüft werden, ob durch die elektronische Archivierung dessen Nutzungsrechte verletzt würden.

\section{Ergebnis}

Damit bleibt festzuhalten, dass bei wissenschaftlichen Primärdaten grundsätzlich kein urheberrechtlicher oder leistungsrechtlicher Schutz entstehen wird. Ausnahmsweise kann jedoch ein leistungsrechtlicher Schutz gem. \ 72 Abs. 1 UrhG an Röntgenbildern oder gem. \87b UrhG an Datensammlungen, wie etwa Tabellen bestehen. Letzterer setzt jedoch voraus, dass im Einzelfall eine wesentliche Investition im Sinne des $\int 87$ a UrhG getätigt worden ist. Sofern dies der Fall ist, ist derjenige Inhaber des Leistungsschutzrechts, der die besagte Investition getätigt hat. In der Regel dürfte dies die archivierende Einrichtung selbst sein, da sie in der Regel das finanzielle Risiko für die Datenbankerstellung trägt. Zu beachten ist aber, dass Forschungsprojekte oftmals über sog. Drittmittel finanziert werden, so dass im Einzelfall geprüft werden muss, ob die fördernde Einrichtung nicht die Investition getätigt hat.

\section{B. Rechtliche Probleme bei der Speicherung und Bearbeitung wissenschaftlicher Primärdaten}

\section{Vorüberlegungen}

Im Rahmen der digitalen Langzeitarchivierung ist es in der Regel notwendig, die zu archivierenden Dateien zu kopieren, da die Archivierung auf speziellen Servern bzw. Datenträgern vollzogen wird und mit den ursprünglichen Dateien weiterhin gearbeitet werden muss. Darüber hinaus werden im Rahmen der digitalen Langzeitarchivierung Dateien nicht nur auf einem, sondern zumeist auf mindestens zwei unterschiedlichen Datenträgern archiviert, die unabhängig voneinander bestehen und sich in der Regel an unterschiedlichen Orten befinden, so dass im Falle der Beschädigung oder Zerstörung des einen Datenträgers, immer noch mindes- 
tens ein kompletter Datensatz zur Verfügung steht. Des Weiteren kann es im Laufe der Jahre notwendig sein, dass Dateiformat der gespeicherten Daten zu ändern, da das ursprüngliche Format inzwischen veraltet ist und man somit nicht mehr bzw. nur noch eingeschränkt auf die Datei zugreifen kann. Darüber hinaus wird die archivierende Einrichtung ihre Daten auch anderen Forschern und Forschungseinrichtungen zur Verfügung stellen, was beispielsweise durch die Verfügbarmachung der Daten im Internet oder anderen Netzwerken möglich ist. Im Folgenden soll deshalb untersucht werden, welche urheberrechtlichen Probleme derartige Maßnahmen mit sich bringen können und wie ein solches Vorgehen durch die archivierende Einrichtung juristisch sicher gestaltet werden kann. Aufbauend auf den so gewonnenen Ergebnissen soll dann dargelegt werden, ob bzw. inwieweit die momentane Gesetzeslage diesbezüglich geändert werden muss und wie eine entsprechende Vereinbarung zur Einholung der erforderlichen Nutzungsrechte ausgestaltet sein müsste.

\section{Urheberrechtliche und leistungsrechtliche Zulässigkeit der Kopie und Verarbeitung der Daten}

Wie oben festgestellt, wird der Fall, dass an wissenschaftlichen Primärdaten ein urheberrechtlicher Schutz entsteht, nur selten eintreten. Wenn überhaupt, kommt ein urheberrechtlicher Schutz an Datenbankwerken, in denen die Daten eingefügt wurden, in Betracht. Da jedoch die Möglichkeit der Entstehung eines urheberrechtlichen Schutzes nicht grundsätzlich ausgeschlossen ist, stellt sich die Frage, ob und unter welchen Voraussetzungen die Kopie eines Werkes, das ausnahmsweise doch urheberrechtlichen Schutz erlangt hat, zulässig ist. Die gleiche Frage stellt sich für Werke, die einen leistungsrechtlichen Schutz genießen, was, vor allem bei Datenbanken, aber auch bei digitalisierten Röntgenbildern der Fall sein kann.

\section{Betroffene Nutzungsrechte}

Im Rahmen der urheber- und leistungsrechtlichen Zulässigkeit der elektronischen Archivierung stellt sich dabei zunächst die Frage, welche Nutzungsrechte hiervon betroffen sein können.

a. Vervielfältigungsrecht gem. \16 UrhG

In Betracht kommt zunächst eine Beeinträchtigung des Vervielfältigungsrechts gem. \16 UrhG. Der Wortlaut der Norm definiert das Vervielfältigungsrecht als „das Recht, Vervielfältigungsstücke des Werkes herzustellen, gleichviel ob vorübergehend oder dauerhaft, in welchem Verfahren und in welcher Zahl“. Unter einer Vervielfältigung versteht man dabei ,jede körperliche Festlegung eines Wer- 
Kapitel 1 - Urheberrechtliche Problematik

kes, die geeignet ist, das Werk den menschlichen Sinnen auf irgendeine Weise unmittelbar oder mittelbar wahrnehmbar zu machen“ “.73

i. Tangierung des Vervielfältigungsrechts im Rahmen der elektronischen Langzeitarchivierung

Die Kopie einer Datei im Rahmen eines Downloads ${ }^{74}$ bzw. ihre Speicherung auf einem anderen Datenträger ${ }^{75}$ stellt demzufolge eine Vervielfältigung im Sinne des $\int 16$ UrhG dar. ${ }^{76}$ Dabei ist die Anzahl der gefertigten Kopien unerheblich, so dass schon die Erstellung einer einzigen Kopie unter den Vervielfältigungsbegriff des $\int 16$ UrhG fällt. ${ }^{77}$ Eine Vervielfältigung liegt hingegen nicht allein dadurch vor, dass die Daten von mehreren Bildschirmen angezeigt werden können, ohne dass sie heruntergeladen werden, da es insoweit an einer körperlichen Festlegung fehlt. ${ }^{78}$ Sollten die Daten also beispielsweise lediglich in den Räumen der archivierenden Einrichtung von mehreren Bildschirmen aus eingesehen werden können, stellte dies für sich genommen noch keine Vervielfältigung dar. Dieser Fall stellt jedoch zugegebenermaßen die Ausnahme dar. Wären die Daten jedoch, was in den vorliegenden Fällen wahrscheinlicher ist, online von anderen Computern einsehbar, würde mit dem Einsehen der Daten durch den Nutzer auch dann eine Vervielfältigung einhergehen, wenn diese vom Nutzer nicht (dauerhaft) heruntergeladen werden können, da in diesem Fall zumindest im Arbeitsspeicher des Nutzers eine Zwischenspeicherung erfolgt. ${ }^{79}$ War dies früher noch umstritten, stellt heute der Wortlaut von $\int 16 \mathrm{UrhG}$ durch die Formulierung „...gleichviel ob vorübergehend oder dauerhaft..." klar, dass auch derartige Zwischenspeicherungen vom Vervielfältigungsbegriff des $\int 16$ UrhG erfasst werden. ${ }^{80}$ Eine Vervielfältigung liegt darüber hinaus erst Recht dann vor, wenn die archivierten Daten von Dritten heruntergeladen werden können, da der Nutzer der Datenbank in diesem Fall eine Kopie der jeweiligen Datenbank erhalten würde.

${ }^{73}$ BT-Drucks. IV/270, 47; BGH NJW 1955, 1276; Dreyer in Dreyer/Kotthoff/Meckel, \ 16 Rn. 6; Dustmann in Fromm/Nordemann, \16 Rn. 9; Heerma in Wandtke/Bullinger, \16 Rn. 2.

${ }^{74} \mathrm{Vgl}$. OLG Karlsruhe MMR 2000, 233.

75 Dabei kommen sowohl die Übertragung einer elektronischen Datei auf einen Datenträger als auch die Digitalisierung einer analogen Datei mittels eines elektronischen Scans in Betracht.

76 BGH GRUR 1999, 325, 327; OLG Frankfurt a.M. CR 1997, 275, 276; KG GRUR 2002, 252, 253; Loewenheim in Loewenheim, \20 Rn. 4, 11; Kroitzsch in Möhring/Niccolini, \16 Rn. 4; Kotthoff, GRUR 1997, 597, 601

77 BGHZ 18, 44, 46; Dustmann in Fromm/Nordemann, \16 Rn. 10; Dreyer in Dreyer/Kotthoff/Meckel, \16 Rn. 10; Loewenheim in Loewenheim, $\mathbb{\$} 20$ Rn. 4.

78 Thum in Wandtke/Bullinger, \87b Rn. 40; Kotthoff, GRUR 1997, 597, 599.

${ }^{79} \mathrm{Vgl}$. OLG Hamburg ZUM 2001, 512, 513; ebenso Schulze in Dreier/Schulze, $\$ 16$ Rn. 13; Heerma in Wandtke/Bullinger, $\mathbb{S} 16$ Rn. 16; Dannecker, K\&R 1999, 529, 536; zur Frage, inwieweit in derartigen Fällen außerdem das Recht der öffentlichen Zugänglichmachung betroffen wäre, siehe unten S. $31 \mathrm{ff}$.

${ }^{80}$ So auch OLG Hamburg ZUM 2001, 512, 513; LG München I ZUM-RD 2003, 607, 610; Heerma in Wandtke/Bullinger, \16 Rn. 16; Klickermann, MMR 2007, 7; Spindler, GRUR 2002, 105, 107. 
Die Kopie oder Speicherung einer urheber- oder leistungsrechtlich geschützten Datei im Rahmen der Langzeitarchivierung stellt demzufolge eine Vervielfältigung im Sinne des $\int 16$ UrhG dar. Gem. $\int 15$ Abs.1 Nr. 1 UrhG steht dieses Recht jedoch ausschließlich dem Urheber zu. Das Gleiche gilt für den Datenbankhersteller gem. \87b Abs. 1 Alt.1 UrhG. Dabei bezieht sich dessen Wortlaut auf den Vervielfältigungsbegriff des $\int 16$ UrhG, ${ }^{81}$ so dass es im Ergebnis zunächst keinen Unterschied macht, ob das in Frage stehende Werk eine leistungsrechtlich geschützte Datenbank i.S.d. \87a UrhG oder eine urheberrechtlich geschütztes Datenbankwerk i.S.d. $\int 4$ Abs. 2 UrhG darstellt.

ii. Bestimmung des wesentlichen Teils einer Datenbank

$\mathrm{Zu}$ beachten ist allerdings, dass sich das ausschließliche Vervielfältigungsrecht des Datenbankherstellers gem. \ 87b Abs. 1 S. 1 UrhG auf die gesamte Datenbank oder wesentliche Teile davon bezieht. Demzufolge wäre sein Vervielfältigungsrecht nicht berührt, wenn im Rahmen der Langzeitarchivierung lediglich ein unwesentlicher Teil der Datenbank vervielfältigt würde, ${ }^{82}$ sofern es sich dabei nicht um eine wiederholte und systemtische Vervielfältigung i.S.v. \87b Abs. 1 S. 2 UrhG handeln würde. ${ }^{83}$

In diesem Zusammenhang stellt sich daher die Frage, ab wann ein wesentlicher Teil der Datenbank vorliegt und wann es sich noch um einen unwesentlichen Teil handelt. Relevant ist dies vor allem für die Fälle, in denen die archivierende Einrichtung nicht gleichzeitig Datenbankherstellerin ist. Sofern für die Archivierungszwecke auch die Nutzung eines unwesentlichen Teils genügen sollte, wäre zumindest die Archivierung unwesentlicher Teile der Datenbank ohne Zustimmung des Datenbankherstellers möglich. Die Datenbankrichtlinie stellt in diesem Zusammenhang klar, dass sich das Merkmal der Wesentlichkeit nicht nur auf den quantitativen Anteil des genutzten Teils an der Datenbank bezieht. Dies wird durch Erwägungsgrund 42 der Datenbankrichtlinie deutlich, wonach der Datenbankhersteller seine Rechte auch dem Benutzer entgegenhalten kann, ,der durch seine Handlungen einen qualitativ oder quantitativ erheblichen Schaden für die Investition verursacht". 84

Der Teil einer Datenbank kann demzufolge sowohl in quantitativer als auch in qualitativer Hinsicht wesentlich i.S.d. $\int 87 \mathrm{~b}$ UrhG sein. ${ }^{85}$ Allerdings enthalten

${ }^{81}$ Dreier in Dreier/Schulze, \87b Rn. 3; Vogel in Schricker/Loewenheim, \87b Rn. 30; 22, 27; Decker in Möhring/Niccolini, \87b Rn. 3-5; Thum in Wandtke/Bullinger, $\int 87 b$ Rn. 34; Nack, GRUR Int. 2004, 227, 229.

82Thum in Wandtke/Bullinger, \87b Rn. 5; Leistner, GRUR Int. 1999, 819, 831; Raue/Bensinger, MMR 1998, 507, 511.

${ }^{83}$ Das Gleiche gilt entsprechend für das Recht der öffentlichen Zugänglichmachung, auf welches auf den S. 31 ff. eingegangen wird.

${ }^{84}$ So auch die Gesetzesbegründung des Regierungsentwurfes: BR-Drucks. 966/96, 47.

85 Ebenso EuGH MMR 2005, 29, 33 Tz. 68; BGH GRUR 2005, 940, 942; Thum in Wandtke/Bullinger, \87b Rn. 12; 16; Derclaye, IIC 2005, 2, 26; Haberstumpf, GRUR 2003, 14, 27; 
weder das Gesetz noch die Datenbankrichtlinie diesbezüglich eine Definition, so dass die Abgrenzung anhand einer teleologischen Auslegung des $\ 87 \mathrm{~b}$ UrhG bzw. der Datenbankrichtlinie erfolgen muss. ${ }^{86}$ Wie oben bereits dargelegt, besteht dieser darin, einen Anreiz für die Erstellung von Informationssystemen zu bieten und seine diesbezüglich getätigten wesentlichen Investitionen zu schützen. ${ }^{87}$ Aus diesem Grund ist zur Beantwortung der Frage, ob es sich bei dem genutzten Teil einer Datenbank um einen wesentlichen oder unwesentlichen Teil handelt, der quantitative Umfang des genutzten Teils mit dem Gesamtumfang der Datenbank zu vergleichen. Denn sofern der entnommene Teil in seinem Umfang einen quantitativ erheblichen Anteil am Gesamtumfang der Datenbank ausmacht, war die Investition, die die diesen Teil betrifft, im gleichen Verhältnis zur Gesamtinvestition erheblich. ${ }^{88}$ Durch die Nutzung des betreffenden Teils würde die Amortisierung der für die Datenbank getätigten Gesamtinvestition gefährdet werden und zwar unabhängig davon, ob diese sich in dem genutzten Teil konkret niedergeschlagen hat oder nicht. ${ }^{89}$

Dem wird teilweise entgegengehalten, dass eine solche Abgrenzung in den Fällen zu Wertungswidersprüchen führen würde, in denen der verwendete Teil der Datenbank für sich genommen keinen sui generis Schutz genießen würde, in seinem Umfang aber einen quantitativ erheblichen Anteil an der Datenbank hätte. ${ }^{90}$ Dem kann jedoch nicht zugestimmt werden. Denn auch wenn der verwendete Teil keinen sui generis Schutz genießt, weil er beispielsweise aus selbst erzeugten Daten besteht, kann seine Verwendung dennoch die Amortisierung der Gesamtinvestition gefährden. Gerade diese Gefährdung der Investition ist aber gerade, vor der die Datenbankrichtlinie den Datenbankhersteller schützen möchte. Sollte die Investition im Einzelfall hingegen nicht wesentlich sein, so entfällt der sui generis Schutz der $\iint 87$ a ff. UrhG ohnehin von vornherein, so dass es auf die Wesentlichkeit des verwendeten Teils nicht mehr ankäme.

Sofern im Rahmen einer elektronischen Langzeitarchivierung Datenbanken archiviert werden sollen, würde ein Eingriff in das Vervielfältigungsrecht des Datenbankherstellers demzufolge nur dann nicht vorliegen, wenn der Umfang des verwendeten Teils in quantitativer Hinsicht unwesentlich zu dem Gesamtumfang der Datenbank wäre, aus dem der besagte Teil entnommen worden ist. Wann dies der Fall ist, muss anhand der Umstände des jeweiligen Einzelfalls entschieden

Kotthoff, GRUR 1997, 597, 602; Leistner, GRUR Int. 1999, 819, 832; Raue/Bensinger, MMR 1998, $507,511$.

${ }^{86}$ Haberstumpf, GRUR 2003, 14, 27; Leistner, GRUR Int. 1999, 819, 832; Nolte, ZUM 2003, 540, 546 f.

${ }^{87}$ Siehe hierzu die Nachweise in Fn. 58.

${ }^{88}$ EuGH GRUR 2009, 572, 577 Tz. 59; EuGH MMR 2005, 29, 33 Tz. 70, 81; BGH GRUR 2005, 857, 859; Thum in Wandtke/Bullinger, \87b Rn. 12; Leistner, GRUR Int. 1999, 819, 832; im Ergebnis auch Nolte, ZUM 2003, 540, 547, siehe auch Sendrowski, GRUR 2005, 369, 375; im Ergebnis auch KG MMR 2001, 171, 172.

89 So auch Leistner, K\&R 1997, 457, 460; Haberstumpf, GRUR 2003, 24, 27.

90 Thum in Wandtke/Bullinger, \87b Rn. 14. 
werden. ${ }^{91}$ Als Abgrenzungskriterium kann dabei jedoch die Frage dienen, ob der verwendete Teil dazu geeignet wäre, das Nutzungsinteresse an der geschützten Datenbank zu ersetzen und damit die Amortisierung der vom Datenbankhersteller getätigten Investition zu gefährden. ${ }^{92}$

Aber auch sofern man bei einer solchen Prüfung zu dem Ergebnis kommt, dass der zu archivierende Teil in quantitativer Hinsicht keinen wesentlichen Teil der Datenbank ausmacht, ist ferner zu prüfen, ob er nicht dennoch einen wesentlichen Teil in qualitativer Hinsicht darstellt. Ein qualitativ wesentlicher Teil liegt dabei dann vor, wenn die Beschaffung, Überprüfung oder Darstellung der verwendeten Daten für sich genommen eine wesentliche Investition darstellen würde, wenn der Verwender lediglich diese Daten zu einer Datenbank zusammengestellt hätte. ${ }^{93}$ Dabei spielt der diesen Daten innewohnende Wert keine Rolle, da der Schutzgegenstand im Rahmen der $\iint 87$ a ff. UrhG gerade nicht der Wert der einzelnen Daten ist, sondern die für die Datenbankerstellung getätigten Investitionen. ${ }^{94}$ Demzufolge kann der Teil einer Datenbank, der für sich genommen in quantitativer Hinsicht keinen wesentlichen Teil der Gesamtdatenbank darstellt, ${ }^{95}$ dennoch einen ,wesentlichen Teil“ i.S.d. \ 87b UrhG darstellen, sofern die Beschaffung, Erhebung oder Darstellung der zur Archivierung vorgesehenen Daten für sich genommen eine wesentliche Investition erfordert haben. Wann dies der Fall ist, muss anhand der oben beschriebenen Kriterien ermittelt werden. ${ }^{96}$

$\mathrm{Zu}$ beachten ist, dass auch unwesentliche Teile einer Datenbank nicht völlig schutzlos sind. Da der Schutzumfang des $\int 87 \mathrm{~b}$ Abs. 1 UrhG nicht -wie sonst im UrhG- von qualitativen sondern inhaltlich quantitativen Vorgaben abhängt, regelt Abs.1 S. 2 die Entnahme unwesentlicher Teile einer Datenbank, um den Schutz von Abs. 1 S. 1 nicht leerlaufen zu lassen. ${ }^{97}$

Sollte es sich bei dem zu archivierenden Teil bzw. den zu archivierenden Teilen einer Datenbank ausnahmsweise um unwesentliche Teile handeln, ist aus Sicht der archivierenden Einrichtung zu beachten, dass gem. $\int 87 b$ Abs. 1 S. 2 UrhG der Vervielfältigung, Verbreitung oder öffentlichen Wiedergabe eines nach Art oder Umfang wesentlichen Teils der Datenbank die wiederholte und systematische Vervielfältigung, Verbreitung oder öffentliche Wiedergabe von nach Art und Umfang unwesentlichen Teilen der Datenbank gleichsteht, sofern diese Handlun-

\footnotetext{
${ }^{91}$ So auch Kotthoff, GRUR 1997, 597, 602; Raue/Bensinger, MMR 1998, 507, 510 will dagegen die Grenze für einen quantitativ wesentlichen Umfang bei 50\% der Datenbank ziehen.

92 Leistner, GRUR Int.1999, 819, 832; Kotthoff, GRUR 1997, 597, 602; Klein, GRUR 2005, 377, 383; vgl. auch BGH GRUR 2005, 857, 859 .

${ }^{93}$ EuGH MMR 2005, 29, 33; Thum in Wandtke/Bullinger, \87b Rn. 17; Sendrowski, GRUR 2005, $369,375$.

${ }^{94}$ EuGH MMR 2005, 29, 33; Thum in Wandtke/Bullinger, \ 87b Rn. 16; Benecke, CR 2004, 608, 612; zustimmend auch Sendrowski, GRUR 2005, 369, 375; Leistner, K\&R 2007, 457, 460 f.

${ }_{95}$ Dies kommt vor allem dann in Betracht, wenn die Gesamtdatenbank sehr umfangreich ist.

${ }^{96}$ Siehe zu den Voraussetzungen einer wesentlichen Investition S. 17 ff.

${ }^{97}$ Caychowski in Fromm/Nordemann, $\int 87 b$ Rn. 21; Vogel in Schricker/Loewenheim, \87b Rn. 52.
} 
Kapitel 1 - Urheberrechtliche Problematik

gen einer normalen Auswertung der Datenbank zuwiderlaufen oder die berechtigten Interessen des Datenbankherstellers unzumutbar beeinträchtigen.

Wie sich aus dem Wortlaut ergibt, müssen die Tatbestandsmerkmale ,wiederholt und systematisch“ in diesem Zusammenhang kumulativ vorliegen, weshalb eine wiederholte Nutzung ohne systematisches Vorgehen aus Sicht der archivierenden Einrichtung unschädlich wäre. ${ }^{98}$ Die archivierende Einrichtung dürfte demzufolge einen oder mehrere unwesentliche Teile einer Datenbank nicht vervielfältigen, sofern diese Vervielfältigungen planmäßig und nach sachlogischen Kriterien erfolgten. ${ }^{99}$ Ein solches Vorgehen wäre vor allem dann anzunehmen, wenn die Einrichtung mehrere unwesentliche Teile einer Datenbank archivieren würde, welche in einem inhaltlichen Zusammenhang stünden. ${ }^{100}$ Darüber hinaus müssten die entnommenen unwesentlichen Teile in ihrer Summe außerdem einen wesentlichen Teil nach Abs. 1 S. 2 ausmachen. ${ }^{101}$

$\mathrm{Da}$ die vorliegend in Betracht kommenden wissenschaftlichen Datenbanken in der Regel zum Zwecke der wissenschaftlichen Nutzung erstellt worden sein werden, stellt sich jedoch die Frage, ob eine systematische und wiederholte Vervielfältigung unwesentlicher Teile zum Zwecke der Archivierung der normalen Auswertung überhaupt zuwiderliefe, da diese in der Regel ebenfalls wissenschaftliche Zwecke verfolgt. Da die archivierten Teile in der Regel jedoch ein Konkurrenzprodukt zu der ursprünglichen Datenbank darstellen würden, würde die Entnahme schon deshalb der normalen Nutzung zuwiderlaufen, weil die archivierende Einrichtung sich hierdurch eigene Aufwendungen ersparen würde. ${ }^{102}$

Selbst wenn die wiederholte und systemtische Vervielfältigung unwesentlicher Teile im Rahmen der Archivierung der normalen Auswertung der Datenbank im Einzelfall nicht zuwider laufen sollte, wäre sie dennoch unzulässig, wenn sie eine unzumutbare Beeinträchtigung berechtigter Interessen des Datenbankherstellers zur Folge hätten. Dies wäre etwa dann der Fall, wenn der Datenbankhersteller aufgrund der mit der Archivierung einhergehenden Nutzung den Ausfall von Werbeeinnahmen erleiden würde, da Interessenten nicht mehr seine Datenbank frequentieren, sondern auf die archivierten Daten zugreifen würden. ${ }^{103}$

Sofern die für eine Archivierung vorgesehenen Datensätze einen urheberrechtlichen Schutz genießen, ist durch ihre Archivierung auf jeden Fall das Vervielfältigungsrecht betroffen, so dass geprüft werden muss, ob dieses bei der archivierenden Einrichtung liegt oder erst noch eingeholt werden muss. Sofern die besagten Datensätze hingegen ausschließlich einen leistungsrechtlichen Schutz nach

\footnotetext{
${ }^{98}$ Cyychowski in Fromm/Nordemann, \87b Rn. 23; Vogel in Schricker/Loewenheim, \87b Rn. 54.

${ }_{99}$ Vgl. Vogel in Schricker/Loewenheim, \87b Rn. 54; Loewenheim in Loewenheim, \43 Rn. 17.

100 Vogel Schricker/Loewenheim, \87b Rn 54.

101 EuGH GRUR 2005, 244, 249; so auch schon KG GRUR-RR 2004, 228, 235; OLG Dresden ZUM 2001, 597; Haberstumpf, GRUR 2003, 14, 28.

102 OLG Dresden, ZUM 2001, 595, 597; Vogel in Schricker/Loewenheim, J 87b Rn. 57.

103 Vgl. LG Berlin CR 1999, 388, 389; Caychowski in Fromm/Nordemann, S 87b Rn. 29; Loewenheim in Loewenheim, $\int 43 \mathrm{Rn} .17$.
} 
$\int 87 b$ UrhG genießen, ist zu beachten, dass das Vervielfältigungsrecht sich nur auf die gesamte Datenbank und wesentliche Teile davon bezieht. Die Vervielfältigung unwesentlicher Teile dieser Datenbank würde demzufolge nicht das Vervielfältigungsrecht des Datenbankherstellers tangieren und wäre daher auch ohne seine Zustimmung möglich, sofern nicht die Voraussetzungen von $\int 87 \mathrm{~b}$ Abs. 1 S. 2 UrhG vorliegen. ${ }^{104}$

Es ist dabei in einem ersten Schritt zu prüfen, ob der zur Archivierung vorgesehene Teil der in Frage stehenden Datenbank für sich genommen geeignet wäre, der Datenbank Konkurrenz zu machen. Ist dies der Fall, so kann davon ausgegangen werden, dass der zur Archivierung vorgesehene Teil einen in quantitativer Hinsicht wesentlichen Teil der Datenbank darstellt. Sofern dies nicht der Fall ist, ist weiter zu prüfen, ob die zur Archivierung vorgesehenen Daten eine wesentliche Investition erfordert haben. Dabei sind allerdings weder der Wert der Daten ${ }^{105}$ noch die zu ihrer Erzeugung angefallenen Kosten zu berücksichtigen, da Letztere insoweit keine „Investition“ im Sinne der $\iint 87$ a ff. UrhG darstellen. Aus dem Gesagten folgt, dass es im Einzelfall schwierig ist, zu bestimmen, ob der Teil einer Datenbank wesentlich ist oder nicht. Auch wird die Archivierung nur eines Teils einer Datenbank aus Sicht der archivierenden Einrichtung oftmals wenig wissenschaftlichen Nutzen mit sich bringen. Von daher sollte grundsätzlich versucht werden, die erforderlichen Nutzungsrechte einzuholen. ${ }^{106}$

Im Falle der Langzeitarchivierung müsste demnach anhand der oben aufgestellten Kriterien ${ }^{107}$ geprüft werden, ob ein urheber- oder leistungsrechtlicher Schutz an den Daten bzw. den Datensammlungen oder Lichtbildern entstanden ist. In einem zweiten Schritt muss sodann festgestellt werden, wer Urheber bzw. Datenbankhersteller oder Lichtbildner ist. Sofern dies nicht die archivierende Einrichtung selbst ist, muss zum Zwecke der Archivierung das entsprechende Nutzungsrecht vom Rechteinhaber eingeholt werden, sofern keine gesetzliche Schranke eingreift oder der urheberrechtliche bzw. leistungsrechtliche Schutz nicht abgelaufen ist. ${ }^{108}$

\section{b. Bearbeitungsrecht gem. \23 UrhG}

Ferner ist hinsichtlich der juristischen Zulässigkeit einer digitalen Langzeitarchivierung entscheidend, inwiefern das Verändern der Daten im Rahmen einer Migration oder Dateikonvertierung urheber- oder leistungsschutzrechtliche Relevanz aufweisen kann. In Betracht kommt in diesem Zusammenhang eine Verletzung

\footnotetext{
${ }^{104}$ Die Archivierung unwesentlicher Teile wird in der Praxis jedoch selten eine Rolle spielen, da die archivierende Einrichtung wenig Interesse daran haben dürfte, lediglich Teile einer Datenbank zu archivieren. Ausgeschlossen werden kann dies indes nicht.

105 So aber KG MMR 2001, 171, 172.

106 Zur Erlangung der Nutzungsrechte siehe unten S. 34 ff.

107 Siehe oben S. $6 \mathrm{ff}$.

108 Dazu siehe unten. S. 35 ff.
} 
Kapitel 1 - Urheberrechtliche Problematik

des Bearbeitungsrechts des Urhebers gem. \23 UrhG.109 Zwar bedarf gem. \23 Satz 1 UrhG erst die Veröffentlichung oder Verbreitung eines bearbeiteten Werkes der Einwilligung des Urhebers. Nach Satz 2 ist jedoch bereits für die Herstellung der Bearbeitung oder die Umgestaltung eines Datenbankwerkes die Zustimmung des Urhebers erforderlich. In diesem Zusammenhang ist umstritten, ob das Bearbeitungsrecht nach $₫ 23$ UrhG ebenfalls dem Datenbankhersteller zusteht. ${ }^{110}$

Dagegen spricht zunächst, dass das Bearbeitungsrecht in $\int 87 \mathrm{~b}$ UrhG nicht aufgeführt ist und sich der Schutzumfang nur auf die Nutzung der in der Datenbank enthaltenen Rechte in unveränderter Form richtet. ${ }^{111}$ Für eine entsprechende Anwendung von $\ 23$ S. 2 UrhG auf Datenbanken i.S.d. $\int 87$ a UrhG spricht jedoch, dass mit einer Bearbeitung oder Umgestaltung eine wesentliche oder eine systematische und wiederholte unwesentliche Entnahme oder Weiterverwendung verbunden ist, die gem. \87b Abs. 1 S. 2 UrhG der Nutzung eines wesentlichen Teils der Datenbank gleichsteht. ${ }^{112}$

Aus diesem Grund ist $\ 23$ S. 2 UrhG dahingehend richtlinienkonform auszulegen, dass er auch auf Datenbanken i.S.d. \87a UrhG Anwendung findet. Beim Lichtbildner ergibt sich die Anwendbarkeit von $₫ 23$ UrhG hingegen unproblematisch aus dem Verweis in $\ 72$ Abs. 2 UrhG auf die Vorschriften des ersten Teils des UrhG, wozu auch $₫ 23$ UrhG zählt. ${ }^{113}$ Damit stellt sich im Folgenden für die urheberrechtliche Zulässigkeit und juristisch sichere Ausgestaltung der digitalen Langzeitarchivierung von wissenschaftlichen Primärdaten die Frage, ob eine Migration der archivierten Daten bzw. Datenbanken eine Bearbeitung oder Umgestaltung i.S.d. $\$ 23$ Satz 2 UrhG darstellt.

Unter einer Bearbeitung versteht man dabei eine Veränderung des Originalwerkes, bei der das Werk geändert bzw. verändert wird, wobei die Veränderung nicht völlig unerheblich sein darf.114 Bei der Frage, ob im Falle einer Migration oder Konvertierung der Datei eine Bearbeitung im Sinne des $\ 23$ UrhG vorliegt, muss danach differenziert werden, ob sich bei der Überführung in ein anderes Dateiformat der geistige Inhalt des Werkes ändert. Ist dies nicht der Fall, so liegt grundsätzliche keine Bearbeitung nach $\ 23 \mathrm{UrhG}$, sondern lediglich eine Vervielfältigung nach $\int 16$ UrhG vor, da es nicht zu einer Änderung des geistigästhetischen Gesamteindrucks des Werkes kommt, vor dem \23 UrhG gerade

\footnotetext{
${ }^{109} \mathrm{Vgl}$. Euler, AfP 2008, 474, 479.

${ }^{110}$ Für ein Bearbeitungsrecht des Datenbankherstellers: Cyychowski in Fromm/Nordemann, \87b Rn. 20; Dreier in Dreier/Schulze, $\int 87 b$ Rn. 3; a.A. Thum in Wandtke/Bullinger, \87b Rn. 10.

111 Vogel in Schricker, \87b Rn. 19.

112 Dreier in Dreier/Schulze, $\int 87 b$ Rn. 3.

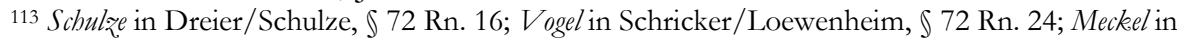
Dreyer/Kotthoff/Meckel, $\int 72$ Rn. 11, 12.

114 BGH GRUR 2002, 532, 534; GRUR 1990, 669, 673 - Bibelreproduktion; GRUR 1994, 41,43 Videozweitauswertung; Loewenheim in Schricker/Loewenheim, $\ 23$ Rn. 7; A. Nordemann in Fromm/Nordemann, $\ 23$ Rn. 8.
} 
schützen möchte. ${ }^{115}$ Handelt es sich hingegen um eine verlustbehaftete Konvertierung, so wäre das Bearbeitungsrecht nach \23 UrhG zumindest dann betroffen, wenn die Konvertierung eine weit reichende Beeinträchtigung des Werkes nach sich zöge. ${ }^{116}$ Dies wird allerdings in den vorliegend relevanten Fällen von Datenbankwerken und vor allem bei digitalisierten Röntgenbildern, bei denen es ja gerade auf eine hohe Qualität ankommt, wohl auszuschließen sein, so dass das Bearbeitungsrecht des $\int 23$ UrhG bei der Archivierung wissenschaftlicher Primärdaten in der Regel nicht betroffen sein wird.

\section{c. Recht der öffentlichen Zugänglichmachung gem. \19a UrhG}

Des Weiteren könnte das Recht der öffentlichen Zugänglichmachung gem. \19a UrhG betroffen sein. Zu bedenken ist, dass die Dateien nicht lediglich kopiert und archiviert werden, sondern dass ein wichtiges Motiv für eine digitale Langzeitarchivierung auch die Verfügbarmachung gewonnener Daten für außenstehende Wissenschaftler und Forschungseinrichtungen im Rahmen von nationalen und internationalen Kooperationen und ein damit einhergehender Datenaustausch ist. Damit stellt sich aber die Frage, ob bzw. ab wann dadurch das Recht der öffentlichen Zugänglichmachung betroffen ist. Gem. \19a UrhG ist das Recht der öffentlichen Zugänglichmachung ,das Recht, das Werk drahtgebunden oder drahtlos der Öffentlichkeit in einer Weise zugänglich zu machen, dass es Mitgliedern der Öffentlichkeit von Orten und zu Zeiten ihrer Wahl zugänglich ist“. Dabei wird der Begriff der öffentlichen Zugänglichmachung auch durch die Formulierung „...̈̈ffentlich wiederzugeben...“ in $\int 87 \mathrm{~b}$ UrhG erfasst. ${ }^{117}$ Die folgenden Überlegungen beziehen sich damit sowohl auf Datenbankwerke als auch auf Datenbanken i.S.v. \ 87 a UrhG ${ }^{118}$ sowie auf Lichtbilder nach $\int 72 \mathrm{I}$, der auf den ersten Teil des UrhG und damit auf $\int 19$ a UrhG verweist. Sofern Personen und Institutionen auf die archivierten Daten zugreifen können, wird dies über offene oder geschlossene Netzwerke geschehen, so dass die Daten unabhängig von Ort und Zeit anderen zugänglich sind. Für die Frage, ob das Recht nach \19a UrhG betroffen ist, kommt es also entscheidend darauf an, unter welchen Vorausset-

115 BGH GRUR 2002, 532, 534; GRUR 1990, 669, 673; Euler, AfP 2008, 474, 481; a.A. Hoeren, Rechtsfragen zur Langzeitarchivierung (LZA) und zum Anbieten von digitalen Dokumenten durch Archivbibliotheken unter besonderer Berücksichtigung von Online-Hochschulschriften, 2005, S. 7 f., abrufbar unter: http://www.langzeitarchivierung.de/downloads/mat/hoeren.pdf . 116 Upmeier, in: Neuroth/Liegmann/Oßwald/Scheffel/Jehn/Strathmann (Hrsg.), Nestor, Eine kleine Enzyklopädie der digitalen Langzeitarchivierung, Version 2.3, 2010, S. 2-6, abrufbar unter: http://www.langzeitarchivierung.de/publikationen/handbuch/handbuch.htm; Heckmann/Weber, AfP 2008, 269, 274.; Katzenberger, GRUR 1990, 94, 97; offen lassend Kleckermann, MMR 2007, 7, 8.

117 Loewenheim in Schricker/Loewenheim, \87b Rn. 51; ders. in Loewenheim, \43 Rn. 20; Kotthoff in Dreyer/Kotthoff/Meckel, \87b Rn. 9; Dreier in Dreier/Schulze, \87b Rn. 3; Dannecker, K\&R 1999, 529, 536.

118 Wobei hinsichtlich der öffentlichen Zugänglichmachung von wesentlichen oder unwesentlichen Teilen einer Datenbank auf die Ausführungen der S. 25 ff. entsprechend verwiesen wird. 
Kapitel 1 - Urheberrechtliche Problematik

zungen eine Zugänglichmachung an Mitglieder der Öffentlichkeit vorliegt und wann es sich lediglich um eine interne Zugänglichmachung handelt.

i. Begriff der Öffentlichkeit im Sinne des $₫ 19 \mathrm{a}$ UrhG

Bei der Bestimmung des Begriffs der Öffentlichkeit im Rahmen von \19a UrhG kann auf die Legaldefinition in $\int 15$ Abs. 3 UrhG zurückgegriffen werden. ${ }^{119} \mathrm{Nach}$ $\int 15$ Abs. 3 UrhG ist eine Wiedergabe dann öffentlich, ,wenn sie für eine Mehrzahl von Mitgliedern der Öffentlichkeit bestimmt ist“. Wer zur Öffentlichkeit gehört, regelt in diesem Zusammenhang $\int 15$ Abs. 3 Satz 2 UrhG. Danach gehört zur Öffentlichkeit jeder, der nicht mit demjenigen, der das Werk verwertet, oder mit anderen Personen, denen das Werk in unkörperlicher Form wahrnehmbar oder zugänglich gemacht wird, durch persönliche Beziehungen verbunden ist. Eine persönliche Verbundenheit liegt in diesem Zusammenhang dann vor, wenn die Beziehungen zum Verwerter oder untereinander stark genug sind, um das Bewusstsein einer persönlichen Verbundenheit zu begründen. ${ }^{120}$ Sofern eine juristische Person das Werk verwertet, kann unter den Teilnehmern aufgrund der persönlichen Beziehung zu der oder den für die juristische Person handelnden Personen eine persönliche Verbundenheit zustande kommen.121 Demzufolge ist im Einzelfall zu prüfen, durch wen und welchem Kreis die Daten zugänglich gemacht werden sollen.

ii. Zugänglichmachung der archivierten Daten im Rahmen der elektronischen Langzeitarchivierung

Geht man davon aus, dass das jeweilige Institut bzw. die jeweilige Forschungsstelle, die von ihr gewonnenen Daten selbst archiviert und zum Abruf bereit hält, liegt hierin keine öffentliche Zugänglichmachung vor, solange nur die Mitarbeiter dieses Institutes oder der Forschungsstelle auf die Dateien zugreifen können. Unter Berücksichtigung der Größe der Einrichtung wäre im Regelfall eine persönliche Beziehung der Mitarbeiter zu der Person, die die Daten archiviert und damit verwertet, grundsätzlich anzunehmen. Etwas anderes würde jedoch gelten, wenn die Daten nicht durch die Forschungsstelle selbst, sondern zentral durch die Einrichtung, der sie angehört, archiviert und allen Mitarbeitern dieser Einrichtung zugänglich gemacht würden.

Dies wäre beispielsweise der Fall, wenn eine Universität Forschungsdaten für sämtliche ihrer angestellten Wissenschaftler bereit hielte. In diesem Fall würde die Universität Verwerter der Datenbanken sein und es käme somit darauf an, ob die

119 Dustmann in Fromm/Nordemann, S 19a Rn. 12; v. Ungern-Sternberg in Schricker/Loewenheim, $\int 19 a$ Rn. 48; Dreyer in Dreyer/Kotthoff/Meckel, \19a Rn. 9.

${ }_{120}$ Dreier in Dreier/Schulze, \15 Rn. 43; Dustmann in Fromm/Nordemann, $\ 15$ Rn. 34; v. Ungern-

Sternberg in Schricker/Loewenheim, \15 Rn. 76; Heerma in Wandtke/Bullinger, \15 Rn. 18.

121 BGH GRUR 1975, 33, 34; Dreier in Dreier/Schulze, $\ 15$ Rn. 43; v. Ungern-Sternberg in Schricker/Loewenheim, \15 Rn. 73. 
Mitarbeiter in enger Verbundenheit zu den Personen stünden, die für die Universität die Verwertung vornähmen. Dies dürfte aufgrund der großen Anzahl von Wissenschaftlern, die eine Universität beschäftigt, jedoch selbst dann zu verneinen sein, wenn die Daten nicht allen Wissenschaftlern, sondern nur denen einer bestimmten Fachrichtung zugänglich gemacht würden. Der Fall, dass die archivierten Daten nur den Mitarbeitern einer einzelnen Forschungsstelle zur Verfügung stehen, wird außerdem eher die Ausnahme darstellen. Denn gerade im Rahmen der Langzeitarchivierung wissenschaftlicher Daten soll ja unter anderem erreicht werden, dass diese Daten anderen Wissenschaftlern und Forschungseinrichtungen zukommen, um die Forschung auf einem bestimmten Gebiet weiter voranzutreiben. Aus diesem Grund werden die archivierten Daten in der Regel auch außenstehenden Wissenschaftlern zur Verfügung gestellt werden, so dass es, sofern man überhaupt noch einen abgrenzbaren Personenkreis annimmt, an der persönlichen Verbundenheit fehlen und damit das Tatbestandsmerkmal der Öffentlichkeit regelmäßig erfüllt sein wird. Demnach wird also das Recht der öffentlichen Zugänglichmachung im Rahmen der digitalen Langzeitarchivierung wissenschaftlicher Primärdaten ebenfalls grundsätzlich betroffen sein.

\section{d. Zwischenergebnis}

Im Rahmen der digitalen Langzeitarchivierung ist, wie gezeigt, das Recht der Vervielfältigung nach \16 UrhG betroffen, sofern ein urheberrechtlich oder leistungsrechtlich geschütztes Werk kopiert wird. Dieses Nutzungsrecht steht dabei nicht nur dem Urheber eines Werkes zu, sondern gem. \ 72 UrhG und $\int 87 b$ UrhG auch dem Lichtbildner sowie dem Datenbankhersteller.Das Recht der Bearbeitung gem. \ 23 UrhG wird indes, wenn überhaupt, nur in den seltensten Fällen relevant sein. Sofern ein schutzfähiges Werk besteht, ${ }^{122}$ liegt mangels geistig schöpferischer Tätigkeit in der überwiegenden Zahl dieser Fälle kein Datenbankwerk oder ein anderes urheberrechtlich geschütztes Werk vor, sondern meist nur Datenbanken nach $\int 87$ a UrhG, die lediglich einen leistungsrechtlichen Schutz genießen.

Im Rahmen einer richtlinienkonformen Auslegung ist die Vorschrift des \23 S. 2 UrhG zwar auch auf diese anwendbar. Solange die Migration oder Konvertierung der Dateien jedoch keine erhebliche qualitative Beeinträchtigung nach sich ziehen, ist keine Bearbeitung im Sinne des $\int 23$ UrhG gegeben. Dabei ist in den vorliegenden Fällen nicht davon auszugehen, dass die Daten durch die Konvertierung oder Migration eine erhebliche qualitative Beeinträchtigung erfahren, da dies das Interesse der archivierenden Einrichtung an der Datenerhaltung konterkarieren würde.

Das Recht der öffentlichen Zugänglichmachung nach \19a UrhG wird indes in den meisten Fällen betroffen sein, da ein Ziel der Langzeitarchivierung die Ver-

122 Wie oben auf S. 10 festgestellt, wird dies äußerst selten sein. 
Kapitel 1 - Urheberrechtliche Problematik

breitung der gewonnenen Daten an andere Wissenschaftler und Forschungseinrichtungen ist. Eine Ausnahme käme jedoch dann in Betracht, wenn die Daten durch eine Forschungsstelle selbst archiviert würden und nur die Mitarbeiter der Forschungsstelle Zugriff auf die archivierten Dateien hätten. Allerdings wäre auch in diesen Fällen stets zu prüfen, ob die Zahl der Mitarbeiter nicht doch so groß ist, dass es an einer persönlichen Verbundenheit zum Verwerter mangelt und damit eine Zugänglichmachung gegenüber der Öffentlichkeit gem. \19a UrhG vorliegt.

\section{Erlangung der Erlaubnis zur Nutzung}

Sofern im Einzelfall durch die Archivierung einer Datenbank, eines Datenbankwerkes oder eines Lichtbildes eines oder mehrere der oben aufgeführten Nutzungsrechte tangiert werden, muss die archivierende Einrichtung prüfen, ob die Verwertung des Werkes das Verwertungsrecht des Urhebers verletzt oder ob die Nutzung gerechtfertigt ist. Grundsätzlich hat der Urheber gem. \ 15 Abs. 1 UrhG das ausschließliche Recht, sein Werk zu verwerten. Gleiches gilt aufgrund der Verweisung in $\int 72$ UrhG auf den ersten Teil des UrhG für den Lichtbildner.

Bei Datenbanken steht dem Datenbankenhersteller gem. \87b hingegen das ausschließliche Recht zu, die Datenbank und wesentliche Teile davon zu vervielfältigen, zu verbreiten und öffentlich wiederzugeben. Sofern die archivierende Einrichtung nicht selbst Inhaberin der erforderlichen Nutzungsrechte wäre, würde die Nutzung des Werkes somit grundsätzlich eine Verletzungshandlung darstellen, die zivil- und auch strafrechtliche Konsequenzen nach sich ziehen könnte. ${ }^{123}$ Die Nutzung des Werkes wäre hingegen dann nicht unrechtmäßig, sofern sich die archivierende Einrichtung auf eine urheberrechtliche Schranke berufen könnte (dazu a). Dies sind Vorschriften, die den Inhalt des Urheberrechts oder den leistungsrechtlichen Schutz zugunsten der Allgemeinheit, der Kulturwirtschaft oder auch einzelner Nutzer beschränken. ${ }^{124}$ Sofern im Einzelfall die Voraussetzungen einer urheberrechtlichen Schranke nicht gegeben sind, muss die archivierende Einrichtung hingegen die erforderlichen Nutzungsrechte vom Rechteinhaber einholen.

Dies wird in den meisten Fällen der Urheber bzw. der Datenbankhersteller oder Lichtbildner sein, sofern er die Rechte nicht bereits an einen Dritten übertragen hat. Dabei geht das Gesetz in $\int 31$ Abs. 1 UrhG ausdrücklich davon aus, dass Nutzungsrechte an einem Werk einem anderen eingeräumt werden können. Dies folgt für die Rechte an Lichtbildern aus dem Verweis in $\int 72$ UrhG. Auch das Recht des Datenbankherstellers ist als verwandtes Schutzrecht frei übertragbar. ${ }^{125}$ Dazu muss aber zunächst die Identität des Rechteinhabers ausfindig gemacht

\footnotetext{
123 Vgl. nur $\int 97$ UrhG oder zur Strafbarkeit \106 UrhG; ausführlich zu den Konsequenzen einer unrechtmäßigen Verwertung des Werkes siehe unten S. 133 ff.

124 Rehbinder, Rn. 432.

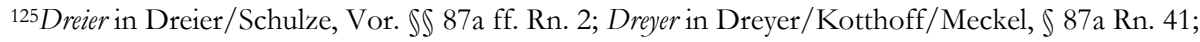
siehe auch Art. 7 Abs. 3 der Datenbankrichtlinie 96/9/EG.
} 
werden. Dies dürfte in der Regel noch recht unproblematisch sein, da die Daten an der archivierenden Einrichtung erhoben worden sind und es sich daher bei dem Inhaber oftmals um einen an der Einrichtung forschenden Wissenschaftler handelt, so dass die Kontaktaufnahme zum Rechteinhaber grundsätzlich möglich sein wird. Dies gilt erst Recht, wenn es sich beim Rechteinhaber um eine andere Institution handelt, die ein bestimmtes Forschungsprojekt der archivierenden Einrichtung finanziert hat und daher Datenbankhersteller ist. Darüber hinaus müsste der Rechteinhaber der Einräumung der relevanten Rechte aber auch zustimmen, weshalb die archivierende Einrichtung bei der Durchführung der digitalen Langzeitarchivierung auf dessen Kooperationsbereitschaft angewiesen wäre.

Sofern keine Schranke greift, stellt sich damit die Frage, ob nicht unter Umständen eine öffentlich-rechtliche Pflicht zur Nutzungsrechtseinräumung für den Urheber bzw. den Inhaber der Leistungsschutzrechte besteht (dazu b.). In diesem Fall müsste der Rechteinhaber eine entsprechende Vereinbarung mit der Universität schließen. Ist jedoch auch dies nicht der Fall, bleibt nur noch die Möglichkeit, dass der Inhaber der Verwertung freiwillig zustimmt, wobei hier stets geprüft werden muss, ob eine zivilrechtliche Verpflichtung zur Nutzungsrechtseinräumung nicht aufgrund eines bestehenden Arbeitsverhältnisses zwischen Rechteinhaber und Universität besteht. Darüber hinaus stellt sich die Frage, welche formalen und materiellen Anforderungen an eine solche Rechteeinräumung zu stellen sind (dazu c.).

\section{a. Gesetzliche Schranken}

Zunächst ist zu prüfen, ob die Rechte des Urhebers bzw. des Inhabers der Leistungsschutzrechte einer gesetzlichen Schranke unterliegen. Dabei ist zum einen zwischen den urheberrechtlichen Schranken der $\iint 44 a$ ff. UrhG (dazu i und ii) und der zeitlichen Begrenzung des Urheberrechtsschutzes (dazu iii) auf der einen und den speziellen leistungsschutzrechtlichen Schranken des $\int 87 \mathrm{c}$ UrhG auf der anderen Seite zu unterscheiden (dazu iv). Im Rahmen der urheberrechtlichen Schranken kommen hinsichtlich der Vervielfältigung des Werkes grundsätzlich die Schranken der $\iint 44 a, 53,55 a$ UrhG in Betracht. Das Recht der öffentlichen Zugänglichmachung könnte dagegen durch die Schranke des \52a UrhG beschränkt sein.

i. Schranke des $₫ 44 \mathrm{a}$ UrhG

Zunächst könnte hinsichtlich der Vervielfältigung der urheber- oder leistungsrechtlich geschützten Daten die Schranke des $\int 44 a$ UrhG greifen. Diese bezieht sich ihrem Wortlaut nach jedoch nur auf vorübergehende Vervielfältigungshandlungen, die flüchtig oder begleitend sind. Sinn und Zweck der Vorschrift ist es, nicht jeden technischen Vervielfältigungsvorgang, der im Wege der Onlineübermittlung geschützter Werke in Zwischenspeichern oder Routern erforderlich ist, 
Kapitel 1 - Urheberrechtliche Problematik

unter das Vervielfältigungsrecht des Urhebers fallen zu lassen. ${ }^{126} \mathrm{Im}$ Rahmen der digitalen Langzeitarchivierung wird die zu archivierende Datei beim Speichern auf dem Datenträger jedoch gerade dauerhaft vervielfältigt. ${ }^{127}$ Dabei können sicherlich auch flüchtige Vervielfältigungen auftreten, so etwa, wenn die Datei von einem Mitarbeiter der archivierenden Einrichtung mittels eines internen Netzwerkes eingesehen und in diesem Rahmen in dem Arbeitsspeicher seines Computers zwischengespeichert wird. Hinsichtlich dieser Vervielfältigungen könnte \44a UrhG somit durchaus greifen. Die im Mittelpunkt dieser Betrachtung stehende Speicherung der zu archivierenden Datei auf einem anderen Speichermedium ist indes von dauerhafter Natur, weshalb \44a UrhG diesbezüglich keine Anwendung findet.

\section{ii. Schranke des $\int 53 \mathrm{UrhG}$}

In Betracht kommt ferner die Schranke des $\int 53$ UrhG. Dabei scheidet Abs. 1 der Vorschrift von vornherein aus, da er seinem Wortlaut nach lediglich auf Vervielfältigungen zum privaten Gebrauch durch eine natürliche Person abstellt. Ein privater Gebrauch liegt jedoch nur dann vor, wenn das Vervielfältigungsstück von der Person, welche es herstellt oder herstellen lässt, zur Befriedigung rein persönlicher Bedürfnissen, also zu außerberuflichen und außererwerbswirtschaftlichen Zwecken gebraucht wird. ${ }^{128}$ Sofern hier überhaupt die Vervielfältigungshandlung einer natürlichen Person vorliegt und nicht einer juristischen Person, wird die vervielfältigte Datei gerade nicht zu außerberuflichen Zwecken, sondern im Rahmen der beruflichen Tätigkeit des Wissenschaftlers benötigt. Es fehlt also an der Tatbestandsvoraussetzung des privaten Gebrauchs.

\section{(1) $\int 53 A b s .2 N r .1 U r b G$}

Allerdings könnte $\int 53$ Abs. 2 UrhG Anwendung finden. Gem. \53 Abs. 2 Nr.1 UrhG ist es zulässig, einzelne Vervielfältigungsstücke zum eigenen wissenschaftlichen Gebrauch herzustellen, oder herstellen zu lassen, soweit die Vervielfältigung zu diesem Zweck geboten ist. Dabei ist das Merkmal des wissenschaftlichen Gebrauchs, d.h. einem methodisch-systematisch ausgerichteten Streben nach Erkenntnis, ${ }^{129}$ in den vorliegend relevanten Fällen unproblematisch gegeben, bezieht sich die vorliegende Untersuchung doch ausschließlich auf wissenschaftliche Pri-

${ }^{126}$ Dreier in Dreier/Schulze, \44a Rn. 1; Loewenheim in Schricker/Loewenheim, \44a Rn. 1; Dreyer in Dreyer/Kotthoff/Meckel, $₫ 44 a$ Rn. 5.

127 Siehe dazu auch die Ausführungen auf S. 23 ff.

128 BGH GRUR 1997, 459, 461 - CB-infobank I; 1981, 355, 358 - Video-Recorder; 1978, 474, 475 Vervielfältigungsstücke (zum früheren Begriff des persönlichen Gebrauchs, der aber mit dem Begriff des privaten Gebrauchs gleichgesetzt wird); siehe auch BT-Drucks. IV/270, S. 70; Loewenheim in Loewenheim, $₫ 31$ Rn. 26; ders. in Schricker/Loewenheim, $\ 53$ Rn. 14; Dreyer in Dreyer/Kotthoff/Meckel, \53 Rn. 15.

${ }^{129}$ Loewenheim in Schricker/Loewenheim, $\ 53$ Rn. 40; Dreyer in Dreyer/Kotthoff/Meckel, $\ 53$ Rn. 51; Lüft in Wandtke/Bullinger, \53 Rn. 25. 
märdaten. Jedoch ist zweifelhaft, ob das Vervielfältigungsstück bei der elektronischen Langzeitarchivierung zum „eigenen“ wissenschaftlichen Gebrauch hergestellt wird.

Wie oben bereits dargestellt, sollen die Daten nicht lediglich archiviert werden, damit anschließend ausschließlich die jeweilige Forschungsstelle darauf zugreifen kann. Vielmehr wird die archivierende Einrichtung in der Regel einen Datenaustausch mit anderen Wissenschaftlern und Forschungseinrichtungen auf nationaler und internationaler Ebene anstreben. Die Schranke des $\int 53$ Abs. 2 Satz 1 Nr. 1 UrhG greift aber nicht, sofern die Vervielfältigungsstücke nicht ausschließlich für den internen Gebrauch hergestellt werden. ${ }^{130}$ Demzufolge sind Vervielfältigungen im Rahmen der digitalen Langzeitarchivierung zumindest dann nicht nach \53 Abs. 2 Nr.1 UrhG zulässig, wenn die Daten auch anderen Wissenschaftlern zur Verfügung gestellt werden sollen. Ein anderes Ergebnis ergibt sich hingegen, sofern die Daten lediglich für den internen Gebrauch archiviert werden sollen. Dabei wird man einen solchen aber schon dann ablehnen müssen, wenn die Daten nicht nur für die Wissenschaftler des Instituts zur Verfügung stehen sollen, welches sie archiviert, sondern beispielsweise für alle Wissenschaftler der Universität, der das Institut angehört. ${ }^{131}$

\section{(2) $\int 53 A b s .2 N r .2 U r b G$}

Des Weiteren kommt die Schranke des $\int 53$ Abs. 2 Nr. 2 UrhG in Betracht. Danach ist die Herstellung einzelner Vervielfältigungsstücke zur Aufnahme in ein eigenes Archiv zulässig, wenn und soweit die Vervielfältigung zu diesem Zweck geboten ist und als Vorlage für die Vervielfältigung ein eigenes Werkstück benutzt wird. Dabei ist jedoch zu beachten, dass die besagte Vorschrift gem. \53 Abs. 5 Satz 1 UrhG keine Anwendung auf Datenbankwerke findet, deren Elemente einzeln mit Hilfe elektronischer Mittel zugänglich sind. ${ }^{132}$ Sofern es sich also bei den archivierten Dateien um Datenbankwerke handelt, sind deren Elemente mit Hilfe elektronischer Hilfsmittel zugänglich. Damit findet 53 Abs. 2 Satz 1 Nr. 2 UrhG auf diese aber von vornherein keine Anwendung. Zu prüfen bleibt demnach nur, ob die Schranke auf Lichtbilder wie etwa Röntgenbilder Anwendung findet, die archiviert werden sollen.

Dabei kommt es zunächst darauf an, ob es sich bei dem Langzeitarchiv, in das die Daten aufgenommen werden sollen, um ein Archiv im Sinne des $\int 53$ Abs. 2 Satz 1 Nr. 2 UrhG handelt. Darunter versteht man eine nach sachlichen Gesichtspunkten vorgenommene Sammlung und Aufbewahrung von Geistesgut jeglicher

\footnotetext{
${ }^{130}$ Loewenheim in Loewenheim, \ 31 Rn. 26; ders. in Schricker/Loewenheim, \53 Rn. 41; Lüft in Wandtke/Bullinger, \53 Rn. 24; Dreyer in Dreyer/Kotthoff/Meckel, \ 53 Rn. 39; siehe auch BTDrucks. 10/837, S.9.

131 Vgl. BGH GRUR 1997, 459, 461 - CB-infobank I; Lüft in Wandtke/Bullinger, \ 53 Rn. 24;

Loewenheim in Schricker/Loewenheim, $\int 53$ Rn. 41.

132 Siehe dazu auch Euler, AfP 2005, 474, 477.
} 
Kapitel 1 - Urheberrechtliche Problematik

Art. ${ }^{133}$ Sofern wissenschaftliche Primärdaten archiviert werden, stellt dies eine solche Sammlung von Geistesgut dar, die auch nach sachlichen Gesichtspunkten erfolgt. Demzufolge handelt es sich bei einem solchen elektronischen Archiv um ein solches im Sinne des $\int 53$ Abs. 2 Satz 1 Nr. 2 UrhG. Wie aber auch schon im Rahmen von $\int 53$ Abs. 2 Satz 1 Nr. 1 UrhG ist auch hier wieder problematisch, dass es sich um ein „eigenes“ Archiv handeln muss. Dies ist aber wiederum nur dann der Fall, wenn es sich um ein betriebsinternes Archiv handelt, da ein Archiv, das auch Dritte benutzen können, nicht unter die Schranke des $\int 53$ Abs. 2 Satz 1 Nr. 2 fällt. ${ }^{134}$ Sofern die archivierten Daten also auch außenstehenden Wissenschaftlern und Forschungseinrichtungen zur Verfügung gestellt werden, handelt es sich bei dem Langzeitarchiv demnach nicht mehr um ein „eigenes“ Archiv, so dass die Schranke des $\int 53$ Abs. 2 Satz 1 Nr. 2 UrhG in den meisten Fällen keine Anwendung finden wird. Etwas anderes könnte sich hingegen dann ergeben, wenn die Daten ausschließlich den Mitarbeitern einer einzelnen Forschungsstelle zur Verfügung stünden und damit ein betriebsinternes Archiv vorläge. Dazu müssten jedoch auch die übrigen Tatbestandsvoraussetzungen der Vorschrift vorliegen.

Nach dem Wortlaut der Norm müsste für die Archivierung zum einen ein ,eigenes Werkstück“ verwendet werden. Dies wäre im Regelfall wohl noch gegeben, da sich die als Vorlage dienenden Werkstücke, wie beispielsweise Röntgenbilder, regelmäßig im Eigentum der archivierenden Einrichtung befinden werden.

Darüber hinaus müsste die Archivierung auch geboten sein. ${ }^{135}$ In der Gesetzesbegründung wurde dafür als Beispiel die Aufnahme eines Bibliothekenbestandes auf Mikrofilm genannt, um Platz zu sparen. ${ }^{136}$ Die Archivierung darf nach der Begründung aber beispielsweise nicht dazu dienen, den Bibliotheksbestand zu erweitern. ${ }^{137}$ Der deutsche Gesetzgeber hat insoweit also keinen Gebrauch von der Möglichkeit des Art. 5 Abs. 2 c) der Europäischen Richtlinie zur Harmonisierung des Urheberrechts gemacht. Sofern erhobene Primärdaten archiviert werden, soll dies zur langfristigen Sicherstellung dieser Daten für Forschung und Wissenschaft dienen, womit klassische Archivierungszwecke verfolgt würden. Solange die Archive also nicht zu gewerblichen Zwecken unter Verfolgung kommerzieller

133 BGH GRUR 1999, 324, 327 - Elektronische Pressespiegel; 1997, 459, 461 - CB-infobank I;

Dreier in Dreier/Schulze, \53 Rn. 27; Loewenheim in Schricker/Loewenheim, \53 Rn. 45; ders. in Loewenheim, \31 Rn. 39.

134 BGH GRUR 1997, 459, 461 - CB-infobank I; LG Hamburg CR 1996, 734, 735; Dreier in Dreier/Schulze, \53 Rn. 27; Dreyer in Dreyer/Kotthoff/Meckel, \53 Rn. 60; Loewenheim in Schricker/Loewenheim, \53 Rn. 45; Lüft in Wandtke/Bullinger, \53 Rn. 30; Euler, AfP 2008, 474, 477.

135 Euler, AfP 2008, 474, 477.

136 BT-Drucks. IV/270 S. 73; Euler, AfP 2008, 474, 477.

${ }_{137}$ BT-Drucks. IV/270 S. 73; Euler, AfP 2008, 474, 477. 
Ziele angelegt werden, dürfte die Vervielfältigung auch geboten sein.138 Somit würde die Schranke des $\int 53$ Abs. 2 Nr. 2 UrhG zumindest hinsichtlich der Vervielfältigung von Lichtbildern greifen, sofern das Archiv nur zu betriebsinternen Zwecken genutzt würde. Da dies in der Regel aber nicht der Fall sein wird, sondern die Daten gerade auch anderen Wissenschaftlern zugänglich gemacht werden sollen, wird $\int 53$ Abs. 2 Nr. 2 UrhG in den meisten Fällen der elektronischen Langzeitarchivierung nicht als Schranke für die Vervielfältigung von Röntgenbildern oder anderen wissenschaftlichen Lichtbildern in Betracht kommen.

\section{iii. Dauer des urheberrechtlichen Schutzes}

Hinsichtlich des urheberrechtlichen Schutzes ist jedoch ferner zu beachten, dass dieser zeitlich begrenzt ist, da das Urheberrecht gem. \64 UrhG siebzig Jahre nach dem Tod des Urhebers erlischt. Dabei beginnt die Frist gem. $\int 69$ UrhG mit Ablauf des Jahres, in dem der Urheber verstirbt. Sofern also ausnahmsweise ein urheberrechtlich geschütztes Werk etwa in Form eines Datenbankwerkes vorliegt und die Universität nicht Inhaberin der Nutzungsrechte ist, sollte im Einzelfall geprüft werden, ob der Urheber nicht bereits länger als siebzig Jahre verstorben ist. Sofern dies der Fall ist, könnte das Werk auch ohne Einholung von Nutzungsrechten und ohne das Vorliegen einer Schranke archiviert werden.

iv. Beschränkung des leistungsrechtlichen Schutzes des Datenbankherstellers

Das Leistungsschutzrecht des Datenbankherstellers wird ebenfalls gesetzlich beschränkt. Dabei sind die Schranken der \$S 45 ff. UrhG auf dessen Rechte nicht anwendbar, da diese sich ausschließlich auf das Urheberrecht und nicht auf verwandte Schutzrechte beziehen. ${ }^{139}$ Vielmehr sieht das Gesetz in $\int 87 \mathrm{c}$ UrhG spezielle Schranken für die Vervielfältigung eines nach Art oder Umfang wesentlichen Teils einer Datenbank unter bestimmte Voraussetzungen als zulässig an. Darüber hinaus werden die Rechte des Datenbankherstellers durch \87d UrhG zeitlich begrenzt. Sofern nicht die archivierende Einrichtung nicht selbst Datenbankherstellerin ist, wäre somit zu prüfen, ob es der Zustimmung des Datenbankherstellers zur Archivierung überhaupt bedarf oder ob seine Rechte nicht einer der Schranken des $\int 87 \mathrm{c}$ UrhG unterliegen oder gem. $\int 87 \mathrm{~d}$ UrhG bereits erloschen sind.

Dabei greift die Schranke des $\int 87 c$ UrhG jedoch nicht bei allen Datenbanken. Vielmehr folgt aus Art. 9 der Datenbankrichtlinie im Rahmen einer richtlinienkonformen Auslegung von $\int 87$ c UrhG, dass die dort aufgeführten Schranken nur auf bereits der Öffentlichkeit zur Verfügung gestellten Datenbanken Anwendung

138 Vgl. Dreyer in Dreyer/Kotthoff/Meckel, \ 53 Rn. 54; Loewenheim in Schricker/Loewenheim, \ 53 Rn. 46; Lüft in Wandtke/Bullinger, $\int 53$ Rn. $28 \mathrm{f}$.

139 OLG Dresden ZUM 2001, 595, 597; Kotthoff in Dreyer/Kotthoff/Meckel, \87c Rn. 1; Caychows$k i$ in Fromm/Nordemann, $\int 87 c$ Rn. 18; Dreier in Dreier/Schulze, $\int 87 c$ Rn. 1; Vogel in Schricker/Loewenheim, $\int 87 \mathrm{c}$ Rn. 1. 
Kapitel 1 - Urheberrechtliche Problematik

finden. ${ }^{140}$ Demzufolge beziehen sich die nachfolgenden Ausführungen nur auf solche Datenbanken, die vor ihrer Archivierung bereits öffentlich zugänglich gemacht worden sind. Dabei folgt aus $\int 87 \mathrm{~b}$ UrhG, dass eine solche öffentliche Zurverfügungstellung nur durch den Datenbankhersteller bzw. nur mit dessen Genehmigung erfolgen kann. ${ }^{141}$ Insofern stellt sich zunächst die Frage, was unter einer öffentlichen Zurverfügungstellung zu verstehen ist, wobei ein Rückgriff auf den Öffentlichkeitsbegriff des $\int 6$ UrhG möglich ist. ${ }^{142}$ Danach wäre die Datenbank der Öffentlichkeit zugänglich gemacht, wenn vom Datenbankhersteller die Möglichkeit für die Allgemeinheit geschaffen wurde, das Werk wahrzunehmen. ${ }^{143}$ Demzufolge müsste im Einzelfall jeweils geprüft werden, ob dies auf die zur Archivierung vorgesehene Datenbank zutrifft. Nur wenn dies der Fall ist, kommt überhaupt die Anwendbarkeit der Schranken des $\int$ 87c Abs.1 Nr.1-3 UrhG in Betracht.

\section{(1) Schranke des $\int 87 c$ Abs.1 Nr. 1 UrbG}

Gem. $\int 87$ c Abs. 1 Nr. 1 UrhG ist eine Vervielfältigung eines nach Art oder Umfang wesentlichen Teils einer Datenbank zum privaten Gebrauch zulässig. Dies gilt gem. $\int 87 c$ Abs. 1 Nr. 1 Halbsatz 2 UrhG nicht für eine Datenbank, deren Elemente einzeln mit Hilfe elektronischer Mittel zugänglich sind. Danach findet die Schranke ihrem Wortlaut nach schon keine Anwendung auf die hier relevanten Fälle, da es sich in der Regel um Datenbanken handeln wird, auf die mit Hilfe elektronischer Mittel zugegriffen wird. Daneben handelt es sich bei der Langzeitarchivierung durch die archivierende Einrichtung auch nicht um einen privaten Gebrauch, da ein solcher nur dann vorliegt, wenn das Vervielfältigungsstück von der Person, die es herstellt oder herstellen lässt, zu außerberuflichen und außererwerbswirtschaftlichen Zwecken, also nur der Befriedigung rein persönlicher Interessen gebraucht werden soll. ${ }^{144}$ Dies ist bei einer Langzeitarchivierung im universitären Bereich allerdings gerade nicht der Fall. ${ }^{145}$

\footnotetext{
${ }^{140}$ Loewenheim in Loewenheim, $\int 43$ Rn. 22; Dreier in Dreier/Schulze, $\int 87 c$ Rn. 4; Kotthoff in

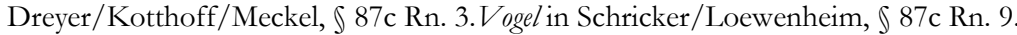

141 EuGH GRUR 2005, 244, 249 Tz. 62; Czychowski in Fromm/Nordemann, J 87c Rn. 3; Dreier in

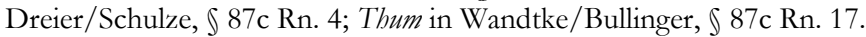

142 Czychowski in Fromm/Nordemann, $\int$ 87c Rn. 2; Leistner, Der Rechtsschutz von Datenbanken im deutschen und europäischen Recht, 2000, S. 198, 202 Fn. 948; Kotthoff in Dreyer/Kotthoff/Meckel, s 87c Rn. 3;

143 LG Frankfurt GRUR 1987, 168, 169 - Krankheiten auf Rezept; KG NJW 1995, 3392, 3393 Botho Strauß; OLG Frankfurt ZUM 1996, 697, 701 - Yellow Submarine; Marquardt in Wandtke/Bullinger, $\int 6$ Rn. 5; siehe zum Begriff der Öffentlichkeit auch $W$. Nordemann in Fromm/Nordemann, $\int 6$ Rn. 10.

144 BGH GRUR 1981, 355, 358 - Video-Recorder; Dreier in Dreier/Schulze, \ 87c Rn. 6 mit Verweis auf $\int 53$ Rn. 7; Dreyer in Dreyer/Kotthoff/Meckel, \53 Rn. 15; Kotthoff in

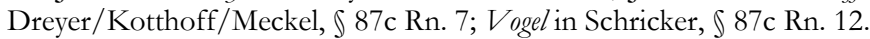

145 Siehe dazu auch die Ausführungen auf S.36.
} 


\section{(2) Schranke des $\int 87 c$ Abs. 1 Nr. 2 UrbG}

Des Weiteren kommt die Schranke des $\int 87$ c Abs. 1 Nr. 2 UrhG in Betracht. Danach ist eine Vervielfältigung zum eigenen wissenschaftlichen Gebrauch zulässig, wenn und soweit die Vervielfältigung zu diesem Zweck geboten ist und der wissenschaftliche Gebrauch nicht zu gewerblichen Zwecken erfolgt.

Dabei liegt ein wissenschaftlicher Gebrauch bei einem methodischsystematischen Streben nach Erkenntnis vor. ${ }^{146}$ Wie oben bereits dargestellt, dient die Archivierung der hier behandelten Datenbanken, dem methodischsystematischen Streben nach Erkenntnis, ist doch Sinn und Zweck der Langzeitarchivierung, die Daten für die weitere Forschung bereit zu halten und darauf auch in Zukunft zugreifen zu können. ${ }^{147}$ Demnach läge das Merkmal des wissenschaftlichen Gebrauchs unproblematisch vor. Allerdings verlangt $\int 87 \mathrm{c}$ Abs. $1 \mathrm{Nr}$. 2 UrhG zusätzlich, dass die Vervielfältigung zum ,eigenen wissenschaftlichen Gebrauch“ erfolgt. Dies ist der Fall, wenn die Vervielfältigung innerhalb einer geschlossenen Einheit stattfindet und keine Weitergabe an Dritte beabsichtigt ist. ${ }^{148}$ Somit würde $\int 87$ c Abs. 1 Nr.2 UrhG, wie auch \53 Abs. 2 Nr. 2 UrhG, nur dann greifen, wenn die archivierende Einrichtung nicht beabsichtigt, die Datenbank nach der Archivierung Außenstehenden zugänglich zu machen.

Schließlich müsste die Vervielfältigung der zu archivierenden Datenbank nach dem Wortlaut des $\int 87$ c Abs.1 Nr.2 UrhG auch geboten sein, wobei die Formulierung mit der in $\int 53$ UrhG übereinstimmt, weshalb auf die diesbezüglichen Literaturansichten zurückgegriffen werden kann. ${ }^{149}$ Geboten wäre die Vervielfältigung zum Zwecke der Archivierung demnach, wenn die wissenschaftliche Arbeit dies erfordert und der Erwerb oder die Ausleihe des Exemplars unzumutbar erscheint oder nicht problemlos möglich wäre. ${ }^{150}$ Die hier in Frage stehenden Datenbanken sind zum einen für die wissenschaftliche Arbeit der archivierenden Einrichtung erforderlich, da sich auf der Grundlage dieser Daten oftmals erst neue Erkenntnisse, sei es im naturwissenschaftlichen oder auch geisteswissenschaftlichen Bereich, ziehen lassen und eine langfristige Archivierung dieser Daten damit unumgänglich erscheint. Des Weiteren ist es Forschungseinrichtungen gerade in Zeiten knapper Budgets und angespannter Haushaltslagen, insbesondere unter Berücksichtigung der in Art. 5 Abs. 3 GG verankerten Wissenschaftsfreiheit nicht zu-

\footnotetext{
146 Dreyer in Dreyer/Kotthoff/Meckel, $\int 53$ Rn. 51; W. Nordemann in Fromm/Nordemann, $\ 53$ Rn. 19; Loewenheim in Schricker/Loewenheim, \53 Rn. 40.

${ }^{147}$ Vgl. oben S. 36

148 BGHZ 134, 250, 257 f. - CB Infobank I; Decker in Möhring/Niccolini, \53 Rn. 18; Dreier in Dreier/Schulze, $\int 87$ c Rn. 9; W. Nordemann in Fromm/Nordemann, SS 53 Rn. 16; siehe auch BT-Drucks. 10/837 S.9.

${ }^{149}$ Crychowski in Fromm/Nordemann, $\int 87 c$ Rn. 8; Kotthoff in Dreyer/Kotthoff/Meckel, $\int 87 c$ Rn. 8; Vogel in Schricker/Loewenheim, $\int 87 c$ Rn. 14; Dreier in Dreier/Schulze, $\int 87 c$ Rn. 10; Decker in Möhring/Niccolini, $₫ 87 \mathrm{c}$ Rn. 4.

150 Dreier in Dreier/Schulze, $\int 53$ Rn. 23; Loewenheim in Schricker/Loewenheim, $\ 53$ Rn. 42; Lüft in

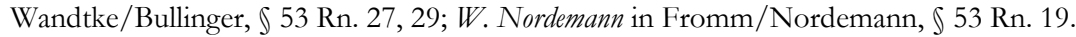


mutbar und darüber hinaus wohl oftmals auch nicht möglich, die entsprechenden Datenbanken bzw. die erforderlichen Rechte käuflich zu erwerben. Die Archivierung von Datenbanken wäre in den hier relevanten Fällen demnach grundsätzlich auch geboten. Sofern die archivierte Datenbank also nicht außenstehenden zugänglich und auch sonst kein gewerblicher Gebrauch von ihr gemacht werden soll, wäre die Vervielfältigung im Rahmen der Archivierung durch $\int 87 \mathrm{c}$ Abs.1 Nr. 2 UrhG gerechtfertigt. Wie aber schon im Rahmen von $₫ 53$ Abs. 2 Nr. 1 UrhG angemerkt, wird dies in den meisten Fällen nicht zutreffen, weshalb die Schrankenregelung im Rahmen der elektronischen Langzeitarchivierung grundsätzlich nicht greifen wird.

\section{(3) Schranke des $\int 87 c$ Abs.1 Nr.3}

Ferner könnte das Leistungsschutzrecht des Datenbankherstellers in den vorliegenden Fällen durch $\int 87 \mathrm{c}$ Abs. 1 Nr. 3 UrhG beschränkt werden. Danach ist die Vervielfältigung für die Benutzung zur Veranschaulichung des Unterrichts zulässig, sofern sie nicht zu gewerblichen Zwecken erfolgt. Da die Vervielfältigungen im Rahmen einer Langzeitarchivierung nicht oder zumindest nicht ausschließlich der Benutzung im Unterricht bzw. in Vorlesungen dienen, kommt die Schranke des $\int 87$ c Abs. 1 Nr. 3 UrhG im Rahmen der Archivierung von Datenbanken grundsätzlich nicht in Betracht. Aus diesem Grund braucht hier auch noch nicht auf die Frage eingegangen werden, ob von $\ 87$ c Abs. 1 Nr. 3 UrhG auch Vorlesungen an Hochschulen erfasst sind. ${ }^{151}$

\section{(4) Zwischenergebnis}

Sofern nicht die archivierende Einrichtung selbst Datenbankherstellerin ist, kommt als Schranke für eine Vervielfältigung, wie sie im Rahmen der Langzeitarchivierung zur Speicherung der Datenbank auf dem Datenträger erforderlich ist, lediglich die Schranke des $₫ 87$ c Abs. 1 Nr. 2 in Betracht. Dies gilt aber nur unter der Voraussetzung, dass die betreffende Datenbank zuvor bereits der Öffentlichkeit zugänglich gemacht worden ist. Des Weiteren greift die Schranke nur dann, wenn die archivierende Einrichtung nicht beabsichtigt, die Datenbank nach der Archivierung Außenstehenden zugänglich zu machen, da ansonsten kein eigener Gebrauch vorläge. Dies wird aber grundsätzlich nicht der Fall sein, da die Daten auch Fremdforschern zur Verfügung gestellt werden sollen, so dass die Schranke des $\int 87 \mathrm{c}$ Abs.1 Nr. 2 UrhG theoretisch zwar in Betracht kommt, praktisch jedoch im Rahmen der elektronischen Langezeitarchivierung von Datenbanken keine große Rolle spielen wird.

151 Siehe dazu unten S. 106. 


\section{(5) Wesentliche Teile der Datenbank}

$\mathrm{Zu}$ beachten ist indes, dass die Schranke des $₫ 87$ c Abs. 1 Nr. 2 UrhG nach dem klaren Wortlaut der Vorschrift nicht zur Vervielfältigung der gesamten Datenbank berechtigt. Vielmehr ist expressis verbis nur die Vervielfältigung eines „nach Art und Umfang wesentlichen Teils der Datenbank zulässig“. Dabei ist umstritten, ob sich die Schranke auch auf die gesamte Datenbank bezieht oder nur auf wesentliche Teile, wie der Wortlaut zunächst vermuten lässt. In diesem Zusammenhang ist zunächst festzuhalten, dass unwesentliche Teile der Datenbank ohnehin erlaubnisfrei genutzt werden dürfen, da $\int 87 \mathrm{~b}$ Abs. 1 Satz 1 UrhG dem Datenbankhersteller lediglich das ausschließliche Recht der Vervielfältigung, Verbreitung oder öffentlichen Wiedergabe der Datenbank insgesamt oder einen nach Art oder Umfang wesentlichen Teil der Datenbank einräumt, unwesentliche Teile der Datenbank jedoch außen vor lässt. ${ }^{152}$ Eine Ausnahme gilt gem. \87b Abs. 1 S. 2 UrhG lediglich dann, wenn unwesentliche Teile wiederholt und systematisch vervielfältigt, verbreitet oder öffentlich wiedergegeben werden und diese Handlungen einer normalen Auswertung der Datenbank zuwiderlaufen oder die berechtigten Interessen des Datenbankherstellers unzumutbar beeinträchtigen. ${ }^{153}$ In diesen Fällen findet darüber hinaus die Vorschrift des $\int 87 \mathrm{c}$ UrhG keine Anwendung. ${ }^{154}$

Teilweise wird die Ansicht vertreten, dass die Schranke des \87c UrhG auch bei einer Vervielfältigung der gesamten Datenbank Anwendung finden würde. Als Begründung wird angeführt, die Formulierung in Art. 9 der Datenbankrichtlinie, die durch $\int 87 \mathrm{c}$ in nationales Recht umgesetzt wurde, diene lediglich einer Klarstellung, da eine frühere Fassung der Richtlinie lediglich Schranken in Bezug auf unwesentliche Teile von Datenbanken gewährte. ${ }^{155}$ Dem kann jedoch nicht gefolgt werden. Gegen eine solche Annahme spricht nämlich, dass die Datenbankrichtlinie in allen sonstigen Vorschriften auch die „Gesamtheit“ erwähnt und nur in Art. 9 lediglich von dem ,wesentlichen Teil“ der Datenbank spricht. Auch der effektive Schutz des Datenbankherstellers, der durch Art. 9 erreicht werden soll, spricht gegen eine Ausdehnung der Schranke auf die gesamte Datenbank. ${ }^{156}$

152 Vogel in Schricker/Loewenheim, \87c Rn. 2; Decker in Möhring/Niccolini, \87c Rn. 1; Dreier in Dreier/Schulze, \87b Rn. 9; Loewenheim in Loewenheim, \43 Rn. 22; Kotthoff in

Dreyer/Kotthoff/Meckel, $\int 87 c$ Rn. 4; Thum in Wandtke/Bullinger, $\int 87 c$ Rn. 10; siehe auch bereits oben S. 25 sowie die Nachweise in Fn. 82.

153 Siehe dazu S. $27 \mathrm{f}$

154 Vogel in Schricker/Loewenheim, \87c Rn. 10.

155 Siehe Art. 8 Abs. 4 und 5 des 1. Richtlinienvorschlags, KOM (92) 24 endg. - SYN 393 v. 13.5.1992 sowie Art. 11 Abs. 5 und 6 des 2. Richtlinienvorschlags, KOM (93) 464 endg. - SYN 393 v. 4.10.1993; Decker in Möhring Niccolini, der seine Ansicht allerdings nicht begründet; Raue/Bensinger, MMR 1998, 507, 512.

156 So auch Leistner, S. 200 f.; ebenso Gaster, CR 1997, 669, 674; 717, 721; Dreier in Dreier/Schulze, $\mathbb{S}$ 87c Rn. 3; Vogel in Schricker/Loewenheim, \87c Rn. 9; Thum in Wandtke/Bullinger, $\int 87 c$ Rn. 11, der allerdings einräumt, dass es Ausnahmen geben kann, in denen sich die Schranke auf die gesamte Datenbank bezieht. Dies allerdings auch nur im Rahmen von $\int 87 \mathrm{c}$ Abs.2; im Ergebnis ebenso Caychowski in Fromm/Nordemann, $\int$ 87c Rn. 5. 
Demzufolge dürfte auch bei Eingreifen der Schranke des $₫ 87$ c UrhG nicht die komplette Datenbank archiviert werden. Dies bedürfte vielmehr der Genehmigung durch den Datenbankhersteller. Im Rahmen der Langzeitarchivierung besteht jedoch zugegebenermaßen ein Interesse der Universität daran, sämtliche Daten bzw. komplette Datenbanken zu archivieren. Da es allerdings in dem Fall, in dem der Datenbankhersteller die Archivierung nicht genehmigt, für die Universität wohl immer noch besser ist, wenigstens einen wesentlichen Teil der Datenbank zu archivieren, als von einer Archivierung der Datenbank ganz abzusehen, kann die Schranke des $₫ 87$ c UrhG in (wenn auch zugegebenermaßen wenigen) Ausnahmefällen für die Langzeitarchivierung der Datenbanken relevant werden. In diesen Fällen ist entscheidend, was unter einem „wesentlichen Teil“" zu verstehen ist bzw. ab wann die Datenbank als Gesamtheit betroffen ist. ${ }^{157}$ Dabei bietet die Datenbankrichtlinie selbst keine Anhaltspunkte für die Bestimmung des wesentlichen Teils. Grundsätzlich wird es jedoch wohl kaum ausreichend sein, wenn die Datenbank beispielsweise bis auf einen oder nur sehr wenige Datensätze vervielfältigt wird. In diesen Fällen würde genau genommen zwar nicht die gesamte Datenbank vervielfältigt werden. Würde man dies aber ausreichen lassen, liefe die Begrenzung der Schranke auf wesentliche Teile quasi ins Leere. Ab wann noch von einem wesentlichen Teil die Rede ist und ab wann die Schwelle zur Gesamtheit erreicht ist, lässt sich demzufolge nicht pauschal beantworten, sondern ist von Fall zu Fall anhand qualitativer als auch quantitativer Merkmale unter Berücksichtigung des Schutzzwecks von Art. 9 der Datenbankrichtlinie zu entscheiden.

\section{(6) Quellenangabe}

Schließlich ist zu beachten, dass auch bei Eingreifen der Schranke des $\ 87$ c Abs. 1 Nr.2 die ursprüngliche Quelle des archivierten Teils der Datenbank gem. $\int 87 \mathrm{c}$ Abs. 1 Satz 2 UrhG anzugeben ist.

\section{(7) Dauer des leistungsrechtlichen Schutzes}

Auch wenn für die Archivierung der jeweiligen Datenbank im konkreten Fall keine Schranke greift, kann die Nutzung durch die Universität auch ohne Zustimmung des Datenbankherstellers zulässig sein, wenn die Dauer des leistungsrechtlichen Schutzes abgelaufen ist. Die Rechte des Datenbankherstellers erlöschen gem. $\int 87 d$ Satz 1 UrhG fünfzehn Jahre nach der Veröffentlichung der Datenbank. Der Schutz erlischt ferner gem. \87d Satz 1 Alt. 2 UrhG fünfzehn Jahre nach der Herstellung der Datenbank, wenn diese nicht innerhalb dieser Frist veröffentlicht worden ist. Soll also eine Datenbank archiviert werden, für die im konkreten Fall keine der Schranken des $\ 87 \mathrm{c}$ UrhG greift, so ist in einem ersten Schritt zu prü-

${ }^{157}$ Zur Abgrenzung des wesentlichen vom unwesentlichen Teil einer Datenbank siehe ausführlich oben S. $25 \mathrm{ff}$. 
fen, ob diese Datenbank bereits veröffentlicht worden ist. Ist dies der Fall, kommt es ferner darauf an, ob seit dem Zeitpunkt der Veröffentlichung bereits fünfzehn Jahre vergangen sind. Sofern dies zutrifft, kann die Datenbank genutzt und damit auch archiviert und öffentlich zugänglich gemacht werden. Ist die Datenbank hingegen nicht veröffentlicht worden, so ist zu prüfen, ob seit der Herstellung der Datenbank bereits fünfzehn Jahre vergangen sind. Bei der Fristberechnung ist dabei jedoch zu beachten, dass sich die Berechnung der Frist gem. \87d Satz 2 UrhG nach der Vorschrift des $\ 69$ UrhG richtet. Dies hat zur Konsequenz, dass die Frist erst mit dem Ablauf des Kalenderjahres zu laufen beginnt, in dem das für den Beginn der Frist maßgebende Ereignis eingetreten ist. In den vorliegenden Fällen ist das also entweder die Veröffentlichung oder die Herstellung der Datenbank.

Ferner ist zu beachten, dass sich die Dauer des Schutzes von Datenbanken verlängert, die kontinuierlich ergänzt oder gepflegt werden, da gem. \87a Abs. 1 Satz 2 UrhG eine Datenbank, deren Inhalt nach Art oder Umfang wesentlich geändert wird, als neue Datenbank gilt, sofern diese Änderung eine nach Art oder Umfang wesentliche Investition erfordert. ${ }^{158}$ Sind also wesentliche Änderungen im Sinne des $₫ 87$ a UrhG vorgenommen worden, so beginnt jeweils mit Ablauf des Jahres, in dem die wesentliche Änderung vorgenommen wurde, für die neue Datenbank eine neue Frist zu laufen. In diesem Zusammenhang ist umstritten, ob die Gesamtheit der neuen Datenbank von dem Neubeginn der Schutzfrist erfasst is $\mathrm{t}^{159}$ oder nur diejenigen Teile, die neu hinzugefügt worden sind. ${ }^{160}$ Nach einer vermittelnden Ansicht soll hingegen die Gesamtheit der neuen Datenbank erfasst sein, bei der Beurteilung, ob ein wesentlicher Teil gem. \87b Abs.1 Satz 1 UrhG genutzt worden ist, jedoch nur auf die Teile der Datenbank abgestellt werden, die noch nicht länger als 15 Jahre veröffentlicht sind bzw. deren Herstellung noch nicht länger als 15 Jahre zurückliegt, sofern sie noch nicht veröffentlicht worden sind. ${ }^{161}$ Gegen die beiden letztgenannten Ansichten spricht jedoch zum einen, dass zwischen der ,alten“ Datenbank und der Erneuerung nicht immer eine klare Trennung möglich ist. Aus diesem Grund wird es im Einzelfall schwer zu beurteilen sein, ob von der jeweiligen Nutzung auch der neue Teil der Datenbank oder nur deren Ursprungsbestand genutzt wird. Zum anderen verlangt auch der Schutzzweck der $\iint 87$ a ff. UrhG vor dem Hintergrund der Datenbankrichtlinie, dass sich die 15-Jahresfrist auch auf den Altbestand der Datenbank bezieht. Sofern der Altbestand der Datenbank trotz der neuen Investitionen, die der Hersteller in sie getätigt hat, genutzt werden dürfte, weil die Frist des $\int 87 \mathrm{~d}$ UrhG für

\footnotetext{
${ }^{158}$ Dreier in Dreier/Schulze, \87d Rn. 6; vgl. auch Art. 10 Abs. 3 der Datenbankrichtlinie.

${ }_{159}$ So Decker in Möhring/Niccolini, \87d Rn. 5; Thum in Wandtke/Bullinger, \87d Rn. 12.

${ }^{160}$ So Gaster, Rechtsschutz von Datenbanken, Rn. 651 f.; Bensinger, Sui-generis Schutz für Datenban-

ken - Die EG-Datenbankrichtlinie vor dem Hintergrund des nordischen Rechts, 1999, S. 269.

161 Dreier in Dreier/Schulze, \87d Rn. 8; Vogel in Schricker/Loewenheim, \87d Rn. 7; Leistner, S.

213.
} 
diesen nicht neu beginnen würde, bestünde die Gefahr, dass sich die neu getätigten Investitionen des Datenbankherstellers nicht amortisieren würden. ${ }^{162}$ Denkbar ist dies etwa für den Fall, in dem die Änderung ihrer Art nach zwar wesentlich ist und auch nicht unerhebliche Investitionen erfordert, den Umfang der Datenbank jedoch nicht wesentlich vergrößert. In diesem Fall würden Nutzer dazu verleitet werden, die alte Version der Datenbank kostenlos zu nutzen und dafür auf die Änderung zu verzichten. Dies liefe aber dem Schutzgegenstand der \$S87a ff. UrhG zuwider, der gerade in dem Schutz der getätigten Investitionen auf die Erstellung und Erhaltung der Datenbank und der Amortisation dieser Investitionen besteht. Schließlich sprechen auch der Wortlaut von \87a Abs.1 Satz 2 UrhG sowie der von Art. 10 Abs. 3 der Datenbankrichtlinie für ein solches Ergebnis. Besagen diese doch gerade, dass eine ihrem Inhalt nach Art oder Umfang wesentlich geänderte Datenbank als neue Datenbank gilt. Daraus folgt, dass eine komplett neue Datenbank entsteht und nicht zu dem Altbestand lediglich ein neuer Teil hinzugefügt wird. Andernfalls hätte der Gesetzgeber den Wortlaut von $\ 87 \mathrm{a}$ Abs.1 Satz 2 UrhG dahingehend formulieren können, dass der geänderte oder neu hinzugefügte Teil den gleichen Schutz wie der Altbestand genieße. Aus den genannten Gründen ist deshalb davon auszugehen, dass die Schutzfrist des $\ 87 \mathrm{~d}$ UrhG hinsichtlich der gesamten Datenbank und nicht nur an den neu hinzugefügten oder geänderten Teilen neu beginnt. ${ }^{163}$

\section{v. Zwischenergebnis}

Hinsichtlich der Vervielfältigung von wissenschaftlichen Primärdaten zum Zwecke der digitalen Langzeitarchivierung kommt für urheberrechtlich geschützte Werke, wenn überhaupt, nur die Schranke des $₫ 53$ Abs. 2 Nr. 2 UrhG in Betracht. Diese wird gem. $\ 53$ Abs. 5 Nr. 2 UrhG jedoch kaum Anwendung finden, da in den meisten der - verhältnismäßig wenigen - Fälle, in denen überhaupt ein urheberrechtlicher Schutz an wissenschaftlichen Primärdaten entsteht, ${ }^{164}$ Datenbankwerke vorliegen, deren Elemente einzeln mit Hilfe elektronischer Mittel zugänglich sind. Auf diese ist die Schranke des $₫ 53$ Abs. 2 Nr. 2 UrhG gem. \53 Abs. $5 \mathrm{Nr}$. 2 UrhG jedoch nicht anwendbar. Somit bliebe die Anwendbarkeit der Schranke von vornherein auf Lichtbilder gem. \ 72 UrhG oder sonstige urheberrechtliche Werke beschränkt. Dabei wird eine Anwendbarkeit des $₫ 53$ Abs. 2 Satz 1 Nr. 2 UrhG auf diese Werke grundsätzlich daran scheitern, dass die archivierten Daten auch Dritten, nämlich Wissenschaftlern anderer Forschungseinrichtungen und Universitäten zugänglich gemacht und nicht nur für den internen Gebrauch archiviert werden sollen. Abschließend lässt sich damit festhalten, dass $\ 53$ Abs. 2 Satz 1 Nr. 2 UrhG auf die Vervielfältigung der vorliegend relevanten Primärdaten nur in seltenen Fällen anwendbar ist. Hinsichtlich des leistungsrechtlichen Schut-

162 Vgl. auch Haberstumpf, GRUR 2003, 14, $30 \mathrm{f}$.

163 So auch Thum in Wandtke/Bullinger, $\ 87$ d Rn. 12; Haberstumpf, GRUR 2003, 14, 31.

164 Siehe dazu oben S. 6 ff. 
zes des Datenbankherstellers ist zu beachten, dass hier nur die Schranke des $\int 87 \mathrm{c}$ Abs. 1 Nr. 2 UrhG in Betracht kommt. Diese wird jedoch in den meisten Fällen nicht greifen, verlangt sie doch, dass die Datenbank zuvor öffentlich zur Verfügung gestellt worden ist, was zumeist nicht der Fall sein wird. Selbst wenn die Schranke des $\int 87$ c Abs. 1 Nr. 2 UrhG aber greifen sollte, wäre danach auch nur die Vervielfältigung eines wesentlichen Teils und nicht der kompletten Datenbank gerechtfertigt. Da die archivierende Einrichtung aber regelmäßig eine vollständige Archivierung der Datenbank anstreben wird, käme die Schranke des \87c Abs. 1 Nr. 2 UrhG lediglich als Notlösung in Betracht.

b. Öffentlich-rechtliche Pflicht zur Nutzungsrechtsübertragung

Des Weiteren käme eine öffentlich-rechtliche Pflicht des Rechteinhabers zur Nutzungsrechtseinräumung in Betracht. Im Gegensatz zu den urheberrechtlichen Schranken müsste sich die öffentliche Einrichtung in diesen Fällen zwar die erforderlichen Nutzungsrechte erst einräumen lassen. Sofern jedoch diesbezüglich eine Pflicht des Rechteinhabers bestünde, könnte der Abschluss einer entsprechenden Vereinbarung gegebenenfalls gerichtlich durchgesetzt werden. Eine solche Ablieferungspflicht besteht beispielsweise im „Gesetz über die Deutsche Nationalbibliothek“" (DNBG) ${ }^{165}$. Dieses sieht jedoch nur Ablieferungspflichten gegenüber der Deutschen Nationalbibliothek vor. Eine vergleichbare Regelung, die eine Ablieferungspflicht gegenüber anderen Einrichtungen vorsieht, existiert hingegen nicht. Demzufolge besteht für die Inhaber von Nutzungsrechten an wissenschaftlichen Primärdaten zumindest keine öffentlich-rechtliche Pflicht, diese anderen Einrichtungen als der DNB einzuräumen.

c. Einräumung der Nutzungsrechte durch den Rechteinhaber

Sofern die archivierende Einrichtung nicht Inhaberin der erforderlichen Nutzungsrechte ist und keine der oben genannten Schrankenregelungen greift, bleibt ihr letztlich nur die Möglichkeit, sich die jeweiligen Rechte vom Rechteinhaber einräumen zu lassen. In Betracht kommt dabei zum einen eine Vereinbarung, Kraft derer sich der Rechteinhaber im Vorfeld verpflichtet, der Universität die zur Vervielfältigung, Bearbeitung und öffentlichen Zugänglichmachung erforderlichen Rechte an den noch herzustellenden Daten zu übertragen. ${ }^{166}$ Für bereits hergestellte Werke kann eine entsprechende Vereinbarung auch nachträglich geschlossen werden. Dabei jedoch bestünde zunächst das Problem, den Rechteinhaber ausfindig zu machen. Dies gilt insbesondere für urheber- oder leistungsrechtlich geschützte Daten, die bereits vor einem längeren Zeitraum erstellt wurden, da die Rechteinhaber zwischenzeitlich Arbeitgeber und Wohnort gewechselt haben können, so dass zu ihrem Auffinden ein unter Umständen hoher Rechercheaufwand

165 Vgl. zum DNBG Heckmann/Weber, AfP 2008, 269.

166 Vgl. Euler, AfP 2008, 474, 477. 
nötig ist. Dieser entfällt auch dann nicht, wenn der Rechteinhaber mittlerweile verstorben ist. Vielmehr sind die Rechte dann gem. \1922 BGB auf seine Rechtsnachfolger übergegangen, da das Urheberrecht gem. $\int 28$ Abs. 1 UrhG vererblich ist, so dass die Erben wiederum ausfindig gemacht werden müssten. Ist der jeweilige Rechteinhaber schließlich ausfindig gemacht worden, muss mit diesem eine Vereinbarung getroffen werden, die den Übergang der durch die Archivierung betroffenen Nutzungsrechte regelt. In diesem Zusammenhang stellt sich die Frage, auf welche Art die Nutzungsrechte durch den ihren Inhaber übertragen werden können.

\section{i. Rechteübertragung kraft ausdrücklicher Vereinbarung}

Das Urheberrecht sieht in $\$ 29$ UrhG vor, dass die Nutzungsrechte, die der Urheber an seinem Werk erlangt, übertragbar sind. Dabei hat der Urheber gem. \31 Abs. 1 Satz 2 UrhG die Möglichkeit, das Nutzungsrecht als ausschließliches oder einfaches Recht zu übertragen. Ferner kann er die Übertragung räumlich, zeitlich und inhaltlich beschränken.

\section{(1) Einräumung als ausschließliches oder einfaches Nutzungsrecht}

Räumt der Urheber dem Erwerber ein einfaches Nutzungsrecht ein, so ist dieser berechtigt, das Werk auf die ihm gestattete Art zu nutzen. Er kann jedoch nicht andere von der Nutzung ausschließen. ${ }^{167}$ Die Einräumung eines einfachen Nutzungsrechtes stellt damit lediglich eine positive Nutzungserlaubnis dar. Überträgt der Urheber dem Erwerber das Recht jedoch als ausschließliches Nutzungsrecht, so darf der Erwerber das Recht nicht nur auf die ihm erlaubte Art nutzen, sondern darüber hinaus andere (auch den Urheber selbst) von der Nutzung ausschlieBen. ${ }^{168}$ Des Weiteren darf der Erwerber eines ausschließlichen Nutzungsrechtes seinerseits Dritten einfache Nutzungsrechte einräumen. Für die vorliegend in Betracht kommenden Fälle würde der archivierenden Einrichtung bereits die Einräumung der benötigten Rechte als einfache Nutzungsrechte genügen. Zum einen ist der Einrichtung hauptsächlich daran gelegen, die Daten auf lange Sicht zu sichern und anderen Wissenschaftlern und Forschungsstellen zugänglich zu machen, so dass es für sie zunächst ohne Belang ist, ob auch andere die Daten urheberrechtlich verwerten dürfen. Zum anderen wird die archivierende Einrichtung die Rechte in der Regel auch selbst ausüben wollen und nicht Dritten eine Lizenz einräumen.

Wird ein Nutzungsrecht übertragen, so kann der Inhaber die Übertragung gem. \31 I 2 UrhG räumlich, zeitlich und inhaltlich beschränken.

\footnotetext{
167 Schricker/Loewenheim in Schricker/Loewenheim, \31 Rn. 15; Spautz in Möhring/Niccolini, \ 31 Rn. 39; Wandtke/Grunert in Wandtke/Bullinger, \31 Rn. 28.

168 J.B. Nordemann in Fromm/Nordemann, \ 31 Rn. 92; Kotthoff in Dreyer/Kotthoff/Meckel, \ 31

Rn. 104; Spautz in Möhring/Niccolini, \31 Rn. 39; Wandtke/Grunert in Wandtke/Bullinger, \ 31

Rn. 29.
} 


\section{(2) Räumliche Beschränkung}

Der Urheber kann dem Erwerber das Nutzungsrecht örtlich beschränkt übertragen. Der Erwerber darf das Werk dann zwar (als ausschließliches oder einfaches Recht) nutzen, jedoch nur an dem vom Urheber festgelegten Ort bzw. Staat. So kann der Urheber Nutzungsrechte an einem Werk beispielsweise mit der Beschränkung übertragen, dass die jeweilige Nutzung nur im Raum der EU, nicht jedoch darüber hinaus erlaubt ist. In Bezug auf die vorliegend relevanten Fälle könnte sich die archivierende Einrichtung somit die erforderlichen Nutzungsrechte räumlich beschränkt einräumen lassen, da sie die angestrebten Verwertungshandlungen wohl nur im Inland durchführen wird. Dies hätte den Vorteil, dass sich der Rechteinhaber unter Umständen schneller dazu bereit erklären würde, eine entsprechende Vereinbarung zu schließen. ${ }^{169}$

\section{(3) Zeitliche Beschränkung}

Der Rechteinhaber kann die Rechteübertragung ferner zeitlich beschränken. In diesem Fall wird das jeweilige Nutzungsrecht nur für den vereinbarten Zeitraum übertragen. Eine zeitlich beschränkte Nutzungsrechtseinräumung wäre für die archivierende Einrichtung hingegen nahezu wertlos, da gerade hinsichtlich des Rechts der öffentlichen Zugänglichmachung aber auch der Vervielfältigung im Rahmen der Migration, ein vorwiegendes Interesse der archivierenden Einrichtung an einer zeitlich unbeschränkten Rechteeinräumung besteht, um eine effektive Archivierung der Daten auf lange Sicht vornehmen zu können.

\section{(4) Inhaltliche Beschränkung}

Schließlich kann der Urheber das zu übertragende Nutzungsrecht auch inhaltlich beschränken. So kann er beispielsweise die Art und Weise des Vertriebswegs oder der Werkvermittlung festlegen. Diesbezüglich könnte man einem unschlüssigen Rechteinhaber eine Vereinbarung anbieten, wonach die Nutzungsrechte lediglich im Rahmen der Archivierung und des Austauschs mit anderen Wissenschaftlern und Forschungsinstituten, nicht jedoch zu kommerziellen Zwecken ausgeübt werden dürfen und ihn damit zu einer Rechteübertragung bewegen.

\section{ii. Rechteübertragung aufgrund eines Arbeitsverhältnisses}

Eine besondere Konstellation kann sich ergeben, wenn der Inhaber der Nutzungsrechte zur archivierenden Einrichtung in einem Angestelltenverhältnis steht. In diesen Fällen kann der Arbeitgeber die Nutzungsrechte zum einen durch eine ausdrückliche Rechteübertragungsklausel im Arbeitsvertrag erlangt haben (dazu

$169 \mathrm{Zu}$ berücksichtigen ist dabei jedoch, dass gerade bei dem Recht der öffentlichen Zugänglichmachung im Internet eine räumliche Beschränkung kaum möglich ist, da ein Werk, dass in Deutschland im Internet öffentlich zur Verfügung gestellt wird, weltweit heruntergeladen werden kann. 
Kapitel 1 - Urheberrechtliche Problematik

(1)). Sofern dies nicht der Fall ist, kommt eine stillschweigende Übertragung der Nutzungsrechte durch Abschluss des Arbeitsvertrages in Betracht (dazu (2)).

\section{(1) Ausdrückliche Rechteübertragungsklausel}

Sofern die archivierende Einrichtung in ihren Arbeitsverträgen entsprechende Rechteübertragungsklauseln eingefügt hat, nach denen der Arbeitnehmer ihr die zur Langzeitarchivierung und einer damit verbundenen öffentlichen Zugänglichmachung im Internet erforderlichen Nutzungsrechte an seinen Werken überträgt, wäre sie Inhaber dieser Rechte geworden. Da Arbeitsverträge grundsätzlich schriftlich abgeschlossen werden, wäre dabei auch grundsätzlich das Formerfordernis des \ 40 I 1 UrhG erfüllt, sofern man dessen Anwendung in diesen Fällen nicht ohnehin für nicht erforderlich erachtet, da der Arbeitnehmer bei Abschluss des Vertrags in der Regel weiß, welches Werk er erstellen wird. ${ }^{170}$

$\mathrm{Zu}$ beachten ist jedoch, dass gem. \31 Abs. 4 (alte Fassung) UrhG Rechte an Nutzungsarten, die zum Zeitpunkt des Vertragsschlusses noch nicht bekannt waren, nicht übertragen werden konnten. Dies könnte in den vorliegenden Fällen insbesondere für das Recht der öffentlichen Zugänglichmachung im Internet relevant sein. Dieses gilt nach überwiegender Ansicht in Literatur und Rechtsprechung seit 1995 als bekannt. ${ }^{171}$ Demzufolge wären Klauseln in Arbeitsverträgen, die vor 1995 geschlossen worden sind und durch die sich der Arbeitnehmer verpflichtet, der Forschungseinrichtung auch „unbekannte Nutzungsrechte an seinem Werk" einzuräumen, zumindest hinsichtlich der zu diesem Zeitpunkt unbekannten Nutzungsarten und damit auch dem Recht der öffentlichen Zugänglichmachung im Internet grundsätzlich unwirksam. Damit stellt sich die Frage, ob $\int 31$ IV a.F. UrhG auf angestellte Urheber überhaupt Anwendung findet. Zu beachten ist dabei, dass das Urheberrechtsgesetz gem. \ 132 Abs. 1 S. 1 mit Ausnahme der $\iint 42$ und 43 UrhG keine Anwendung auf Verträge findet, die vor dem 1.1.1966 geschlossen worden sind. Daraus folgt, dass die Anwendung von \ 31 IV a.F. UrhG auf solche Arbeitsverträge von vornherein ausgeschlossen ist, die vor diesem Zeitpunkt geschlossen wurden. In diesen Fällen wäre also eine stillschweigende Einräumung der Onlinerechte aufgrund des Arbeitsverhältnisses möglich, sofern das Onlinenutzungsrecht zur Erfüllung des Betriebszwecks der Forschungseinrichtung benötigt wird.

$170 \mathrm{Vgl}$. Dreier in Dreier/Schulze, \ 43 Rn. 19 m.w.N.

171 OLG Hamburg ZUM 2000, 870, 873; KG GRUR 2002, 252; Wandtke/ Grunert in Wandtke/Bullinger, \ 31 Rn. 57; Schack, Rn. 551; Hoeren, CR 1995, 710, 713; Ehrmann/Fischer, GRUR Int. 2008, 284, 286 (die für den rechtswissenschaftlichen Bereich allerdings von einer Bekanntheit seit 1993 ausgehen). 
(a) Meinungsstand hinsichtlich der Anwendbarkeit von $\int 31$ Abs. 4 a.F. UrhG auf Arbeitsverträge

Die Anwendbarkeit urheberrechtlicher Vorschriften im Arbeitsverhältnis wird grundsätzlich durch $\int 43$ UrhG geregelt, wonach die Vorschriften des 2. Unterabschnittes von Abschnitt 5 des ersten Teils des Urheberrechtsgesetzes auch anzuwenden sind, wenn der Urheber das Werk in Erfüllung seiner Verpflichtung aus einem Arbeits- oder Dienstverhältnis geschaffen hat, soweit sich aus dem Inhalt oder dem Wesen des Arbeits- oder Dienstverhältnisses nichts anderes ergibt. Die Vorschrift erfasst dabei sowohl Angestellte in privaten Unternehmen als auch Angestellte im öffentlichen Dienst. ${ }^{172}$

Die Vorschrift des \31 IV würde nach dem Wortlaut von \43 UrhG also keine Anwendung auf Vereinbarungen in Arbeitsverträgen finden, wenn sich dies aus dessen Wesen oder Inhalt ergäbe. Dies wird in der Literatur jedoch kontrovers beurteilt.

Nach Teilen der Literatur soll \ 31 IV a.F. UrhG auch im Arbeitsverhältnis uneingeschränkt gelten, so dass der Arbeitgeber sich nicht durch Abschluss des Arbeitsvertrages Nutzungsrechte einräumen lassen konnte, die zu diesem Zeitpunkt noch unbekannt waren. ${ }^{173}$ Sofern Arbeitsverträge also vor 1995 geschlossen wurden, wäre nach dieser Ansicht eine Klausel unwirksam, nach der Nutzungsrechte an den entstehenden Werken des Arbeitnehmers für noch unbekannte Nutzungsarten auf den Arbeitgeber übergehen sollen.

Andere Stimmen in der Literatur wollen hingegen \ 31 IV UrhG im Arbeitsverhältnis generell keine Anwendung finden lassen, da der Arbeitnehmerurheber aufgrund seines Lohnanspruches gegen den Arbeitgeber nicht des gleichen Schutzes bedürfe, wie ein freischaffender Urheber. ${ }^{174}$

Nach einer vermittelnden Ansicht ist dagegen stets auf den Einzelfall abzustellen, weshalb die Anwendbarkeit von \31 IV a.F. UrhG im Arbeitsverhältnis nicht generell bejaht oder abgelehnt werden könne. Vielmehr stellt \ $31 \mathrm{IV}$ a.F. UrhG nach dieser Ansicht zwar eine zwingende Norm des Urheberrechtsgesetzes dar, ${ }^{175}$ die jedoch vertraglich abbedungen werden konnte. ${ }^{176}$

\section{(b) Eigene Stellungnahme}

Bei der Beurteilung, ob \31 IV a.F. UrhG im Arbeitsverhältnis Anwendung finden soll, ist stets zu prüfen, ob der angestellte Urheber im Einzelfall des Schutzes des $\int 31 \mathrm{IV}$ a.F. UrhG bedarf oder nicht. Die genannten Ansichten vermögen

\footnotetext{
172 A. Nordemann in Fromm/Nordemann, $\int 43$ Rn. 12, 10.

173 Wandtke in Wandtke/Bullinger, \ 43 Rn. 67; ders., Die Rechte der Urheber und ausübenden

Künstler im Arbeits- und Dienstverhältnis, Berlin 1993, S. 79.

174 Mathis, Der Arbeitnehmer als Urheber, Frankfurt a.M. 1988, S. 125 f.

175 Vgl. BGH GRUR 1991, 133, 135; BGH GRUR 1988, 296, 298.

176 BGH GRUR 1991, 133,135 - Videozweitauswertung I; A. Nordemann in Fromm/Nordemann, $\int 43$ Rn. 46; Katzenberger, Elektronische Printmedien und Urheberrecht, Stuttgart 1996, S. 126 f.
} 
Kapitel 1 - Urheberrechtliche Problematik

hingegen nicht zu überzeugen, da sie zu pauschal sind und nicht die Besonderheiten des Einzelfalls berücksichtigen. Auf der einen Seite liefe nämlich eine ausnahmslose Anwendung von \ 31 IV a.F. UrhG in Arbeitsverhältnissen dem Sinn und Zweck der Vorschrift zuwider. Dieser besteht vor allem darin, dem Urheber die Entscheidung darüber vorzubehalten, ob bzw. gegen welche Vergütung das Werk auf eine neu entdeckte Nutzungsart genutzt werden darf. ${ }^{177}$ Dabei soll vor allem der freischaffende Urheber geschützt werden, der kein festes Gehalt bezieht, sondern auf die Erträge aus der Verwertung seins Werkes angewiesen ist. ${ }^{178}$ Sofern ein Urheber sich jedoch in einem Arbeitsverhältnis befindet und ein festes Gehalt bezieht, kann dieser Schutz unter Umständen überflüssig sein, weshalb in diesen Fällen keine Grund für eine Anwendung von \ 31 IV UrhG besteht. ${ }^{179}$

Aus dem gleichen Grund ist auch die Gegenansicht abzulehnen, wonach \ 31 IV a.F. UrhG im Arbeitsverhältnis grundsätzlich keine Anwendung finden soll, da damit Urheber benachteiligt würden, die sich zwar in einem Angestelltenverhältnis befinden, aber trotzdem das wirtschaftliche Risiko für die Verwertung ihres Werkes tragen. ${ }^{180}$

Es kommt damit stets darauf an, ob sich aus dem jeweiligen Arbeitsverhältnis die Nichtanwendbarkeit von \ 31 IV a.F. ergibt. Gerade bei öffentlich-rechtlichen Forschungseinrichtungen, wie etwa wissenschaftlichen Akademien oder Universitäten ist dabei zunächst zu berücksichtigen, dass die Forschungsprojekte solcher Einrichtungen auf mehrere Jahrzehnte angelegt sein können und die Urheber daher nicht nur kurzfristig, sondern in der Regel für einen sehr langen Zeitraum angestellt werden. Von daher sind die Fälle der dort beschäftigten Autoren von vornherein nicht mit dem vom BGH entschiedenen Fall Videozweitauswertung I vergleichbar, in dem es sich bei dem angestellten Urheber um einen angestellten Regisseur handelte, dessen Beschäftigungsdauer also im Gegensatz zu den Angestellten einer öffentlich-rechtlichen Forschungseinrichtung ungleich kürzer war. Unabhängig davon ist weiter zu berücksichtigen, dass Angestellte im öffentlichen Dienst neben ihrem gesetzlichen Rentenanspruch einen zusätzlichen Versorgungsanspruch erlangen. ${ }^{181}$

Demzufolge ist der angestellte Urheber einer öffentlich-rechtlichen Forschungseinrichtung wirtschaftlich in besonderem Maße abgesichert und demzufolge gerade nicht auf die Erträge aus der Verwertung seines Werkes angewiesen. Schließlich ist zu beachten, dass die Vergütung von Angestellten im öffentlichen Dienst nicht individuell ausgehandelt wird, sondern sich aus den Besoldungsgruppen des Tarifvertrags der Länder ergibt. Die Einordnung der Angestellten in die

\footnotetext{
177 BT-Drucks. IV/270 S. 56; ebenso BGH GRUR 1991, 133, 135; Ehrman/Fischer, GRUR Int. 2008, 284,285

178 BT-Drucks. IV/270 S. 62; Mathis, S. 125 f.

${ }^{179}$ Vgl. BT-Drucks. IV/270, S. 62.

${ }^{180}$ Vgl. BGH GRUR 1991, 133.

${ }^{181}$ Betriebsrente der Versorgungsanstalt des Bundes und der Länder (VBL) für tariflich Beschäftigte im öffentlichen Dienst.
} 
Besoldungsgruppen richtet sich dabei unter anderem nach der Art Ihrer Tätigkeit, so dass es auch nicht im Belieben des Leiters der Forschungseinrichtung steht, in welche Besoldungsgruppe er seine Angestellten einstuft. Vielmehr hat er sich dabei an die gesetzlichen Vorgaben zu halten. Demzufolge hätte ein Urheber, der beispielsweise im Jahre 1980 seinen Arbeitsvertrag mit einer öffentlich-rechtlichen Forschungseinrichtung geschlossen hat, keine höhere Vergütung verlangen können, wenn zu diesem Zeitpunkt das Recht der öffentlichen Zugänglichmachung im Internet bereits bekannt gewesen wäre und er dieses ausdrücklich auf seinen Arbeitgeber übertragen hätte.

Darüber hinaus wird aufgrund des Bekanntwerdens einer urheberrechtlichen Nutzungsart und der damit verbundenen Möglichkeit, diese zu übertragen, auch nicht die Vergütungshöhe in den einzelnen Besoldungs- bzw. Tarifgruppen erhöht. Angestellte Urheber in öffentlich-rechtlichen Forschungseinrichtungen bedürfen damit gerade nicht des Schutzes aus $\ 31 \mathrm{IV}$ a.F. UrhG. Demzufolge ist nicht ersichtlich, warum die Vorschrift auf sie Anwendung finden sollte. Vielmehr folgt in diesen Fällen aufgrund der hohen wirtschaftlichen Absicherung durch den Arbeitgeber und der durch den Tarifvertrag vorgegebenen Höhe der Vergütung „etwas anderes“ i.S.d. \43 UrhG aus dem Wesen des Angestelltenverhältnisses. Dass eine solche Lösung auch dem Sinn und Zweck von \31 IV UrhG entspricht, folgt auch aus der Gesetzesbegründung, nach der „der wirtschaftlich gesicherte Arbeitnehmer oder Beamte, der kein wirtschaftliches Risiko für sein Schaffen trägt, der Schutzbestimmung in der Regel nicht bedarf ${ }^{\prime \prime}{ }^{182}$ Des Weiteren ist zu beachten, dass auch der BGH in der Entscheidung Videozweitauswertung I zwar feststellt, dass $₫ 31$ IV UrhG zu „den zwingenden Normen des Urheberrechtsgesetzes gehört", seine Aussage allerdings dahingehend einschränkt, dass \31 IV UrhG „grundsätzlich“ nicht von $₫ 43$ UrhG verdrängt wird und damit zu erkennen gibt, dass es durchaus Fallkonstellationen geben kann, in denen von diesem Grundsatz eine Ausnahme gemacht werden muss. Ob \31 IV UrhG generell nicht von $\int 43$ UrhG verdrängt werden kann, hat das Gericht hingegen offen gelassen. ${ }^{183}$

Damit sind Klauseln in Arbeitsverträgen, von Angestellten von öffentlichrechtlichen Forschungseinrichtungen, nach denen der Urheber auch Rechte an noch nicht bekannten Nutzungsarten auf den Arbeitgeber überträgt, als wirksam zu erachten. Etwas anderes wird sich jedoch in der Regel für Angestellte von privatrechtlichen Unternehmen ergeben. Zunächst ist die Höhe des Arbeitsentgeltes bei diesen grundsätzlich verhandelbar, so dass der Arbeitnehmer für die Übertragung des Rechts an einer Nutzungsart theoretisch eine höhere Vergütung verlangen kann. Des Weiteren ist bei einem privatrechtlichen Unternehmen die Arbeitsplatzsituation ungleich weniger abgesichert als dies bei öffentlich-rechtlichen Arbeitgebern der Fall ist. Dies folgt zum einen aus der Möglichkeit, dass der Arbeit-

182 BT-Drucks. IV/270 S. 62.

183 Vgl. BGH GRUR 1991, 133, 135. 
Kapitel 1 - Urheberrechtliche Problematik

geber zahlungsunfähig wird oder sich seine wirtschaftliche Lage derart verschlechtert, dass er einzelnen Mitarbeitern aus betriebsbedingten Gründen kündigt. Zum anderen bieten privatrechtliche Arbeitgeber in der Regel auch keine zusätzliche Versorgung für das Rentenalter an. ${ }^{184}$ Daraus folgt, dass der Arbeitnehmer eines privatrechtlichen Unternehmens grundsätzlich wirtschaftlich schlechter abgesichert ist als im Falle der Beschäftigung bei einer öffentlich-rechtlichen Einrichtung, weshalb er des Schutzes von $\int 31$ Abs. IV a.F. UrhG in der Regel bedarf und dieser damit auf Arbeitsverträge mit privatrechtlichen Unternehmen in der Regel Anwendung findet.

\section{(2) Stillschweigende Rechteübertragung aufgrund des Arbeitsvertrags}

Auch wenn der Arbeitsvertrag zwischen der archivierenden Einrichtung und dem Rechteinhaber keine ausdrückliche Rechteübertragungsklausel enthält, könnte eine im Arbeitsvertrag stillschweigend vereinbarte Pflicht des Arbeitnehmers zur Übertragung auch der Nutzungsrechte an zum Zeitpunkt des Vertragsschlusses unbekannten Nutzungsarten bestehen. Aufgrund des Arbeitsverhältnisses ist der Arbeitnehmer verpflichtet, die Nutzungsrechte auf seinen Arbeitgeber zu übertragen, die er in Erfüllung seiner Verpflichtungen aus dem Arbeits- oder Dienstvertrag erworben hat. ${ }^{185}$ Dabei erfolgt die Einräumung der Nutzungsrechte regelmäßig im Voraus bei Abschluss des Arbeits- oder Dienstvertrages, ${ }^{186}$ spätestens jedoch mit Ablieferung des Werkes. ${ }^{187}$ Sofern der Urheber eines Werkes bzw. der Datenbankhersteller oder Lichtbildner in einem Angestellten- oder Dienstverhältnis zur archivierenden Einrichtung stand, wäre er dieser gegenüber also grundsätzlich zur Übertragung der Nutzungsrechte verpflichtet.

Insofern gilt nichts anderes als im Rahmen einer ausdrücklichen Rechteübertragung. Zumindest die stillschweigende Übertragung des Rechts der öffentlichen Zugänglichmachung im Internet könnte in diesen Fällen jedoch wieder an \31 IV a.F. UrhG scheitern, sofern der Rechteinhaber nicht Angestellter einer öffentlichrechtlichen Forschungseinrichtung ist und er aus diesem Grund des Schutzes von \31 IV a.F. UrhG bedarf. ${ }^{188}$

Darüber hinaus ist auch im Rahmen von Arbeitsverhältnissen die sog. Zweckübertragungslehre zu beachten, weshalb die erforderlichen Nutzungsrechte, unabhängig von der Anwendbarkeit von \ 31 IV a.F. UrhG, nur dann stillschweigend

\footnotetext{
${ }^{184}$ Eine Ausnahme bilden hier allerdings die Betriebsrenten, die jedoch überwiegend von großen Unternehmen gewährt werden.

185 BGH GRUR 1991, 523, 525; 1952, 257, 258 - Krankenhauskartei; Dreier in Dreier/Schulze, \43

Rn. 18; Dreyer in Dreyer/Kotthoff/Meckel, $\int 43$ Rn. 7, 13; A. Nordemann in

Fromm/Nordemann, \ 43 Rn. 1; Rojahn in Schricker/Loewenheim, \ 43 Rn. 37 f.; Wandtkee, GRUR 1999, 390, 392.

186 Dreier in Dreier/Schulze, \43 Rn. 19; Rojahn in Schricker/Loewenheim, \43 Rn. 43.

187 BGH GRUR 1974, 480, 483 - Hummelrechte; A. Nordemann in Fromm/Nordemann, $\int 43$ Rn. 30.

188 Siehe dazu bereits oben $51 \mathrm{ff}$.
} 
übertragen worden wären, wenn die archivierende Einrichtung diese Nutzungsrechte zur Erfüllung ihres betrieblichen Zwecks benötigt. Damit kommt es für die Frage, ob bzw. in welchem Umfang Forschungseinrichtungen die Onlinenutzungsrechte stillschweigend erlangt haben können, neben der Frage des \31 IV a.F. UrhG entscheidend darauf an, worin der Betriebszweck der archivierenden Einrichtung besteht und ob diese die Onlinenutzungsrechte an den von ihr herausgegebenen Werken zu dessen Erfüllung benötigt.

(a) Berücksichtigung von Art. 5 Abs. 3 GG

Zu beachten ist dabei zunächst, dass aufgrund der durch Art. 5 Abs. 3 GG verfassungsrechtlich garantierten Wissenschaftsfreiheit die gerade genannten Grundsätze für angestellte Urheber nicht auf Hochschulprofessoren übertragen werden können, da die Veröffentlichung von Forschungsergebnissen nicht mehr deren Aufgabenbereich zu rechnen ist. ${ }^{189}$ Handelt es sich bei dem Urheber des Datenbankwerkes oder dem Datenbankhersteller also um einen Hochschulprofessor ${ }^{190}$, so ist die Universität an der er lehrt, nicht allein aufgrund des Arbeitsvertrags Inhaberin der entsprechenden Nutzungsrechte geworden. Auch besteht aufgrund des Arbeitsverhältnisses keine diesbezügliche Verpflichtung, sofern der Vertrag keine entsprechende Klausel enthält. Das Gleiche gilt für wissenschaftliche Mitarbeiter, sofern es sich bei den zu archivierenden Daten bzw. Datenbanken oder Datenbankwerken um ihre eigene wissenschaftliche Arbeit handelt, da auch in diesen Fällen der Schutzbereich von Art. 5 Abs. 3 GG eröffnet ist. ${ }^{191}$ Daraus folgt, dass eine Pflicht zur Übertragung der Nutzungsrechte aufgrund des Arbeitsverhältnisses von vornherein nur dann in Betracht kommt, wenn der Rechtsinhaber diese in Ausübung einer weisungsgebundenen Tätigkeit erlangt hat. ${ }^{192}$ Denkbar ist dies vor allem bei Forschungseinrichtungen, wie etwa wissenschaftlichen Akademien oder den Einrichtungen der Max-Planck-Gesellschaft. Bei deren angestellten Forschern ist die Publikation von Forschungsergebnissen nämlich in der Regel Bestandteil ihrer geschuldeten Tätigkeit. In diesen Fällen käme eine Übertragung von Nutzungsrechten an urheberrechtlich oder leistungsrechtlich geschützten Datenbankwerken oder Datenbanken also grundsätzlich in Betracht.

189 BGH GRUR 1991, 523, 525 - Grabungsmaterialien; 1985, 529, 530 - Happening; OLG Karlsruhe GRUR 1988, 536, 537 - Hochschulprofessor; Dreier in Dreier/Schulze, \ 43 Rn. 12; Rojabn in Schricker/Loewenheim, \43 Rn. 31, 62; A. Nordemann in Fromm/Nordemann, \43 Rn. 43; Ehrmann/Fischer, GRUR Int. 2008, 284, 287.

${ }^{190}$ Das gleiche gilt für außerplanmäßige, Honorar- und Gastprofessoren, siehe Dreier in Dreier/Schulze, $\ 43$ Rn. 12; Rojahn in Schricker/Loewenheim, \ 43 Rn. 63.

191 Dreier in Dreier/Schulze, $\ 43$ Rn. 12; Wandtke in Wandtke/Bullinger, $\ 43$ Rn. 40; so wohl auch Rojahn in Schricker/Loewenheim, \43 Rn. 63.

192 A. Nordemann in Fromm/Nordemann, $\ 43$ Rn. 9; Dreier in Dreier/Schulze, $\ 43$ Rn. 9; Rojahn in Schricker, $\int 43$ Rn. 63; Rebbinder in FS Hubmann, 1985, S. 359, 370. 
(b) Unwirksamkeit der Verpflichtung aufgrund der Vorschrift des \ $31 \mathrm{IV}$ a.F. UrhG

Sofern ein stillschweigender Erwerb der Onlinenutzungsrechte nicht schon an der gerade beschriebenen Wertung des Art. 5 Abs. 3 GG scheitert, könnte diesbezüglich \31 IV a.F. UrhG entgegenstehen, sofern das Arbeitsverhältnis vor 1995 geschlossen worden ist. Auf Arbeitsverträge von privatrechtlichen Forschungseinrichtungen findet $\ 31$ Abs. 4 a.F. UrhG selbst bei ausdrücklichen Rechteübertragungsklauseln Anwendung, weshalb bei diesen ein stillschweigender Erwerb der Onlinerechte für Arbeitsverträge, die vor 1995 geschlossen wurden, konsequenterweise von vornherein ausscheidet. Bei öffentlich-rechtlichen Forschungseinrichtungen findet $\ 31 \mathrm{IV}$ a.F. UrhG nach der hier vertretenen Auffassung jedoch zumindest bei einer ausdrücklichen Rechteübertragung grundsätzlich keine Anwendung, da der im öffentlichen Dienst angestellte Urheber insofern ausnahmsweise nicht schutzbedürftig ist und sich daher aus dem Wesen des Arbeitsverhältnisses gem. \43 UrhG die Nichtanwendbarkeit von \31 IV a.F. UrhG ergibt. Etwas anderes könnte sich jedoch im Rahmen einer stillschweigenden Rechteübertragung ergeben, sofern in diesem Fall eine höhere Schutzbedürftigkeit des Arbeitnehmers anzunehmen ist.

Auch hier ist wiederum zu berücksichtigen, dass der angestellte Urheber einer öffentlich-rechtlichen Forschungseinrichtung in besonders hohem Maße wirtschaftlich abgesichert ist und für sein Schaffen kein Risiko trägt. Des Weiteren gilt auch im Rahmen einer stillschweigend vereinbarten Verpflichtung zur Übertragung von Nutzungsrechten bzw. der Vereinbarung einer diesbezüglichen Pflicht, dass es auf die Höhe der Vergütung keinen Einfluss gehabt hätte, wenn die Parteien eine ausdrückliche Klausel in den Vertrag eingefügt hätten. Denn auch in diesem Fall wäre der Urheber in die tarifvertraglich geregelte bzw. gesetzlich vorgeschriebene Besoldungsgruppe eingeordnet worden, die für seine Tätigkeit vorgesehen ist. Somit findet $\ 31$ IV a.F. UrhG auch im Rahmen von stilschweigenden Vereinbarungen aufgrund der Regelung des $\ 43 \mathrm{UrhG}$ und des besonderen Wesens des Arbeitsverhältnisses keine Anwendung auf Arbeitsverträge öffentlichrechtlicher Forschungseinrichtungen. Demzufolge kann für den Arbeitnehmer einer öffentlich-rechtlichen Forschungseinrichtung grundsätzlich eine Pflicht zur Übertragung der Onlinenutzungsrechte bestehen, wenn diese Nutzungsart zum Zeitpunkt des Vertragsschlusses noch unbekannt war und der Arbeitsvertrag diesbezüglich keine ausdrückliche Vereinbarung enthält.

(c) Erforderlichkeit des Übergangs der Onlinerechte zur Erfüllung des Betriebszwecks

Aus dem oben Gesagten folgt zwar, dass der Erwerb von Nutzungsrechten an zum Zeitpunkt der Begründung des Arbeitsverhältnisses noch unbekannten Nutzungsarten zumindest bei öffentlich-rechtlichen Forschungseinrichtungen entgegen $\int 31$ Abs. 4 a.F. UrhG grundsätzlich auch stillschweigend möglich ist. Das 
Bestehen einer solchen Pflicht des Arbeitnehmers hängt allerdings zusätzlich davon ab, ob die Einrichtung diese Rechte zur Erfüllung ihres Betriebszwecks benötigt. Dies folgt aus der sog. Zweckübertragungslehre, die ihren Niederschlag unter anderem in $\$ 31$ Abs. 5 UrhG gefunden hat und auch im Rahmen von Arbeitsverträgen Anwendung findet. ${ }^{193}$ Nach der sog. Zweckübertragungslehre bestimmt sich der Umfang der eingeräumten Nutzungsrechte nach dem Vertragszweck, sofern die Parteien diesbezüglich keine ausdrückliche Vereinbarung getroffen haben. ${ }^{194}$ Dabei wird die Zweckübertragungslehre im Rahmen von Arbeitsverhältnissen jedoch aufgrund der Regelung des $₫ 43$ UrhG modifiziert. ${ }^{195}$ Der Arbeitgeber erlangt bei einer nicht ausdrücklichen Rechteübertragung nur diejenigen Nutzungsrechte, die er zur Erfüllung seines betrieblichen Zwecks benötigt. ${ }^{196}$ Nach diesem Betriebszweck richtet sich ferner auch der Umfang der zu übertragenen Nutzungsrechte. Demzufolge kommt es für die Beantwortung der Frage, ob eine öffentlich-rechtliche Forschungsanstalt die zur elektronischen Langzeitarchivierung erforderlichen Nutzungsrechte von ihren Angestellten bereits aufgrund des Arbeitsverhältnisses erlangt hat, darauf an, ob diese Einrichtungen Onlinenutzungsrechte zur Erfüllung ihres betrieblichen Zwecks benötigen.

Dies beurteilt sich nach dem Zweck von öffentlich-rechtlichen Forschungseinrichtungen. Dieser liegt zum einen in der Forschung und der Durchführung wissenschaftlicher Arbeiten. Zum anderen besteht er jedoch auch vor allem in der Zugänglichmachung der gewonnenen Forschungsergebnisse, da nur durch den dadurch bewirkten Austausch gewonnener Erkenntnisse mit anderen Forschungseinrichtungen eine effektive wissenschaftliche Forschung möglich ist. ${ }^{197}$ Dass dies zweifellos zu den Aufgaben wissenschaftlicher Forschungseinrichtungen gehört, geht auch aus den einzelnen Satzungen der Einrichtungen hervor. So beschreibt beispielsweise die Satzung der Akademie der Wissenschaften zu Göttingen, dass ihre Aufgabe darin besteht, ,in eigener Arbeit und im Zusammenwirken mit den gelehrten Körperschaften des In- und Auslandes der Wissenschaft zu dienen““.198 Ein solcher Austausch mit anderen Forschungseinrichtungen im In- und Ausland wäre jedoch nahezu unmöglich, wenn die Einrichtungen ihre Forschungsergebnisse nicht in analoger oder digitaler Form publizieren und anderen Institutionen damit die Möglichkeit des Zugriffs auf diese Erkenntnisse geben würden. Um

\footnotetext{
193 Wandtke in Wandtke/Bullinger, \4 43 Rn. 55; Ehrmann/Fischer, GRUR Int. 2008, 284, 287.

194 Wandtke/Grunert in Wandtke/Bullinger, \ 31 Rn. 39; Frey/Rudolph, ZUM 2007, 13, 22; siehe auch BT-Drucks. 16/1828, S. 45.

195 A. Nordemann in Fromm/Nordemann, $\ 43$ Rn. 45.

196 Wandtke in Wandtke/Bullinger, $\ 43$ Rn. 58; A. Nordemann in Fromm/Nordemann, $\ 43$ Rn. 45, 27.

197 Vgl. Meusel, S. 24 Rn. 40.

198 Siehe $\int 1$ der Satzung der Akademie der Wissenschaften zu Göttingen, Bek. v. 1.3.1972 (Nds. MBl., S. 569; siehe auch Meusel, S. 100 Rn. 118 m.w.N.; vgl. auch die Beschreibung des Akademienprogramms auf der Homepage der Union der Deutschen Akademien der Wissenschaften, abrufbar unter http://www.akademienunion.de/akademienprogramm
} 
diesen betrieblichen Zweck zu erfüllen, benötigen die Forschungseinrichtungen auf jeden Fall das Verlagsrecht, also das Recht der Vervielfältigung und das Recht der Verbreitung des Werkes in analoger Form. Allerdings lässt sich mit Printpublikationen nur ein Bruchteil der Interessenten erreichen, die über das Internet Zugang zu den kostenlosen Onlineversionen des gleichen Werkes erlangen würden. Hinzu kommt, dass in Zeiten sinkender Budgets und nicht unerheblichen Einsparungsmaßnahmen im öffentlich-rechtlichen Bereich, viele Bibliotheken die Anschaffung einiger wissenschaftlicher Werke nicht mehr finanzieren können, da deren Verkaufspreis oftmals sehr hoch ist. Daraus folgt, dass öffentlich-rechtliche Forschungseinrichtungen das Recht der Onlineverwertung zur Erreichung ihres Betriebszwecks, nämlich des Austauschs der gewonnenen Erkenntnisse mit anderen Institutionen im In- und Ausland, benötigen. Dies gilt gerade auch für die öffentliche Zugänglichmachung von Forschungsdaten im Rahmen der elektronischen Langzeitarchivierung, da die Publikation dieser Daten zum einen finanziell wohl nahezu unmöglich wäre und zum anderen der Zugriff auf diese Daten für Fremdforscher wesentlich schwerer wäre als dies im Rahmen eines digitalen Abrufs von einer Onlineplattform der Fall ist. Daraus könnte man den Schluss ziehen, dass diese Rechte bei Angestellten von öffentlich-rechtlichen Forschungseinrichtungen aufgrund des Arbeitsvertrags stillschweigend übergegangen sind. $\mathrm{Zu}$ beachten ist dabei jedoch, dass der Betriebszweck bereits bei Vertragsschluss bestanden haben muss. Hat er sich hingegen nach Abschluss des Vertrags geändert, so ist ein stillschweigender Rechteübergang nur dann anzunehmen, wenn die Änderung für den Arbeitnehmer bei Vertragsschluss erkennbar war. ${ }^{199}$

In den vorliegend in Betracht kommenden öffentlich-rechtlichen Forschungseinrichtungen stellt die Bereitstellung von Forschungsdaten im Internet oder einem anderen Netzwerk jedoch keine Änderung des Betriebszwecks dar. Es wurde vielmehr die Art und Weise, mit der der Betriebszweck erreicht werden soll, um die Verwendung neuer Technologien erweitert. Dies stellt jedoch keine Änderung des Betriebszwecks an sich dar, was schon an dem oben genannten Beispiel der Aufgabenbeschreibung in der Satzung der Akademie der Wissenschaften zu Göttingen aus dem Jahr 1972 deutlich wird. Im Mittelpunkt steht nach wie vor die Erlangung von wissenschaftlichen Erkenntnissen und deren Austausch mit anderen Wissenschaftlern und wissenschaftlichen Einrichtungen.

Bei Arbeitsverträgen von öffentlich-rechtlichen Forschungseinrichtungen ist daher davon auszugehen, dass auch bei Fehlen einer ausdrücklichen Rechteübertragungsklausel das Recht der Onlineverwertung sowie das Recht der Vervielfältigung und Verbreitung stillschweigend mit dem Zeitpunkt des Vertragsschlusses auf den Arbeitgeber übergegangen ist, da es zur Erreichung des Betriebszwecks erforderlich ist.

199 A. Nordemann in Fromm/Nordemann, \43 Rn. 30; Rojahn in Schricker/Loewenheim, $\int 43$ Rn. 54; Schmechel-Gaumé, K\&R 2001, 74, 76. 
Ob die betreffenden Nutzungsrechte räumlich oder zeitlich beschränkt und ob als einfache oder ausschließliche Nutzungsrechte einzuräumen sind, hängt wiederum davon $a b$, in welchem Umfang die betreffende Forschungseinrichtung die Nutzungsrechte zur Erreichung ihres betrieblichen Zweckes benötigt.

Hinsichtlich der Frage der zeitlichen Beschränkung ist dabei zu berücksichtigen, dass Forschungseinrichtungen langfristig arbeiten und ihre Forschungsergebnisse dauerhaft anderen Wissenschaftlern zur Verfügung stellen möchten. Denn nur durch eine dauerhafte Zurverfügungstellung ist es den Einrichtungen möglich, einen möglichst umfangreichen Erkenntnisaustausch untereinander und damit eine effektive Forschung zu erreichen. Insofern erfordert der Betriebszweck öffentlich-rechtlicher Forschungseinrichtungen eine zeitlich unbeschränkte Rechteeinräumung der oben genannten Nutzungsrechte.

In Bezug auf die räumliche Beschränkung ist wiederum zu berücksichtigen, dass zu einer effektiven Forschungstätigkeit ein Wissensaustausch mit möglichst vielen anderen Forschern und Forschungseinrichtungen erforderlich ist, was jedoch nur dann erreicht werden kann, wenn die von der Forschungseinrichtung publizierten Werke weltweit zugänglich gemacht werden. Dies gilt auch oder gerade für Onlinepublikationen, da vor allem im wissenschaftlichen Bereich der Trend immer mehr dazu übergeht, sich die gewünschten Informationen zunächst online zu recherchieren. Aus diesem Grund ist zur Erfüllung des Betriebszwecks öffentlich-rechtlicher Forschungseinrichtungen erforderlich, dass diese das Onlinenutzungsrecht als räumlich unbeschränktes Recht eingeräumt bekommen. ${ }^{200}$

Des Weiteren stellt sich die Frage, ob die Forschungseinrichtungen das Onlinenutzungsrecht als ausschließliches oder einfaches Nutzungsrecht von ihren Arbeitnehmern erlangen. Grundsätzlich wird in diesem Zusammenhang die Ansicht vertreten, dass der Arbeitgeber im Zweifel die Nutzungsrechte als ausschließliche Rechte erlangt, da er ein Interesse daran hat, andere von der Nutzung des Werkes auszuschließen. ${ }^{201}$ Dabei könnte jedoch im Falle von öffentlich-rechtlichen Forschungseinrichtungen etwas anderes gelten. Aufgrund der Tatsache, dass diese ihre Publikationen oftmals kostenlos im Internet als sogenannte Open Access Publikationen zur Verfügung stellen, stellt sich die Frage, ob sie wirklich ein Interesse daran haben, andere von der Nutzung dieses Werkes auszuschließen, da ihnen ja keine finanziellen Einbußen drohen. Zu beachten ist jedoch, dass Onlinepublikationen den Forschungseinrichtungen eine effektive Möglichkeit geben, sich der Öffentlichkeit zu präsentieren und ihre Arbeitsergebnisse darzulegen, was gerade in Zeiten von Haushalts- und Budgetkürzungen für Forschungseinrichtun-

\footnotetext{
${ }^{200}$ Darüber hinaus wäre es schwer, dass Recht der Onlinenutzung räumlich beschränkt auszuüben, da Werke, die beispielsweise auf einer deutschen Website zugänglich gemacht werden, von jedem Teil der Welt abrufbar sind. Dies wäre höchstens anhand sogenannter IP-Filter möglich, was jedoch für den Verwerter des jeweiligen Werkes sehr aufwendig und unter Umständen sehr kostspielig wäre; vgl. auch Dreier in Dreier/Schulze, \$137 l UrhG Rn. 29.

${ }^{201} W$ andtke in Wandtke/Bullinger, $\ 43$ Rn. 73.
} 
gen ein effektiver Weg ist, um den Erhalt von öffentlichen Geldern zu rechtfertigen. Diese sind wiederum erforderlich, um den Betriebszweck erfüllen zu können. Aus diesem Grund haben die Forschungseinrichtungen ein Interesse daran, mit den online publizierten Werken in Verbindung gebracht zu werden, was jedoch nicht der Fall ist, sofern ein Dritter die Werke online zugänglich macht. In diesen Fällen muss es den Forschungseinrichtungen also möglich sein, andere von der Onlinenutzung auszuschließen. Dies gilt auch, sofern es sich bei dem geschützten Werk nicht um ein Buch oder eine ähnliche Publikation handelt, sondern um Forschungsdaten bzw. Datenbanken oder Datenbankwerke in denen diese Daten enthalten sind. Zum einen ist nicht ausgeschlossen, dass diese Daten nicht doch nur gegen Entgelt Dritten zur Verfügung gestellt werden sollen und die Forschungseinrichtung damit eine Möglichkeit erhält, ihre Forschungskosten zumindest teilweise zu amortisieren. Zum anderen kann auch bei der Veröffentlichung von Forschungsdaten ein Interesse der Forschungseinrichtung bestehen, mit der Gewinnung der damit verbundenen Erkenntnisse in Verbindung gebracht zu werden. Von daher ergibt sich auch bei öffentlich-rechtlichen Forschungseinrichtungen aufgrund des Betriebszwecks das Erfordernis, das Onlinenutzungsrecht als ausschließliches Nutzungsrecht zu erlangen.

Öffentlich-rechtliche Forschungseinrichtungen erlangen demzufolge auch bei Arbeitsverträgen, die keine ausdrückliche Rechteübertragungsklausel beinhalten und die vor 1995 geschlossen worden sind, einen Anspruch auf Übertragung des Rechts der Onlinenutzung als ausschließliches und räumlich sowie zeitlich unbeschränktes Nutzungsrecht.

(d) Dingliche Übertragung der Nutzungsrechte

Auch wenn eine Pflicht des Arbeitnehmers einer öffentlich-rechtlichen Forschungseinrichtung zu bejahen ist, aufgrund des dem deutschen Zivilrecht zugrunde liegenden Trennungsprinzips gehen die Nutzungsrechte jedoch nicht bereits durch diese rein schuldrechtliche Verpflichtung auf die jeweilige Forschungseinrichtung über. Vielmehr bedarf es dazu der dinglichen Verfügung durch den Urheber, die allerdings mit der Verpflichtung zeitlich zusammenfallen kann. Damit stellt sich die Frage, ob der Urheber diese Verfügung bei Abschluss des Arbeitsvertrages stillschweigend vorgenommen hat oder ob er die betreffenden Rechte hierfür ausdrücklich auf die Forschungseinrichtung übertragen muss. Sofern man eine ausdrückliche Rechteübertragung verlangt, müssten die Forschungseinrichtungen ihren Anspruch aus dem Arbeitsvertrag auf Einräumung der Onlinerechte zunächst gegen den Urheber geltend machen. Bis zur Einräumung der Nutzungsrechte dürften sie diese jedoch nicht nutzen, da andernfalls zivil- und strafrechtliche Konsequenzen drohen würden. ${ }^{202} \mathrm{Zu}$ beachten ist in diesem Zusammenhang insbesondere, dass es nach Ansicht der Rechtsprechung

202 Vgl. \$S 97, 106 UrhG. 
nicht rechtsmissbräuchlich ist, wenn ein Rechteinhaber den rechtswidrigen Nutzer seines Werkes auf Unterlassung verklagt, obwohl er diesem gegenüber zur Einräumung der Nutzungsrechte verpflichtet ist. ${ }^{203}$

$\mathrm{Ob}$ die Nutzungsrechte stillschweigend bei Abschluss des Arbeitsvertrages übergegangen sind oder ob es einer ausdrücklichen Übertragungshandlung des Urhebers bedarf, lässt sich hingegen nicht pauschal beantworten. Vielmehr hängt die Lösung auch hier wieder davon ab, ob der Urheber im Einzelfall ein Interesse daran hat, dass die Nutzungsrechte zunächst bei ihm verbleiben und er die Übertragung der Rechte ausdrücklich vornehmen muss. ${ }^{204}$ Auch hier ist wieder die im Vergleich geringere Schutzbedürftigkeit des angestellten Autors im öffentlichen Dienst zu berücksichtigen. Da die Vergütung des Urhebers sich nach den vorgegebenen Besoldungsgruppen richtet und er darüber hinaus aufgrund seiner Anstellung einen zusätzlichen Rentenanspruch und damit eine besondere wirtschaftliche Absicherung erlangt, besteht für ihn im Gegensatz zu freischaffenden Urhebern oder solchen, bei denen die Vergütung individuell auf Grundlage der einzuräumenden Nutzungsrechte vereinbart wird, keinerlei wirtschaftliches Risiko bei der Verwertung seines Werkes. Aus diesem Grund läuft es auch nicht seinen Interessen zuwider, wenn durch den Arbeitsvertrag nicht nur die Verpflichtung zur Rechteübertragung noch unbekannter Nutzungsrechte begründet wird, sondern die dingliche Übertragung dieser Nutzungsrechte ebenfalls stillschweigend aufgrund des Arbeitsvertrags erfolgt. ${ }^{205}$ Dafür spricht letztlich auch, dass öffentlichrechtliche Forschungseinrichtungen und damit auch die Vergütung des Urhebers durch Steuergelder getragen werden, so dass ein hohes Allgemeininteresse an einer umfangreichen Verwertung der von den Mitarbeitern der Forschungsstellen in Ausübung ihrer Tätigkeit geschaffenen Werke besteht. Aus diesem Grund kann es auch keine Rolle spielen, ob die Nutzungsart noch während des Arbeitsverhältnisses bekannt geworden ist oder erst danach, da dies für den Arbeitnehmer keinen Unterschied macht. ${ }^{206}$

d. Erwerb der erforderlichen Rechte aufgrund der Rechtsübertragungsfiktion des $\int 1371 \mathrm{UrhG}$

Sofern ein Erwerb des Onlinerechtes nicht schon im Rahmen einer ausdrücklichen oder stillschweigenden Rechteübertragung erfolgt ist, könnten Forschungseinrichtungen die Onlinerechte an den für die Archivierung vorgesehenen Datenbanken oder Datenbankwerken schließlich aufgrund der Rechtsübertragungsfikti-

\footnotetext{
203 Vgl. BGH NJW 2002, 896, 899.

204 Rojahn in Schricker, \43 Rn. 20; Schmechel-Gaumé, K\&R 2001. 74, 76.

205 Zumindest für die Einräumung von Rechten an Nutzungsarten, die während des Arbeitsverhältnisses bekannt werden: Rojahn in Schricker/Loewenheim, \43 Rn. 54; Schmechel-Gaumé, K\&R $2001,74,76$.

206 Zumindest für Nutzungsrechte an Nutzungsarten, die noch während des Arbeitsverhältnisses neu entstanden sind: Sack in Münchener Handbuch zum Arbeitsrecht, Bd. 1, \ 100 Rn. 43.
} 
Kapitel 1 - Urheberrechtliche Problematik

on des $\int 1371$ Abs. 1 UrhG erlangt haben. Dies kommt insbesondere für privatrechtliche Forschungseinrichtungen in Betracht, da bei diesen der Erwerb des Onlinenutzungsrechts aufgrund des Arbeitsvertrags zumeist an der Vorschrift des $\int 31$ Abs. 4 a.F. UrhG scheitern wird. ${ }^{207}$ Aber auch für die übrigen Forschungseinrichtungen kann es für den Erwerb bestimmter Nutzungsrechte, vor allem des Onlinenutzungsrechts, auf die Vorschrift des \1371 UrhG ankommen. Dies gilt beispielsweise für solche Fallkonstellationen, in denen der Rechteinhaber nicht in einem Arbeitsverhältnis zur archivierenden Einrichtung stand, aufgrund dessen die benötigten Nutzungsrechte auf sie übergegangen sind.

Dies setzt voraus, dass alle zum Zeitpunkt des jeweiligen Vertragsschlusses relevanten Nutzungsrechte auf die archivierende Einrichtung als ausschließliche sowie räumlich und zeitlich unbeschränkte Rechte übertragen worden sind und der Rechteinhaber die nach Vertragsschluss bekannt gewordenen Rechte, namentlich das Recht der öffentlichen Zugänglichmachung im Internet, nicht vor dem 1.1.2008 an einen Dritten übertragen hat. Des Weiteren würde die Rechtsübertragungsfiktion gem. \ 1371 Abs. 1 S. 2 UrhG ausscheiden, wenn der Rechteinhaber bis zum Ablauf des 31.12.2008 seinen Widerspruch erklärt hätte.

\section{i. Wesentliche Nutzungsrechte}

In diesem Zusammenhang stellt sich zunächst die Frage, welche Rechte in Bezug auf die vorliegend in Betracht kommenden Werke als wesentlich anzusehen sind. $\mathrm{Da}$ bis vor wenigen Jahren lediglich die konventionelle Vervielfältigung und Verbreitung von Sprachwerken als Printpublikation üblich war, werden sich viele Forschungseinrichtungen, sofern die von ihnen geschlossenen Arbeitsverträge überhaupt eine Rechteübertragungsklausel enthalten, lediglich das Recht der analogen Vervielfältigung sowie das Recht der Verbreitung des Werkes in analoger Form (Verlagsrecht) einräumen lassen haben. Rechte an anderen Nutzungsarten, wie beispielsweise der Nutzung des Werkes auf CD-ROM, welche gerade ab Anfang der 90er Jahre stark an Bedeutung gewonnen hat, sind von den vertraglichen Vereinbarungen zwischen Arbeitnehmer und Arbeitgeber hingegen oftmals nicht erfasst. Würde es sich bei dem genannten Recht aber um ein wesentliches Recht i.S.d. \137 1 UrhG handeln, würde dessen Übertragungsfiktion für Werke, deren Rechte erst nach Bekanntwerden der Nutzungsart CD-ROM auf den Verlag übertragen wurden, nur dann greifen, wenn die Rechteübertragung dieses Recht ebenfalls umfassen würde, da $\int 1371$ UrhG voraussetzt, dass ,,alle wesentlichen Nutzungsrechte“ übertragen wurden. Aus diesem Grund soll im Folgenden zunächst geklärt werden ob bzw. unter welchen Voraussetzungen das Recht der CD-ROMNutzung in den vorliegend relevanten Fällen als wesentliches Recht i.S.d. \ 1371 UrhG anzusehen ist.

${ }^{207}$ Siehe dazu oben S. $51 \mathrm{ff}$. 


\section{(1) Bekanntwerden der Nutzungsart „CD-ROM“}

Diesbezüglich ist zunächst zu prüfen, ob diese Nutzungsart vor der Nutzung des Werkes im Internet bekannt geworden ist. Wäre dies nicht der Fall, würde sich die Frage, ob die Nutzung des Werkes auf CD-ROM ein wesentliches Recht i.S.d. \$ 1371 UrhG darstellt erübrigen, da die Übertragung des Rechts an einer nicht bekannten Nutzungsart nach alter Rechtslage gem. \31 Abs. 4 a.F. UrhG nicht möglich war. ${ }^{208}$ Hinsichtlich des Zeitpunktes des Bekanntwerdens der CD-ROMNutzung werden jedoch unterschiedliche Ansichten vertreten. So soll die Nutzung von Fachzeitschriften durch CD-ROMs nach teilweise vertretener Ansicht bereits zwischen 1985 und 1988 bekannt geworden sein. ${ }^{209}$ Nach anderer Ansicht liegt der Zeitpunkt des Bekanntwerdens hingegen erst zu Beginn der 90er Jahre. ${ }^{210}$ Auch hinsichtlich der Nutzung von Büchern und Publikumszeitschriften wird der Zeitpunkt unterschiedlich beurteilt. ${ }^{211}$ Allerdings haben die genannten Ansichten gemein, dass das Recht der Nutzung des Werkes auf CD-ROM zumindest vor 1995 und damit vor der Onlinenutzung bekannt geworden ist. ${ }^{212}$ Demzufolge hinge die Rechtsübertragungsfiktion des \ 1371 UrhG davon ab, ob das Recht der CD-ROM-Nutzung seinerzeit übertragen wurde, sofern dies ein wesentliches Nutzungsrecht darstellt.

Der Wortlaut der Norm enthält dabei weder Anhaltspunkte hinsichtlich der Frage was unter den wesentlichen Nutzungsrechten zu verstehen ist, noch anhand welcher Kriterien diese zu bestimmen sind. ${ }^{213}$ Auch aus der Gesetzesbegründung zu \137 l UrhG geht lediglich hervor, dass diejenigen Nutzungsrechte wesentlich sind, mit denen der Vertragspartner den Verwertungszweck umfassend erreicht, nicht jedoch, dass alle denkbaren Verwertungsformen eines Werkes erfasst sind. ${ }^{214}$ Vielmehr wurde die nähere Definition dieses Tatbestandsmerkmals der Rechtsprechung und Literatur überlassen. ${ }^{215}$

\footnotetext{
208 Siehe hierzu oben S. 51 ff.

${ }^{209}$ Haberstumpf, Handbuch des Urheberrechts, 2. Aufl. 2000, Rn. 407; nach Katzenberger, AfP 1997, 434, 440 gilt diese Nutzungsart hingegen bereits seit 1988 als bekannt.

${ }^{210}$ Dreier in Lehmann (Hrsg.), Internet- und Multimediarecht (Cyberlaw), 1997, S. 134; Hoeren, CR $1995,710,713$.

211 Vgl. Heckmann in Spindler (Hrsg.), Rechtliche Rahmenbedingungen von Open Access - Publikationen, 2006, S. 130 m.w.N.

212 A.A. Schulze in FS Beier, 1996, S. 403, 405, wonach der Zeitpunkt des Bekanntwerdens erst im Jahre 1995 liegt.

213Jani in Wandtke/Bullinger, $\int 1371$ Rn. 7.

214 BT-Drucks. 16/1828, S.33.

215 cholz in Mestmäcker/Schulze, Urheberrechtskommentar, Stand: 47. Aktualisierungslieferung August 2008, Bd. 1.2, \137 1 Rn. 13.
} 
(2) Auslegung des Merkmals ,wesentlich“

Die Frage, welche Rechte vorliegend vom Tatbestandsmerkmal der „wesentlichen Nutzungsrechte" erfasst sind, ist somit anhand einer Auslegung von \137 1 Abs. UrhG zu beantworten, wobei hier eine grammatikalische Auslegung, wie gerade gezeigt, nicht weiterhilft. Die Definition der Wesentlichkeit lässt sich jedoch unter Umständen vor dem Hintergrund des Sinn und Zwecks des \137 l UrhG im Rahmen einer teleologischen Auslegung ermitteln. ${ }^{216}$ Dieser besteht zunächst darin, dem Erwerber von Nutzungsrechten an einem Werk auch die Nutzung auf neue, erst nach Vertragsschluss bekannt gewordene Arten zu ermöglichen.

Nach der Gesetzesbegründung ist in diesem Zusammenhang darauf abzustellen, „ob im konkreten Fall alle diejenigen Rechte übertragen wurden, die für eine umfassende Verwertung nach dem jeweiligen Vertragszweck notwendig sind“. ${ }^{217}$ Es kommt folglich im Einzelfall darauf an, ob die archivierende Einrichtung das Werk, in den vorliegenden Fällen also die Datenbank bzw. das Datenbankwerk oder das Lichtbild, auch ohne das Recht der Nutzung auf CD-ROM nach dem jeweiligen Vertragszweck verwerten konnte. Sofern eine Verwertung nach dem Vertragszweck ohne das Recht der CD-ROM-Nutzung möglich gewesen wäre, würde dieses Recht kein wesentliches Recht i.S.d. \137 1 UrhG darstellen, so dass, unter der Voraussetzung der übrigen Tatbestandsvoraussetzungen, dessen Fiktion grundsätzlich zugunsten der archivierenden Einrichtung greifen würde. In den Fällen, in denen das Werk hingegen nicht ohne das Recht der CD-ROM Nutzung vollumfänglich verwertet werden konnte, wäre es hingegen als wesentliches Recht im Sinne des $\$ 1371$ UrhG anzusehen und die Rechtsübertragungsfiktion würde folglich nur dann greifen, wenn der Rechteinhaber der archivierenden Einrichtung seinerzeit auch das Recht zur Verbreitung als CD-ROM übertragen hätte, was jedoch eher selten zutreffen wird.

Vor dem Hintergrund der unterschiedlichen in Wissenschaft und Forschung verbreiteten Publikationsmedien kann die Frage, ob das Recht der Nutzung durch CD-ROM zu den „wesentlichen Nutzungsrechten“ gehört, jedoch nicht pauschal beantwortet werden. Es ist vielmehr danach zu differenzieren, ob es sich im Einzelfall um Zeitschriftenjahrgänge, Monographien oder aber, wie vorliegend, Datenbanken oder Datenbankwerken handelt, da bei diesen Werken zum einen teilweise unterschiedliche Vertragszwecke bestehen werden und zum anderen ihre Verwertung auf unterschiedliche Arten erfolgen kann.

Im Rahmen von wissenschaftlichen Publikationen ist es bereits seit Anfang der 90er Jahre üblich, diese auch auf CD-ROM zu verbreiten, wobei dies jedoch nicht generell für alle Arten wissenschaftlicher Publikationen gilt. Ist die Nutzung von Zeitschriftenbänden oder periodisch erscheinenden Werken auf CD-ROM schon seit Ende der 80er Jahre üblich, hat sie sich bei Monographien erst in den letzten

${ }^{216}$ Vgl. Zippelius, Juristische Methodenlehre, 10. Aufl. 2006, S. 49 f.

${ }_{217}$ BT-Drucks. 16/1828, S. 33; Jani in Wandtke/Bullinger, \$137 1 Rn. 8; siehe auch Frey/Rudolph, ZUM 2007, 13, $21 \mathrm{f}$. 
Jahren und auch nur zum Teil durchgesetzt. Da die Lagerung von periodisch erscheinenden Werken einen erheblichen Raumbedarf mit sich bringt und gerade wissenschaftliche Fachzeitschriften oftmals nur im Abonnement erhältlich sind, ist es für viele Nutzer wesentlich effektiver, diese Werke auf CD-ROM zu nutzen. Hinzu kommt, dass Nutzer von Zeitschriftenjahrgängen und periodisch erscheinenden Werken, wie etwa juristischen Kommentaren, oftmals nur Auszüge des Werkes benötigen und die erforderlichen Stellen mit Hilfe der elektronischen Suchfunktion wesentlich schneller finden kann als in der Printversion des Werkes.

Dies gilt erst Recht für die vorliegend im Fokus stehenden Datenbanken und Datenbankwerke. Gerade bei diesen ist eine digitale Nutzung auf CD-ROM wesentlich praktikabler als eine solche in analoger Form. Dies gilt insbesondere für besonders umfangreiche Datenbanken oder Datenbankwerke wie beispielsweise solchen aus dem Bereich der Astrophysik. Aufgrund deren oftmals immensen Umfangs wäre eine Veröffentlichung in Printform zum einen finanziell nahezu unmöglich und zum anderen auch nicht im Interesse des Nutzers, da es diesem in der Regel nur darauf ankommt, auf die entsprechenden Daten punktuell zugreifen zu können. Das Bedürfnis, das jeweilige Werk „am Stück“ durchlesen zu können, wie dies unter Umständen bei Monographien der Fall ist, besteht bei Datenbanken und Datenbankwerken jedoch gerade nicht.

Dabei ist jedoch stets zu berücksichtigen, ob der Vertragszweck im Einzelfall auch die Publikation des jeweiligen Werkes umfasst. Dies wird bei einem Autorenvertrag zwischen dem Autor eines Zeitschriftenbeitrags unschwer zu bejahen sein, wird der Vertrag doch gerade deshalb geschlossen, um die Publikation des Werkes zu ermöglichen. Das Gleiche gilt für Verträge zwischen dem Autor einer Monographie und dem Verlag. Im Gegensatz dazu ist weder die Publikation der von angestellten Wissenschaftlern erstellten Datenbanken und Datenbankwerken zwingend, noch dass deren Publikation Teil des Vertragszwecks ist. So erfasst der Zweck des Vertrags zwischen einem Mediziner und einer Universitätsklinik nicht zwangsläufig auch die Publikation der Daten, die er im Rahmen seiner medizinischen Forschung an der Universität erzeugt und zu Datenbanken oder sogar Datenbankwerken zusammenstellt.

Etwas anderes könnte man hingegen für die Publikation der Forschungsergebnisse annehmen, die jedoch nicht im Fokus der vorliegenden Arbeit stehen, da es sich insoweit nicht mehr um Primärdaten handelt. Allerdings ist zu beachten, dass bei der Veröffentlichung der Forschungsergebnisse oftmals auch die Veröffentlichung der Daten einhergeht, die diesen Ergebnissen zugrunde liegen. Im Einzelfall ist daher stets zu prüfen, ob sich der Zweck des Vertrags zwischen dem Rechteinhaber und der archivierenden Einrichtung auch auf die Publikation der im Rahmen der forschenden Tätigkeit erzeugten Daten erstreckt. Ist dies jedoch der Fall, so ist davon auszugehen, dass dieser Zweck bei wissenschaftlichen Datenbanken, die in dem betreffenden Zeitraum zwischen 1989 und 1995 erstellt wurden, nur dann vollumfänglich erreicht werden konnte, wenn sie auch als CD- 
ROM genutzt wurden. Hat in den vorliegend relevanten Fallkonstellationen der Datenbankhersteller oder Urheber eines Datenbankwerkes der archivierenden Einrichtung also nur das Recht der Vervielfältigung in analoger Form sowie das Recht der Verbreitung eingeräumt, so stellt dies für den Zeitraum zwischen 1989 und 1995 nicht die Einräumung aller wesentlichen Nutzungsrechte dar und die Fiktion des $₫ 1371$ UrhG würde insoweit für die oben genannten Werke nicht greifen.

Sofern sich der Vertragszweck hingegen nicht auch auf die Publikation erstreckt, sondern beispielsweise auf eine interne Verwendung in der Forschungsstelle, wäre er hingegen auch ohne eine Nutzung der Daten auf CD-ROM erreichbar, so dass das besagte Recht in diesen Fallkonstellationen nicht als wesentliches Nutzungsrecht i.S.d. \137 1 UrhG anzusehen wäre und die Rechtsübertragungsfiktion demzufolge auch dann greifen würde, wenn lediglich das Recht der Vervielfältigung und Verbreitung auf die archivierende Einrichtung übertragen worden wäre.

Für den Zeitraum vor 1989 stellen das Recht der Vervielfältigung und das Recht der Verbreitung für Datenbanken bzw. Datenbankwerke hingegen grundsätzlich ,alle wesentlichen Nutzungsrechte“ i.S.d. \$137 1 UrhG dar. Hinsichtlich dieses Zeitraums würde die Rechtsübertragungsfiktion somit greifen, wenn der Rechteinhaber der archivierenden Einrichtung diese Rechte als räumlich und zeitlich unbegrenzte Rechte eingeräumt hätte.

ii. „räumlich unbegrenzte“ Rechteeinräumung

Für den Eintritt der Fiktion der Rechteübertragung verlangt \137 1 UrhG weiter, dass die zu diesem Zeitpunkt wesentlichen Nutzungsrechte sowohl räumlich als auch zeitlich unbegrenzt übertragen worden sind. In diesem Zusammenhang stellt sich die Frage, ob unter einer ,räumlich unbegrenzten“ Übertragung eine weltweite Einräumung der wesentlichen Nutzungsrechte erforderlich ist, oder ob eine solche auch dann gegeben ist, wenn sich die Beschränkung aus den Grundsätzen der im deutschen Urheberrecht geltenden Zweckübertragungslehre ergibt.

Eine räumlich unbegrenzte Rechteeinräumung im Sinne des \137 1 UrhG liegt unproblematisch dann vor, wenn die wesentlichen Nutzungsrechte ausdrücklich weltweit eingeräumt wurden. ${ }^{218} \mathrm{Im}$ Schrifttum wird hierzu vertreten, dass $\ 1371$ UrhG darüber hinaus jedoch auch dann Anwendung finden soll, wenn eine räumliche Beschränkung zwar nicht ausdrücklich vereinbart wurde, sich aber aufgrund der sog. Zweckübertragungslehre ergibt. Dasselbe gelte außerdem für Vereinbarungen, die ausdrücklich eine begrenzte Einräumung vorsehen, sofern sich die Beschränkung auf den Raum beziehe, der sich auch aus den Grundsätzen der Zweckübertragungslehre ergeben würde. ${ }^{219}$ Teilweise wird sogar vertreten, dass

218 J.B. Nordemann in Fromm/Nordemann, \ 1371 Rn. 10.

219 Schulze, UFITA 2007, 641, 689; J.B. Nordemann in Fromm/Nordemann, \137 1 Rn. 10. 
bereits eine auf das Gebiet der Bundesrepublik Deutschland begrenzte Einräumung räumlich unbegrenzt im Sinne des $\ 1371$ Abs. 1 sei. ${ }^{220}$ Diese Ansichten vermögen jedoch aus folgenden Gründen nicht zu überzeugen.

\section{(1) Vorüberlegungen}

Nach dem Grundsatz der Zweckübertragungslehre soll die Einräumung von Nutzungsrechten nur in dem Umfang erfolgen, den der mit dem Vertrag verfolgte Zweck erfordert. ${ }^{221}$ Ausschlaggebend für eine solche Auslegung ist dabei der dem Urheberrecht anhaftende Grundgedanke, den Urheber möglichst weitgehend an den Erträgen der Verwertung beteiligt werden und die Nutzungsrechte aus diesem Grund weitestgehend bei ihm verbleiben sollen. ${ }^{222}$ Bei der Zweckübertragungslehre handelt es sich demnach um einen Auslegungsgrundsatz für den Umfang der durch Vertrag eingeräumten Nutzungsrechte. ${ }^{223}$ Dieser Auslegungsgrundsatz kommt somit auch zum Tragen, wenn es darum geht, den räumlichen Umfang einer Rechteeinräumung zu bestimmen, sofern dies nicht oder nicht hinreichend durch die Parteien erfolgt ist.

\section{(2) Auswirkungen in Bequg auf das Merkmal „räumlich unbegrenzte Rechteeinräu- mung"}

Demzufolge müssen pauschale Formulierungen wie etwa „ohne räumliche Begrenzung“" nach dem mit dem Vertragsschluss verfolgten Zweck ausgelegt werden. ${ }^{224}$ Etwas anderes würde hingegen gelten, wenn die Parteien ausdrücklich eine „weltweite Einräumung“ vereinbart hätten, da dann aufgrund der hinreichenden Spezifikation des Wortlautes kein Platz mehr für eine Auslegung bestünde. Somit kann der Umfang einer Rechteübertragung aufgrund der Zweckübertragungslehre räumlich begrenzt sein, obwohl die Parteien eine „,räumlich unbegrenzte“ Übertragung vereinbart haben.

In Fällen, in denen die Parteien eine unbegrenzte Rechteeinräumung vereinbaren und sich eine Begrenzung erst im Rahmen einer solchen Auslegung ergibt, soll nach Ansicht eines großen Teils der Literatur \ 137 l UrhG trotzdem anwendbar sein. Fraglich ist aber, ob ein solches Ergebnis einer grammatikalischen und teleo-

${ }^{220}$ Jani in Wandtke/Bullinger, $\ 1371$ Rn. 10; Kotthoff in Dreyer/Kotthoff/Meckel, $\ 1371$ Rn. 7;

Berger, GRUR 2005, 907, 911; a.A. Schulze in Dreier/Schulze, \ 1371 Rn. 29, der mindestens eine

Einräumung auf das Gebiet der EU verlangt; ebenso J.B. Nordemann in Fromm/Nordemann, S

1371 Rn. 10.

221 J.B. Nordemann in Fromm/Nordemann, \ 31 Rn. 109 m.w.N.

222 Schulze in Dreier/Schulze, \31 Rn. 110, J.B. Nordemann in Fromm/Nordemann, \31 Rn. 108 m.w.N.

223 BGH GRUR 1998, 680, 682; Kotthoff in Dreyer/Kotthoff/Meckel, $₫ 31$ Rn. 131; J.B. Nordemann in Fromm/Nordemann, \31 Rn. 108.

${ }^{224}$ J.B. Nordemann in Fromm/Nordemann, Urheberrecht, 10. Aufl. 2008, \ 31 Rn. 145. 
Kapitel 1 - Urheberrechtliche Problematik

logischen Auslegung der Norm standhält oder unter Umständen zu unbilligen Ergebnissen führen könnte.

\section{(a) Grammatikalische Auslegung}

Aus dem Wortlaut der Norm lässt sich ein solches Ergebnis zunächst nicht herleiten. Dieser besagt gerade nicht, dass die Parteien eine räumlich unbegrenzte Rechteübertragung vereinbart haben müssen; vielmehr müssen die Rechte tatsächlich räumlich unbegrenzt übertragen worden sein. Der Wortlaut knüpft folglich an den tatsächlichen Umfang der Rechteübertragung und nicht deren Wortlaut an.

Dies bedeutet zum einen, dass eine auf das Gebiet der Bundesrepublik Deutschland begrenzte Einräumung konsequenterweise keine unbegrenzte Einräumung i.S.d. \137 1 Abs. 1 UrhG darstellen kann. Eine solche Auslegung hätte nämlich zur Folge, dass grundsätzlich immer eine räumlich unbegrenzte Einräumung vorläge, da eine Beschränkung des Verbreitungsrechtes innerhalb der BRD aufgrund des Erschöpfungsgrundsatzes schon nicht möglich ist.225 Dies folgt aus $\int$ 17 Abs. 2 UrhG, wonach die Weiterverbreitung des Werkes zulässig ist, sofern das Original oder Vervielfältigungsstücke mit Zustimmung des zur Verbreitung Berechtigten im Gebiet der Europäischen Union im Wege der Veräußerung in Verkehr gebracht wurden. ${ }^{226}$

Darüber hinaus folgt aus dem oben Gesagten auch, dass eine räumlich unbegrenzte Einräumung i.S.d. \137 1 UrhG auch dann nicht vorliegt, wenn die Rechteeinräumung auf ein bestimmtes Gebiet wie beispielsweise das der EU begrenzt ist, sei es aufgrund der ausdrücklichen Parteivereinbarung oder aufgrund einer Auslegung nach den Grundsätzen der Zweckübertragungslehre. ${ }^{227}$ Aus dem Wortlaut von \ 1371 UrhG lässt sich folglich nicht schließen, dass eine aufgrund der Zweckübertragungslehre begrenzte Rechteeinräumung für den Tatbestand der Norm ausreicht. Auf der anderen Seite geht aus dem Wortlaut aber auch nicht eindeutig hervor, dass eine weltweite Rechteeinräumung erforderlich ist.

\section{(b) Teleologische Auslegung}

Auch im Rahmen einer teleologischen Auslegung ergibt sich nichts anderes. Der Sinn und Zweck des $\ 1371$ UrhG besteht darin, die zum Zeitpunkt des Vertragsschlusses unbekannten Nutzungsrechte auf den Vertragspartner übergehen zu lassen, sofern davon auszugehen ist, dass der Urheber dem Vertragspartner auch

\footnotetext{
${ }^{225}$ J.B. Nordemann in Fromm/Nordemann, Urheberrecht, 10. Aufl. 2008, \ 137 1 Rn. 10; Schulze in Dreier/Schulze, Urheberrechtsgesetz, 3. Aufl. 2008, \ 137 1 Rn. 29; ders., UFITA 2007, 641, 689. 226 Vgl. Schulze in Dreier/Schulze, Urheberrechtsgesetz, 3. Aufl. 2008, \ 31 Rn. 31; J.B. Nordemann in Fromm/Nordemann, Urheberrecht, 10. Aufl. 2008, \ 137 1 Rn. 11.

227 So aber Kotthoff in Dreyer/Kotthoff/Meckel, Urheberrecht, 2. Aufl. 2009, \$ 137 1 Rn. 7; Schulze in Dreier/Schulze, Urheberrechtsgesetz, 3. Aufl. 2008, \ 137 1 Rn. 29; J.B. Nordemann in Fromm/Nordemann, Urheberrecht, 10. Aufl. 2008, \ 137 l Rn. 10; Jani in Wandtke/Bullinger, Praxiskommentar zum Urheberrecht, 3. Aufl. 2009, \$ 137 l Rn. 10.
} 
diese Nutzungsrechte übertragen hätte, sofern sie zum Zeitpunkt des Vertragsschlusses ebenfalls bekannt gewesen wären. ${ }^{228}$ Davon kann ausgegangen werden, wenn der Urheber dem Vertragspartner alle wesentlichen Nutzungsrechte ausschließlich sowie räumlich und zeitlich unbegrenzt eingeräumt hat. ${ }^{229}$ Dies gilt aber wiederum gerade nicht, wenn der Urheber mit dem Verlag gar keine Absprache hinsichtlich der räumlichen Beschränkung getroffen hat oder nur pauschal eine „räumlich unbegrenzte“ Rechteübertragung vereinbart wurde. In diesen Fällen liegt nämlich gerade kein Indiz dafür vor, dass der Urheber dem anderen auch die zu diesem Zeitpunkt unbekannten Rechte eingeräumt hätte.

Eine solche stillschweigende oder seinem Wortlaut nach unbegrenzte Rechteeinräumung könnte allerdings auch dafür sprechen, dass der Rechteinhaber dem Vertragspartner auch die Rechte an unbekannten Nutzungsarten eingeräumt hätte. Dabei ist jedoch zu bedenken, dass dem Urheber in Kenntnis der Zweckübertragungslehre durchaus bewusst gewesen sein kann, dass die Vereinbarung nach dem Vertragszweck auszulegen ist und seine Erklärung damit nicht eine weltweite Rechteeinräumung zur Folge hat. Damit lässt sich aus dem Schweigen der Vertragsparteien auf jeden Fall nicht mit genügender Sicherheit schließen, dass der Rechteinhaber auch die unbekannten Nutzungsrechte übertragen wollte. Von daher spricht auch der Sinn und Zweck der Norm dafür, eine räumlich unbegrenzte Rechteeinräumung nur dann anzunehmen, wenn die Rechteeinräumung weltweit erfolgt ist.

\section{(c) Unbilliges Ergebnis}

Darüber hinaus könnte die von der Literatur vertretene Ansicht auch zu unbilligen Ergebnissen führen. Hätte der Urheber nämlich beispielsweise sowohl einem Verlag in den USA als auch einem deutschen Verlag die zum damaligen Zeitpunkt wesentlichen Nutzungsrechte nach der Parteivereinbarung ,räumlich unbegrenzt“ eingeräumt oder hätte es diesbezüglich keine bzw. nur eine stillschweigende Vereinbarung gegeben, so hätten beide Verlage nach der Zweckübertragungslehre die Rechte nur für den Raum erlangt, der zur Erreichung des mit dem jeweiligen Vertrag verfolgten Zwecks erforderlich ist. Unterstellt man, dass dies für den USamerikanischen Verlag das Gebiet der USA, für den deutschen Verlag der deutschsprachige Raum ist, so hätten beide die Nutzungsrechte für diese Gebiete erlangt. Würde man dies jedoch, der Ansicht der Literatur folgend, für die Anwendbarkeit des $\ 1371$ UrhG genügen lassen, hätte dies zur Folge, dass beide Verlage zum 1.1.2009 das Recht der Onlineverwertung des Werkes erlangt hätten. Nach überwiegender Auffassung in der Literatur hätten beide Verlage dieses Recht auch als ausschließliches Recht für das Gebiet erlangt, für das zuvor die

\footnotetext{
228 Siehe dazu bereits oben die Nachweise in Fn. 217.

${ }^{229}$ J.B. Nordemann in Fromm/Nordemann, Urheberrecht, 10. Aufl. 2008, \ 137 1 Rn. 12; vgl. Jani in Wandtke/Bullinger, Praxiskommentar zum Urheberrecht, 3. Aufl. 2009, § 137 1 Rn. 10.
} 
Kapitel 1 - Urheberrechtliche Problematik

wesentlichen Rechte eingeräumt wurden. ${ }^{230}$ Problematisch daran ist aber, dass sich das Recht der Internetnutzung, dass zumindest momentan in den Hauptanwendungsbereich von $\int 1371$ UrhG fällt, nicht bzw. nur schwer territorial begrenzen lässt. ${ }^{231}$ So kann ein Werk, dass beispielsweise in Deutschland im Internet veröffentlicht wird, weltweit abgerufen werden und umgekehrt. Es käme damit zu territorialen Überschneidungen, wenn das Recht der Onlineverwertung als ausschließliches Recht bei unterschiedlichen Inhabern läge. ${ }^{232}$

Dieses Ergebnis versucht ein Teil der Literatur dadurch zu vermeiden, dass die Nutzungsrechte an der neuen Nutzungsart im Zweifel beim Urheber verbleiben sollen, in denen ihre Auswertung nur von einer einzigen Person sinnvoll verwertet werden können. ${ }^{233}$ Damit käme diese Ansicht zwar in einer Vielzahl der einschlägigen Fälle zu dem gleichen Ergebnis, dass auch erzielt würde, sofern man \ 1371 UrhG von vornherein nicht anwendete, steht doch in der überwiegenden Zahl der Fälle gerade das Recht der Onlineverwertung im Mittelpunkt. Damit könnten sich Verlage allerdings fast nie sicher sein, dass der Anwendungsbereich von $\int 1371$ UrhG auf die von ihnen verlegten Werke eröffnet ist. Denn oftmals wird sich gar nicht oder nur schwer feststellen lassen, ob der Urheber noch mit einem Dritten einen Vertrag geschlossen hat, nach dessen Wortlaut die Rechte räumlich unbegrenzt übergehen sollen.

Aus den genannten Argumenten folgt, dass \137 l UrhG nicht einschlägig ist, wenn die Parteien zwar eine räumlich unbegrenzte Rechteeinräumung vereinbart haben, aufgrund der Zweckübertragungslehre die Übertragung der Rechte aber nur für ein bestimmtes Gebiet erfolgt. ${ }^{234}$

iii. Zeitlich unbegrenzte Einräumung der wesentlichen Nutzungsrechte

Schließlich müsste der Rechteinhaber die wesentlichen Nutzungsrechte nicht nur räumlich sondern auch zeitlich unbegrenzt auf die archivierende Einrichtung übertragen haben. Die Fiktion von \137 1 UrhG würde demnach nicht zugunsten der archivierenden Einrichtung greifen, wenn diese zwar alle wesentlichen Nutzungsrechte als ausschließliche Nutzungsrechte ohne räumliche Beschränkung erlangt hätte, diese Rechteübertragung jedoch auf einen bestimmten Zeitraum begrenzt wäre. Dabei ist es ebenfalls als zeitliche Begrenzung anzusehen, wenn die Rechteübertragung nur für eine bestimmte Anzahl von Auflagen erfolgt. ${ }^{235}$ Aus diesem Grund ist im Einzelfall stets zu prüfen, ob die Nutzungsrechtsübertragung aus-

\footnotetext{
${ }^{230}$ J.B. Nordemann in Fromm/Nordemann, Urheberrecht, \ 137 1 Rn. 18; Berger, GRUR 2005, 907, 911; Schulze, UFITA 2007, 641, 692; a.A. Spindler/Heckmann, ZUM 2006, 620, 626.

231 Denkbar wären etwa sog. IP-Filter, wie sie auch von google-books verwendet werden.

232 So auch Schulze in Dreier/Schulze, $\int 137$ 1 Rn. 29; ders., UFITA 2007, 641, 689.

233 Schulze in Dreier/Schulze, $\int 1371$ Rn. 29.

${ }^{234}$ Im Ergebnis wohl auch Schippan, ZUM 2008, 844, 851; zu einem anderen Ergebnis würde man hingegen gelangen, wenn der Vertragspartner nach Spindler/Heckmann, ZUM 2006, 620, 626 die Nutzungsrechte lediglich als einfache Nutzungsrechte erlangen würde.

235 Vgl. Wandtke/Grunert in Wandtke/Bullinger, \ 31 Rn. 11.
} 
drücklich im Vertrag beschränkt worden ist oder sich eine solche Begrenzung unter Umständen bei einer Auslegung der Vereinbarung nach der Zweckübertragungslehre ergibt. $\mathrm{Da}$ in den vorliegenden Fällen jedoch zumeist ein Arbeitsverhältnis zwischen der archivierenden Einrichtung und dem Rechteinhaber bestanden haben wird, wird die Rechteübertragung regelmäßig ohne zeitliche Begrenzung erfolgt sein. 236

\section{iv. Zwischenergebnis}

Damit ist bei der Beantwortung der Frage, ob bei einem Werk alle zum Zeitpunkt des Vertragsschlusses wesentlichen Nutzungsrechte an den Vertragspartner übertragen wurden, zunächst danach zu differenzieren, ob die Übertragung der Rechte vor Bekanntwerden der CD-ROM-Nutzung erfolgt ist oder erst danach. Sofern diese Nutzungsart zum Zeitpunkt des Vertragsschlusses bereits bekannt gewesen ist, ist bei wissenschaftlichen Werken, die periodisch erscheinen, das Recht der CD-ROM-Nutzung grundsätzlich als wesentliches Nutzungsrecht anzusehen, so dass $\ 1371$ UrhG in diesen Fällen nur einschlägig ist, wenn dieses Recht ebenfalls als ausschließliches Recht räumlich und zeitlich unbegrenzt übertragen wurde. Bei Monographien genügt es hingegen, wenn das Verlagsrecht ausschließlich und unbegrenzt übertragen worden ist. Bei wissenschaftlichen Datenbanken oder Datenbankwerken ist hingegen im Einzelfall zu prüfen, ob der Zweck des Vertrags zwischen dem Rechteinhaber und der archivierenden Einrichtung die Publikation dieser Daten erfasst. Nur wenn dies der Fall ist, muss auch bei diesen Werken davon ausgegangen werden, dass sich der Vertragszweck nur durch die Nutzung der Datenbank auf CD-ROM vollumfänglich verwerten lässt. Etwas anders gilt hingegen, wenn der Vertragszweck nicht die Veröffentlichung der Daten, sondern nur die interne Verwendung erfasst, wie etwa der Nutzung für andere Forschungsvorhaben der archivierenden Einrichtung. Dieser Zweck lässt sich nämlich auch ohne Verwertung des Werkes auf CD-ROM erreichen, weshalb das diesbezügliche Recht in diesen Fällen nicht als wesentliches Recht i.S.d. \137 1 UrhG anzusehen ist.

Die von $\ 1371$ UrhG geforderte „räumlich unbegrenzte“ Übertragung ist dabei aber nur dann anzunehmen, wenn die wesentlichen Nutzungsrechte auch tatsächlich unbegrenzt eingeräumt worden sind. Sofern die Parteien eine räumlich unbegrenzte Übertragung vereinbaren, sich aber aus der Zweckübertragungslehre eine räumliche Begrenzung ergibt, scheidet die Anwendbarkeit von \137 l UrhG hingegen aus.

236 Siehe dazu bereits oben S. 59. 
Kapitel 1 - Urheberrechtliche Problematik

v. Konsequenzen für die vorliegenden Fallkonstellationen

Sofern die archivierende Einrichtung sich auf die Rechtsübertragungsfiktion des $\$ 1371 UrhG stützen möchte, muss sie damit zunächst prüfen, ob das Recht der Nutzung des Werkes auf CD-ROM im konkreten Einzelfall zu den „wesentlichen Nutzungsrechten“ i.S.d. \ 1371 UrhG zu rechnen ist. Bei den vorliegend im Fokus stehenden Datenbanken und Datenbankwerken wird man dabei in der Regel davon ausgehen können, dass die Erreichung des Vertragszwecks nicht die Verwertung des Werkes auf CD-ROM erfordert, so dass es grundsätzlich nur darauf ankommt, ob das Recht der Vervielfältigung in analoger Form sowie das Recht der Verbreitung auf die archivierende Einrichtung in dem genannten Umfang übergegangen sind. Dabei ist in den vorliegenden Fällen zu berücksichtigen, dass die Rechteinhaber der zu archivierenden Werke in der Regel in einem Angestelltenverhältnis zur archivierenden Einrichtung gestanden haben werden. Aufgrund dieses Arbeitsverhältnisses werden die genannten Rechte, mit Ausnahme der Fälle, in denen der Rechteinhaber Hochschulprofessor und die archivierende Einrichtung die Hochschule ist, an der er tätig war, ${ }^{237}$ regelmäßig bereits aufgrund des Arbeitsverhältnisses als ausschließliche Nutzungsrechte ohne zeitliche Beschränkung zur weltweiten Nutzung auf die archivierende Einrichtung übergegangen sein.

Damit bliebe letztlich nur zu prüfen, ob der Rechteinhaber gegenüber der archivierenden Einrichtung rechtzeitig den Widerspruch nach $\int 1371$ Abs.1 S. 1 Hs. 2 UrhG erklärt hat oder die Onlinenutzungsrechte gem. \137 1 Abs. 1 S. 4 UrhG nach deren Bekanntwerden aber vor Inkrafttreten der Norm am 1.1.2008 auf einen Dritten übertragen hat.

Sofern dies nicht der Fall ist, hätte die archivierende Einrichtung das Recht der Onlinenutzung des Werkes gem. \137 1 Abs. 1 UrhG als ausschließliches Nutzungsrecht erlangt. ${ }^{238}$

\section{vi. Anspruch auf angemessene Vergütung}

Sofern der Rechteinhaber der Universität die benötigten Rechte überträgt, hat er gem. \ 32 Abs. 1 Satz 1 UrhG einen Anspruch auf die vereinbarte Vergütung. Sofern die Höhe der Vergütung nicht bestimmt ist, gilt gem. \32 Abs. 1 Satz 2 UrhG die angemessene Vergütung als vereinbart. Wann dies der Fall ist, regelt wiederum \32 Abs. 2 Satz UrhG. Danach ist die Vergütung dann angemessen, wenn sie im Zeitpunkt des Vertragsschlusses dem entspricht, was im Geschäftsverkehr nach Art und Umfang der eingeräumten Nutzungsmöglichkeit, insbesondere nach Dauer und Zeitpunkt der Nutzung üblicherweise zu leisten ist. Erfolgt die Übertragung der Nutzungsrechte jedoch aufgrund einer Verpflichtung aus dem Dienst- oder Arbeitsvertrag, so ist die spätere Verwertung des Werkes durch

\footnotetext{
237 Siehe oben S. 55.

238 Jani in Wandtke/Bullinger, $\ 1371$ Rn. 24; a.A.: Spindler/Heckmann, ZUM 2006, 620, 626.
} 
den Arbeitgeber jedoch bereits bei der Vereinbarung des Arbeitslohnes berücksichtigt worden. ${ }^{239}$ Der Arbeitnehmer hat in diesem Fall somit keinen zusätzlichen Vergütungsanspruch für die Übertragung der Nutzungsrechte. Dies gilt, wie oben dargelegt, auch dann, wenn aufgrund des Arbeitsvertrags Rechte an solchen Nutzungsarten auf eine öffentlich-rechtliche Forschungseinrichtung übergegangen sind, die zum Zeitpunkt des Vertragsschlusses noch unbekannt waren. Bei privatrechtlichen Forschungseinrichtungen kommt dagegen eine rechtsgeschäftliche Übertragung von Rechten an unbekannten Nutzungsarten von vornherein nicht in Betracht, da die Vorschrift des $₫ 31$ Abs. 4 a.F. UrhG auf diese Anwendung findet. Sofern die Forschungseinrichtung in diesen Fällen die Onlinenutzungsrechte nach $\ 1371$ Abs. 1 UrhG erlangt, ist zu beachten, dass dem Rechteinhaber eine Vergütungsanspruch nach $\ 137$ l Abs. 5 UrhG zusteht. Dieser kann jedoch gem. \ 1371 Abs. 5 S. 3 UrhG nur durch eine Verwertungsgesellschaft und nicht den Rechteinhaber selbst geltend gemacht werden.

\section{vii. Form des Vertrags}

Sofern mit dem Rechteinhaber ein entsprechender Lizenzvertrag geschlossen wird, weil die archivierende Einrichtung die erforderlichen Nutzungsrechte nicht anderweitig erlangen kann, stellt sich für sie die Frage, welchen formalen Anforderungen ein solcher Vertrag entsprechen muss. Dabei gilt im Zivilrecht der allgemeine Grundsatz, dass Verträge keiner besonderen Form bedürfen. ${ }^{240}$ Eine Ausnahme findet sich jedoch in $₫ 40$ Abs. 1 Satz 1 UrhG. Danach bedarf ein Vertrag, durch den sich der Urheber zur Einräumung von Nutzungsrechten an künftigen Werken verpflichtet, die überhaupt nicht näher oder nur der Gattung nach bestimmt sind, der schriftlichen Form. Was unter der Schriftform zu verstehen ist, definiert \126 BGB. Aus Gründen der Rechtssicherheit sollte aufgrund der höheren Beweiskraft aber nach Möglichkeit stets ein schriftlicher Vertrag geschlossen werden, da die archivierende Einrichtung im Falle einer Rechteverwertung und eines damit unter Umständen einhergehenden Rechtsstreits den Beweis dafür erbringen müsste, dass sie die Inhaberin der erforderlichen Rechte ist.

\section{e. Formulierungsvorschlag für eine Nutzungsrechtsvereinbarung}

Beim Abschluss einer Rechtevereinbarung ist zu beachten, dass eine in der Praxis häufig verwendete Formulierung nach der ,der Urheber/Datenbankhersteller alle an dem Werk bestehenden Nutzungsrechte an die Universität abtritt“ für den vorliegenden Zweck ungenügend wäre, da es an der erforderlichen Bestimmtheit

\footnotetext{
${ }^{239}$ Dreier/Schulze-Dreier, $₫ 43$ Rn 30; A. Nordemann in Fromm/Nordemann, $₫ 43$ Rn. 58; Rojahn in Schricker/Loewenheim, \ 43 Rn. 64; Kolle, GRUR 1985, 1016, 1024; Sundermann, GRUR 1988, 350, 354; a.A. Wandtke in Wandtke/Bullinger, \ 43 Rn. 138.

240 Scbulze in Dreier/Schulze, $₫ 31$ Rn. 22; Abrens in Prütting/Wegen/Weinreich, BGB Kommentar, 6. Aufl. 2011, $₫ 125$ Rn. 1.
} 
Kapitel 1 - Urheberrechtliche Problematik

mangeln würde. ${ }^{241}$ Sind bei der Einräumung eines Nutzungsrechts die Nutzungsarten nämlich nicht ausdrücklich bezeichnet, so bestimmt sich gem. \31 Abs.5 Satz 1 UrhG nach dem von beiden Partnern zugrunde gelegten Vertragszweck, auf welche Nutzungsarten es sich erstreckt. ${ }^{242}$ Aus diesem Grund sollte die Vereinbarung möglichst genau beschreiben, auf welche Nutzungsarten sich die Rechteeinräumung erstreckt, um spätere Auseinandersetzungen über die Zulässigkeit einer Nutzung von vornherein auszuschließen. Dabei ist zu unterscheiden, ob die Rechte für ein bereits hergestelltes Werk übertragen werden sollen oder für erst in der Zukunft entstehende Werke. Bei Letzterem bietet es sich an, eine entsprechende Klausel in die standardmäßig verwendeten Arbeitsverträge der betroffenen Forschungseinrichtungen einzufügen, sofern dieses nicht schon geschehen ist.

f. Formulierungsvorschlag für bereits hergestellte Werke

Im Folgenden soll kurz dargestellt werden, wie eine Vereinbarung über die Übertragung bereits hergestellter Werke formuliert werden könnte:

Zwischen

\section{Lizenzvertrag}

Herrn/Frau <Name des Urhebers/Datenbankherstellers etc. $>$ -im Folgenden „Lizenzgeber“-

und der Universität Göttingen, vertreten durch den Präsidenten $<$ Name des amtierenden Universitätspräsidenten>

- im Folgenden „Lizenznehmerin“-

wird folgender Vertrag geschlossen:

Vertragsgegenstand

Gegenstand dieser Vereinbarung ist die Übertragung der in \ 2 benannten Nutzungsrechte an dem im Anhang befindlichen Werk (siehe Anlage). Anmerkung: Da es sich in den vorliegenden Fällen zumeist um Datenbanken handeln wird, die nicht obne weiteres anhand ibres Titels identifiziert werden können, empfieblt es sich, einen Ausdruck des jeweiligen Werkes als Anlage dem Vertrag beizufügen.

$\int 2$

Rechteübertragung

2.1 Der Lizenzgeber räumt der Lizenznehmerin das Recht der Vervielfältigung, insbesondere der Speicherung auf Datenträgern (insbes. CD-ROM, DVD, HD-

\footnotetext{
241 BGH GRUR 1996, 121, 122 - Pauschale Rechtseinräumung, J.B. Nordemann in

Fromm/Nordemann, \31 Rn. 123; Schulze in Dreier/Schulze, \ 31 Rn. 111; Schricker/Loewenheim in Schricker/Loewenheim, \ 31 Rn. 69.

242 Siehe zur Zweckübertragungslehre bereits die Darstellungen auf den S. 56 ff.
} 
DVD, Blu-Ray-Disc) und in Datenbanken, der Bearbeitung des Werkes, insbesondere der Umwandlung in ein anderes Format, auch wenn dies qualitative Einschränkungen zur Folge haben sollte, und der öffentlichen Zugänglichmachung des Werkes, insbesondere in offenen und geschlossenen elektronischen Netzwerken, sowie das Recht, das Werk zum Download und Ausdruck durch Dritte bereitzuhalten, ein.

2.2 Die in $\int 2.1$ bezeichneten Rechte werden der Lizenznehmerin ohne räumliche Beschränkung für die Dauer des gesetzlichen Urheberrechtsschutzes zum Zwecke der Langzeitarchivierung und des nicht kommerziellen wissenschaftlichen Austauschs als einfache Nutzungsrechte eingeräumt.

2.3 Der Lizenzgeber stimmt einer Übertragung der bezeichneten Rechte an Dritte durch die Lizenznehmerin zu.

Rechte Dritter

3.1 Der Lizenznehmer erklärt, dass er über die in $\int 2$ benannten Rechte verfügen darf und durch die Rechtseinräumung keine Rechte Dritter verletzt werden.

3.2 Sollten durch die Einräumung der Rechte oder die Ausübung durch die Lizenznehmerin dennoch Rechte verletzt werden, so verpflichtet sich der Lizenzgeber, die Lizenznehmerin von Ansprüchen Dritter, die aufgrund dieser Verletzung entstehen, freizustellen.

\section{$\int 4$ \\ Vergütungsanspruch}

Der Lizenzgeber erhält für die Einräumung der oben bezeichneten Rechte eine Vergütung von <Höhe der Vergütung>. Eine Regelung, wonach kein Vergütungsanspruch besteht, wäre gem. $\int 32$ Abs. 3 Satz 1 UrhG unwirksam.

$<$ Unterschrift der Vertragsparteien $>$

g. Formulierungsvorschlag für künftige Werke

Sofern die zu archivierenden Werke erst noch entstehen sollen, bietet es sich an, eine entsprechende Klausel von vornherein in den Arbeitsvertrag aufzunehmen. Dies hat zum einen den Vorteil, dass man den Lizenzgeber eher zur Übertragung von Nutzungsrechten bewegen kann, da er ja ein grundsätzliches Interesse am Zustandekommen des Arbeitsverhältnisses hat. Somit wäre es unter Umständen sogar möglich, die Nutzungsrechte als ausschließliche Nutzungsrechte zu erlangen. Zum anderen folgt die Pflicht zur Übertragung zwar, wie bereits dargestellt, grundsätzlich aus dem Arbeitsverhältnis. Da jedoch, wie oben ausgeführt, die 
Kapitel 1 - Urheberrechtliche Problematik

Rechteübertragungen stets nach der sog. Zweckübertragungsregel auszulegen sind, bringt eine ausdrückliche Rechteübertragungsklausel im Arbeitsvertrag für alle Beteiligten ein erhöhtes $\mathrm{Maß}$ an Rechtssicherheit mit sich, da nicht immer Einigkeit darüber herrschen wird, welche Rechte die archivierende Einrichtung zur Erfüllung ihres betrieblichen Zwecks benötigt. ${ }^{243}$ Darüber hinaus, besteht die Pflicht zur Übertragung der Nutzungsrechte aufgrund des bloßen Bestehens eines Arbeits- oder Dienstverhältnisses nicht für Hochschulprofessoren und unter bestimmten Umständen auch nicht für wissenschaftliche Mitarbeiter. Insofern bietet sich eine Klausel, durch die die zur Archivierung erforderlichen Nutzungsrechte explizit eingeräumt werden, durchaus an. Die gleiche Vorgehensweise könnte beim Abschluss mit Dritten favorisiert werden, die ein bestimmtes Forschungsprojekt finanzieren, da aufgrund derartiger Drittmittelfinanzierungen -wie oben dargestellt- die finanzierende Einrichtung zumeist als Datenbankhersteller i.S.d. \$ 87a UrhG anzusehen sein wird, ${ }^{244}$ sofern im Rahmen des Projektes schutzfähige Datenbanken entstehen.

Für eine derartige Klausel bietet sich folgende Formulierung an:

\title{
Rechteeinräumung
}

Abs. $1<$ Name des Vertragspartners $>$ überträgt $<$ Name der Einrichtung $>$ die im Rahmen seiner Tätigkeit/im Rahmen des Projektes (Kommentar: hier kommt es darauf an, ob die Klausel in einen Arbeitsvertrag oder in einen Vertrag mit einer finanzierenden Institution eingefügt werden soll) entstehenden Nutzungsrechte an den entstehenden Werken oder Datenbanken an die Universität Göttingen. Dies gilt insbesondere für das Recht der Vervielfältigung, insbesondere der Speicherung auf Datenträgern (insbes. CD-ROM, DVD, HD-DVD, Blu-Ray-Disc) und in Datenbanken, der Bearbeitung des Werkes, insbesondere der Umwandlung in ein anderes Format, auch wenn dies qualitative Einschränkungen zur Folge haben sollte, und der öffentlichen Zugänglichmachung des Werkes, insbesondere dieses in offenen und geschlossenen elektronischen Netzwerken zum Download und Ausdruck durch Dritte bereitzuhalten.

\begin{abstract}
Abs.2 Die in $\int 1.1$ bezeichneten Rechte werden der Universität Göttingen ohne räumliche Beschränkung für die Dauer des gesetzlichen Urheberrechtsschutzes zum Zwecke der Langzeitarchivierung und des nicht kommerziellen wissenschaftlichen Austauschs als einfache Nutzungsrechte eingeräumt. (Kommentar: Natürlich wäre es auch möglich, eine weitergehende Rechteübertragung zu vereinbaren. Allerdings bestünde dann die Gefahr, dass der Vertragspartner von der Unterzeichnung abgeschreckt würde.)
\end{abstract}

\footnotetext{
${ }^{243}$ Vgl. Dreier in Dreier/Schulze, \43 Rn. 20; Rojabn in Schricker/Loewenheim, $\int 43$ Rn. 52 f.; $A$. Nordemann in Fromm/Nordemann, \43 Rn. 27, 45; einschränkend hingegen Zöllner in FS Hubmann, S. 523, 531.

${ }^{244}$ Siehe dazu die Ausführungen auf S. 21.
} 
Abs.3 Der Lizenzgeber stimmt einer Übertragung der bezeichneten Rechte an Dritte durch die Lizenznehmerin zu.

\section{Ergebnis}

Wie die obigen Ausführungen zeigen, wird in den Fällen der digitalen Langzeitarchivierung von wissenschaftlichen Daten, die einem urheber- oder leistungsrechtlichen Schutz unterliegen, nur selten eine gesetzliche Schranke zu Gunsten der archivierenden Einrichtung greifen. Dies liegt in erster Linie daran, dass die Dateien in der Regel auch Wissenschaftlern, die nicht an der archivierenden Einrichtung beschäftigt sind, zugänglich gemacht werden sollen, um so im Rahmen von internationalen und nationalen Kooperationen Daten und wissenschaftlichen Erkenntnisse auszutauschen. Ein solcher Austausch ist aber für Forschungseinrichtungen notwendig, um auf wissenschaftlicher Ebene konkurrenzfähig zu bleiben. Dies ist gerade in Zeiten von existenzieller Bedeutung, in denen Forschungsprojekte oftmals nur mit Hilfe von Drittmittelgebern realisiert werden können. Von daher müsste von Seiten des Gesetzgebers die Schranke des \53 Abs. 2 UrhG dahingehend erweitert werden, dass die Archivierung nicht nur für eigene Zwecke erfolgen darf, sondern die archivierten Daten auch anderen Wissenschaftlern zur Verfügung gestellt werden dürfen. Eine ähnliche Problematik besteht für Gedächtnisinstitutionen, wie der Deutschen Nationalbibliothek, die zwar urheberrechtlich geschützte Werke archiviert, diese jedoch nicht öffentlich zugänglich machen darf. Zu beachten ist dabei jedoch, dass die Einschränkung von \53 Abs. 5 UrhG auf der europäischen Datenbankrichtlinie beruht und der nationale Gesetzgeber daher in seinem Entscheidungsspielraum beschränkt ist. Von daher ist nicht anzunehmen, dass der nationale Gesetzgeber eine Schranke schafft, kraft derer auch Datenbankwerke zu Archivzwecken vervielfältigt und öffentlich zugänglich gemacht werden dürfen. Da in den Fällen von wissenschaftlichen Primärdaten jedoch in den seltensten Fällen ein Datenbankwerk entstehen wird, ${ }^{245}$ wird \53 Abs. 5 UrhG wohl ohnehin kaum eine praktische Rolle für die Langzeitarchivierung wissenschaftlicher Primärdaten spielen.

Änderungsbedarf besteht aus Sicht von Archiven und wissenschaftlichen Forschungseinrichtungen wohl auch hinsichtlich der Schranke des $\int 87$ c UrhG, da dieser sich nur auf wesentliche Teile der Datenbank bezieht, jedoch nicht die gesamte Datenbank erfasst. Da auch diese Norm der Umsetzung der Datenbankrichtlinie dient, wäre aber auch hier ein Handeln auf europäischer Ebene erforderlich, da der nationale Gesetzgeber an die Richtlinie gebunden ist und diese eine Vervielfältigung der gesamten Datenbank gerade nicht vorsieht. Abzuwarten bleibt, wie sich die Antworten auf das Grünbuch „Urheberrechte in der wissens-

245 Siehe oben S 15. 
Kapitel 1 - Urheberrechtliche Problematik

bestimmten Gesellschaft" der Europäischen Kommission ${ }^{246}$ in diesem Zusammenhang auswirken werden. Letztendlich wäre aber eine Archivierung der Datenbanken und der öffentlichen Zugänglichmachung von Datenbanken aufgrund der nach $\int 87 \mathrm{~d}$ UrhG beschränkten Schutzdauer nach 15 Jahren möglich, sofern die Datenbank nicht in ihrem Inhalt nach Art oder Umfang wesentlich geändert wird und damit gem. \87a Abs. 1 S. 2 UrhG eine neue Datenbank entsteht. Damit bleibt festzuhalten, dass in den Fällen, in denen an wissenschaftlichen Primärdaten ein urheberrechtlicher- oder leistungsrechtlicher Schutz entsteht und die archivierende Einrichtung nicht Rechteinhaberin ist und auch keine Verpflichtung des Rechteinhabers zur Übertragung der Nutzungsrechte besteht, eine Gesetzesänderung aufgrund der europäischen Vorgaben nur schwer möglich ist. Forschungseinrichtungen sollten daher versuchen, sich frühzeitig die benötigten Rechte einräumen zu lassen und ihre Arbeitsverträge entsprechend gestalten um möglichen Missverständnissen von vornherein vorzubeugen.

\section{Rechtliches Verhältnis zwischen archivierender Einrichtung und Nutzern der Daten}

Wie bereits oben dargelegt wurde, werden wissenschaftliche Forschungseinrichtungen in der Regel nicht nur ein Interesse an der Archivierung der erlangten Daten haben. Vielmehr werden die meisten Einrichtungen bemüht sein, die Daten auch anderen Wissenschaftlern und Forschungseinrichtungen, sei es entgeltlich oder unentgeltlich, zur Verfügung zu stellen, da letztlich nur durch einen solchen Informationsaustausch ein effektives wissenschaftliches Arbeiten möglich ist. Dabei werden die Einrichtungen jedoch bemüht sein, dass die Daten nicht unkontrolliert für jedermann abrufbar sind. Dies folgt zum einen aus dem Bedürfnis, einem eventuellen Missbrauch dieser Daten vorzubeugen. Zum anderen besteht auch in datenschutzrechtlicher Hinsicht eine Verpflichtung der archivierenden Einrichtung, dafür Sorge zu tragen, dass personenbezogene Daten nicht in unbefugte Hände gelangen. ${ }^{247}$ Um einer Weitergabe von abgerufenen Daten durch Fremdforscher entgegenzuwirken, kommen dabei zunächst technische Schutzmechanismen wie etwa sog. Digital Rights Management Systeme (DRMS) in Betracht, die dem Fremdforscher die Weitergabe der Daten erschweren bzw. dafür sorgen, dass der Empfänger die Dateien nicht verwerten kann. Darüber hinaus ist jedoch vor allem entscheidend, welche rechtlichen Möglichkeiten der archivierenden Einrichtung zur Verfügung stehen, wenn ein Fremdforscher Daten weitergibt oder derartige technische Sicherungsmaßnahmen aufhebt oder umgeht.

\footnotetext{
$246 \operatorname{KOM}(2008)$ 466/3; abrufbar unter: http://ec.europa.eu/internal_market/copyright/docs/copyright-infso/greenpaper_de.pdf (Stand: 02.12.2011).

247 Dazu unten S. 144 ff.
} 


\section{Technische Schutzmaßnahmen}

Hinsichtlich technischer Schutzmechanismen käme die Verwendung eines Digital Rights Management Systems in Betracht. Mit Hilfe dieser Systeme könnten die Dateien auf der einen Seite zwar Fremdforschern zur Verfügung gestellt und durch diese auch genutzt werden, sofern dies im Sinne der jeweiligen archivierenden Einrichtung ist. Auf der anderen Seite könnte aber verhindert werden, dass diese Fremdforscher die Dateien unbefugt kopieren und an Dritte weiterleiten. Mit Hilfe von DRM-Systemen kann also sowohl der Zugang zur jeweiligen Datei kontrolliert werden als auch die Nutzung der Datei selbst, wozu beispielswiese auch Kopierschutzsysteme gehören. ${ }^{248}$

\section{Funktionsweise von DRM-Systemen}

Eine Zugangskontrolle kann zum einen durch entsprechende Kontrollmechanismen an der Datenquelle erreicht werden. ${ }^{249}$ Dies wäre in den vorliegenden Fällen die Internetplattform, auf der die archivierten Dateien anderen Forschern und Forschungseinrichtungen zum Download bereitgestellt werden. Der Abruf der archivierten Daten müsste dabei so gestaltet werden, dass diese nicht von Jedermann von der Onlineplattform der archivierenden Einrichtung heruntergeladen werden können, sondern der jeweilige Nutzer sich zunächst mittels eines Benutzernamens und eines Passwortes anmelden müsste um Zugang zu den archivierten Daten zu erhalten. Solche Systeme werden beispielsweise auch von Anbietern digitaler Zeitschriften oder Musikdateien verwendet. Da die entsprechenden Zugangsdaten vom Langzeitarchivierungsverbund vergeben würden, könnte man auf diesem Weg darüber hinaus sicherstellen, dass bestimmte Daten nur ausgewählten Nutzern zur Verfügung stehen. Dies ist insbesondere für personenbezogene medizinische Daten relevant. ${ }^{250}$ Zusätzlich käme eine Verschlüsselung der archivierten Daten mittels eines kryptographischen Verfahrens in Betracht. Dabei wird der Inhalt der Bits der jeweilige Datei mittels eines bestimmten Algorithmus so verändert, dass der ursprüngliche Inhalt der Datei nicht mehr gelesen werden kann. ${ }^{251}$ Damit der Empfänger der Datei den Inhalt lesen kann, müsste er diese zunächst mit Hilfe eines entsprechenden Schlüssels zurücktransformieren und so die Originalinformation wiederherstellen. ${ }^{252} \mathrm{Die}$ archivierende Einrichtung könnte damit durch die Vergabe des jeweiligen Schlüssels kontrollieren, wer die Datei öffnen kann. Die Vergabe des Schlüssels müsste wiederum nicht manuell erfolgen. Vielmehr könnte sowohl die Verschlüsselung als auch Entschlüsselung durch einen

\footnotetext{
${ }^{248}$ Arlt, Digital Rights Management Systeme, 2006, S. 15 f.

${ }^{249}$ Arlt, S. 25.

250 Siehe dazu unten S. 165 ff.

251 Trayer, Technische Schutzmaßnahmen und elektronische Rechtewahrnehmungssysteme, 2003, S. 34; Hornung, Die digitale Identität, 2006, S. 70 f.

252 Zum Unterschied zwischen asymmetrischen und symmetrischen Verschlüsselungsverfahren siehe oben S. 197 ff. sowie Hornung, S. 71m.w.N.
} 
Lizenzserver verwaltet werden. ${ }^{253}$ Sobald die Datei jedoch einmal entschlüsselt ist, wären das Anfertigen einer Kopie und deren Weitergabe problemlos möglich, so dass auch hiergegen ein Schutzmechanismus eingebaut werden muss. Dies könnte erreicht werden, indem die Dateien zusätzlich mit einem elektronischen Kopierschutz versehen werden, so dass nicht nur das Öffnen der Datei sondern auch ihre spätere Nutzung durch die archivierende Einrichtung kontrolliert werden könnte. Solche Kopierschutzmaßnahmen sind vor allem aus der Musik- und Filmindustrie bekannt. ${ }^{254}$ Durch Verwendung der genannten Kopierschutzmechanismen könnte verhindert werden, dass Fremdforscher, die zum Download der archivierten Datei befugt sind, selbige unbefugt kopieren und weitergeben. Darüber hinaus kann auch festgelegt werden, dass die Datei nur bis zu einer begrenzten Anzahl kopiert werden kann. Sofern der Fremdforscher versucht, die Datei zu kopieren, werden zunächst die Metadaten, also die Zusatzinformationen gelesen, aus denen hervorgeht, ob bzw. wie oft die Datei kopiert werden darf. Die Kopie wird nur dann erstellt, wenn dies nach besagten Zusatzinformationen erlaubt ist bzw. die Maximalanzahl der erlaubten Kopien noch nicht erreicht ist. ${ }^{255}$

Schließlich kann die Datei zusätzlich mit einem sogenannten digitalen Wasserzeichen versehen werden. Dabei wird der digitale Inhalt der Datei derart mit Metadaten verknüpft, dass eine anschließende Entfernung des Wasserzeichens nicht mehr möglich ist, ohne den digitalen Inhalt zu beschädigen. ${ }^{256}$ Diese Metadaten sind für das menschliche Auge oder Ohr nicht wahrnehmbar und geben, wenn sie ausgelesen werden, unter anderem Auskunft über den Urheber bzw. den Inhaber verwandter Schutzrechte geben. Durch Verwendung derartiger Wasserzeichen kann eine unbefugte Weitergabe der Daten zwar nicht unmittelbar verhindert werden. Allerdings können digitale Wasserzeichen als Beweis dienen, wenn in Frage steht, ob die archivierende Einrichtung wirklich Inhaberin der geltend gemachten Rechte ist. Entscheidende Bedeutung hat dies vor allem bei der Geltendmachung von Unterlassungs- und Beseitigungsansprüchen, auf die im Folgenden noch eingegangen wird. ${ }^{257}$

\section{Rechtlicher Schutr.}

Sofern die archivierende Einrichtung ihre Datenbanken oder Datenbankwerke nach den gerade geschilderten Methoden schützt, stellt dies eine technische Maßnahme im Sinne des $\int 95 a$ Abs. 1 UrhG dar. Dies hätte zur Folge, dass die technische Schutzmaßnahme durch den Nutzer der Daten nicht umgangen werden dürf-

\footnotetext{
253 Wolter, JurPC Web-Dok. 160/2008, Abs. 20.

254 Gottschalk, GRUR Int. 2002, 95, 104 f.; Arlt, S. 18.

255 Bechtold, Vom Urheber- zum Informationsrecht, 2002, S. 34; Arlt, S. 15 f.

256 Dittman, Digitale Wasserzeichen, 2000, S. 2; Bechtold, S. 54.

257 Siehe unten S. 133.
} 
te. Sofern ein Dritter dies dennoch täte, stünde dies einer Verletzung urheberrechtlicher Nutzungsrechte gleich ${ }^{258}$.

\section{Zwischenergebnis}

Die Weitergabe der Daten durch Fremdforscher kann also zum einen durch technische Maßnahmen erreicht werden. Zunächst kann durch die Zuweisung von Nutzerdaten, anhand derer sich der Bezieher der gewünschten Daten beim Onlineportal der archivierenden Einrichtung identifizieren und anmelden muss und ohne die ein Download der Dateien nicht möglich ist, erreicht werden, dass Unbefugte nicht auf den Bestand der archivierten Daten zugreifen können. Auf der anderen Seite müssen die benötigten Daten auch nicht manuell verschickt werden, sondern die registrierten Nutzer können sich die benötigten Dateien unter Verwendung ihrer Nutzerdaten selbst herunterladen. Die Verhinderung der unbefugten Weitergabe der Dateien wird dadurch allein jedoch noch nicht erreicht. Dies ist jedoch insbesondere mit Hilfe von Kopierschutzmaßnahmen im Rahmen von DRM-Systemen möglich. Darüber hinaus können die Daten mit sog. digitalen Wasserzeichen versehen werden, durch den die archivierende Einrichtung im Falle der prozessualen Geltendmachung eines Beseitigungs-, Unterlassungs- oder Schadensersatzanspruches einen Beweisvorteil hinsichtlich der Rechteinhaberschaft erlangen würde.

\section{Rechtlicher Schutz vor der Weitergabe durch Fremdforscher}

Neben der technischen Komponente ist ferner entscheidend, ob und wie der Langzeitarchivierungsverbund der Weitergabe der Dateien rechtlich vorbeugen bzw. entgegentreten kann. Da an den einzelnen Daten oder Datenpaketen grundsätzlich kein urheber- oder leistungsrechtlicher Schutz entstehen wird, muss der LZA-Verbund versuchen, durch vertragliche Vereinbarungen mit den Nutzern der Daten die unbefugte Weitergabe zu unterbinden. Eine solche Vereinbarung hätte aufgrund der nur relativen Wirkung von Schuldverhältnissen zwar keine Wirkung gegenüber Dritten, die die Daten vom Fremdforscher erlangen. Die archivierende Einrichtung könnte jedoch gegebenenfalls aufgrund vertraglicher Ansprüche Rückgriff bei dem vertragsbrüchigen Fremdforscher nehmen. Dabei kommt vor allem der Abschluss eines Lizenzvertrages zwischen der archivierenden Einrichtung und den einzelnen Fremdforschern in Betracht. Daneben besteht die Möglichkeit, dass einzelne Daten bzw. Datenpakete einem urheber- oder leistungsrechtlichen Schutz nach den Vorschriften des UrhG unterliegen. ${ }^{259}$

\footnotetext{
258 Siehe dazu S. $133 \mathrm{ff}$.

${ }^{259}$ Dazu siehe oben Frage 1 S. 6 ff.
} 


\section{Weitergabeverbot aufgrund vertraglicher Vereinbarung}

Im Rahmen eines Lizenzvertrages könnte die archivierende Einrichtung mit den Fremdforschern eine Verschwiegenheitsklausel vereinbaren, die diesen die Weitergabe der erlangten Daten untersagt. Zur effizienten Durchsetzung dieser Verschwiegenheitsklausel sollte ferner eine Vertragsstrafe für den Fall eines Verstoßes vorgesehen werden. ${ }^{260}$

Wie oben bereits im Rahmen der technischen Schutzmechanismen festgestellt, müsste der Langzeitarchivierungsverbund den Abruf der Daten nicht Jedermann gestatten, sondern könnte den Abruf der Daten auf diejenigen Fremdforscher beschränken, die einem entsprechenden Lizenzvertag zugestimmt hätte und damit von der archivierenden Einrichtung die entsprechenden Zugangsdaten zur Verfügung gestellt bekommen würde. Im Rahmen eines solchen Lizenzvertrages könnte die Einrichtung dem Fremdforscher zum einen das Recht einräumen, die Daten abzurufen und im Rahmen seiner wissenschaftlichen Tätigkeit zu verwenden. $\mathrm{Ob}$ der Abruf dabei unentgeltlich erfolgen soll oder ob eine entsprechende Nutzungsgebühr beim Abruf der Daten fällig wird, ist dabei für die rechtliche Würdigung einer solchen Vereinbarung zunächst nicht von Belang. Zum anderen könnte der jeweilige Partner eines solchen Lizenzvertrags durch Einfügen einer Verschwiegenheitsklausel dazu verpflichtet werden, die Forschungsdaten nicht bzw. nur innerhalb seines Forschungsprojektes weiterzugeben. Derartige Klauseln sind vor allem im Rahmen von sog. „Know-how-Verträgen“, aber auch Forschungs- und Entwicklungsverträgen üblich. ${ }^{261}$

Darüber hinaus müssten für den Fall der Verletzung einer solchen Klausel, entsprechende Konsequenzen vereinbart werden. Diese müssten dabei eine abschreckende Wirkung auf den Vertragspartner haben, so dass dieser vor einem Verstoß gegen die Vereinbarung zurückschrecken würde. Dies könnte durch Vereinbarung einer durch den Fremdforscher im Falle des Verstoßes zu zahlenden Vertragsstrafe bewirkt werden. Dadurch würde zum einen erreicht, dass der Fremdforscher zur Einhaltung der Verschwiegenheitsverpflichtung angehalten wird, zum anderen würde der archivierenden Einrichtung für den Fall der unbefugten Weitergabe durch den Vertragspartner die Schadloshaltung erleichtert werden. ${ }^{262} \mathrm{Im}$ Fall einer Vertragsverletzung stünden der Einrichtung unter Umständen zwar auch unabhängig von einer solchen Klausel Schadensersatzansprüche gegen den Fremdforscher nach $\ 280$ I S. 1 BGB zu. Allerdings müsste sie aufgrund der Beweislastverteilung im Zivilprozess den ihr entstandenen Schaden nachweisen, ${ }^{263}$ der allerdings teilweise nur schwer zu beziffern sein wird. Mitunter kann es sogar gänzlich an einem materiellen Schaden fehlen. Bei Vereinbarung

\footnotetext{
${ }^{260}$ Vgl. Möffert in Schütze/Weipert (Hrsg.), Münchener Vertragshandbuch, Bd. 3 Wirtschaftsrecht II, 6. Aufl. 2009, IV. 2 Anm. 18.

261 Vgl. Möffert in Schütze/Weipert, IV. 1.

262 BGH NJW 1983, 385, 387; Medicus in Schwab/Wegen/Weinreich, Vor \$S 339 bis 345 Rn. 1.

263 Musielak in Musielak, ZPO. 8. Aufl. 2011, Einl. Rn. 37.
} 
einer Vertragsstrafe müsste die Einrichtung hingegen lediglich die unbefugte Weitergabe durch den Fremdforscher nachweisen.

Neben der Vereinbarung einer Vertragsstrafe empfiehlt sich aus Sicht der archivierenden Einrichtung die Verwendung einer Freistellungsklausel, kraft derer der Forscher verpflichtet ist, die Einrichtung gegebenenfalls von Ansprüchen Dritter, die durch die Weitergabe entstanden sind, freizustellen. ${ }^{264}$

Im Folgenden soll daher geprüft werden, ob die Vereinbarung einer solchen Verschwiegenheitsklausel in Verbindung mit einer Vertragsstrafe einer gerichtlichen Überprüfung standhalten würden. Dabei stehen vor allem die $\iint 305 \mathrm{ff}$. BGB im Mittelpunkt.

\section{a. Zulässigkeit nach den $\iint 305$ ff. BGB}

Die Unzulässigkeit derartiger Klauseln könnte sich vor allem aus den \$§ 305 ff. BGB ergeben. Da es sich bei den in Frage stehenden Klauseln um Vertragsbedingungen handelt, die für eine Vielzahl von Verträgen vorformuliert werden und die den jeweiligen Fremdforschern in der Regel von der archivierenden Einrichtung gestellt werden, handelt es sich um Allgemeine Geschäftsbedingungen i.S.d. \ 305 Abs. 1 S. 1 BGB, so dass der Schutzbereich der \305 ff. BGB grundsätzlich eröffnet ist. 265

\section{i. Überraschende Klausel nach \305c BGB}

Die Vereinbarung der genannte Klauseln könnte zunächst eine überraschende Klausel im Sinne des $\int 305$ c Abs. 1 BGB darstellen. Danach würde die Vereinbarung eines Weitergabeverbotes und einer Vertragsstrafe bei Zuwiderhandlung in Allgemeinen Geschäftsbedingungen nicht Vertragsbestandteil des Vertrages werden, wenn sie nach den Umständen, insbesondere nach dem äußeren Erscheinungsbild des Vertrags, so ungewöhnlich wäre, dass der Vertragspartner, hier also der Fremdforscher oder eine fremde Forschungseinrichtung, mit ihr nicht zu rechnen bräuchte. Eine ungewöhnliche Klausel ist dabei dann anzunehmen, wenn mit ihr nach den Umständen vernünftigerweise nicht zu rechnen ist, da sie nach den gesamten Umständen und dem äußeren Erscheinungsbild des Vertrages von den Erwartungen abweicht, die der redliche Geschäftsverkehr typischerweise an den Vertragsinhalt knüpft. ${ }^{266}$ Überraschend ist eine Klausel dann, wenn zwischen ihrem Inhalt und den berechtigten Erwartungen des Kunden eine solche erhebli-

\footnotetext{
264 BGH NJW 2002, 2382; Brinkmann in Prütting/Wegen/Weinreich, \ 157 Rn. 42; zu einem Entwurf für eine entsprechende Klausel siehe unten S. 138.

265 Vgl. Grüneberg in Palandt, BGB, 71. Aufl. 2012, \ 305 Rn. 8 ff; J. Becker in Bamberger/Roth (Hrsg.), Kommentar zum Bürgerlichen Gesetzbuch Bd. 1, 2. Aufl. 2007, \ 305 Rn. 16 ff.; K.P. Berger in Prütting/Wegen/Weinreich, \305 Rn. 4 ff.

266 BGH NJW-RR 2004, 780, 781; NJW 2000, 1179, 1181; Grüneberg in Palandt, \ 305c Rn. 3; H. Schmidt in Bamberger/Roth, Bd. 1, \305c Rn. 11; K.P. Berger in Prütting/Wegen/Weinreich, 』 305c Rn. 5.
} 
Kapitel 1 - Urheberrechtliche Problematik

che Diskrepanz besteht, dass der Klausel ein Überrumpelungseffekt innewohnt. ${ }^{267}$ Abzustellen ist dabei auf den vertragstypischen Kundenkreis, ${ }^{268}$ in den hier relevanten Fällen also Fremdforschern bzw. fremden Forschungseinrichtungen. Diesbezüglich ist zwischen der Verschwiegenheitsklausel und der Vereinbarung einer Vertragsstrafe zu differenzieren.

\section{(1) Verschwiegenheitsklausel}

Das Verbot der Weitergabe von wissenschaftlichen Daten ist nicht unüblich, so dass Forscher, die an fremden Forschungseinrichtungen Daten abrufen, erwarten können, dass ein solches Verbot besteht. Dies gilt insbesondere vor dem Hintergrund, dass zur Erhebung dieser Daten oftmals erhebliche Forschungsmittel aufgewendet werden mussten und die Universität damit ein für Außenstehende erkennbares Interesse daran hat, dass sie die Weitergabe der Daten kontrollieren kann. Somit wohnt dem Weitergabeverbot auch kein Überrumpelungseffekt inne; vielmehr wird ein durchschnittlicher Wissenschaftler oder wissenschaftlicher Mitarbeiter mit einer solchen Klausel rechnen. Darüber hinaus ist zu berücksichtigen, dass ein erheblicher Teil der Daten aus dem medizinischen Bereich stammt, so dass es sich oftmals für den Vertragspartner erkennbar um medizinische personenbezogene Daten handeln wird und die archivierende Einrichtung bezüglich der Weitergabe dieser datenschutzrechtlich sensiblen Daten besondere Sorgfaltspflichten zu treffen hat. ${ }^{269}$ Damit stellt eine derartige Klausel keine überraschende Klausel im Sinne des $\int 305$ c Abs. 1 BGB dar.

\section{(2) Vertragsstrafe}

Als überraschende Klausel könnte sich indes die Vereinbarung einer Vertragsstrafe für den Fall einer Zuwiderhandlung darstellen, da die Vertragspartner mit einer solchen Klausel nicht unbedingt rechnen brauchen und es aus diesem Grund Fallkonstellationen geben kann, in denen der Vereinbarung einer Vertragsstrafe ein Überrumpelungseffekt anhaftet. Aus diesem Grund sollten die archivierenden Einrichtungen derartige Klauseln sowohl durch die gewählte Drucktype als auch eine drucktechnisch herausgestellte Überschrift, aus der der Klauselinhalt eindeutig hervorgeht, hervorheben, so dass erwartet werden kann, dass der Verwender von ihr Kenntnis nimmt. Darüber hinaus sollte ein ausdrücklicher Hinweis ${ }^{270}$ in die AGB auf die drohende Vertragsstrafe aufgenommen werden und die Klausel neben ihrer optischen Hervorhebung auch verständlich verfasst sein, so dass ihre

\footnotetext{
267 BGH NJW-RR 2002, 485; NJW 2001, 1416 f.; K.P. Berger in Prütting/Wegen/Weinreich, \305c Rn. 6.

268 BGH NJW-RR 2001, 439, 440; NJW 1994, 2145; NJW 1981, 117, 118 ; Ulmer/Schäfer in: Ulmer/Brandner/Hensen, $\int 305 c \mathrm{Rn} 14$.

269 Siehe hierzu S. $163 \mathrm{ff}$.

270 BGH NJW 1992, 1822, 1823; 1985, 848, 849; BGH NJW 1978, 1519, 1520; Grüneberg in Palandt, \305c Rn. 4; Ulmer/Schäfer in Ulmer/Brandner/Hensen, J 305c Rn. 24.
} 
inhaltliche Bedeutung und Tragweite erkennbar ist, und eine überraschende Wirkung von vornherein vermieden wird. ${ }^{271} \mathrm{Da}$ die Vereinbarung einer Vertragsstrafe gerade abschreckend wirken soll, um die Weitergabe der Daten zu verhindern, wird ihre Hervorhebung im Vertrag regelmäßig auch im Interesse der archivierenden Einrichtung liegen.

ii. Unwirksamkeit der Verschwiegenheitsklausel nach den SS 307 - 309 BGB

Soweit die Klauseln Vertragsbestandteil geworden sind, stellt sich ferner die Frage, ob sie einer Prüfung nach $\int 307$ BGB standhalten. Ob hierbei die $\iint 308$ und 309 BGB Anwendung finden, hängt davon ab, ob es sich bei dem Fremdforscher bzw. der Forschungseinrichtung, die Vertragspartei ist, um einen Unternehmer im Sinne der $\iint 310$ Abs. 1, 14 BGB oder einen Verbraucher gem. \ 13 BGB handelt. Bei den in Betracht kommenden Forschungseinrichtungen wird es sich vorliegend in der Regel um juristische Personen des privaten oder öffentlichen Rechts handeln, so dass die Unternehmereigenschaft grundsätzlich gegeben ist. Die Wirksamkeit der Ihnen gestellten AGB ist damit gem. \ 310 Abs. 1 BGB nur an \ 307 BGB zu messen ${ }^{272}$. Bei Fremdforschern ist danach zu differenzieren, ob diese den Vertrag mit dem Langzeitarchivierungsverbund im Rahmen ihrer selbständigen beruflichen Tätigkeit schließen oder in ihrer Funktion als Angestellte. Dabei wird grundsätzlich Letzteres der Fall sein, weshalb diese Fremdforscher regelmäßig als Verbraucher im Sinne des $\int 13$ BGB anzusehen sein werden. Die Ihnen gestellten AGB unterfallen damit auch der Prüfung der $\iint 508$ und 309.

Unabhängig davon, ob die $\iint 308$ und 309 BGB im jeweiligen Fall Anwendung finden oder ob sie nach $\int 310$ Abs.1 BGB ausgeschlossen sind, ist ein Verstoß gegen die \$S 308 und 309 BGB durch Vereinbarung einer Verschwiegenheitsklausel in Form einer Verschwiegenheitsklausel nicht ersichtlich. Somit bleibt zu prüfen, ob ein derartiges Verbot gegen \307 BGB verstoßen würde. Dabei ist auf den ersten Blick keine Abweichung von wesentlichen Grundgedanken einer gesetzlichen Regelung ersichtlich. Es stellt sich jedoch die Frage, ob durch die Vereinbarung einer Verschwiegenheitsklausel dem Vertragspartner nicht urheberoder leistungsschutzrechtliche Schranken abbedungen werden, die die Nutzung der jeweiligen Datenbank oder eines Datenbankwerkes eigentlich erlauben würden. Zur Beantwortung dieser Frage kommt es zunächst darauf an, ob in den vorliegenden Fällen überhaupt Schranken auf die Nutzung von Datenbanken oder

\footnotetext{
271 BGH NJW 1996, 191, 192; WM 1984, 1056, 1058; Ulmer/Schäfer in Ulmer/Brandner/Hensen, S 305c Rn. 24.

272 Siehe dazu auch die Ausführungen auf S. 84 ff204.
} 
Datenbankwerken in Betracht kämen (dazu (3) ${ }^{273}$. Sofern diese Frage zu bejahen ist, muss in einem weiteren Schritt geklärt werden, ob urheberrechtliche Schranken abdingbar sind (dazu (4).

\section{(3) In Betracht kommende Schranken}

Eine derartige Lizenzvereinbarung könnte jedoch die gesetzlichen Schranken der $\iint 44 a$ ff. UrhG aushebeln und daher unzulässig sein. Daher ist zunächst zu prüfen, ob die Nutzung einzelner Daten durch einen Fremdforscher, die dieser vom LZA erlangt hat, überhaupt durch eine gesetzliche Schranke gedeckt sein könnte. Im Gegensatz zu den Ausführungen auf den Seiten 35 ff. geht es also nicht um die Frage, ob erhobene Daten aufgrund einer Schranke archiviert werden dürfen, sondern ob Daten, die bereits archiviert worden sind, aufgrund einer gesetzlichen Schrankenbestimmung von Fremdforschern genutzt werden dürfen. Somit ist zunächst zu untersuchen, welche Schranken im Einzelnen in Betracht kämen. Aus dem Katalog der $\$ \int 44 a$ UrhG kommen für die vorliegend relevanten Fälle dabei grundsätzlich die $\iint 51,52 \mathrm{a}, 52 \mathrm{~b}$ sowie $\ 53 \mathrm{UrhG}$ in Betracht. Wie oben bereits festgestellt, kommen ein urheberrechtlicher Schutz von wissenschaftlichen Primärdaten und damit das Bedürfnis einer gesetzlichen Schranke dabei eigentlich nur für Datenbankwerke in Betracht.

\section{(a) $\int 51 \mathrm{UrhG}$}

Die Vervielfältigung, Verbreitung und öffentliche Wiedergabe eines bereits veröffentlichten Werkes ist gem. \51 S.1 UrhG zum Zwecke des Zitats zulässig, sofern die Nutzung in ihrem Umfang durch ihren besonderen Zweck gerechtfertigt ist. Dabei enthalten die Nr.1-3 einen nicht abschließenden Katalog mit Fällen, in denen die Nutzung des Werkes durch $₫ 51 \mathrm{~S} .1 \mathrm{UrhG}$ insbesondere gedeckt ist.

Die Vorschrift differenziert in diesem Zusammenhang zwischen dem wissenschaftlichen Großzitat in Nr. 1, dem Kleinzitat in Nr. 2 und dem Musikzitat in Nr. 3 , wobei Letzteres in den vorliegenden Fällen von vornherein ausscheidet. $\mathrm{Zu}$ beachten ist, dass die Schranke des $\$ 51$ UrhG nur in den Fällen erforderlich ist, in denen die genutzte Datei bzw. das genutzte Datenpaket selbst eine persönliche geistige Schöpfung darstellt und damit Werkqualität aufweist. ${ }^{274}$

Damit ist ihre Anwendbarkeit nur dann relevant, wenn nicht nur das Langzeitarchiv insgesamt, sondern die genutzte Datei aus diesem Archiv für sich genommen eine geistige Schöpfungshöhe im Sinne des $\ 2$ Abs. 2 UrhG aufweist, was, wenn überhaupt, nur der Fall ist, sofern eine archivierte Datensammlung ein Datenbankwerk im Sinne des $₫ 4$ UrhG darstellt. ${ }^{275} \mathrm{Zu}$ berücksichtigen ist, dass

\footnotetext{
273 Im Gegensatz dazu behandelten die Ausführungen auf S. 35 ff. die Frage, ob sich die archivierende Einrichtung gegenüber dem ursprünglichen Rechteinhaber auf urheber- oder leistungsrechtliche Schranken berufen kann.

274 Dustmann in Fromm/Nordemann, $\mathbb{\int} 51$ Rn. 13.

275 Siehe oben S. $10 \mathrm{ff}$.
} 
$\int 51 \mathrm{UrhG}$ nur bereits veröffentlichte Werke zum Gegenstand hat. Ein Werk ist gem. \6 Abs. 1 UrhG dann veröffentlicht, wenn es mit Zustimmung des Berechtigten der Öffentlichkeit zugänglich gemacht worden ist, so dass theoretisch jedermann davon Kenntnis nehmen kann ${ }^{276}$. In den vorliegend im Mittelpunkt stehenden Fallkonstellationen können die archivierten Daten von Fremdwissenschaftlern abgerufen werden. Dabei wird die archivierende Einrichtung zwar regelmäßig verlangen, dass sich die Bezieher der Daten vor dem Abruf registriert und eine entsprechende Lizenzvereinbarung abschließt. Dies ändert jedoch nichts daran, dass theoretisch Jedermann von den Daten Kenntnis nehmen kann, so dass es sich bei den Daten bzw. Datensammlungen, die die Voraussetzungen des Werkbegriffes erfüllen, um veröffentlichte Werke im Sinne des $\int 51$ UrhG handelt. $^{277}$

Die Nutzung ist gem. \51 S. 2 Nr. 1 UrhG insbesondere dann zulässig, wenn einzelne Werke nach der Veröffentlichung in ein selbständiges wissenschaftliches Werk zur Erläuterung des Inhalts aufgenommen werden. Das Regelbeispiel von Nr. 1 käme demzufolge insbesondere dann in Betracht, wenn Dritte ein archiviertes Datenbankwerk vervielfältigen, verbreiten oder öffentlich wiedergeben möchten, um den Inhalt einer eigenen Publikation zu erläutern bzw. ihre Erkenntnisse und Schlussfolgerungen anhand der genutzten Daten zu untermauern. Zu denken wäre in diesem Zusammenhang etwa an eine Tabelle mit einzelnen Forschungsdaten, die vollständig vom Fremdforscher genutzt wird, indem dieser sie in ein eigenes Werk einfügt und deren Anordnung oder Zusammenstellung ausnahmsweise eine geistige Schöpfungshöhe aufweist. Dabei ist von der Schranke des $\int 51 \mathrm{Nr} .1$ UrhG nicht nur die Nutzung von Teilen des Werkes, sondern des ganzen Werkes erfasst $^{278}$. Eine Einschränkung kann sich lediglich dann ergeben, wenn zur Erreichung des Zitatzwecks auch die Nutzung von denjenigen Teilen eines größeren Werkes genügt, deren Aufnahme es zur Erläuterung des zitierenden Werkes bedarf. ${ }^{279}$

\section{Regelbeispiel des $\int 51$ S. 2 Nr. 1 UrbG}

Der Fremdforscher könnte sich allerdings nur dann auf das Regelbeispiel des $\int 51$ S. $2 \mathrm{Nr} .1 \mathrm{UrhG}$ berufen, wenn es sich bei dem Werk, in das er die Daten aufnimmt, um ein selbständiges wissenschaftliches Werk handelt und dieses unabhängig vom zitierten Werk ist. ${ }^{280}$ Unter Wissenschaft ist dabei vor dem Hintergrund von Art. 5 Abs. 3 GG, ,jede ernsthafte, methodisch gekennzeichnete Suche

\footnotetext{
276 W. Nordemann in Fromm/Nordemann. S 6 Rn. 10.

277 Siehe auch OLG München ZUM 1990, 95, wonach eine Veröffentlichung unter bestimmten Umständen sogar dann vorliegt, wenn Werkstücke an einen ausgewählten Kreis von Empfängern versendet worden sind.

278 Dreier in Dreier/Schulze, \ 51 Rn. 10.

279 Dreier in Dreier/Schulze, \51 Rn. 10.

280 Dustmann in Fromm/Nordemann, \51 Rn. 19; Schricker/Spindler in Schricker/Loewenheim \51 Rn. 20.
} 
nach Erkenntnis“ zu verstehen. ${ }^{281}$ Dabei wird die Nutzung von archivierten Primärdaten in den vorliegend relevanten Fallkonstellationen regelmäßig vor einem wissenschaftlichen Hintergrund erfolgen, so dass die Daten in nahezu allen Fällen in ein wissenschaftliches Werk eingefügt werden. Des Weiteren wird es sich auch regelmäßig um ein vom Zitat unabhängiges Werk handeln, da es nur schwer vorstellbar ist, dass ein Fremdforscher die zitierte Datenbank lediglich abwandelt, zumal dies bei Daten, anders als beispielsweise bei einem Aufsatz, auch nur schwer möglich erscheint. Hinsichtlich des Merkmals „selbständig“ stellt sich indes die Frage, ob das Werk, in das die Daten eingefügt werden, seinerseits urheberschutzfähig sein muss. ${ }^{282}$ Gegen ein solches Erfordernis spricht zunächst, dass es sich bei den in $\int 51$ S. 2 Nr.1-3 genannten Fällen nur um Regelbeispiele handelt. Die Generalklausel in $\int 51$ S. 1 UrhG verlangt im Gegensatz zu Satz 2 jedoch gerade nicht die Selbständigkeit des aufnehmenden Werkes, was grundsätzlich gegen das Erfordernis einer eigenen urheberrechtlichen Schutzfähigkeit des Werkes spricht, in welches das Zitat eingefügt wird. ${ }^{283}$ Für ein solches Erfordernis spricht hingegen der Sinn und Zweck der Vorschrift. Dieser besteht darin, das eigene geistige Schaffen des Zitierenden und damit den kulturellen und wissenschaftlichen Fortschritt zu fördern. ${ }^{284}$ Damit erscheint es geboten, die Schranke nur demjenigen zugutekommen zu lassen, der auch tatsächlich eine eigene geistige Schöpfung erschafft und damit durch seine geistige Leistung einen Beitrag zum wissenschaftlichen Fortschritt leistet. ${ }^{285}$ Insofern kann sich ein Fremdforscher nur dann auf die Zitatschranke des $\int 51$ UrhG berufen, wenn das Werk, in das die Datei aufgenommen wird, urheberrechtliche Schutzfähigkeit aufweist.

Darüber hinaus müsste der Zweck der Aufnahme der Erläuterung des Inhalts des aufnehmenden Werkes dienen. ${ }^{286}$ Dies wird in dem Großteil, der hier im Vordergrund stehenden Fallkonstellationen zutreffen, ist es doch nur schwer vorstellbar, Daten oder Tabellen in ein Werk aufzunehmen, ohne dass diese der Erläuterung des Inhalts dienen. Vielmehr werden die Daten ja grundsätzlich gerade deshalb eingefügt, um den Inhalt zu belegen oder ihn zu erläutern.

\footnotetext{
281 Dreier in Dreier/Schulze, \51 Rn. 8.

282 So unter anderem BGH GRUR 1994, 800, 802; OLG Köln GRUR 1994, 47, 48; KG GRUR 1970, 616, 617; Schricker/Spindler in Schricker/Loewenheim, $\int 51$ Rn. 20; Dustmann in Fromm/Nordemann, \5 51 Rn. 19; Götting in Loewenheim, \ 31 Rn. 167.

283 So OLG Thüringen ZUM 2008, 523, 525; zumindest nicht ablehnend Dreier in Dreier/Schulze, $\int 51$ Rn. 6,

284 BGH GRUR 1973, 216, 217; 1986, 59, 60; Lüft in Wandtke/Bullinger, \ 51 Rn. 1.

285 Götting in Loewenheim, \ 31 Rn. 167; Schricker/Spindler in Schricker/Loewenheim, \51 Rn. 20.

286 Dreier in Dreier/Schulze, $\int 51$ Rn. 3; Schricker/Spindler in Schricker/Loewenheim \51 Rn. 14; Dustmann in Fromm/Nordemann, $\int 51$ Rn. 23.
} 
Regelbeispiel des $\int 51$ S. 2 Nr. 2 UrbG

Auch beim Kleinzitat muss das übernehmende Werk unabhängig vom zitierten Werk und darüber hinaus selbständig sein, was nach der hier gefolgten Ansicht bedeutet, dass es selbst urheberschutzfähig sein muss. ${ }^{287}$ Der Zitatzweck geht hingegen über den des Nr. 1 hinaus, da die Aufnahme von „Stellen eines Werkes nach der Veröffentlichung in ein selbständiges Sprachwerk“" gem. \51 S. 2 Nr. 2 UrhG zulässig ist, ohne dass der Wortlaut vorschreibt, dass dies zur Erläuterung des Inhaltes erfolgen muss. ${ }^{288}$

Eine Einschränkung im Vergleich zum Großzitat erfährt das Kleinzitat jedoch dadurch, dass es nach seinem ausdrücklichen Wortlaut nur die Aufnahme in ein Sprachwerk rechtfertigt. Für die Nutzung von wissenschaftlichen Primärdaten ist dies allerdings insofern irrelevant, als deren Aufnahme in andere Werke als Sprachwerke nur schwer vorstellbar ist. Darüber hinaus käme bei der Nutzung von wissenschaftlichen Primärdaten eine Rechtfertigung über $\int 51$ S. 2 Nr. 2 UrhG nur dann in Betracht, wenn der Fremdwissenschaftler lediglich Teile der Datenbank in ein anderes Werk aufnimmt. Sofern eine Datenbank, die im Langzeitarchiv gespeichert ist, von einem Fremdforscher in ein anderes Werk aufgenommen werden soll, wird dieser in der überwiegenden Anzahl der Fälle das komplette Datenbankwerk aufnehmen und nicht nur Teile davon. Eine Rechtfertigung der Nutzung von wissenschaftlichen Primärdaten in Form der Vervielfältigung oder Verbreitung ist daher zwar grundsätzlich möglich, aus den genannten Gründen wird $\int 51$ S. 2 Nr. 2 UrhG jedoch nur in seltenen Fällen Anwendung finden.

(b) $\int 52 \mathrm{a} U \mathrm{rhG}$

Eine öffentliche Zugänglichmachung des empfangenen Datenbankwerkes durch den Fremdforscher könnte hingegen durch \52a UrhG gerechtfertigt sein. Dabei ist jedoch zu beachten, dass die Vorschrift nicht die öffentliche Zugänglichmachung des gesamten Werkes gestattet. Vielmehr sieht der Wortlaut von \52a Abs. $1 \mathrm{Nr} .1 \mathrm{UrhG}$ nur die öffentliche Zugänglichmachung „,kleiner Teile“ eines veröffentlichten Werkes vor. In der Regel wird der Wissenschaftler jedoch das gesamte Datenbankwerk nutzen wollen. Bei besonders umfangreichen Datenbankwerken kann aber unter Umständen auch die Nutzung lediglich kleiner Teile genügen, weshalb die Schranke des $\int 52 \mathrm{a}$ Abs. 1 Nr. 1 in den vorliegenden Fällen nicht von vornherein ausscheidet. Darüber hinaus ist nach Nr. 2 auch die öffentliche Zugänglichmachung von Werken geringen Umfangs zulässig, so dass sowohl die Schranke in Nr. 1 als auch die in Nr. 2 in grundsätzlich in Betracht kommen, sofern die jeweiligen Tatbestandsvoraussetzungen vorliegen. Ob dies der Fall ist, soll im Folgenden untersucht werden.

\footnotetext{
287 Siehe dazu bereits oben S. 87.

288 BGH GRUR 1973, 216, 218; OLG Hamburg GRUR 1970, 38.
} 


\section{$\int 52 a A b s .1 N r .1 \mathrm{UrbG}$}

Die öffentliche Zugänglichmachung veröffentlichter kleiner Teile eines Werkes, Werke geringen Umfangs, sowie einzelne Beiträge aus Zeitungen oder Zeitschriften ist gem. $\int 52 \mathrm{a}$ Abs. $1 \mathrm{Nr} .1 \mathrm{UrhG}$ zur Veranschaulichung im Unterricht an Schulen, Hochschulen, nichtgewerblichen Einrichtungen der Aus- und Weiterbildung ausschließlich für den abgegrenzten Kreis von Unterrichtsteilnehmern zulässig. Dabei kommt für die öffentliche Zugänglichmachung von wissenschaftlichen Primärdaten grundsätzlich wohl die Veranschaulichung im Unterricht an Hochschulen in Betracht. Daneben ist auch die Verwendung im Unterricht an Schulen, insbesondere in naturwissenschaftlichen Fächern, denkbar.

Entscheidend für den Umfang der zulässigen Zugänglichmachung ist, ob es sich bei dem jeweils betroffenen Datenbankwerk um „ein Werk geringen Umfangs" handelt. Ist dies nicht der Fall, so gestattet \52a Abs. 1 Nr. 1 UrhG lediglich die Zugänglichmachung „kleiner Teile“ des Werkes. Ob ein Werk geringen Umfangs im Einzelfall vorliegt, richtet sich danach, ob das betreffende Werk im Rahmen einer Gesamtbetrachtung aller möglichen Werke umfänglich zu den Kleinsten gehört. ${ }^{289}$ Dies wurde von der Rechtsprechung beispielsweise für kurze Artikel, Kurzgeschichten ${ }^{290}$, Novellen ${ }^{291}$, Tonfolgen, Gedichte, Liedertexte und Lieder bejaht. ${ }^{292}$ Demzufolge kann ein Datenbankwerk, welches wissenschaftliche Primärdaten enthält, ein Werk geringen Umfangs darstellen. Da jedoch gerade im naturwissenschaftlichen Bereich Datenbanken einen beträchtlichen Umfang erreichen können, gilt dies nicht zwangsläufig. Es ist daher stets auf den jeweiligen Einzelfall abzustellen. Handelt es sich nicht um ein Werk geringen Umfangs, so erlaubt \52a Abs. 1 UrhG nur die öffentliche Zugänglichmachung kleiner Teile, wobei hinsichtlich der Frage, bis zu welchem Umfang noch ein kleiner Teil vorliegt, in Rechtsprechung und Literatur unterschiedliche Ansichten vertreten werden. Teilweise soll die Obergrenze bei einem Umfang von 10\% des Gesamtumfangs liegen, 293 andere Stimmen in der Literatur wollen die Grenze hingegen bei 20\% ziehen. ${ }^{294}$ Nach anderer Ansicht kann hingegen keine starre Grenze gezogen werden; vielmehr komme es stets auf eine Betrachtung im Einzelfall an. ${ }^{295}$ Sofern ein Datenbankwerk zur Veranschaulichung im Unterricht an Hochschulen öffentlich zugänglich gemacht werden soll, wird ein Umfang von nur $20 \%$ jedoch wohl ohnehin zu gering sein, um den veranschaulichenden Effekt zu erzielen, weshalb die Schranke des $\int 52 \mathrm{a}$ Abs. 1 Nr.1 UrhG für die öffentliche Zugänglichmachung

\footnotetext{
289 Dustmann in Fromm/Nordemann, $\int 52 \mathrm{a}$ Rn. 8.

290 BGH GRUR 1972, 432, 433.

291 RGZ 80, $78 \mathrm{f}$.

292 Dunstmann in Fromm/Nordemann, $\int 52 \mathrm{a}$ Rn. 8.

293 OLG Karlsruhe GRUR 1987, 818; Loewenheim in Schricker/Loewenheim, \ 52a Rn. 7, \53 Rn.

51; $v$. Bernuth, ZUM 2003, 438, 440.

294 Lüft in Wandtke/Bullinger, \52a Rn. 5.

295 Dustmann in Fromm/Nordemann, $\int 52 \mathrm{a}$ Rn. 7.
} 
von wissenschaftlichen Primärdaten kaum Anwendung finden wird, sofern es sich nicht um ein Werk geringen Umfangs handelt.

Des Weiteren müsste das Datenbankwerk bzw. der Teil des Datenbankwerkes, der öffentlich zugänglich gemacht werden soll, nach dem ausdrücklichen Wortlaut von $\int 52 \mathrm{a}$ Abs. $1 \mathrm{Nr} .1 \mathrm{UrhG}$ bereits veröffentlicht worden sein, wobei die Norm an den Veröffentlichungsbegriff von $\int 6$ Abs. 1 UrhG anknüpft. ${ }^{296}$ Das Datenbankwerk wäre danach veröffentlicht, wenn es mit Zustimmung des Berechtigten der Öffentlichkeit zugänglich gemacht worden ist. Zur Öffentlichkeit gehört dabei gem. $\int 15$ Abs. 3 S. 2 UrhG jeder, der nicht mit demjenigen der das Werk verwertet, oder mit anderen Personen, denen das Werk, wie hier, in unkörperlicher Form wahrnehmbar oder zugänglich gemacht wird, durch persönliche Beziehungen verbunden ist. Geht man, wie hier, von der Fallkonstellation davon aus, dass die archivierende Einrichtung als Berechtigte i.S.d. \ 6 Abs. 1 UrhG die archivierten Daten anderen Forschungseinrichtungen und Fremdwissenschaftlern zum Abruf bereitstellt, so wären diese potentiellen Nutzer weder durch eine persönliche Beziehung zu der archivierenden Einrichtung noch durch eine solche Beziehung untereinander verbunden, so dass die Daten der Öffentlichkeit bereits zugänglich gemacht worden wären, bevor sie der Fremdforscher erhält und seinerseits anderen öffentlich zugänglich macht.

Schließlich ist zu beachten, dass Absatz 1 Nr.1 nur die öffentliche Zugänglichmachung „für den bestimmt abgegrenzten Kreis von Unterrichtsteilnehmern“ zulässt. Sollte $\int 52$ Abs. 1 Nr. 1 UrhG also im Einzelfall doch für die öffentliche Zugänglichmachung wissenschaftlicher Primärdaten einschlägig sein, so muss mit Hilfe von Kontrollsystemen, wie etwa Passwörtern, sichergestellt werden, dass nur diejenigen Studenten bzw. Schüler auf das Werk zugreifen können, die an der entsprechenden Vorlesung oder Unterrichtseinheit auch teilnehmen, für die das Werk benötigt wird. ${ }^{297}$

Des Weiteren wäre die öffentliche Zugänglichmachung nur dann nach \52a UrhG zulässig, ,,soweit dies zu dem jeweiligen Zweck geboten und zur Verfolgung nicht kommerzieller Zwecke gerechtfertigt ist“. Die öffentliche Zugänglichmachung ist im Rahmen von Nr. 1 dann nicht geboten, wenn das Werk ohne erheblichen Aufwand offline angeboten werden könnte. ${ }^{298}$ Dies wird bei Datenbankwerken jedoch regelmäßig der Fall sein, da die Daten zumeist auf Papier gedruckt und den Teilnehmern des Unterrichts oder der Vorlesung offline angeboten werden können, so dass eine öffentliche Zugänglichmachung von wissenschaftlichen Primärdaten für die Veranschaulichung im Unterricht oder in der Vorlesung grundsätzlich regelmäßig nicht geboten sein wird. Eine Ausnahme von diesem Grundsatz könnte jedoch dann gelten, wenn das Datenbankwerk bzw. kleine Teile eines solchen nur schwer offline zur Verfügung gestellt werden können. Da Nr. 1 je-

\footnotetext{
296 Dreyer in Dreyer/Kotthoff/Meckel, \52a Rn. 8.

297 Dustmann in Fromm/Nordemann, $\int 52 \mathrm{a}$ Rn. 11.

298 Lüft in Wandtke/Bullinger, S 52a Rn. 9.
} 
doch sowieso nur auf die öffentliche Zugänglichmachung kleiner Teile eines Datenbankwerkes bzw. von Datenbankwerken mit geringem Umfang Anwendung findet, ist dies nur schwer vorstellbar. Zu denken ist jedoch beispielsweise an Vorlesungen mit großer Hörerzahl, in der es mit unverhältnismäßigen Kosten und einem nicht unerheblichen Zeitaufwand verbunden wäre, Teile des Datenbankwerkes analog oder digital offline zur Verfügung zu stellen. Insofern wäre in diesen Fällen die öffentliche Zugänglichmachung eines Datenbankwerkes mit geringem Umfang bzw. des kleinen Teils eines Datenbankwerkes mit nicht geringem Umfang von $₫ 52 \mathrm{a}$ Abs. $1 \mathrm{Nr} .1 \mathrm{UrhG}$ gedeckt.

\section{$\int 52 a$ Abs. 1 Nr. 2 UrbG}

Im Gegensatz zu Nr. 1, der die öffentliche Zugänglichmachung von Werken zur Veranschaulichung des Unterrichts regelt, lässt Nr. 2 die öffentliche Zugänglichmachung von Werken zu Zwecken der eigenen wissenschaftlichen Forschung zu. Dabei dürfen veröffentlichte Teile eines Werkes und Werke geringen Umfangs für einen bestimmt abgegrenzten Kreis von Personen für deren eigene wissenschaftliche Forschung öffentlich zugänglich gemachte werden, soweit die zu dem jeweiligen Zweck geboten und zur Verfolgung nicht kommerzieller Zwecke gerechtfertigt ist. Im Gegensatz zu Nr. 1 dürfen demnach nicht nur „kleine Teile“ öffentlich zugänglich gemacht werden. Vielmehr folgt aus der Beschränkung auf „Teile“ lediglich die Einschränkung, dass das Gesamtwerk durch die öffentliche Zugänglichmachung des zugänglich gemachten Teils nicht überflüssig werden darf.. ${ }^{299}$ Der Begriff der „wissenschaftlichen Forschung“ wird dabei wie der „wissenschaftliche Gebrauch" als methodische und auf Erkenntnisfindung ausgerichtete Tätigkeit definiert. 300

Sofern das Datenbankwerk einen geringen Umfang i.S.d. \52a Abs. 1 Nr. 2 UrhG hat, ${ }^{301}$ dürfte der Fremdwissenschaftler oder eine fremde Forschungseinrichtung das Datenbankwerk einem bestimmt abgegrenzten Kreis von Personen öffentlich zugänglich machen, die die Daten für ihre eigene wissenschaftliche Forschung benötigen. Dabei hatte der Gesetzgeber vor allem kleine Forschungsteams vor Augen, die im Rahmen des gleichen Forschungsprojektes tätig sind. ${ }^{302}$ Nicht erfasst sein soll hingegen beispielsweise die Zugänglichmachung des Werkes an alle Forschungseinrichtungen einer Universität. ${ }^{303}$ Wie auch im Rahmen der öffentlichen Zugänglichmachung unter Nr. 1 müssten auch in diesen Fällen technische Vorkehrungen getroffen werden, um den zugelassenen Nutzerkreis ausschließlich auf diese Personen zu beschränken, ${ }^{304}$ wobei auch hier wieder die Ver-

\footnotetext{
${ }^{299}$ Lüft in Wandtke/Bullinger, \52a Rn. 12; Dreier in Dreier/Schulze, \52a Rn. 9.

300 Statt vieler Dreier in Dreier/Schulze, \ 52a Rn. 10; \ 53 Rn. 23.

301 Siehe dazu bereits oben S. 90.

302 Dustmann in Fromm/Nordemann, \52a Rn. 14.

303 BT-Drucks 15/837, S. 34.

304 BT-Drucks. 15/538, S. 78; Lüft in Wandtke/Bullinger, \52a Rn. 13.
} 
wendung von Passwörtern in Betracht kommt. ${ }^{305}$ Sollte es sich bei dem Datenbankwerk hingegen nicht um ein Werk geringen Umfangs handeln, dürften Teile des Datenbankwerkes öffentlich zugänglich gemacht werden. Dies macht natürlich nur Sinn, sofern der Empfänger der Daten nicht auf das vollständige Datenbankwerk angewiesen ist. Auch in den Fällen von Nr. 2 muss die öffentliche Zugänglichmachung geboten sein, wobei diese auch hier wiederum entfällt, wenn das Datenbankwerk ohne erheblichen Aufwand offline beschafft werden könnte. ${ }^{306}$ Dies wird in den meisten Fällen jedoch der Fall sein, da das Datenbankwerk, anders als in den Fällen der Nutzung im Unterricht an Hochschulen, aufgrund der zumeist geringen Dateigröße in der überwiegenden Anzahl der Fälle den Empfängern auch offline oder per Email übermittelt werden kann. Sollte die öffentliche Zugänglichmachung ausnahmsweise einmal doch geboten sein, so ist weiter zu beachten, dass dies nur zur Verfolgung nicht kommerzieller Zwecke zulässig ist. Dabei ist in den Fällen von Nr. 2 nicht auf die organisatorische Struktur der Forschungseinrichtung abzustellen, sondern auf die Forschungstätigkeit als solche, für die das Datenbankwerk genutzt wird. ${ }^{307}$ Sofern im Einzelfall die wissenschaftliche Forschung im Rahmen einer Auftragsforschung stattfindet, läge ein kommerzieller Zweck vor, so dass die öffentliche Zugänglichmachung in diesem Fall nicht gem. \52a Abs. 1 Nr. 2 UrhG gerechtfertigt wäre.

\section{Zwischenergebnis}

Damit kommen sowohl $\int 52 \mathrm{a}$ Abs. 1 Nr. 1 als auch $\int 52 \mathrm{a}$ Abs. 1 Nr. 2 UrhG grundsätzlich als Schranke für Fremdforscher in Betracht, die archivierte Daten in Form eines Datenbankwerkes von der archivierenden Einrichtung erlangt haben und diese anderen öffentlich zugänglich machen wollen. Allerdings werden sich Nutzer von wissenschaftlichen Primärdaten in der Praxis wohl nur selten auf $\int$ 52a UrhG stützen können, da es sowohl in den Fällen von Nr. 1 als auch Nr. 2 zum einen oftmals an der Gebotenheit der öffentlichen Zugänglichmachung fehlen wird und zum anderen für die öffentliche Zugänglichmachung gem. \52a Abs. 4 UrhG eine Vergütung zu zahlen ist. Sollten die Tatbestandsvoraussetzungen von Nr.1 oder Nr. 2 im Einzelfall doch einmal vorliegen, so ist zu beachten, dass nach Nr. 1 nur die Zugänglichmachung kleiner Teile des Werkes oder Werke geringen Umfangs, nach Nr. 2 nur die Zugänglichmachung von Teilen des Werkes oder Werken geringen Umfangs zulässig ist. Die öffentliche Zugänglichmachung eines vollständigen Datenbankwerkes, welches einen erheblichen Umfang hat, ist demzufolge weder nach \52a Abs. 1 Nr. 1 UrhG noch nach \52a Abs. 1 Nr. 2 UrhG zulässig.

\footnotetext{
305 Lüft in Wandtke/Bullinger, \52a Rn. 13.

306 Lüft in Wandtke/Bullinger, $\int 52 \mathrm{a}$ Rn. 14.

307 Siehe Erwägungsgrund 42 der Richtlinie 2001/29/EG; Lüft in Wandtke/ Bullinger, \52a Rn. 14.
} 
(c) $\int 52 \mathrm{~b} \mathrm{UrhG}$

Als weitere Schranke für die Weitergabe von wissenschaftlichen Primärdaten kommt unter Umständen \52b UrhG in Betracht. Dieser erlaubt öffentlich zugänglichen Bibliotheken, Museen und Archiven, die keinen unmittelbaren oder mittelbaren wirtschaftlichen Zweck verfolgen, in den Räumen ihrer Einrichtung an eigens dafür eingerichteten elektronischen Leseplätzen veröffentlichte Werke zur Forschung und für private Studien zugänglich zu machen. Auch wenn die Schranke vornehmlich der digitalen Zugänglichmachung von Printpublikationen dienen soll, ${ }^{308}$ ist seine Anwendung auf Werke, wie beispielsweise den vorliegend relevanten elektronischen Daten, nicht von vornherein ausgeschlossen. Dies folgt aus dem Wortlaut der Norm, die lediglich von veröffentlichten Werken spricht, den Werkbegriff ansonsten aber nicht einschränkt. ${ }^{309}$

Damit könnten die oben genannten Einrichtungen Daten und Datenbanken, die sie von der archivierenden Einrichtung erlangt haben, an den elektronischen Leseplätzen zugänglich machen, sofern die Voraussetzungen von \52b UrhG vorlägen. Die Vorschrift erlaubt die Zugänglichmachung an Leseplätzen jedoch nur unter der Voraussetzung, dass dem keine vertraglichen Regelungen entgegenstehen. Dabei genügt eine Regelung unter Verwendung von Allgemeinen Geschäftsbedingungen, so dass individuelle Verhandlungen nicht erforderlich sind. ${ }^{310}$ Eine solche Vereinbarung wird jedoch regelmäßig im Rahmen des Lizenzvertrags vorliegen, den die archivierende Einrichtung mit der Einrichtung geschlossen hat, der sie die Daten zur Verfügung gestellt hat. ${ }^{311}$ Von daher ist es den Bibliotheken, Museen und Archiven versagt, ihnen zur Verfügung gestellte wissenschaftliche Primärdaten bzw. Datenbankwerke über $\int 52 \mathrm{~b}$ UrhG zu nutzen, sofern der Lizenzgeber ihnen dies im Rahmen des Lizenzvertrags untersagt oder nur zu bestimmten Bedingungen gestattet. ${ }^{312}$ Demzufolge kommt eine Zugänglichmachung oder Weitergabe von wissenschaftlichen Primärdaten durch Bibliotheken, Museen oder Archiven unter Berufung auf $\int 52 \mathrm{~b}$ UrhG in den vorliegenden Fällen grundsätzlich nicht in Betracht. ${ }^{313}$

(d) $\int 53$ Abs. 2 S.1 Nr. 1 UrhG

Eine weitere Schranke könnte sich ferner aus $\int 53$ UrhG ergeben. Wie bereits erwähnt, wird ein urheberrechtlicher Schutz an wissenschaftlichen Primärdaten höchstens dann entstehen, wenn mehrere Daten derart zusammengestellt worden

\footnotetext{
308 Jani in Wandtke/Bullinger, \52b Rn. 4.

309 So auch Jani in Wandtke/Bullinger, $\int 52 \mathrm{~b}$ Rn. 4.

310 Jani in Wandtke/Bullinger, \52b Rn. 27; siehe auch Spindler, NJW 2008, 9, 13.

311 BT-Drucks. 16/1828, S 26; vgl. auch Spindler/Heckmann, GRUR Int. 2008.

312 Dustmann in Fromm/Nordemann, \52b Rn. 11; Spindler, NJW 2008, 9, 11; ders./Heckmann, GRUR Int. 2008, 271, 275; Hoeren, MMR 2007, 615, 617.

313 Darüber hinaus wäre die Einsehbarkeit von Daten an elektronischen Leseplätzen, anders als zum Beispiel bei Aufsätzen oder Monographien, wenig praktikabel.
} 
sind, dass ein Datenbankwerk gem. \4 UrhG entsteht. Bei der vorliegend im Fokus stehenden elektronischen Langzeitarchivierung kann auf den Inhalt von Datenbankwerken, sofern solche entstehen, zwangsläufig elektronisch zugegriffen werden. Damit sind aber die Einschränkungen in $₫ 53$ Abs. 5 S. 1 UrhG zu beachten. Danach finden die Absätze 1, 2 S. 1 Nr. 2 bis 4 sowie Abs. 3 Nr. 2 von vornherein keine Anwendung. Absatz 2 S. 1 Nr. 1 sowie Abs. 3 Nr. 1 UrhG finden gem. $\int 53$ Abs. 5 S. 2 UrhG hingegen nur mit der Maßgabe Anwendung, dass der wissenschaftliche Gebrauch sowie der Gebrauch im Unterricht nicht zu gewerblichen Zwecken erfolgen.

Die Herstellung oder das Herstellenlassen einzelner Vervielfältigungsstücke von archivierten Datenbankwerken wäre gem. \53 Abs. 2 Nr. 1 UrhG zum eigenen wissenschaftlichen Gebrauch zulässig, wenn und soweit die Vervielfältigung zu diesem Zweck geboten wäre und sie keinen gewerblichen Zwecken dienen würde. Die letztgenannte Einschränkung wurde dabei im Rahmen des „Gesetzes zur Regelung des Urheberrechts in der Informationsgesellschaft"314 vom 26.10.2007 eingefügt, so dass sich die Voraussetzung der nicht gewerblichen Nutzung im Rahmen von Abs. 2 S. 1 Nr. 1 UrhG unabhängig von Abs. 5 S. 2 ergibt.

\section{Eigener Gebrauch}

Ein eigener Gebrauch nach $\ 53$ Abs. 2 UrhG liegt dann vor, wenn die Herstellung der Vervielfältigungsstücke zur eigenen Verwendung erfolgt und nicht zur Weitergabe an Dritte. ${ }^{315}$ Dabei zählen die Angestellten eines Unternehmens nicht als Dritte, so dass die Vervielfältigung der Datei und Weitergabe an die Angestellten einer juristischen Person oder Behörde als „eigener Gebrauch“ angesehen werden kann. ${ }^{316}$ Sofern ein Fremdwissenschaftler die von der archivierenden Einrichtung erhaltenen Daten also lediglich für sich oder einen Kollegen vervielfältigt, liegt darin ein eigener Gebrauch i.S.d. $\ 53$ Abs. 2 Nr. 2 UrhG. Das Gleiche gilt, sofern eine Forschungseinrichtung die Daten für ihre angestellten Wissenschaftler vervielfältigt.

\section{Wissenschaftlicher Gebrauch}

Darüber hinaus müsste die Vervielfältigung zum wissenschaftlichen Gebrauch erfolgen. Dieser liegt vor, wenn die Vervielfältigung vor dem Hintergrund einer wissenschaftlichen, also methodisch und auf Erkenntnisfindung ausgerichteten Tätigkeit hergestellt wird. ${ }^{317} \mathrm{Da}$ im Mittelpunkt der vorliegenden Untersuchung wissenschaftliche Primärdaten stehen, wird eine Vervielfältigung solcher Daten

\footnotetext{
314 BGBl. 2007 Teil I, S. 2513.

315 BT-Drucks. 10/837, S. 9; Dreier in Dreier/Schulze, \53 Rn. 18; W. Nordemann in

Fromm/Nordemann, \53 Rn. 16.

316 BT-Drucks. 10/837 S. 9; vgl. auch BGH GRUR 1997, 459, 461.

317 Dreier in Dreier/Schulze, \& 53 Rn. 23.
} 
stets vor einem wissenschaftlichen Hintergrund erfolgen, so dass auch dieses Tatbestandsmerkmal bei der Vervielfältigung der Datenbank durch den Fremdforscher im Regelfall erfüllt sein wird.

\section{Gebotenheit der Vervielfältigung}

Die Vervielfältigung müsste darüber hinaus jedoch auch geboten sein. Dies wäre etwa dann nicht der Fall, wenn es der Forschungseinrichtung oder dem Fremdwissenschaftler zumutbar wäre, die Daten selbst von der archivierenden Einrichtung zu erwerben. ${ }^{318}$ In diesem Zusammenhang wird man danach differenzieren müssen, ob die archivierende Einrichtung die archivierten Daten Fremdforschern und fremden Forschungseinrichtungen kostenlos zur Verfügung stellt oder ob sie dafür eine Gebühr erhebt.

\section{Kostenloser Emwerb bei der archivierenden Einrichtung möglich}

In den Fällen, in denen die archivierende Einrichtung ihre Daten kostenlos zur Verfügung stellt, wird es stets zumutbar sein, die Daten direkt bei der Einrichtung zu beziehen. Zwar könnte man dem entgegensetzen, dass die archivierende Einrichtung, sofern sie die Daten kostenlos zur Verfügung stellt, auch damit einverstanden wäre, wenn Fremdwissenschaftler diese untereinander kopieren würden. $\mathrm{Zu}$ beachten ist jedoch, dass die archivierende Einrichtung dann nicht die Möglichkeit hätte, mit den Beziehern ihrer archivierten Daten einzelne Lizenzvereinbarungen abzuschließen. Dies ist jedoch gerade in Bezug auf den Umgang mit archivierten personenbezogenen Daten relevant. ${ }^{319}$ Darüber hinaus wird seitens der archivierenden Einrichtung zumeist auch ein Interesse daran bestehen, dass die Daten stets über ihr Internetportal abgerufen werden, um dessen Bekanntheitsgrad zu steigern. Ein solches Interesse kann sich gerade aus der Tatsache ergeben, dass die Forschungseinrichtungen dadurch die Möglichkeit erhalten, über ihre Internetpräsenz ihre Bekanntheit und Reputation zu steigern. Darüber hinaus finanzieren sich Internetportale, in denen Daten oder Software kostenlos angeboten werden, oftmals über Werbeanzeigen, die in diesen Portalen geschaltet werden. Die Höhe der Werbeeinahmen richtet sich dabei jedoch regelmäßig nach der Anzahl der Nutzer der jeweiligen Internetseite.

\section{Vergütungspflicht für Datenerwerb}

Sofern die Einrichtung für den Zugang der Daten hingegen eine Vergütung verlangt, könnte der direkte Erwerb von der archivierenden Einrichtung zumindest dann unverhältnismäßig sein, wenn die anfallenden Kosten und der dafür erforderliche Zeitaufwand zu hoch wären. Da der Abruf der Daten regelmäßig über ein Onlinenetzwerk erfolgen wird, kann jedoch davon ausgegangen werden, dass der

\footnotetext{
${ }^{318}$ Lift in Wandtke/Bullinger, $\ 53$ Rn. 27; Loewenheim in Schricker/Loewenheim, $\int 53$ Rn. 42; $W$.

Nordemann in Fromm/Nordemann, \53 Rn. 19.

319 Siehe dazu S. 139 ff.
} 
erforderliche Zeitaufwand nicht zu hoch sein wird. Hinsichtlich der anfallenden Kosten könnte aber eine Unzumutbarkeit dann vorliegen, wenn die archivierende Einrichtung nicht eine gesonderte Vergütung für die abgerufenen Daten verlangt, sondern die Nutzer des Internetportals aufgrund des abzuschließenden Lizenzvertrags für einen bestimmten Zeitraum eine regelmäßige Vergütung zahlen müssten, obwohl sie nur ein einziges Mal die Dienste der archivierenden Einrichtung in Anspruch nehmen möchten. ${ }^{320}$ In diesem Fall wäre es wohl unzumutbar, dass ein Wissenschaftler oder eine Forschungseinrichtung diese Kosten tragen müsste, obwohl er nur Interesse an einem einzigen Datensatz hat. Zu beachten ist jedoch, dass eine solche längerfristige Bindung unter Umständen dann nicht mehr unverhältnismäßig wäre, wenn der Fremdforscher auch noch weitere Datenbankwerke der gleichen Einrichtung benötigt und diese ebenfalls vervielfältigen möchte.

\section{Kein gewerblicher Zweck}

Schließlich dürfte die Vervielfältigung gem. $\ 53$ Abs. 2 S. 1 Nr. 1 a.E. UrhG keinen gewerblichen Zwecken dienen. Sofern die Vervielfältigung also dazu bestimmt ist, sie für eine Verlagspublikation oder im Rahmen einer Auftragsforschung zu nutzen, würde dies zu einem gewerblichen Zweck dienen und die Vervielfältigung damit nicht mehr von $₫ 53$ Abs. $2 \mathrm{~S}$. $1 \mathrm{Nr}$. 1 UrhG gedeckt sein. ${ }^{321}$ Hingegen ist ein gewerblicher Zweck wohl abzulehnen, wenn die Vervielfältigung für den alltäglichen Forschungsbetrieb des Wissenschaftlers oder der Forschungseinrichtung genutzt wird und für die Durchführung des Forschungsvorhabens somit keine gesonderte Vergütung durch einen Auftraggeber gezahlt wird. ${ }^{322}$

\section{Zwischenergebnis}

Wie gezeigt, kann die Vervielfältigung eines Datenbankwerkes, das aus wissenschaftlichen Primärdaten besteht, grundsätzlich von $\ 53$ Abs. 2 S. 1 Nr.1 UrhG gerechtfertigt sein. Die Schrankenregelung wird jedoch in den meisten Fällen nicht einschlägig sein, weil es dem Wissenschaftler bzw. der Forschungseinrichtung, die die Vervielfältigung für ihre wissenschaftliche Tätigkeit vornimmt, in der Regel zumutbar sein wird, das Datenbankwerk selbst bei der Einrichtung zu erwerben, welche die Nutzungsrechte an dem betreffenden Werk hält. Damit fehlt es in diesen Fällen jedoch an der von $₫ 53$ Abs. 2 S. 1 Nr. 1 UrhG geforderten Gebotenheit der Vervielfältigung.

\footnotetext{
320 So ist es beispielsweise bei vielen Datenbanken juristischer Fachliteratur auch nicht möglich, lediglich den gerade benötigten Aufsatz oder Auszug aus einem Kommentar zu erwerben. Vielmehr muss der Nutzer einen Vertrag für eine festgesetzte Laufzeit abschließen, der ihn für diesen Zeitraum berechtigt, auf die Datenbank zuzugreifen. Im Gegenzug muss er aber auch für diesen Zeitraum, unabhängig davon, ob er die Datenbank nutzt oder nicht, monatlich Lizenzgebühren entrichten.

321 BT-Drucks. 16/1828, S. 41; so auch Dreier in Dreier/Schulze, \53 Rn. 23.

322 Vgl. BT-Drucks. 16/1828 S. 41; siehe auch Art. 5 Abs. 3 a) der RL 2001/29/EG.
} 


\section{(a) \53a UrhG}

Des Weiteren könnte eine Weiterreichung von Datensätzen durch Fremdwissenschaftler von der Schranke des \53a UrhG gedeckt sein. Die Norm unterscheidet dabei zwischen der in Satz 1 geregelten Vervielfältigung und Übermittlung von geschützten Werken in analoger Form im Wege des Post- oder Faxversands und der Vervielfältigung und Übermittlung in sonstiger elektronischer Form in Satz 2. Dabei privilegiert $\int 53 \mathrm{a}$ UrhG seinem klaren Wortlaut nach nur öffentliche Bibliotheken, so dass sich natürliche Personen und sonstige Forschungseinrichtungen, die die Daten an Dritte übermitteln wollen, von vornherein nicht auf die Schranke berufen können. Unerheblich ist hingegen, ob sich die jeweilige Bibliothek in öffentlicher oder privater Hand befindet. ${ }^{323} \mathrm{Da}$ es jedoch möglich erscheint, dass auch öffentliche Bibliotheken wissenschaftliche Primärdaten von archivierenden Einrichtungen zur Verfügung gestellt bekommen, stellt sich die Frage, ob diese die Daten gem. \53a UrhG an Dritte weitergeben dürfen.

\section{$\int 53 a$ Abs. 1 S. 1 UrbG}

Sofern die Datensätze nicht elektronisch, sondern in analoger Form vervielfältigt und übermittelt werden, wäre dies gem. \53a Abs. 1 S. 1 UrhG zulässig, sofern die betreffenden Datenbankwerke in Zeitungen oder Zeitschriften als Beitrag in Zeitungen oder Zeitschriften erschienen sind oder es sich bei dem übermittelten Datensatz, um kleine Teile eines erschienen Werkes handelt, wobei eher Letzteres der Fall sein wird.

\section{In Zeitungen und Zeitschriften erschienene Beiträge}

Die Schranke des $\int 53 \mathrm{a}$ Abs. 1 S. 1 UrhG könnte zunächst auf Datenbankwerke Anwendung finden, die als Beitrag in Zeitungen oder Zeitschriften erschienen sind. Denkbar wäre dies etwa im Rahmen von wissenschaftlichen Publikationen in Fachzeitschriften oder -zeitungen.

\section{Kleine Teile eines erschienenen Werkes}

Darüber hinaus ist die Nutzung kleiner Teile bereits erschienener Werke gem. \$ 53a Abs. 1 S. 1 UrhG zulässig. Damit wäre es öffentlichen Bibliotheken also grundsätzlich erlaubt, kleine Teile von Datenbankwerken auf Einzelbestellung zu vervielfältigen und zu übermitteln. Zwar ergibt sich der Wert einer Datenbank zumeist erst aus ihrer Gesamtheit. Es ist jedoch denkbar, dass Wissenschaftlern in bestimmten Fällen bereits Teile eines Datenbankwerkes genügen oder sie zunächst prüfen möchten, ob das betreffende Datenbankwerk für ihr eigenes Forschungsvorhaben brauchbar ist, bevor sie das komplette Datenbankwerk erwerben. Dies gilt insbesondere in den Fällen, in denen dieses nur entgeltlich bezogen werden kann. Damit $\int 53 a$ UrhG in diesen Fällen greift, muss das Datenbankwerk

\footnotetext{
323 Nordemann-Schiffel in Fromm/Nordemann, \ 53a Rn. 5.
} 
jedoch nach dem Wortlaut der Norm bereits erschienen sein. Der Begriff des Erscheinens richtet sich dabei nach $\$ 6$ Abs. 2 UrhG. Damit gilt das Werk dann als erschienen, wenn mit Zustimmung des Berechtigten Vervielfältigungsstücke nach ihrer Herstellung in genügender Anzahl der Öffentlichkeit angeboten oder in Verkehr gebracht worden sind. Unproblematisch wäre dies in den Fällen, in denen es in einer erschienenen Publikation abgedruckt wäre. Dies wird jedoch nur selten der Fall sein, da die Daten in der weit überwiegenden Anzahl der Fälle, wenn überhaupt, von der archivierenden Einrichtung lediglich in elektronischer Form auf einem Speichermedium zum Abruf bereit gehalten werden. Damit stellt sich die Frage, ob die Daten durch die Speicherung auf einem Internetserver bereits als erschienen gelten. Die Daten werden zwar mit Zustimmung des Berechtigten der Öffentlichkeit angeboten werden, indem es Dritten ermöglicht wird, sich die archivierten Daten herunterzuladen. ${ }^{324}$ Allerdings verlangt $\int 6$ Abs. 2 UrhG darüber hinaus die Anfertigung von Vervielfältigungsstücken in "genügender Anzahl“. Dabei müssen die Vervielfältigungen durch die Nutzer, die sich die Daten herunterladen, unberücksichtigt bleiben, da die Vervielfältigung nach dem Wortlaut der Norm zeitlich vor dem Angebot an die Öffentlichkeit bzw. dem In-VerkehrBringen zu erfolgen hat. ${ }^{325}$ Damit liegen vor dem Angebot an die Öffentlichkeit eigentlich keine Vervielfältigungsstücke in genügender Zahl vor, so dass das Werk nicht als erschienen im Sinne des $₫ 6$ Abs. 2 UrhG gelten würde. ${ }^{326}$

Nach teilweise vertretener Ansicht in der Literatur soll ein im Internet veröffentlichtes Werk deswegen erst dann als erschienen anzusehen sein, wenn das Zugänglichmachen im Internet von einer gewissen Dauer ist, da erst dann das Kriterium der „,bleibenden“ Zugänglichmachung erfüllt sei. ${ }^{327}$ Begründet wird dies damit, dass es beim bloßen Zugänglichmachen auf einem Internetserver an der Verselbständigung des Werkes fehle. ${ }^{328}$ Darüber hinaus spreche gegen ein Erscheinen, dass die auf dem Server bereitgestellte Datei nicht die Sphäre des Anbieters verlasse und die später von den Erwerbern hergestellten Vervielfältigungsstücke aufgrund der mangelnden Erschöpfung des Verbreitungsrechts noch nicht verkehrsfähig seien. ${ }^{329}$ Teilweise wird jedoch eingeräumt, dass ein Erscheinen zumindest dann vorliege, wenn ein Hersteller von Datenträgern diese einem Datenbankbetreiber (sog. ,host") zur Nutzung im Rahmen eines Kauf- oder Mietvertrags anbietet und überlässt. 330

\footnotetext{
324 W. Nordemann in Fromm/Nordemann, \6 Rn. 21; Süßenberger/C_yychowski, GRUR 2003, 489, 491.

325 Süßenberger/Caychowski, GRUR 2003, 489, 491.

326 So Schack, GRUR 2007, 639, 644.

327 Dreier in Dreier/Schulze, $\int 6$ Rn. 16; Dreyer in Dreyer/Kotthoff/Meckel, $\mathbb{} 6$ Rn. 62.

328 Katzenberger in Schricker/Loewenheim, \6 Rn. 56.

329 Schack, GRUR 2007, 639, 645.

330 Katzenberger in Schricker/Loewenheim, \6 Rn. 56.
} 
Trotz der genannten Argumente ist ein Werk, das auf einem Internetserver zum Abruf bereitgestellt wird, dennoch als erschienen anzusehen. ${ }^{331}$ Dafür spricht zunächst die Tatsache, dass der Gesetzgeber zum Zeitpunkt der Schaffung der Norm im Jahr 1965 die technischen Möglichkeiten von Printpublikationen vor Auge hatte. Bei diesen ist es jedoch gerade nötig, dass das Werk zunächst in genügender Anzahl vervielfältigt und anschließend der Öffentlichkeit angeboten wird, damit sie dem interessierten Verkehrskreis frei zur Verfügung stehen. ${ }^{332}$ Bei Werken, die zum Abruf im Internet bereit gestellt werden, hat jedoch ab dem Zeitpunkt der Bereitstellung prinzipiell jedermann die Möglichkeit, das Werk zu vervielfältigen und herunterzuladen, so dass es dem Interessentenkreis auch ohne die vorherige Herstellung mehrerer Vervielfältigungsstücke grundsätzlich frei zur Verfügung steht. ${ }^{333}$

Es ist zwar einzuräumen, dass die auf dem Server abgelegte Datei nicht die Sphäre des Urhebers verlässt, wohl aber die von den Nutzern hergestellten Vervielfältigungsstücke. Dies ist aber faktisch gesehen das Gleich als würde ein Verlag Bücher vervielfältigen und diese anschließend öffentlich anbieten. Denn auch in diesem Fall müsste ja nicht das Originalmanuskript die Sphäre des Urhebers verlassen, sondern nur dessen Kopien in Form von Büchern. Des Weiteren spricht für die Annahme des Erscheinens auch ein Vergleich mit sog. print-on-demandWerken. ${ }^{334}$ Dass diese als erschienen gelten, sofern sie der Öffentlichkeit angeboten werden, wird nämlich auch von der Gegenansicht nicht bestritten. ${ }^{335}$ Beim print-on-demand wird das Werk jedoch auch zunächst der Öffentlichkeit angeboten und erst dann eine Vervielfältigung erstellt, wenn eine entsprechende Bestellung beim Anbieter erfolgt. ${ }^{336}$ Bis zur ersten Vervielfältigung existiert somit in der Regel auch beim print-on-demand nur die digitale Datei, die dem Verlag als Druckvorlage dient. Von daher ergibt sich in diesen Fällen im Gegensatz zu Wer-

331 So auch Ablberg in Möhring/Niccolini, \ 6 Rn. 29; Dreier in Dreier/Schulze, $\ 6$ Rn. 16; Dreyer in

Dreyer/Kotthoff/Meckel, \6 Rn. 2; Katzenberger in Schricker/Loewenheim, \6 Rn. 55; W. Nordemann in Fromm/Nordemann, \6 Rn. 21; Dietz, GRUR Int. 1975, 341, 343; Goe-

bel/Hackemann/Scheller, GRUR 1986, 355, 359; Hubmann, GRUR 1980, 537, 541; Süßenberger/Caychowski, GRUR 2003, 489, 491.

332 W. Nordemann in Fromm/Nordemann; Süßenberger/Cyychowski, GRUR 2003, 489, 492.

333 Marquardt in Wandtke/Bullinger, \& 6 Rn. 2; Maaßen, ZUM 19925, 338, 343; Süßenberger/Csychowski, GRUR 2003, 489, 492.

${ }^{334}$ Zur Frage des Erscheinens beim print-on-demand: Melichar, Printing on Demand-Eine Bestandsaufnahme, in FS Dittrich, S. 229, 232 f.

335 Möhring/Niccolini, \6 Rn. 23; anders hingegen bei einer bloßen, an die Öffentlichkeit gerichteten Werbeaufforderung, Bücher zu subskribieren, wenn mit der Herstellung der Bücher gewartet wird, bis eine ausreichende Anzahl an Bestellungen vorliegt; siehe dazu Ulmer, Urheber- und Verlagsrecht, 3. Auflage 1980, \32 III 2; v. Gamm, Urheberrechtsgesetz, 1968, \6 Rn. 8; Schiefler, Veröffentlichung und Erscheinen nach dem neuen Urheberrechtsgesetz, UFITA 48 (1966), 81, 94.

${ }^{336}$ Etwas anderes gilt hingegen dann, wenn eine „Startauflage“ von einigen wenigen Exemplaren gedruckt wird. Dies wird zwar teilweise, jedoch nicht zwangsläufig beim print-on-demand so praktiziert. 
ken, die zum Download angeboten werden, lediglich der Unterschied, dass die Vervielfältigung beim print-on-demand auf Papier und nicht digital erfolgt.

Darüber hinaus kann auch grundsätzlich nicht verlangt werden, dass das Werk für einen bestimmten Zeitraum zum Abruf bereitgehalten wird. Selbst bei einer vergleichsweise kurzen Bereithaltung von nur einigen Tagen oder Stunden kann das Werk von Nutzern vervielfältigt und heruntergeladen werden. Da dies weltweit und damit für nahezu jedermann möglich ist, der Zugang zum Internet hat, ergibt sich automatisch ein nicht unerheblicher Kreis von potentiellen Interessenten. Andernfalls käme man zu dem widersprüchlichen Ergebnis, dass ein Werk zwar erschienen wäre, wenn es lediglich in einer Auflage von beispielsweise 50 Exemplaren erscheint, nicht jedoch, wenn es mehrere Wochen zum Abruf im Internet bereit steht, obwohl davon auszugehen ist, dass das Werk in dieser Zeit mindestens genauso häufig heruntergeladen würde. Darüber hinaus könnte die Bestimmung, wie lange ein Werk im Internet zum Abruf bereit stehen muss, damit es als erschienen gilt, nur willkürlich erfolgen. $\mathrm{Zu}$ dem gleichen Ergebnis kommt man ebenfalls über eine analoge Anwendung des $\ 6$ Abs. 2 S. 2 UrhG. ${ }^{337}$ Danach gilt ein Werk der bildenden Künste nämlich auch dann als erschienen, wenn das Original oder ein Vervielfältigungsstück des Werkes mit Zustimmung des Berechtigten bleibend der Öffentlichkeit zugänglich ist.

Insofern ist nach der hier vertretenen Ansicht ein Werk bereits dann erschienen, wenn es zum Download im Internet bereitgestellt worden ist. In diesen Fällen wäre es somit denkbar, dass die Schranke des $₫ 53$ a UrhG auf die Vervielfältigung und Übermittlung kleiner Teile eines Datenbankwerkes Anwendung findet.

Wo die Grenze hinsichtlich der Beurteilung gezogen werden muss, ob noch ein „kleines Werk" vorliegt, wird auch im Rahmen von $\int 53$ a UrhG unterschiedlich beurteilt. Insoweit kann auf die Ausführungen zu $\int 52 \mathrm{a}$ Abs. $1 \mathrm{Nr} .1 \mathrm{UrhG}$ verwiesen werden. ${ }^{338}$ Dabei gilt auch in den Fällen des $₫ 53$ a Abs. 1 UrhG, dass es grundsätzlich dahingestellt bleiben kann, ob die Grenze bei 10\% oder 20\% gezogen wird, da der Teil des Datenbankwerkes nach beiden Ansichten für Interessenten zu gering sein dürfte. Des Weiteren ist zu beachten, dass $₫ 53$ a Abs. 1 UrhG nur in den Fällen greift, in denen die Nutzung durch den Besteller nach $\ 53$ UrhG zulässig wäre. Dies sind im Rahmen der vorliegend im Vordergrund stehenden Datenbankwerke grundsätzlich nur die Fälle des $\ 53$ Abs. 1 oder Abs. 2 S. 1 Nr. 1 UrhG. ${ }^{339}$ Diese Schranke des $\ 53$ Abs. 2 S. 1 Nr. 1 UrhG findet jedoch, wie oben festgestellt in den vorliegend relevanten Sachverhaltskonstellationen nur selten Anwendung, da es in der Regel an der Gebotenheit der Vervielfältigung fehlen wird. ${ }^{340}$ Sofern die Voraussetzungen von $₫ 53$ a Abs. 1 S. 1 im Einzelfall doch vorliegen sollten, ist zu beachten, dass die Übermittlung nur im Wege des

\footnotetext{
337 Vgl. Marquardt in Wandtke/Bullinger, \6 Rn. 29.

338 Siehe oben S. 90.

${ }^{339}$ Siehe dazu bereits oben S. 94.

340 Siehe oben S. 95 ff.
} 
Post- und Faxversands zulässig wäre. Dies ist jedoch ein weiterer Grund, weshalb die Schranke des $\int 53$ a Abs. 1 S. 1 UrhG in den vorliegend relevanten Fallkonstellationen nur selten Anwendung findet, wird der Lizenznehmer die Daten in der Regel dem Dritten doch in elektronischer Form weiterleiten.

Eine solche Vervielfältigung und Übermittlung von Teilen des Datenbankwerkes in sonstiger elektronischer Form wäre hingegen nach $\int 53$ a Abs. 1 S. 2 ausschließlich als grafische Datei und zur Veranschaulichung des Unterrichts oder für Zwecke der wissenschaftlichen Forschung zulässig, soweit dies zur Verfolgung nicht gewerblicher Zwecke gerechtfertigt ist. Demzufolge wäre es grundsätzlich möglich, dass öffentliche Bibliotheken kleine Teile von erschienenen Datenbankwerken als grafische Datei, wie beispielsweise in Form einer pdf-Datei versenden würden. ${ }^{341}$ Dies wäre zunächst unproblematisch, da die Darstellung eines Datenbankwerkes als pdf grundsätzlich ausreichend wäre. Darüber hinaus müssten die Vervielfältigung und der Versand in elektronischer Form jedoch zur Veranschaulichung des Unterrichts oder für Zwecke der wissenschaftlichen Forschung erfolgen. Die Vervielfältigung und der Versand in elektronischer Form wäre also von \ 53a Abs. 1 S. 2 UrhG gedeckt, sofern die Kopie dazu beitragen würde, den Unterrichtsstoff besser oder verständlicher darzustellen. ${ }^{342}$ Dies wäre grundsätzlich denkbar, da auch Teile einer Datenbank dazu geeignet sein können, bestimmte wissenschaftliche Zusammenhänge oder Phänomene zu verdeutlichen. Die Kopie dürfte jedoch nur für den Teilnehmerkreis der Unterrichtsveranstaltung zugänglich sein. Unerheblich ist hingegen, ob der Unterricht tatsächlich in der Schule stattfindet oder ob es sich um einen öffentlichen oder privaten Schulträger handelt. ${ }^{343}$ Allerdings dürfte die Schule nicht auf die Erzielung von Gewinnen ausgerichtet sein, da der Versand in elektronischer Form gem. Satz 2 zu nicht gewerblichen Zwecken erfolgen darf, wobei es jedoch nicht auf die organisatorische Struktur der Einrichtung als solche ankommt, sondern die konkrete Tätigkeit. ${ }^{344}$

Des Weiteren erlaubt $\ 53 \mathrm{a}$ Abs. 1 S. 2 die Versendung in elektronischer Form zum Zwecke der wissenschaftlichen Forschung. Dies wird wohl in der überwiegenden Zahl der hier relevanten Fälle zutreffen, stehen im Vordergrund dieser Untersuchung doch wissenschaftliche Primärdaten. Deshalb werden die Bezieher dieser Daten wohl hauptsächlich zum Zwecke der wissenschaftlichen Forschung benötigen. Auch hier ist jedoch wieder zu beachten, dass die Forschung nicht zu gewerblichen Zwecken dienen darf, weshalb Satz 2 in den Fällen der Auftragsfor-

\footnotetext{
${ }^{341}$ Nordemann-Schiffel in Fromm/Nordemann, \53a Rn. 13; Hoeren, MMR 2007, 615, 618; vgl. auch Jani in Wandtke/Bullinger, $\int 53 a$ Rn. 26.

342 Jani in Wandtke/Bullinger, \53a Rn. 27; Nordemann-Schiffehn Fromm/Nordemann, \53a Rn. 14.

343 Nordemann-Schiffel in Fromm/Nordemann, \53a Rn. 14; einschränkend v. Bermuth, ZUM 2003, 438, 441, nach dem Privatschulen nur dann in den Anwendungsbereich fallen, wenn sie öffentlich zugänglich sind.

${ }^{344}$ Jani in Wandtke/Bullinger, $\ 53$ a Rn. 28; Hoeren, MMR 2007, 515, 518.
} 
schung nicht greift. ${ }^{345}$ Demzufolge wäre die Übermittlung kleiner Teile von Datenbankwerken oder Datenbankwerken, die bereits in Zeitschriften oder Zeitungen erschienen sind, in elektronischer Form grundsätzlich von $\int 53$ a Abs.1 S. 2 UrhG privilegiert.

$\mathrm{Zu}$ beachten ist im Einzelfall jedoch, dass Satz 3 die Privilegierung dahingehend einschränkt, dass eine Vervielfältigung und Übermittlung in elektronischer Form nur zulässig ist, wenn der Zugang zu den Beiträgen oder kleinen Teilen eines Werkes den Mitgliedern der Öffentlichkeit nicht offensichtlich von Orten und zu Zeiten ihrer Wahl mittels einer vertraglichen Vereinbarung zu angemessenen Bedingungen ermöglicht wird. ${ }^{346}$ Durch diese Einschränkung soll sichergestellt werden, dass Verlage oder Lizenznehmer, die das betreffende Werk bereits in elektronischer Form anbieten, durch die Schranke des $\ 53 \mathrm{a}$ UrhG nicht beeinträchtigt werden. ${ }^{347}$ Sofern das Datenbankwerk bzw. kleine Teile davon im Einzelfall bereits im Internet angeboten werden, wäre eine Versendung in elektronischer Form demnach ausgeschlossen. ${ }^{348}$ Bedenkt man, dass gerade öffentlich getragene Forschungseinrichtungen ihre archivierten Daten oftmals im Internet Dritten, insbesondere Fremdforschern zur Verfügung stellen werden, wird der Anwendungsbereich von $₫ 53$ a Abs. 1 S. 2 UrhG in der Praxis relativ klein ausfallen. Das Onlineangebot der archivierenden Einrichtungen wird in der Regel nämlich auch "offensichtlich“" sein. Dies ist nach dem Bericht des Rechtsausschusses dann der Fall, wenn es in einer zentral administrierten Datenbank der Bibliotheken und Verlage verzeichnet ist. ${ }^{349} \mathrm{Da}$ die Archivierung und die damit verbundene öffentliche Zugänglichmachung zum großen Teil von Universitäten oder Universitätsbibliotheken durchgeführt werden wird, ist davon auszugehen, dass die archivierten Daten und Datenbankwerke in den angegebenen Katalogen verzeichnet und damit der Öffentlichkeit „offensichtlich“ zugänglich ist. Damit scheidet $₫ 53$ a Abs. 1 S. 2 UrhG in diesen Fällen jedoch aus.

\section{(b) $\int 69 \mathrm{~d} \mathrm{UrhG}$}

Des Weiteren könnten in den vorliegenden Fällen die Schranken der $\$ \int 69 \mathrm{~d}$ und 69e UrhG einschlägig sein. Dabei folgt aus den $\$ \int 69 \mathrm{~b}$ und 69c UrhG, dass es sich dafür bei den Daten um Computerprogramme handeln müsste. Unter den Begriff des Computerprogramms fallen gem. \69a UrhG Programme in jeder Gestalt, einschließlich des Entwurfsmaterials. Was jedoch genau unter einem Computer-

\footnotetext{
345 Nordemann-Schiffel in Fromm/Nordemann, \53a Rn. 14; Jani in Wandtke/Bullinger, \53a Rn. 28; Hoeren, MMR 2007, 615, 618; dies gilt jedoch ebenso für die Fälle des Satzes 1 aufgrund der Tatsache, dass die Vervielfältigung durch den Besteller von $\ 53$ UrhG gedeckt sein müsste, der wiederum die Vervielfältigung zum wissenschaftlichen Gebrauch nur zu nicht gewerblichen Zwecken gestattet.

346 Vgl. Spindler, NJW 2008, 9, 14.

347 Vgl. Sprang/Ackermann, K\&R 2008, 7, 8; Spindler, NJW 2008, 9, 14.

348 Spindler, NJW 2008, 9, 14.

${ }^{349}$ BeschlE Rausschuss 2. Korb - BT-Drucks. 16/5939, S. 45.
} 
programm zu verstehen ist, definiert das Gesetz hingegen nicht. ${ }^{350}$ Fest steht jedoch, dass Datenbanken und einzelne Daten nicht unter den Begriff des Computerprogramms i.S.d. \ 69a UrhG fallen, was sich bereits aus den $\iint 4$ und $87 \mathrm{a}$ UrhG ergibt. ${ }^{351}$ Aus diesem Grund kommen sowohl die Schranken des $\int 69 \mathrm{~d}$ als auch die Schranke des $\int$ 69e UrhG im Rahmen der elektronischen Langzeitarchivierung von vorliegend im Fokus stehenden wissenschaftlichen Primärdaten nicht in Betracht.

\section{(c) $\int 87 b$ i.V.m. 87e UrhG}

Als weitere Schranke kommt die Vorschrift des $\int 87 b$ i.V.m. $\int 87$ e UrhG in Betracht, sofern eine Datenbank i.S.d. \87a UrhG vorliegt. Danach ist eine vertragliche Vereinbarung unwirksam, durch die sich der zum Gebrauch einer Datenbank Berechtigte gegenüber dem Datenbankhersteller dazu verpflichtet, die Vervielfältigung, Verbreitung oder öffentliche Wiedergabe von nach Art und Umfang unwesentlichen Teilen der Datenbank zu unterlassen, soweit diese Handlungen weder einer normalen Auswertung der Datenbank zuwiderlaufen noch die berechtigten Interessen des Datenbankherstellers unzumutbar beeinträchtigen. Aus $\int 87 \mathrm{e}$ in Verbindung mit $\int 87 \mathrm{~b}$ UrhG folgt damit, dass die Nutzung unwesentlicher Teile eines Datenbankwerkes erlaubt ist, sofern es sich nicht um eine wiederholte und systematische Nutzung handelt. ${ }^{352}$ Genau genommen handelt es sich bei $\int 87 \mathrm{e}$ UrhG nicht um eine urheberrechtliche Schranke, da durch die $\iint 87 \mathrm{~b}$ und $87 \mathrm{e}$ UrhG nicht die bestehenden Nutzungsrechte des Rechteinhabers beschränkt werden, sondern die Nutzungsrechte des Datenbankherstellers gem. \ 87b Abs. 1 S. 1 UrhG von vornherein nur für die Nutzung des wesentlichen Teils der Datenbank entstehen. Sofern der Lizenznehmer also nur bestimmte Daten aus einer Datenbank vervielfältigen wollte, würde dies aufgrund der Regelung des $\int 87 \mathrm{~b}$ UrhG keine Verletzung der Nutzungsrechte des Datenbankherstellers darstellen, sofern es sich nicht gem. \87 Abs. 1 S. 2 UrhG um eine systematische und wiederholte Vervielfältigung von unwesentlichen Teilen der Datenbank handeln würde. ${ }^{353}$

(d) $\int 87 \mathrm{c}$ UrhG

Sofern der Lizenznehmer einen nach Art und Umfang wesentlichen Teil der Datenbank vervielfältigt, könnte außerdem eine der Schranken des $\int 87$ c UrhG einschlägig sein. Danach ist die Vervielfältigung eines wesentlichen Teils einer Da-

\footnotetext{
350 Dreier in Dreier/Schulze, \69a Rn. 12; Kotthoff in Dreyer/Kotthoff/Meckel, \ 69a Rn. 4; Loewenbeim in Schricker/Loewenheim, S 69a Rn 2; Caychowski in Fromm/Nordemann, S 69a Rn. 5.

351 Caychowski in Fromm/Nordemann, $\int$ 69a Rn. 12; Grützmacher in Wandtke/Bullinger, $\int 69 a$ Rn. 16; Dreier in Dreier/Schulze, $\int$ 69a Rn. 12; vgl. auch Art. 1 Abs. 3 und Art. 2 a) der DatenbankRichtlinie.

352 Loewenheim in Loewenheim, \ 43 Rn. 22.

353 Zur Frage, wann ein unwesentlicher Teil einer Datenbank vorliegt, vergleiche die Ausführungen auf S. 43.
} 
tenbank zulässig zum privaten Gebrauch, zum eigenen wissenschaftlichen Gebrauch sowie für die Benutzung zur Veranschaulichung im Unterricht.

$$
\int 87 c \text { Abs. } 1 \text { S. } 1 \text { Nr. } 1 \text { UrbG }
$$

Die Vervielfältigung eines wesentlichen Teils einer Datenbank durch den Lizenznehmer wäre gem. \87c Abs. 1 S. 1 Nr. 1 UrhG zum privaten Gebrauch zulässig. ${ }^{354}$ Aus $\int 87$ c Abs. 1 S. 1 Nr. 1 Hs. 2 UrhG folgt jedoch, dass die Schranke nur die Vervielfältigung von nicht-elektronischen Datenbanken erfasst und daher in den vorliegenden Fällen keine Anwendung findet. Abgesehen davon würden die sonstigen Tatbestandsvoraussetzungen regelmäßig nicht erfüllt sein, da es sich bei den Lizenznehmern in der Regel nicht um natürliche, sondern juristische Personen oder Personengesellschaften handeln wird, und damit schon kein privater Gebrauch vorliegen kann. ${ }^{355}$

$$
\int 87 c \text { Abs. } 1 \text { S. } 1 \text { Nr. } 2 \text { UrbG }
$$

In Betracht kommt jedoch die Schranke des $\int 87$ c Abs. 1 S. 1 Nr. 2 UrhG, wonach die Vervielfältigung wesentlicher Teile einer Datenbank zum eigenen wissenschaftlichen Gebrauch zulässig ist, soweit die Vervielfältigung zu diesem Zweck geboten ist und der wissenschaftliche Gebrauch nicht zu gewerblichen Zwecken erfolgt. Aus der letztgenannten Voraussetzung folgt, dass sich Forschungsabteilungen von Wirtschaftsunternehmen oder Forschungseinrichtungen, die mit Gewinnerzielungsabsicht arbeiten, von vornherein nicht auf die Schranke des $\int 87 \mathrm{c}$ Abs. 1 S. 1 Nr. 1 UrhG berufen können. ${ }^{356}$ Darüber hinaus müsste die Vervielfältigung durch den Lizenznehmer zum wissenschaftlichen Gebrauch erfolgen. Wie oben bereits festgestellt, wird die Vervielfältigung der hier im Vordergrund stehenden Datenbanken grundsätzlich dem wissenschaftlichen Gebrauch, also dem methodischen systematischen Streben nach Erkenntnis dienen, da die Lizenznehmer die Vervielfältigungen in der Regel vornehmen werden, um die in der Datenbank enthaltenen Daten in ihren eigenen Forschungsvorhaben zu verwenden ${ }^{357}$.

Darüber hinaus müsste der Lizenznehmer die Datenbankinhalte jedoch zum eigenen wissenschaftlichen Gebrauch vervielfältigen. Dies ist der Fall, wenn die Vervielfältigung innerhalb einer geschlossenen Einheit stattfindet und keine Wei-

\footnotetext{
${ }^{354}$ Zur Anwendbarkeit der Schranken des \87c UrhG im Verhältnis zwischen der archivierenden Einrichtung und dem Nutzungs- oder Leistungsschutzrechteinhaber der zu archivierenden Werke siehe bereits oben S. $40 \mathrm{ff}$.

355 Vgl. BGH GRUR 1978, 474, 475; Vogel in Schricker/Loewenheim, \87c Rn. 12 (der auf Loewenheim in Schricker/Loewenheim, $\int 53$ Rn. 14 verweist); Dreier in Dreier/Schulze, $\int 87 c$ Rn. 6 (mit Verweis auf 53 Rn. 7); Cyychowski in Fromm/Nordemann, $\ 87$ c Rn. 7 (mit Verweis auf W. Nordemann in Fromm/Nordemann, $\$ 53$ Rn. 7).

356 Vgl. Loewenheim in Loewenheim, \43 Rn. 23; Vogel in Schricker/Loewenheim, \87c Rn. 16.

357 Siehe zum Merkmal des wissenschaftlichen Gebrauchs die Ausführungen auf S. 41.
} 
tergabe an Dritte beabsichtigt ist. ${ }^{358}$ Die Schranke des $\int 87$ c Abs. 1 Nr.2 UrhG greift daher in den vorliegenden Fällen nur dann, wenn der Lizenznehmer nicht beabsichtigt, die vervielfältigten Inhalte der Datenbank Außenstehenden, wie etwa anderen Forschungseinrichtungen zugänglich zu machen. Vielmehr dürfte er die Vervielfältigung nur vornehmen, um mehreren Wissenschaftlern der gleichen Forschungseinrichtung das gleichzeitige Arbeiten mit den Datensätzen zu ermöglichen. 359

Die Vervielfältigung der Datensätze wäre allerdings nur dann geboten, wenn die wissenschaftliche Arbeit des Lizenznehmers dies erfordert und der Erwerb der Daten bzw. weiterer Lizenzen unzumutbar erscheint oder nicht problemlos möglich wäre. ${ }^{360}$ Gegen die Gebotenheit der Vervielfältigung wesentlicher Teile einer Datenbank ließe sich daher anführen, dass der Lizenznehmer weitere Lizenzen vom Lizenzgeber unproblematisch erwerben könnte und sich damit eine Vervielfältigung wesentlicher Teile der Datenbank erübrigen würde. Dies wäre zumindest dann der Fall, wenn der Lizenzgeber die Lizenzen kostenlos zur Verfügung stellen würde. ${ }^{361}$ Etwas anderes ergibt sich jedoch für die Fälle, in denen der Lizenzerwerb entgeltlich erfolgt. Hier würden für den Lizenzgeber erhebliche Mehrkosten durch den Erwerb mehrerer Lizenzen eines Datensatzes entstehen. Da jedoch gerade bei nicht gewerblichen Forschungseinrichtungen in der Regel nur begrenzte finanzielle Mittel zur Verfügung stehen, wäre dies gerade bei Projekten, in denen eine hohe Anzahl von Forschern tätig sind und demzufolge auch dementsprechend viele Lizenzen erforderlich wären, kaum finanzierbar. ${ }^{362}$

Aus diesem Grund ist in diesen Fällen von einer Gebotenheit der Vervielfältigung auszugehen. Demzufolge findet die Schranke des $₫ 87$ c Abs. 1 S. 1 Nr. 2 UrhG grundsätzlich Anwendung, wenn der Lizenznehmer sich wesentliche Teile der Datenbank vervielfältigt, die er vom Lizenzgeber erhalten hat. Zu beachten ist dabei jedoch, dass in diesem Fall gem. \87c Abs. 1 S. 2 UrhG die Quelle der Datenbank deutlich anzugeben ist. Der Lizenznehmer hat also den Namen des Datenbankherstellers sowie den Namen der Datenbank zu nennen, aus der die Teile stammen. 363

\footnotetext{
358 BGHZ 134, 250, 257 f. - CB Infobank I; Decker in Möhring/Niccolini, \53 Rn. 18; Dreier in Dreier/Schulze, \87c Rn. 9; $W$. Nordemann in Fromm/Nordemann, IS 53 Rn. 16; siehe auch BT-Drucks. 10/837, S.9.

359 Vgl. dazu auch die Ausführungen auf S. 41.

360 Dreier in Dreier/Schulze, \87c Rn. 10 (mit Verweis auf $\int 53$ Rn. 23); Vogel in Schricker, \87c Rn. 13 (mit Verweis auf Loewenheim in Schricker, $\int 53$ Rn. 23); Lüft in Wandtke/Bullinger, $\int 53 \mathrm{Rn}$. 27, 29; Crychowski in Fromm/Nordemann, $\int 87 \mathrm{c}$ Rn. 8 (mit Verweis auf W. Nordemann in Fromm/Nordemann, \53 Rn. 19).

361 Vgl. auch die Ausführungen auf S. 96.

362 Vgl. auch die parallele Problematik im Rahmen von \53 Abs. 2 S. 1 Nr. 1 auf S. 95 ff.

363 Vogel in Schricker/Loewenheim, \87c Rn. 16; Thum in Wandtke/Bullinger, \87c Rn. 36.
} 


\section{$\int 87 c$ Abs. 1 S. 1 Nr. 3 UrbG}

Als letzte Schranke im Rahmen von $\int 87$ c UrhG käme die Benutzung zur Veranschaulichung des Unterrichts in Betracht. Wie oben bereits dargelegt, ist es denkbar, dass ein Lizenznehmer die vom Lizenzgeber erlangten Daten oder Teile davon zur Veranschaulichung des Unterrichts nutzen möchte. Sofern er dafür den wesentlichen Teil 364 einer Datenbank verwendet, könnte in diesen Fällen die Schranke des $\int 87$ c Abs. 1 S. 1 Nr. 3 UrhG greifen.

In diesem Zusammenhang ist umstritten, ob sich auch Hochschulen auf die Schranke berufen können, wenn die Vervielfältigung zum Gebrauch in der Vorlesung erfolgt. ${ }^{365} \mathrm{Da}$ der Wortlaut von $\int 87 \mathrm{c}$ UrhG -im Gegensatz zur Parallelvorschrift des $\int 53$ Abs. 3 Nr. 1 UrhG- insofern keine Einschränkungen enthält, könnte man dies durchaus in Betracht ziehen. ${ }^{366}$

Zu berücksichtigen ist jedoch, dass der Unterricht an Hochschulen und Universitäten gerade nicht im Katalog der Parallelvorschrift des $\int 53$ Abs. 3 Nr. 1 UrhG aufgezählt wird. Da jedoch der Gesetzgeber von der Möglichkeit der Richtlinie keinen Gebrauch gemacht hat, die privilegierten Einrichtungen in $\int 87 \mathrm{c}$ UrhG zu beschränken, ${ }^{367}$ ist davon auszugehen, dass der bestehende Katalog der Parallelvorschrift des $\int 53$ Abs. 3 Nr. 1 UrhG nicht erweitert werden sollte. Es gibt nämlich keine Anhaltspunkte dafür, dass die Gründe, die den Gesetzgeber dazu bewogen haben, den Unterricht an Hochschulen und Universitäten nicht unter die Schranke des $\int 53$ Abs. 3 Nr. 1 UrhG zu fassen, im Rahmen des $\int$ 87c Abs. 1 Nr. 2 UrhG nicht bestehen. ${ }^{368}$ Vielmehr bestünde auch hier das Problem der Unüberschaubarkeit des nutzenden Personenkreises, wenn die Schranke auch für den Unterricht an Hochschulen greifen würde. Darüber hinaus spricht für ein solches Ergebnis auch die Tatsache, dass die möglichst parallele Ausgestaltung der Schranken des Urheberrechts und denen des Leistungsschutzrechts das erklärte Ziel der Richtliniengeber war. ${ }^{369}$ Demzufolge fällt der Unterricht an Hochschulen und Universitäten nicht unter $\int 87$ c Abs. 1 Nr. 3 UrhG. Vielmehr muss sich bei der Bestimmung der von $\int 87 \mathrm{c}$ UrhG privilegierten Einrichtungen am Katalog des $\int 53$ Abs. 3 UrhG orientiert werden. ${ }^{370}$

\footnotetext{
364 Zur Frage, wann ein solcher wesentlicher Teil vorliegt, siehe oben S. 43.

${ }^{365}$ Vgl. Thum in Wandtke/Bullinger, \87c Rn. 33.

366 Vgl. Vogel in Schricker/Loewenheim, \87c Rn. 19.

367 Siehe Erwägungsgrund 51 der Datenbankrichtlinie.

368 Vgl. Leistner, Der Datenschutz von Datenbanken im deutschen und europäischen Recht, S. 316.

${ }^{369}$ Gaster, Der Rechtsschutz von Datenbanken, Rn. 600 - 602; Leistner, Der Datenschutz von Datenbanken im deutschen und europäischen Recht, S. 316.

370 So auch Thum in Wandtke/Bullinger, \87c Rn. 33; Crychowski in Fromm/Nordemann, \87c Rn. 11; Leistner, Der Datenschutz von Datenbanken im deutschen und europäischen Recht, S. 316; Haberstumpf, GRUR 2003, 14, 30; Vogel in Schricker/Loewenheim, \87c Rn. 19; a.A. Kotthoff in Dreyer/Kotthoff/Meckel, \87c Rn. 9, da der Wortlaut von \87c Abs. 1 Nr. 3 gerade keine Einschränkung hinsichtlich der Bildungseinrichtung enthalte.
} 
Wie auch bei Nr. 2 muss es sich darüber hinaus auch im Rahmen des $\int 87$ c Abs. 1 S. 1 Nr. 3 UrhG um eine nichtgewerbliche Einrichtung handeln, weshalb beispielsweise entgeltliche Privatschulen nicht von der Norm erfasst werden. ${ }^{371}$ Darüber hinaus müsste die Vervielfältigung nach dem Wortlaut der Norm zur Veranschaulichung des Unterrichts erfolgen. Dies ist der Fall, wenn die Datenbankteile bzw. die in ihnen enthaltenen Daten in den Unterricht eingebunden werden und damit zur Veranschaulichung des Stoffes beitragen sollen. ${ }^{372}$ Auch hier ist wiederum zu beachten, dass der Lizenznehmer gem. \87c Abs. 1 S. 2 UrhG die Quelle der Datenbankteile deutlich anzugeben hat.

\section{(4) Abdingbarkeit dieser Schranken durch vertragliche Vereinbarung}

Wenn urheberrechtliche Schranken in den vorliegenden Fällen auch nur in einem verhältnismäßig kleinen Umfang greifen werden, stellt sich dennoch die Frage, ob bzw. inwiefern die archivierenden Einrichtungen diese Schranken durch Lizenzvereinbarungen abbedingen können, die sie mit anderen Forschungseinrichtungen oder Fremdforschern schließen.

(a) Vertretene Ansichten in der Literatur

Die Zulässigkeit einer vertraglichen Abbedingung urheberrechtlicher Schranken wird in der Literatur uneinheitlich beantwortet. Nach teilweise vertretener Ansicht soll zumindest eine individualvertragliche Abbedingung urheberrechtlicher Schranken grundsätzlich zulässig sein, sofern das Gesetz die Schranke nicht ausdrücklich für unabdingbar erklärt. ${ }^{373}$ Eine Abbedingung urheberrechtlicher Schranken mittels AGB soll hingegen dann unzulässig sein, wenn die Schranke „von einem überragenden, insbesondere von einem selbst explizit grundrechtlich verbürgten Allgemeininteresse getragen ist" ${ }^{\text {", }} \mathrm{da}$ in diesen Fällen eine unangemessene Benachteiligung nach $\int 307$ Abs. 1 BGB vorliege. ${ }^{374}$ Dagegen soll beispielsweise die vertragliche Abbedingung einer Schranke, die lediglich ein Marktversagen korrigiert, auch im Rahmen von AGB zulässig. ${ }^{375}$

Nach einer anderen im Schrifttum vertretenen Ansicht sind urheberrechtliche Schranken hingegen zwingend und zwar auch dann, wenn das Gesetz die Abding-

371 Thum in Wandtke/Bullinger, $\int 87 c$ Rn. 33; Vogel in Schricker/Loewenheim, \87c Rn. 19.

372 Vogel in Schricker/Loewenheim, $\int 87 \mathrm{c}$ Rn. 20; vgl. auch Thum in Wandtke/Bullinger, $\int 87 c$ Rn. 32.

373 Dreier in Dreier/Schulze, Vor $\$ ₫ 44 a$ ff. Rn. 9; Götting in Schricker/Loewenheim, \95b Rn. 20; a.A. Guibault in Hugenholtz (Hrsg.), Copyright and Electronic Commerce, S. 125, 155 f., die eine Abbedingung nur im Schul-, Bibliotheks-, Archiv- und Museumsbereich für möglich hält.

${ }^{374}$ Dreier in Dreier/Schulze, Vor \$S 44a ff. Rn. 9; Dreier/Senftleben in Lejeune, S. 116 f. Rn. 44; Hohagen in FS Schricker, S. 362 f.

375 Dreier in Dreier/Schulze, Vor \$S 44a ff. Rn. 9; Dreier/Senftleben in Lejeune (Hrsg.), Der ECommerce-Vertrag nach amerikanischem Recht, 2001, S. 117. 
barkeit nicht ausdrücklich ausschließt. ${ }^{376}$ Lediglich in Einzelfällen, solle eine Abbedingung zulässig sein, so etwa das durch AGB von Videotheken vereinbarte Verbot, die entliehenen Videofilme oder DVDs zu kopieren. ${ }^{377}$

\section{(b) Eigene Stellungnahme}

Bei der Beurteilung der Zulässigkeit urheberrechtlicher Schranken ist zunächst zu prüfen, ob das Gesetz eine solche Abbedingung ausdrücklich untersagt oder sich die Unzulässigkeit im Rahmen einer teleologischen oder europarechtskonformen Auslegung der Schrankenregelungen ergibt. Sofern dies nicht der Fall ist, würde die Abbedingung nach dem in Art. 2 Abs. 1 GG verankerten Grundsatz der Privatautonomie grundsätzlich zulässig sein. Dabei könnte sich eine Unzulässigkeit allerdings aus den Vorschriften des allgemeinen Zivilrechts, namentlich der SS 138 und 307 ff. BGB ergeben.

\section{Gesetzliches Verbot hinsichtlich konketer Schranken}

Ein konkretes Verbot der vertraglichen Abbedingung besteht lediglich hinsichtlich $\operatorname{der} \iint 55 \mathrm{a}^{378}, 69 \mathrm{~d}$ Abs. 2 und Abs. $3{ }^{379}, \int 69 \mathrm{e}^{380}$ sowie der $\iint 87 \mathrm{e}$ und $95 \mathrm{~b}$ Abs. 1 UrhG ${ }^{381}$. Hinsichtlich der übrigen Schranken schließt das Gesetz eine vertragliche Abbedingung hingegen nicht ausdrücklich aus. Daraus könnte man im Umkehrschluss folgern, dass eine vertragliche Abdingbarkeit für die übrigen Schranken stets zulässig ist. Dabei ist jedoch zu beachten, dass die genannten Vorschriften jeweils der Umsetzung europäischer Richtlinien dienen, die ausdrücklich vorsehen, dass die genannten Schranken nicht vertraglich abbedungen werden dürfen. ${ }^{382}$ Wenn der deutsche Gesetzgeber in Umsetzung dieser Richtlinien in nationales Recht die Abdingbarkeit ausgeschlossen hat, so folgt daraus mithin nicht zwangsläufig im Umkehrschluss, dass die übrigen Schranken abdingbar sind. Vielmehr ist davon auszugehen, dass der Gesetzgeber bei der Schaffung der Vorschriften die Umsetzung der jeweiligen Richtlinie vor Augen hatte, zu der er verpflichtet ist. Es würde damit zu weit gehen, anzunehmen, der Gesetzgeber hätte eine abschließende Regelung hinsichtlich der Abdingbarkeit urheberrechtlicher oder leistungsrechtlicher Schranken beabsichtigt. ${ }^{383}$ Somit kann also nicht allein aus einem Um-

\footnotetext{
376 Vgl. Schack, Rn. 81; ders., ZUM 2002, 497, 503; Guibault, Copyright Limitations and Contract, 2002, S. 291.

377 Vgl. Schack, ZUM 2002, 497, 503 Fn. 55, wobei bei DVDs oftmals ein technischer Kopierschutz installiert ist.

378 Siehe $\int 55 \mathrm{a}$ S. 3 UrhG.

379 Siehe $\int 69 \mathrm{~g}$ Abs. 2 UrhG.

380 Siehe $\int 69 \mathrm{~g}$ Abs. 2 UrhG.

381 Siehe $\int 95$ b Abs. 1 S. 2 UrhG.

382 Vgl. Art. 6 Abs. 1, Art. 8, Art. 15 der Datenbankrichtlinie (RL 96/9/EG v. 11.3.1996); Art. 5 Abs. 2 und 3, Art. 6 und Art. 9 Abs. 1 der RL 91/250/EWG v. 14.5.1991.

383 Dagegen hat der belgische Gesetzgeber im Rahmen der Umsetzung der Datenbankrichtlinie die vertragliche Abbedingung für sämtliche gesetzliche Schranken im Urheberrecht für unwirksam
} 
kehrschluss zu den $\iint 55 \mathrm{a}, 69 \mathrm{~g}$ und 87 e UrhG die vertragliche Abdingbarkeit der übrigen Schrankenvorschriften geschlossen werden. ${ }^{384}$ Auf der anderen Seite können die genannten Normen und das in ihnen enthaltene Abbedingungsverbot nicht analog auf die übrigen Schranken übertragen werden. ${ }^{385}$ Die genannten Vorschriften belegen, dass der Gesetzgeber sich der Möglichkeit der vertraglichen Abbedingung urheberrechtlicher Schranken und der damit einhergehenden Probleme bewusst war. Damit fehlt es hinsichtlich der übrigen Schranken jedoch an einer planwidrigen Regelungslücke.

\section{Teleologische Auslegung der gesetzlichen Schrankenregelungen}

Auch wenn die Schrankenregelungen bis auf die oben genannten Ausnahmen kein ausdrückliches Verbot der Abbedingung enthalten, könnte sich ein solches aus dem Sinn und Zweck der Schrankenregelungen im Rahmen einer teleologischen Auslegung ergeben. In diesem Zusammenhang ist zunächst festzustellen, welchen Zweck die Schrankenregelungen verfolgen. Diesbezüglich weichen die Ansichten innerhalb der Literatur und Rechtsprechung teilweise voneinander ab.

Grundsätzlich lässt sich jedoch zunächst festhalten, dass die Schrankenregelungen einen Ausgleich zwischen den Interessen des Urhebers und denen der Allgemeinheit herstellen sollen. ${ }^{386} \mathrm{Zu}$ beachten ist in diesem Zusammenhang, dass das Urheberrecht als verfassungsrechtlich geschütztes Eigentum i.S.v. Art. 14 GG anzusehen ist. ${ }^{387}$ Durch die urheberrechtlichen Schranken wird in dieses Recht des Urhebers auf sein (geistiges) Eigentum aus Art. 14 Abs. 1 S. 1 GG eingegriffen. ${ }^{388}$ Dadurch soll ein Ausgleich zwischen den Interessen des Urhebers an der Wahrung seines geistigen Eigentums und dem damit verbundenen Schutz seiner geistigen Leistung auf der einen Seite und den Zugangs- und Nutzungsinteressen von Endnutzern und Werkvermittlern auf der anderen Seite hergestellt werden. ${ }^{389}$ Der Schutz dieser Zugangs- und Nutzungsinteressen folgt in diesem Zusammenhang ebenfalls aus Art. 14, da dieser nicht nur den Schutz der urheberrechtlichen Nutzungsrechte garantiert, sondern gleichzeitig in Abs. 2 die Sozialgebundenheit die-

erklärt; vgl. Visscher/Michaux, Précis du droit d'auteur et des droits voisins, Brüssel 2000, S. 102 .

384 So auch Hohagen in Ohly/Bodewig/Dreier/Götting/Haedicke/Lehmann (Hrsg.), FS Schricker, S. 361; vgl. auch Schack, ZUM 2002, 497, 502.

385 Schack, ZUM 2002, 497, 502; vgl. auch Gibault, Copyright Limitations and Contracts, 2002, S. 220, nach der das Schweigen des Gesetzgebers zur Abdingbarkeit der übrigen Schranken sowohl für als auch gegen deren vertragliche Abdingbarkeit sprechen könnte.

386 Vgl. BVerfG GRUR 1980, 44, 46; Melichar in Schricker/Loewenheim, Vor SS 44a ff. Rn. $1 \mathrm{ff}$; Dreier in Dreier/Schulze, Vor $\iint 44$ ff. Rn. 1; Wandtke/Lüft in Wandtke/Bullinger, Vor $\iint 44 a f f$. Rn. 1; Dreyer in Dreyer/Kotthoff/Meckel, Vor $\iint 54$ a ff. Rn. 1; J.B. Nordemann in Fromm/Nordemann, Vor \$S 44a ff. Rn. 1; Hohagen in FS Schricker, S. 353, 357 f.

387 BVerfG GRUR 1972, 481.

388 Wandtke, GRUR 2002, 1, 6.

389 Vgl. BGH GRUR 1997, 459, 462; Dreier in Dreier/Schulze, Vor $\iint 4_{44}$ ff. Rn. 1; J.B. Nordemann/W. Nordemann in Fromm/Nordemann, Vor $\iint 44$ ff. UrhG. 
ser Nutzungsrechte vorschreibt. ${ }^{390}$ Nach Ansicht des Gesetzgebers „muss der Urheber insbesondere dort im Interesse der Allgemeinheit freien Zugang zu seinen Werken gewähren, wo dies unmittelbar der Förderung der geistigen und kulturellen Werte dient, die ihrerseits Grundlage für sein Werkschaffen sind“.391 Die urheberrechtlichen Schranken sollen damit also vor allem den Belangen der Allgemeinheit Rechnung tragen. ${ }^{392}$

Daneben wird in der Literatur der Sinn und Zweck einiger Schranken vereinzelt auch in einer Korrektur eines Marktversagens gesehen. Gemeint ist damit, dass es bei bestimmten Nutzungen praktisch kaum möglich ist, beim Rechteinhaber zunächst eine Lizenz einzuholen. Als Beispiel sei nur die Kopie eines wissenschaftlichen Aufsatzes oder von Teilen einer wissenschaftlichen Monographie genannt. Wollte der Nutzer sich vor jeder Kopie, die er beispielsweise für seine wissenschaftliche Tätigkeit benötigt, zunächst eine Lizenz einholen, stellte einen wohl kaum zu bewerkstelligen Aufwand dar. Außerdem könnte der Rechteinhaber kaum die unzähligen Lizenzanfragen bearbeiten. Aus diesem Grund liegt nach dieser Ansicht ein Marktversagen vor, das durch bestimmte Schranken, insbesondere die Schranken des $\int 53$ UrhG, korrigiert werden soll. ${ }^{393}$

Sofern der Lizenznehmer jedoch vertraglich dazu verpflichtet wird, die Daten nicht $\mathrm{zu}$ vervielfältigen und an Dritte weiterzugeben, läuft dies nicht dem Zugangs- und Nutzungsinteresse der Allgemeinheit zuwider, da aufgrund der relativen Wirkung von Schuldverhältnissen lediglich der Lizenznehmer daran gehindert wird, eine Vervielfältigung vorzunehmen. Da er den gewünschten Datensatz jedoch vom Lizenzgeber erhalten hat, ist seinem Informationsinteresse insoweit genüge getan. Jeder Dritte darf diese Daten hingegen im Rahmen der gesetzlichen Schranken vervielfältigen, da er durch die Vereinbarung zwischen Lizenznehmer und -geber nicht berührt wird. Dem ließe sich zwar entgegenhalten, dass Dritte gar keine Gelegenheit zu einer Vervielfältigung erhalten, wenn der Lizenznehmer sie diesen gar nicht zur Verfügung stellen dürfte. Allerdings ist dabei zu beachten, dass der Zweck von \53 UrhG nicht darin besteht, Inhaber eines Werkstückes dazu zu verpflichten, dieses Dritten zum Zwecke der Vervielfältigung zur Verfügung stellen. Selbst wenn dem Lizenznehmer die Weitergabe also nicht untersagt

390 Vgl. BVerfG GRUR 1972, 481, 484; Depenheuer in v. Mangoldt/Klein/Starck, GG Kommentar, 6. Aufl. 2010, Art. 14 Rn. 218; Hofmann in Schmidt-Bleibtreu/Hofmann/Hopfauf, GG Kommentar zum Grundgesetz, 11.Aufl. 2008, Art. 14 Rn. 50; Jarass in Jarass/Pieroth (Hrsg.), GG Kommentar, 11. Aufl. 2011, Art. 14 Rn. 2, 35; Dreier in Dreier/Schulze, Vor \$S 44a ff. Rn. 1; Wandtke, GRUR 2002, 1, 6.

391 BT-Drucks. IV/270, S. 63.

392 Vgl. BT-Drucks. IV/270 S. 30; ebenso Melichar in Schricker/Loewenheim, Vor SS 44a Rn. 2, 4; J.B. Nordemann/W. Nordemann in Fromm/Nordemann, Vor $\$ \$ 44 a$ ff. Rn. 1, 2; Wandtke, GRUR 2002, 1, 6 .

393 Vgl. BGH GRUR 1965, 104, 108; so auch BGH GRUR 1997, 459, 462, der jedoch betont, dass dies nicht der einzige Zweck sei; Dreier in Dreier/Schulze, \53 Rn. 1; Dreier in Lejeune, S. 117 Fn. 110a. 
wäre, würde daraus nicht folgen, dass er das Werk tatsächlich auch Dritten zwecks Vervielfältigung zur Verfügung stellen würde. Sofern man den Zweck von \53 UrhG darin sieht, ein Marktversagen zu korrigieren, folgt auch hieraus nicht, dass dieser Zweck durch eine Abbedingungsklausel im Lizenzvertrag unterlaufen würde. Die Tatsache, dass die Parteien einen Lizenzvertrag geschlossen haben, belegt ja gerade, dass im Verhältnis von Lizenznehmer und Lizenzgeber ein solches Marktversagen nicht vorliegt. Von daher bedarf es in diesem Verhältnis auch keiner Korrektur, so dass die Abbedingung urheberrechtlicher Schranken den vom Gesetzgeber verfolgten Zweck (sofern man den Zweck einzelner urheberrechtlicher Schranken überhaupt in der Korrektur eines Marktversagens sieht) ebenfalls nicht konterkariert. Demzufolge ergibt sich bei einer teleologischen Auslegung der Schrankenbestimmungen nicht die Unzulässigkeit der Abbedingung dieser Schranken.

\section{Gesetzliches Verbot aufgrund der Regelung in $\int 95 b$ Abs. 1 S. 2 UrbG}

Allerdings könnte sich ein gesetzliches Verbot der vertraglichen Abbedingung aus \ 95b Abs. 1 S. 2 UrhG zumindest hinsichtlich der in Abs. 1 der Vorschrift genannten Schranken ergeben. Verwender technischer Schutzmaßnahmen sind gem. $\int 95 \mathrm{~b}$ Abs. $1 \mathrm{~S} .1 \mathrm{UrhG}$ dazu verpflichtet, sicherzustellen, dass die Begünstigten der dort genannten Schranken trotz der getroffenen technischen Schutzmaßnahmen von den Schrankenbestimmungen in dem erforderlichen Maße Gebrauch machen können. ${ }^{394}$ Sofern das Datenbankwerk also durch technische Maßnahmen geschützt ist und der Lizenzgeber gleichzeitig der Verwender dieser technischen Schutzmaßnahme ist, wäre eine Vereinbarung, nach der der Lizenznehmer beispielsweise auf die Schranke des $₫ 52 \mathrm{a}$ UrhG verzichtet, gem. \95b Abs. 1 S.2 UrhG unwirksam, da er durch diese Vereinbarung den Lizenzgeber von seiner Pflicht nach $\int 95 \mathrm{~b}$ Abs. 1 S. 1 UrhG befreien würde. ${ }^{395}$ Sollte der Lizenzgeber seine Daten also mit technischen Schutzmaßnahmen ${ }^{396}$ versehen, müsste er sicherstellen, dass der Lizenznehmer von den in \95b Abs. 1 S. 1 UrhG genannten Schranken Gebrauch machen kann, da der Katalog des $\int 95$ Abs. 1 UrhG die vorliegend in Betracht kommenden Schranken des $\ 52 \mathrm{a}$ und $\ 53$ UrhG beinhaltet. $^{397}$

\footnotetext{
394 Siehe dazu auch Berger, ZUM 2004, 257, 261.

395 Vgl. Dreyer in Dreyer/Kotthoff/Meckel, \95b Rn. 36; Wandtke/Ohst in Wandtke/Bullinger, \95b

Rn. 36; Caychowski in Fromm/Nordemann, \95b Rn. 14.

396 Siehe dazu bereits oben S. 78.

${ }^{397}$ Vgl. $\$ 95$ b Abs. 1 S. 1 Nr. 5 und 6.
} 
Sofern die Datensätze nicht mit einer solchen elektronischen Maßnahme versehen wären, würde $\int 95 \mathrm{~b}$ UrhG hingegen keine direkte Anwendung finden. In diesen Fällen könnte jedoch eine analoge Anwendung der Vorschrift in Betracht kommen, so dass die in $\int 95 \mathrm{~b}$ Abs. 1 S. 2 UrhG genannten Schranken ${ }^{398}$ generell nicht vertraglich abbedungen werden dürften.

\section{Vergleichbare Interessenlage}

Dazu bedürfte es zunächst einer vergleichbaren Interessenlage in den Fällen, in denen ein Werk durch technische Maßnahmen geschützt ist und solchen, in denen ein solcher Schutz nicht besteht. In diesem Zusammenhang ist zunächst der Hintergrund der Regelung des $\int 95 \mathrm{~b}$ Abs. $1 \mathrm{UrhG}$ zu berücksichtigen. Dieser liegt vor allem in Art. 6 Abs. 4 Unterabsatz 1 der sog. Info-Soc-RL ${ }^{399}$, wonach die Mitgliedstaaten sicherstellen müssen, dass die Rechteinhaber den Begünstigten die Mittel zur Verfügung stellt, die erforderlich sind, um die im nationalen Recht gem. Artikel 5 Absatz 2 Buchstaben a), c),d), oder e) oder Absatz 3 Buchstaben a), b) oder e) vorgesehenen Ausnahmen oder Beschränkungen wahrzunehmen. ${ }^{400} \int 95 \mathrm{~b}$ Abs. $1 \mathrm{~S} 1 \mathrm{UrhG}$ dient damit dem Ausgleich zwischen dem Rechtsschutz technischer Maßnahmen gem. \95a Abs. 1 UrhG $^{401}$ und den urheberrechtlichen Schrankenregelungen. ${ }^{402}$

Damit diese „Balance“ nicht durch vertragliche Vereinbarungen wieder aufgehoben wird, erklärt \95b Abs. 1 S.2 UrhG eine vertragliche Abbedingung von \ 95b Abs.1 S.1 UrhG für unwirksam. Dementsprechend könnte man dahingehend argumentieren, dass in den Fällen, in denen das Werk nicht durch technische Maßnahmen geschützt ist, gerade kein Ausgleich hergestellt werden muss und es somit an einer vergleichbaren Interessenlage fehlt. $\mathrm{Zu}$ bedenken ist jedoch, dass der Gesetzgeber durch die Regelung in $\int 95 \mathrm{~b}$ Abs. 1 S.1 UrhG deutlich gemacht hat, dass die dort genannten Schranken vom Rechteinhaber nicht ausgeschlossen werden sollen. Dann kann es jedoch keinen Unterschied machen, ob der Ausschluss direkt durch eine vertragliche Vereinbarung erfolgt oder indirekt, indem der nach $\int 95 \mathrm{~b}$ Abs. 1 S. 1 UrhG Verpflichtete sich durch vertragliche Vereinbarung dieser Pflicht entledigt. In beiden Fällen läuft der von einer Schranke Begünstigte Gefahr, aufgrund einer vertraglichen Vereinbarung seine Schranken aufzugeben, so dass in beiden Sachverhaltskonstellationen eine vergleichbare Interessenlage gegeben ist.

\footnotetext{
${ }^{398}$ Dabei erfasst der Katalog des $₫ 95 b$ Abs. 1 S. 2 UrhG nicht alle Schranken der $\$ ₫ 44 a$ ff. UrhG. 399 Siehe RL 2001/29/EG; vgl. Götting in Schricker/Loewenheim, \95b Rn. 1.

400 Vgl. Art. 6 Abs. 4 Unterabsatz 1 der RL 2001/29/EG.

${ }^{401}$ Der wiederum der Umsetzung von Art. 6 Abs. 1 der Info-Soc-RL dient; vgl. Wandtke/Ohst in Wandtke/Bullinger, \$ 95a Rn. 1.

402 Götting in Schricker/Loewenheim, \95b Rn. 1.
} 


\section{Planwidrigkeit der Regelungslücke}

Eine vergleichbare Interessenlage allein genügt hingegen noch nicht für eine analoge Anwendung. Vielmehr muss die bestehende Regelungslücke planwidrig, d.h. sie darf vom Gesetzgeber nicht gewollt sein. Die betreffende Norm kann nur dann analog angewendet werden, wenn davon auszugehen ist, dass der Gesetzgeber für die vergleichbare Sachverhaltskonstellation eine Regelung getroffen hätte, die der Vorschrift entspricht, die analog angewendet werden soll. Dabei kann davon ausgegangen werden, dass der Gesetzgeber sich bei Verabschiedung des \ 95b UrhG bewusst gewesen ist, dass das Problem einer vertraglichen Abbedingung nicht nur im Rahmen von Werken besteht, die durch technische Maßnahmen geschützt sind, sondern dass eine vertragliche Abbedingung urheberrechtlicher Schranken auch in Betracht kommt, wenn das betreffende Werk nicht durch technische Maßnahmen geschützt ist. Dafür spricht ferner, dass die Vorschriften der $\iint 69 \mathrm{~g}$ und $87 \mathrm{e}$ zum Zeitpunkt des Inkrafttretens von $\int 95 \mathrm{~b}$ UrhG im Jahre 2003 bereits bestanden.

Dies bedeutet, dass der Gesetzgeber sich bei der Schaffung des \95b UrhG bewusst gewesen ist, dass sich Begünstigte durch vertragliche Vereinbarung mit dem Rechteinhaber zum Verzicht auf die ihnen zustehenden urheberrechtlichen Schranken verpflichten könnten. Andernfalls hätte er die $\iint 69 \mathrm{~g}$ und \87e UrhG nicht geschaffen. Die Tatsache, dass er die Unwirksamkeit der vertraglichen Abbedingung urheberrechtlicher Schranken aber weder bei Schaffung der $\iint 59 \mathrm{~g}$ und 87e UrhG noch im Rahmen der Verabschiedung von $\int 95 \mathrm{~b}$ UrhG und der beiden Gesetzesreformen zur Regelung des Urheberrechts in der Informationsgesellschaft geregelt hat, zeigt, dass der Gesetzgeber die Regelungslücke bewusst nicht geschlossen hat. Mangels einer Planwidrigkeit der Regelungslücke ist $\int 95 \mathrm{~b}$ Abs. 1 S. 2 UrhG damit nicht analog auf die vertragliche Abbedingung der in $\int 95 \mathrm{~b}$ Abs. 1 S.1 UrhG genannten Schranken anzuwenden. Damit ist die vertragliche Abbedingung urheberrechtlicher Schranken nach \95b Abs. 1 S. 2 UrhG nur dann unwirksam, sofern der Lizenzgeber die Daten mit technischen Schutzmaßnahmen versehen hat. Ist dies nicht der Fall, ergibt sich eine solche Unwirksamkeit weder aus $\int 95 b$ Abs. 1 S. 2 UrhG direkt, noch -mangels Vorliegen einer planwidrigen Regelungslücke- im Rahmen einer analogen Anwendung dieser Vorschrift.

\section{Richtlinienkonforme Auslegung}

Des Weiteren könnte die Unwirksamkeit einer vertraglichen Abbedingung aus einer richtlinienkonformen Auslegung der nationalen Schrankenregelung vor dem Hintergrund der sog. Info-Soc-Richtlinie ${ }^{403}$ folgen, die in Artikel 5 Vorgaben für die Beschränkungen der Rechte des Urhebers enthält. Aus dem Inhalt der Richtlinie folgt jedoch auch nicht eindeutig, dass vertragliche Abbedingungen urheberrechtlicher Schranken generell unwirksam sein sollen. Vielmehr hat der europäi-

${ }^{403}$ RL 2001/29/EG. 
sche Gesetzgeber in Erwägungsgrund 45 gerade deutlich gemacht, dass er dies den nationalen Gesetzgebern überlässt. Dort heißt es nämlich, dass „die in Artikel 5 Absätze 2, 3 und 4 vorgesehenen Ausnahmen und Beschränkungen [...] nicht die vertraglichen Beziehungen zur Sicherstellung eines gerechten Ausgleichs für die Rechtsinhaber entgegenstehen sollen, soweit dies nach innerstaatlichem Recht zulässig ist". Die Unzulässigkeit einer vertraglichen Abbedingung ergibt sich damit auch nicht im Rahmen einer richtlinienkonformen Auslegung.

\section{Umkebrschluss aus \95a UrbG}

Die generelle Wirksamkeit der Abbedingung urheberrechtlicher Schranken könnte sich indes aus einem Umkehrschluss aus \95a UrhG ergeben. Nach \95a Abs. 1 UrhG dürfen technische Schutzmaßnahmen nämlich nicht ohne Zustimmung des Rechtsinhabers umgangen werden. Dies bedeutet, betrachtet man $\ 95$ a isoliert, dass beispielsweise der Erwerber einer Musik CD, die mit einem technischen Kopierschutz versehen ist, keine Privatkopie von dieser CD anfertigen dürfte, solange der Rechteinhaber nicht seine Zustimmung dazu gegeben hat. Damit wäre durch $\ 95 \mathrm{a}$ UrhG die gesetzliche Schranke des $\ 53$ Abs. 1 UrhG ausgeschlossen. Wenn das Gesetz es jedoch zulässt, dass ein Rechteinhaber anderen die Möglichkeit verwehrt, sein Werk auf eine Art und Weise zu nutzen, die eine gesetzliche Schranke ausdrücklich erlaubt, so müsste dies doch erst Recht gelten, wenn der Rechteerwerber, diesem Schrankenverzicht ausdrücklich zugestimmt hat. Denn in den Fällen des $\int 95 a$ UrhG erfolgt der Ausschluss der Schranken ja gerade nicht durch vertragliche Vereinbarung, sondern durch die Installation eines elektronischen Kopierschutzes. ${ }^{404}$

$\mathrm{Zu}$ beachten ist demgegenüber jedoch, dass ein Rechtsinhaber, der technische Maßnahmen anwendet, wie bereits oben erwähnt, gem. \95b Abs. 1 UrhG verpflichtet ist, die notwendigen Mittel zur Verfügung zu stellen, um von den in der Norm genannten Schrankenregelungen Gebrauch zu machen. Somit kann aus $\mathbb{S}$ 95a UrhG aufgrund der Einschränkung in \95b UrhG nicht ohne Weiteres der Schluss gezogen werden, dass urheberrechtliche Schranken durch vertragliche Vereinbarung abdingbar sind.

\section{Grundsatr, der Privatautonomie}

Sofern die Schrankenregelungen kein ausdrückliches Verbot einer vertraglichen Abdingbarkeit enthalten und sich ein solches auch nicht aus einem Umkehrschluss oder einer analogen Anwendung der $\iint 55 \mathrm{a}, 69 \mathrm{~g}$ und $87 \mathrm{e}$ UrhG ergibt, spricht für eine Abdingbarkeit der Grundsatz der Privatautonomie, der seinen Ursprung

\footnotetext{
${ }^{404} \mathrm{Da}$ es dem Erwerber eines Mediums beim Kauf nicht immer bewusst ist, dass dieses durch einen elektronischen Kopierschutz gesichert ist, kann dem auch nicht entgegengehalten werden, dass der Käufer der Abbedingung seiner Schranken stillschweigend durch den Kauf der CD zustimmt. Darüber hinaus kommt dieser Kaufvertrag auch nicht zwischen dem Käufer und dem Rechteinhaber sondern dem Verkäufer zustande.
} 
in Art. 2 S. 1 GG hat. ${ }^{405}$ Danach steht es den Parteien zum einen frei, mit wem sie kontrahieren und welchen Inhalt die vertragliche Vereinbarung hat. ${ }^{406}$ Somit würde der Grundsatz der Vertragsfreiheit für die Zulässigkeit einer vertraglich vereinbarten Abdingbarkeit sprechen. Dabei ist insbesondere zu berücksichtigen, dass es dem Nutzer grundsätzlich freisteht, ob er eine Lizenzvereinbarung mit dem Rechteinhaber abschließt und dadurch auf bestimmte urheberrechtliche Schranken verzichtet oder ob er eine solche Vereinbarung ablehnt und sich damit zwar weiterhin auf die gesetzlichen Schranken berufen kann, im Gegenzug jedoch nicht die Nutzungsrechte erhält, die er durch Abschluss der Lizenzvereinbarung erlangt hätte.

$\mathrm{Zu}$ beachten ist jedoch, dass der Grundsatz der Privatautonomie nicht unbegrenzt gilt. So sind beispielsweise sittenwidrige Rechtsgeschäfte gem. \138 BGB nichtig. Das Gleiche gilt gem. \134 BGB für Rechtsgeschäfte, die gegen ein gesetzliches Verbot verstoßen und gem. \125 S. 1 BGB für die Fälle, in denen die geschlossenen Verträge nicht einer gesetzlich vorgeschriebenen Form entsprechen. Auch das UrhG enthält teilweise Beschränkungen der Privatautonomie. Als Beispiel sei hierfür $\int 32$ UrhG genannt, wonach der Urheber nicht vertraglich auf seinen in $\int 32$ UrhG verankerten Vergütungsanspruch verzichten kann. ${ }^{407}$

Auf der anderen Seite spricht gegen die Zulässigkeit der Abdingbarkeit, dass sich der Rechteerwerber in den meisten Fällen in einer zumindest faktischen Zwangslage befinden wird. Es steht ihm zwar frei, auf eine Lizenzvereinbarung zu verzichten, sofern ihm deren Inhalt nicht zusagt. Da der jeweilige Rechteinhaber jedoch in der Regel der einzige sein wird, der ihm die benötigten Nutzungsrechte an dem betroffenen Werk einräumen kann, besteht ein Machtgefälle vom Lizenzgeber zum Lizenznehmer. Dies gilt auch in den hier im Vordergrund stehenden Fällen, in denen eine Universität oder Forschungseinrichtung ihre gewonnenen und archivierten Daten zum Abruf bereitstellt. Sofern andere Forschungseinrichtungen an diesen Daten interessiert sind oder diese für ihre eigene Forschung benötigen, sind sie auf die archivierende Einrichtung angewiesen, da andere Forschungseinrichtungen meistens wohl nicht über die gleichen Daten verfügen werden. Der Rechteinhaber hat damit in der Regel eine stärkere Machtposition als der potentielle Lizenznehmer und somit eine bessere Ausgangslage für die Vertragsverhandlungen. Dies könnte dafür sprechen, den Lizenznehmer dahingehend zu schützen, dass er nicht wirksam auf seine Schranken verzichten kann, selbst wenn er eine dahingehend lautende Vereinbarung mit dem Rechteinhaber schließt. Ein solches Machtgefälle ist auch der Hintergrund von $\ 32$ UrhG, der dem

\footnotetext{
405 Jarass in Jarass/Pieroth, Art. 2 Rn. 4; Hillgruber in Umbach/Clemens (Hrsg.), Grundgesetz, 2002, Band 1, Art. 2 I Rn. 94; Hofmann in Schmidt/Bleibtreu/Klein (Hrsg.), Kommentar zum Grundgesetz, 10. Aufl. 2004, Art. 2 Rn. 33.

406 Vgl. Hillgruber in Umbach/Clemens, Art. 2 I Rn. 95 f.

${ }^{407}$ Siehe $₫ 32$ Abs. 3 S. 1 UrhG.
} 
-gegenüber dem Verwerter wirtschaftlich unterlegenen- Urheber einen Vergütungsanspruch sichert, der gem. \ 32 Abs. 3 S. 1 UrhG auch nicht vertraglich ab bedungen werden kann. ${ }^{408}$ Aus dem Grundsatz der Vertragsfreiheit folgen damit sowohl Argumente für als auch gegen die Möglichkeit einer vertraglichen Abbedingung urheberrechtlicher Schranken.

\section{Zwischenergebnis}

Demzufolge folgt die generelle Unzulässigkeit der Abbedingung urheberrechtlicher Schranken weder ausdrücklich aus dem Gesetz noch aus einer europarechtskonformen Auslegung der Schrankenregelungen oder einer analogen Anwendung von $\int 95 \mathrm{~b}$ UrhG. Auf der anderen Seite kann die generelle Abdingbarkeit urheberrechtlicher Schranken auch nicht aus einem Umkehrschluss zu \95a UrhG geschlussfolgert werden. Die generelle Abdingbarkeit urheberrechtlicher Schranken ergibt sich vielmehr aus dem Grundsatz der Privatautonomie. Demzufolge ist die Abbedingung urheberrechtlicher Schranken grundsätzlich möglich und nicht von vornherein ausgeschlossen. Wie gezeigt, gilt die Privatautonomie jedoch nicht unbeschränkt. Vielmehr sind die konkreten vertraglichen Vereinbarungen an den allgemeinen zivilrechtlichen Vorschriften zu messen. Welche Vorschriften dabei in den vorliegenden Fällen in Betracht kommen und unter welchen Umständen eine Vertragsklausel aufgrund einer dieser Vorschriften unwirksam sein kann, soll im Folgenden untersucht werden.

(c) Unwirksamkeit aufgrund von Vorschriften des allgemeinen Zivilrechts Da sich aus den Vorschriften des Urheberrechtsgesetzes keine stichhaltigen Argumente für oder gegen die Zulässigkeit einer vertraglichen Abbedingung von urheberrechtlichen Schranken ziehen lassen, ist zu prüfen, ob sich die Unwirksamkeit einer solchen Abbedingung aus den allgemeinen zivilrechtlichen Vorschriften ergeben könnte. Namentlich kommen dabei vor allem \138 BGB sowie

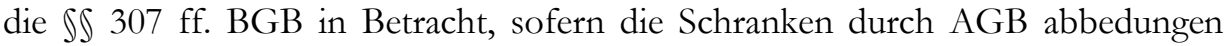
werden sollen. Es ist also zunächst danach zu differenzieren, ob es sich um eine individualvertragliche Vereinbarung handelt oder um eine Vereinbarung im Rahmen von AGB. 409

\section{Ausschluss durch individualvertragliche Vereinbarung}

Aufgrund des oben beschriebenen Grundsatzes der Vertragsfreiheit ist grundsätzlich eine Abdingbarkeit durch eine individualvertragliche Vereinbarung als zulässig zu erachten. ${ }^{410}$ Wie jedoch bereits oben erwähnt, findet der Grundsatz der Vertragsfreiheit seine Grenzen in den gesetzlichen Bestimmungen. In Betracht käme

\footnotetext{
408 Vgl. Kotthoff in Dreyer/Kotthoff/Meckel, \32 Rn. 3; Schulze in Dreier/Schulze, \ 32 Rn. 1.

${ }^{409}$ So auch Hohagen in FS Schricker, S. 361.

${ }^{410}$ Ebenso Dreier/Senftleben in Lejeune, S .113 Rn. 40.
} 
daher vor allem eine Unwirksamkeit nach \138 BGB, sofern eine solche Vereinbarung gem. \ 138 Abs. 2 BGB einen Fall des Wuchers darstellen würde oder gem. \138 Abs. 1 BGB sittenwidrig wäre.

Wucher

Gem. \138 Abs. 2 BGB wäre die Abbedingung urheberrechtlicher Schranken nichtig, wenn der Lizenzgeber die Zwangslage, die Unerfahrenheit, den Mangel an Urteilsvermögens oder die erhebliche Willensschwäche des Lizenznehmers ausbeuten würde und der Verzicht des Lizenznehmers auf bestimmte urheberrechtliche Schranken in einem auffälligen Missverhältnis zur Leistung des Lizenzgebers stünde. Die Gegenleistung wird dabei regelmäßig die Einräumung von Nutzungsrechten an der jeweiligen Datenbank bzw. dem Datenbankwerk durch den Lizenzgeber sein. In subjektiver Hinsicht käme in den vorliegenden Fällen unter Umständen die Ausnutzung einer Zwangslage des Lizenznehmers in Betracht. Wie bereits oben dargelegt, wird eine solche Zwangslage für den Lizenznehmer zumeist vorliegen, da er die jeweiligen Datensätze in der Regel nur von dem Lizenzgeber erhalten kann und dieser damit quasi eine Monopolstellung inne hat.

Dies könnte jedoch dahingestellt bleiben, sofern in objektiver Hinsicht kein auffälliges Missverhältnis zwischen Leistung und Gegenleistung vorläge. Dabei stellt sich zunächst die Frage, ob der Verzicht auf die urheberrechtlichen Schranken eine Gegenleistung zur Einräumung von Nutzungsrechten durch den Lizenzgeber darstellt, wie dies von $\int 138$ Abs. 2 BGB vorausgesetzt wird. ${ }^{411}$ Dies wird jedoch grundsätzlich zu bejahen sein, da der Lizenzgeber gerade deshalb auf seine Schranken verzichtet, um die von ihm begehrten Nutzungsrechte vom Vertragspartner eingeräumt zu bekommen. Insofern stellt sich die Frage, ob Leistung und Gegenleistung in einem auffälligen Missverhältnis stehen. Dabei ist der objektive Wert von Leistung und Gegenleistung zum Zeitpunkt des Vertragsschlusses zugrunde zu legen.

Bei der Ermittlung des objektiven Wertes des Lizenzgebers kann dabei auf die üblicherweise für vergleichbare Daten gezahlte Lizenzgebühr abgestellt werden. Schwieriger ist es jedoch, den Wert der Gegenleistung des Lizenznehmers zu ermitteln. Dieser setzt sich aus der gezahlten Lizenzgebühr sowie dem objektiven Wert des Verzichtes auf die urheberrechtlichen Schranken zusammen. Bei Letzterem stellt sich die Frage, wie dessen Wert ermittelt werden kann. Dies erscheint auf den ersten Blick schwierig, da Schranken, anders als z.B. Nutzungsrechte, nicht rechtsgeschäftlich erworben werden, so dass ihr Wert nicht anhand des für sie marktüblich gezahlten Preises errechnet werden kann. Zu beachten ist jedoch, dass die $\iint 45 a, 46,47,49,52 a$, 53a einen Anspruch des Urhebers gegen den Nutzer auf Zahlung einer angemessenen Vergütung begründen. ${ }^{412}$ Bei diesen Fällen

\footnotetext{
411 Vgl. BGH NJW 1982, 2767.

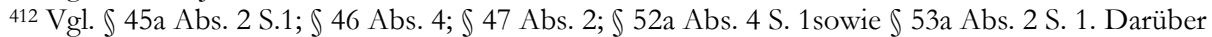
hinaus steht dem Urheber gem. \54 Abs. 1 UrhG auch ein Vergütungsanspruch zu, wenn nach
} 
handelt es sich um sogenannten gesetzliche Lizenzen, ${ }^{413}$ weshalb der Wert des Verzichts auf eine der genannten Schranken nach der Höhe der „Lizenzgebühr“ bemessen werden kann, die der Nutzer dem Urheber hätte zahlen müssen, wenn er das Werk auf Grundlage einer dieser Schranken genutzt hätte. Letztlich kann die Höhe einer angemessenen Vergütung jedoch nur ein Indiz sein, da zu berücksichtigen ist, dass der Vergütungsanspruch bei jeder Nutzung aufgrund der jeweiligen Schranke entsteht und davon auszugehen ist, dass ein Nutzer dasselbe Werk mehrere Male auf Grundlage derselben Schranke genutzt hätte.

Verzichtet der Nutzer nun endgültig auf diese Schranke, so liegt der Wert dieses Verzichts nicht in der Höhe einer angemessenen Vergütung für eine einzige Nutzung des Werkes. Somit kann also auch bei den Schranken, die einen Vergütungsanspruch des Urhebers vorsehen, nicht genau gesagt werden, welchen Wert der Verzicht auf diese Schranken hat. Dies ist unter Umständen jedoch auch gar nicht erforderlich. Damit ein auffälliges Missverhältnis i.S.d. \ 138 Abs. 2 BGB vorliegt, müsste der Wert der Gegenleistung414 des Lizenznehmers nämlich den Marktpreis der vom Lizenznehmer erworbenen Nutzungsrechte um 100\% oder mehr übersteigen, ${ }^{415}$ was in den vorliegend relevanten Fällen grundsätzlich nicht zutreffen wird. Zwar wird der Marktwert der Nutzungsrechte an dem Datenbankwerk bzw. der Datenbank abhängig von der Nachfrage von Fall zu Fall divergieren, regelmäßig jedoch so hoch, dass er den Wert des Schrankenverzichts um ein vielfaches übersteigen wird. Ein auffälliges Missverhältnis könnte sich daher höchstens dann ergeben, wenn die Höhe der Lizenzgebühr weit über dem Marktwert der eingeräumten Lizenz liegt. Dies ist jedoch kein spezifisches Problem, das aus der Abbedingung urheberrechtlicher Schranken folgt. Das Gleiche gilt für die Schranken, die keinen Vergütungsanspruch für den Urheber begründen.

Hier lässt sich der objektive Wert zwar noch schwieriger ermitteln, jedoch ist hier e contrario erst Recht davon auszugehen, dass der Wert des Schrankenverzichts so niedrig sein wird, dass ein auffälliges Missverhältnis regelmäßig nicht gegeben sein wird. Demzufolge wird regelmäßig allein dadurch, dass der Lizenznehmer nicht nur die Lizenzgebühr zahlt, sondern zusätzlich auf bestimmte urheberrechtliche Schranken verzichtet, kein auffälliges Missverhältnis zwischen der Leistung des Lizenznehmers und der des Lizenzgebers bestehen, so dass eine derartige Vereinbarung nicht nach $\int 138$ Abs. 2 BGB nichtig wäre.

der Art des Werkes zu erwarten ist, dass es nach $₫ 53$ Abs. 1 bis 3 UrhG vervielfältigt wird. Dieser Anspruch richtet sich jedoch nicht gegen den Nutzer des Werkes, sondern den Hersteller von Geräten, die zu derartigen Vervielfältigungen benutzt werden. Von daher eignet sich die Höhe der zu zahlenden Vergütung nicht dazu, den Wert des Verzichts auf die Schranke des $₫ 53$ Abs. 1 bis 3 UrhG zu ermitteln.

413 Vgl. Dreier in Dreier/Schulze, Vor \$S 44a ff. Rn. 11.

414 Also der Wert der zu zahlenden Lizenzgebühr zuzüglich des Wertes des Verzichts auf eine oder mehrere Schranken.

${ }^{415}$ So BGH NJW 2004, 3553, 3554; NJW 2000, 1254, 1255; NJW-RR 1989, 1068. 


\section{Sittenwidrigkeit nach $\int 138$ Abs. 1 BGB}

In Betracht käme jedoch eine Nichtigkeit nach \ 138 Abs. 1 BGB, sofern der Lizenzvertrag durch die Vereinbarung einer Schrankenabbedingung sittenwidrig wäre. Dabei ist nach Ansicht der Rechtsprechung ein Rechtsgeschäft dann sittenwidrig, „wenn es gegen das Anstandsgefühl aller billig und gerecht Denkenden verstößt". ${ }^{416}$ Dabei bezweckt auch Absatz 1 den schwächeren Vertragspartner gegen eine wirtschaftliche oder intellektuelle Überlegenheit des anderen zu schützen. ${ }^{417}$ Dabei käme in den vorliegenden Fällen zum einen eine Sittenwidrigkeit wegen eines auffälligen Missverhältnisses zwischen Leistung und Gegenleistung in Betracht. Zum anderen dient $\int 138$ Abs. 1 BGB jedoch auch als sog. „Einfallstor“ für das im Grundgesetz verkörperte Wertsystem. ${ }^{418}$ Daher könnte die vertragliche Abbedingung urheberrechtlicher Schranken auch dann sittenwidrig sein, sofern dadurch grundrechtlich geschützte Interessen des Lizenznehmers verletzt würden.

\section{Auffälliges Missverbältnis}

Die Abbedingung urheberrechtlicher Schranken wäre gem. \ 138 Abs. 1 BGB sittenwidrig, wenn dadurch das Verhältnis zwischen Leistung und Gegenleistung im Lizenzvertrag in einem auffälligen Missverhältnis stünden. ${ }^{419}$ Diese Fälle werden zwar vor allem von Abs. 2 erfasst. Sofern jedoch nicht alle Tatbestandsmerkmale des Wuchertatbestandes erfüllt sind, kann das Rechtsgeschäft, bei dem ein auffälliges Missverhältnis besteht, auch nach Abs. 1 nichtig sein. ${ }^{420}$ Wie oben bereits festgestellt, wird ein solches Missverhältnis aber in der Regel nicht durch die Abbedingung urheberrechtlicher Schranken entstehen. Aus diesem Grund werden die hier in Betracht kommenden Lizenzverereinbarungen aufgrund nicht wegen eines Missverhältnisses von Leistung und Gegenleistung nach \138 Abs. 1 UrhG nichtig sein, sofern sie eine Verschwiegenheitsklausel enthalten, durch die dem Lizenznehmer urheberrechtliche Schranken abbedungen werden.

\section{Unwirksamkeit aufgrund der Verletzung grundrechtlich verbürgter Interessen}

Eine vertragliche Abbedingung wäre jedoch dann sittenwidrig, wenn dadurch grundrechtlich verbürgte Interessen des Lizenznehmers verletzt würden. Die Grundrechte wirken zwar grundsätzlich nur als Abwehrrechte des Bürgers gegenüber dem Staat. Nach dem modernen Verständnis der Grundrechte sind diese jedoch darüber hinaus als objektive Werteordnung zu verstehen, die ihren Einfluss im Privatrecht im Rahmen der zivilrechtlichen Generalklauseln erlangen. ${ }^{421}$ Diese Vorschriften sind, sofern der Schutzbereich von Grundrechten eröffnet ist, im

\footnotetext{
416 Statt vieler BGH NJW 2004, 2668, 2670 m.w.N.; BAG NZA 2006, 1354, 1355 f.

417 BGH NJW 1981, 1206; Wendtland in Bamberger/Roth, Bd. 1, \138 Rn. 2.

418 Ständige Rspr. des BVerfG, siehe nur BVerfG NJW 1958, 257; NJW 1990, 1469, 1470.

419 Wendtland in Bamberger/Roth, Bd. 1, J 138 Rn. 21.

420 Wendtland in Bamberger/Roth, Bd. 1, J 138 Rn. 41.

${ }^{421}$ BVerfG NJW 2001, 957, 958.
} 
Lichte dieser Grundrechte auszulegen und anzuwenden, was vor allem bei zivilrechtlichen Generalklauseln oftmals der Fall ist. ${ }^{422}$ Bei der Abwägung, ob eine Sittenwidrigkeit nach $\int 138$ BGB vorliegt, ist dabei die Bedeutung der einzelnen Grundrechte zu berücksichtigen. ${ }^{423}$ Vor diesem Hintergrund ist im Rahmen von \138 Abs. 1 BGB zu prüfen, ob durch die Abbedingung urheberrechtlicher Schranken Grundrechte des Lizenznehmers verletzt werden. Sofern dies der Fall ist, müsste diese Grundrechtsbeeinträchtigung im Rahmen der gerade dargestellten mittelbaren Drittwirkung von Grundrechten, bei der Frage der Sittenwidrigkeit berücksichtigt werden. Bei den vorliegend in Betracht kommenden Schranken der $\iint 52 a, 53$ Abs. 2 S. 1 Nr. 1, Nr. 3 sowie $\ 53$ a Abs. 1 UrhG und $\ 87$ c Abs. 1 UrhG sind insbesondere die in Art. 5 Abs. 1 S.1 UrhG verankerte Informationsfreiheit sowie die Freiheit der Wissenschaft, Forschung und Lehre nach Art. 5 Abs. 3 GG zu berücksichtigen.

Insofern ist im Rahmen von $\int 138$ Abs. $1 \mathrm{UrhG}$ zu prüfen, ob die Vereinbarung eines Weitergabeverbotes und die damit einhergehende Abbedingung der einschlägigen urheberrechtlichen Schranken eine Verletzung von Art. 5 Abs. 3 GG darstellen könnte. Wie die Informationsfreiheit, kann auch die Wissenschaftsfreiheit im Verhältnis von Privatrechtssubjekten Drittwirkung entfalten. ${ }^{424}$ Dabei fallen die Tätigkeiten der vorliegend in Betracht kommenden Lizenznehmer, namentlich Universitäten und sonstige Forschungseinrichtungen, sowohl unter den Wissenschafts- als auch dem Forschungsbegriff, da diese den ernsthaften und planmäßigen Versuch zur Ermittlung der Wahrheit beinhalten. ${ }^{425}$ Darüber hinaus wird gerade in den Fällen, in denen Universitäten den Lizenzvertrag mit der archivierenden Einrichtung abschließen, der Schutzbereich der wissenschaftlichen Lehre eröffnet sein, da sie ihre durch Forschung gewonnenen Erkenntnisse grundsätzlich weitergeben. ${ }^{426}$ Dem steht auch nicht entgegen, dass es sich bei den vorliegend in Betracht kommenden Grundrechtsträgern oftmals um juristische Personen, wie etwa Universitäten oder auch privatrechtliche Gesellschaften handeln wird, da auch diese Träger des Grundrechts aus Art. 5 Abs. 3 GG sein können. ${ }^{427}$ Damit ist der Schutzbereich von Art. 5 Abs. 3 S.1 GG in den vorliegenden Fallkonstellationen grundsätzlich eröffnet.

Sofern es den Lizenznehmern aufgrund der Abbedingung in der Lizenzvereinbarung beispielsweise rechtlich nicht möglich wäre, die vom Lizenzgeber empfangenen Daten für Unterricht und Vorlesung öffentlich zugänglich zu machen,

\footnotetext{
422 BVerfG NJW 2001, 957, 958; NJW 1958, 257.

${ }^{423}$ Vgl. BVerfG NJW 2001, 957, 958; ZUM 2000, 149, 157; Jarass in Jarass/Pieroth, Art. 1 Rn. 54.

424 Starck in v. Mangoldt/Klein/Starck, Art 5 Abs. 3 Rn. 411; Jarass in Jarass/Pieroth, Art. 5 Rn. 130.

${ }^{425}$ Vgl. BVerfG NJW 1978, 1621; Jarass in Jarass/Pieroth, Art.5 Rn. 121 f; Zöbeley in Um-

bach/Clemens, Art.5 Rn. 242.

426 Vgl. BVerfG NJW 1973, 1176, 1179; Jarass in Jarass/Pieroth, Art. 5 Rn. 123

427 BVerfGE 15, 256, 261 f.; 21, 362, 373 f.; 31, 314, 322; Jarass in Jarass/Pieroth, Art. 5 Rn. 125.

Starck in v. Mangoldt/Klein/Starck, Art. 5 Abs. 3 Rn. 408.
} 
könnte dies einen Eingriff in die Freiheit der Lehre darstellen. ${ }^{428} \mathrm{Zu}$ beachten ist jedoch zum einen, dass es vorliegend nur um die öffentliche Zugänglichmachung von Daten bzw. Datensätzen geht. Diese kann zwar dazu dienen, den Unterrichtsbzw. Vorlesungsstoff zu untermauern, allerdings nicht in dem Maße, in dem dies durch die auszugsweise öffentliche Zugänglichmachung von Lehrbüchern oder Aufsätzen erreicht wird. Zu beachten ist in diesem Zusammenhang außerdem, dass die Forschungseinrichtung eine zusätzliche Lizenz vom Lizenzgeber einholen könnte, nach der es ihr gestattet ist, die Datensätze oder Teile davon, zum Zwecke der Lehre öffentlich zugänglich zu machen. ${ }^{429}$ Somit ist ein Eingriff in die Freiheit der Lehre durch die Abbedingung von $\ 52 \mathrm{a}$ UrhG für die vorliegenden Fälle abzulehnen.

Darüber hinaus ist zu bedenken, dass die Lizenzvereinbarung in den vorliegenden Fällen auch die Vervielfältigung des Lizenznehmers zum eigenen wissenschaftlichen Gebrauch untersagen könnte. In diesem Fall würde der Lizenznehmer zwar den gewünschten Datensatz erhalten, dürfte diesen jedoch weder analog noch digital vervielfältigen. Eine derartige Beschränkung könnte insbesondere dann einen unzulässigen Eingriff in die Freiheit von Wissenschaft und Forschung darstellen, wenn es sich beim Lizenznehmer um eine Forschungseinrichtung bzw. allgemein um eine juristische Person handelt, die mehrere Wissenschaftler beschäftigt. In diesen Fällen wird nämlich das grundsätzliche Bedürfnis bestehen, die Daten zu vervielfältigen, damit alle Wissenschaftler der Forschungseinrichtung bzw. des jeweiligen Forschungsprojektes mit ihnen gleichzeitig arbeiten können.

Wie oben gezeigt, wäre eine solche Vervielfältigung grundsätzlich auch von $\mathbb{S}$ 53 Abs. 2 Nr. 1 UrhG gedeckt. Eine Verpflichtung des Lizenznehmers, auf derartige Vervielfältigungen zu verzichten, könnte daher seine Wissenschaftsfreiheit aus Art. 5 Abs. 3 GG verletzen, da ein wissenschaftliches Arbeiten mit diesen Daten nicht durch mehrere Personen zur gleichen Zeit erfolgen könnte. Sofern Forschungseinrichtungen und Universitäten die empfangenen Daten aufgrund eines entsprechenden Verbotes in der Lizenzvereinbarung nicht für ihre eigenen Mitarbeiter und Wissenschaftler vervielfältigen dürften, läge darin nämlich eine nicht unerhebliche Beeinträchtigung ihrer wissenschaftlichen Tätigkeit. Denn gerade bei großen Datenpaketen bzw. großen Forschungsprojekten wird es erforderlich sein, dass mehrere Datensätze zur Verfügung stehen, damit mehrere Wissenschaftler gleichzeitig mit diesen Daten arbeiten können. Den Lizenznehmern bliebe zwar auch hier wieder die Möglichkeit, weitere Lizenzen zu erwerben, was jedoch in Zeiten sinkender Budgets und knapper Haushaltsmittel in Anbetracht der nicht geringen Anzahl erforderlicher Lizenzen oftmals kaum möglich sein wird. Demzufolge liegt in der vertraglichen Abbedingung der Schranke des $₫ 53$ Abs. 2 Nr.1 zumindest ein Eingriff in die Wissenschafts- und Forschungsfreiheit. Dieser Ein-

${ }^{428}$ Vgl. Jarass in Jarass/Pieroth, Art. 5 Rn. 128.

${ }^{429}$ Vgl. auch Gounalakis, Elektronische Kopien für Unterricht und Forschung ( $(52 \mathrm{a}$ UrhG) im Lichte der Verfassung, 2003, S. 47 f. 
griff könnte hingegen gerechtfertigt sein. Der Schrankenvorbehalt von Art. 5 Abs. 2 GG findet dabei auf Abs. 3 allerdings keine Anwendung. ${ }^{430}$ Dies bedeutet jedoch nicht, dass die Wissenschaftsfreiheit vollkommen unbeschränkt gilt. Sie findet ihre Grenzen vielmehr in der Verfassung selbst, ${ }^{431}$ in den vorliegenden Fällen also vor allem in der Wahrung des durch Art. 14 GG grundrechtlich geschützten Eigentumsinteresse des Rechteinhabers. Sofern der Lizenznehmer sich auf die Schranke des $\int 53$ Abs. 2 Nr. 1 UrhG berufen könnte, würde dies eine Beeinträchtigung des Schutzbereichs von Art.14 Abs. 1 GG darstellen. ${ }^{432}$ Wie jedoch oben bereits dargelegt, folgt aus Art. 14 GG nicht nur der Schutz des Eigentums, sondern gem. Art. 14 Abs. 2 GG ebenso dessen Sozialgebundenheit. Aufgrund dieser Sozialgebundenheit hat der Urheber bestimmte Einschränkungen als Ausgleich zwischen seinem Eigentumsinteresse und den Interessen der Allgemeinheit hinzunehmen, namentlich die Nutzung des Werkes durch Dritte im Rahmen der Schrankenregelungen. Demzufolge wird der auf der Abbedingung von \53 UrhG beruhende Eingriff in die Wissenschaftsfreiheit des Lizenznehmers nicht durch das Eigentumsrecht des Lizenzgebers gerechtfertigt, da der Urheber einen solchen Eingriff gerade dulden muss. Demzufolge würde die Abbedingung der Schranke des $\int 53$ Abs. 2 Nr. 1 UrhG den Lizenznehmer in seiner Wissenschaftsfreiheit verletzen.

Ein Eingriff in die Freiheit der Lehre durch die Abbedingung des $\int 52 \mathrm{a}$ UrhG ist hingegen abzulehnen, da die Lehrtätigkeit der Hochschule bzw. des lehrenden Wissenschaftlers nicht wesentlich dadurch beeinträchtigt wird, dass die Daten den Hörern der Lehrveranstaltung nicht öffentlich zugänglich gemacht werden dürfen.

Die Abbedingung der vorliegend in Betracht kommenden Schranken könnte außerdem einen unzulässigen Eingriff in die nach Art. 5 Abs. 1 S. 1 GG grundrechtlich garantierte Informationsfreiheit darstellen. Sofern durch die Abbedingung bestimmter Schranken sowohl die Wissenschafts- als auch die Informationsfreiheit verletzt würden, läge ein Fall der sog. Grundrechtskonkurrenz vor, ${ }^{433}$ in deren Rahmen die Informationsfreiheit hinter der spezielleren Wissenschaftsfreiheit zurücktreten würde.434 In Betracht käme aber auch eine Verletzung der Informationsfreiheit von Drittnutzern, also solchen Personen, die die Daten vom Lizenznehmer erlangen könnten, wenn diesem die Weitergabe nicht aufgrund der Verschwiegenheitsklausel untersagt wäre. Relevant wäre dies insbesondere in den Fällen, in denen die Schranke des $\int 52 \mathrm{a}$ UrhG im Einzelfall doch einschlägig ist und der Lizenznehmer die Daten eigentlich den Unterrichts- oder Vorlesungsteil-

\footnotetext{
430 BVerfG NJW 1971, 1645, 1646; NJW 1978, 1621; Kannengießer in Schmidt-

Bleibtreu/Hofmann/Hopfauf, Art. 5 Rn. 21; Jarass in Jarass/Pieroth, Art. 5 Rn. 131.

431 Vgl. Starck in v. Mangoldt/Klein/Starck, Art. 1 Abs. 3 Rn. 319; Jarass in Jarass/Pieroth, Vorb. vor

Art. 1 Rn. 48.

432 Gounalakis, S. 10.

433 Vgl. Jarass in Jarass/Pieroth, Vorb.vor Art. 1 Rn. 17.

434 Jarass in Jarass/Pieroth, Art. 5 Rn. 120.
} 
nehmern zugänglich machen dürfte. ${ }^{435} \mathrm{Da}$ bei diesen im Gegensatz zum Lizenznehmer nicht zwangsläufig der Schutzbereich von Art. 5 Abs. 3 GG eröffnet ist, wäre Art. 5 Abs. 1 GG grundsätzlich anwendbar. Auch wenn die Informationsfreiheit grundsätzlich ein Abwehrrecht gegenüber dem Staat darstellt, 436 ist allgemein anerkannt, dass sie auch Ausstrahlungswirkung auf privatrechtliche Verhältnisse haben und damit privatrechtliche Normen beeinflussen kann. ${ }^{437}$ Als Beispiel seien hierfür die Entscheidungen des Bundesverfassungsgerichtes zur Duldungspflicht des Vermieters bei Anbringung einer Parabolantenne durch den Mieter genannt. ${ }^{438}$ Demzufolge könnte aufgrund dieser Ausstrahlungswirkung der Informationsfreiheit in den zivilrechtlichen Bereich eine Sittenwidrigkeit der vertraglichen Abbedingung der hier in Betracht kommenden Schranken folgen. Dies setzt jedoch zunächst voraus, dass bei Abschluss einer solchen Vereinbarung in den Schutzbereich von Art. 5 Abs. 1 ohne Rechtfertigung eingegriffen würde.

Nach Art. 5 Abs. 1 GG hat jeder das Recht, sich aus allgemein zugänglichen Quellen ungehindert zu unterrichten. Dabei spricht die Tatsache, dass die hier im Mittelpunkt stehenden wissenschaftlichen Primärdaten eventuell nur entgeltlich zugänglich gemacht werden, nicht von vornherein gegen das Vorliegen einer allgemein zugänglichen Quelle in Form der Onlineplattform, von der die Daten heruntergeladen werden können. Eine Informationsquelle ist nämlich nicht nur dann allgemein zugänglich, wenn sie kostenlos zur Verfügung steht, sondern auch dann, ,wenn sie geeignet und bestimmt ist, der Allgemeinheit, also einem individuell nicht bestimmbaren Personenkreis, Informationen zu verschaffen“".439 Dabei sind als Quellen i.S.d. Art. 5 GG alle Träger von Informationen einzustufen, weshalb auch Onlineplattformen eine Quelle im Sinne des Art. 5 GG darstellen, auf denen Daten zum Abruf bereitgehalten werden. ${ }^{440}$ Geschützt sind dabei sowohl die Entgegennahme von Informationen als auch das aktive Beschaffen der selbigen, ${ }^{441}$ womit auch das durch $₫ 53$ Abs. 2 S. 1 Nr.1 UrhG erlaubte Kopieren eines Werkes zum eigenen wissenschaftlichen Gebrauch eine Informationsbeschaffung aus allgemein zugänglichen Quellen im Sinne von Art. 5 Abs. 1 S. 1 GG darstellt.

Damit ist der Schutzbereich der Informationsfreiheit in den vorliegenden Fällen grundsätzlich eröffnet. Da der Lizenznehmer aufgrund der Lizenzvereinbarung keine weiteren Vervielfältigungen anfertigen dürfte, kommt ein Eingriff in den Schutzbereich von Art. 5 Abs.1 grundsätzlich in Betracht. Dabei ist auch ein wirksamer Grundrechtsverzicht des Lizenznehmers durch Abschluss der Lizenz-

\footnotetext{
${ }^{435}$ Dass dies grundsätzlich möglich ist, wurde bereits auf S. 89 ff. dargelegt.

436 Kannengießer in Schmidt-Bleibtreu/Hofmann/Hopfauf, Art. 5 Rn. 10; Jarass in Jarass/Pieroth, Art. 5 Rn. 20.

${ }^{437}$ BVerfGE 90, 27, 33; Jarass in Jarass/Pieroth, Art. 5 Rn 21; zur Drittwirkung von Grundrechten siehe auch oben S. 120.

438 Siehe BVerfG NJW 1993, 1253; BVerfGE 90, 27, 36 f.

439 BVerfG NJW 1970, 235, 237; NJW 1984, 1741, 1743; Jarass in Jarass/Pieroth, Art. 5 Rn. 16.

440 Vgl. BVerfG NJW 1970, 235, 237; Jarass in Jarass/Pieroth, Art. 5 Rn. 15.

441 BVerfG NJW 1970, 235, 237; Jarass in Jarass/Pieroth, Art. 5 Rn. 17.
} 
vereinbarung abzulehnen. Zwar wird in der Literatur teilweise die Ansicht vertreten, dass der Grundrechtsträger in den Eingriff bestimmter Grundrechte einwilligen könne. ${ }^{442}$ Dies gilt allerdings nicht für die durch Art. 5 GG verbürgten Grundrechte. ${ }^{443}$ Darüber hinaus würde ein Grundrechtsverzicht hier wohl schon an der erforderlichen Freiheit des Lizenznehmers scheitern. ${ }^{444}$ Damit ist ein Eingriff in den Schutzbereich hier nicht aufgrund eines wirksamen Verzichts des Lizenznehmers abzulehnen.

Für die hier einschlägigen Sachverhaltskonstellationen ist jedoch zu berücksichtigen, dass der Lizenznehmer auf die ihm zustehenden urheberrechtlichen Schranken nur im Gegenzug zur Einräumung der von ihm begehrten Lizenz verzichtet. Dies bedeutet jedoch, dass der Lizenznehmer die Daten zumindest zum eigenen Gebrauch erlangt und diese folglich als Grundlage seiner wissenschaftlichen Tätigkeit verwenden kann. Damit erlangt er jedoch die Informationen, weshalb ein Eingriff in den Schutzbereich der Informationsfreiheit durch die Abbedingung der Schranken abzulehnen ist. Dagegen spricht auch nicht, dass die Einräumung der Lizenz in der Regel entgeltlich erfolgen wird, da Art. 5 Abs. 1 gerade kein Recht auf unentgeltliche Information gibt. ${ }^{445}$ Ein Eingriff in die Informationsfreiheit könnte allenfalls auf Seiten von Dritten bestehen, da der Lizenznehmer diesen die Daten und damit die darin verkörperten Informationen aufgrund des Weitergabeverbotes nicht mehr zukommen lassen dürfte. Auch hinsichtlich dieser „Drittnutzer“ ist jedoch eine Verletzung der Informationsfreiheit abzulehnen.

Zum einen haben sie die Möglichkeit, die Daten bei der archivierenden Einrichtung zu beziehen, so dass die Informationsaufnahme nicht endgültig verhindert wird. Dem könnte man zwar entgegenhalten, dass ein Eingriff in die Informationsfreiheit auch durch die Beschränkung allein eines bestimmten Informationsmediums gegeben sein kann. ${ }^{446}$ Letztlich handelt es sich bei den Speichermedien des Lizenznehmers, auf denen die Daten gespeichert werden, aber schon nicht um öffentlich zugängliche Quellen im Sinne von Art. 5 Abs. 1 GG, da sie nicht dazu bestimmt und wohl auch nicht technisch geeignet sind, die Daten der Allgemeinheit zugänglich zu machen. ${ }^{447}$ Demzufolge würde durch ein Weitergabeverbot und die damit einhergehende Abbedingung der oben genannten urheberrechtlichen Schranken weder auf Seiten des Lizenznehmers noch auf Seiten Dritter ein

\footnotetext{
${ }^{442}$ Vgl. Jarass in Jarass/Pieroth, Vorb. vor Art. 1 Rn. 36.

443 Vgl. Starck in v. Mangoldt/Klein/Starck, Art. 1 Abs. 3 Rn. 301; Jarass in Jarass/Pieroth, Vorb. vor Art. 1 Rn. 36.

${ }_{444}$ Vgl. BVerwG MMR 2004, 114, 115; Dreier in Dreier (Hrsg.), Grundgesetz, Bd. 1 2. Aufl. 2004,

Vorb. Vor Art. 1 Rn. 131; Jarass in Jarass/Pieroth, Vorb. vor Art. 1 Rn. 36.

445 BVerfG AP Nr. 1 zu 169 GVG; BVerwG NJW 1968, 1393, 1394; Clemens in Umbach/Clemens,

Grundgesetz Mitarbeiterkommentar und Handbuch Bd. 1, Art. 5 Rn. 52; Kannengießer in

Schmidt-Bleibtreu/Hofmann/Hopfauf, Art. 5 Rn. 10; Starck in v. Mangoldt/Klein/Starck, Art.

5 Abs. 1, 2 Rn. 53.

446 BVerfG NJW 1963, 755, 756; Jarass in Jarass/Pieroth, Art. 5 Rn. 19.

${ }^{447} \mathrm{Vgl}$. Kannengießer in Schmidt-Bleibtreu/Hofmann/Hopfauf, Art. 5 Rn. 8.
} 
Verletzung der von Art. 5 Abs. 1 GG geschützten Informationsfreiheit einhergehen.

Damit liegt eine Verletzung grundrechtlich verbürgter Interessen in den vorliegenden Fällen zumindest dann vor, wenn der Lizenznehmer die Daten auch nicht zum eigenen wissenschaftlichen Gebrauch vervielfältigen darf, da dies einen unzulässigen Eingriff in die durch Art. 5 Abs. 3 GG garantierte Wissenschaftsfreiheit darstellen würde. Demzufolge wäre die Lizenzvereinbarung grundsätzlich gem. \138 Abs. 1 BGB nichtig, sofern sich aus $\ 139$ BGB nicht etwas anderes ergibt. ${ }^{448}$ Danach ist bei Nichtigkeit des Teils eines Rechtsgeschäftes das ganze Rechtsgeschäft nichtig, wenn nicht anzunehmen ist, dass es auch ohne den nichtigen Teil vorgenommen worden wäre. Zumindest der Lizenznehmer hätte das Rechtsgeschäft auch ohne den nichtigen Teil vorgenommen, da er ohne diesen die Daten überhaupt nicht erlangen würde.

Anders verhält es sich hingegen mit dem Lizenzgeber. Bei diesem kann nicht ohne weiteres angenommen werden, dass er die Lizenzvereinbarung geschlossen hätte, dass das Weitergabeverbot bzw. die darin enthaltene Abbedingung bestimmter urheberrechtlicher Schranken nichtig ist. Allerdings wäre es rechtsmissbräuchlich, wenn der Lizenznehmer sich in diesen Fällen auf die Nichtigkeit der gesamten Lizenzvereinbarung berufen würde, da der Lizenzgeber hierdurch risikolos besonders harte Abbedingungsklauseln in die Vereinbarung aufnehmen könnte. ${ }^{449}$ In diesem Fall könnte der Lizenzgeber nämlich völlig risikolos besonders harte Abbedingungsklauseln in die Vereinbarung einfügen, da er wüsste, dass die Vereinbarung insgesamt nichtig wäre, sofern sich der Lizenznehmer auf die Sittenwidrigkeit der Abbedingungsklausel berufen würde. Umgekehrt würde der Lizenznehmer von einer solchen Lösung wohl auch davon abgeschreckt werden, die Sittenwidrigkeit überhaupt geltend zu machen, da er auf die Wirksamkeit des Lizenzvertrags angewiesen ist, sofern er die Daten erlangen möchte. Hinzu kommt, dass die Abbedingungsklausel vom übrigen Vertrag eindeutig abtrennbar ist die Lizenzvereinbarung auch ohne die sittenwidrige Klausel noch sinnvoll ist, ${ }^{450}$ zumal der Lizenzgeber die Lizenzgebühr als synallagmatische Gegenleistung erhält und insofern auch kein unbilliges Ergebnis entsteht.

Damit stellt sich die Frage, ob eine solche Abbedingung gänzlich unwirksam wäre oder ob sie dahingehend ausgelegt werden müsste, dass Vervielfältigungen für eigene wissenschaftliche Zwecke entgegen dem Wortlaut der Vereinbarung doch erlaubt sind. Für die erste Möglichkeit spricht, dass dadurch die Parteivereinbarung weitestgehend erhalten bliebe. Allerdings hätte eine solche Lösung zur Konsequenz, dass Lizenzgeber bedenkenlos sehr strenge Abbedingungsklauseln in ihre Vereinbarungen einfügen könnten, da die Klausel im schlimmsten Fall verfas-

\footnotetext{
448 Vgl. BGH NJW 1989, 26; Wendtland in Bamberger/Roth, Bd. 1, \138 Rn. 32; Ellenberger in Palandt, $\int 138$ Rn. 19.

449 Wendtland in Bamberger/Roth, Bd. 1, \138 Rn. 29; Ellenberger in Palandt, \138 Rn. 21.

${ }^{450}$ Vgl. BGH NJW 1965, 2147; 2001; 815, 817; Wendtland in Bamberger/Roth, Bd. 1, \138 Rn. 32.
} 
sungskonform ausgelegt würde und damit weitestgehend erhalten bliebe. Aus diesem Grund spricht mehr dafür, die Abbedingungsklausel insgesamt für nichtig zu erklären. Nur dann würden die Lizenzgeber dazu angehalten werden, keine Klauseln in die Vereinbarung aufzunehmen, die den Lizenznehmer in seinen grundrechtlich verbürgten Interessen verletzen. ${ }^{451}$

\section{Zwischenergebnis}

Sofern die Abbedingungsklausel dem Lizenznehmer auch die Vervielfältigung zu eigenen wissenschaftlichen Zwecken untersagt, liegt darin ein unzulässiger Eingriff in die Wissenschaftsfreiheit aus Art. 5 Abs. 3 GG, weshalb eine solche Abbedingung gem. \138 Abs. 1 BGB nichtig wäre. ${ }^{452}$ Dies hat jedoch nicht die Gesamtnichtigkeit des Lizenzvertrags zur Folge. Vielmehr ist davon auszugehen, dass die Nichtigkeit nur die Abbedingungsklausel betrifft, da die Parteien ein Interesse an der Beibehaltung des übrigen Lizenzvertrages haben. Darüber hinaus wäre es rechtsmissbräuchlich, wenn sich der Lizenzgeber auf die Nichtigkeit des gesamten Vertrags berufen würde. Die Abbedingungsklausel ist hingegen als insgesamt nichtig anzusehen und nicht etwa geltungserhaltend zu reduzieren.

Damit stellt sich die anfangs aufgeworfene Frage, ob die Vereinbarung einer Verschwiegenheitsklausel, aufgrund derer es dem Lizenznehmer untersagt wird, die empfangenen Daten an Dritte weiterzugeben, einer Prüfung nach den $\$ \int 307$ ff. BGB standhielte. Dies soll im Folgenden untersucht werden.

(d) Abbedingung durch Vereinbarung in Allgemeinen Geschäftsbedingungen (AGB)

In der Praxis wird der Abschluss von Lizenzvereinbarungen in der Regel nicht individualvertraglich erfolgen, sondern im Rahmen von AGB, so dass sich in diesen Fällen eine Unwirksamkeit aus den $\$ ₫ 307$ ff. BGB ergeben könnte. Diese haben gegenüber $₫ 138$ BGB den Vorrang, sofern sich die Sittenwidrigkeit nicht aus anderen als den in den $\iint 307 \mathrm{ff}$. BGB genannten Gründen ergibt. ${ }^{453}$ Sofern in den vorliegend relevanten Fällen AGB vorliegen und wirksam in den Vertrag mit einbezogen worden sind, würden diese grundsätzlich nach Maßgabe der $\$ ₫ 307 \mathrm{ff}$. BGB auf ihre Wirksamkeit hin überprüft werden. Dabei wird regelmäßig der Lizenzgeber die AGB stellen. Dies folgt schon daraus, dass der Vertragsschluss in der Regel über eine vom Lizenzgeber eingerichtete Onlineplattform erfolgen wird und die Lizenznehmer den AGB per Mausklick zustimmen muss. Bei dem Lizenzgeber wird es sich dabei in der Regel um eine juristische Person oder Personengesellschaft und damit eine Unternehmerin im Sinne des $₫ 14$ BGB handeln. ${ }^{454}$

\footnotetext{
451 Vgl. BGH NJW 1958, 1772; Ellenberger in Palandt, \138 Rn. 19; Wendtland in Bamberger/Roth, Bd. 1, \138 Rn. 32.

452 So auch Hohagen in FS Schricker, S. 362 f.

453 Ellenberger in Palandt, \138 Rn. 16; Wendtland in Bamberger/Roth, Bd. 1, \138 Rn. 10.

${ }^{454}$ Vgl. Ellenberger in Palandt, $\ 14$ Rn. 2; Schmidt-Räntsch in Bamberger/Roth, Bd. 1, $\ 14$ Rn. 5 f.
} 
Das Gleiche gilt für den Lizenznehmer. Selbst wenn dieser eine natürliche Person sein sollte (was wohl eher die Ausnahme darstellt), wäre der Zweck des Vertragsschlusses in nahezu allen Fällen seiner selbständigen oder beruflichen Tätigkeit als Wissenschaftler zuzurechnen, womit er ebenfalls als Unternehmer im Sinne des $₫ 14$ BGB tätig wäre. Das Gleiche gilt für privatrechtlich oder öffentlichrechtlich organisierte Forschungseinrichtungen, da auch diese Unternehmer im Sinne des $₫ 14 \mathrm{BGB}$ sind. ${ }^{455}$ Des Weiteren ist zu beachten, dass gem. $₫ 310$ Abs. 1 S. 1 BGB die Anwendbarkeit des $\ 305$ Abs. 2 und 3 sowie der $\int \mathbb{S} 308$ und 309 BGB ausgeschlossen ist, sofern es sich beim Lizenznehmer um einen Unternehmer, eine juristische Person des öffentlichen Rechts oder um öffentlich-rechtliches Sondervermögen handelt. Da jedoch davon auszugehen ist, dass die Daten von einer Onlineplattform bezogen werden können, wie dies etwa auch bei digitalisierten Zeitschriftenbeiträgen der Fall ist, wird der Lizenznehmer im Rahmen seiner Registrierung die AGB des Lizenzgebers per Mausklick akzeptieren. Daher wird der Lizenzgeber in der Regel Verwender der AGB sein, so dass es vorliegend auf die Vermutungsregel des $₫ 310$ Abs. 3 Nr. 1 BGB nicht ankommt. Der Anwendungsbereich der Vorschriften über die AGB-Kontrolle ist somit in den vorliegenden Fällen hinsichtlich der Lizenzvereinbarungen grundsätzlich eröffnet.

\section{Unwirksamkeit gem. \305c Abs. 1 BGB}

Wie bereits oben festgestellt, stellt die Vereinbarung einer Verschwiegenheitsklausel grundsätzlich keine überraschende Klausel i.S.d. \305c Abs. 1 BGB dar. ${ }^{456}$ Etwas anderes könnte sich jedoch unter Umständen aufgrund der Tatsache ergeben, dass durch diese Klausel urheberrechtliche Schranken abbedungen werden. Vor dem Hintergrund, dass durch den Lizenzvertrag Nutzungsrechte erworben und die rechtlichen Befugnisse des Lizenznehmers demnach erweitert werden sollen, könnte die Abbedingung urheberrechtlicher Schranken eine objektiv ungewöhnliche Klausel darstellen, da dies dem Leitbild des Vertrags zuwiderliefe, der ja eigentlich eine Erweiterung der Nutzungsbefugnisse beweckt. ${ }^{457}$ Auf der anderen Seite kann man dem entgegenhalten, dass der Lizenzvertrag die Nutzungsmöglichkeiten des Lizenznehmers nicht zwangsläufig erweitern, sondern ihm lediglich eine bestimmte Art oder bestimmte Arten der Nutzung ermöglichen soll, für die ihm der Lizenzgeber die erforderlichen Nutzungsrechte einräumt. Damit würde eine Abbedingung urheberrechtlicher Schranken dem Leitbild des Vertrags nicht zuwiderlaufen. Darüber hinaus ist es ja gerade Sinn und Zweck eines Lizenzvertrages, den Rahmen der Nutzung festzulegen, die dem Lizenznehmer gestattet wird. Dazu gehört jedoch auch, die Grenzen der erlaubten Nutzung zu definieren, auch wenn dadurch Nutzungen untersagt werden, die das Gesetz dem Lizenznehmer grundsätzlich gestatten würde oder diese von vornherein nicht

\footnotetext{
455 Vgl. Ellenberger in Palandt, $\int 14$ Rn. 2; Schmidt-Räntsch in Bamberger/Roth, Bd. 1, $\int 14$ Rn. 5 f. 456 Siehe oben S. 84.

457 Vgl. BGH NJW 1993, 779, 780; Grünberg in Palandt, \305c Rn. 3.
} 
verbieten. Deutlich wird dies beispielsweise bei sog. Know-how-Verträgen. Diese gestatten dem Know-how-Nehmer die Nutzung des (gesetzlich nicht geschützten) Wissens des Vertragspartners, legen jedoch gleichzeitig die Grenzen dieser Nutzung fest. ${ }^{458}$ Dementsprechend widerläuft die Abbedingung urheberrechtlicher Schranken aus Sicht eines objektiven Empfängers nicht dem vertraglichen Leitbild, so dass eine derartige Klausel im Rahmen eines Lizenzvertrags nicht ungewöhnlich ist und damit keine überraschende Klausel darstellt.

\section{Unwirksamkeit gem. \307}

Eine Unwirksamkeit nach $₫ 307$ Abs. 1 BGB würde vorliegen, wenn die Abbedingung urheberrechtlicher Schranken den Lizenznehmer entgegen den Geboten von Treu und Glauben unangemessen benachteiligen würde. Die Vorschrift findet entgegen $\ 308$ und $\int 309$ BGB auch Anwendung, wenn die AGB, wie in den vorliegend relevanten Fällen, gegenüber einem Unternehmer oder einer juristischen Person des öffentlichen Rechts gestellt werden. ${ }^{459}$ Eine solche unangemessene Benachteiligung wäre gem. \307 Abs. 2 Nr. 1 BGB im Zweifel anzunehmen, wenn die Abbedingung mit wesentlichen Grundgedanken der Regelung nicht vereinbar ist, von der abgewichen wird. Als gesetzliche Regelung kommt dabei gem. Art. 2 EGBGB jede Rechtsnorm in Betracht. ${ }^{460}$ Vorliegend könnte die Abbedingung urheberrechtlicher Schranken mit den wesentlichen Grundgedanken der $\int \mathbb{S} 44 \mathrm{a}$ ff. UrhG in Widerspruch stehen. Wie jedoch oben bereits festgestellt, ist grundsätzlich von einer Abdingbarkeit dieser Schranken auszugehen, sofern das Gesetz nichts Gegenteiliges bestimmt, weshalb ihrer Abbedingung grundsätzlich nicht die wesentlichen Grundgedanken der $\int S 44 \mathrm{a}$ ff. UrhG entgegenstehen. 461

$\mathrm{Zu}$ berücksichtigen sind jedoch auch hier wieder eventuelle Verletzungen grundrechtlicher verbürgter Interessen, da die Klausel in diesem Fall ebenfalls wesentlichen (nämlich verfassungsrechtlichen) Grundgedanken zuwiderliefe und damit eine Unwirksamkeit nach $\ 307$ BGB zur Folge hätte. ${ }^{462}$ Damit lässt sich mit Verweis auf die oben gemachten Erkenntnisse feststellen, dass eine Klausel, nach deren Inhalt der Lizenznehmer die Daten bzw. Datensätze nicht für eigene wissenschaftliche Zwecke vervielfältigen dürfte, nach \307 I, II Nr. 1 BGB unwirksam wäre. An einer Vervielfältigung und Weitergabe der Daten innerhalb der Forschungseinrichtung, die als Lizenznehmerin auftritt, wird der Lizenzgeber in der Regel auch keinen Anstoß nehmen, so dass eine solche Vervielfältigung wohl in den seltensten Fällen ausdrücklich untersagt sein wird. Zu beachten ist allerdings, dass die Klausel insgesamt nichtig ist, sofern sie allgemein die Vervielfältigung und

\footnotetext{
458 Vgl. Stumpf/ Groß, Der Lizenzvertrag, 8. Aufl. 2005, Rn. 16.

${ }^{459}$ Grüneberg in Palandt, $\ 307$ Rn. 39.

460 Fuchs in Ulmer/Brandner/Hensen, \ 307 Rn. 207.

461 So auch Hobagen in FS Schricker, S. 364 f.; siehe auch die Ausführungen oben auf S. 109 ff.

462 Dreier/Senftleben in Lejeune, S. 117; Dreier in Dreier/Schulze, Vor $\$ \int 44$ ff. Rn. 9.
} 
Weitergabe der Daten untersagt, ohne klarzustellen, dass Vervielfältigungen zu den gerade genannten Zwecken hiervon nicht erfasst sind.

Denn im Gegensatz zu individualvertraglichen Vereinbarungen, bei denen man die Erklärungen der Parteien gegebenenfalls verfassungskonform auslegen kann, ist eine solche Auslegung zu Lasten des Lizenznehmers aufgrund der Bestimmung in $\int 305$ Abs. 2 BGB im Rahmen von AGB nicht zulässig. Vielmehr gehen Zweifel zu Lasten des Verwenders, so dass die Klausel im Zweifel insgesamt nichtig ist. Damit würde sich der Inhalt des Vertrags gem. \ 306 Abs. 2 BGB insoweit nach den gesetzlichen Bestimmungen richten, was in den vorliegenden Fällen bedeutete, dass die Schranken der $\iint 44 \mathrm{ff}$. UrhG Anwendung fänden. Der übrige Vertrag, insbesondere die Einräumung der vom Lizenznehmer begehrten Nutzungsrechte bliebe indes gem. \306 Abs. 1 BGB wirksam. Demzufolge sollte der Lizenzgeber die Klausel dahingehend präzisieren, dass die Vervielfältigung zum eigenen wissenschaftlichen Gebrauch nicht von dieser Klausel umfasst ist. Ungenügend und damit nichtig wäre hingegen eine Klausel, die die Vervielfältigung der Datensätze insoweit untersagt, als dadurch keine grundrechtlichen Interessen des Lizenznehmers verletzt werden. ${ }^{463}$

\section{(5) Unwirksamkeit der Vertragsstrafe}

Hinsichtlich der Vertragsstrafe könnte sich dabei eine Unwirksamkeit vor allem aus $₫ 309$ Abs. 1 Nr. 6 BGB ergeben, sofern die $\int \ 308$ und 309 BGB anwendbar sind. Ist ihre Anwendbarkeit hingegen ausgeschlossen, könnte ein Verstoß gegen die genannten Normen jedoch eine Unwirksamkeit nach \307 BGB indizieren. ${ }^{464}$ Darüber hinaus kommt eine Unwirksamkeit nach der Generalklausel des \307 BGB in Betracht, sofern die Vertragsstrafe den Fremdforscher unangemessen benachteiligt.

(a) Unwirksamkeit gem. \309 Nr.6 BGB

Nach \309 Abs. 1 Nr. 6 BGB ist eine Vereinbarung in AGB unwirksam, sofern dadurch die Zahlung einer Vertragsstrafe für den Fall der Nichtabnahme oder verspäteten Abnahme der Leistung, des Zahlungsverzugs oder für den Fall versprochen wird, dass der andere Vertragsteil sich vom Vertrag löst. Insofern wäre die Vereinbarung der Vertragsstrafe nicht nach $\ 309$ Abs. 1 Nr. 6 BGB unwirksam, sofern daraus deutlich hervorginge, dass die Vertragsstrafe nur für den Fall gezahlt werden muss, dass der Fremdforscher die Daten weitergibt.

\footnotetext{
463 BGH WM 2003, 798, 800; BGHZ 93, 29, 48; BGH WM 1996. 967, 975; vgl. Schmidt in Ulmer/Brandner/Hensen, \ 306 Rn. 14.

464 BGH NJW 1988, 1785, 1788; BGH NJW 1984, 1750, 1751; Grüneberg in Palandt, \ 307 Rn. 40; K.P. Berger in Prütting/Wegen/Weinreich, $\int 307$ Rn. 30; siehe auch die Nachweise in Fn. 680.
} 
(b) Uniwirksamkeit gem. \307 BGB

Eine Unwirksamkeit der Vereinbarung einer Vertragsstrafe könnte sich damit nur noch aus \307 BGB ergeben und zwar unabhängig davon, ob der Vertragspartner Unternehmer oder Verbraucher ist. Es kommt gem. \307 Abs. 1 BGB somit darauf an, ob die Vereinbarung einer Vertragsstrafe den Vertragspartner, also den Fremdforscher oder die Forschungseinrichtung, unangemessen benachteiligt. Dies wäre wiederum gem. \307 Abs. 2 BGB anzunehmen, wenn die Vereinbarung einer Vertragsstrafe mit wesentlichen Grundgedanken der Regelung, von der abgewichen wird, nicht zu vereinbaren ist (Nr.1) oder wesentliche Rechte oder Pflichten, die sich aus der Natur des Vertrags ergeben, so eingeschränkt würden, dass die Erreichung des Vertragszwecks gefährdet würde (Nr.2).

Durch die Vereinbarung einer Vertragsstrafe wird jedoch gerade nicht von dem Grundgedanken einer gesetzlichen Regelung abgewichen. Vielmehr sehen die \S 339-345 BGB die grundsätzliche Möglichkeit einer Vertragsstrafe ausdrücklich vor. Des Weiteren folgt aus einem Umkehrschluss zu $\ 309$ Nr.6 BGB, dass in der Regel von der Wirksamkeit der Klausel auszugehen sein wird. Des Weiteren besteht der Sinn und Zweck eines Vertrags, durch den der Langzeitarchivierungsverbund Fremdforschern die Nutzung und den Abruf der archivierten Daten gestattet, darin, den Fremdforschern den wissenschaftlichen Dialog und fremde Forschungsprojekte durch die Bereitstellung der bereits erlangten Kenntnisse zu fördern. Dazu ist hingegen nicht erforderlich, dass der Fremdforscher die Daten seinerseits an andere Forscher weitergibt, die außerhalb seines Forschungsprojektes bzw. seiner Forschungseinrichtung stehen. Der Vertragszweck würde durch die Vereinbarung einer Vertragsstrafe damit auch nicht gefährdet. Die Vereinbarung einer Vertragsstrafe in AGB wäre demzufolge grundsätzlich nicht nach den

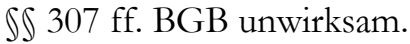

Etwas anderes würde sich hingegen ergeben, wenn die Vertragsstrafe außer Verhältnis zur Pflichtverletzung stehen würde, da sie anderenfalls ausnahmsweise doch nach $₫ 307$ Abs. 1 unwirksam wäre. 465

\section{b. Zwischenergebnis zu CII1}

Somit bleibt festzuhalten, dass sowohl die Vereinbarung einer Verschwiegenheitsklausel als auch einer Vertragsstrafe für den Fall der Zuwiderhandlung grundsätzlich keine überraschende Klauseln im Sinne des $₫ 305$ c BGB darstellen, sofern die Vereinbarung der Vertragsstrafe inhaltlich verständlich sowie optisch hervorgehoben worden ist. Darüber hinaus wären die besagten Klauseln auch nicht nach den $\$ \int 307 \mathrm{ff}$. BGB unwirksam, sofern aus der Verschwiegenheitsklausel klar hervorgeht, dass der Lizenznehmer die Daten zum eigenen wissenschaftlichen Gebrauch vervielfältigen darf.

465 BGH NJW 2003, 1805; 2000, 2106, 2107; 1987, 380; Fuchs in Ulmer/Brandner/Hensen, \ 309

Nr. 6 Rn. 28; Medicus/Stürner in Prütting/Wegen/Weinreich, \343 Rn. 15. 
Damit wäre es möglich, durch Abschluss eines Lizenzvertrages Fremdforscher auch dann an einer Weitergabe der Daten zu hindern, wenn ein urheberrechtlicher oder leistungsrechtlicher Schutz der Daten nicht gegeben sein sollte.

Die Vereinbarung einer Verschwiegenheitsklausel stellt darüber hinaus auch keine Gefährdung des Vertragszwecks nach $\ 307$ Abs. 2 Nr. 2 BGB dar. Denn Grundlage des Nutzungsvertrages wird die Förderung der Forschung anderer Wissenschaftler sein, denen die von der archivierenden Einrichtung gewonnenen Daten zum Abruf bereitgestellt werden. Dieser Vertragszweck wird jedoch nicht dadurch gefährdet, dass dem Fremdforscher die Weitergabe an Personen untersagt wird, mit denen er nicht zusammenarbeitet. Solange sich das Weitergabeverbot also nicht auch auf Kollegen des Fremdforschers bezieht, die mit ihm unmittelbar zusammen arbeiten, werden seine forschende Tätigkeit, und damit die Grundlage des Vertrages, nicht gefährdet. Ein Weitergabeverbot würde damit unter diesen Umständen auch einer Prüfung nach $\ 307$ BGB standhalten.

\section{Rechtlicher Schutz aufgrund gesetzlicher Regelungen}

Auch wenn ein effektiver Schutz durch den Abschluss von Lizenzverträgen erreicht werden kann, stellt sich für die archivierende Einrichtung die Frage, inwieweit die Daten einem gesetzlichen Schutz unterliegen, sofern sie im Einzelfall doch urheberrechtsfähig sein sollten. Bedeutung kann dies vor allem in den Fällen haben, in denen entweder kein Lizenzvertrag geschlossen wurde oder die geschlossene Vereinbarung unwirksam ist. In diesen Fällen könnte der Fremdforscher kraft Gesetzes zur Unterlassung der Weitergabe verpflichtet sein, sofern die Weitergabe der Daten eine Verletzung der an ihnen bestehenden Nutzungsrechte darstellen.

a. Vervielfältigung gem. $\int 16 \mathrm{UrhG}$

Sofern der Fremdforscher die Daten Dritten zukommen lässt, wird er im Regelfall eine Kopie der jeweiligen Datei erstellen und diese an den Dritten senden. Dieses Kopieren der Datei bzw. ihre Speicherung auf einem anderen Datenträger würde dabei eine Vervielfältigung im Sinne des $\ 16$ UrhG darstellen. ${ }^{466}$ Gem. $\ 15$ Abs. 1 Nr. 1 UrhG hat jedoch der Urheber das ausschließliche Recht der Vervielfältigung. Die archivierende Einrichtung wird sich dabei regelmäßig das Nutzungsrecht der Vervielfältigung zumindest als einfaches Nutzungsrecht eingeräumt haben lassen, um die Datei archivieren zu können. ${ }^{467}$ Die archivierende Einrichtung wird den Nutzern ihrer Daten dieses Recht in der Regel jedoch oder zumin-

\footnotetext{
466 BGH, GRUR 1999, 325, 327; OLG Frankfurt a.M. CR 1997, 275, 276; KG GRUR 2002, 252, 253; OLG München MMR 1998, 365, 367; Dreyer in Dreyer/Kotthoff/Meckel, Urheberrecht, \ 16 Rn. 6, 11; Loewenheim in Loewenheim, $\ 20$ Rn. 4, 11; Kroitzsch in Möhring/Niccolini, \16 Rn. 4; Siehe auch bereits oben S. 23 sowie die Nachweise in Fn. 76.

467 Zur Möglichkeit der Nutzungsrechteerlangung siehe die Ausführungen oben auf S. $47 \mathrm{ff}$.
} 
dest nur zum eigenen Download der Daten einräumen. Demzufolge würde der Fremdforscher eine Urheberrechtsverletzung begehen, sofern er die Datei einem Dritten zukommen ließe und sie zu diesem Zweck kopieren bzw. auf einem anderen Datenträger speichern würde. ${ }^{468}$

b. Verbreitung gem. \17 UrhG

Des Weiteren könnte von der Weitergabe an einen Dritten das Verbreitungsrecht gem. \17 Abs. 1 UrhG betroffen sein. Das Verbreitungsrecht ist nach \17 Abs.1 das Recht, das Original oder Vervielfältigungsstücke des Werkes der Öffentlichkeit anzubieten oder in Verkehr zu bringen. Sofern also ein urheberrechtsfähiges Werk vorliegt und der Fremdforscher dieses Dritten zukommen lässt, könnte dies eine Verbreitung i.S.d. Urheberrechtsgesetzes darstellen. Allerdings steht auch das Verbreitungsrecht gem. \ 15 Abs. 1 Nr.2 UrhG ausschließlich dem Urheber zu. Zunächst könnte es sich bei der Weitergabe von Datenbanken oder Datenbankwerken um ein Angebot an die Öffentlichkeit gem. \17 Abs. 1 Alt. 1 UrhG handeln.

Dabei kommt es nicht auf die Anzahl der Personen an, denen ein solches Angebot unterbreitet wird. Vielmehr kann ein Angebot an die Öffentlichkeit bereits dann vorliegen, wenn es sich lediglich an eine einzige Person richtet, sofern der Anbieter zu dieser keine persönliche Beziehung hat. ${ }^{469}$ Damit scheiden Angebote an Freunde, Bekannte oder Angestellte aus. ${ }^{470}$ Sofern der Dritte die Daten also lediglich an Kollegen weitergibt, wird keine Verbreitung im Sinne des $\int 17$ Abs. 1 UrhG vorliegen. Anders wird es jedoch wohl sein, wenn der Dritte die Daten an Forscher weitergibt, mit denen er nicht zusammenarbeitet und auch sonst keine persönliche Beziehung zu ihnen hat. ${ }^{471}$ In diesem Fall wird also regelmäßig ein Angebot an die Öffentlichkeit und damit eine Verbreitung im Sinne des $₫ 17$ Abs. 1 UrhG vorliegen. Sofern kein Angebot an die Öffentlichkeit vorliegt, kann die Weitergabe ein Inverkehrbringen im Sinne des $\int 17$ Abs. 1 Alt. 2 darstellen. Dies ist jede Handlung, durch die Werkstücke aus der internen Betriebssphäre der Öffentlichkeit zugeführt werden. ${ }^{472}$ Dabei genügt die Überlassung eines einzelnen Exemplars, sofern die Überlassung gegenüber der Öffentlichkeit erfolgt. ${ }^{473}$

\footnotetext{
${ }^{468} \mathrm{Zu}$ den rechtlichen Konsequenzen siehe unten S. 133.

${ }^{469}$ Heerma in Wandtke/Bullinger, $\ 17$ Rn. 10; Loewenheim in Schricker/Loewenheim, \ 17 Rn. 12 f.; so bereits zur Rechtslage vor 2003: BGH GRUR 1982, 102, 103; NJW 1991, 1234, 1235; OLG Köln GRUR 1992, 312, 313; KG GRUR 2000, 49; siehe auch BT-Drucks. 15/38 S. 17.

${ }^{470}$ KG GRUR 1983, 174, 175 -Videoraubkassetten; Loewenheim in Schricker/Loewenheim, \ 17 Rn. 11.

$471 \mathrm{Zu}$ denken wäre etwa an Forscher anderer Forschungseinrichtungen oder anderer Forschungsabteilungen.

472 EuGH GRUR 2008, 604; BGH GRUR 1991, 316, 317 - Einzelangebot; OLG Hamburg GRUR 1972, 375, 376 - Polydor II; Dreyer in Dreyer/Kotthoff/Meckel, \17 Rn. 12; Loewenheim in Schricker/Loewenheim, \17 Rn. 14.
} 
c. Öffentliche Zugänglichmachung gem. \19 UrhG

Durch die Weitergabe der Daten an Dritte kann darüber hinaus auch das Recht der öffentlichen Zugänglichmachung gem. \ 19a UrhG betroffen sein. Darunter versteht man gem. \19a UrhG das Recht, das Werk drahtgebunden oder drahtlos der Öffentlichkeit in einer Weise zugänglich zu machen, dass es Mitgliedern der Öffentlichkeit von Orten und zu Zeiten ihrer Wahl zugänglich ist. Dies wäre beispielsweise dann der Fall, wenn der Fremdforscher die von der archivierenden Einrichtung erlangten Daten auf einer Homepage zum Abruf für Dritte bereithielte. ${ }^{474}$

d. Zwischenergebnis

Die Weitergabe einer urheberrechtlich geschützten Datei bzw. einer Datenbank oder eines Datenbankwerkes, bringt zumindest eine unzulässige Vervielfältigung im Sinne von $\int 16$ Abs. 1 I UrhG mit sich, da der Fremdforscher in der Regel eine Kopie erstellen wird. Etwas anderes wäre der Fall, wenn er die Datei oder Datensammlung lediglich zum Download im Internet oder einem anderen Netzwerk bereithielte, da die Vervielfältigung dann von dem jeweiligen Dritten vorgenommen würde. Des Weiteren kann daneben eine unzulässige Verbreitung nach \ 17 Abs. 1 UrhG oder öffentliche Zugänglichmachung gem. \19a UrhG vorliegen.

\section{Rechtsfolgen der unerlaubten Verwertung des Werkes}

Sofern der Fremdforscher die urheber- oder leistungsrechtlich geschützten Dateien unbefugt an Dritte weiterleiten würde, hätte dies sowohl zivil- als auch strafrechtliche Konsequenzen. Vor dem Hintergrund der Frage, wie eine Weitergabe an Dritte verhindert werden kann, soll hier vorwiegend auf die zivilrechtlichen Konsequenzen eingegangen werden.

a. Anspruch auf Unterlassung und Schadensersatz

Der archivierenden Einrichtung stünde gegen den Fremdforscher gem. \97 Abs. 1 Satz 1 Alt. 1 UrhG zunächst ein Anspruch auf Beseitigung der Beeinträchtigung zu. Dieser kann zum Beispiel auf das Entfernen der zum Download bereitgestellten Datei auf einer Homepage durch den Fremdforscher gerichtet sein, der die Verletzung begangen hat. Wichtiger für die vorliegenden Fälle ist jedoch, dass der archivierenden Einrichtung außerdem bei Vorliegen einer Wiederholungsgefahr gem. \ 97 Abs. 1 Satz 1 Alt. 2 UrhG ein Anspruch auf Unterlassung zustände.

${ }^{473}$ Loewenheim in Schricker/Loewenheim, \17 Rn. 15; Dreyer in Dreyer/Kotthoff/Meckel, $\ 17$ Rn.

13.

${ }^{474}$ Vgl. Dreyer in Dreyer/Schulze, \19a Rn. 8; v. Ungern-Sternberg in Schricker/Loewenheim, \19a Rn.

1. 
Dabei indiziert die Rechtsverletzung grundsätzlich die Wiederholungsgefahr. ${ }^{475}$ Der Verletzer kann die Wiederholungsgefahr jedoch durch die Abgabe einer sog. strafbewehrten Unterlassungserklärung ausräumen. ${ }^{476}$ Darunter versteht man das Versprechen des Verletzers, der archivierenden Einrichtung im Falle einer nochmaligen Verletzung eine angemessen hohe Vertragsstrafe zu zahlen. ${ }^{477}$ Sofern sie dieses Versprechen annimmt, kann die archivierende Einrichtung im Falle einer nochmaligen Verletzung die Zahlung der vereinbarten Vertragsstrafe verlangen und den Fremdforscher erneut auf Unterlassen in Anspruch nehmen, da die Wiederholungsgefahr in diesem Fall wieder aufleben würde. ${ }^{478}$ Die Unterlassungserklärung und die darin enthaltene Vertragsstrafe stellen somit ein probates Mittel dar, um zu vermeiden, dass der Fremdforscher die Verletzung nochmals begeht. Des Weiteren wird ein Hinweis auf die soeben dargestellten Konsequenzen die Empfänger der archivierten Daten größtenteils wohl von vornherein davon abhalten, diese unbefugt weiterzugeben.

Die Inanspruchnahme des Verletzers kann jedoch problematisch werden, sofern die Person, die die Daten tatsächlich weitergegeben hat, nicht ermittelt werden kann. Denkbar ist dies etwa in den Fällen, in denen die archivierende Einrichtung die Daten nicht einer einzelnen natürlichen Person zur Verfügung stellt, sondern einer anderen Forschungseinrichtung, und ein Angestellter dieser Einrichtung die Daten unbefugt weitergibt. Gerade bei großen Forschungseinrichtungen mit vielen Mitarbeitern stellt sich dann das Problem, dass die Person, die die Daten weitergegeben hat, nur schwer oder mitunter sogar gar nicht ermittelt werden kann. Dies ist etwa dann der Fall, wenn zwar festgestellt werden kann, dass die Datei von einer bestimmte IP-Adresse einem Dritten zugesandt wurde, jedoch im Nachhinein nicht mehr ermittelt werden kann, welche Person zur fraglichen Zeit an dem betreffenden Computer gearbeitet hat. Allerdings kann der aus $₫ 97$ Abs. 1 UrhG folgende Unterlassungs- und Beseitigungsanspruch in diesen Fällen unter Umständen gem. $\int 99$ UrhG gegenüber dem Inhaber des Unternehmens selbst geltend gemacht werden, womit es auf die Identität des Mitarbeiters, der die Daten tatsächlich weitergegeben hat, nicht mehr ankäme.

\footnotetext{
${ }^{475}$ BGH GRUR 1997, 929, 930 ; BGH 1961, 138, 140 ; Wild in Schricker/Loewenheim, \ 97 Rn. 123; v. Wolff in Wandtke/Bullinger, \97 Rn. 36.

476 BGH GRUR 1997, 379, 380; Meckel in Dreyer/Kotthoff/Meckel, \ 97 Rn. 42; v. Wolff in Wandtke/Bullinger, \97 Rn. 37; J.B. Nordemann in Fromm/Nordemann, \97 Rn. 32.

477 V. Wolff in Wandtke/Bullinger, $\mathbb{S} 97$ Rn. 38; Meckel in Dreyer/Kotthoff/Meckel, $\mathbb{S} 97$ Rn. 42; Wild in Schricker/Loewenheim, \97 Rn. 123.

478 BGH GRUR 1980, 241, 242 - Rechtsschutzbedürfnis; Meckel in Dreyer/Kotthoff/Meckel, $\$ 97$ Rn. 44; v. Wolff in Wandtke/Bullinger, $₫ 97$ Rn. 40.
} 
b. Inhaber des Unternehmens

Inhaber des Unternehmens im Sinne des $\int 99$ UrhG ist derjenige, unter dessen Namen der Betrieb geführt wird und der damit nach außen die Verantwortung übernimmt. ${ }^{479}$ Wer dies im Einzelfall ist, hängt von der jeweiligen Einrichtung ab. Sofern es sich beispielsweise um die Fakultät einer Universität handelt, wird Letztere zumeist Trägerin der Fakultät und damit Inhaberin des Unternehmens sein.

\section{c. Arbeitnehmer oder Beauftragter}

Der die Verletzung begehende Fremdforscher müsste gem. \99 UrhG Arbeitnehmer oder Beauftragter des Unternehmens sein. Arbeitnehmer in diesem Sinne ist eine Person, die aufgrund eines Beschäftigungsverhältnisses, wie einem Dienstoder Werkvertrag, aber auch Auftrag, verpflichtet ist, in dem Geschäftsbetrieb Dienste zu leisten. ${ }^{480}$ Dabei fallen insbesondere Angestellte, Arbeiter, Beamte, freiberufliche Mitarbeiter sowie Auszubildende und Praktikanten unter den Arbeitnehmerbegriff des $\int 99$ UrhG, sofern sie weisungsabhängig sind. ${ }^{481}$ Dabei wird das Merkmal des Arbeitnehmers regelmäßig unproblematisch vorliegen, da die Fremdforscher in der überwiegenden Zahl der Fälle in einem Arbeitsverhältnis zur Forschungseinrichtung stehen werden, zumindest jedoch ein Auftragsverhältnis zugrunde liegen wird.

d. Verletzung innerhalb des Unternehmens

Die Rechtsverletzung müsste gem. \99 UrhG innerhalb des Unternehmens begangen worden sein. Dies wäre dann der Fall, wenn der Angestellte oder Beauftragte die Rechtsverletzung im Rahmen seiner Obliegenheiten begangen hätte; eine Verletzung, die nur bei Gelegenheit und im eigenen Interesse erfolgt ist, reicht dagegen nicht aus. ${ }^{482}$ In den vorliegenden Fällen wird die Verletzungshandlung im Regelfall darin bestehen, dass ein unbefugt handelnder Fremdforscher die Daten an andere Forscher bzw. Forschungseinrichtungen weitergibt. Damit wird die Rechtsverletzung in den meisten Fällen im Zusammenhang mit seiner beruflichen Tätigkeit begehen, so dass die Rechtsverletzung zumeist „innerhalb des Unternehmens" begangen werden wird.

Im Großteil der Fälle, in denen ein Fremdforscher die Daten unbefugt weitergibt, werden die Voraussetzungen des \99 UrhG somit vorliegen. Demnach müsste nicht erst aufwendig nach dem eigentlichen Verletzer gesucht werden. Vielmehr könnte man sich gleich an das Unternehmen halten, dessen Mitarbeiter oder Beauftragter die Verletzung begangen hat und dieses zur Abgabe einer straf-

${ }^{479}$ Meckel in Dreyer/Kotthoff/Meckel, \ 99 Rn. 5; Wild in Schricker/Loewenheim, \ 99 Rn. 2.

480 BGH GRUR 1993, 37, 39 - Seminarkopien; Meckel in Dreyer/Kotthoff/Meckel, I 99 Rn. 4; Wild in Schricker/Loewenheim, \& 99 Rn. 5.

481 J.B. Nordemann in Fromm/Nordemann, $\int 99$ Rn. 4; Köhler, GRUR 1991, 344, 346.

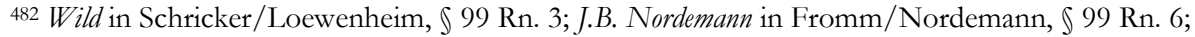
Köhler, GRUR 1991, 344, 352. 
bewehrten Unterlassungserklärung bewegen. Aufgrund der bereits oben beschriebenen abschreckenden Wirkung der damit einhergehenden Vertragsstrafe würde das Risiko einer erneuten Weitergabe an Dritte damit weitestgehend eingeschränkt.

\section{Urheber-oder leistungsrechtlicher Schutz an dem Langzeitarchiv insgesamt}

Ein urheber- oder leistungsrechtlicher Schutz könnte an den Daten darüber hinaus entstehen, sobald sie in das Langzeitarchiv der archivierenden Einrichtung aufgenommen und damit Bestandteil dieses Archives geworden sind. Das Langzeitarchiv, also die Gesamtheit der archivierten Daten, ist zwar nicht als Datenbankwerk im Sinne des $\int 4$ Abs. 2 UrhG einzuordnen, da es ja gerade auf Vollständigkeit angelegt ist und es somit wieder an der schöpferisch gestaltenden Tätigkeit mangelt. ${ }^{483}$

Ein elektronisches Langzeitarchiv wird jedoch regelmäßig eine Datenbank im Sinne des $\int 87 \mathrm{a}$ UrhG darstellen. Zum einen handelt es sich um eine Sammlung voneinander unabhängiger Daten, die einzeln mit Hilfe elektronischer Mittel zugänglich sind. Zum anderen erfordert das Sammeln der an der Universität entstandenen Daten sowie deren ständige Pflege, wozu unter anderem auch die regelmäßigen Umformatierungen in aktuelle Dateiformate zählen, einen nicht unerheblichen Arbeitsaufwand. Damit sind bei der Beschaffung, Überprüfung und vor allem Darstellung der einzelnen Daten im Archiv wesentliche Investitionen erforderlich. ${ }^{484}$ Das Langzeitarchiv unterliegt damit dem sui-generis Schutz der

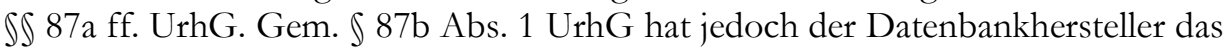
ausschließliche Recht, die Datenbank insgesamt oder einen nach Art oder Umfang wesentlichen Teil der Datenbank zu vervielfältigen, zu verbreiten und öffentlich wiederzugeben. ${ }^{485}$ Damit könnte die Vervielfältigung einer Datei, die für sich genommen zwar nicht schutzfähig ist, jedoch Teil der geschützten Datenbank ist, eine Nutzungsrechtsverletzung darstellen.486 Der archivierenden Einrichtung stünden somit grundsätzlich die oben dargestellten Ansprüche nach den \$S 97 ff. UrhG zu. Zu beachten ist jedoch, dass eine einzelne Datei keinen wesentlichen Teil einer so umfangreichen Datenbank wie dem Langzeitarchiv darstellt ${ }^{487}$. Der sui generis Schutz des Datenbankherstellers besteht jedoch gem. \87b Abs. Satz 1 UrhG gerade nicht an unwesentlichen Teilen. Zwar steht gem. \87b Abs. 1 Satz 2

\footnotetext{
483 BGH GRUR 1999, 923, 924 - Tele-Info-CD; OLG Hamburg GRUR 2000, 319, 320 - Börsendaten; OLG Düsseldorf MMR 1999, 729, 731; Dreier in Dreier/Schulze, \4 Rn. 20.

${ }^{484} \mathrm{Zu}$ den Voraussetzungen einer Datenbank an den einzelnen archivierten Datenpaketen siehe bereits S. $15 \mathrm{ff}$.

485 Zum Schutz von Datenbanken siehe EuGH GRUR 2007, 688; BGH GRUR 2007, 685; siehe auch Spindler, K\&R 2008, 565, 571.

486 Zum Eingriff in das Vervielfältigungsrecht des Datenbankherstellers siehe auch EuGH GRUR 2007, 688, 690 Tz. 22.

${ }^{487} \mathrm{Zu}$ den Abgrenzungskriterien siehe oben S. 25 ff.
} 
der Nutzung eines wesentlichen Teils die wiederholte und systematische Nutzung von nach Art und Umfang unwesentlichen Teilen gleich, wodurch verhindert werden soll, dass der Schutz nach Abs. 1 Satz 1 durch eine gezielte und häufige Nutzung unwesentlicher Teile umgangen wird. ${ }^{488}$ Allein durch die Weitergabe von Daten durch einen Fremdforscher ist eine solche wiederholte und systematische Nutzung allerdings noch nicht gegeben.

\section{Ergebnis}

Damit bleibt festzuhalten, dass der archivierenden Einrichtung Unterlassungsansprüche zustünden, sofern ein Fremdforscher die Daten an Dritte weitergeben würde und an den Daten ein urheberrechtlicher oder leistungsrechtlicher Schutz entstanden ist. Da dies jedoch nur selten der Fall sein wird, sollten zunächst technische Schutzmaßnahmen ergriffen werden, wie etwa die Einrichtung einer $\mathrm{Zu}$ gangsbeschränkung zu dem Datenarchiv sowie die Installation eines Kopierschutzes. Des Weiteren sollten mit den Fremdforschern, denen der Abruf der archivierten Daten gewährt wird, entsprechende Lizenzverträge geschlossen werden. Darin sollte dem Vertragspartner die Weitergabe der Daten an Dritte, die nicht zum Kreis seiner Forschungsstelle bzw. seines Forschungsprojektes gehören, im Rahmen einer Verschwiegenheitsklausel untersagt werden. Zur Durchsetzung dieser Klausel empfiehlt sich die Vereinbarung einer entsprechenden Vertragsstrafe bei Zuwiderhandlung. Zu beachten ist dabei, dass eine solche Klausel sowohl optisch hervorgehoben als auch eindeutig formuliert werden muss, damit sie einer Prüfung nach den $\$ \int 305 \mathrm{ff}$. BGB standhält.

Darüber hinaus müsste die Vertragsstrafe auch in einem angemessenen Verhältnis zur begangenen Pflichtverletzung stehen. Bezüglich der Verschwiegenheitsklausel ist zu beachten, dass diese dem Lizenznehmer unter Umständen bestimmte urheberrechtliche Schranken abbedingt. Aus diesem Grund ist diese Klausel so zu formulieren, dass aus ihr klar hervorgeht, dass der Lizenznehmer die Daten zum eigenen wissenschaftlichen Gebrauch vervielfältigen darf. Relevant ist dies insbesondere dann, wenn es sich bei dem Lizenznehmer nicht um eine natürliche Person, sondern eine andere Forschungseinrichtung handelt, da diese die bezogenen Daten in mehrfacher Ausfertigung benötigen, da regelmäßig mehrere Wissenschaftler gleichzeitig mit den Daten arbeiten müssen. Sofern die Verschwiegenheitsklausel ausnahmslos die Vervielfältigung und Weitergabe der Daten vorsieht, wäre dies nicht mit der in Art. 5 Abs. 3 GG verankerten Wissenschaftsfreiheit vereinbar, was eine Nichtigkeit der Verschwiegenheitsklausel nach \307 Abs. 1 BGB bzw. im Falle einer individualvertraglichen Vereinbarung gem. $₫ 138$ BGB zur Folge hätte. Demzufolge können sich die archivierenden Einrichtungen durch Abschluss von Lizenzverträgen, die eine Verschwiegenheits-

${ }^{488}$ EuGH GRUR 2005, 244, 251 Tz. 86; Thum in Wandtke/Bullinger, \87b Rn. 60; Cyychowski in Fromm/Nordemann, \87b Rn. 21, 23. 
vereinbarung enthalten, effektiv davor schützen, dass die Daten von Fremdforschern unbefugt an Dritte weitergeleitet werden, sofern die Klausel präzise genug formuliert ist.

IV. Anlage Entwurf einer Verschwiegenheitsklausel

\X Verschwiegenheitsverpflichtung

X.1 Der Vertragspartner verpflichtet sich zur Geheimhaltung über die ihm vom $<$ Name der archivierenden Einrichtung $>$ zur Verfügung bzw. zum Abruf bereit gestellten Daten und diese keinem Dritten zugänglich zu machen. Zur Erfüllung dieser Geheimhaltungspflicht hat der Vertragspartner alle notwendigen und zumutbaren Maßnahmen zu ergreifen.

X.2 Der Vertragspartner darf die Daten nur denjenigen Mitarbeitern zugänglich machen, die diese Daten im Rahmen ihrer Forschungstätigkeit zwingend benötigen. Diese Mitarbeiter sind vom Vertragspartner gemäß den Regelungen dieser Geheimhaltungsvereinbarung schriftlich zu verpflichten. Der Vertragspartner hat $<$ Name der archivierenden Einrichtung $>$ auf Anforderung unverzüglich Auskunft über die mit diesen Mitarbeitern getroffenen Geheimhaltungsregelungen zu erteilen und Kopien entsprechender Geheimhaltungsdokumente auszuhändigen.

X.3 Für den Fall, dass der Vertragspartner gegen die Geheimhaltungsverpflichtung verstößt, hat er $<$ Name der archivierenden Einrichtung $>$ eine Vertragsstrafe in Höhe von XX $€$ zu zahlen. Darüber hinaus stellt der Vertragspartner $<$ Name der archivierenden Einrichtung $>$ von Ansprüchen Dritter frei, die diese aufgrund der unbefugten Weitergabe der Daten durch den Vertragspartner gegen <Name der archivierenden Einrichtung $>$ geltend machen. Die Geltendmachung von sonstigen Schadensersatzansprüchen bleibt davon unberührt.

X.4 Sind einzelne der vorstehenden Ziffern oder Teile dieser Ziffern nichtig, so bleibt die Vereinbarung im Übrigen wirksam und an die Stelle der unwirksamen Regelung tritt entweder die gesetzliche Vorschrift oder, sofern eine solche Vorschrift nicht existiert, eine solche Regelung, die die Parteien nach Treu und Glauben zulässigerweise getroffen hätten, wenn ihnen die Nichtigkeit bekannt gewesen wäre. 



\section{Kapitel 2 - Datenschutzrechtliche Problematiken}

\section{A. Datenschutzrechtliche Zulässigkeit der Langzeitarchivierung wissenschaftlicher Daten mit Personenbezug}

Sofern es sich bei den zu archivierenden Daten um personenbezogene Daten handelt, stellt sich neben den urheberrechtlichen Problematiken die Frage nach der datenschutzrechtlichen Zulässigkeit einer solchen Archivierung. Datenschutzrechtliche Vorschriften finden sich dabei sowohl in Form des Bundesdatenschutzgesetzes (BDSG) als auch auf Länderebene in Form der einzelnen Landesdatenschutzgesetze. Darüber hinaus können im Einzelfall auch die Bestimmungen des Sozialgesetzbuches X (SGB X) einschlägig sein. Des Weiteren ist bei medizinischen Forschungsdaten unter Umständen die berufliche Schweigepflicht des behandelnden Arztes zu berücksichtigen.

I. Anwendbarkeit der Datenschutzgesetze

Sofern es um die Zulässigkeit nach den Vorschriften der Datenschutzgesetze geht, stellt sich zunächst die Frage, ob das BDSG oder eines der Landesdatenschutzgesetze in den Fällen der elektronischen Langzeitarchivierung Anwendung findet. 
Dies hängt im Einzelfall zunächst davon ab, welche Einrichtung die Archivierung vornimmt.

\section{Anwendbarkeit der Landesdatenschutzgesetze}

Die einzelnen Landesdatenschutzgesetze finden Anwendung, sofern eine öffentliche Einrichtung des jeweiligen Landes, der Gemeinden und Landkreise oder sonstige der Aufsicht des Landes unterstehende Körperschaften, Anstalten oder Stiftungen des öffentlichen Rechts die Verarbeitung der personenbezogenen Daten vornimmt. ${ }^{489}$ Der Anwendungsbereich der einzelnen Landesdatenschutzgesetze ist daher grundsätzlich eröffnet, sofern eine öffentliche Einrichtung wie beispielsweise eine Universität oder eine Forschungseinrichtung in der Rechtsform einer Anstalt oder einer Stiftung des öffentlichen Rechts die personenbezogenen Daten verarbeitet. 490

Darüber hinaus finden die einzelnen Landesdatenschutzgesetze auch Anwendung auf nicht-öffentliche Einrichtungen bzw. privatrechtliche Vereinigungen, wenn diese Aufgaben der öffentlichen Verwaltung wahrnehmen. ${ }^{491}$ Insofern würden das jeweilige Landesdatenschutzgesetz auch dann Anwendung finden, wenn die Archivierung nicht von einer Universität oder einer anderen öffentlichen Einrichtung eines Landes vorgenommen würde, sondern beispielsweise von einem sog. Langzeitarchivierungsverbund, also dem Zusammenschluss mehrerer öffentlicher oder auch nicht-öffentlicher Stellen zu einer privatrechtlichen Vereinigung, wie beispielsweise einer Gesellschaft des bürgerlichen Rechts (GbR). Voraussetzung dafür ist jedoch, dass die archivierende Einrichtung Aufgaben der öffentlichen Verwaltung wahrnimmt und keine öffentliche Stelle des Bundes beteiligt ist. Im letztgenannten Fall wäre nämlich gem. $\ 2$ Abs. 3 Nr. 1 BDSG der Anwen-

489 Vgl. \2 Abs. 1 Landesdatenschutzgesetz Baden-Württemberg (LDSG B-W) v. 18.09.2000, zuletzt geändert durch Gesetz v. 18.11.2008; \ 2 Abs. 1 Bayerisches Datenschutzgesetz (BayDSG) v. 23.07.1993, zuletzt geändert durch Gesetz v. 25.10.2000; \& 2 Abs. 1 Berliner Datenschutzgesetz (BlnDSG) v. 17.12.1990, zuletzt geändert durch Gesetz v. 30.11.2007; § 2 Abs. 1 Brandenburgisches Datenschutzgesetz (BBDSG); \1 Abs. 2 Bremisches Datenschutzgesetz (BremDSG) vom 04.03.2003; \2 Abs. 1 Hamburgisches Datenschutzgesetz (HmbDSG) v.05.07.1990, zuletzt geändert durch Gesetz v. 17.02.2009; 3 Abs. 1 Hessisches Datenschutzgesetz (HDSG) v. 07.01.1999; \2 Abs. 1 Landesdatenschutzgesetz Mecklenburg-Vorpommern (LDSG M-V) v. 28.03.2002; \2 Abs. 1 S. 1 Niedersächsisches Datenschutzgesetz (NDSG); 2 Datenschutzgesetz Nordrhein-Westfalen (DSG NRW) v. 15.3.1988, zuletzt geändert durch Gesetz v. 30.05.2000; \ 2 Abs. 1 Landesdatenschutzgesetz Rheinland-Pfalz (LDSG R-P) v. 05.07.1994; \ 2 Abs. 1 Saarländisches Gesetz zum Schutz personenbezogener Daten (SDSG) v. 24.03.1993, zuletzt geändert durch Gesetz v. 19.05.2008; Gesetz zum Schutz personenbezogener Daten der Bürger des Landes Sachsen-Anhalt (DSG-LSA) v. 12.03.1992, zuletzt geändert durch Gesetz v. 18.11.2005; \2 Abs. 1 Sächsisches Datenschutzgesetz (SächsDSG) v. 10.07.2003; \ 3 Landesdatenschutzgesetz Schleswig-Holstein (LDSG S-H) v. 09.02.2000; \2 Abs. 1 Thüringer Datenschutzgesetz (ThürDSG) v. 29.10.1991, zuletzt geändert durch Gesetz v. 14.09.2001.

490 Vgl. Tinnefeld/Ehmann/Gerling, Einführung in das Datenschutzrecht, 4. Aufl. 2005, S. 260.

491 Siehe z.B. \3 Abs. 1 S. 2 LDSG-LSA; \2 Abs. 1 S. 2 BlnDSG; $\ 2$ Abs. 2 S. 1 HmbDSG. 
dungsbereich des BDSG eröffnet, sofern die Vereinigung länderübergreifend tätig würde oder dem Bund gem. $\ 2$ Abs. 3 Nr. 2 die absolute Mehrheit der Anteile oder der Stimmen zustünde. Dabei wird grundsätzlich eine länderübergreifende Tätigkeit anzunehmen sein, sofern die Daten nicht nur für interne Zwecke archiviert werden, sondern zusätzlich anderen Forschungsstellen bundesweit oder sogar international zugänglich gemacht werden sollen. Dies wird aufgrund der heutigen technischen Möglichkeiten sowie der zunehmenden staatenübergreifenden Verknüpfung von Forschungseinrichtungen der Regelfall sein. Sofern sich also öffentliche Stellen der Länder und des Bundes zu einer Vereinigung privaten Rechts zusammenschließen, wären nicht die Landesdatenschutzgesetze, sondern gem. \2 Abs. 3 BDSG das BDSG anwendbar. Bei dem Zusammenschluss mehrerer öffentlicher Einrichtungen der Länder zu einer privatrechtlichen Vereinigung bliebe es hingegen bei der Anwendbarkeit der Landesdatenschutzgesetze.

\section{Anwendbarkeit des BDSG}

Der Anwendungsbereich des BDSG richtet sich nach $₫ 1$ BDSG. Gem. $\int 1$ Abs. 2 BDSG findet das Gesetz Anwendung auf die Erhebung, Verarbeitung und Nutzung personenbezogener Daten durch öffentliche Stellen des Bundes sowie öffentliche Stellen der Länder, soweit der Datenschutz nicht durch Landesgesetz geregelt ist und die Voraussetzungen von $₫ 1$ Abs. 2 Nr. 2 a) und b) BDSG vorliegen. Für die Erhebung personenbezogener Daten durch nicht öffentliche Stellen richtet sich die Anwendbarkeit nach \$ 1 Abs.3 BDSG. Da der Datenschutz, wie oben gezeigt, ausnahmslos durch alle Länder gesetzlich geregelt ist, wird der Anwendungsbereich des BDSG also im Rahmen der Langzeitarchivierung personenbezogener Daten nur dann Anwendung finden, wenn diese durch eine Einrichtung des Bundes, eine nicht-öffentliche Stelle, wie beispielsweise eine private Forschungseinrichtung erfolgt oder gem. \2 Abs. 3 BDSG durch eine privatrechtliche Vereinigung, der öffentliche Stellen des Bundes und der Länder angehören. ${ }^{492}$

Im letztgenannten Fall folgt die Anwendbarkeit des BDSG aus $\$ 1$ Abs. 2 Nr. 3. Danach findet dieses nämlich auch Anwendung auf nichtöffentliche Stellen, soweit diese Daten unter Einsatz von Datenverarbeitungsanlagen verarbeiten, nutzen oder dafür erheben oder die Daten in oder aus nicht automatisierten Dateien verarbeiten, nutzen oder dafür erheben, es sei denn, die Erhebung, Verarbeitung oder Nutzung der Daten erfolgt ausschließlich für persönliche oder familiäre Tätigkeiten. Die Anwendung des BDSG auf nichtöffentliche Stellen hängt also davon ab, ob diese Daten automatisiert oder dateigebunden verarbeitet werden. ${ }^{493}$

\footnotetext{
$492 \mathrm{Zu}$ denken wäre z.B. an die Max-Planck-Gesellschaft e.V. oder kommerzielle Forschungseinrichtungen; zum Zusammenschluss von öffentlichen Einrichtungen der Länder und des Bundes und der daraus resultierenden Konsequenzen für die Anwendbarkeit der unterschiedlichen datenschutzrechtlichen Regelungen siehe bereits oben S. 140.

${ }^{493}$ Gola/Schomerus, BDSG Kommentar, 10. Aufl. 2010, \$1 Rn. 20.
} 
Bei der Archivierung personenbezogener Daten werden diese gespeichert und in der Regel auch an Dritte übermittelt, wie zum Beispiel Fremdforscher. Auch wenn es sich in den vorliegenden Fällen um elektronische Dateien handelt, folgt daraus jedoch nicht zwangsläufig, dass diese unter Einsatz von Datenverarbeitungsanlagen oder für eine Verarbeitung durch solche Anlagen erhoben worden sind. Dies gilt gerade auch im Hinblick auf ältere Daten, die zu Zeiten erhoben wurden, zu denen die Verwendung von Datenverarbeitungsanlagen noch nicht so selbstverständlich war wie heute bzw. in denen solche Anlagen noch gar nicht verwendet wurden. Insbesondere besteht eine Erhebung nicht darin, dass bereits gewonnene Daten lediglich in Datenverarbeitungsanlagen eingetippt werden, da unter Erheben gem. $\int 3$ Abs. 3 BDSG das Beschaffen von Daten zu verstehen ist. ${ }^{494}$

Sofern die Daten jedoch im Internet oder anderen Netzwerken zum Abruf bereitgestellt werden, stellt dies eine Übermittlung und damit gem. $\ 1$ Abs. Nr. 3 BDSG grundsätzlich eine Datenverarbeitung dar. ${ }^{495}$ Das Gleiche gilt für die Speicherung der Daten in digitaler Form. Sowohl die Übermittlung an Fremdforscher als auch die Speicherung erfolgen dabei durch den Einsatz von Computern, mithin Datenverarbeitungsanlagen i.S.d. \1 Abs. 2 Nr. 3 BDSG. ${ }^{496}$ Das Vorliegen einer automatisierten Datenverarbeitung erfordert neben dem Einsatz von Datenverarbeitungsanlagen bei der Erhebung, Verarbeitung oder Nutzung jedoch auBerdem die Möglichkeit einer automatisierten Auswertung der Daten. ${ }^{497}$

Dass eine automatisierte Auswertung auch tatsächlich erfolgt, ist hingegen nicht erforderlich. ${ }^{498}$ Eine solche Möglichkeit ist dann gegeben, wenn wesentliche Verfahrensschritte, wie etwa das Lesen der Daten, in programmgesteuerten Einrichtungen ablaufen. ${ }^{499}$ Dieses Kriterium folgt aus der Notwendigkeit, den erhöhten Risiken Rechnung zu tragen, die in einem solchen Verfahren auftreten. Sofern eine automatisierte Auswertung der Daten jedoch trotz Einsatz einer Datenverarbeitungsanlage nicht möglich ist, wie etwa bei der Datenverarbeitung mittels eines Faxgerätes, ${ }^{500}$ besteht mangels eines erhöhten Risikos kein Grund für eine besondere Regelung. ${ }^{.01}$

\footnotetext{
${ }^{494}$ So auch entsprechend Gola in Gola/Schomerus, \ 3 Rn. 24.

${ }^{495}$ Entsprechend Gola in Gola/Schomerus, \ 3 Rn. 32.

496 Theoretisch wäre zwar auch an eine manuelle Übermittlung unter Ausschluss von Datenverarbeitungsanlagen denkbar. Im Mittelpunkt der vorliegenden Untersuchung steht jedoch der wohl praxisrelevantere Fall, in dem die Daten durch Dritte von beliebigen Orten über ein Netzwerk abgerufen werden können.

${ }^{497}$ Entsprechend Gola in Gola/Schomerus , \ 3 Rn. 15a; Dammann in Simitis (Hrsg.), Bundesdatenschutzgesetz, 7. Aufl. 2011, \3 Rn. 79.

498 Dammann in Simitis, \ 3 Rn. 82; Gola in Gola/Schomerus, \ 3 Rn. 15a.

${ }^{499}$ Dammann in Simitis, \3 Rn. 82; Gola/Schomerus , \3 Rn. 15 a.

500 Dammann in Simitis, $\$ 3$ Rn. 84.

${ }^{501}$ Dammann in Simitis, $\$ 3$ Rn. 79.
} 
Anders verhält es sich hingegen in den vorliegenden Fällen. Zum einen wird der Abruf der Daten durch Fremdforscher automatisch mittels eines Programms erfolgen, welches beispielsweise Patientendaten anhand bestimmter Kriterien, wie etwa des Alters des Patienten oder der Blutgruppe, herausfiltern und dem Nutzer zum Download bereitstellen kann. Selbst wenn ein solcher Abruf durch Fremdforscher im Einzelfall nicht möglich sollte, läge in den Fällen der elektronischen Langzeitarchivierung trotzdem eine automatisierte Verarbeitung vor, da die archivierten Daten maschinell gelesen und damit einer automatischen Auswertung zugänglich gemacht werden können. 502

Unabhängig davon, ob die archivierten Daten für Fremdforscher zum Abruf bereit gehalten werden, liegt demzufolge aufgrund der Speicherung der Daten in elektronischen Datenverarbeitungsanlagen eine automatisierte Verarbeitung i.S.d. \ 3 Abs. 2 BDSG vor, weshalb das BDSG in den vorliegenden Fällen regelmäßig auch dann anwendbar sein wird, wenn es sich bei der archivierenden Einrichtung um eine nicht-öffentliche Stelle handelt. Dabei sind unter nicht-öffentlichen Stellen gem. \2 Abs. 4 S. 1 BDSG natürliche und juristische Personen, Gesellschaften und andere Personenvereinigungen des privaten Rechts zu verstehen, soweit sie nicht unter $₫ 2$ Abs. 1 bis 3 BDSG fallen. Sofern eine nicht-öffentliche Stelle hoheitliche Aufgaben der öffentlichen Verwaltung wahrnimmt, ist auch sie gem. $\int 2$ Abs. 4 S. 2 BDSG als öffentliche Stelle anzusehen. Dies wäre dann der Fall, wenn der betroffene Hoheitsträger die Aufgabe auch hoheitlich oder schlicht-hoheitlich erfüllen könnte, er sich jedoch einer „eigenen“ privatrechtlichen Vereinigung bedient, wie dies zum Beispiel bei beliehenen Unternehmern wie Schornsteinfegern ${ }^{503}$ oder Notaren ${ }^{504}$ der Fall ist. ${ }^{505}$ Reine Finanzbeteiligungen des Bundes und der Länder fallen hingegen nicht unter $\$ 2$ Abs. 3 BDSG. ${ }^{506}$

\section{Zwischenergebnis}

Im Rahmend der Langzeitarchivierung personenbezogener Daten kommt je nach Einzelfall die Anwendbarkeit des BDSG oder der einzelnen Landesdatenschutzgesetze in Betracht. Dabei findet das BDSG auf nicht-öffentliche Stellen oder Stellen des Bundes Anwendung, die Landesdatenschutzgesetze hingegen auf öffentliche Einrichtungen der Länder sowie nicht-öffentliche Stellen, die Aufgaben der Länder wahrnehmen. Dies kann insbesondere dann der Fall sein, wenn sich beispielsweise Universitäten und Staatsbibliotheken oder andere dem Land unterstehende Einrichtungen einen Langezeitarchivierungsverbund in Form einer privatrechtlichen Rechtsform gründen.

\footnotetext{
502 Vgl. Dammann in Simitis, \ 3 Rn. 82.

503 BGH NJW 1991, 568.

504 BGH NJW 1974,

505 Wedde in Roßnagel, Handbuch Datenschutzrecht, 2003, 4.3 Rn. 34; Tinnefeld/Ehmann/Gerling, S. 269.

506 Gola/Schomerus, \ 2 Rn. 5; Simitis in Simitis, \ 2 Rn. 41.
} 
Bei der Prüfung der datenschutzrechtlichen Zulässigkeit der Archivierung personenbezogener Daten sind daher im Einzelfall entweder die Wirksamkeitsvoraussetzungen des BDSG oder die eines Landesdatenschutzgesetzes in Betracht zu ziehen. Zu beachten ist darüber hinaus im Rahmen von medizinischen Forschungsdaten, dass der Arzt, der den Probanden behandelt bzw. untersucht, der ärztlichen Schweigepflicht unterliegt. Diese wird zum einen aus $\ 203$ StGB hergeleitet, zum anderen auf das Persönlichkeitsrecht des Patienten gestützt. ${ }^{507}$ Der Zweck der Schweigepflicht wird außerdem mit dem Vertrauensverhältnis zwischen Arzt und Patienten begründet. ${ }^{508}$ Die ärztliche Schweigepflicht umfasst dabei neben der Privatsphäre des Kranken auch dessen Krankendaten.

Auch wenn es sich bei den Probanden eines medizinischen Versuches nicht zwangsläufig um einen Kranken handelt, besteht demzufolge die ärztliche Schweigepflicht auch im Verhältnis des Arztes zu einem nicht erkrankten Probanden, da auch in diesem Fall ein vergleichbares Vertrauensverhältnis besteht.

\section{B. Rechtliche Einschränkungen im Rahmen der Archivierung wissenschaftlicher Primärdaten mit Personenbezug}

Da bei wissenschaftlichen Primärdaten generell nur selten ein urheber- oder leistungsrechtlicher Schutz entsteht, ${ }^{509}$ ist bei der Frage nach dem zulässigen Inhalt medizinischer Forschungsdaten vor allem entscheidend, inwiefern die Daten dem Bundesdatenschutzgesetz (BDSG), dem Niedersächsischen Datenschutzgesetzes (NDSG) oder dem Sozialgesetzbuch X (SGB X) unterliegen.

I. Zulässigkeit der Archivierung medizinischer personenbezogener Forschungsdaten nach den Vorschriften des BDSG und der Landesdatenschutzgesetze

Die Erhebung, Verarbeitung und Nutzung medizinischer personenbezogener Forschungsdaten ist damit gem. \4 Abs. 1 BDSG nur zulässig, soweit das BDSG oder eine andere Rechtsvorschrift dies erlaubt oder anordnet oder der Betroffene eingewilligt hat. ${ }^{510}$ Die mit der Langzeitarchivierung der Daten zwangsläufig ein-

\footnotetext{
${ }^{507}$ Deutsch, AcP 192 (1992), 162 ff; Scbirmer in Roßnagel, Handbuch des Datenschutzrechts, 2003, 7.12 Rn. 22; Ulsenheimer in Laufs/Kern (Hrsg.), Handbuch des Arztrechts, 4. Aufl. 2010, \ 66 Rn. 15.

508 Schirmer in Roßnagel, 7.12 Rn. 23; Ulsenbeimer in Laufs/Kern, \ 65 Rn. 8, 16.

509 Siehe dazu oben S. $10 \mathrm{ff}$.

${ }^{510}$ Sofern eines der Landesdatenschutzgesetze einschlägig sein sollte, folgt das Gleiche aus deren Bestimmungen. Siehe \4 Abs. 1 LDSG B-W; \4 Abs. 1 BBDSG; Art. 15 Abs. 1 BayDSG; $\int 6$ Abs. 1 BlnDSG; $\$ Abs. 1 BremDSG; \5 Abs. 1 HmbDSG; $\ 7$ Abs. 1 HDSG; $\int 4$ Abs. 3 LDSG M-V; \ 4 Abs. 1 NDSG; \4 Abs. 1 DSG NRW; \5 Abs. 1 LDSG R-P; 4 Abs. 1 SDSG; \ 4
} 
hergehende Speicherung der Daten stellt dabei gem. $\int 3$ Abs. 4 S. 1 BDSG eine Verarbeitung dar. Des Weiteren wird in der Regel mit der Archivierung auch die Zugänglichmachung der Daten für andere Forscher einhergehen, da ein derartiger Daten- und damit Wissensaustausch elementar für ein effektives wissenschaftliches Arbeiten ist. Eine derartige Zugänglichmachung der Daten für Dritte würde jedoch gem. \ 3 Abs. 4 S. 1 Nr. 3 b) BDSG eine Übermittlung darstellen.

Die archivierende Einrichtung bedarf zur Archivierung personenbezogener Daten demnach sowohl nach den Vorschriften des BDSG als auch der Landesdatenschutzgesetze entweder der Einwilligung des Betroffenen oder des Vorliegens einer Erlaubnisnorm. Aus Gründen der Rechtssicherheit sollte die archivierende Einrichtung nach Möglichkeit jedoch stets die Einwilligung des Betroffenen einholen, da andernfalls die Gefahr besteht, dass eine Norm, die die jeweilige Verarbeitung oder Nutzung der Daten im konkreten Fall vermeintlich erlaubt, im Endeffekt aber nicht greift. Darüber hinaus können sich auch Beweisschwierigkeiten ergeben, die durch die vorherige Einwilligung des Betroffenen weitestgehend vermieden werden können.

\section{Rechtliche Anforderungen an eine Einverständniserklärung des Probanden}

Damit stellt sich die Frage, wie eine solche Einwilligungserklärung nach den Vorgaben des BDSG bzw. den Landesdatenschutzgesetzen inhaltlich ausgestaltet sein muss und ob sie einer bestimmten Form bedarf. Ferner ist zu klären, ob für die Einwilligung im Rahmen der Datenschutzgesetze die gleichen Anforderungen gelten, wie für eine Einwilligung, die den Arzt von seiner Schweigepflicht entbindet.

a. Einwilligung des Betroffenen

Sofern der Betroffenen einwilligt, ist die Verarbeitung personenbezogener Daten sowohl gem. \4 Abs. 1 BDSG als auch nach den jeweiligen Landesdatenschutzgesetzen $^{511}$ auch in den Fällen erlaubt, in denen dies nicht ausdrücklich durch eine andere Rechtsvorschrift vorgesehen ist. Aus Gründen der Rechtssicherheit sollte mit Probanden daher grundsätzlich immer eine entsprechende Vereinbarung geschlossen werden, da ansonsten bei jedem Datensatz geprüft werden müsste, ob die Tatbestandsvoraussetzungen der in Betracht kommenden Erlaubnisnorm auch tatsächlich vorliegen. Darüber hinaus kann die Einwilligung des Probanden unter Umständen weiter gehen, als die eventuell einschlägigen Erlaubnisnormen.

Abs. 1 DSG-LSA; \ 4 Abs. 1 SächsDSG; \ 4 Abs. 2 S. 1 SDSG; \11 Abs. 1 LDSG S-H; \ 4

Abs. 1 ThürDSG.

511 Siehe dazu die Paragraphenangaben in Fn. 510. 


\section{i. Form}

Gem. \4a BDSG bedarf die Einwilligung des Betroffenen der Schriftform. Auch die Landedatenschutzgesetze verlangen ausnahmslos die Erklärung der Einwilligung in Schriftform. ${ }^{512}$ Die Einwilligung muss demzufolge gem. \126 Abs. 1 BGB vom Betroffenen eigenhändig durch Namensunterschrift oder mittels notariell beglaubigten Handzeichens unterzeichnet werden. Eine Kopie oder ein Fax genügen demzufolge nicht. ${ }^{513}$ Ebenso wenig eine eingescannte und ausgedruckte Unterschrift oder eine Email. ${ }^{514}$ Die Einwilligung muss vielmehr die Originalunterschrift des Verfassers tragen, um die Schriftform zu wahren. ${ }^{515}$ Dabei kann die schriftliche Form gem. \126 Abs. 3 BDSG durch die elektronische Form ersetzt werden, sofern dies nicht gesetzlich ausgeschlossen ist, was jedoch im Rahmen von $\int 4$ a BDSG und der Landesdatenschutzgesetze nicht der Fall ist, sondern in einigen Gesetzen sogar ausdrücklich vorgesehen wird. ${ }^{516}$ Sofern die Schriftform durch die elektronische Form ersetzt werden soll, hat der Betroffene seinen Namen hinzufügen und das elektronische Dokument mit einer qualifizierten elektronischen Signatur ${ }^{517}$ nach dem Signaturgesetz zu versehen.

Darüber hinaus hat die verantwortliche Stelle die Erklärungen der Betroffenen zu protokollieren und der Einwilligungstext muss dem Betroffenen jederzeit zugänglich sein. ${ }^{518}$ Sofern die Einwilligung des Probanden weder der Schriftform noch einer zulässigen elektronischen Form entspricht, ist sie gem. \125 BGB grundsätzlich nichtig, es sei denn, dass wegen besonderer Umstände eine andere Form angemessen ist. ${ }^{519}$ Dies wird unter anderem bei einer Geschäftsbeziehung von längerer Dauer, bei telefonisch durchgeführten Meinungsumfragen oder im Falle einer besonderen Eilbedürftigkeit angenommen. ${ }^{520} \mathrm{Im}$ Rahmen der vorliegend relevanten Fälle der elektronischen Langzeitarchivierung könnte dabei in Einzelfällen die zweite Fallkonstellation in Betracht kommen, so etwa bei telefoni-

512 Vgl. \ 4 Abs. 3 S. 1 LDSG B-W; Art. 15 Abs. 3 S. 1 BayDSG; \ 6 Abs. 4 BlnDSG; \ 3 Abs. 4 Nr. 1 BremDSG; \4 Abs. 2 S. 1 BBDSG; $₫ 5$ Abs. 2 S. 1 HmbDSG; 77 Abs. 2 S. 1 HDSG; $\ 7$ Abs. 1 LDSG M-V; \ 4 Abs. 2 S. 1 NDSG; \ 4 Abs. 1 S. 3 LDSG NRW; \5 Abs. 3 S. 1 LDSG R-P; \ 4 Abs. 2 S. 2 DSG-LSA; \ 4 Abs. 4 S. 1 SächsDSG; \12 Abs. 1 LDSG S-H; \ 4 Abs. 3 S. 2 ThürDSG.

513 Ellenberger in Palandt, $\int 126$ Rn. 8; Wendtland in Bamberger/Roth, Bd. 1, \126 Rn. 6.

514 Simitis in Simitis, $\int 4 a$ Rn. 38; Ellenberger in Palandt, $\int 126$ Rn. 8; Wendtland in Bamberger/Roth, Bd. 1, \126 Rn. 8, 11.

515 Ellenberger in Palandt, $\int 126$ Rn. 8; Wendtland in Bamberger/Roth, Bd. 1, \126 Rn. 8.

516 So etwa in $₫ 4$ Abs. 5 SächsDSG; $\int 4$ Abs. 3 S. 2 Alt. 2 ThürDSG.

${ }^{517} \mathrm{Zu}$ den Anforderungen an eine qualifizierte elektronische Signatur siehe unten S.198.

518 Simitis in Simitis, \4a Rn. 38.

${ }^{519}$ Golal Schomerus, \4a Rn. 13; Roßnnagel/Holznagel/Sonntag, Handbuch Datenschutzrecht Rn. 40; Simitis in Simitis, $\ 4$ a Rn. 26; vgl. auch $\ 7$ Abs. 2 S. 1 HDSG; $\ 4$ Abs. 2 S. 1 NDSG; $\ 4$ Abs. 2 S. 2 ThürDSG.

${ }^{520}$ Gola/Schomerus, \ 4a Rn. 13; Holznagel/Sonntag in Roßnagel, 4.8 Rn. 29; Simitis in Simitis, \4a Rn. 61. 
schen Befragungen einer Forschungsstelle zu Versuchszwecken. ${ }^{521}$ Dies wird aber die Ausnahme darstellen, so dass grundsätzlich nicht wegen besonderer Umstände auf das Schriftformerfordernis verzichtet werden kann.

\section{ii. Ausnahmen vom Schriftformerfordernis}

Eine weitere Ausnahme vom Schriftformerfordernis, ergibt sich im Rahmen der wissenschaftlichen Forschung, aus \4a Abs. 2 S. 1 BDSG, ${ }^{522}$ wonach ebenfalls ein besonderer Umstand im Sinne des Absatzes 1 vorliegt, sofern durch das Schriftformerfordernis die Erreichung des Forschungswecks erheblich beeinträchtigt würde. Dies gilt nicht nur für Forschungseinrichtungen sondern vielmehr für den Gesamtbereich der wissenschaftlichen Forschung, so dass eine institutionelle Anbindung nicht erforderlich ist.

Zu beachten ist jedoch, dass $\int 4$ a Abs. 2 S. 1 BDSG aufgrund seines Ausnahmecharakters, der sowohl am Wortlaut als auch der Intention des Gesetzgebers festgemacht wird, möglichst restriktiv anzuwenden sind. ${ }^{523}$ Demzufolge sollte aus Gründen der Rechtssicherheit stets eine schriftliche Einwilligung des Probanden eingeholt werden. Auch wenn besondere Umstände der Norm vorliegen, bedarf es dennoch zumindest einer mündlichen Erklärung des Betroffenen.524 Stillschweigende oder mutmaßliche Einwilligungen genügen hingegen in keinem Fall, da anderenfalls der Betroffene übergangen würde. ${ }^{525}$ Das Einwilligungserfordernis scheidet darüber hinaus auch nicht deshalb aus, weil es dem Betroffenen an der Einwilligungsfähigkeit fehlt. In diesen Fällen ist vielmehr auf das Einverständnis des gesetzlichen Vertreters abzustellen.526 Gesetzliche Vertreter können dabei gem. \1629 BGB die Eltern des Betroffenen sein, sofern dieser noch minderjährig ist. Wurde für den Probanden ein Betreuer nach \ 1897 BGB bestellt, so kann, je nach Reichweite der Betreuung, dieser für die Abgabe der Einwilligung zustän-

521 Vgl. Gola in Gola/Schomerus, \ 4 a Rn. 16.

522 Die Landesdatenschutzgesetze enthalten ebenfalls diese Ausnahme, wenn auch mit teilweise unterschiedlicher Reichweite: Siehe $\int 4$ Abs. 4 LDSG B-W; Art. 15 Abs. 2 S. 3 BayDSG; 30 Abs. 1 BlnDSG; \ 28 Abs.1 BBDSG; \19 Abs. 1 BremDSG; \ 27 Abs. 1 HmbDSG; 』 33 Abs. 1 HDSG; 34 Abs. 3 LDSG M-V; \ 25 Abs. 2 Nr. 3 NDSG; 28 Abs. 2 Nr. 3 LDSG NRW; 30 Abs. 4 LDSG R-P; \ 30 Abs. 4 SDSG; \ 27 Abs. 4 DSG-LSA; \ 36 Abs. 4 SächsDSG; \ 22 Abs. 3 Nr. 2, 3 i.V.m. Abs. 4 LDSG S-H; 4 Abs. 4 ThürDSG.

523 Roßsnagel/ Pfitzmann/Garstka, Modernisierung des Datenschutzrechts: Gutachten im Auftrag des Bundesministeriums des Inneren, 2001, S. 94; Simitis in Simitis, \4a Rn. 44; vgl. auch LG Darmstadt, RDV 1999, 28, 29; dies gilt auch im Rahmen der Landesdatenschutzgesetze: siehe nur Kommentar zum NDSG, \25 Zu Abs. 6 Nr. 24.

524 So auch Gola/Schomerus, $\int 4$ a Rn. 16.

525 Wohlgemuth/Gerloff, Datenschutzrecht, 3. Aufl. 2005, Rn. 182; Kroll, Datenschutz im Arbeitsverhältnis, 1981, S. 181.

526 Bizer, Forschungsfreiheit, S. 260; Holznagel/Sonntag in Roßnagel, 4.8 Rn. 21; Wolters, Datenschutz und medizinische Forschungsfreiheit, 1988, S. 42.

${ }^{527}$ Kommentar zum NDSG, \ 4, Zu Abs. 2 Nr. 5; siehe auch Hinweise zum ThürDSG, Zu \ 4, 4.3, wonach der Einwilligende die Bedeutung seiner Entscheidung überblicken können muss. 
dig sein. Für die besagte Einwilligungsfähigkeit ist jedoch nicht erforderlich, dass der Betroffene geschäftsfähig ist. Es kommt vielmehr auf den jeweiligen Reifezustand des Betroffenen an. Als Anhaltspunkte können dabei verschiedene gesetzliche Regelungen dienen, die die Einsichtsfähigkeit Minderjähriger voraussetzen, wie beispielsweise $\ 5$ des Gesetzes über die religiöse Kindererziehung (RelKErzG). ${ }^{527}$

\section{iii. Persönliche Abgabe}

Des Weiteren stellt sich die Frage, ob der Proband die Einwilligung persönlich abgeben muss oder ob er sich zu dieser Erklärung auch eines Stellvertreters bedienen kann. Dabei steht das oben angesprochene Schriftformerfordernis einer Vertretung nicht von vornherein entgegen, da der Vertreter grundsätzlich im Namen des Vertretenen unterschreiben darf. ${ }^{528}$ Für die persönliche Abgabe der Einwilligung könnte aber das Informationsbedürfnis des Probanden sprechen. ${ }^{529}$ Für die Möglichkeit einer Stellvertretung spricht jedoch, dass auch in diesem Fall der Vertretungsberechtigte umfassend aufgeklärt werden müsste und dem Informationsbedürfnis damit genüge getan wird. ${ }^{530} \mathrm{Zu}$ beachten ist aber, dass nur der Betroffene selbst, hier also der Proband, in der Lage ist, die Auswirkungen der Verarbeitung seiner Daten zu beurteilen und auf dieser Grundlage zu entscheiden, ob er der Datenverarbeitung zustimmen möchten oder nicht. Dies spricht wiederum dafür, dass nur eine höchstpersönliche Abgabe in Betracht den Anforderungen von $\int 4$ a BDSG entspricht. ${ }^{531}$ Um nicht dem Risiko einer unwirksamen Einwilligung und daraus resultierenden Haftungsansprüchen ausgesetzt zu sein, sollte die archivierende Einrichtung daher stets eine höchstpersönliche Einwilligung des Probanden einholen. Dies wird im Rahmen von wissenschaftlichen Forschungsvorhaben in der Regel unproblematisch sein, da die Forschungseinrichtungen grundsätzlich im Kontakt zu den Betroffenen stehen. Schwierigkeiten könnten sich aber bezüglich solcher Daten ergeben, die bereits vor längerer Zeit erhoben wurden, da die Kontaktdaten der Betroffenen dementsprechend veraltet sein oder nicht mehr vorliegenwerden.

\footnotetext{
${ }^{527}$ Kommentar zum NDSG, \4, Zu Abs. 2 Nr. 5; siehe auch Hinweise zum ThürDSG, Zu \4, 4.3, wonach der Einwilligende die Bedeutung seiner Entscheidung überblicken können muss. ${ }_{528}$ BGHZ 45, 193, 195; Ellenberger in Palandt, $\$ 126$ Rn. 9; Wendtland in Bamberger/Roth, Bd. 1, \$ 126 Rn. 9; vgl. auch Kommentar zum NDSG, \4, Zu Abs. 2 Nr. 5.

${ }^{529}$ Siehe dazu unten S. Fehler! Textmarke nicht definiert..

${ }^{530}$ Holznagel/ Sonntag in Roßnagel, 4.8 Rn. 27.

531 Simitis in Simitis, \4a Rn. 30 m.w.N.; Podlech/Pfeifer, RDV 1998, 139, 152; so auch Kommentar zum NDSG, $₫ 4 \mathrm{Zu}$ Abs. 2 Nr. 6.
} 


\section{iv. Erkennbarkeit}

Schließlich ist zu beachten, dass die Einwilligung für den Probanden erkennbar sein muss. Der Proband muss also erkennen können, dass er eine Willensbekundung abgibt. ${ }^{532}$ Daran kann es fehlen, wenn dieser die Einwilligung zusammen mit anderen Erklärungen abgibt und sich aus diesem Grund über den Inhalt und die Tragweite der einzelnen Erklärung gar nicht im Klaren ist. ${ }^{533}$ Aus diesem Grund verlangt $\int 4 \mathrm{a}$ Abs. 1 S. 4 BDSG, dass die Einwilligungserklärung im äußeren Erscheinungsbild hervorzuheben ist, wenn sie zusammen mit anderen Erklärungen schriftlich erteilt werden soll. Erreicht werden kann dies zum Beispiel durch einen schriftlichen Hinweis auf die Einwilligung im selben Dokument oder eine visuelle Hervorhebung, wie etwa einer Einrahmung oder einem Fettdruck. ${ }^{534}$ Geeignet ist auch die Verwendung eines besonders gekennzeichneten getrennten Formulars. ${ }^{535}$

\section{v. Inhalt der Einwilligung}

Neben der Form der Einwilligung ist für die Wirksamkeit ferner entscheidend, welchen inhaltlichen Anforderungen die Einwilligung genügen muss.

\section{(1) Ausreichende Information des Probanden}

Erforderlich ist zunächst, dass die Einwilligung des Probanden in Kenntnis der Sachlage erfolgt. Dies folgt sowohl aus $\int 4$ a Abs. 1 S. 2 BDSG als auch aus Art. 2 h) der Datenschutzrichtlinie ${ }^{536}$ (DSRL). Gem. \4a Abs. 1 S. 2 BDSG ist der Betroffene auf den vorgesehenen Verwendungszweck der Daten sowie auf die Möglichkeit und die Rechtsfolgen einer Verweigerung der Einwilligung und der Möglichkeit des Widerrufs hinzuweisen. ${ }^{537}$ Diese Aufzählung ist dabei keineswegs als abschließend zu verstehen, sondern enthält vor dem Hintergrund des Wortlautes der DSRL und dem Sinn und Zweck der Informationspflicht lediglich Beispiele

532 OLG Celle, NJW 1980, 347, 348; Holznagel in Roßnagel, 4.8 Rn. 41; Simitis in Simitis, \ 4a Rn. 40; siehe auch Kommentar zum NDSG, \4 Zu Abs. 2 Nr. 6; Hinweise zum ThürDSG, Zu \ 4, 4.3.

533 Holznagel in Roßnagel, 4.8 Rn. 41; Simitis in Simitis, \4a Rn. 41.

534 Dies gilt auch im Anwendungsbereich der Landesdatenschutzgesetze, siehe nur Art. 15 Abs. 4 BayDSG; Kommentar zum NDSG, \4 Zu Abs. 2 Nr. 6; Hinweise zum ThürDSG, Zu \ 4, 4.3.

535 Holznagel/Sonntag in Roßnagel, 4.8 Rn. 43; Gola/Schomerus, \4a Rn. 13; Simitis in Simitis, $\ 4$ a Rn. 41; H. Geiger, CR 1985, 72, 76.

536 Richtlinie 95/46/EG zum Schutz natürlicher Personen bei der Verarbeitung personenbezogener Daten und zum freien Datenverkehr.

536 Richtlinie 95/46/EG zum Schutz natürlicher Personen bei der Verarbeitung personenbezogener Daten und zum freien Datenverkehr.

537 Siehe auch OLG Hamm NJW-RR 1986, 927, 931; im Anwendungsbereich der Landesdatenschutzgesetze folgt dies aus $₫ 4$ Abs. 2 LDSG B-W; Art. 15 Abs. 2 BayDSG; $₫ 6$ Abs. 3 BlnDSG; \4 Abs. 2 BBDSG; \3 Abs. 3 BremDSG; \5 Abs. 2 S. 2 HmbDSG; $\ 7$ Abs. 2 HDSG; $\int 8$ Abs. 1 S. 3, 4 LDSG M-V; \4 Abs. 2 S. 3, 4 NDSG; \ 4 Abs. 1 S. 3 LDSG NRW; \5 Abs. 2 S. 2, 3 DSG R-P; \ 4 Abs. 1 S. 4 SDSG; \ 4 Abs. 2 S. 1 DSG-LSA; \ 4 Abs. 3 S. 2 SächsDSG; \} 1 2 Abs. 2 LDSG S-H; $\int 4$ Abs. 3 S. 1 ThürDSG (wobei dieser nur eine Aufklärung über die Rechtsfolgen der Verweigerung voraussetzt, wenn der Betroffene diese verlangt). 
für besonders wichtige Angaben. 538 Da die Einwilligung nur soweit reichen kann, wie die Kenntnis des Probanden von den wesentlichen Informationen, sollte die Belehrung den konkreten Zweck der Verarbeitung benennen, den Namen und die Anschrift des Verantwortlichen oder dessen Vertretungsberechtigten für die Datenverarbeitung enthalten, über geplante Übermittlungen der Daten an Dritte informieren und darüber hinaus erkennen lassen, welche Daten, in welcher Art und über welchen Zeitraum gespeichert werden sollen. Des Weiteren ist der Proband über die Konsequenzen, die eine Verweigerung der Einwilligung für ihn nach sich ziehen würde sowie über alle sonstigen für die Beurteilung des Datenverarbeitungsvorgangs erforderlichen Informationen zu belehren. ${ }^{539}$ Der Proband ist dabei vor Beginn der Datenerhebung zu informieren, ${ }^{540}$ so dass im Rahmen des medizinischen Forschungsvorhabens die Einwilligungen und die damit verbundenen Information der Probanden vor Beginn der Versuchsreihe eingeholt bzw. vorgenommen werden sollten.

\section{(2) Ausreichende Bestimmtheit der Einwilligung}

Die Einwilligung des Probanden muss des Weiteren so formuliert sein, dass man ihr entnehmen kann, unter welchen Bedingungen die Daten nach Vorstellung des Probanden verarbeitet werden dürfen und an welche Stellen sie gegebenenfalls übermittelt werden. ${ }^{541}$ Dabei gilt, dass die Erklärung umso umfangreicher sein muss, je komplexer die Datenverarbeitung ist. ${ }^{542}$ Speziell im Rahmen der vorliegend relevanten Fallkonstellationen von medizinischen Forschungsvorhaben ist außerdem zu beachten, dass es sich dabei zumeist um personenbezogene Angaben über die Gesundheit des Probanden handeln wird, so dass er gem. \4a Abs.3i.V.m. \ 3 Abs. 9 BDSG ausdrücklich auf deren Verarbeitung Daten hingewiesen werden muss. ${ }^{543}$

538 Holznagel/Sonntag in Roßnagel, 4.8 Rn. 44; anders hingegen Hinweise zum ThürDSG, Zu \4, 4.5, wonach der in $\$ 4$ Abs. 5 ThürDSG enthaltene Katalog abschließend sein soll. Zu beachten ist jedoch, dass der Katalog wörtlich aus der DSRL übernommen worden ist.

539 OLG Hamm, NJW-RR 1986, 927, 931; OLG Karlsruhe, NJW-RR 1988, 302, 304; Holznagel/Sonntag in Roßnagel, 4.8 Rn. 45; Simitis in Simitis, \4a Rn. 72.

${ }^{540}$ Holznagel/Sonntag in Roßnagel, 4.8 Rn. 48; Gola/Schomerus, \ 4a Rn. 11; Simitis in Simitis, \ 4a Rn. 70.

541 \4 Abs. 2 LDSG B-W; Art. 15 Abs. 2 BayDSG; \6 Abs. 3 S. 1 BlnDSG; \ 4 Abs. 2 S. 3 BBDSG; \ 3 Abs. 3 S. 2 BremDSG; \5 Abs. 2 S. 2 HmbDSG; \ 7 Abs. 2 S. 2, 4, 5 HDSG; $₫ 8$ Abs. 1 S. 2 , 4 LDSG M-V; \ 4 Abs. 2 S. 2, 4 NDSG; \4 Abs. 1 S. 4 LDSG NRW; \5 Abs. 2 S. 2 DSG R-P; \4 Abs. 1 S. 4 SDSG; \ 4 Abs. 2 S. 1, 2 DSG-LSA; \ 4 Abs. 3 S. 1 SächsDSG; \ 12 Abs. 2 S. 1 LDSG S-H; $₫ 4$ Abs. 3 S. 1 ThürDSG

542 Holznagel/ Sonntag in Roßnagel, 4.8 Rn. 49.

543 Dies gilt ebenso, sofern nicht das BDSG, sondern ein Landesdatenschutzgesetz einschlägig sein sollte. Siehe $₫ 33$ Abs. 1 Nr. 2 LDSG B-W; Art. 15 Abs. 7 Nr. 2 BayDSG; \6a Abs. 2 BlnDSG; \4a Nr. 1 BBDSG; \ 3 Abs. 2 Nr. 2 BremDSG; \5 Abs. 2 S. 2 Nr. 2 HmbDSG; \ 7 Abs. 2 S. 2 i.V.m. Abs. 4 HDSG; \ 8 Abs. 1 S. 2 i.V.m. \ 7 Abs. 2 LDSG M-V; \ 4 Abs. 2 S. 2 NDSG; \ 4 Abs. 3 Nr.1 LDSG NRW; \5 Abs. 4 i.V.m. \3 Abs. 9 LDSG R-P; \4 Abs. 2 SDSG; \26 Abs. 


\section{(3) Freie Entscheidung des Probanden}

Schließlich muss die Einwilligung des Probanden gem. $\int 4$ Abs. 1 S. 1 BDSG auf seiner freien Entscheidung beruhen, so dass sie unwirksam ist, wenn sie durch Androhung rechtswidriger Nachteile bewirkt wurde. ${ }^{544}$ Die Forschungsstelle darf jedoch von der Einwilligung abhängig machen, ob sie die betreffende Person als Probanden annimmt oder nicht, sofern der Proband im Einzelfall dadurch nicht in eine Zwangssituation gedrängt wird. ${ }^{545} \mathrm{Da}$ Probanden aber grundsätzlich freiwillig an dem Forschungsvorhaben teilnehmen, wird hinsichtlich der Daten, die im Rahmen wissenschaftlicher Versuchsreihen erlangt wurden, regelmäßig eine freiwillige Einwilligung vorliegen. ${ }^{546}$ Dennoch ist im Einzelfall darauf zu achten, dass die Probanden nicht unter Druck gesetzt und zur Abgabe der Einwilligung gedrängt werden, da es dann an der notwendigen Freiwilligkeit fehlen würde.

\section{Auswirkungen des Widerrufs der Einwilligung}

Nachdem oben erläutert wurde, welche Anforderungen an eine wirksame Einwilligung zu stellen sind und unter welchen Voraussetzungen eine Archivierung personenbezogener Daten auch ohne Einwilligung zulässig sein kann, soll im Folgenden dargestellt werden, welche Konsequenzen ein Widerruf der Einwilligung durch den Betroffenen mit sich bringt.

\section{a. Rechtliche Möglichkeit des Zurückziehens der Einverständniserklärung}

Der Betroffene ist an seine Einwilligung nicht endgültig gebunden, sondern kann diese widerrufen. ${ }^{547}$ Hinsichtlich der Folgen des Widerrufs ist danach zu differenzieren, ob dieser vor oder nach der Datenerhebung erfolgt.

\section{b. Widerruf vor Datenerhebung}

Widerruft der Betroffene seine Einwilligung noch bevor die ersten Untersuchungen oder Tests durchgeführt worden sind, in deren Zusammenhang seine Daten erhoben werden, so steht die archivierende Einrichtung so, als hätte sie die Einwilligung niemals erhalten. Dies bedeutet, dass sie die personenbezogenen Daten des Betroffenen gem. \4 Abs. 1 NDSG bzw. \4 BDSG nicht speichern oder anderweitig verwerten darf, sofern nicht eine gesetzliche Vorschrift dies erlaubt.

1 Nr. 2 DSG-LSA; \ 4 Abs. 2 Nr. 2 SächsDSG; \ 11 Abs. 3 Nr. 1 LDSG S-H; \ 4 Abs. 5 Nr. 2 ThürDSG.

544 BVerfG NJW 1992, 1875 f; Gola/Schomerus, \4a Rn.6; Holznagel/Sonntag in Roßnagel, 4.8 Rn. 54; Simitis in Simitis, $\mathbb{\int}$ a Rn. 62.

$545 \mathrm{Vgl}$. Holinagel/Sonntag in Roßnagel, 4.8 Rn. 54; Simitis in Simitis, \ 4a Rn. 62.

546 Etwas anderes könnte jedoch gelten, wenn es sich um Forschungsreihen zur Entwicklung eines Medikamentes gegen eine unheilbare Krankheit handelt und sich die Teilnahme für den Probanden quasi als „letzte Chance“ darstellt, geheilt zu werden bzw. sein Leiden zu mindern.

547 Gola/Schomerus, \4a Rn. 18; siehe auch die diesbezügliche Hinweispflicht in $\ 13$ Abs. 2 Nr. 4 TMG. 


\section{c. Widerruf nach Datenerhebung}

Sofern die Verarbeitung von personenbezogenen Daten des Probanden allerdings bereits begonnen hat, gilt sein Widerrufsrecht unter Umständen nicht unbegrenzt. Vielmehr scheidet die Möglichkeit eines Widerrufes dann aus, wenn die Daten bereits anonymisiert worden sind. Sofern nämlich eine vollständige Anonymisierung vorgenommen wurde, fehlt es bereits am Personenbezug der (anonymisierten) Daten, so dass in diesen Fällen das Recht auf informationelle Selbstbestimmung nicht mehr betroffen ist. ${ }^{548} \mathrm{Da}$ gerade im Rahmen von medizinischen Forschungsvorhaben eine Anonymisierung nicht immer möglich ist, sondern die Daten oftmals lediglich pseudonymisiert werden, besteht in einer Vielzahl von Fällen weiterhin ein Personenbezug, da die Zuordnung der Daten zu dem Betroffenen ohne einen verhältnismäßig hohen Aufwand möglich ist. ${ }^{549}$ Widerruft ein Betroffener seine Einwilligung in einem solchen Fall wirksam, stellt sich die Frage, welche Auswirkungen dies für die bereits erfolgten und künftigen Datennutzungen hat.

Ein solcher Widerruf würde dabei -anders als zum Beispiel die Anfechtung einer Willenserklärung- ex-nunc, also mit Wirkung für die Zukunft wirken. ${ }^{550}$ Die zuvor erhobenen und gespeicherten Daten des Betroffenen dürften damit von der archivierenden Einrichtung nicht weiterverwendet werden. Konkret bedeutet dies, dass die Daten zum einen nicht mehr an Fremdwissenschaftler weitergeleitet werden dürften. Zum anderen müssten die entsprechenden Datensätze vollständig gelöscht werden, da sie nicht mehr erforderlich wären. ${ }^{551}$ Würden die Daten hingegen trotz des Widerrufs der Einwilligung weiterverwendet werden bzw. weiter auf den Speicherplätzen der archivierenden Einrichtung gespeichert bleiben, ohne dass die Merkmale, die für den Personenbezug erforderlich sind, gelöscht würden, läge eine unzulässige Datenverarbeitung nach \4 Abs. 1 BDSG vor, wodurch die archivierende Einrichtung unter Umständen Schadensersatzansprüchen des Betroffenen nach den $\iint 7,8$ BDSG bzw. \18 NDSG ausgesetzt wäre.

\section{Zwischenergebnis}

Widerruft der Betroffene seine Einwilligung, so entfällt diese für die Zukunft. Dies hat zur Folge, dass die bis zu diesem Zeitpunkt erfolgten Datenverarbeitungen zwar von der Einwilligung gedeckt sind, die Daten des Betroffenen ab dem Zeitpunkt des Widerrufs jedoch nicht mehr verarbeitet werden dürfen, es sei denn, dass eine Rechtsvorschrift dies ausdrücklich erlaubt.

\footnotetext{
548 Roßnagel/Scholz, MMR 2000, 721, 723.

549 Roßnagel/Scholz, MMR 2000, 721, $725 \mathrm{f}$.

550 Gola/Schomerus, S 4 a Rn. 18.

551 Holznagel/Sonntag in Roßnagel, 4.8 Rn. 67; siehe auch \ 20 Abs. 2 Nr.2 BDSG; \ 35 Abs. 2 Nr. 3 BDSG.
} 


\section{Erlaubnistatbestände des BDSG und der Landesdatenscbutzgesetže}

$\mathrm{Da}$ es teilweise aus organisatorischen Gründen nicht möglich ist, eine Einverständniserklärung des Betroffenen einzuholen, ist für die Langzeitarchivierung personenbezogener Daten ferner entscheidend, ob eine Archivierung ausnahmsweise auch ohne Einwilligung des Betroffenen zulässig ist. Dies wäre der Fall, wenn eine gesetzliche Erlaubnisnorm einschlägig wäre. Ob bzw. wann dies der Fall ist, soll im Folgenden geprüft werden.

a. Zulässigkeit der Archivierung aufgrund einer Erlaubnisnorm des BDSG

Auch wenn eine Einwilligung aus datenschutzrechtlicher Sicht für datenverarbeitende Stelle aus den oben genannten Fällen am vorteilhaftesten ist, kann es im Einzelfall auf das Vorliegen einer Erlaubnisnorm des BDSG oder der Landesdatenschutzgesetze ankommen. Denkbar ist dies etwa für den Fall, dass Daten archiviert werden sollen, die bereits in der Vergangenheit erhoben worden sind und für die entweder keine Einwilligung der Betroffenen vorliegt und auch nicht eingeholt werden kann oder deren Einwilligung nicht die Speicherung in einem Langzeitarchiv und die Übermittlung an Fremdforscher erfasst. In diesen Fällen ist zu prüfen, ob die Archivierung aufgrund einer Vorschrift des BDSG erlaubt ist. Die Erlaubnisnormen für die Datenverarbeitung durch nicht-öffentliche Stellen finden sich in den $\iint 27$ ff. BDSG. Dabei könnte sich die Zulässigkeit der Speicherung und Übermittlung personenbezogener Daten im Rahmen der elektronischen Langzeitarchivierung aus $\ 28$ oder $\ 29$ BDSG ergeben.

\section{i. Zulässigkeit nach $\ 28$ BDSG}

Die Datenverarbeitung zum Zwecke der elektronischen Archivierung und Zugänglichmachung für Fremdwissenschaftler könnte nach \28 BDSG zulässig sein. Dabei finden die Abs. 1 und 2 sowohl auf die Datenerhebung als auch die Datenverarbeitung oder Nutzung Anwendung. \28 Abs. 1 BDSG enthält dabei drei Tatbestandsvarianten. Diese haben gemein, dass die verarbeitende Stelle die Daten stets ,als Mittel zur Erfüllung eigener Geschäftszwecke“ verarbeitet. Die Datenverarbeitung muss demnach zur Erfüllung bestimmter anderer eigener Zwecke der Daten verarbeitenden Stelle erfolgen, wie zum Beispiel der Abwicklung von eingegangenen Verträgen oder der Betreuung von Kunden. ${ }^{552}$ Dies ist bei einer Datenverarbeitung durch die archivierende Stelle jedoch eher unwahrscheinlich, steht doch die Speicherung und Zugänglichmachung der Daten gerade im Mittelpunkt dieser Einrichtungen und bildet damit das eigentliche „Geschäftsinteresse“. Der Erlaubnistatbestand des $₫ 28$ Abs. 1 S. 1 BDSG setzt jedoch voraus, dass die Verarbeitung lediglich einen Mittel zum Zweck darstellt, um einen dahinterstehenden Geschäftszweck zu erreichen. ${ }^{553}$ Aus diesem Grund scheidet die Zulässig-

552 Gola/Schomerus, \ 28 Rn. 4.

${ }^{553}$ Gola/Schomerus, $\$ 28$ Rn. 4. 
keit der Nutzung personenbezogener Daten aufgrund der Erlaubnisnorm des $₫ 28$ BDSG im Rahmen der elektronischen Langzeitarchivierung von wissenschaftlichen Primärdaten grundsätzlich aus.

\section{ii. Zulässigkeit nach \29 BDSG}

Die Zulässigkeit der Verarbeitung personenbezogener Daten im Rahmen der elektronischen Langzeitarchivierung könnte sich hingegen aus \29 BDSG ergeben. Danach ist das geschäftsmäßige Erheben, Speichern, oder Verändern personenbezogener Daten zulässig, wenn dies zum Zweck der Übermittlung geschieht. Der Tatbestand des $\ 29$ BDSG erfasst also die Fälle, in denen im Gegensatz zu \28 BDSG die Datenverarbeitung für den Selbstzweck erfolgt. ${ }^{554}$ Zwar werden die archivierten Daten in der Regel auch Fremdforschern zugänglich gemacht, was eine Übermittlung darstellt, so dass die Tatbestandsvoraussetzungen von $\int 29$ BDSG auf den ersten Blick vorliegen. Zu beachten ist jedoch, dass $₫ 29$ Abs. 1 BDSG voraussetzt, dass die Daten genau zu diesem Zweck erhoben, gespeichert oder verändert werden.

Die Erhebung der zu archivierenden Daten erfolgte jedoch zum Zweck der Forschung und Wissenschaft, nicht aber, um diese an andere zu übertragen. Auch aus der Aufzählung des $\ 29$ Abs. 1 S. 1 BDSG, wonach die Norm ,insbesondere im Rahmen der Werbung, der Tätigkeit von Auskunfteien, dem Adresshandel oder der Markt- und Meinungsforschung" einschlägig ist, folgt nicht ausdrücklich die Anwendbarkeit auf die vorliegenden Fälle. Zwar ist die Aufzählung in Abs. 1 nicht abschließend, ${ }^{555}$ jedoch folgt aus den dargestellten Tätigkeitsbereichen, dass die Übermittlung der Daten im Mittelpunkt steht. Dies ist bei der Speicherung der Daten durch eine wissenschaftliche Einrichtung, die die Daten archiviert, jedoch gerade nicht der Fall, weshalb auch $₫ 29$ BDSG in den vorliegenden Fällen grundsätzlich nicht einschlägig sein wird. Die Zulässigkeit der Datenverarbeitung nach \ 29 BDSG würde darüber hinaus auch aus anderen Gründen scheitern, verlangt die Vorschrift doch, dass die Daten entweder aus allgemein zugänglichen Quellen entnommen werden könnten ( $\mathbb{\$} 29$ Abs. 1 S. 1 Nr. 2 BDSG) oder kein Grund zu der Annahme besteht, dass der Betroffene ein schutzwürdiges Interesse an dem Ausschluss der Speicherung hat (\$29 Abs. 1 S. 1 Nr. 1 BDSG).

$\mathrm{Da}$ es sich jedoch in den vorliegenden Fällen zum großen Teil um medizinische Forschungsdaten und damit um besondere Arten personenbezogener Daten i.S.d. $\int 3$ Abs. 9 BDSG handeln wird da sie Angaben über die Gesundheit des Betroffenen machen. Diese Daten hat der Gesetzgeber jedoch gerade unter einen besonderen Schutz gestellt. Daraus folgt dann aber konsequenterweise, dass bei sensiblen Daten grundsätzlich ein schutzwürdiges Interesse des Betroffenen im Sinne des $₫ 29$ Abs. 1 S. 1 Nr. 1 BDSG besteht. ${ }^{556}$ Damit scheidet eine Zulässig-

\footnotetext{
554 Hoeren in Roßnagel, 4.6. Rn. 56.

555 Gola/Schomerus, $\int 29$ Rn. 8.

556 Hoeren in Roßnagel, 4.6 Rn. 65.
} 
keit der Archivierung der Daten aufgrund von \29 BDSG selbst dann aus, wenn dessen Anwendungsbereich in den vorliegenden Fällen ausnahmsweise doch eröffnet wäre.

\section{iii. Zwischenergebnis zu a}

Die Zulässigkeit der Verarbeitung personenbezogener Daten durch die archivierende Einrichtung ist somit weder nach \28 noch nach \29 BDSG zulässig. Ferner sind auch keine anderen Vorschriften im Sinne des $\int 4$ Abs. 1 BDSG ersichtlich, die die Verarbeitung erlauben. Darüber hinaus ist zu beachten, dass die archivierende Einrichtung die personenbezogenen Daten gem. \40 Abs. 3 BDSG nur veröffentlichen dürfte, wenn der Betroffene eingewilligt hat, oder die Veröffentlichung für die Darstellung von Ereignissen der Zeitgeschichte unerlässlich wäre. Da die Zugänglichmachung der Daten für andere Forschungseinrichtungen jedoch eine solche Veröffentlichung darstellt, 557 wäre diese ohne die Einwilligung des Betroffenen wohl generell ausgeschlossen, da die Veröffentlichung von personenbezogenen Forschungsdaten in der Regel nicht zur Darstellung von Ereignissen der Zeitschichte unerlässlich ist. Folglich bedarf die archivierende Einrichtung, sofern das BDSG Anwendung findet, für die Archivierung personenbezogener Daten grundsätzlich der Einwilligung durch den Betroffenen.

b. Zulässigkeit der Archivierung aufgrund einer landesdatenschutzrechtlichen Vorschrift

Sofern die archivierende Einrichtung in den Anwendungsbereich eines Landesdatenschutzgesetzes fällt, stellt sich auch hier die Frage, ob die Datenverarbeitung unter Umständen durch eine datenschutzrechtliche Vorschrift erlaubt ist.

In den vorliegend relevanten Fällen der elektronischen Langzeitarchivierung von wissenschaftlichen Primärdaten könnte sich die Zulässigkeit der Nutzung personenbezogener Daten in diesem Zusammenhang daraus ergeben, dass es sich um die Durchführung wissenschaftlicher Forschungsvorhaben handelt. Für diese Fälle enthalten ausnahmslos alle Landesdatenschutzgesetze eine Ausnahmevorschrift. $558 \mathrm{Da}$ die Landesdatenschutzgesetze grundsätzlich die gleichen Anforderungen an die Verarbeitung personenbezogener Daten im Rahmen der wissenschaftlichen Forschung stellen, soll hier anhand der Erlaubnisnorm des $\int 25$ NDSG exemplarisch untersucht werden, ob diese Vorschrift die Langzeitarchivierung personenbezogener Daten sowie deren Zugänglichmachung an Dritte rechtfertigt. Danach sind für die Verarbeitung personenbezogener Daten zur Durchführung von wissenschaftlichen Forschungsvorhaben die $\iint$ 9-15 NDSG nach Maßgabe der Absätze 2 bis 5 des $\int 25$ NDSG anzuwenden. Somit ist zunächst zu

${ }^{557}$ Zum Veröffentlichungsbegriff siehe oben S. 133.

558 Siehe \35 LDSG B-W; Art. 23 BayDSG; \30 BlnDSG; \28 BBDSG; \19 BremDSG; \27

HmbDSG; \33 HDSG; \34 LDSG M-V; 『28 LDSG NRW; \30 DSG R-P; \30 SDSG; \ 27

DSG-LSA; \36 SächsDSG; \22 LDSG S-H; \25 ThürDSG. 
prüfen, ob die Voraussetzungen des $₫ 10$ Abs. 1 NDSG in Bezug auf die zu archivierenden Daten vorliegen. Gem. $\ 10$ Abs. 1 NDSG ist das Speichern, Verändern oder Nutzen von personenbezogenen Daten zulässig, wenn es zur Erfüllung der Aufgaben der öffentlichen Stellen erforderlich ist und die Daten für diese Zwecke erhoben worden sind.

\section{iv. Erforderlichkeit der Datenarchivierung}

Damit stellt sich zunächst die Frage, ob die Archivierung von personenbezogenen Forschungsdaten zur Erfüllung der Aufgaben einer Universität oder einer anderen Forschungseinrichtung erforderlich ist. Sofern dies nicht der Fall ist und der Forschungszweck dies gestattet, sind die Merkmale, mit denen ein Personenbezug hergestellt werden kann, gem. \25 Abs. 4 NDSG ${ }^{559}$ zu löschen, so dass in diesem Fall schon keine Archivierung personenbezogener Daten mehr vorläge. Diesbezüglich kommt es also stets auf die Aufgaben der archivierenden Einrichtung im Einzelfall an. So ergeben sich die beispielsweise die Aufgaben niedersächsischer Universitäten aus $\ 3$ des Niedersächsischen Hochschulgesetzes ${ }^{560}$ (NHG). Danach gehört es unter anderem die Pflege und Entwicklung der Wissenschaften und Künste durch Forschung, Lehre, Studium und Weiterbildung in einem freiheitlichen, demokratischen und sozialen Rechtsstaat zu ihren Aufgaben. Des Weiteren sollen die Hochschulen gem. \3 Abs.1 S.1 Nr. 4 NHG den Wissens- und Technologietransfer zu fördern. Dazu gehören auch das Erheben und die Archivierung von Forschungsdaten. Auch die Bereitstellung solcher Daten für Fremdforscher ist erforderlich, um den Wissenstransfer mit anderen Forschungseinrichtungen zu fördern.

In diesem Zusammenhang ist es teilweise auch nicht möglich, diese Daten zu anonymisieren, weshalb eine Archivierung in nicht-anonymisierter Form durchaus erforderlich sein kann. Allerdings wird dies wohl nicht den Regelfall darstellen, so dass im Einzelfall genau geprüft werden muss, ob nicht die Archivierung anonymisierter oder zumindest pseudonymisierter Daten für den jeweiligen Forschungszweck genügt. Grundsätzlich wird die Archivierung personenbezogener Daten jedoch nicht erforderlich sein, sondern eine Archivierung ohne die Merkmale genügen, mit denen ein Personenbezug hergestellt werden kann.

\footnotetext{
${ }^{559}$ Kommentar zum NDSG, $\ 25$ Zu Abs. 4; Ebenso die Vorschriften der übrigen Landesdatenschutzgesetze. Siehe \35 Abs. 1 LDSG B-W; Art. 23 Abs. 1, Abs. 3 S. 1 BayDSG; \30 Abs. 1 Nr. 2, Abs. 2 S. 1 BlnDSG; $\ 28$ Abs. 2 BBDSG; \19 Abs. 3 Hs. 1 BremDSG; 27 Abs. 1 S. 1 , Abs. 3 S. 1 HmbDSG; \33 Abs. 2 HDSG; \ 34 Abs. 1 S. 1, 2 LDSG M-V; 28 Abs. 1 S. 1, 2, Abs. 2 LDSG NRW; \ 30 Abs. 3 S. 1 LDSG R-P; \30 Abs. 4 S. 1 SDSG; \27 Abs. 3 S. 1 DSGLSA; \36 Abs. 1 S. 1 Hs. 1 SächsDSG; \22 Abs. 4 LDSG S-H; \25 Abs. 3 S. 1 ThürDSG. 560 (Nds. GVBl. S. 286 - VORIS 22210 -) zuletzt geändert durch Artikel 1 des Gesetzes vom 21. November 2006 (Nds. GVBl. S.538).
} 
v. Erhebung der Daten zum gleichen Zweck

Darüber hinaus müssten die Daten aber gem. \10 Abs. 1 S.1 NDSG561 auch zu diesem Zweck erhoben worden sein. Der Zweck der Datenverarbeitung erfolgt dabei aus der jeweiligen Fachaufgabe. ${ }^{562}$ Die im Rahmen von Forschungsvorhaben gewonnenen Daten werden zu dem Zweck erhoben, die Forschung zu fördern und in diesem Zusammenhang die Daten auch zu speichern bzw. auf lange Sicht $\mathrm{zu}$ archivieren. Dennoch ist dies nicht zwangsläufig so. Es sind ebenso Fälle denkbar, in denen nicht die wissenschaftliche Forschung Zweck der Erhebung war, sondern eine Forschungsstelle sich erst nachträglich der Daten bedient und diese für ihre wissenschaftliche Arbeit nutzt. Denkbar ist auch, dass es sich bei der archivierenden und der datenerhebenden Forschungsstelle um zwei unterschiedliche Einrichtungen bzw. unterschiedliche Forschungsvorhaben handelt. In diesen Fällen bedürfte es wiederum gem. $\int 10$ Abs.2 Nr.1 NDSG für die Datenverarbeitung der Einwilligung des Betroffenen, sofern die Voraussetzungen der Nr. 2 oder Nr. 3 nicht vorlägen.

Dies gilt jedoch nur, wenn der Zweck, zu dem die Daten archiviert werden, der wissenschaftlichen Forschung dient. Sollen hingegen Daten, die zum Zwecke der wissenschaftlichen Forschung erhoben wurden, für andere Zwecke als dem der wissenschaftlichen Forschung verarbeitet werden, fände $\int 10$ Abs. 2 Nr. 2, 3 hingegen keine Anwendung, da gem. \ 25 Abs. 3 NDSG die in den vorliegenden Fällen für wissenschaftliche Zwecke erlangten Daten nur für Zwecke der wissenschaftlichen Forschung verarbeiteten werden dürfen. Sollten die Daten also für einen anderen Zweck als dem der wissenschaftlichen Forschung archiviert oder übermittelt werden, kämen aufgrund der endgültigen Zweckbindung des $\ 25$ Abs. 3 NDSG die Tatbestände des $\int 10$ Abs. 2 Nr. 2 und 3 nicht in Betracht, 563 es bedürfte damit auf jeden Fall der Einwilligung des Betroffenen.

In den Fällen hingegen, in denen die Daten zwar zum Zwecke der wissenschaftlichen Forschung verarbeitet werden sollen, jedoch $\mathrm{zu}$ einem anderen Zweck oder einem anderen Forschungsvorhaben erhoben worden sind, würde sich die Zulässigkeit er Verarbeitung hingegen nach \25 Abs. 2 NDSG richten, wonach die Verarbeitung in diesen Fällen nur erlaubt dann erlaubt wäre, wenn der Betroffene eingewilligt hat, eine Rechtsvorschrift dies vorsieht oder Art und Verarbeitung der Daten darauf schließen lassen, dass ein schutzwürdiges Interesse der Betroffenen der Verarbeitung der Daten für das Forschungsvorhaben nicht entgegensteht oder das öffentliche Interesse an der Durchführung des Forschungsvor-

\footnotetext{
561 So auch $₫ 35$ Abs. 1 S. 3 LDSG B-W; Art. 23 Abs. 1 BayDSG; \30 Abs. 3 S. 1 BlnDSG; $₫ 28$

Abs. 1 S. 1 BBDSG; $\$ 19$ Abs. 4 BremDSG; $₫ 27$ Abs. 1 S. 1 Hs. 1 HmbDSG; $₫ 33$ Abs. 3 S. 1 HDSG; \ 34 Abs. 4 LDSG M-V; \28 Abs. 2 Hs. 1 LDSG-NRW; \ 30 Abs. 1 LDSG R-P; \ 30 Abs. 1 S. 1 SDSG; $\ 27$ Abs. 1 DSG-LSA; \36 Abs. 3 SächsDSG; \22 Abs. 1 LDSG S-H; \25 Abs. 1 ThürDSG.

562 Kommentar zum NDSG, \ 10 Zu Abs.1 Nr. 2.

563 Kommentar zum NDSG, \$ 25 Zu Abs.4 Nr. 19.
} 
habens das schutzwürdige Interesse der Betroffenen erheblich überwiegt. Dabei wird grundsätzlich keine Rechtsvorschrift im Sinne von \25 Abs.2 Nr.2 NDSG einschlägig sein, weshalb im Einzelfall eine Abwägung gem. \ 25 Abs. 2 Nr. 3 NDSG getroffen werden muss. Nur wenn im Rahmen dieser Abwägung das Interesse der Betroffenen der Bereitstellung nicht entgegensteht oder das öffentliche Interesse an der Bereitstellung das schutzwürdige Interesse des Betroffenen erheblich überwiegt, wäre die Verarbeitung für andere Zwecke zulässig. Auch im Falle des $₫ 25$ Abs. 2 NDSG ist die Datenverwendung gem. \25 Abs. 3 NDSG allerdings wiederum nur für Zwecke der wissenschaftlichen Forschung zulässig ist. Die elektronische Langzeitarchivierung wissenschaftlicher Primärdaten wird dabei jedoch grundsätzlich zu diesem Zweck erfolgen.

$\mathrm{Zu}$ beachten ist ferner, dass $\ 25$ Abs. 4 NDSG auch hinsichtlich der bereits archivierten Daten Anwendung findet. Aus diesem Grund sind die Merkmale, mit deren Hilfe ein Bezug auf eine bestimmte natürliche Person hergestellt werden kann, gesondert zu speichern, sobald der Stand des Forschungsvorhabens es gestattet. ${ }^{564}$ Dabei darf eine Reanonymisierung nur mit einem unverhältnismäßig großen Aufwand an Zeit, Kosten und Arbeitskraft einer bestimmten oder bestimmbaren natürlichen Person möglich sein. ${ }^{565}$ Des Weiteren sind die zur Identifizierung des Betroffenen geeigneten Merkmale gem. Abs. 4 zu löschen, sobald der Forschungszweck dies zulässt. ${ }^{566}$

\section{c. Zwischenergebnis}

Somit ist die Speicherung und Übermittlung von Forschungsdaten gem. \10 Abs. 1 S. 1 NDSG grundsätzlich auch ohne Einwilligung des Betroffenen möglich. Die Tatbestandsvoraussetzungen der Norm sind jedoch unter Umständen dann nicht einschlägig, wenn die Daten entweder zu einem anderen Zweck erhoben worden sind als dem der wissenschaftlichen Forschung oder im Rahmen eines anderen Forschungsvorhabens gewonnen wurden. Da dies im Einzelfall teilweise nur

564 So auch $₫ 35$ Abs. 2 S. 1 LDSG B-W; Art. 23 Abs. 3 S. 1 BayDSG; \ 30 Abs. 2 BlnDSG; $₫ 28$ Abs. 2 S. 1 BBDSG; $\mathbb{1} 19$ Abs. 3 BremDSG; \27 Abs. 3 S. 1 HmbDSG; $₫ 33$ Abs. 2 S. 1 HDSG; \34 Abs. 2 S. 7 LDSG M-V; \28 Abs. 3 S. 1 LDSG-NRW; \30 Abs. 3 S. 1 LDSG R-P; \30 Abs. 4 S. 1 SDSG; \27 Abs. 3 S. 1 DSG-LSA; \36 Abs. 2 Hs. 2 SächsDSG; 22 Abs. 5 S. 1 LDSG S-H; $₫ 25$ Abs. 3 S. 1 ThürDSG.

${ }^{565}$ Kommentar zum NDSG, Zu $\ 25$, Zu Abs. 4 Nr. 19; siehe auch die Legaldefinition in $\ 6$ Abs. 3 BDSG; siehe auch Hinweise zum ThürDSG, Zu \3,3.9.

${ }^{566}$ So auch $₫ 35$ Abs. 2 S. 1 LDSG B-W; Art. 23 Abs. 3 S. 1 BayDSG; $₫ 30$ Abs. 2 BlnDSG (der jedoch eine endgültige Löschung der Identifikationsmerkmale erst nach Erreichen des Forschungszwecks verlangt und nicht, sobald dieser es zulässt); \28 Abs. 2 S. 1 BBDSG; \19 Abs. 3 BremDSG (auch hier wird eine Löschung erst nach Erreichen des Forschungszwecks verlangt); $₫ 27$ Abs. 3 S. 1 HmbDSG; $₫ 33$ Abs. 2 S. 1 HDSG (mit dem gleichen Unterschied wie beim BBDSG und dem BremDSG); \34 Abs. 2 S. 7 LDSG M-V; \28 Abs. 3 S. 1 LDSG-NRW; \30 Abs. 3 S. 1 LDSG R-P; \30 Abs. 4 S. 1 SDSG; \27 Abs. 3 S. 1 DSG-LSA; \36 Abs. 2 Hs. 2 SächsDSG; $₫ 22$ Abs. 5 S. 1 LDSG S-H; $₫ 25$ Abs. 3 S. 1 ThürDSG. 
schwer zu bestimmen sein wird, sei auch hier wieder angemerkt, dass aus diesem Grund stets die Einwilligung des Betroffenen eingeholt werden sollte, da die Datenverarbeitung dann bereits nach $\int 4$ Abs. 1 Nr. 2 NDSG bzw. \25 Abs. 2 Nr. 1 zulässig wäre. Des Weiteren ist zu beachten, dass die Daten unter den Voraussetzungen des \25 Abs. 4 NDSG bzw. der vergleichbaren Vorschriften der übrigen Landesdatenschutzgesetze ${ }^{567}$ gegebenenfalls anonymisiert werde müssen. Die Erlaubnisnormen der Landesdatenschutzgesetze greifen darüber hinaus auch im Anwendungsbereich des BDSG. 568

Die Verwertung personenbezogener Daten kann also im Anwendungsbereich der Landesdatenschutzgesetze im Gegensatz zu dem des BDSG, grundsätzlich gesetzlich erlaubt sein. Da die archivierende Einrichtung jedoch die Beweislast für das Vorliegen der Tatbestandsvoraussetzungen der Erlaubnisnorm trifft und eine solche auch nicht in allen Fällen einschlägig ist, sollte stets die Einwilligung des Betroffenen eingeholt werden. Darüber hinaus spricht für eine solche Vorgehensweise, dass selbst im Falle einer gesetzlichen Erlaubnis der Datenverwertung, die archivierende Einrichtung ihre Datenbestände ständig dahingehend prüfen müsste, ob der jeweilige Stand der Forschung es bei einzelnen Datensätzen zulieBe, dass die Merkmale, mit denen ein Personenbezug hergestellt werden kann, gesondert gespeichert oder sogar gelöscht werden. Bedenkt man den Umfang des Datenvolumens, der gerade bei Universitäten anfallen wird, ist ein solcher administrativer Aufwand von den archivierenden Einrichtungen aber wohl kaum zu bewältigen.

\section{Einschränkung durch das SGB X}

Neben den rechtlichen Beschränkungen hinsichtlich des Inhalts der zu archivierenden Daten durch das BDSG kommt ferner eine Anwendung der Vorschriften des SGB X in Betracht. Das Speichern von Sozialdaten ist gem. \67b Abs. 1 SGB $\mathrm{X}$ zulässig, soweit dies durch eine Vorschrift des SGB X oder eine andere Rechtsvorschrift erlaubt oder vorgeschrieben ist oder der Betroffene eingewilligt hat.

\section{Anwendbarkeit des $\int 67 b$ SGB X auf die vorliegenden Fälle}

Dazu müsste es sich bei den Forschungsdaten gem. \67b SGB X zunächst um Sozialdaten im Sinne des SGB X handeln. Diese definiert das Gesetz in $\int 67$ Abs. 1 S. 1 SGB X als „Einzelangaben über persönliche oder sachliche Verhältnisse einer bestimmten oder bestimmbaren natürlichen Person (Betroffener), die von einer in $\int 35$ des Ersten Buches genannten Stelle im Hinblick auf ihre Aufgaben nach diesem Gesetzbuch erhoben, verarbeitet oder genutzt werden“. Zwar wird es sich bei den Forschungsangaben mit Personenbezug teilweise um Einzelangaben über persönliche oder sachliche Verhältnisse einer bestimmten oder bestimmba-

567 Siehe die Angaben in Fn. 566.

568 Gola/ Schomerus, \ 4 Rn. 7. 
ren Person handeln. Fraglich ist jedoch, ob die archivierende Einrichtung, die im Einzelfall diese Daten erhebt, eine in $\$ 35$ SGB X genannte Stelle ist. Dabei kommen vorliegend grundsätzlich weder die in den $\$ \subseteq 18-29$ SGB I genannten Leistungsträger noch die in $\ 35$ SGB I genannten sonstigen Stellen in Betracht. Eine Ausnahme könnte sich jedoch dann ergeben, wenn Leistungen im Rahmen eines Forschungsprojektes in Verbindung mit der gesetzlichen Krankenversicherung erbracht werden. Letztere sind nämlich gem. \21 i.V.m. \12 SGB I Leistungsträger im Sinne des $₫ 35$ SGB I. Damit fallen sie in den Anwendungsbereich des $\$ 67b SGB X. Sofern also Daten archiviert werden sollen, die im Rahmen von Leistungen der gesetzlichen Krankenversicherungen erhoben wurden, kann es sich dabei durchaus um Sozialdaten handeln. Damit wäre das Speichern dieser Daten gem. \67b Abs. 1 SGB X in einem Langzeitarchiv nur dann zulässig, wenn eine Rechtsvorschrift dies erlauben würde oder der Betroffene in die Verarbeitung eingewilligt hat.

\section{Zulässigkeit der Archivierung aufgrund der Erlaubnisnorm des \67c SGBX}

Als Erlaubnisnorm könnte in diesen Fällen allerdings \ 67 c SGB X greifen. Die Zulässigkeit der Speicherung könne sich dabei entweder aus $\$ 67c Abs. 1 oder Abs. 2 SGB X ergeben.

\section{a. Zulässigkeit nach $\ 67 \mathrm{c}$ Abs. 1 SGB X}

Das Speichern, Verändern oder Nutzen der Sozialdaten wäre gem. \67c Abs. 1 SGB X zulässig, wenn es zur Erfüllung der in der Zuständigkeit der verantwortlichen Stelle liegenden gesetzlichen Aufgaben nach SGB X erforderlich wäre für die Zwecke erfolgen würde, für die die Daten erhoben wurden. Dabei ist zu beachten, dass Adressat der Norm die jeweilige gesetzliche Krankenkasse ist, die bei der Erhebung der Daten Leistungen erbracht hat. Die Archivierung fällt aber nicht zwangsläufig in deren gesetzliche Aufgaben. Des Weiteren müsste die Speicherung gem. \67 Abs. 1 SGB X auch zu dem Zweck erfolgen, zu dem sie erhoben wurden. Zweck der Erhebung von Daten durch die gesetzlichen Krankenversicherungen wird jedoch in der Regel nicht die Archivierung dieser Daten und Zugänglichmachung für Fremdforscher sein. Damit scheidet eine Zulässigkeit der elektronischen Langzeitarchivierung von Sozialdaten nach $\ 67 \mathrm{c}$ Abs. 1 SGB X aus.

\section{b. Zulässigkeit nach $\ 67 \mathrm{c}$ Abs. 2 SGB X}

Als Erlaubnisnorm käme außerdem $₫ 67$ Abs. 2 SGB X in Betracht. Danach dürfen Sozialdaten von der Stelle, die diese erhoben hat, für andere Zwecke gespeichert, verändert oder genutzt werden, wenn die Daten für die Erfüllung von Aufgaben nach anderen Rechtsvorschriften des SGB X erforderlich sind als denjenigen, für die sie erhoben wurden, der Betroffene im Einzelfall eingewilligt hat oder es zur Durchführung eines bestimmten Vorhabens der wissenschaftlichen For- 
schung oder Planung im Sozialleistungsbereich erforderlich ist und die Voraussetzungen des $\ 75$ Abs. 1 SGB X vorliegen. Zum einen ist jedoch keine andere Rechtsvorschrift aus dem SGB X ersichtlich, zu deren Erfüllung es der Speicherung der Sozialdaten in einem Langzeitarchiv bedarf. Zum anderen ist die Langzeitarchivierung wissenschaftlicher Primärdaten auch nicht zur Durchführung eines bestimmten Vorhabens der wissenschaftlichen Forschung oder Planung im Sozialleistungsbereich erforderlich. Somit blieb auch hier nur die Möglichkeit einer Einwilligung durch den Betroffenen.

c. Anforderungen an eine Einwilligung gem. \67b SGB X

Wie bereits oben festgestellt, ist die Einholung einer Einwilligung beim Betroffenen schon aufgrund der Vorschriften des BDSG und der Landesdatenschutzgesetze anzuraten. Damit stellt sich jedoch die Frage, ob an die Wirksamkeit einer Einwilligung im Rahmen des $\$ 67b SGB X strengere Anforderungen zu stellen sind, als im Rahmen der Datenschutzgesetze. Die Voraussetzungen einer wirksamen Einwilligung sind dabei in $\ 67 \mathrm{~b}$ Abs. 2 SGB X geregelt. Danach ist der Betroffene auf den Zweck der vorgesehenen Verarbeitung oder Nutzung sowie auf die Folgen der Verweigerung der Einwilligung hinzuweisen.

Die Einwilligung des Betroffenen ist gem. 2 SGB X nur wirksam, wenn sie auf dessen freier Entscheidung beruht. Schließlich bedürfen die Einwilligung und der Hinweis gem. Satz 3 der Schriftform, soweit nicht wegen besonderer Umstände eine andere Form angemessen ist. Soll die Einwilligung zusammen mit anderen Erklärungen schriftlich erteilt werden, so ist die Einwilligungserklärung gem. \67b Abs. 2 S. 4 SGB X im äußeren Erscheinungsbild der Erklärung hervorzuheben. Demnach ergeben sich hinsichtlich der Anforderungen an die Einwilligungen im Rahmen des SGB X keine strengeren Anforderungen als im Rahmen der Datenschutzgesetze. Sofern also eine nach den Vorschriften des BDSG oder der Landesdatenschutzgesetze wirksame Einwilligung ${ }^{569}$ des Betroffenen vorliegt, ist die Speicherung auch gem. \67b Abs.1 SGB X zulässig, sofern dieser im Einzelfall Anwendung finden sollte.

\section{d. Zwischenergebnis zu b}

Die Zulässigkeit der Speicherung von Sozialdaten, die im Rahmen von wissenschaftlichen Forschungsprojekten anfallen, ergibt sich aus $\ 67$ c SGBX. Eine andere Rechtsvorschrift, aus der die Zulässigkeit der Speicherung von Sozialdaten in einem elektronischen Langzeitarchiv hervorginge, ist ebenfalls nicht ersichtlich. Demzufolge wäre auch im Anwendungsbereich des SGB X die Speicherung der Daten nur zulässig, wenn die Betroffenenihre Einwilligung erteilt hätten. Bezüglich der inhaltlichen und formalen Anforderungen gilt dabei das Gleiche wie im Rahmen der Datenschutzgesetze.

${ }^{569}$ Siehe dazu die Ausführungen oben auf S.145. 


\section{Entbindung des Arztes von seiner Schweigepflicht}

Sofern der Betroffene in die Nutzung seiner Daten eingewilligt hat, stellt sich weiter die Frage, ob er dadurch den Arzt, der die Untersuchungen im Rahmen des Forschungsvorhabens durchgeführt hat, von der zivilrechtlich begründeten ärztlichen Schweigepflicht entbunden hat oder ob es dazu einer weiteren Einwilligung bedarf. Dabei ist zunächst festzuhalten, dass ein Vorrang der datenschutzrechtlichen Regelungen vor der ärztlichen Schweigepflicht nicht besteht, sondern diese vielmehr parallel nebeneinander bestehen. ${ }^{570}$ Der Arzt darf die Daten seiner Patienten preisgeben, sofern sich der Proband damit einverstanden erklärt. Dabei kann der Proband nicht nur über das „Ob“ sondern auch das „Wie“ der Weitergabe seiner Daten entscheiden. ${ }^{571}$ Fraglich ist, ob in der Einwilligung nach $\ 4$ Abs. 1 BDSG bzw. \4 Abs. 1 NDSG gleichzeitig eine Entbindung des Arztes von seiner Schweigepflicht zu sehen ist. Im Gegensatz zur Einwilligung im Rahmen des Datenschutzrechtes muss die Aufhebung der Schweigepflicht nicht ausdrücklich erfolgen und bedarf außerdem keiner bestimmten Form. Eine konkludente Einwilligung oder schlüssiges Verhalten können damit genügen. ${ }^{572}$ Damit stellen sich an die Aufhebung der ärztlichen Schweigepflicht deutlich geringere Anforderungen als im Rahmen des NDSG und des BDSG. Damit ist davon auszugehen, dass der Proband den Arzt, der die Untersuchung bzw. das Forschungsvorhaben leitet, konkludent durch Einwilligung in die Nutzung seiner personenbezogenen Daten von dessen Schweigepflicht entbindet. Dabei reicht diese Entbindung so weit, wie auch die Einwilligung in die datenschutzrechtlich relevante Nutzung der Daten erfolgt ist.

\section{Ergebnis}

Wie gezeigt, wird der Inhalt von personenbezogenen Forschungsdaten, die in einem elektronischen Langzeitarchiv abgelegt werden sollen, vor allem durch das BDSG, die Landesdatenschutzgesetze sowie im Einzelfall auch durch die Vorschriften des SGB X rechtlich eingeschränkt, was gerade im Hinblick auf medizinische Forschungsdaten eine wichtige Rolle spielt. Als Erlaubnisnorm im Anwendungsbereich des NDSG kommt grundsätzlich \25 Abs. 1, 2 in Verbindung mit $\int 10$ Abs. 1 NDSG in Betracht, wobei im Einzelfall jedoch stets geprüft werden muss, ob die Archivierung und Bereitstellung der Daten für Fremdforscher auch Zweck der Erhebung der Daten war. Die Erlaubnisnormen des BDSG und des SGB X werden jedoch im Regelfall nicht vorliegen, weshalb stets eine Einwilligung des Betroffenen eingeholt werden sollte. Sofern eine solche vorliegt, ist die Speicherung der personenbezogenen Daten in einem elektronischen Langzeitarchiv, sowie die Bereitstellung der Daten für andere Forscher zulässig, sofern sich

\footnotetext{
${ }^{570}$ Schirmer in Roßnagel, 7.12 Rn. 26.

571 Schirmer in Roßnagel, 7.12 Rn. 41.

${ }^{572}$ Schirmer in Roßnagel, 7.12 Rn. 42.
} 
die Einwilligung ausdrücklich hierauf bezieht. Dabei stellen die oben genannten Vorschriften an die Wirksamkeit der Einwilligung die gleichen Anforderungen. Die Einholung einer Einwilligungserklärung hat dabei außerdem den Vorteil, dass sie in der Regel weitreichender ist und der archivierenden Einrichtung damit mehr Befugnisse einräumt als ein gesetzlicher Erlaubnistatbestand. Darüber hinaus ist eine Einwilligung aber vor allen aus Gründen der Beweissicherheit für die an der Archivierung beteiligten Stellen vorteilhaft und daher in jedem Fall anzuraten.

\section{Technische und organisatorische Zugangssicherungsmaßnahmen bei der Langezeitarchivierung personenbezogener Daten}

Neben der Frage bezüglich der rechtlichen Zulässigkeit der Archivierung von wissenschaftlichen Primärdaten mit Personenbezug, ist ferner entscheidend, welche technischen Maßnahmen die archivierende Einrichtung ergreifen muss. Dies ist insbesondere im Hinblick auf etwaige Schadensersatzansprüche entscheidend, die gegen die archivierende Einrichtung entstehen können.

I. Gesetzliche Vorgaben zur Anonymisierung und Pseudonymisierung

Relevant ist dabei vor allem die Frage, inwieweit eine gesetzliche Pflicht für die archivierende Einrichtung besteht, Merkmale gesondert zu speichern, mit denen ein Personenbezug zu den Daten hergestellt werden kann (dazu1.). Sofern dies der Fall ist, stellt sich ferner die Frage, ob diese Merkmale von der archivierenden Einrichtung selbst gespeichert werden dürfen oder ob dies von einer außenstehenden Einrichtung vorgenommen werden muss (dazu 2.)

\section{Pflicht zur gesonderten Speicherung von Merkmalen, anband derer ein Personenbezug hergestellt werden kann}

Werden personenbezogene Daten zur Durchführung von wissenschaftlichen Forschungsvorhaben verarbeitet, so sind gem. $\int \S 3$ a S. 2, 40 Abs. 2 BDSG die Merkmale zu löschen, mit deren Hilfe ein Bezug auf eine bestimmte natürliche Person hergestellt werden kann, sobald der Forschungszweck dies zulässt. ${ }^{573}$ Sofern dies nicht möglich ist, sind diejenigen Merkmale gesondert zu speichern, mit denen Einzelangaben über persönliche oder sachliche Verhältnisse einer bestimmten oder bestimmbaren Person zugeordnet werden können. Gleiches folgt auf landesrechtlicher Ebene aus $\ 25$ Abs. 4 Hs. 2 NDSG, ${ }^{574}$ wonach die Merkmale, mit

\footnotetext{
573 Scholz in Simitis, $₫ 3$ a Rn. 45.

574 Ebenso die übrigen Landesdatenschutzgesetze: Siehe \35 Abs. 2 S. 1, 2 LDSG B-W; \23 Abs. 3

S. 1, 2 BayDSG; $₫ 30$ Abs. 2 BlnDSG; $₫ 28$ Abs. 2 S. 1, 2 BBDSG; \19 Abs. 3 BremDSG; $\ 27$

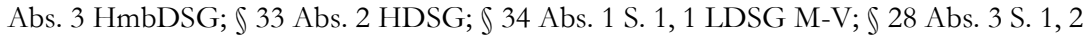


denen ein Personenbezug zu den einzelnen Daten hergestellt werden kann, gesondert zu speichern sind, sobald der Stand des Forschungsvorhabens dies gestattet. Dabei geht aus den genannten Vorschriften allerdings nicht hervor, in welcher Form diese Merkmale gespeichert werden müssen. In diesem Zusammenhang stellt sich jedoch insbesondere die Frage, ob die archivierende Einrichtung die besagten Merkmale selbst speichern darf oder ob dies durch einen Dritten geschehen muss.

\section{Pflicht zur separaten Speicherung aufgrund teleologischer Auslegung}

Eine solche Pflicht zur „externen“ Speicherung der zur Identifikation geeigneten Merkmale könnte sich jedoch unter Umständen im Rahmen einer teleologischen Auslegung aus dem Sinn und Zweck von $₫ 40$ Abs. 2 BDSG bzw. \25 Abs. 4 NDSG ergeben.

Sowohl $₫ 40$ Abs. 2 BDSG als auch $₫ 25$ Abs. 4 NDSG konkretisieren den „Grundsatz der Erforderlichkeit“ im Datenschutzrecht, wonach die Speicherung personenbezogener Daten nur zulässig ist, solange und soweit anonymisierte Daten für den Zweck des Vorhabens nicht ausreichen bzw. der angestrebte Zweck ohne die Erhebung oder Speicherung personenbezogener Daten nicht erreicht werden kann. ${ }^{575}$ Der Grundgedanke ist somit, dass eine Zuordnung der Daten zu dem jeweiligen Betroffenen verhindert werden soll, weshalb die zur Identifizierung dienenden Merkmale nach Möglichkeit gelöscht werden müssen. Da die Möglichkeit der Identifizierung der Betroffenen jedoch gerade bei medizinischen Langzeitprojekten oftmals unumgänglich ist, hat die Löschung im Rahmen der Verarbeitung zu wissenschaftlichen Zwecken gem. \40 Abs. 2 BDSG bzw. auf landesrechtlicher Ebene $\ 25$ Abs. 4 NDSG erst dann zu erfolgen, wenn der Forschungszweck dies zulässt. Bis zu dieser Anonymisierung sind die zur Identifizierung erforderlichen Merkmale allerdings gesondert zu speichern. Hintergrund des \25 Abs. 4 Hs. 1 NDSG ist es also, dass auch in den Fällen, in denen die zur Identifizierung dienenden Merkmale nicht gelöscht werden können, die im Forschungsbetrieb verwendeten Daten trotzdem keinen Rückschluss auf ihren Inhaber zulassen und die Betroffenen damit vor den Gefahren geschützt werden, die sich aus einer langen Aufbewahrung ergeben können. ${ }^{576}$ Aus diesem Grund sind die Daten aufzuspalten und ihr personenbezogener Teil gesondert zu speichern.

Dieser Zweck kann grundsätzlich auch dann erreicht werden, wenn die personenbezogenen Merkmale von der gleichen Einrichtung gespeichert werden, wie der nichtpersonenbezogene Teil, solange sichergestellt ist, dass Unbefugte nicht

LDSG-NRW; \30 Abs. 3 S. 1, 2 LDSG R-P; \30 Abs. 4 SDSG; \ 27 Abs. 3 S. 1, 2 DSG-LSA; 』 36 Abs. 2 SächsDSG; \ 22 Abs. 1, Abs. 5 LDSG S-H; \ 25 Abs. 3 S. 1, 2 ThürDSG.

575 Kommentar zum NDSG, \ 25 Zu Abs. 4 Nr. 19.; Gola/ Schomerus, \ 3a Rn. 4; Roßnagel, DuD 1999, 253; siehe auch den Bericht der Bundesregierung zur Umsetzung des IuKDG, BT-Drucks.

14/1191, S. 13.

576 Gola/Schomerus, \40 Rn. 14; Simitis in Simitis, BDSG, \40 Rn. 74. 
an die Merkmale gelangen können. Demzufolge spricht auch der Sinn und Zweck von \25 Abs. 4 NDSG grundsätzlich nicht gegen eine Speicherung der Merkmale, mit denen ein Personenbezug hergestellt werden kann, durch die archivierende Einrichtung selbst. Es kommt also vielmehr darauf an, dass die jeweilige Einrichtung die erforderlichen Maßnahmen trifft, um zu verhindern, dass Unbefugte Zugriff auf die zur Identifizierung der Betroffenen erforderlichen Merkmale erlangen..$^{57}$

\section{Zwischenergebnis}

Sowohl der Wortlaut als auch der Sinn und Zweck von \40 Abs. 2 BDSG sowie der Landesdatenschutzgesetze, wie dem hier als Beispiel gewählten $\ 25$ Abs. 4 NDSG sprechen demzufolge nicht dagegen, dass die personenbezogenen Daten sowie die zur Zuordnung erforderlichen Merkmale durch dieselbe Einrichtung erfolgt.

\section{Organisationspflichten der archivierenden Einrichtung}

Die archivierenden Einrichtungen haben sowohl im Rahmen des BDSG ${ }^{578}$ als auch im Rahmen der Landesdatenschutzgesetze die erforderlichen technischen und organisatorischen Maßnahmen zu ergreifen, um eine rechtmäßige Verarbeitung der Daten sicherzustellen. Aus diesem Grund sind in diesem Zusammenhang vor allem die Vorschriften des 99 S. 1 i.V.m. der Anlage zu \9 S. 1 BDSG sowie $\$ 7 Abs. 2 NDSG zu beachten, die spezielle Maßnahmen für automatisierte Verfahren vorschreiben und auf die im Folgenden näher eingegangen werden soll.

Die archivierende Einrichtung hat gem. \9 BDSG die technischen und organisatorischen Maßnahmen zu treffen, die erforderlich sind, um die Ausführung der Vorschriften des BDSG, insbesondere die in der Anlage zum BDSG genannten Anforderungen, zu gewährleisten. Erforderlich sind Maßnahmen gem. \9 S. 2 BDSG in diesem Zusammenhang jedoch nur, wenn ihr Aufwand in einem angemessenen Verhältnis zu dem angestrebten Schutzzweck steht. Welche konkreten Maßnahmen dies sind, besagt die Vorschrift hingegen nicht. Das Bundesdatenschutzgesetz enthält insoweit jedoch eine Reihe von Vorschriften, die die vorzunehmenden Maßnahmen konkretisieren. So haben öffentliche und nicht öffentliche Stellen, die personenbezogene Daten verarbeiten, gem. \4f Abs. 1 BDSG schriftlich einen Datenschutzbeauftragten zu bestellen. Dieser hat gem. \4g Abs. 1 BDSG auf die Einhaltung der Vorschriften des BDSG und anderer Vorschriften über den Datenschutz hinzuwirken. Unabhängig von der Langzeitarchivierung von Forschungsdaten wird ein solcher Datenschutzbeauftragter in den jeweiligen Einrichtungen, die die Archivierung vornehmen bzw. in deren Auftrag diese erfolgt, regelmäßig schon bestellt worden sein, da diese Stellen bereits vorher per-

577 Worin diese Maßnahmen bestehen können, dazu siehe S. 165.

578 Siehe $₫ 9$ BDSG. 
sonenbezogene Datenverarbeitet haben werden, wie etwa Arbeitnehmerdaten. Eine weitere organisatorische Maßnahme findet sich in $\int 5$ BDSG, wonach es den bei der Datenverarbeitung beschäftigten Personen untersagt ist, personenbezogene Daten unbefugt zu erheben, zu verarbeiten oder zu nutzen. Sind die Personen bei einer nicht-öffentlichen Stelle angestellt, sind sie bei der Aufnahme ihrer Tätigkeit auf das Datengeheimnis zu verpflichten. Demzufolge werden durch $₫ 5$ S. 1 BDSG die Angestellten selbst, durch \5 S. 2 BDSG hingegen die archivierende Stelle selbst verpflichtet. ${ }^{579} \mathrm{Zu}$ den Angestellten i.S.d. \5 BDSG zählen dabei neben Arbeitnehmern auch Praktikanten oder freie Mitarbeiter sowie Mitarbeiter, die im Rahmen der Auftragsdatenverarbeitung Daten verarbeiten. ${ }^{580}$ Sofern die archivierende Einrichtung die Archivierung teilweise oder insgesamt durch Dritte vornehmen lässt, und diese damit Zugang zu personenbezogenen Daten erhalten, muss sie demzufolge entsprechende Vereinbarungen ${ }^{581}$ mit ihrem Auftragnehmer treffen. Weitere zu treffende Maßnahmen sind in der Anlage zu \9 S. 1 BDSG aufgeführt. Danach hat die archivierende Einrichtung „Maßnahmen zu treffen, die je nach der Art der zu schützenden personenbezogenen Daten oder Datenkategorien geeignet sind,

1. Unbefugten den Zutritt zu Datenverarbeitungsanlagen, mit denen personenbezogene Daten verarbeitet oder genutzt werden, zu verwehren (Zutrittskontrolle),

2. zu verhindern, dass Datenverarbeitungssysteme von Unbefugten genutzt werden können (Zugangskontrolle),

3. zu gewährleisten, dass die zur Benutzung eines Datenverarbeitungssystems Berechtigten ausschließlich auf die ihrer Zugriffsberechtigung unterliegenden Daten zugreifen können, und dass personenbezogene Daten bei der Verarbeitung, Nutzung und nach der Speicherung nicht unbefugt gelesen, kopiert, verändert oder entfernt werden können (Zugriffskontrolle),

4. zu gewährleisten, dass personenbezogene Daten bei der elektronischen Übertragung oder während ihres Transports oder ihrer Speicherung auf Datenträger nicht unbefugt gelesen, kopiert, verändert oder entfernt werden können, und dass überprüft und festgestellt werden kann, an welche Stellen eine Übermittlung personenbezogener Daten durch Einrichtungen zur Datenübertragung vorgesehen ist (Weitergabekontrolle),

5. zu gewährleisten, dass nachträglich überprüft und festgestellt werden kann, ob und von wem personenbezogene Daten in Datenverarbeitungssysteme eingegeben, verändert oder entfernt worden sind (Eingabekontrolle),

\footnotetext{
579 Vgl. Gola/Schomerus, \5 Rn. 2.

580 Gola/Schomerus, $\int 5 \mathrm{Rn}, 2$.

581 Ausführlich zum Inhalt des Vertrags zwischen archivierender Einrichtung und Auftragnehmer S. 180 .
} 
6. zu gewährleisten, dass personenbezogene Daten, die im Auftrag verarbeitet werden, nur entsprechend den Weisungen des Auftraggebers verarbeitet werden können (Auftragskontrolle),

7. zu gewährleisten, dass personenbezogene Daten gegen zufällige Zerstörung oder Verlust geschützt sind (Verfügbarkeitskontrolle),

8. zu gewährleisten, dass zu unterschiedlichen Zwecken erhobene Daten getrennt verarbeitet werden können."

$\mathrm{Zu}$ beachten ist dabei jedoch, dass dieser Katalog aufgrund des ausdrücklichen Wortlautes nur auf die automatisierte Datenverarbeitung oder -nutzung Anwendung findet. Eine solche liegt gem. \3 Abs. 2 BDSG dann vor, wenn personenbezogene Daten unter Einsatz von Datenverarbeitungsanlagen erhoben, verarbeitet oder genutzt werden. Wie bereits oben festgestellt, liegt eine automatisierte Datenverarbeitung nicht schon deshalb vor, weil vorliegend elektronische Daten im Fokus der Betrachtung stehen. Trotzdem wird im Rahmen der elektronischen Langzeitarchivierung in der Regel eine solche vorliegen. ${ }^{582}$

Um den oben genannten Maßnahmenkatalog zu erfüllen, sollte die archivierende Einrichtung daher automatische Zugriffskontrollen einrichten, sowie Berechtigungsausweise und Personenkontrollen einführen, um zu verhindern, dass Unbefugte Zugang zu den EDV-Anlagen erhalten. ${ }^{583}$ Um einen Zugang Unbefugter zu den Daten an sich zu verhindern, sollte die archivierende Einrichtung ferner ihre Systeme mit Passwörtern ausstatten, die ein Eindringen in das System verhindern. ${ }^{584}$

Darüber hinaus sollte protokolliert werden, welcher Mitarbeiter zu welchem Zeitpunkt auf die Daten zugegriffen bzw. Daten in das System eingegeben hat. ${ }^{585}$ Um die Wirkung einer solchen Protokollierung zu gewährleisten, ist ferner eine regelmäßige Auswertung der Protokolle erforderlich. ${ }^{586}$ Um ein unbefugtes Kopieren, Verändern oder Lesen der Datenträger zu verhindern, sollten die gespeicherten Daten außerdem verschlüsselt werden. Des Weiteren sind konkrete Regeln hinsichtlich der Vernichtung nicht mehr benötigter Datenträger aufzustellen. ${ }^{587}$ Dies dürfte insbesondere für die Fälle relevant werden, in denen eine Anonymisierung $^{588}$ personenbezogener Daten vorgenommen wird und die Datenträger zerstört werden, auf denen die zur Identifizierung erforderlichen Merkmale gespeichert sind. Sofern ein Fall der Auftragsdatenverarbeitung vorliegt, hat der Auftragnehmer zu gewährleisten, dass die Daten nur entsprechend den Weisungen des Auftraggebers verarbeitet werden. ${ }^{589}$

\footnotetext{
582 Siehe dazu oben S. 167 f.

${ }^{583}$ Gola/Schomerus, \$ 9 Rn. 22.

584 Gola/Schomerus, \$ 9 Rn. 23.

585 Golal Schomerus, $\$ 9$ Rn. 24, 26.

586 Vgl. Gola/Schomerus, \9 Rn. 24.

587 Vgl. Gola/Schomerus, § 9 Rn. 25.

588 Siehe dazu auch S. $163 \mathrm{ff}$.

${ }^{589}$ Gola/Schomerus, $\$ 9$ Rn. 27.
} 
Der Auftraggeber wiederum hat darauf zu achten, dass die Weisungen hinsichtlich des Umgangs mit den Daten hinreichend bestimmt sind und durch Kontrollen sicher zu stellen, dass der Auftragnehmer diese auch befolgt. ${ }^{590} \mathrm{Um}$ die Daten vor einer zufälligen Zerstörung, wie etwa durch Wasser- oder Brandschäden zu verhindern, sind entsprechende Sicherungskopien anzufertigen und entsprechend zu verwahren. Weitere Maßnahmen können etwa die Installation von Notstromaggregaten und die Erstellung von Katastrophenplänen sein. ${ }^{591} \mathrm{Maß}$ nahmen nach den Landesdatenschutzgesetzen.

Auch die Landesdatenschutzgesetze sehen bei automatisierten Datenverarbeitungsverfahren umfangreiche technische und organisatorische Maßnahmen vor, die die archivierende Einrichtung zu ergreifen hat. So hat die Einrichtung gem. $\int 7$ Abs. 1 NDSG ${ }^{592}$ die technischen und organisatorischen Maßnahmen zu treffen, die erforderlich sind, um eine den Vorschriften des NDSG entsprechende Verarbeitung personenbezogener Daten sicherzustellen. Der Aufwand für die Maßnahmen muss dabei unter Berücksichtigung des Standes der Technik in einem angemessenen Verhältnis zu dem angestrebten Zweck stehen. Insoweit ergibt sich nichts anderes als im Rahmen des BDSG. Sofern die Datenverarbeitung im Rahmen eines automatisierten Verfahrens erfolgt, was, wie gezeigt, im Rahmen der elektronischen Langzeitarchivierung regelmäßig der Fall sein wird, ist darüber hinaus $\int 7$ Abs. 2 NDSG einschlägig. Danach hat die Einrichtung Maßnahmen zu ergreifen, „die je nach Art der Daten und ihrer Verwendung geeignet sind,

1. Unbefugten den Zugang zu den Verarbeitungsanlagen zu verwehren (Zugangskontrolle),

2. zu verhindern, dass Datenträger unbefugt gelesen, kopiert, verändert oder entfernt werden können (Datenträgerkontrolle),

3. die unbefugte Eingabe in den Speicher sowie die unbefugte Kenntnisnahme, Veränderung oder Löschung gespeicherter Daten zu verhindern (Speicherkontrolle),

4. zu verhindern, dass Datenverarbeitungssysteme mit Hilfe von Einrichtungen zur Datenübertragung von Unbefugten benutzt werden können (Benutzerkontrolle),

5. zu gewährleisten, dass die zur Benutzung eines Datenverarbeitungssystems Berechtigten ausschließlich auf die ihrer Zugriffsberechtigung unterliegenden Daten zugreifen können (Zugriffskontrolle),

\footnotetext{
${ }^{590}$ Vgl. Gola/Schomerus, \9 Rn. 27; \11 Rn. 2.

${ }^{591}$ Gola/Schomerus, $\int 9$ Rn. 28.

592 Im Rahmen der übrigen Landesdatenschutzgesetze ergeben sich weitgehend die gleichen Voraussetzungen, wenn auch in unterschiedlichem Umfang, aus $\$ 9$ Abs. 3 LDSG B-W; Art. 7 Abs. 2 BayDSG; $\ 5$ Abs. 2 BlnDSG; $₫ 10$ Abs. 2 BBDSG; $\int 7$ Abs. 4 S. 2 BremDSG; $\int 8$ Abs. 2 HmbDSG; \10 Abs. 2 S.2 HDSG; \\ 21 Abs. 2, 22 Abs. 1-4 LDSG M-V; \ 10 Abs. 2 LDSGNRW; 9 Abs. 2 S. 2 LDSG R-P; \11 Abs. 2 SDSG; \6 Abs. 2 DSG-LSA; 9 Abs. 2 SächsDSG; $\int 6$ Abs. 1-5 LDSG S-H; $\int 9$ Abs. 2 ThürDSG.
} 
6. zu gewährleisten, dass überprüft und festgestellt werden kann, welche Daten zu welcher Zeit an wen übermittelt worden sind (Übermittlungskontrolle),

7. zu gewährleisten, dass überprüft und festgestellt werden kann, welche Daten zu welcher Zeit von wem in Datenverarbeitungssysteme eingegeben worden sind (Eingabekontrolle),

8. zu gewährleisten, dass personenbezogene Daten gegen zufällige Zerstörung oder Verlust geschützt sind (Verfügbarkeitskontrolle),

9. zu gewährleisten, dass Daten, die im Auftrag verarbeitet werden, nur entsprechend den Weisungen der Auftraggeber verarbeitet werden können (Auftragskontrolle),

10. zu gewährleisten, dass bei der Übertragung von Daten sowie beim Transport von Datenträgern diese nicht unbefugt gelesen, kopiert, verändert oder gelöscht werden können (Transportkontrolle),

11. die innerbehördliche oder innerbetriebliche Organisation so zu gestalten, dass sie den besonderen Anforderungen des Datenschutzes gerecht wird (Organisationskontrolle).“

Dieser Katalog ist jedoch nicht als abschließend zu verstehen. Es kommt im Einzelfall vielmehr auf eine Abwägung der Sensibilität der Daten, dem damit einhergehenden Grad der Schutzbedürftigkeit sowie dem Aufwand an, der für die Sicherungsmaßnahmen betrieben werden muss. ${ }^{593}$

Gerade im Rahmen von medizinischen Forschungsdaten mit Personenbezug ist jedoch zu berücksichtigen, dass es sich dabei zumeist um besonders sensible Daten handelt. ${ }^{594} \mathrm{Um}$ den oben genannten Anforderungen gerecht zu werden, sollte die archivierende Einrichtung daher zumindest alle vorhandenen Datenverarbeitungsanlagen erfassen und den Personenkreis festlegen, der Zugang zu diesen Anlagen erhalten darf.

In diesem Zusammenhang sind auch die Zugangsbedingungen der einzelnen zugriffsberechtigten Personen zu bestimmen. ${ }^{595}$ Des Weiteren ist eine Bestandsaufnahme der vorhandenen Daten vorzunehmen und zu bestimmen, welche Mitarbeiter in welchem Umfang Zugang zu diesen Daten erhalten sollen. ${ }^{596}$ Ersteres wird in den vorliegend relevanten Fällen von Forschungsdaten regelmäßig schon im Rahmen der jeweiligen wissenschaftlichen Forschungsvorhaben erfolgt sein. Um zu Gewährleisten, dass Unbefugte keine Kenntnis von den Daten erlangen oder die Datenträger unbefugt ändern oder entfernen können, sollte die archivierende Einrichtung die Datenverarbeitungsanlagen mit Zugangssperren wie Pass-

\footnotetext{
593 Kommentar zum NDSG, $\int 7$ Zu Abs. 2 Nr. 5.

594 Vgl. \4 Abs. 2 S. 2 NDSG, aus dem sich die hohe Sensibilität personenbezogener Daten über die Gesundheit des Betroffenen ergibt ; entsprechend auch \ 3 Abs. 9 BDSG.

595 Kommentar zum NDSG, $\int 7$ Zu Abs. 2 Nr. 1.

${ }^{596}$ Kommentar zum NDSG, $\int 7$ Zu Abs. 2 Nr. 2.
} 
wörtern oder biometrischen Identifikationsverfahren versehen. Möglich ist auch der Nachweis der Berechtigung mittels spezieller Chipkarten.597 Sofern Daten übermittelt werden, muss dies zum einen protokolliert werden, um nachvollziehen zu können, welche Daten wohin gesendet worden sind. Zum anderen sollten sensible personenbezogene Daten nur verschlüsselt übermittelt werden. ${ }^{598}$ Eine Verschlüsselung der Daten ist auch erforderlich, um zu gewährleisten, dass die Daten beim Transport der Datenträger nicht unbefugt gelesen oder kopiert werden können. ${ }^{599}$ Bei der Frage, welchen Anforderungen die Verschlüsselungsmethode entsprechen muss, wird man sich an der Sensibilität der Daten und dem aktuellen Stand der Technik orientieren müssen. Schließlich muss die archivierende Einrichtung Maßnahmen ergreifen, die es erlauben, festzustellen, von welchem Arbeitsplatz die einzelnen Daten in das System eingegeben wurden. Auf diese Weise kann Manipulation des Datenbestandes vorgebeugt werden, da der Verantwortliche damit rechnen muss, identifiziert zu werden. ${ }^{600}$ Neben diesen technischen Sicherungsmaßnahmen hat die archivierende Einrichtung gem. $\int 7$ Abs. 2 Nr. 11 NDSG die Organisationskontrolle zu gewährleisten. Diese umfasst beispielsweise das automatische Abschalten von Bildschirmen, die Prüfung und Aufbewahrung von Benutzungsprotokollen sowie die Überwachung von Wartungs- und Administrationsarbeiten. ${ }^{601} \mathrm{Als}$ Grundsatz lässt sich dabei jedoch festhalten, dass die technischen Maßnahmen gegenüber den organisatorischen vorzugswürdig sind. ${ }^{602}$

Auch wenn sich die Landesdatenschutzgesetze grundsätzlich nur an öffentliche Einrichtungen richten, sind diese Maßnahmen auch dann zu ergreifen, wenn die eigentlichen Archivierungsmaßnahmen durch eine beauftragte natürliche Person oder juristische Person des Privatrechts vorgenommen würde, da die den Auftrag erteilende öffentliche Stelle im datenschutzrechtlichen Sinne verantwortlich bliebe. ${ }^{603}$ Aus diesem Grund sollte die öffentliche Stelle, die den Auftrag erteilt, den Auftragnehmer zur Durchführung der oben genannten Maßnahmen verpflichten. ${ }^{604}$

\section{Ergebnis}

Unabhängig davon, ob das BDSG oder eines der Landesdatenschutzgesetze einschlägig ist, sind personenbezogene Forschungsdaten zu anonymisieren, sobald der Forschungszweck dies zulässt. Sollte eine Anonymisierung nicht möglich sein,

\footnotetext{
${ }^{597}$ Kommentar zum NDSG, $\int 7$ Zu Abs. 2 Nr. 3, 4 und 5; dies ist auch im Rahmen der Gewährleistung der Authentizität relevant. Siehe dazu unten S. $197 \mathrm{ff}$.

598 Kommentar zum NDSG, $\int 7$ Zu Abs. 2 Nr. 6.

${ }^{599}$ Kommentar zum NDSG, $\int 7$ Zu Abs. 2 Nr. 10.

${ }^{600}$ Kommentar zum NDSG, $\int 7$ Zu Abs. 2 Nr. 7; siehe dazu auch unten S. 197 ff.

601 Kommentar zum NDSG, $\int 7$ Zu Abs. 2 Nr. 11.

602 So auch Kommentar zum NDSG, $\int 7$ Zu Abs. 1 Nr. 3.

603 Siehe etwa $\int 6$ Abs. 1 S. 1 NDSG.

604 Zum Inhalt des Vertrags zwischen archivierender Stelle und Auftragnehmer siehe S. $180 \mathrm{ff}$.
} 
so hat die archivierende Stelle die Merkmale, mit denen der Personenbezug der einzelnen Daten hergestellt werden kann, gesondert zu speichern. Dabei ist jedoch nicht erforderlich, dass diese Merkmale durch einen Dritten gespeichert werden. Vielmehr hat die archivierende Einrichtung dafür zu sorgen, dass Unbefugte keinen Zugriff zu den Merkmalen erhalten.

Da es sich bei der elektronischen Langzeitarchivierung in der Regel um eine automatisierte Datenverarbeitung handeln wird, sind darüber hinaus die besonderen Vorschriften von \9 S. 1 i.V.m. der Anlage zu \9 S. 1 BDSG bzw. \ 7 Abs. 2 NDSG oder die entsprechenden Vorschriften der anderen Landesdatenschutzgesetze zu beachten. Aus diesen folgt vor allem, dass die archivierende Einrichtung technische Maßnahmen zu ergreifen hat, um einen unbefugten Zugriff oder eine unbefugte Veränderung der Daten zu verhindern. Neben diesen technischen Maßnahmen hat sie außerdem organisatorische Maßnahmen zu ergreifen. Zu beachten ist im Rahmen des Anwendungsbereichs der Landesdatenschutzgesetze, dass diese Maßnahmen auch dann ergriffen werden müssen, wenn die eigentliche Archivierung von einem beauftragten Dritten erfolgt, der keine öffentliche Stelle i.S.d. Landesdatenschutzgesetze darstellt. Zwar finden die Vorschriften der Landesdatenschutzgesetze auf ihn keine Anwendung, jedoch bleibt die den Auftrag erteilende öffentliche Stelle verantwortliche Stelle i.S.d. Landesdatenschutzgesetze, weshalb sie den Auftragnehmer im Rahmen des Auftragsverhältnisses dazu verpflichten sollte, die besagten Maßnahmen zu ergreifen und ihr die Möglichkeit einzuräumen, dies jederzeit überprüfen zu können.

\section{Rechtsfolgen des Verstoßes gegen einzelne Bestimmungen des BDSG, des SGB X oder der Landesdatenschutzgesetze}

Wurden in den vorangegangenen Abschnitten die datenschutzrechtlichen Anforderungen an eine elektronische Langzeitarchivierung gestellt, stellt sich die Frage, welche Konsequenzen ein Verstoß gegen die datenschutzrechtlichen Bestimmungen hat. Dabei kann sowohl im Anwendungsbereich des BDSG als auch der Landesdatenschutzgesetze zwischen strafrechtlichen Konsequenzen bzw. Ordnungswidrigkeiten und zivilrechtlichen Schadensersatzansprüchen differenziert werden. Im Folgenden soll vor allem der Inhalt des Schadensersatzanspruches näher beleuchtet werden. Da die Vorschriften des BDSG und der Landesdatenschutzgesetze diesbezüglich annähernd gleich ausgestaltet sind, wird auf eine getrennte Betrachtung der Vorschriften verzichtet. 


\section{Strafrechtliche Konsequenzen und Ordnungswidrigkeiten}

Sofern gegen die Vorschriften des BDSG vorsätzlich oder fahrlässig verstoßen wird, stellt dies gem. \43 BDSG eine Ordnungswidrigkeit dar. Eine Straftat liegt gem. $\ 44$ Abs. 1 BDSG hingegen vor, wenn die in $\ 43$ Abs. 2 BDSG beschriebenen Handlungen vorsätzlich und gegen Entgelt oder in der Absicht begangen werden, sich oder einen anderen zu bereichern oder einen anderen zu schädigen. Für das Vorliegen eines Vorsatzes genügt bereits Eventualvorsatz. Dieser ist gegeben, wenn der Täter die Möglichkeit des Verstoßes gegen die Bestimmungen des BDSG erkennt, diese jedoch billigend in Kauf nimmt und trotzdem handelt. ${ }^{605}$ $\mathrm{Zu}$ beachten ist allerdings, dass die Tat gem. \44 Abs. 2 S.1 BDSG nur auf Antrag verfolgt wird. Antragsberechtigt sind neben dem Betroffenen gem. $\ 44$ Abs. 2 S. 2 BDSG die verantwortliche Stelle, der Bundesbeauftragte für den Datenschutz und die Informationsfreiheit sowie die Aufsichtsbehörde. Auch nach $\ 29$ Abs. 1 NDSG $^{606}$ handelt ordnungswidrig, wer personenbezogene Daten, die nicht allgemein zugänglich sind, entgegen $\int 5$ NDSG zu einem anderen als dem zur jeweiligen rechtmäßigen Aufgabenerfüllung gehörenden Zweck verarbeitet oder offenbart oder sich durch Vortäuschung falscher Tatsachen verschafft oder an sich oder andere übermitteln lässt. Der Ordnungswidrigkeitenkatalog hat damit einen wesentlich geringeren Umfang als der des $₫ 43$ BDSG. Ein weiterer Unterschied zu $₫ 43$ BDSG besteht darin, dass nach $₫ 29$ NDSG nur eine Ordnungswidrigkeit bei vorsätzlichem Handeln vorliegt und nicht bereits bei Fahrlässigkeit. Dies lässt sich zwar nicht dem Wortlaut entnehmen, folgt jedoch aus den $\ 10$ i.V.m. \ 2 OWiG, ${ }^{607}$ wonach als Ordnungswidrigkeit nur vorsätzliches Handeln geahndet werden kann, sofern das Gesetz fahrlässiges Handeln nicht ausdrücklich mit Geldbuße bedroht. Dies ist jedoch im Falle des $\$ 29$ NDSG gerade nicht der Fall.

Eine Straftat begeht gem. \28 NDSG608hingegen, „wer gegen Entgelt oder in der Absicht, sich oder einen anderen zu bereichern oder einen anderen zu schädi-

${ }^{605}$ BGH NStZ 2000, 583, 584; Joecks in Joecks/Miebach (Hrsg.), Münchener Kommentar zum Strafgesetzbuch, Band 1 2003, \16 Rn. 29.

${ }^{606}$ Die übrigen Landesdatenschutzgesetze klassifizieren eine unbefugte Verarbeitung bis auf wenige Ausnahmen ebenfalls als Ordnungswidrigkeit. Dabei weichen die Tatbestandsvoraussetzungen jedoch teilweise voneinander ab. Siehe $\int 40$ LDSG B-W; Art. 37 Abs. 1 und Abs. 2 BayDSG; $\$ 38 Abs. 1 BBDSG; $₫ 38$ BremDSG; \33 Abs. 1 HmbDSG; \41 HDSG; § 34 Abs. 1 LDSGNRW; \ 36 Abs. 1 SDSG; \31a Abs. 1 und Abs. 2 DSG-LSA (der teilweise auch fahrlässiges Handeln genügen lässt); \38 Abs. 1 SächsDSG; \44 Abs. 1 LDSG S-H. Keine Ordnungswidrigkeitentatbestände sehen hingegen das BlnDSG, das LDSG M-V, das LDSG R-P sowie das ThürDSG vor.

${ }^{607}$ So auch Kommentar zum NDSG, \ 29 Zu Abs. 1 Nr. 6.

608 Art. 37 Abs. 3 BayDSG; \38 Abs. 3 BBDSG; \37 BremDSG; \32 HmbDSG; \40 HDSG; \42 Abs. 1 und Abs. 2 LDSG M-V (der allerdings für eine Strafbarkeit keine Bereicherungs- oder Schädigungsabsicht voraussetzt. Diese stellt vielmehr den Qualifikationstatbestand des Abs. 2 dar. Es genügt damit für eine Strafbarkeit vorsätzliches Verhalten des Täters.); \33 Abs. 1 LDSG-NRW; \35 Abs. 1 SDSG; \31 Abs. 1 und Abs. 2 DSG-LSA; \39 SächsDSG; \44 Abs. 
gen, personenbezogene Daten, die nicht allgemein zugänglich sind, unbefugt erhebt, speichert, verändert, löscht, übermittelt oder nutzt oder durch Vortäuschung falscher Tatsachen ihre Weitergabe an sich oder andere veranlasst". Wie auch das BDSG stellt also auch das NDSG für das Vorliegen einer Straftat darauf ab, ob die unbefugte Datenverarbeitung mit Bereicherungs- oder Schädigungsabsicht erfolgte. Ein wichtiger Unterschied besteht hingegen darin, dass im Rahmen des NDSG gem. \28 Abs. 2 bereits der Versuch strafbar ist.

Sollte die archivierende Einrichtung also ohne Einwilligung des Betroffenen und ohne, dass eine Vorschrift dies erlauben würde, personenbezogene Forschungsdaten elektronisch archivieren, würde dies eine unbefugte Datenverarbeitung und damit eine Ordnungswidrigkeit nach \43 Abs. 2 Nr. 1 BDSG darstellen, sofern dies einschlägig wäre. Sollten die Daten darüber hinaus für Fremdwissenschaftler in einem Netzwerk, wie beispielsweise dem Internet zugänglich gemacht werden, so wäre daneben der Tatbestand des $\int 43$ Abs. 2 Nr. 2 BDSG erfüllt, da in diesem Fall ein automatisiertes Verfahren vorläge. Des Weiteren läge nach \ 43 Abs. 2 Nr. 6 eine Ordnungswidrigkeit vor, wenn die Daten und die Merkmale, anhand derer eine Zuordnung zu den Betroffenen möglich ist, entgegen \40 Abs. 2 BDSG zusammengeführt würden. Sofern eine der genannten Handlungen vorsätzlich und gegen Entgelt oder mit Gewinnerzielungs- oder Schädigungsabsicht erfolgt, liegt gem. \44 Abs. 1 BDSG eine Straftat vor. Denkbar wäre dies in den vorliegend einschlägigen Fällen unter anderem dann, wenn personenbezogene Forschungsdaten entgeltlich anderen Wissenschaftlern angeboten würden.

Da der Katalog des \29 NDSG einen wesentlich geringeren Umfang hat, als der des $\int 43$ BDSG, wird der Tatbestand einer Ordnungswidrigkeit im Rahmen der elektronischen Langzeitarchivierung im Anwendungsbereich des NDSG bei einem Verstoß gegen einzelne datenschutzrechtliche Vorschriften der Tatbestand der Ordnungswidrigkeit seltener als im Rahmen des BDSG verwirklicht werden. Sofern die Daten jedoch vorsätzlich unbefugt gespeichert oder übermittelt würden, läge im Anwendungsbereich des NDSG ebenfalls eine Straftat vor. ${ }^{609}$

Sofern die archivierende Stelle es also für möglich hält, dass die Speicherung der Forschungsdaten oder deren Zugänglichmachung an Fremdforscher weder durch die Einwilligung der jeweiligen Betroffenen noch durch eine gesetzliche Norm gestattet ist, sollte sie die Datenverarbeitung im Zweifel unterlassen, da sie ansonsten Gefahr läuft, eine Ordnungswidrigkeit oder sogar Straftat zu begehen. Die Verwendung von Daten, bei denen die Merkmale gelöscht wurden, mit denen sie

1-3 ThürDSG (der ebenfalls keine Bereicherungs- oder Schädigungsabsicht voraussetzt und damit vorsätzliches Handeln genügen lässt). Keinen Straftatbestand enthält hingegen das LDSG SH.

${ }^{609}$ Ebenso nach den in Fn. 608 genannten Vorschriften der übrigen Landesdatenschutzgesetze, mit Ausnahme des LDSG S-H, das wie festgestellt, keinen Straftatbestand enthält. 
den Betroffenen zugeordnet werden können, bleibt hiervon allerdings ausgenommen, da diese Daten schon keine personenbezogenen Daten darstellen und der Anwendungsbereich des BDSG bzw. der Landesdatenschutzgesetze damit gar nicht eröffnet ist. ${ }^{610}$

\section{Zivilrechtliche Konsequenzen}

Neben den oben dargestellten strafrechtlichen Konsequenzen, die eine unbefugte Datenverarbeitung nach sich ziehen kann, stellt sich für die archivierende Einrichtung in einem solchen Fall vor allem das Problem möglicher zivilrechtlicher Konsequenzen. Deren Ausmaß und Umfang hängen unter anderem davon ab, ob das BDSG oder ein Landesdatenschutzgesetz einschlägig sind, weshalb im Folgenden eine getrennte Betrachtung der jeweiligen Konsequenzen erfolgt.

\section{Zivilrechtliche Konsequenzen im Anwendungsbereich des BDSG}

Im Rahmen des BDSG kommen als zivilrechtliche Konsequenzen bei einer unzulässigen Verarbeitung personenbezogener Forschungsdaten durch die archivierende Stelle Ansprüche des Betroffenen auf Schadensersatz (dazu a) sowie auf Löschung oder Sperrung der Daten in Betracht (dazu b).

\section{a. Schadensersatzanspruch des Betroffenen}

Die für die archivierende Einrichtung wohl schwerwiegendste zivilrechtliche Konsequenz folgt aus den $\$ \$ 7$ und 8 BDSG, die einen Schadensersatzanspruch des Betroffenen gegen die verantwortliche Stelle begründen. Verantwortliche Stelle ist dabei gem. $\ 3$ Abs. 7 BDSG jede Person oder Stelle, die personenbezogene Daten für sich selbst erhebt, verarbeitet oder nutzt oder dies durch andere im Auftrag vornehmen lässt. Aufgrund der letztgenannten Alternative ist daher auch im Rahmen der Auftragsdatenverarbeitung die den Auftrag erteilende Stelle Anspruchsgegner. ${ }^{611}$ Dabei erfasst der Anspruch aus $\int 7$ BDSG sowohl die unzulässige oder unrichtige Datenverarbeitung, -erhebung oder -nutzung in einem nicht automatisierten als auch in einem automatisierten Verfahren durch eine öffentliche oder eine nicht-öffentliche Stelle. Diese hat dem Betroffenen den dadurch entstandenen Schaden zu ersetzen. Voraussetzung für den Haftungsanspruch ist dabei jeder Verstoß gegen eine Vorschrift bezüglich der Verarbeitung personenbezogener Daten und nicht nur eine Verarbeitung ohne Rechtfertigungsgrund oder Einwilligung des Betroffenen. ${ }^{612}$

Demzufolge kann für den Betroffenen auch ein Schadensersatzanspruch entstehen, wenn die Datenverarbeitung zwar mit seiner Einwilligung erfolgt ist bzw.

\footnotetext{
${ }^{610}$ Vgl. nur $\ \int 1$ 1, 3 Abs. 1 BDSG; $\int \ 1,3$ Abs. 1 NDSG; siehe auch Gola/Schomerus, $\ 3$ Rn. 43.

${ }^{611} \mathrm{Zu}$ den daraus resultierenden Konsequenzen für das Vertragsverhältnis zwischen archivierender

Einrichtung und Auftragnehmer siehe unten S. $186 \mathrm{ff}$.

${ }^{612}$ Gola/Schomerus, \& 7 Rn. 3.
} 
diese gesetzlich gerechtfertigt war, die verantwortliche Stelle bei der Verarbeitung jedoch gegen die zu ergreifenden Organisationspflichten verstoßen hat. Sofern die verantwortliche Stelle die nach den Umständen des Falles gebotene Sorgfalt beobachtet hat, entfällt gem. $\ 7$ S. 2 BDSG jedoch die Schadensersatzpflicht. Die Beweislast für die Einhaltung der gebotenen Sorgfalt trägt dabei die archivierende Einrichtung, wie sich aus dem Wortlaut von $₫ 7$ S. 2 BDSG ergibt. Was genau unter der gebotenen Sorgfalt zu verstehen ist, geht aus dem Gesetz hingegen nicht ausdrücklich hervor. Als Anhaltspunkt kann jedoch der Katalog des \11 BDSG herangezogen werden, auf den im Folgenden noch ausführlich eingegangen wird. ${ }^{613}$ Darüber hinaus kann sich die archivierende Einrichtung an den umfangreichen Vorschlägen des Bundesamtes für Datenschutz in der Informationstechnologie (BSI) hinsichtlich zu ergreifender Sicherungsmaßnahmen orientieren. ${ }^{614}$ Auch wenn diese keine gesetzliche Wirkung entfalten, können sie zumindest als Maßstab für die üblicherweise zu ergreifenden Maßnahmen beim Umgang mit personenbezogenen Daten dienen.

Eine verschuldensunabhängige Haftung sieht hingegen $\ 8$ BDSG für die Fälle vor, in denen dem Betroffenen durch eine unrichtige oder unzulässige Datenerhebung, -verarbeitung oder -nutzung in einem automatisierten Verfahren ein Schaden entsteht. Anspruchsgegner kann nach dem klaren Wortlaut jedoch nur eine öffentliche Stelle sein, so dass private Forschungseinrichtungen grundsätzlich nicht aus $\int 8$ BDSG in Anspruch genommen werden können. Daraus folgt, dass $\int 7$ und $\ 8$ nicht in einem Alternativverhältnis zueinander stehen, sondern auch nebeneinander einschlägig sein können. Für den Betroffenen hat der Anspruch gem. $\int 8$ BDSG allerdings den Vorteil, dass sich die öffentliche Stelle nicht exkulpieren kann. Ein weiterer Vorteil des $₫ 8$ BDSG ist, dass der Betroffene gem. Abs. 2 auch eine Entschädigung in Geld für einen erlittenen immateriellen Schaden verlangen kann. Dies ist im Rahmen von $₫ 7$ BDSG hingegen nicht möglich, da eine Entschädigung in Geld für einen Schaden, der nicht Vermögensschaden ist, gem. $\$ 253$ Abs. 1 nur in den gesetzlich bestimmten Fällen gefordert werden kann. Eine solche Geldentschädigung sieht $\int 7$ für den Fall eines immateriellen Schadens im Gegensatz zu $\int 8$ BDSG jedoch gerade nicht vor. Der Betroffene könnte im Rahmen der Anspruchsgrundlage des $₫ 7$ BDSG demzufolge ausschließlich den Ersatz des materiellen Schadens verlangen.

Die Entstehung eines solchen materiellen Schadens aufgrund einer unrechtmäßigen Datenverarbeitung ist zwar grundsätzlich möglich, wird aber in den vorliegenden Fällen wohl eher die Ausnahme als die Regel darstellen. Die Entstehung eines immateriellen Schadens kommt aber insbesondere bei der unrechtmäßigen Verarbeitung von medizinischen Forschungsdaten in Betracht, da hierdurch nicht

${ }^{613}$ Siehe dazu S. 181.

${ }^{614}$ Handbuch für die sichere Anwendung der Informationstechnik, 1992, abrufbar unter: http://www.bsi.bund.de/literat/kriterie.htm. 
unwesentlich in die Intimsphäre des Betroffenen eingegriffen werden kann. Damit ist die Haftung aus $\ 8$ BDSG auf der einen Seite aufgrund der verschuldensunabhängigen Haftung und der Entschädigungspflicht für einen immateriellen Schaden zwar strenger als diejenige aus $₫ 7$ BDSG. Auf der anderen Seite ist jedoch zu beachten, dass die Ansprüche nach $\ 8$ Abs. 1 und Abs. 2 jedoch in ihrer Höhe gem. $\ 8$ Abs. 3 auf $130.000 €$ begrenzt sind. Dies gilt gem. Abs. 3 S. 2 selbst dann, wenn auf Grund desselben Ereignisses an mehrere Personen Schadensersatz zu leisten ist, der insgesamt den Höchstbetrag von 130.000 Euro übersteigt.

\section{b. Löschung oder Sperrung der Daten}

Neben einem Anspruch des Betroffenen auf Schadensersatz hat eine unzulässige Datenverarbeitung unter Umständen die weitere Konsequenz, dass die verantwortliche Stelle gem. \20 Abs. 2 bzw. Abs. 3 BDSG die Daten zu löschen oder zu sperren hat. Voraussetzung dafür ist gem. $\ 20$ Abs. 2 Nr. 1 sowohl eine unzulässige Speicherung als auch die Verarbeitung in einem automatisierten Verfahren oder die Speicherung der personenbezogenen Daten in einer nicht automatisierten Datei. Da im Rahmen der elektronischen Langzeitarchivierung regelmäßig eine automatisierte Datenverarbeitung vorliegen wird, ${ }^{615}$ müsste die archivierende Einrichtung die Daten demzufolge gem. \20 Abs. 2 Nr. 1 BDSG löschen, wenn die Speicherung der Daten unzulässig war. Die Daten können gelöscht werden, indem sie überschrieben werden oder indem die Verknüpfung zwischen den Daten mit Informationswert und jenen getrennt gespeicherten Daten, die die Identifizierung ermöglichen, aufgehoben wird. ${ }^{616}$ Entscheidend ist dabei, dass der Vorgang irreversibel ist, die Daten bzw. der Personenbezug also endgültig nicht mehr hergestellt werden können. ${ }^{617}$

Sofern der Löschung allerdings gesetzliche Aufbewahrungsfristen ${ }^{618}$ entgegenstehen, was insbesondere bei medizinischen Untersuchungsdaten in Betracht kommt (Nr. 1), Grund zur Annahme besteht, dass durch die Löschung schutzwürdige Interessen des Betroffenen verletzt werden (Nr. 2) oder eine Löschung wegen der besonderen Art der Speicherung nicht oder nur mit unverhältnismäßigem Aufwand in Betracht kommt (Nr. 3) tritt gem. \20 Abs. 3 BDSG an die Stelle der Löschung eine Sperrung der Daten. In diesem Fall sind die Daten entsprechend zu kennzeichnen bzw. elektronisch zu sperren ${ }^{619}$ und dürfen nicht mehr übermittelt oder genutzt werden ${ }^{620}$. Dies bedeutet für die vorliegend relevanten Fälle, dass die Daten grundsätzlich weder Fremdwissenschaftlern zugänglich gemacht werden noch im Rahmen eigener Forschungsprojekte genutzt werden dürf-

\footnotetext{
615 Siehe dazu ausführlich S. 167.

${ }^{616}$ Dammann in Simitis/Dammann, EG-RL Art. 12 Rn. 16; Gola/Schomerus, \$ 20 Rn. 9.

${ }^{617}$ Siehe entsprechend Kommentar zum NDSG, \ 20 Zu Abs. 2 Nr. 2.

${ }^{618}$ Hinsichtlich bestehender gesetzlicher Archivierungspflichten siehe S. $191 \mathrm{ff}$.

${ }^{619}$ Vgl. Zapatka, DuD 1977, 82.

${ }^{620}$ Gola/ Schomerus, \$ 20 Rn. 14.
} 
ten. Ausnahmsweise wäre die Übermittlung oder Nutzung allerdings gem. \ 20 Abs. 7 BDSG auch im Falle einer Sperrung ohne die Einwilligung des Betroffenen zulässig, wenn dies zu wissenschaftlichen Zwecken unerlässlich wäre. Es genügt demzufolge nicht, dass die Übermittlung oder Nutzung zur Erreichung des wissenschaftlichen Zwecks lediglich erforderlich ist. ${ }^{621}$ Vielmehr müsste die Erreichung des wissenschaftlichen Zwecks ohne die Übermittlung oder Nutzung nahezu ausgeschlossen sein.622 Der Ausnahmetatbestand des \20 Abs. 7 BDSG wird sich im Rahmen der wissenschaftlichen Langzeitarchivierung von daher eher selten vorliegen, da es schwer vorstellbar ist, dass die Übermittlung oder Nutzung einzelner personenbezogener Forschungsdaten die Erreichung des Zwecks der Langzeitarchivierung auszuschließen. Dieser besteht ja gerade darin, wissenschaftliches Arbeiten durch die Bereithaltung der erforderlichen Daten zu fördern. Dies ist jedoch auch möglich, wenn einzelne Daten gesperrt sind.

Darüber hinaus wäre die Nutzung gesperrter Daten auch dann zulässig, wenn dazu die Einwilligung des Betroffenen eingeholt würde. ${ }^{623}$ Dies wird in den relevanten Fällen jedoch schwer möglich sein, da andernfalls bereits für die Speicherung die Zustimmung des Betroffenen eingeholt worden wäre. Darüber hinaus dürfte es bei umfangreichen Datenbeständen auch praktisch kaum möglich sein, die Aufenthaltsorte aller Betroffenen ausfindig zu machen. Sofern es sich hingegen nur um die Daten einzelner Betroffener handelt, wäre diese Möglichkeit allerdings vorzuziehen, da sie der archivierenden Einrichtung die größtmögliche Rechtssicherheit verschafft.

\section{Zivilrechtliche Konsequenzen im Anwendungsbereich der Landesdatenschutagesetze}

Auch die Landesdatenschutzgesetze sehen für den Fall einer unrechtmäßigen Datenverarbeitung einen Schadensersatzanspruch des Betroffenen vor und verpflichten die verantwortliche Stelle, unrechtmäßig gespeicherte Daten zu löschen oder zu sperren. Dies wird im Folgenden wieder am Beispiel des Niedersächsischen Datenschutzgesetzes dargestellt. Auf die vergleichbaren Vorschriften der übrigen Landesdatenschutzgesetze wird in den Fußnoten entsprechend verwiesen.

a. Schadensersatzanspruch gem. I 18 NDSG

Sofern das NDSG Anwendung findet, hat der Betroffene im Falle einer nach datenschutzrechtlichen Vorschriften unzulässigen Datenverarbeitung gem. \18 Abs. $1 \mathrm{NDSG}^{624}$ einen Anspruch auf Ersatz des daraus entstehenden Scha-

621 Gola/Schomerus, \20 Rn. 31.

622 OVG Münster NJW 1988, 90, 91.

${ }^{623}$ Die Speicherung würde dadurch jedoch nicht rückwirkend zulässig werden, da die Datenschutzgesetze die Möglichkeit der Genehmigung einer nach den datenschutzrechtlichen Vorschriften unzulässigen Maßnahme nicht vorsehen. Siehe dazu auch S. 144 ff.

${ }^{624}$ Im Rahmen der übrigen Landesdatenschutzgesetze folgt der Schadensersatzanspruch des Betroffenen aus \25 LDSG B-W; Art. 14 Abs. 1 S. 1 BayDSG; 18 Abs. 1 S. 1 BlnDSG; \ 20 Abs. 1 
dens. Dabei kann sich die verantwortliche Stelle gem. \18 Abs. 1 Hs. 2 NDSG ebenfalls nur im Falle einer nicht automatisierten Datenverarbeitung exkulpieren, indem sie nachweist, dass sie die Unzulässigkeit nicht zu vertreten hat ${ }^{625}$. Im Unterschied zu den Schadensersatzansprüchen des BDSG kann der Betroffene, wie sich aus $\llbracket 18$ Abs. 1 S. 2 NDSG ${ }^{626}$ ergibt, auch bei einer Verletzung im Rahmen einer nicht automatisierten Datenverarbeitung eine Geldentschädigung als Ersatz für einen immateriellen Schaden verlangen. Voraussetzung dafür ist jedoch eine schwere Verletzung des Persönlichkeitsrechts, wobei das Gesetz offen lässt, wann eine solche vorliegt. ${ }^{627}$ Abzustellen ist in diesem Zusammenhang vor allem auf den objektiven Umfang der Beeinträchtigung und der Reichweite des Bildes vom Betroffenen, welches sich aufgrund der Verletzung im privaten Bereich oder sogar in der Öffentlichkeit abzeichnet. ${ }^{628}$ Im Rahmen der Auftragsdatenverarbeitung ist zu beachten, dass Schadensersatzansprüche in diesen Fällen nicht gegen den Auftragnehmer, sondern den Auftraggeber zu richten sind. Dies ergibt sich aus $₫ 6$ Abs. 1 S. 2 NDSG. ${ }^{629}$

S. 1 BBDSG; \23 Abs. 1 S. 1 BremDSG; \20 Abs. 1 S. 1 HmbDSG; \20 Abs. 1 S. 1 HDSG; $\$ 27 Abs. 1 und Abs. 2 LDSG M-V; \20 Abs. 1 S. 1 LDSG-NRW; \21 Abs. 1 LDSG R-P (jedoch nur für die Fälle einer automatisierten Datenverarbeitung); \24 Abs. 1 und Abs. 1 SDSG; \18 Abs. 1 S. 1 DSG-LSA; \ 23 Abs. 1 S. 1 SächsDSG; \30 Abs. 1 S. 1 LDSG S-H (allerdings nur im Falle eines automatisierten Verfahrens); $₫ 18$ Abs. 1 S. 1 ThürDSG.

${ }^{625}$ Ebenso Art. 14 Abs. 2 S. 1 BayDSG; eine Exkulpationsmöglichkeit auch im Falle einer automatisierten Verarbeitung sieht hingegen $₫ 25$ Abs. 1 S. 2 LDSG B-W vor; $\ 20$ Abs. 1 S. 3 BBDSG, der ebenfalls eine generelle Exkulpationsmöglichkeit vorsieht; \23 Abs. 1 S. 2 BremdDSG; \20 Abs. 1 S. 3 HmbDSG; \27 Abs. 2 LDSG M-V; \20 Abs. 2 S. 3, 4 LDSG-NRW; \ 21 Hs. 2 LDSG R-P; \24 Abs. 2 S. 1, 2 SDSG; $\ 18$ Abs. 1 S. 2 DSG-LSA; anders hingegen $\ 18$ BlnDSG; \20 HDSG, die gar keine Exkulpationsmöglichkeit für die verantwortliche Stelle vorsieht; \18 Abs. 1 S. 2 SächsDSG sieht eine Exkulpationsmöglichkeit zwar unabhängig von der Art der Datenverarbeitung, jedoch nur im Fall eines unabwendbaren Ereignisses vor; eine verschuldensunabhängige Haftung im Falle einer automatisierten Verarbeitung sieht ebenfalls $\ 30$ Abs. 1 S. 1 LDSG S-H vor; $\int 18$ Abs. 1 S. 2 ThürDSG, wonach eine Exkulpation jedoch auch im Rahmen einer automatisierten Verarbeitung zulässig ist.

${ }^{626}$ So auch $\ 25$ Abs. 2 LDSG B-W; Art. 14 Abs. 2 S. 2 BayDSG (allerdings nur für den Fall einer automatisierten Datenverarbeitung, wie sich aus der systematischen Stellung in Abs. 2 ergibt); $\$$ 18 Abs. 1 S. 3 BlnDSG; $\ 20$ Abs. 1 S. 2 BBDSG; $\ 23$ Abs. 2 S. 2 BremDSG (auch nur in den Fällen einer automatisierten Verarbeitung); $₫ 20$ Abs. 1 S. 2 HmbDSG; \20 Abs. 1 S. 2 HDSG; \27 Abs. 3 LDSG M-V; \20 Abs. 1 S. 2 LDSG-NRW; 『21 Abs. 2 LDSG R-P; \ 24 Abs. 1 S. 2 SDSG (ebenfalls nur im Falle einer automatisierten Datenverarbeitung); $₫ 18$ Abs. 2 S. 2 DSGLSA (jedoch nur im Falle einer automatisierten Datenverarbeitung; \30 Abs. 2 LDSG S-H; anders hingegen $₫ 18$ ThürDSG, der keine Entschädigung in Geld für einen erlittenen immateriellen Schaden vorsieht.

${ }^{627}$ Vgl. Kommentar zum NDSG, \18 Zu Abs. 1 Nr. 4.

${ }^{628}$ Kommentar zum NDSG, $\ 18 \mathrm{Zu}$ Abs. 1 Nr. 4.

${ }^{629} \mathrm{Zu}$ den Auswirkungen von $₫ 6$ Abs. 1 S. 2 NDSG auf den Inhalt des Vertrags mit dem Auftragnehmer siehe auch S. $180 \mathrm{ff}$. 
b. Löschung oder Sperrung der Daten

Auch im Anwendungsbereich der Landesdatenschutzgesetze sind die verantwortlichen Stellen verpflichtet, unzulässig gespeicherte Daten zu löschen. Dies folgt aus \17 Abs. 2 Nr. 1 NDSG ${ }^{630}$. Das Gleiche gilt gem. \17 Abs. 2 Nr. 2, wenn die Kenntnis der Daten für die Aufgabenerfüllung der verarbeitenden Stelle nicht mehr erforderlich ist. Dies wird bei den hier im Vordergrund stehenden Fällen jedoch eher selten der Fall sein, da die Daten ja gerade deshalb archiviert und anderen zur Verfügung gestellt werden sollen, weil ihre Kenntnis für die Aufgabenerfüllung der Forschungseinrichtungen erforderlich ist. Nichtsdestotrotz ist natürlich nicht auszuschließen, dass die Voraussetzungen von Nr. 2 in Ausnahmefällen vorliegen können, wahrscheinlicher ist jedoch in den vorliegenden Fällen die Tatbestandserfüllung von Nr. 1.

Wie auch im Rahmen des BDSG, sind die Daten in diesem Fall gem. \17 Abs. 3 NDSG $^{631}$ anstelle der Löschung zu sperren, solange und soweit ihre Richtigkeit von den Betroffenen bestritten wird und sich weder die Richtigkeit noch die Unrichtigkeit feststellen lässt (Nr. 1), wenn die Betroffenen an Stelle der Löschung unzulässig gespeicherter Daten die Sperrung verlangen oder die weitere Speicherung im Interesse der Betroffenen geboten ist (Nr. 2), oder solange sie auf Grund gesetzlicher Aufbewahrungsvorschriften nicht gelöscht werden dürfen (Nr. 3). Wie bereits bei den Ausführungen zum BDSG erwähnt, kommt dabei im Rahmen der elektronischen Langzeitarchivierung vor allem die Tatbestandsalternative des Abs. 3 Nr. 3 in Betracht. Die gesperrten Daten sind gem. $\int 17$ Abs. 3 S. 2 NDSG mit einem Sperrvermerk zu versehen. In automatisierten Verfahren ist die Sperrung gem. $\ 17$ Abs. 3 S. 2 Hs. 2 NDSG durch zusätzliche technische Maßnahmen zu gewährleisten. In Betracht kommen dabei auch hier die bereits oben genannten prorammtechnischen Maßnahmen. Gesperrte Daten dürfen auch im Rahmen des NDSG nicht mehr weiter verarbeitet werden. Eine Ausnahme sieht $₫ 17$ Abs. 3 S. 3 Hs. 3 NDSG jedoch für den Fall vor, dass dies zur Behebung einer bestehenden Beweisnot oder aus sonstigen im überwiegenden Interesse der speichernden Stelle oder Dritter liegenden Gründen unerlässlich ist oder die Betroffenen eingewilligt haben. In Betracht kommt dabei vor allem ein überwiegendes Interesse der archivierenden Einrichtung. Dies wird man in Anlehnung an \20 Abs. 7 BDSG zumindest dann

\footnotetext{
${ }^{630}$ Ebenso \23 Abs. 1 LDSG B-W; Art. 12 Abs. 1 BayDSG; \17 Abs. 3 BlnDSG; $\int 17$ Abs. 2 S. 1 BBDSG; \22 Abs. 2 BremDSG; \19 Abs. 3 HmbDSG; \19 Abs. 3 und Abs. 4 HDSG; \ 19 Abs. 3 LDSG-NRW; \19 Abs. 2 LDSG R-P; \21 Abs. 3 S. 1 SDSG; \16 Abs. 1 S. 1 DSGLSA; \20 Abs. 1 SächsDSG; \28 Abs. 2 LDSG S-H; $₫ 16$ Abs. 1 ThürDSG.

${ }^{631}$ Ebenso \24 Abs. 1 i.V.m. \ 23 Abs. 4 LDSG B-W; Art. 12 Abs. 2 BayDSG; \17 Abs. 2 BlnDSG; \17 Abs. 3 BBDSG; $\ 22$ Abs. 2 BremDSG; $\ 19$ Abs. 2 S. 1 HmbDSG; 19 Abs. 2 HDSG; \ 25 Abs. 1 LDSG M-V; \ 19 Abs. 2 S. 1 LDSG-NRW; \ 19 Abs. 3 LDSG R-P; 21 Abs. 2 S. 1 SDSG; \16 Abs. 3 S. 1 DSG-LSA; \21 Abs. 1 i.V.m. \20 Abs. 4 SächsDSG; \28 Abs. 3 i.V.m. Abs. 2 S. 3 LDSG S-H; \15 Abs. 1 i.V.m. \16 Abs. 4 ThürDSG.
} 
annehmen können, wenn die Verarbeitung zu wissenschaftlichen Zwecken unerlässlich ist, die Erreichung des Zwecks also ohne die Verarbeitung nahezu ausgeschlossen wäre. ${ }^{632}$

\section{E. Rechtliche Anforderungen an Verträge über die Archivierung personenbezogener Forschungsdaten}

Wie bereits erwähnt, sehen die Datenschutzgesetze die grundsätzliche Möglichkeit vor, Dritte mit der Datenverarbeitung zu beauftragen. In den Fällen der elektronischen Langzeitarchivierung wird dies vor allem bei kleineren Forschungseinrichtungen relevant sein, da diese oftmals nicht über die notwendigen technischen und personellen Voraussetzungen verfügen, die eine effektive Archivierung und Bereitstellung der Daten im Internet erfordern und deshalb einen IT-Dienstleister mit der Archivierung beauftragen müssen. Denkbar ist auch, dass sich mehrere Forschungsstellen $\mathrm{zu}$ einem Langzeitarchivierungsverbund zusammenschließen, der dann die Archivierung im Auftrag der beteiligten Forschungsstellen übernimmt. Aus diesem Grund soll im Folgenden untersucht werden, welche Anforderungen an den Inhalt eines solchen Vertrags zu stellen ist.

\section{Ausgangslage}

Die Archivierung personenbezogener Forschungsdaten, die von einer Forschungsstelle erhoben werden, muss nicht zwangsläufig durch die Forschungsstelle selbst durchgeführt werden. Denkbar ist beispielsweise die Archivierung durch einen Langzeitarchivierungsverbund, der aus mehreren Forschungsstellen besteht und der die Archivierung der Daten der beteiligten Forschungsstellen übernimmt. Darüber hinaus ist auch denkbar, dass eine Forschungsstelle ein Drittunternehmen mit der Archivierung beauftragt. Damit stellt sich die Frage, ob hinsichtlich der Archivierung von personenbezogenen Forschungsdaten besondere Anforderungen an den Vertrag zwischen Forschungsstelle und dem beauftragten Fremdunternehmen zu stellen sind. Dabei ist zum einen festzustellen, ob bzw. inwiefern das NDSG spezielle Vorschriften für die Verträge zwischen der verarbeitenden Stelle und einem Dritten beinhaltet. Darüber hinaus ist zu prüfen, ob der Universität aufgrund der mittelbaren Wirkung datenschutzrechtlicher Bestimmungen anzuraten ist, bestimmte Regelungen in einen solchen Vertrag aufzunehmen.

Neben den generellen Voraussetzungen einer rechtmäßigen Archivierung personenbezogener Daten kommt es bei einer Archivierung durch ein Fremdunternehmen zusätzlich darauf an, ob damit eine Übermittlung im datenschutzrechtlichen Sinne einhergeht und die Einwilligung des Betroffenen sich damit auch auf diese Übermittlung erstrecken muss. Dies könnte deshalb der Fall sein, weil die

${ }_{632}$ Vgl. OVG Münster NJW 1988, 90, 91. 
archivierende Einrichtung dem Auftragnehmer die Daten zwangsläufig zur Verfügung stellen muss, damit dieser sie archivieren kann. Sowohl nach dem BDSG als auch den Landesdatenschutzgesetzen setzt eine Übermittlung jedoch eine Weitergabe an „Dritte“ oder eine Einsicht in die Daten durch Dritte voraus. Sofern ein Fremdunternehmen jedoch mit der Archivierung beauftragt würde, wäre dieses Unternehmen sowohl nach dem BDSG als auch den Landesdatenschutzgesetzen nicht „Dritter“. ${ }^{633}$ Damit liegt beispielsweise keine Übermittlung im datenschutzrechtlichen Sinne vor, wenn die EDV-Abteilung einer Klinik die Forschungsdaten einer zur Klinik gehörenden Forschungsabteilung archiviert. Das Gleiche, wenn die Archivierung durch ein von der Forschungsabteilung beauftragtes Fremdunternehmen durchgeführt wird. Insofern bedürfte die Weiterleitung der Daten an das mit der Archivierung beauftragte Unternehmen keiner Einwilligung des Betroffenen.

\section{Gesetzliche Vorschriften hinsichtlich des Vertragsinhalts}

Die Tatsache, dass eine Forschungseinrichtung mit der Archivierung der Dateien ein Fremdunternehmen beauftragt, ändert zwar nichts an der Tatsache, dass die Forschungsstelle verarbeitende Stelle im Sinne der Datenschutzgesetze anzusehen ist. Dies folgt auf Bundesebene aus $₫ 11$ Abs. 1 BDSG, auf Landesebene aus \10 NDSG ${ }^{634}$.Somit ist zu klären, welche Voraussetzungen der Vertragsinhalt zwischen der archivierenden Einrichtung und dem Auftragnehmer hinsichtlich der Verarbeitung personenbezogener Daten enthalten muss. Zum einen ist daher zu prüfen, ob bestimmte Vorschriften direkt Vorgaben hinsichtlich des Vertragsinhaltes enthalten (dazu 1). Zum anderen ist jedoch auch denkbar, dass sich solche Anforderungen unter Umständen auch indirekt aus dem Gesetz ergeben (dazu b).

\section{Ausdrückliche Bestimmungen}

Zunächst ist zu prüfen, ob einzelne Datenschutzgesetze ausdrückliche Vorgaben für den Inhalt des Auftrags bzw. des Vertragsverhältnisses beinhalten. Dabei ist auch hier wieder danach zu differenzieren, ob das BDSG oder einzelne Landesdatenschutzgesetze Anwendung finden.

${ }^{633}$ \3 Abs. 8 S. 3 BDSG; \ 3 Abs. 5 LDSG B-W; Art. 4 Abs. 10 S. 2 BayDSG; \ 4 Abs. 3 Nr. 3 BlnDSG; \ 3 Abs. 4 Nr. 3 c) BBDSG; \ 2 Abs. 3 Nr. 3 BremDSG; \4 Abs. 4 HmbDSG; \ 2 Abs. 5 HDSG; § 3 Abs. 6 S. 2 LDSG M-V; \3 Abs. 4 S. 2 NDSG; \ 3 Abs. 4 S. 2 LDSG-NRW; \3 Abs. 4 S. 3 LDSG R-P; \3 Abs. 5 SDSG; \ 2 Abs. 9 S. 2 DSG-LSA; 22 Abs. 5 Nr. 3 LDSG S-H; $\ 3$ Abs. 6 S. 3 ThürDSG; nicht ganz eindeutig ist hingegen $₫ 3$ Abs. 4 SächsDSG

${ }^{634}$ Die übrigen Landesdatenschutzgesetze sehen dies in den $\iint 3$ Abs. 3 LDSG B-W; Art. 4 Abs. 9 BayDSG; IS 3 Abs. 1 S. 1 BlnDSG; 3 Abs. 4 Nr. 1 BBDSG; 2 Abs. 3 Nr. 1; 9 Abs. 1 BremDSG; 4 Abs. 3 HmbDSG; 2 Abs. 3 HDSG; 10 NDSG; 3 Abs. 3 LDSG NRW; 3 Abs. 3 LDSG R-P; 5 Abs. 1 SDSG; 2 Abs. 8; 8 Abs. 1 S. 1 DSG-LSA; 3 Abs. 3; 7 Abs. 1 S. 1 SächsDSG; 2 Abs. 3; 17 Abs. 1 LDSG S-H sowie 3 Abs. 5 ThürDSG vor. 
2. Vorschriften des BDSG

Sofern das BDSG Anwendung findet, würden sich direkte Vorgaben für den Vertragsinhalt zunächst aus $\int 11$ Abs. 2 S. 2 BDSG ergeben. Danach hat der Auftrag $^{635}$ zunächst schriftlich zu erfolgen. Darüber hinaus sind im Vertrag insbesondere die folgenden Punkte zu regeln:

1. Der Gegenstand und die Dauer des Auftrags,

2. der Umfang, die Art und der Zweck der vorgesehenen Erhebung, Verarbeitung oder Nutzung von Daten, die Art der Daten und der Kreis der Betroffenen,

3. die nach $\int 9$ zu treffenden technischen und organisatorischen Maßnahmen,

4. die Berichtigung, Löschung und Sperrung von Daten,

5. die nach Absatz 4 bestehenden Pflichten des Auftragnehmers, insbesondere die von ihm vorzunehmenden Kontrollen,

6. die etwaige Berechtigung zur Begründung von Unterauftragsverhältnissen,

7. die Kontrollrechte des Auftraggebers und die entsprechenden Duldungsund Mitwirkungspflichten des Auftragnehmers,

8. mitzuteilende Verstöße des Auftragnehmers oder der bei ihm beschäftigten Personen gegen Vorschriften zum Schutz personenbezogener Daten oder gegen die im Auftrag getroffenen Festlegungen,

9. der Umfang der Weisungsbefugnisse, die sich der Auftraggeber gegenüber dem Auftragnehmer vorbehält,

10. die Rückgabe überlassener Datenträger und die Löschung beim Auftragnehmer gespeicherter Daten nach Beendigung des Auftrags.

${ }^{635}$ Wobei der „Auftrag“ als Synonym für das Vertragsverhältnis zu verstehen ist und nicht als Auftrag i.S.d. $\int \$ 662$ ff. BGB. 
$\mathrm{Zu}$ beachten ist dabei, dass dieser Katalog nicht als abschließend anzusehen ist. Vielmehr stellen die genannten Punkte, wie aus der Formulierung „insbesondere“ deutlich wird, nur Regelbeispiele dar. Im Einzelfall kann es also durchaus erforderlich sein, weitere Punkte in den Vertrag aufzunehmen.

a. Direkte Vorgaben aufgrund landesdatenschutzrechtlicher Vorschriften Hinsichtlich der Verträge über die Auftragsdatenverarbeitung stellen die Landesdatenschutzgesetze unterschiedliche Anforderungen an den Inhalt, weshalb sie im Folgenden gesondert betrachtet werden sollen. Dabei kann jedoch vorab festgehalten werden, dass sämtliche Datenschutzgesetze für die Vereinbarung im Rahmen der Auftragsdatenverarbeitung die Schriftform vorschreiben.

i. Baden-Württembergisches Landesdatenschutzgesetz

Sofern im Einzelfall das LDSG B-W Anwendung findet, richtet sich der Inhalt des Vertrags zwischen datenverarbeitender Stelle und Auftragnehmer nach $\int 7$. Die Vorschrift sieht dabei in Abs. 2 S. 3 vor, dass insbesondere Gegenstand und Umfang der Datenverarbeitung, die notwendigen technischen und organisatorischen Maßnahmen, etwaige Unterauftragsverhältnisse sowie die Befugnis des Auftraggebers festzulegen sind, dass er hinsichtlich der Verarbeitung personenbezogener Daten dem Auftragnehmer Weisungen erteilen darf. Insofern bestehen also Parallelen zu den Voraussetzungen nach \11 BDSG.

\section{ii. Bayrisches Datenschutzgesetz}

Das BayDSG schreibt in Art. 6 Abs. 2 S. 2 vor, dass Datenerhebung, -verarbeitung oder -nutzung, die technischen und organisatorischen Maßnahmen und etwaige Unterauftragsverhältnisse in der Vereinbarung festzulegen sind. Weitere Voraussetzungen für den Inhalt der Vereinbarung enthält das Gesetz dagegen nicht.

\section{iii. Berliner Datenschutzgesetz}

Auch das Berliner Datenschutzgesetz stellt hinsichtlich des Inhalts der Vereinbarung im Vergleich zum BDSG relativ geringe ausdrückliche Anforderungen an den Vertragsinhalt. So ist gem. \3 Abs. 1 S. 3 BlnDSG der Gegenstand und der Umfang der Datenverarbeitung, der technischen und organisatorischen Maßnahmen und etwaiger Unterauftragsverhältnisse festzulegen.

iv. Brandenburgisches Datenschutzgesetz

Auch nach dem Brandenburgischen Datenschutzgesetz ist gem. $\int 11$ Abs. 2 S. 1 der Gegenstand, der Umfang der Datenverarbeitung, die technischen und organisatorischen Maßnahmen und etwaige Unterauftragsverhältnisse zu regeln. 
v. remisches Datenschutzgesetz

Das Bremische Datenschutzgesetz schreibt in $\int 9$ Abs. 1 S. 3 lediglich vor, dass in der Vereinbarung zwischen Auftraggeber und -nehmer die Datenverarbeitung, die technischen und organisatorischen Maßnahmen nach $\int 7$ und etwaige Unterauftragsverhältnisse festzulegen sind.

\section{vi. Hamburgisches Datenschutzgesetz}

Sofern das Hamburgische Datenschutzgesetz einschlägig ist, muss gem. \ 3 Abs. 1 S. 4 der Auftragnehmer dazu verpflichtet werden, die Daten nur zu dem Zweck zu verarbeiten, zu dem sie ihm überlassen worden sind, sowie nach Erledigung des Auftrags die überlassenen Datenträger zurückzugeben, zu löschen oder zu vernichten und gespeicherte personenbezogene Daten zu löschen, soweit nicht besondere Rechtsvorschriften entgegenstehen. Demzufolge muss die Vereinbarung eine Klausel beinhalten, durch die der Auftragnehmer entsprechend den genannten Vorgaben verpflichtet wird.

\section{vii. Hessisches Datenschutzgesetz}

Das Hessische Datenschutzgesetz regelt die Auftragsdatenverarbeitung in \4. Die Vorschrift besagt in Abs. 2 S. 2 Hs. 2, dass der Gegenstand und der Umfang der Datenverarbeitung, die technischen und organisatorischen Maßnahmen sowie etwaige Unterauftragsverhältnisse in der Vereinbarung mit dem Auftragnehmer festzulegen sind.

viii. Landesdatenschutzgesetz Mecklenburg-Vorpommern

Sofern das Datenschutzgesetz des Landes Mecklenburg-Vorpommern zur Anwendung kommt, hat die Vereinbarung mit dem Auftragnehmer gem. \4 Abs. 1 S. 4 die Art und den Umfang der Verarbeitung sowie erforderlichenfalls ergänzende Weisungen zu technischen und organisatorischen Maßnahmen und etwaige Unterauftragsverhältnisse zu enthalten.

ix. Niedersächsisches Datenschutzgesetz

Das Niedersächsische Datengesetz sieht in $\int 6$ Abs. 3 vor, dass in der Vereinbarung Weisungen zu technischen und organisatorischen Maßnahmen und die Zulassung von Unterauftragsverhältnissen festzuhalten sind.

x. Landesdatenschutzgesetz Nordrhein-Westfalen

Sollte im Einzelfall das Nordrhein-Westfälische Landesdatenschutzgesetz einschlägig sein, sind die ausdrücklichen Anforderungen an den Inhalt der Verarbeitung nicht sehr umfangreich. So verlangt \11 Abs. 1 S. 5 lediglich, dass ergänzende Weisungen zu technischen und organisatorischen Maßnahmen und etwaige 
Unterauftragsverhältnisse festzulegen sind, und dies auch nur dann, wenn es erforderlich sein sollte.

\section{xi. Landesdatenschutzgesetz Rheinland-Pfalz}

Das Rheinland-Pfälzische Landesdatenschutzgesetz schreibt in $\int 4$ Abs. 2 S. 2 hingegen zwingend vor, dass der Gegenstand und der Umfang der Datenverarbeitung, der technischen und organisatorischen Maßnahmen und etwaiger Unterauftragsverhältnisse festzulegen sind.

\section{xii. Landesdatenschutzgesetz des Saarlandes}

Sollte das Landesdatenschutzgesetz des Saarlandes Anwendung finden, würden sich die Anforderungen an den Inhalt der Vereinbarung zwischen Auftraggeber und Auftragnehmer nach $\int 5$ SDSG richten. Dieser setzt in Abs. 1 S. 3 ebenfalls voraus, dass Gegenstand und Umfang der Datenverarbeitung festzulegen sind. Darüber hinaus muss er gem. Abs. 1 S. 4 Weisungen zur Umsetzung der Vorgaben von $\int 11$ SDSG enthalten. Dieser regelt das Verfahren für die Fälle von automatisierten Datenverarbeitungen und schreibt unter anderem die Durchführung einer Vorabkontrolle hinsichtlich der Gefährdung des Rechts auf informationelle Selbstbestimmung und enthält außerdem Vorgaben für technische und organisatorische Maßnahmen ${ }^{636}$ im Rahmen der automatisierten Datenverarbeitung. Sofern der Auftragnehmer Subunternehmer beauftragen möchte, muss die Vereinbarung diesbezüglich gem. $\int 5$ Abs. 1 S. 6 ferner eine ausdrückliche Zustimmung beinhalten.

xiii. Datenschutzgesetz des Landes Sachsen-Anhalt

Auch nach dem Datenschutzgesetz des Landes Sachsen-Anhalt sind gem. \ 8 Abs. 2 S. 2 im Vertrag die Datenerhebung, -verarbeitung oder -nutzung, die technischen und organisatorischen Maßnahmen und etwaige Unterauftragsverhältnisse festzulegen.

xiv. Sächsisches Datenschutzgesetz

Ähnliche Voraussetzungen stellt das Sächsische Datenschutzgesetz. Gem. I 7 Abs. 2 S. 2 sind ebenfalls Gegenstand und Umfang der Datenverarbeitung, die notwendigen zusätzlichen personellen, technischen und organisatorischen Maßnahmen sowie etwaige Unterauftragsverhältnisse festzulegen.

xv. Landesdatenschutzgesetz Schleswig-Holstein

Das Schleswig-Holsteinische Landesdatenschutzgesetz sieht zum einen in $\int 17$ Abs. 3 vor, dass der Auftragnehmer dazu zu verpflichten ist, der datenverarbei-

${ }^{636}$ Allgemein zu technischen und organisatorischen Voraussetzungen siehe S. 163 ff. 
tenden Stelle jederzeit von ihr veranlasste Kontrollen zu ermöglichen, sofern der Auftragnehmer nicht in den Anwendungsbereich des LDSG S-H fällt. Zum anderen verlangt Abs. 5, dass der Auftragnehmer zu verpflichten ist, die Daten nur zu dem Zweck zu verarbeiten, zu dem sie ihm überlassen worden sind und nach Erledigung des Auftrags die ihm von der datenverarbeitenden Stelle überlassenen Datenträger zurückzugeben sowie gespeicherten Daten zu löschen, soweit nicht besondere Rechtsvorschriften entgegenstehen.

xvi. Thüringer Datenschutzgesetz

Auch das Thüringische Datenschutzgesetz sieht in $\ 8$ Abs. 2 S. 2 vor, dass in der Vereinbarung die Datenverarbeitung oder -nutzung, die technischen und organisatorischen Maßnahmen und etwaige Unterauftragsverhältnisse festzulegen sind. Darüber hinaus wird dazu geraten, Vereinbarungen für den Fall von festgestellten Verstößen des Auftragnehmers gegen den Datenschutz oder die Datensicherheit einzufügen, wie etwa Kündigungsklauseln. ${ }^{637}$

b. Indirekte Vorgaben für den Vertragsinhalt aufgrund datenschutzrechtlicher Bestimmungen

Neben den gesetzlich ausdrücklich vorgesehenen Punkten, die der Vertrag bzw. die Vereinbarung mit dem Auftragnehmer beinhalten muss, kann sich das Erfordernis bestimmter weiterer Vertragsinhalte auch indirekt aus datenschutzrechtlichen Normen ergeben. Dabei ist zunächst wieder danach zu unterscheiden, ob das Bundesdatenschutzgesetz oder eines der Landesdatenschutzgesetze Anwendung findet.

\section{i. Indirekte Vorgaben aufgrund einzelner Bestimmungen des BDSG}

Sofern das Bundesdatenschutzgesetz einschlägig ist, könnte sich das Bedürfnis für weitere Punkte, die zum Vertragsinhalt gemacht werden sollten, aus $₫ 7$ BDSG ergeben. Danach ist die datenverarbeitende Stelle dem Betroffenen zum Schadensersatz verpflichtet, sofern diesem durch eine nach den Vorschriften des BDSG oder nach anderen Vorschriften über den Datenschutz unzulässige oder unrichtige Erhebung, Verarbeitung oder Nutzung seiner personenbezogenen Daten ein Schaden entsteht. ${ }^{638}$ Diese Schadensersatzpflicht gem. \7 S. 2 BDSG besteht jedoch nur dann, wenn die datenverarbeitende Stelle die nach den Umständen des Falles gebotene Sorgfalt nicht beachtet hat. Zu dieser Sorgfalt gehört auch, die Datenverarbeitung durch den Auftragnehmer vertraglich hinreichend festzuhalten, so dass sich durch die Vorschrift des $₫ 7$ BDSG indirekt weitere Erfordernisse für den Vertragsinhalt ergeben. Zu beachten ist dabei jedoch, dass das BDSG, im Gegensatz zu den meisten Landesdatenschutzgesetzen, durch den

${ }^{637}$ Hinweise zum ThürDSG, Zu \8, 8.1.

638 Ausführlich zu den Rechtsfolgen einer unzulässigen Datenverarbeitung siehe oben S. 171. 
bereits oben dargestellten Katalog in $₫ 11$ Abs. 2 sehr konkrete und umfangreiche Vorgaben hinsichtlich des Vertragsinhalts enthält. Sofern die datenverarbeitende Stelle die dort genannten Punkte im Vertrag berücksichtigt, dürfte sie in der Regel die gebotene Sorgfalt beachtet haben. Nichtsdestotrotz sollte die datenverarbeitende Stelle eine Freistellungsklausel in den Vertrag aufnehmen, damit sie gegen den Auftragnehmer einen Anspruch auf Freistellung hat, sofern dieser gegen datenschutzrechtliche Bestimmungen verstößt. Dies gilt insbesondere vor dem Hintergrund, dass $\int 8$ BDSG für die Fälle eine verschuldensunabhängige Haftung der verantwortlichen öffentlichen Stelle vorsieht, in denen dem Betroffenen durch eine unzulässige oder unrichtige automatisierte Datenerhebung einen Schaden erleidet. Sofern es sich bei den archivierenden Einrichtungen um öffentlich-rechtliche Forschungsstellen wie Universitäten oder wissenschaftliche Akademien handelt und die Datenverarbeitung in einem automatisierten Verfahren erfolgt, wird diese dem Betroffenen demzufolge verschuldensunabhängig haften, da im Rahmen der elektronischen Langzeitarchivierung regelmäßig eine automatisierte Verarbeitung vorliegen wird. ${ }^{639}$

In der Regel stünde der datenverarbeitenden Stelle zwar auch ein Rückgriffsanspruch gem. \ 280 Abs. 1 BGB gegen den Auftragnehmer zu. ${ }^{640}$ Ein Freistellungsanspruch hätte demgegenüber jedoch den zusätzlichen Vorteil, dass der Auftragnehmer die datenverarbeitende Stelle bereits bei der Abwehr des Anspruchsbegehrens des Betroffenen unterstützen müsste. ${ }^{641}$ Aus diesem Grund sollten die archivierenden Einrichtungen entsprechende Freistellungsklauseln in die Verträge mit ihrem Auftragnehmer aufnehmen.

ii. Indirekte Vorgaben aufgrund landesdatenschutzrechtlicher Bestimmungen

Ebenso wie das BDSG enthalten auch die Landesdatenschutzgesetze Anspruchsgrundlagen auf Schadensersatz für den Fall, dass die datenverarbeitende Einrichtung gegen datenschutzrechtliche Bestimmungen verstößt und der Betroffene dadurch einen Schaden erleidet. Da die Landesdatenschutzgesetze diesbezüglich annähernd gleiche Bestimmungen enthalten, soll im Folgenden wieder exemplarisch das Niedersächsische Datenschutzgesetz betrachtet werden. Hinsichtlich der übrigen Landesdatenschutzgesetze sei auf die entsprechenden Angaben in den Fußnoten verwiesen. Wie oben bereits ausgeführt, ${ }^{642}$ steht dem Betroffenen gem. $\int 18$ NDSG ein Anspruch auf Schadensersatz zu, wenn ihm durch eine nach datenschutzrechtlichen Vorschriften unzulässige Verarbeitung seiner personenbezo-

\footnotetext{
639 Siehe S. 167 f.

${ }^{640}$ Aufgrund der in \ 280 Abs. 1 S. 2 BGB verankerten Beweislastumkehr müsste die datenverarbeitende Stelle dem Auftragnehmer in diesem Rahmen auch kein Vertretenmüssen nachweisen.

${ }^{641}$ BGH NJW 1970, 1594, 1595; NJW 1983 1729, 1730; NJW 2002, 2382.

642 Siehe dazu S. 177 f.
} 
genen Daten ein Schaden entsteht. ${ }^{643} \mathrm{Im}$ Gegensatz zu $\int 7$ BDSG sieht $\int 18$ NDSG jedoch nur im Rahmen der automatisierten Datenverarbeitung eine verschuldensunabhängige Haftung der datenverarbeitenden Stelle vor; im Falle einer nichtautomatisierten Datenverarbeitung besteht der Anspruch demzufolge nur dann, wenn die datenverarbeitende Stelle ein Verschulden trifft. ${ }^{644} \mathrm{Im}$ Falle der Auftragsdatenverarbeitung sind Ansprüche Dritter gem. \6 Abs. 1 S. 2 NDSG jedoch gegen die öffentliche Stelle geltend zu machen, die den Auftrag zur Datenverarbeitung erteilt hat. ${ }^{645}$ Der Auftragnehmer handelt im Außenverhältnis demzufolge nicht eigenverantwortlich, sondern als „verlängerter Arm“ der öffentlichen Einrichtung. ${ }^{646}$ Diese müsste demzufolge auch im Rahmen einer nicht automatisierten Datenverarbeitung haften, wenn nicht sie, sondern den Auftragnehmer ein Verschulden träfe. ${ }^{647}$ Unabhängig davon, ob es sich im Einzelfall um eine automatisierte Datenverarbeitung handelt oder nicht, sollte die datenverarbeitende Einrichtung daher auch in den Fällen, in denen nicht das BDSG, sondern das

${ }^{643}$ Sofern ein anderes Landesdatenschutzgesetz einschlägig ist, folgt der Schadensersatzanspruch des Betroffenen aus \25 Abs. 1 LDSG B-W; Art. 27 Abs. 1 und Abs. 2 BayDSG; $\$ 18$ BlnDSG (dieser sieht ebenfalls eine generell verschuldensunabhängige Haftung der datenverarbeitenden Stelle vor); \20 BBDSG; \23 Abs. 1 und Abs. 2 BremDSG; \20 Abs. 1 HmbDSG; \20 Abs. 1 HDSG, der ebenfalls in beiden Fällen kein Verschulden der datenverarbeitenden Stelle verlangt; \27 Abs. 1 und Abs. 2 DSG M-V; \20 Abs. 1 und Abs. 2 LDSG-NRW; \21 Abs.1 LDSG R-P; \24 Abs. 1 SDSG (der ebenfalls grundsätzlich eine verschuldensunabhängige Haftung vorsieht; \18 Abs. 1 und Abs. 2 DSG-LSA; \23 Abs. 1 SächsDSG (der die Haftung lediglich im Falle eines unabwendbaren Ereignisses entfallen lässt); \30 LDSG S-H (der einen Schadensersatzanspruch jedoch nur im Falle einer unzulässigen oder unrichtigen Datenverarbeitung in einem automatisierten Verfahren vorsieht); $\$ 18$ Abs. 1 ThürDSG (der die Haftung auch im Falle einer automatisierten Datenverarbeitung entfallen lässt, wenn die verantwortliche Stelle die erforderliche Sorgfalt beachtet hat).

${ }^{644}$ Von den in Fn. 643 zitierten landesrechtlichen Vorschriften sehen die Folgende hingegen einen anderen Verschuldensmaßstab vor: $\int \$ 25$ Abs. 1 LDSG B-W; 18 Abs. 1 BlnDSG; 20 Abs. 1 HDSG; 24 Abs. 1 SDSG, die auch im Falle einer nichtautomatisierten Datenverarbeitung eine verschuldensunabhängige Haftung der öffentlichen Stelle vorsehen; \20 Abs. 1 BBDSG, der die Haftung auch im Falle der automatisierten Verarbeitung entfallen lässt, wenn der verarbeitenden Stelle der Entlastungsbeweis gelingt; $\ 23$ Abs. 1 SächsDSG, der die Haftung im Falle eines unabwendbaren Ereignisses entfallen lässt; \30 LDSG S-H, der einen Schadensersatzanspruch nur im Falle einer unzulässigen oder unrichtigen Datenverarbeitung in einem automatisierten Verfahren vorsieht; $₫ 18$ Abs. 1 ThürDSG, der die Haftung auch im Falle einer automatisierten Datenverarbeitung entfallen lässt, wenn die verantwortliche Stelle die erforderliche Sorgfalt beachtet hat und ihr damit keine Fahrlässigkeit zur Last fällt.

${ }^{645}$ Dies gilt auch im Rahmen der anderen Landesdatenschutzgesetze. Siehe $\ 6$ Abs. 1 S. 2 i.V.m. $\ 5$ Abs. 1 S. 1 Nr. 5 LDSG B-W; Art. 6 Abs. 1 S. 2 BayDSG; \18 Abs. 1 S. 1 BlnDSG; $\int 20$ Abs. 1 S. 5 BBDSG; $₫ 9$ Abs. 1 S. 1 i.V.m. \1 Abs. 2 BremDSG; \3 Abs. 1 S. 1 HmbDSG; \ 4 Abs. 1 S. 1 HDSG; \4 Abs. 1 S. 2 LDSG M-V; \11 Abs. 1 S. 2 i.V.m. \5 S. 1 Nr. 5 LDSG-NRW; \ 4 Abs. 1 S. 2 i.V.m. \ 6 Abs. 1 Nr. 6 LDSG R-P; \ 24 Abs. 1 S. 1 i.V.m. \5 Abs. 1 S. 1 SDSG; \ 8 Abs. 1 S. 2 DSG-LSA; $₫ 8$ Abs. 1 S. 2 SächsDSG; $₫ 17$ Abs. 1 S. 2 LDSG S-H; $₫ 8$ Abs. 1 S. 2 ThürDSG.

${ }^{646}$ Kommentar zum NDSG, \ 6 Zu Abs. 1 Nr. 1.

${ }^{647}$ Vgl. auch Kommentar zum NDSG, \6 Zu Abs. 1 Nr. 3. 
NDSG Anwendung findet, in den Vertrag mit dem Auftragnehmer eine Freistellungsklausel einfügen, aufgrund derer sie gegebenenfalls beim Auftragnehmer Regress nehmen kann.

Durch eine derartige Klausel könnte die archivierende Einrichtung sicherstellen, nicht für eine rechtswidrige Verarbeitung der Daten durch den Auftragnehmer in Anspruch genommen zu werden, ohne bei diesem Rückgriff nehmen zu könne. Die Vereinbarung einer solchen Freistellungsklausel macht auch dann Sinn, wenn der Auftragnehmer ein Langzeitarchivierungsverbund ist, an dem die archivierende Einrichtung in der tragenden Rechtsform des Verbundes beteiligt sein sollte. Da dieser wohl in der Rechtsform einer GmbH oder UG organisiert wäre, würde für seine Verbindlichkeiten nämlich nur das Gesellschaftsvermögen haften, ${ }^{648}$ so dass die beteiligten Einrichtungen selbst nicht mit hohen Schadensersatzansprüchen konfrontiert würden.

\section{Ergebnis}

Sowohl im Rahmen des BDSG als auch der einzelnen Landesdatenschutzgesetze muss die Vereinbarung zwischen archivierender Stelle und dem Auftragnehmer auf jeden Fall den Gegenstand und Umfang der Datenverarbeitung, die notwendigen technischen und organisatorischen Maß-nahmen, etwaige Unterauftragsverhältnisse sowie die Befugnis des Auftraggebers festlegen. Dabei ist zu beachten, dass diese Aufzählung nicht abschließend ist, sondern im Einzelfall noch weitere Regelungspunkte hinzutreten können. Dies gilt insbesondere im Anwendungsbereich des BDSG, welches diesbezüglich in $\ 11$ Abs. 2 einen ausführlichen Katalog enthält. Aber auch im Rahmen der Landesdatenschutzgesetze ist zu beachten, dass die Anforderungen an den Inhalt, wie oben gezeigt, teilweise divergieren. Im Zweifel sollte sich die archivierende Einrichtung daher an dem Katalog des $\ 11$ BDSG orientieren. Darüber hinaus ist zu beachten, dass dem Betroffenen im Falle einer unrechtmäßigen Verarbeitung seiner Daten ein Schadensersatzanspruch zusteht, den er im Falle der Auftragsdatenverarbeitung gegen die den Auftrag erteilende Stelle zu richten hat, da diese „Verantwortliche“ im datenschutzrechtlichen Sinne bleibt. Aus diesem Grund sollte die archivierende Einrichtung in ihren Vertrag mit dem Auftragnehmer eine Freistellungsklausel aufnehmen. Das Bedürfnis einer solchen Klausel ergibt sich ferner aus der verschuldensunabhängigen Haftung der archivierenden Einrichtung, die das BDSG sowie die Mehrzahl der Landesdatenschutzgesetze im Rahmen einer automatisierten Datenverarbeitung vorsehen.

${ }^{648}$ Siehe $₫ 13$ Abs. 2 GmbHG 



\section{Kapitel 3 - Haftungsrechtliche Probleme}

Neben der bereits oben behandelten Haftung für eine widerrechtliche Nutzung personenbezogener Daten, stellt sich im Rahmen der elektronischen Langzeitarchivierung wissenschaftlicher Primärdaten weiter die Frage, welche haftungsrechtlichen Probleme sich beispielsweise bei einem Datenverlust oder allgemein einer Datenbeschädigung ergeben können. Relevant wird dies vor allem dann, wenn die Langzeitarchivierung nicht durch die archivierende Einrichtung selbst durchgeführt, sondern an ein Fremdunternehmen ausgelagert wird. Dabei ist zunächst entscheidend, ob wissenschaftliche Einrichtungen generell verpflichtet sind, die von ihnen erlangten Daten zu archivieren (dazu A.), da in diesem Fall ein Datenverlust unter Umständen eine gesetzliche Haftung der archivierenden Einrichtung auslösen würde. Darüber hinaus ist für die archivierenden Einrichtungen aus haftungsrechtlicher Sicht entscheidend, inwieweit sie die Authentizität und Integrität der Daten sicherstellen müssen (dazu B.). Schließlich stellt sich die Frage, wer für den Schaden haften muss, der durch einen Datenverlust im Rahmen von Archivierungsmaßnahmen entsteht (dazu D.). 


\section{A. Rechtliche Pflicht zur Langzeitarchivierung und Verantwortlichkeit}

Sofern eine Pflicht zur Archivierung bestimmter Daten an der Universität bzw. den universitären Einrichtungen besteht, stellt sich die Frage, wer für die Durchführung dieser Archivierung verantwortlich ist. Gesetzliche Aufbewahrungspflichten finden sich dabei vor allem im Bereich der Buchführung ${ }^{649}$, bei Personalsachen $^{650}$, bei Bankunterlagen ${ }^{651}$, Akten der Verwaltung ${ }^{652}$, Gerichtsakten ${ }^{653}$ und für medizinische Dokumentationen ${ }^{654}$. Im Rahmen des vorliegenden Sachverhaltes sind dabei vor allem Aufbewahrungs- und Archivierungspflichten von medizinischen Dokumentationen relevant, da diese auf Daten, die innerhalb der medizinischen Fakultät gewonnen werden, Anwendung finden können. In einem ersten Schritt ist somit zu prüfen, welche Archivierungspflichten für Daten aus dem Bereich der medizinischen Fakultät bestehen. Im Anschluss daran ist festzustellen, ob sich die Verantwortlichkeit für die Langzeitarchivierung dieser Daten aus diesen Vorschriften ergibt und falls dies nicht der Fall ist, wie die Verantwortlichkeit dann bestimmt werden kann.

Eine Archivierungs- und Aufbewahrungspflicht für medizinische Dokumentationen ergibt sich dabei zum einen aus $\int 28$ Röntgenverordnung (RöntgV), \ 42 Strahlenschutzverordnung (StrlSchV), $\int 1$ Gentechnikaufzeichnungsverordnung (GenTAufzV) sowie aus der standesrechtlichen Regelung des $\int 10$ Berufsordnung der Ärztekammer Niedersachsen (BerufsO Ärzte Niedersachsen). Darüber hinaus folgt aufgrund des Persönlichkeitsrechts des Patienten eine ärztliche Dokumentationspflicht als Nebenpflicht aus dem Arzt- oder Krankenhausvertrag. ${ }^{655}$

\section{Dokumentationspflicht gem. \28 RöntgV}

Gem. \ 28 Abs. 3 Satz 2 RöntgV sind Röntgenbilder zehn Jahre lang nach der letzten Untersuchung aufzubewahren. Dabei muss jedoch nicht das Original aufbewahrt werden. Vielmehr sieht der Wortlaut von $\ 28$ Abs. 4 RöntgV vor, dass die Röntgenbilder auch als Wiedergabe auf einem Bildträger oder auf anderen Datenträgern aufbewahrt werden können, wenn sichergestellt ist, dass die Wieder-

\footnotetext{
${ }^{649} \mathrm{Vgl}$. bspw. $\iint 238 \mathrm{ff}$. HGB.

${ }^{650}$ Vgl. bspw. \623 BGB, \ 2 Abs. 1 Satz 3 NachwG.

651 Vgl. bspw. $\int 34 \mathrm{WpHG}$.

652 Vgl. bspw. $\int 29 \mathrm{VwVfG}$.

653 Vgl. bspw. $\int 298$ a ZPO.

${ }^{654} \mathrm{Vgl}$. bspw.S 28 Abs. 4 RöntgV; siehe auch Roßnagel/Fischer-Dieskau/Jandt/Knopp, Langfristige

Aufbewahrung elektronischer Dokumente, 2007, S. 154 f.

655 BGH NJW 1978, 2337, 2338 f., Roßnagel/Fischer-Dieskau/Jandt/Knopp, S. 85;Roßnagel/Schmücker, Beweiskräftige elektronische Archivierung, 2006, S. 27.
} 
gaben der Daten mit den Bildern bildlich übereinstimmen und während der Dauer der Aufbewahrungsfrist verfügbar sind und jederzeit innerhalb angemessener Zeit lesbar gemacht werden können. Demzufolge besteht zwar keine direkte Pflicht, wohl aber die Möglichkeit die Bilder in digitaler Form zu archivieren. Damit stellt sich weiter die Frage, wer für die Archivierung der Röntgenbilder verantwortlich ist. Gem. $\int 15$ Abs. 1 Nr. 4 RöntgV hat der Strahlenschutzverantwortliche dafür zu sorgen, dass die Vorschrift des $₫ 28$ Abs. 1 bis 3 Satz 1 und 2 sowie Abs. 4 bis 6 RöntgV eingehalten werden. Darüber hinaus hat auch der Strahlenschutzbeauftragte gem. $\int 15$ Abs.2 Nr.1 RöntgV dafür zu sorgen, dass die in Abs. 1 Nr. 4 genannten Vorschriften, wozu auch \28 Abs. 4 RöntgV zählt, eingehalten werden. Wer Strahlenschutzverantwortlicher und Strahlenschutzbeauftragter ist, ergibt sich dabei aus $₫ 13$ Abs. 1 RöntgV. Strahlenschutzverantwortlicher ist danach, wer einer Genehmigung nach $\ 3$ bzw. $\ 5$ bedarf oder wer eine Anzeige nach $\ 4$ zu erstatten hat. Sofern es sich bei dem Strahlenschutzverantwortlichen um eine juristische Person handelt, werden seine Aufgaben gem. \13 Abs. 1 Satz 2 RöntgV von der durch Gesetz, Satzung oder Vertrag zur Vertretung berechtigten Person wahrgenommen.

Der Strahlenschutzbeauftragte wird dagegen gem. \13 Abs. 2 RöntgV vom Strahlenschutzverantwortlichen bestellt. Die Vertreter müssen jedoch nicht persönlich für die Einhaltung der in $\ 15$ Abs. $1 \mathrm{Nr}$. 4 RöntgV und damit für die Dokumentation der Röntgenbilder gem. \28 Abs. 4 RöntgV zu sorgen. Vielmehr schreibt $\int 15$ Abs. 1 RöntgV vor, dass dies insbesondere durch geeignete Regelungen des Betriebsablaufs und durch Bereitstellung ausreichenden und geeigneten Personals zu erfolgen hat. Demzufolge sind beispielsweise der Vorstand der Universitätsmedizin, sowie die von ihm ernannten Strahlenschutzbeauftragten, dafür verantwortlich, dass die in der Klinik erstellten Röntgenbilder gem. \28 Abs. 3 Satz 2 RöntgV aufbewahrt werden, was gem. \ 28 Abs. 4 RöntgV auch durch die Wiedergabe auf einem Datenträger mithin einer digitalen Archivierung oder auch Langzeitarchivierung erfolgen kann. Eine ausdrückliche Pflicht zur elektronischen Archivierung besteht hingegen nicht.

\section{Dokumentationspflicht gem. \42 Strahlenschutzverordnung (StrlSchV)}

Eine weitere Dokumentationspflicht ergibt sich aus $₫ 42$ StrlSchV. ${ }^{656}$ Danach sind die Aufzeichnungen nach den $\$ ₫ 40$ und 41 so lange aufzubewahren, bis die überwachte Person das 75. Lebensjahr vollendet hat oder vollendet hätte, mindestens jedoch 30 Jahre nach Beendigung der jeweiligen Beschäftigung. Gem. \42 Abs. 1 Satz 2 StrlSchV sind die Aufzeichnungen jedoch spätestens 95 Jahre nach der Geburt der betroffenen Person zu löschen. Gem. \33 Abs. 1 Nr. 2 b) bb) aaa) StrlSchV hat dabei der Strahlenschutzverantwortliche unter Beachtung des Standes von Wissenschaft und Technik zum Schutz des Menschen und der Umwelt

${ }^{656}$ So auch Scblund in Laufs/Kern, \59 Rn. 3. 
vor den schädlichen Wirkungen ionisierender Strahlung durch geeignete Schutzmaßnahmen, insbesondere durch Bereitstellung geeigneter Räume, Ausrüstungen und Geräte, durch geeignete Regelung des Betriebsablaufs und durch Bereitstellung ausreichenden und geeigneten Personals dafür zu sorgen, dass die Vorschrift des $\ 42$ Abs. 1 StrlSchV eingehalten wird. Ähnlich wie bei der oben angesprochenen Pflicht zur Aufbewahrung von Röntgenbildern besteht also auch im Rahmen des $\ 42$ Abs. 1 Satz 1 StrlSchV zwar keine Pflicht, die Aufzeichnungen elektronisch aufzubewahren. Allerdings werden die Aufzeichnungen heutzutage wohl in den meisten Fällen in elektronischer Form vorliegen. Es besteht somit eine, wenn auch zeitlich begrenzte Pflicht zur Archivierung der Daten. Verantwortlicher ist gem. $\int 33$ Abs. 1 StrlSchV der Strahlenschutzverantwortliche, der den Betriebsablauf entsprechend zu regeln und entsprechendes Personal zur Verfügung zu stellen hat. Strahlenschutzverantwortlicher im Rahmen der StrlSchV ist dabei, wer einer Genehmigung nach $₫ 7$ StrlSchV bedarf. Dies wird in der Regel der Träger der Einrichtung sein, in der mit ionisierenden Strahlen gearbeitet wird. $\mathrm{Da}$ es sich dabei grundsätzlich um eine juristische Person handeln wird, werden die Aufgaben des Strahlenschutzverantwortlichen gem. \31 Abs. 1 Satz 2 StrlSchV durch deren Organe wahrgenommen. Demzufolge sind diese auch für die Archivierung der Aufzeichnungen verantwortlich.

III. Dokumentationspflicht gem. \1 der Verordnung über Aufzeichnungen bei gentechnischen Arbeiten oder über Freisetzungen (GenTAufzV)

Eine Aufzeichnungs- und Aufbewahrungspflicht besteht ferner gem. \1 GenTAufzV für denjenigen, der gentechnische Arbeiten oder Freisetzungen durchführt. Die inhaltlichen Anforderungen an diese Aufforderungen sind in $\ 2$ GenTAufzV geregelt. Die Form der Aufzeichnungen ist in $\ 3$ der Verordnung geregelt. Dieser sieht in Absatz 2 Satz 1 auch die Möglichkeit einer Aufzeichnung auf einem Bildträger oder anderen Datenträgern vor. Die Dauer der Aufbewahrungspflicht hängt dabei gem. $\ 4$ GenTAufzV von der Sicherheitsstufe der durchgeführten Arbeiten ab und liegt zwischen zehn und dreißig Jahren jeweils nach Beendigung der gentechnischen Arbeiten oder der Freisetzungen. Demnach besteht für die Aufzeichnungen eine Pflicht zur Aufbewahrung. Damit stellt sich, wie auch schon bei den vorangegangenen Normen, die Frage, wer für die Archivierung verantwortlich ist. Die Aufbewahrungspflicht gem. \1 GenTAufzV richtet sich seinem Wortlaut nach an denjenigen, der „...gentechnische Arbeiten oder Freisetzungen durchführt...". Dies wären in den vorliegenden Fällen also die Forschungseinrichtungen, die Forschungen im Bereich der Gentechnik durchführen und die durch ihre Organe handeln. Auch hier gilt aber, dass die Organe der 
Einrichtungen die Archivierung nicht persönlich vornehmen müssen, sondern durch Bereitstellung des notwendigen Personals und der erforderlichen Infrastruktur der Archivierungspflicht nachkommen können. ${ }^{657}$

IV. Dokumentationspflicht gem. \10 Berufsordnung der Ärztekammer Niedersachsen

Eine weitere allerdings berufsständische Dokumentationspflicht im medizinischen Bereich folgt aus den Berufsordnungen der Ärztekammern, wie beispielsweise der Berufsordnung der Ärztekammer Niedersachsen. Gem. \10 Abs. 1 BerufsO Ärzte Niedersachsen haben Ärzte über die in Ausübung ihres Berufes gemachten Feststellungen und getroffenen Maßnahmen die erforderlichen Aufzeichnungen zu machen. Dabei sieht Abs. 3 der Norm eine Aufbewahrungspflicht von zehn Jahren vor, soweit nicht nach gesetzlichen Vorschriften eine längere Aufbewahrungspflicht besteht. Darüber hinaus können sich längere Fristen vor allem daraus ergeben, dass die Informationen noch für spätere Behandlungen des Patienten benötigt werden. ${ }^{658}$ Bei Aufzeichnungen auf elektronischen Datenträgern oder anderen Speichermedien sind zwar grundsätzlich zulässig, bedürfen gem. \10 Abs. 5 Satz 1 der Berufsordnung aber besonderer Sicherungs- und Schutzmaßnahmen, um deren Veränderung, Vernichtung oder unrechtmäßige Verwendung zu verhindern. Auch hierbei handelt es sich demnach nicht um eine spezielle Pflicht zur elektronischen Archivierung, sondern einer Pflicht, überhaupt zu archivieren. Dabei ist festzuhalten, dass die Berufsordnung nicht nur Anwendung auf niedergelassene Ärzte Anwendung findet. Vielmehr stellt $\int 22$ der Berufsordnung klar, dass ihre Regeln auch für Ärzte gelten, welche ihre ärztliche Tätigkeit im Rahmen eines privatrechtlichen Arbeitsverhältnisses oder öffentlichrechtlichen Dienstverhältnisses ausüben. Die Berufsordnung findet demzufolge auch auf Ärzte Anwendung, die in öffentlichen Krankenhäusern angestellt sind. Diese sind somit gem. $\int 10$ BerufsO Ärzte Niedersachsen standesrechtlich dazu verpflichtet, die in Ausübung ihres Berufes erhobenen Daten aufzubewahren. Im Rahmen von Daten, die durch ärztliche Untersuchungen gewonnen werden, sind demzufolge die Ärzte für eine Archivierung verantwortlich. Diese Verantwortlichkeit erstreckt sich zwangsläufig auch auf eine elektronische Archivierung, welche heutzutage wohl auch eher den Regelfall als die Ausnahme darstellen wird.

\section{Dokumentationspflicht als Nebenpflicht}

Darüber hinaus besteht eine Dokumentationspflicht in Form einer Nebenpflicht. Diese wird zum Teil aus dem Krankenhausaufnahme- bzw. Arztvertrag, teilweise auch aus dem Persönlichkeitsrecht des Patienten und aus der ärztlichen Behandlungspflicht hergeleitet. Nach Auffassung der Rechtsprechung handelt es sich der

${ }^{657}$ Vgl. BGH NJW 1980, 2810, 2811; Schwarz/Schöpfl in Bamberger/Roth, Bd. 1, \ 31 Rn. 14

${ }_{658}$ Roßnagel/Fischer-Dieskau/Jandt/Knopp, S. 85; Roßnagel/Schmücker, S. 86. 
Dokumentations- und Aufbewahrungspflicht um eine ,selbstverständliche therapeutische Pflicht" ${ }^{\text {".59 }}$ Dabei stellt die Rechtsprechung an die Form der Dokumentation keine speziellen Anforderungen, so dass auch eine digitale Dokumentation zur Erfüllung der Nebenpflicht zulässig ist.660 Die Pflicht zur Dokumentation trifft dabei zum einen den behandelnden Arzt, zum anderen auch das Klinikum als Vertragspartner des Krankenhausaufnahmevertrages. ${ }^{661}$ Darüber hinaus wird in Rechtsprechung und Literatur die überwiegende Auffassung vertreten, dass unabhängig vom Vorliegen eines Behandlungsvertrags eine allgemeine Verpflichtung zur Dokumentation des Behandlungsgeschehens sowie pflegerischer Maßnahmen besteht. ${ }^{662}$

\section{Ergebnis}

Eine Dokumentationspflicht für die vorliegend im Mittelpunkt stehende wissenschaftlichen Primärdaten kann sich demnach vor allem aus $\ 28$ Abs. 4 RöntgV, $\mathbb{S}$ 42 StrlSchV sowie der aus dem Krankenhausaufnahmevertrag abgeleiteten Dokumentationspflicht ergeben. Darüber hinaus folgt aus $\ 1$ GenTAufzV eine Aufzeichnungspflicht für den Fall, dass gentechnische Arbeiten oder Freisetzungen durchgeführt werden. Dabei ist festzuhalten, dass es sich bei diesen Dokumentationspflichten nicht speziell um Pflichten zu einer digitalen Langzeitarchivierung handelt. Allerdings werden die Einrichtungen die betreffenden Daten in den meisten Fällen aufgrund des allgemeinen technischen Fortschrittes in digitaler Form und, wie oben aufgezeigt, für einen längeren Zeitraum speichern, so dass von einer digitalen Langzeitarchivierung dieser Daten gesprochen werden kann. Verantwortlich für die Dokumentation von Röntgenbildern und damit auch, sofern sie durchgeführt wird, für eine digitale Archivierung dieser Daten, sind zum einen je nach Rechtsform der Vorstand bzw. Geschäftsführer des Krankenhauses, zum anderen der Strahlenschutzbeauftragte. Für die Archivierung von Behandlungsund Untersuchungsdaten ist daneben auch der jeweilige behandelnde Arzt verantwortlich.

\footnotetext{
${ }^{659}$ BGH NJW 1978, 2337, 2338 f.; 1986, 2365; 1988, 762, 763; 1987, 1482, 1483.

660 Schlund in Laufs/Kern, \ 55 Rn. 11; Ortner/Geis, MedR 1997, 337, 338.

661 BGH NJW 1988, 762, 763; Kern, MedR 2000, 347, 348.

662 BGH NJW 1986, 2365, 2366; 1988, 762; OLG Zweibrücken MedR 2000, 233, 235; Ublenbruck/Schlund in Laufs/Uhlenbruck, \59 Rn. 2; Kern, MedR 2000, 347, 348; Höftberger, MedR 2000, 505, $508 \mathrm{f}$.
} 


\section{B. Sicherstellung der Authentizität und Integrität der archivierten Daten}

Des Weiteren stellt sich aus haftungsrechtlicher Sicht die Frage, ob die archivierende Einrichtung die Authentizität und Integrität ihrer archivierten Daten sicherstellen muss. Dabei ist zunächst festzuhalten, dass das Gesetz diesbezüglich keine expliziten Regelungen bereit hält. ${ }^{663}$

Etwas anderes ergibt sich hingegen bei der Archivierung personenbezogener Daten. Wie oben bereits festgestellt, haben öffentliche und nicht-öffentliche Stellen, die selbst oder im Auftrag personenbezogene Daten erheben, verarbeiten oder nutzen, die technischen oder organisatorischen Maßnahmen zu treffen, die erforderlich sind, um die Ausführung der Vorschriften der Datenschutzgesetze zu gewährleisten. Welche Maßnahmen dies im Einzelnen sind, ergibt sich dabei insbesondere aus der Anlage zu \9 Satz 1 BDSG. Dieser bezieht sich seinem Wortlaut nach zwar nur auf die automatisierte Nutzung oder Verarbeitung personenbezogener Daten. Selbst wenn ausnahmsweise aber doch eine manuelle Datenverarbeitung vorliegen sollte, könnte die Anlage zu \9 BDSG entsprechende Anwendung finden.664 Darüber hinaus enthalten die Landesdatenschutzgesetze ebenfalls vergleichbare Vorschriften. ${ }^{665}$ Damit ist die archivierende Einrichtung zumindest bei personenbezogenen Daten dazu verpflichtet die Integrität und Authentizität sicherzustellen.

Aber auch bei Daten ohne Personenbezug ergibt sich die Notwendigkeit einer Sicherstellung der Integrität und Authentizität zumindest aus wissenschaftlicher Sicht, da die archivierten Daten für andere Forschungseinrichtungen nahezu wertlos wären, wenn nicht sichergestellt wäre, dass diese nicht manipuliert wurden bzw. eine solche Manipulation für den Verwender der Daten nicht erkennbar wäre. Letztlich ist es ja gerade das Ziel einer Archivierung, die archivierten Werke in ihrem -zumindest inhaltlich- ursprünglichen Zustand zu erhalten. ${ }^{666}$

Zum anderen ist zu beachten, dass die archivierende Einrichtung unter Umständen für Schäden haften müsste, die aufgrund manipulierter Daten bei anderen Forschungseinrichtungen entstehen. Als Anspruchsgrundlage käme dabei vor allem \280 Abs. 1 BGB in Betracht, da zwischen der archivierenden Einrichtung und der Empfängerin der Daten regelmäßig ein Schuldverhältnis in Form eines Vertrags bestehen wird. Sofern die archivierende Einrichtung fehlerhafte Daten zur Verfügung stellen würde, läge darin eine Pflichtverletzung nach

\footnotetext{
${ }^{663}$ Roßnagel/Fischer-Dieskau/S. Jandt/M. Knopp, S. 107.

664 Vgl. Ernestus in Simitis, $\int 9$ Rn. 18.

665 Siehe dazu S. $168 \mathrm{ff}$.

${ }^{666}$ Rojnagel/Fischer-Dieskau/S. Jandt/M. Knopp, S.44.
} 
\280 Abs. 1 S. 1 BGB. Die Universität müsste gem. \280 Abs. 1 BGB für daraus entstehende Schäden allerdings nur dann haften, wenn sie die Pflichtverletzung zu vertreten hätte. Dies liegt gem. \276 I BGB grundsätzlich bei vorsätzlichem oder fahrlässigem Handeln vor. Demzufolge könnte die archivierende Einrichtung unter Umständen einer Haftung entgehen, wenn die Daten von ihr entsprechend gegen eine Manipulation gesichert worden wären oder eine Manipulation für den Empfänger zumindest erkennbar war. Damit stellt sich die Frage, welchen Anforderungen eine solche Verschlüsselung entsprechen müsste. Um die Integrität und Authentizität von Daten sicher zu stellen, sind verschiedene Verfahren denkbar. Dabei kann man zwischen systembezogenen und organisatorischen Sicherungsmitteln, datenträgerbezogenen sowie dokumentbezogenen Sicherungsmitteln unterscheiden. 667

\section{Systembezogene und organisatorische Sicherungsmittel}

Zunächst kann die Integrität und Authentizität der Daten dadurch sichergestellt werden, dass das Archiv so konfiguriert wird, dass nur ein bestimmter Personenkreis auf die Daten zugreifen kann. ${ }^{668}$ Des Weiteren sind auch Beschränkungen der Zugriffsfunktionen möglich. So können die archivierten Dateien anderen Forschungseinrichtungen beispielsweise dergestalt zugänglich gemacht werden, dass diese sie nur lesen oder herunterladen, die Dateien jedoch nicht ändern können. ${ }^{669}$ Darüber hinaus können auch integrierte Protokollierungssysteme als Sicherungsmittel eingesetzt werden. Diese zeichnen auf, wann und vor allem wer auf Dateien im Archiv zugegriffen hat. ${ }^{670}$ Darüber hinaus besteht ferner die Möglichkeit zur Speicherung solche Datenträger zu verwenden, die lediglich einmal beschreibbar sind.

\section{Datenträgerbezogene Sicherungsmittel}

Des Weiteren besteht die Möglichkeit, zur Archivierung Speichermedien zu verwenden, die lediglich einmal beschreibbar sind, so dass eine Veränderung der Daten nach der Speicherung nicht mehr möglich ist. Bei optischen Medien, wie etwa CD-ROM oder DVD wird die Oberfläche bei einer Speicherung dabei irreversibel verändert. Bei Festplattensystemen kann eine einmalige Beschreibbarkeit hingegen durch den Einsatz spezieller Software erreicht werden. ${ }^{671}$

${ }^{667}$ Roßnnagel/Fischer-Dieskau/S. Jandt/M. Knopp, S. 23.

${ }^{668}$ Dies ist bei der Archivierung personenbezogener Daten schon aufgrund der einschlägigen Bestimmungen der Datenschutzgesetze erforderlich, siehe oben S. $165 \mathrm{ff}$.

${ }^{669}$ Roßnagel/Fischer-Dieskau/S. Jandt/M. Knopp, S. 24.

${ }^{670}$ Roßnagel/Fischer-Dieskau/S. Jandt/M. Knopp, S. 24.

${ }^{671}$ Roßnagel/Fischer-Dieskau/S. Jandt/M. Knopp, S. 24. 


\section{Dateibezogene Sicherungsmittel}

Schließlich ist es auch möglich, hinsichtlich Sicherung der Integrität und Authentizität bei den archivierten Dateien selbst anzusetzen. So können die Dateien derart verschlüsselt werden, dass sie von Unbefugten gar nicht erst gelesen werden können. Durch eine Verschlüsselung kann jedoch auch der Schutz vor einer Manipulation der Daten erreicht und damit deren Integrität und Authentizität gesichert werden. Da keine ausdrücklichen Regelungen für diese Fälle bestehen, könnten als Anhaltspunkte für eine sichere Verschlüsselung bereits existierende Vorschriften dienen, die sich auf elektronisch verschlüsselte Dokumente beziehen. Dies sind vor allem die SS 126a BGB und 371a ZPO. Soll die gesetzlich vorgeschriebene Schriftform durch eine elektronische Form ersetzt werden, so muss der Aussteller das Dokument mit einer qualifizierten elektronischen Signatur im Sinne des Signaturgesetzes versehen. Sollen elektronische Dokumente im Zivilprozess die Beweiskraft privater Urkunden haben, so verlangt auch \371a ZPO, dass diese Dokumente mit einer qualifizierten elektronischen Signatur versehen sind. Demnach wird eine qualifizierte elektronische Signatur vom Gesetzgeber als sicher genug erachtet, um derart verschlüsselte elektronische Dokumente als Ersatz für schriftliche Dokumente, sei es im Rahmen der Abgabe einer Willenserklärung oder als Beweisstück vor Gericht, zuzulassen. Auch wenn die archivierten Daten in der Regel nicht zur prozessualen Beweissicherung dienen werden, kann durch eine Signatur die Authentizität und Integrität der Daten und damit das Vertrauen Dritter in die Datenbestände gesichert werden. ${ }^{672}$

\section{Voraussetzungen einer qualifizierten elektronischen Signatur}

Eine qualifizierte elektronische Signatur wird in $\int 2$ Nr.3 SigG definiert als ,elektronische Signatur nach $\int 2$ Nr. 2 SigG, die auf einem zum Zeitpunkt ihrer Erzeugung gültigen qualifizierten Zertifikat beruht und mit einer sicheren Signaturerstellungseinheit erzeugt wurde“. Der Inhalt des gültigen Zertifikates wird wiederum von $\int 7$ SigG vorgeschrieben. Aus dem Zertifikat geht wiederum die Identität des Inhabers der Signaturschlüssels hervor, $\int 7$ Abs. 1 Nr. 1 SigG.

Wird eine Datei elektronisch signiert, so wird sie mit Hilfe eines Algorithmus verschlüsselt (sog. „Private Key“). Für das Entschlüsseln wird wiederum ein anderer Schlüssel verwandt (sog. Public Key) wobei man aus dem einen nicht den anderen Schlüssel errechnen kann. ${ }^{673}$ Durch Verwendung des öffentlichen Schlüssels kann der Empfänger der Datei feststellen, ob die Datei verändert wurde. Damit bietet eine elektronische Signatur zwar keinen Schutz vor einer Veränderung der Datei. Allerdings stellt sie sicher, dass der Verwender der Datei durch Verwenden des öffentlichen Schlüssels feststellen kann, dass die Datei verändert worden ist.

\footnotetext{
672 Roßnagel/Fischer-Dieskau/S. Jandt/M. Knopp, S.108; Roßnnagel/Schmücker, Beweiskräftige elektronische Archivierung, 2006, S. 21.

${ }^{673}$ Beutelspacher/Schwenk/Wolfenstetter, 2005, S. 6 ff.; Roßßnagel/Fischer-Dieskau/S. Jandt/M. Knopp, S.25.
} 
Im Unterschied zu einer symmetrischen Verschlüsselung, bei der zur Ver- und Entschlüsselung derselbe Schlüssel verwandt wird, erfolgt die Erstellung einer elektronischen Signatur somit asymmetrisch. Wird ein Dokument vom Versender (hier also der archivierenden Einrichtung) signiert, so wird aus der Datei ein sog. Hash-Wert gebildet. Dieser wird mit Hilfe des sog. Private Keys verschlüsselt und bildet die Signatur. Der Private Key bleibt auch für den Verwender unbekannt und ist auf einer Chipkarte gespeichert, die von einem sog. Trust Center erstellt wird. Möchte der Empfänger der Datei (in den vorliegenden Fällen also beispielsweise die Forschungseinrichtung, die auf die archivierten Dateien zugreift) deren Integrität überprüfen, so bildet auch er aus dem empfangenen Dokument den sog. Hashwert. Anschließend entschlüsselt er die Signatur mit Hilfe des Public Keys, der für jeden Netzteilnehmer jederzeit abrufbar ist. Nach der Entschlüsselung liegen dem Empfänger zwei Hash-Werte vor. Zunächst der Hash-Wert, den der Empfänger durch Komprimierung des empfangenen Dokumentes erlangt hat. Des Weiteren der Hash-Wert des Ausgangsdokumentes, den er mit Hilfe des Public-Keys entschlüsselt hat. Wurde das Ausgangsdokument nicht verändert, sind die beiden Hash-Werte identisch, andernfalls weichen sie voneinander ab. Somit kann der Empfänger überprüfen, ob der von ihm heruntergeladene Datensatz zwischenzeitlich verändert wurde. Das Signieren der Dateien könnte dabei zum einen von der für die Archivierung zuständigen Stelle oder direkt von den Forschungsprojekten vorgenommen werden, die die Daten im Rahmen ihrer wissenschaftlichen Tätigkeit ermittelt haben. Insofern bestünde die Möglichkeit, die zu archivierenden Dateien vor der Archivierung zu signieren.

\section{Lebensdaner der Signatur vs. Archivierungsdauer}

Problematisch ist an der Verwendung einer elektronischen Signatur jedoch, dass die zur Schlüsselung verwendeten Algorithmen und Parameter nur für einen begrenzten Zeitraum verwendet werden können. Damit würde für Dateien, deren Archivierung und Signatur weit in der Vergangenheit liegt, die Gefahr bestehen, dass die Verschlüsselungstechnik unterlaufen wird und die Dateien trotz der Signatur unbemerkt verändert werden können. Zur Lösung dieses Problems bietet es sich auch hier wieder an, die gesetzlichen Regelungen für elektronische Signaturen als Orientierungspunkte zu Hilfe zu nehmen. So sind Daten mit einer qualifizierten elektronischen Signatur gem. $\int 17 \mathrm{Satz} 1 \mathrm{SigV}$ neu zu signieren, wenn diese für längere Zeit in signierter Form benötigt werden, als die für ihre Erzeugung und Prüfung eingesetzten Algorithmen oder der zugehörigen Parameter geeignet sind. Bevor die Neusignatur erfolgt, müssen die Daten gem. \ 17 Satz 3 SigV unter Einbeziehung aller bereits bestehenden Signaturen mit einem qualifizierten Zeitstempel versehen werden. Die neue Signatur muss gem. \17 Satz $3 \mathrm{SigV}$ mit geeigneten neuen Algorithmen oder zugehörigen Parametern erfolgen, frühere Signaturen einschließen und einen qualifizierten Zeitstempel tragen. Ein solcher Zeitstempel ist gem. $\int 2 \mathrm{Nr}$. $14 \mathrm{SigG}$ die von einem qualifizierten Zertifizierungs- 
dienstanbieter ausgestellte elektronische Bescheinigung darüber, dass ihm die betreffenden elektronischen Daten zu einem bestimmten Zeitpunkt vorgelegen haben. Werden dabei Zeitstempel eingesetzt, die eine qualifizierte elektronische Signatur beinhalten, so ist eine weitere Signatur nicht notwendig, da diese nicht zu einer erhöhten Sicherheit führt. ${ }^{674} \mathrm{Die}$ neue Signatur hat gem. \17 Satz $2 \mathrm{SigV}$ vor dem Zeitpunkt des Ablaufs zu erfolgen. Eine Übersicht der sicherheitsgeeigneten Algorithmen und die zugehörigen Parameter sowie der Zeitpunkt, bis zu dem die Eignung jeweils gilt, veröffentlicht die Bundesnetzagentur im Bundesanzeiger. ${ }^{675}$

\section{Ergebnis}

Eine generelle Pflicht für die Gewährleistung der Integrität und Authentizität der archivierten Daten besteht demzufolge nicht.Etwas anderes gilt jedoch im Rahmen der Archivierung personenbezogener Daten. Hier wird man zumindest eine Pflicht der archivierenden Einrichtung zur Anbringung einer elektronischen Signatur annehmen müssen. Diese folgt im Anwendungsbereich des BDSG aus der Anlage zu $\int 9$ Nr. 5 BDSG, da hiernach gewährleistet werden muss, dass eine Veränderung personenbezogener Daten festgestellt werden kann. Im Anwendungsbereich des BDSG wird die Anlage zu \9 dabei regelmäßig einschlägig sein, da im Rahmen der elektronischen Langzeitarchivierung grundsätzlich eine automatisierte Datenverarbeitung vorliegt. Die Landesdatenschutzgesetze enthalten ebenfalls eine solche Verpflichtung, so dass eine elektronische Signatur auch dann erforderlich ist, wenn nicht das BDSG, sondern ein Landesdatenschutzgesetz einschlägig ist.

Ein praktisches Bedürfnis der Gewährleistung der Integrität und Authentizität der Daten besteht jedoch auch bei nicht personenbezogenen Daten aufgrund der Tatsache, dass die archivierten Daten andernfalls aus wissenschaftlicher Sicht wertlos wären, da ein Vertrauen in ihre Integrität konsequenterweise nicht bestünde. Darüber hinaus könnten sich unter gewissen Umständen auch haftungsrechtliche Probleme für die archivierende Einrichtung ergeben, sofern Dritten aufgrund verfälschter Daten ein Schaden entstünde. Die Gewährleistung einer solchen Integrität kann dabei zum einen durch datei- als auch durch systembezogene Sicherungsmaßnahmen erreicht werden. Welche Methode dabei angewandt wird, hängt letztlich vom jeweils aktuellen technischen Standard ab. Da aber nie ausgeschlossen werden kann, dass derartige Sicherungen nicht doch umgangen werden können, sollten auch diese Daten zusätzlich mit einer qualifizierten elektronischen Signatur nach dem Signaturgesetz versehen werden, wie sie beispielsweise auch \ $126 a$ BGB oder $\ 371$ a ZPO verlangen. Aufgrund des großen und im Laufe der

\footnotetext{
${ }^{674}$ Roßnagel/Schmücker, S. 29; Roßnagel/Pordesch in Roßnagel, A. (Hrsg.): Recht der Multimediadienste, \ $17 \mathrm{SigV}, \mathrm{Rn} .54$.

${ }^{675}$ Roßnagel/Fischer-Dieskau/S. Jandt/M. Knopp, S.63
} 
Jahre stetig anwachsenden Datenvolumens wird die nach $₫ 17 \mathrm{SigV}$ erforderliche regelmäßige Neusignierung indes sehr zeitaufwendig sein, weshalb die archivierende Einrichtung ein System einrichten sollte, welches die Signaturen automatisch erneuert und mit einem qualifizierten Zeitstempel versieht.

\section{Haftung für Datenfehler, die im Rahmen der Archivierung verursacht werden}

Neben den rechtlichen Beziehungen der archivierenden Einrichtung zu Nutzungsrechteinhabern, Betroffenen von personenbezogenen Daten und Dritten, die archivierte Daten von ihr beziehen, sind für die Archivierung außerdem Fragen hinsichtlich der Haftung für einen Datenverlust oder eine Datenbeschädigung von Interesse.

\section{Umsetzung der einzelnen Arbeitsschritte}

Neben der Frage, welche Maßnahmen in den oben dargestellten Arbeitsschritten vorgenommen werden, kommt es für die Frage der Haftung vor allem darauf an, von wem diese Arbeitsschritte ergriffen werden.

Dabei ist zum einen denkbar, dass die archivierende Einrichtung die oben dargestellten Arbeitsschritte vollständig selbst übernimmt. Denkbar wäre aber auch, dass Dritte mit der Wahrnehmung einzelner oder sogar sämtlicher Arbeitsschritte betraut werden. In diesem Zusammenhang stellt sich jedoch die Frage, wer haften muss, wenn im Rahmen der oben genannten Arbeitsschritte Fehler auftreten. Darüber hinaus sind Fälle denkbar, in denen ein Datenverlust nicht aufgrund menschlichen Versagens eintritt, sondern aufgrund mangelhaften Materials, wie etwa defekten Festplatten oder anderen Speichermedien. In diesen Fällen ist zunächst entscheidend, ob der archivierenden Einrichtung überhaupt ein bezifferbarer Schaden entstanden ist und sofern dies der Fall ist, wer für diesen haftet.

Dabei ist danach zu differenzieren, ob der Fehler durch einen Dritten verursacht worden ist (dazu 1.) oder durch interne Fehler im Rahmen der archivierenden Einrichtung (dazu 2.).

\section{Arcbivierung durch Dritte}

Zunächst ist denkbar, dass der Datenfehler durch innere Einflüsse verursacht wird. Verursacht werden kann dies durch menschliches Fehlverhalten (dazu a.), wie beispielsweise dem versehentlichen Löschen eines Datensatzes oder durch Materialfehler (dazu b.), wobei Letzteres auch wiederum auf menschliches Fehlverhalten, wie etwa unterlassener Wartungsarbeiten zurückzuführen sein kann. 
a. Datenfehler aufgrund menschlichen Fehlverhaltens

Ein Datenfehler kann zunächst durch menschliches Fehlverhalten entstehen. Unter Berücksichtigung der einzelnen Archivierungseinheiten und der ihnen zufallenden Aufgaben ist dabei zunächst denkbar, dass eine Datei vollständig gelöscht wird. Dies wäre insbesondere im Rahmen des „Ingest“ denkbar, wo die SIPs in Empfang genommen werden, bevor sie von dort an das „Data Management“ und das „Archival Storage“ weitergesendet werden. Darüber hinaus wäre aber auch denkbar, dass die Daten zwar nicht beschädigt werden, ihnen jedoch die falsche Identifikationsdatei zugeordnet wird und sie dadurch nicht mehr oder nur noch schwer auffindbar sind.

Darüber hinaus könnte ein Datenverlust auch im Rahmen der Migration oder durch Fehler bei der Erneuerung der Hardware durch das „Archival Storage“ entstehen. Tritt ein Datenfehler auf, stellt sich die Frage, wer für diesen Fehler haftet und vor allem gegenüber wem. Dabei ist danach zu differenzieren, ob die Universität die Archivierung selbst vornimmt, oder ob die Archivierung oder zumindest Teile davon an außenstehende Firmen ausgelagert werden.

Sofern die Universität die Archivierung oder zumindest Teile davon an außenstehende Dritte übertragen würde, läge dem ein Vertrag zugrunde, kraft dessen sich der Dritte zur Archivierung beziehungsweise zu einzelnen Arbeitsschritten, die zur Archivierung erforderlich sind, gegen die Zahlung einer Vergütung verpflichten würde. Sofern Datenfehler bei dem Dritten auftreten, käme damit eine Haftung des Dritten gegenüber der Universität gem. \ 280 Abs. 1 BGB in Betracht. Danach kann der Gläubiger Ersatz für den durch die Pflichtverletzung des Schuldners entstandenen Schaden verlangen. Insofern wäre im Einzelfall zu prüfen, ob die Tatbestandsvoraussetzungen des $\ 280$ Abs. 1 Satz 1 BGB vorliegen.

\section{i. Schuldverhältnis}

Zunächst müsste dafür ein Schuldverhältnis vorliegen. Darunter wird nach allgemeiner Ansicht ein Rechtsverhältnis verstanden, das für die einzelnen Parteien Rechte und Pflichten begründet. ${ }^{676}$ Ein solches Schuldverhältnis stellt der Vertrag zwischen der archivierenden Einrichtung und der Fremdfirma dar. Der genaue Vertragstyp kann dabei von Fall zu Fall divergieren. Denkbar sind aber vor allem Dienst-, Werk- oder auch Mietverträge bzw. typengemischte Verträge, die einzelne Elemente der genannten Vertragstypen enthalten. ${ }^{677}$ Sofern es sich bei dem Vertragsverhältnis um einen Werkvertrag handeln sollte, ist zu beachten, dass

${ }^{676}$ Grüneberg in Bamberger/Roth, Bd. 1, \241 Rn. 3 f.; Kramer in Münchener Kommentar BGB, Bd. 2, 5. Aufl. 2007, Einl. Rn. 13.

677 Vgl. Scbmidt in Redeker (Hrsg.), Handbuch der IT-Verträge, Bd. 1, 1.5 Rn. 6; Karger in Redeker, Bd. 1, 1.8 Rn. 4 f; 1.9 Rn. 21 f. 
in diesen Fällen das spezielle Mängelgewährleistungsrecht der $\iint 634$ ff. BGB einschlägig wäre. In diesen Fällen fände $\int 280$ Abs. 1 BGB jedoch über die Verweisnorm des $₫ 634$ Nr. 4 BGB Anwendung678.

\section{ii. Pflichtverletzung}

Des Weiteren müsste der Dritte eine Pflicht aus diesem Vertrag verletzt haben. Die Pflichten, die aus dem Schuldverhältnis folgen, richten sich dabei nach $₫ 241$ Abs. 1 und Abs. 2 BGB. Nach Abs. 1. folgen die Pflichten der Parteien aus dem Schuldverhältnis. In dem Vertrag zwischen der Universität und dem Dritten wird dabei regelmäßig vereinbart sein, dass die Datensätze in einem entsprechenden Zustand erhalten werden. Sofern der Dritte mit der eigentlichen Datenaufbewahrung („Archival Storage“) betraut wird, wäre die Erhaltung der Daten sogar eine Hauptleistungspflicht. Dazu gehört auch die Zuordnung der richtigen Metadaten und der richtigen Identifikationsdatei. Sofern der Dritte mit dem „Ingest“ oder dem Datenmanagement („Administration“) betraut würde, wäre auch dies eine Hauptleistungspflicht. Selbst wenn dies nicht der Fall sein sollte, so stellt der Erhalt der Daten zumindest eine aus $\ 241$ Abs. 2 BGB folgende Treuepflicht dar, wonach die Parteien zur Rücksichtnahme auf die Rechte, Rechtsgüter und Interessen des anderen Teils verpflichtet sind. Die Beschädigung oder sogar Löschung von Daten stellt damit eine Pflichtverletzung im Sinne des $₫ 280$ Abs. 1 Satz 1 BGB dar.

\section{iii. Vertretenmüssen}

Der Vertragspartner müsste diese Pflichtverletzung ferner zu vertreten haben. Dabei bestimmt $\int 276$ BGB, dass der Schuldner Vorsatz und Fahrlässigkeit zu vertreten hat, wenn eine strengere oder mildere Haftung weder bestimmt noch aus dem sonstigen Inhalt des Schuldverhältnisses zu entnehmen ist. Fahrlässig handelt gem. \276 Abs. 2 BGB, ,wer die im Verkehr erforderliche Sorgfalt außer Acht lässt"“. Es kommt also darauf an, dass der Vertragspartner den Datenfehler aufgrund mindestens fahrlässigen Verhaltens herbeigeführt hat.

In diesem Zusammenhang ist zu beachten, dass im Falle eines Prozesses nicht die archivierende Einrichtung das Beweisrisiko hinsichtlich des Vertretenmüssens treffen würde. Vielmehr müsste der Schuldner, hier also den für die Archivierung zuständige Vertragspartner, aufgrund der in $\ 280$ Abs. 1 Satz 2 BGB vorgesehenen Beweislastumkehr beweisen, dass er die Pflichtverletzung gerade nicht zu vertreten hat. Um trotzdem im Falle eines Datenfehlers- oder Verlustes abschätzen zu können, ob ein Anspruch nach $\ 280$ Abs. 1 BGB besteht, soll im Folgenden darauf eingegangen werden, unter welchen Umständen ein Vertretenmüssen vorläge. Dabei ist zu beachten, dass der Vertragspartner die notwendigen Arbeiten

${ }^{678}$ Ggf. in Verbindung mit $\ 283$ S.1 sofern die Nacherfüllung unmöglich ist. 
zumeist nicht selbst vornehmen wird sondern sich dazu seiner Angestellten bedient. Damit stellt sich aber ferner die Frage, ob und wie ihm deren Fehlverhalten zuzurechnen ist.

\section{(1) Eigenes Vertretenmüssen des Vertragspartners}

Dabei kommt zum einen ein eigenes Vertretenmüssen des Vertragspartners in Betracht, wenn dieser seine Angestellten nicht sorgfältig ausgesucht oder den Betriebsablauf nicht sorgfältig organisiert hat. Ist dies der Fall, so hat er die im Verkehr erforderliche Sorgfalt außer Acht gelassen und damit fahrlässig gehandelt, so dass er die Pflichtverletzung zu vertreten hätte. Sofern es sich bei dem Vertragspartner um eine juristische Person handelt, würde dieser ein solches Organisationsverschulden ihres Vorstands in analoger Anwendung des \31 BGB als eigenes Verschulden zur Last fallen. ${ }^{679}$

\section{(2) Vertrtetenmüssen der Angestellten}

Sofern der Vertragspartner seine Angestellten jedoch unter Beachtung der erforderlichen Sorgfalt ausgesucht hat, kommt eine Zurechnung des Vertretenmüssens des Angestellten über $\int 278$ BGB in Betracht. Danach hat der Schuldner ein Verschulden der Personen, deren er sich zur Erfüllung seiner Verbindlichkeit bedient, in gleichem Umfang zu vertreten wie eigenes Verschulden. Handelt ein Angestellter des Vertragspartners also fahrlässig oder vorsätzlich und hat dieser damit die Pflichtverletzung zu vertreten, so würde dieses Vertretenmüssen gem. \278 BGB dem Vertragspartner zugerechnet werden, so dass dieser die Beschädigung des Datensatzes zu vertreten hätte.

\section{(3) Haftungsbeschränkung}

Da der Schuldner gem. \276 Abs. 1 BGB Vorsatz und Fahrlässigkeit nur dann zu vertreten hat, wenn eine strengere oder mildere Haftung nicht bestimmt ist, könnte der Vertragspartner versuchen, sein Vertretenmüssen durch entsprechende Klauseln in seinen Allgemeinen Geschäftsbedingungen (AGB) möglichst weit zu begrenzen. Damit stellt sich die Frage, inwieweit dies möglich ist und wann derartige Klauseln unwirksam wären. Die Wirksamkeit von AGB richtet sich dabei grundsätzlich nach den \$S 307 ff. BGB. Da es sich bei der Universität Göttingen um eine Stiftung und damit um eine juristische Person öffentlichen Rechts handelt, finden die $\iint 308$ und 309 BGB auf AGB, die ihr gegenüber gestellt werden, jedoch gem. \ 310 Abs. 1 Satz 1 BGB keine Anwendung. Vielmehr sind diese AGB nur anhand des $\int 307$ Abs. 1 und Abs. 2 BGB zu prüfen, wobei die $\iint 308$ und 309 BGB jedoch eine gewisse Indizwirkung für eine unangemessene Benach-

${ }^{679}$ Ellenberger in Palandt, $\ 31$ Rn. 2, 3; Schwary/Schöpfl in in Bamberger Roth, Bd. 1, \31 Rn. 1, 3; 
teiligung des Vertragspartners entfalten können. ${ }^{680}$ Zunächst ist festzuhalten, dass eine Freizeichnung für eigenes vorsätzliches Handeln des Vertragspartners schon gem. \276 Abs. 3 BGB unwirksam wäre. Es stellt sich damit die Frage, inwieweit der Vertragspartner die Haftung für eigenes fahrlässiges Verhalten sowie fahrlässiges und vorsätzliches Verhalten seiner Erfüllungsgehilfen einschränken kann.

(a) Ausschluss der Haftung für grob fahrlässiges Verhalten

Unter grob fahrlässigem Verhalten versteht man das außer Acht lassen der im Verkehr erforderlichen Sorgfalt in besonders schwerem und ungewöhnlich hohem Maße. ${ }^{681}$ Die Wirksamkeit einer Klausel, wonach der Vertragspartner grob fahrlässiges Verhalten nicht zu vertreten hat, hängt von $₫ 307$ Abs. 1 und Abs. 2 BGB ab. Bestimmungen in AGB sind gem. $\ 307$ Abs.1 Satz 1 BGB unwirksam, „wenn sie den Vertragspartner des Verwenders entgegen den Geboten von Treu und Glauben unangemessen benachteiligen". Eine solche unangemessene Benachteiligung ist in den vorliegenden Fällen zumindest dann anzunehmen, wenn sich der IT-Dienstleister für eigenes grob fahrlässiges Verhalten freizeichnen möchte. ${ }^{682}$

Dies folgt mittelbar aus $\ 309 \mathrm{Nr}$. 7b BGB, welcher hier gem. \310 Abs. 1 BGB zwar keine direkte Anwendung findet, jedoch eine Indizwirkung für eine unangemessene Benachteiligung der archivierenden Einrichtung entfaltet und damit eine Unwirksamkeit eines solchen Haftungsausschlusses nach $\ 307$ Abs. 1 BGB zur Folge hat. Hinsichtlich des Ausschlusses der Haftung für grob fahrlässiges Verhalten der Mitarbeiter des Vertragspartners würde dies zumindest auch für leitende Angestellte gelten. ${ }^{683}$ Der Ausschluss der Haftung für eine grob fahrlässige Pflichtverletzung durch den Erfüllungsgehilfen wäre zumindest dann unwirksam, wenn dieser auch für Verstöße gegen vertragliche Hauptpflichten gelten sollte. ${ }^{64}$ Eine Freizeichnung für grob fahrlässiges Handeln des IT-Dienstleisters oder seiner Mitarbeiter ist damit in den meisten Fällen gem. \307 Abs. 2 BGB unwirksam.

${ }^{680}$ BGH NJW 1984, 1750, 1751; 1988, 1785, 1788; ZIP 1986; 653, 656; Ulmer in Ulmer/Brandner/Hensen, AGB-Recht, $₫ 310$ Rn. 27; Berger in Prütting/Wegen/Weinreich, $₫ 310$ Rn. 3; Basow in MüKo, $₫ 310$ Rn. 7.

${ }^{681}$ BGH NJW 1988, 1265, 1266; Unberath in Bamberger/Roth, Bd. 1, \276 Rn. 19; Schmidt-Kessel in Prütting/Wegen/Weinreich, $\ 276$ Rn. 20; siehe auch die Legaldefinition in $₫ 45$ Abs. 2 Satz 3 Nr. 3 SGB X.

${ }_{682}$ BGH NJW 1985, 623, 627; NJW-RR 1988, 1437, 1438; 1989, 953, 955; NJW 1999, 942, 943 f.; Fuchs in Ulmer/Brandner/Hensen, \ 307 Rn. 285; \ 310 Rn. 33; Basedow in Münchener Kommentar, Bd. 2, \309 Nr. 7 Rn. 34; Grüneberg in Palandt, \309 Rn. 55; Spindler in Spindler (Hrsg.), Vertragsrecht der Internet-Provider, 2. Aufl. 2004, Teil IV Rn. 330

${ }^{683}$ BGH NJW 1996, 1407; NJW 1985, 914, 916; Becker in Bamberger/Roth, Bd. 1, § 309 Nr. 7 Rn. 47.

${ }^{684}$ BGH NJW 1984, 1350, 1351; 1985, 623, 627; NJW-RR 1989, 953, 955; NJW 1999, 942, 943 f. 
(b) Beschränkung der Haftung auf grobe Fahrlässigkeit und Vorsatz

Ferner könnte der Vertragspartner durch seine AGB die Haftung auf grob fahrlässige Pflichtverletzungen und Vorsatz beschränken wollen, so dass er im Falle von „einfach“ fahrlässigen Pflichtverletzungen durch sich selbst oder einen seiner Angestellten nicht haften müsste. Ein solcher Haftungsausschluss wäre jedoch im nichtkaufmännischen Verkehr gem. \ 309 Nr. 7 b) BGB unwirksam, sofern die Verletzung des Körpers, des Lebens oder der Gesundheit von der Freizeichnung nicht ausdrücklich ausgeschlossen wären. Dabei ist allgemein anerkannt, dass dies im unternehmerischen Geschäftsverkehr ebenso gilt. ${ }^{685}$

Sofern die Verletzung der genannten Rechtsgüter jedoch vom Haftungsausschluss nicht erfasst wäre, würde die Klausel nicht gegen $\int 309 \mathrm{Nr} .7$ a) und b) $\mathrm{BGB}$ verstoßen, so dass dieser nicht als Indiz für eine unangemessene Benachteiligung herangezogen werden könnte. Die Unwirksamkeit einer solchen Klausel könnte sich indes aus $\int 307$ Abs. 2 BGB ergeben. Dieser enthält Regelbeispiele für eine unangemessene Benachteiligung im Sinne des Abs. 1.686 Dabei könnte eine solche Haftungsbeschränkung die Einschränkung wesentlicher Rechte oder Pflichten darstellen, die sich aus der Natur des Vertrages ergeben. Wie oben bereits dargestellt, wird bei der Beauftragung eines Dritten mit der Langzeitarchivierung oder zumindest einzelnen Arbeitsschritten, die dazu erforderlich sind, zumeist ein typengemischter Vertrag vorliegen. In Betracht kommt aber beispielsweise auch ein reiner Mietvertrag gem. \535 BGB, sofern die Universität lediglich Speicherplatz für die archivierten Dateien anmietet. Welche Leistungen von dem Begriff der wesentlichen Pflichten erfasst sind, hängt also von dem konkreten Vertrag ab. Bei einer genauen Betrachtung der höchstrichterlichen Rechtsprechung zu derartigen Freizeichnungen, stellt man fest, dass der BGH ein Verbot der Freizeichnung für die Haftung bei der Verletzung von sogenannten Kardinalpflichten entwickelt hat. ${ }^{687}$ Auch nach der Schuldrechtsreform scheint die Rechtsprechung im Rahmen der $\iint 307$ ff. BGB an diesen Grundsätzen festzuhalten. ${ }^{688}$ Dabei ist zwar zu beachten, dass in den entschiedenen Fällen oftmals $\int 309$ Nr. 7 BGB direkt Anwendung fand, da die Klausel gegenüber Verbrauchern gestellt wurde. Im Rahmen des Haftungsausschlusses für die Verletzung wesentlicher Pflichten differenziert die Rechtsprechung allerdings kaum zwischen dem

\footnotetext{
685 OLG München BB 1993, 1753; OLG Hamm NJW-RR 1996, 969; Grüneberg in Palandt, \ 309 Rn. 48; Stoffels, AGB-Recht, 2. Aufl. 2009, Rn. 980.

${ }^{6} 86$ BGHZ 153, 148, 155; Grüneberg in Palandt, $₫ 307$ Rn. 28; K.P. Berger in Prütting/Wegen/Weinreich, \ 307 Rn. 18; Fuchs in Ulmer/Brandner/Hensen, \307 Rn. 3.

${ }^{687}$ BGH NJW 1956, 1065, 1066; ; BGH NJW 1985, 914, 916; 1165, 1166; 1993, 335; NJW-RR 1993, 560; NJW 1994, 1060, 1063; 1999, 1031, 1032 unter II 1 NJW-RR 2000, 998; NJW 2001, 292, 302 unter XV 2 b.; NJW-RR 2001, 342; NJW 2002, 673, 674; Fuchs in Ulmer/Brandner/Hensen, § 307 Rn. 288; vgl. auch BGHZ 65, 364, 367.

688 BGH NJW 2005, 422, 424; 2005, 1774 unter II.1.; NJW-RR 2005, 1496, 1505 f; Fuchs in Ulmer/Brandner/Hensen, \307 Rn. 289.
} 
Geschäftsverkehr mit Verbrauchern und dem mit Unternehmern. ${ }^{699}$ Insofern ist davon auszugehen, dass die Haftungsfreizeichnung, sofern sie auch die Verletzung wesentlicher Pflichten erfasst, unwirksam ist. Eine Freizeichnung für „einfach“ fahrlässige Pflichtverletzungen nicht wesentlicher Pflichten wäre demnach möglich. Zu beachten ist jedoch, dass die AGB des Vertragspartners die Haftungsbeschränkung ausdrücklich auf unwesentliche Pflichten beziehen müssten. Eine pauschale Haftungsfreizeichnung, wonach der Vertragspartner ,lediglich Vorsatz und grobe Fahrlässigkeit zu vertreten hat", würde hingegen unwirksam sein. Zum einen würde unter eine solche Klausel auch die gerade angesprochene Verletzung wesentlicher Pflichten fallen. Zum anderen würde eine solche Klausel auch eine Haftungsbeschränkung für Schäden aufgrund der Verletzung des Lebens, des Körpers oder der Gesundheit umfassen. Aufgrund des Verbotes der geltungserhaltenden Reduktion von AGB, welches aus $\$ 305$ c Abs. 2 BGB folgt, wäre eine solche Klausel insgesamt unwirksam, so dass auch eine Haftungsbeschränkung für eine fahrlässig verursachte Verletzung von nicht wesentlichen Nebenpflichten von der Unwirksamkeit erfasst würde. ${ }^{690}$ Der Vertragspartner könnte sich ferner auch nicht durch eine sog. salvatorische Klausel behelfen, wonach die Haftung nur ausgeschlossen ist, „,soweit der Ausschluss gesetzlich zulässig ist“ “691

\section{(c) Zwischenergebnis}

Eine Haftungsbeschränkung durch den Vertragspartner auf grob fahrlässige Pflichtverletzungen und Vorsatz ist damit wohl nur hinsichtlich nicht wesentlicher Pflichten möglich. Sofern eine haftungsbeschränkende Klausel einen generellen Haftungsausschluss für leicht fahrlässiges Verhalten vorsieht, ohne die Verletzung wesentlicher Pflichten sowie die Verletzung des Lebens, des Körpers oder der Gesundheit von der Haftungsbefreiung auszunehmen, wäre die Klausel insgesamt unwirksam. Sofern die Pflichtverletzung darin besteht, dass der Vertragspartner Daten, die er archivieren bzw. zum Zwecke einer Archivierung bearbeiten soll, beschädigt oder sogar löscht, wird es sich aber grundsätzlich um die Verletzung einer wesentlichen Pflicht handeln, da der Hauptzweck des Vertrages die ordnungsgemäße Archivierung bzw. Bearbeitung der Daten oder Bereitstellung von Speicherplatz ist.

\footnotetext{
${ }^{689}$ Als Beispiele für das Verbot der Freizeichnung im unternehmerischen Geschäftsverkehr siehe nur BGH NJW 1985, 3016; NJW-RR 1988, 559; NJW 1993, 335; NJW-RR 1993, 560.

${ }^{690}$ BGHZ 93, 29, 48; Spindler in Spindler, Teil IV Rn. 342.

691 BGHZ 93, 29 ,48; NJW 1996, 1407.; LG Köln, CR 2003, 697, 698; Basedow in Münchener Kommentar BGB, Bd. 2, 5. Aufl. 2007, \ 305 Rn. 71; \306 Rn. 29; Schmidt in Bamberger/Roth, Bd. 1, \306 Rn. 17; Spindler in Spindler, Teil IV Rn. 335.
} 


\section{iv. Kausaler Schaden}

Sofern die oben genannten Voraussetzungen vorliegen, muss der IT-Dienstleister der archivierenden Einrichtung den durch die Datenlöschung oder -beschädigung entstandenen Schaden ersetzen. Ein solcher ist grundsätzlich gegeben, wenn sich bei einer Subtraktion des Wertes des (hypothetischen) Vermögens, welches ohne das schädigende Ereignis bestünde, von dem Wert des tatsächlichen Vermögens, eine negative Differenz entsteht. ${ }^{692}$ Der Schaden würde darüber hinaus kausal auf der Pflichtverletzung beruhen, wenn diese nicht hinweg gedacht werden kann, ohne dass der Schaden in seiner konkreten Form entfiele ${ }^{693}$ und der Eintritt des Schadens als Folge der Pflichtverletzung ferner nicht völlig unwahrscheinlich ist und außerhalb der allgemeinen Lebenserfahrung liegt. ${ }^{694}$ Sofern nicht nur einzelne Daten, sondern ganze Datenbanken gelöscht würden, kämen als Schaden zunächst entgangenen Einnahmen in Betracht, die die archivierende Einrichtung durch den kostenpflichtigen Download der Daten durch andere Forschungseinrichtungen erzielt hätte.

Sofern die Daten hingegen für einen unentgeltlichen Abruf bestimmt gewesen sind, wäre das Vorliegen eines Schadens allerdings schwer nachweisbar. Insbesondere könnten in diesem Zusammenhang nicht die Kosten herangezogen werden, die für die Erhebung der Daten angefallen sind. Diese Kosten wären nämlich auch dann entstanden, wenn die Daten nicht gelöscht worden wären, weshalb es am erforderlichen Kausalzusammenhang fehlt. Allerdings könnte die archivierende Einrichtung die Kosten für eine erforderliche Rekonstruktion bzw. die Neuerhebung der Daten als Schaden geltend machen. ${ }^{695}$ Als Schaden kämen schließlich noch Ansprüche Dritter in Betracht, die diesen aufgrund der Löschung gegen die archivierende Einrichtung zustehen. Zu denken sind dabei insbesondere an datenschutzrechtliche Schadensersatzansprüche, sofern es sich bei den gelöschten Daten um solche mit Personenbezug handelt. ${ }^{696}$

\section{v. Schadensausgleich}

Im Rahmen des Schadensausgleichs, der sich nach den $\iint 249 \mathrm{ff}$. BGB richtet, ist zu beachten, dass dieser nach dem Grundsatz der sogenannten „Naturalrestitution“ erfolgt. Danach bestimmt \249 Abs. 1, dass der zum Schadensersatz Verpflichtete den Zustand wiederherzustellen hat, der bestehen würde, wenn der zum Ersatz verpflichtende Umstand nicht eingetreten wäre. Der Geschädigte soll

${ }^{692}$ Oetker in Münchener Kommentar BGB, Bd. 2, \ 249 Rn. 19; Schmidt in Bamberger/Roth, Bd. 1, \ 249 Rn. 11.

${ }^{693}$ C. Schubert in Bamberger/Roth, Bd. 1, \249 Rn. 44; Oetker in Münchener Kommentar, Bd. 1, \ 249 Rn. 98.

${ }^{694}$ BGH NJW 1972, 1943, 1944 f.; NJW 1976, 1143, 1144; ; C. Schubert in Bamberger/Roth, Bd. 1, 』 249 Rn. 46.

${ }^{695}$ BGH NJW 2009, 1066, 1067.

${ }^{696}$ Siehe dazu S. $174 \mathrm{ff}$. 
durch den Schadensersatz folglich nicht besser gestellt werden als er ohne das schädigende Ereignis stünde. Auf der anderen Seite soll der Schädiger - anders als beispielsweise im anglo-amerikanischen Rechtsraum - auch nicht bestraft werden. In den vorliegenden Fällen wäre der Vertragspartner gem. \249 Abs. 1 BGB also verpflichtet, die beschädigten Daten zu reparieren oder diese neu zu erstellen bzw. gem. $\ 249$ Abs. 2 BGB der Universität den dafür erforderlichen Betrag zu zahlen. Sofern die Datensätze jedoch irreparabel zerstört worden sind, wird eine Wiederherstellung allerdings regelmäßig nicht möglich sein. Eine Ausnahme läge allerdings dann vor wenn die Daten beispielsweise noch als Ausdruck verfügbar wären und damit neu in das Computersystem eingepflegt werden könnten. ${ }^{697}$ Sofern dies nicht der Fall ist, wäre eine Wiederherstellung nur durch eine Neugewinnung der Daten denkbar, indem etwa die entsprechende Versuchsreihe neu durchgeführt würde. Zu beachten ist jedoch zum einen, dass hierdurch nicht garantiert wäre, die gleichen Ergebnisse nochmals zu erlangen, eine Wiederherstellung also teilweise von vornherein nicht möglich wäre. In diesen Fällen hätte der IT-Dienstleister der archivierenden Einrichtung gem. \251 Abs. 1 BGB eine angemessene Entschädigung in Geld zu zahlen. ${ }^{698}$

\section{vi. Mitverschulden der Universität}

Des Weiteren ist im Rahmen der Haftung des Vertragspartners entscheidend, ob die Universität ein Mitverschulden trifft. Der Umfang des zu leistenden Schadensersatzes hängt gem. \254 Abs. 1 BGB in den Fällen, in denen die Universität bei der Entstehung dieses Schadens ein Verschulden trifft, davon ab, inwieweit der Schaden von der Universität oder dem Vertragspartner verursacht worden ist. Sofern die Universität also ein Mitverschulden an der Beschädigung oder der Löschung der Daten trifft, würde ihr Mitverschuldensanteil von dem zu zahlenden Schadensersatz des Vertragspartners entsprechend abgezogen werden. Dabei würde der Universität wieder das Verschulden ihrer Mitarbeiter gem. \278 BGB zugerechnet werden. Dies folgt aus $₫ 254$ Abs. 2 Satz 2 BGB, der nach überwiegender Ansicht als Absatz 3 zu lesen ist und sich damit auch auf ein Mitverschulden im Rahmen der Haftungsbegründung gem. \254 Abs. 1 BGB bezieht. ${ }^{699}$

In diesem Zusammenhang stellt sich die Frage, ob die Universität im Rahmen ihrer Sorgfaltspflichten von den zu archivierenden Daten Sicherungskopien erstellen und aufbewahren muss. ${ }^{700}$ Das Bestehen derartiger Kopien würde einen Schaden nämlich regelmäßig nahezu entfallen lassen, da man auf die unversehrten Kopien zurückgreifen könnte und die Informationen damit nicht verloren wären.

\footnotetext{
${ }^{697}$ Vgl. BGH NJW 2009, 1066, 1067.

698 Vgl. BGH NJW 2009, 1066, 1067; Oetker, NJW 1985, 345, 346 f.

699 BGH NJW 1951, 477; Unberath in Bamberger/Roth, Bd. 1, \ 254 Rn. 40; Oetker in Münchener

Kommentar, Bd. 2, \254 Rn. 126; Heinrichs in Palandt, \254 Rn. 48.

700 Zum Erstellen einer Sicherungskopie als Mitwirkungsleistung des Kunden bei Software-

Verträgen siehe Schneider, Handbuch des EDV-Rechts, 3. Auflage 2003, J. Rn. 197.
} 
Eine solche Sorgfaltspflicht zur Erstellung von Sicherungskopien würde dann bestehen, wenn ein verständiger Mensch im eigenen Interesse derartige Sicherungskopien erstellt hätte. Maßgebend ist in diesem Zusammenhang also die vernünftige Verkehrsanschauung. ${ }^{701}$ Dabei ist zu bedenken, dass das Anlegen eines solchen Sicherungsarchives für die Universität jedoch einen erheblichen personellen und wohl auch finanziellen Aufwand darstellen würde. Insbesondere müsste der Universität genügend Speicherplatz zur Verfügung stehen. Bei der Prüfung, ob eine Sorgfaltspflicht zur Erstellung von Sicherungskopien besteht, ist deshalb darauf abzustellen, inwieweit der Vertragspartner mit der Archivierung beauftragt wird. Soll er lediglich bei einzelnen Arbeitsschritten unterstützend tätig werden, so kann von der Universität bzw. dem LZA-Verbund wohl erwartet werden, entsprechende Sicherungskopien bereit zu halten, da sie ja auch die sonstige Archivierung organisiert und damit über die dafür erforderliche Infrastruktur verfügt. Anders wird es sich jedoch wohl verhalten, wenn der Vertragspartner mit der gesamten Archivierung beauftragt wird oder wenn seine Leistung gerade in der Bereitstellung von Speicherplatz besteht, wie etwa bei einem Mietvertrag über Servermodule.

In diesen Fällen gibt die Universität die Archivierung ja gerade deshalb in die Hände eines Dritten, weil es ihr nur schwer oder sogar überhaupt nicht möglich ist, die Archivierung selbst durchzuführen. Würde man nun jedoch von ihr erwarten, zusätzliche Sicherungskopien aufzubewahren, da ihr ansonsten ein Mitverschulden zur Last fiele, könnte sie die Archivierung gleich selbst durchführen. Demzufolge wäre in diesen Fällen das Bereithalten von Sicherungskopien aufgrund des nicht unerheblichen Aufwands, der damit verbunden ist, nicht als Sorgfaltspflicht einzustufen, so dass die Universität im Falle eines Schadens aufgrund der Löschung oder Beschädigung der Datensätze kein Mitverschulden nach $\ 254$ Abs. 1 BGB treffen würde.

Etwas anderes würde jedoch gelten, sofern der Vertragspartner nur Teile der Archivierung vornimmt und die Universität bzw. der LZA-Verbund ihm zu diesem Zweck die Daten zur Verfügung stellt. Da die sonstigen Arbeitsschritte und der damit verbundene Aufwand durch die Universität erledigt würden, würde es als „Herrin“ der Archivierungsmaßnahmen auch zu ihren Sorgfaltspflichten zählen, entsprechende Sicherungsmaßnahmen zu treffen.

b. Datenfehler- oder Verlust aufgrund eines Materialfehlers

Neben der Beschädigung oder Löschung von Datensätzen aufgrund menschlichen Fehlverhaltens können Fehler auch infolge von Materialversagen entstehen. Dies kann zum einen Fehler oder Beschädigungen der Hardware zur Ursache haben, aber auch Softwarefehler, die beispielsweise zum Absturz des Systems führen

${ }^{701}$ BGH NJW 2001, 149, 150; 2004, 3328; 2006, 1426, 1427; NJW-RR, 2006, 965, 966; Oetker in Münchener Kommentar BGB, Bd. 2, \254 Rn. 30; Unberath in Bamberger/Roth, Bd. 1, \254 Rn. 9. 
können. Die Pflichtverletzung besteht in diesen Fällen also nicht darin, dass Daten versehentlich gelöscht oder falsch bearbeitet werden. Eine Pflichtverletzung kann in diesen Fällen jedoch darin bestehen, dass der Vertragspartner seine Geräte nicht ordnungsgemäß gewartet oder keine entsprechenden Datensicherung vorgenommen hat. Darüber hinaus kann der Vertragspartner eine Pflichtverletzung auch dadurch begehen, dass er von den ihm zur Archivierung übermittelten Daten keine Sicherungskopien erstellt hat. Dies wird, wie oben gezeigt, zumindest dann der Fall sein, wenn der Vertragspartner überwiegend oder vollständig allein für die Archivierung zuständig ist. Die Pflicht, die Geräte und Software zu warten und damit den unversehrten Bestand der Daten zu schützen, würde generell als Nebenpflicht aus $\int 241$ Abs. 2 BGB folgen, wonach die Parteien des Schuldverhältnisses zur Rücksicht auf die Rechte, Rechtsgüter und Interessen des anderen Teils verpflichtet sein können. Sofern die Leistung des Vertragspartners jedoch darin besteht, dass er der Universität Speicherplatz zur Verfügung stellt, läge dem ein Mietvertrag zugrunde, so dass die Erhaltung der Speichermodule zum bestimmungsgemäßen Gebrauch gem. $\int 535$ Abs. 1 S. 2 BGB sogar zu seinen Hauptpflichten zählen würde. ${ }^{702}$

In diesem Fall wäre der vertragsmäße Gebrauch die Speicherung der zur Archivierung bestimmten Daten auf den angemieteten Speichermedien. Zu beachten ist, dass der Vertragspartner in diesen Fällen nicht nach $\ 280$ Abs. 1 BGB sondern nach \536a Abs. 1 BGB Schadensersatz verlangen könnte. Bedeutung hat dies im Rahmen des Vertretenmüssens. Sofern die vom Vertragspartner zur Verfügung gestellten Speichermedien nämlich schon bei deren Überlassung mangelhaft wären, würde der Vertragspartner, sofern aufgrund dieses Mangels ein Datenverlust entstünde, der Universität gem. \536a Abs. 1 Alt. 1 BGB und der darin enthaltenen Garantiehaftung auch dann haften, wenn er den Mangel nicht zu vertreten hätte. Sofern der Mangel erst später auftritt, müsste der Vermieter Schadensersatz leisten, sofern er die Umstände, die zur Mangelhaftigkeit der Speichermedien geführt haben - wozu auch die nicht ordnungsgemäße Durchführung von Wartungsarbeiten zählt - zu vertreten hat. Zu beachten ist, dass das Vertretenmüssen von der Universität im Rahmen eines Prozesses bewiesen werden müsste, da die oben angesprochene Beweislastumkehr des $₫ 280$ Abs. 1 Satz 2 BGB im Rahmen des $₫ 536$ a BGB keine Anwendung findet.

\section{Eigene Archivierung}

Sofern der Fehler innerhalb der archivierenden Einrichtung selbst auftritt bzw. durch deren Mitarbeiter verursacht wird, ist auch hier ist wieder danach zu differenzieren, ob der Fehler durch menschliches Fehlverhalten (dazu 1.) entstanden ist oder durch Materialversagen (dazu 2.).

702 Schneider, F. Rn. 216, 221 
a. Datenfehler aufgrund menschlichen Fehlverhaltens

Sofern der Datenfehler innerhalb der Universität auftritt, kommt auch hier zum einen ein menschliches Fehlverhalten in Betracht, wie es bereits unter B.I.1. dargestellt worden ist. Allerdings wäre der Schädiger hier ein Angestellter der Universität, so dass sich eventuelle Schadensersatzansprüche nur gegen diesen selbst richten könnten. Als Anspruchsgrundlage käme auch hier wieder zunächst \280 Abs. 1 Satz 1 BGB in Betracht. Das zugrunde liegende Schuldverhältnis im Sinne des \ 280 Abs. 1 BGB wäre dabei der zugrunde liegende Arbeitsvertrag zwischen der Universität und dem Arbeitnehmer. Dabei handelt es sich um einen Dienstvertrag,

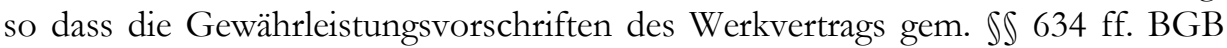
von vornherein ausscheiden. ${ }^{703}$ Die Pflicht des Arbeitnehmers, die Datensätze der Universität nicht zu beschädigen, folgt wiederum gem. \241 Abs. 2 BGB als Nebenpflicht aus dem Arbeitsverhältnis, sofern sich nicht ohnehin eine entsprechende Klausel im Arbeitsvertrag findet.

Sofern die Pflichtverletzung jedoch von einem eigenen Arbeitnehmer erfolgt ist, sind die Grundsätze des sog. innerbetrieblichen Schadensausgleichs zu beachten. Danach hat der Arbeitnehmer zwar gem. \ 276 Abs. 1 BGB Vorsatz und Fahrlässigkeit (und damit auch leichte Fahrlässigkeit) zu vertreten. Nach den Grundsätzen des ,innerbetrieblichen Schadensausgleichs“, der in ständiger Rechtsprechung des Bundesarbeitsgerichts für Verletzungshandlungen und Pflichtverletzungen von Arbeitnehmern im Arbeitsverhältnis angewandt wird, ${ }^{704}$ hat bei leichter Fahrlässigkeit des Arbeitnehmers der Arbeitgeber den Schaden allein zu tragen. ${ }^{705}$ Im Falle von mittlerer Fahrlässigkeit tragen Arbeitnehmer und Arbeitgeber den Schaden anteilig. Lediglich bei grober Fahrlässigkeit und bei vorsätzlichem Handeln trägt der Arbeitnehmer den Schaden allein. Dabei kann jedoch auch bei grob fahrlässigem Handeln des Arbeitnehmers eine Schadensteilung in Betracht kommen, wenn zwischen dem Schadensrisiko und dem Verdienst des Arbeitnehmers ein grobes Missverhältnis besteht. ${ }^{706}$

Sofern der Datenfehler also auf leicht fahrlässigem Verhalten des Arbeitnehmers bei einer betriebsbezogenen Tätigkeit beruht, so steht der archivierenden Einrichtung mangels Vertretenmüssen des Arbeitnehmers kein Anspruch gem. \$ 280 I 1 BGB zu. Vielmehr müsste sie den entstandenen Schaden selbst tragen. Sofern dem Arbeitnehmer zwar keine leichte sondern einfache Fahrlässigkeit zur Last fällt, hätte die archivierende Einrichtung einen Schadensersatzanspruch auf zumindest teilweisen Ersatz des entstandenen Schadens. Lediglich bei vorsätzli-

\footnotetext{
703 Sprau in Palandt, Vorb v $\int 633$ Rn. 2.

704 BAG NJW 2003, 377, 378; NJW 1993, 1732; Weidenkaff in Palandt, \ 611 Rn. 152 ff; Fuchs in Bamberger/Roth (Hrsg.), Bd. 2, 2. Aufl. 2008, J 611 Rn. 93 f.

705 Fuchs in Bamberger/Roth, Bd. 2, \ 611 Rn. 94; Schmidt-Kessel in Prütting/Wegen/Weidenreich, \ 276 Rn. 23; Weidenkaff in Palandt, \ 611 Rn. 157.

706 BAG NZA NJW 1990, 468, 469 f.; NZA 1999, 263; Fuchs in Bamberger/Roth, Bd. 2, J 611 Rn. 94.
} 
chem und grob fahrlässigem Handeln des Arbeitnehmers könnte somit ein Anspruch auf Ausgleich des gesamten Schadens gegen den Arbeitnehmer entstehen. $\mathrm{Zu}$ beachten ist schließlich, dass die Beweislastumkehr des $₫ 280$ Abs. 1 S. 2 BGB gem. $\int 619 \mathrm{a}$ BGB im Arbeitsverhältnis keine Anwendung zu Lasten des Arbeitnehmers findet.

\section{b. Datenfehler aufgrund Materialverschleißes}

Sofern der Datenfehler aufgrund eines Materialfehlers hervorgerufen worden ist, kommt es zunächst darauf an, ob es sich bei der defekten Hardware um Eigentum der Universität handelt oder um gemietete oder geleaste Geräte. Sofern sich die defekte Hardware im Eigentum der Universität befindet, käme ein Anspruch gegen den Verkäufer gem. \S 280 Abs. 1, 433 Abs. 1 im Rahmen des Ersatzes des sog. Mangelfolgeschadens in Betracht. Der Verkäufer müsste den Mangel der Hardware allerdings zu vertreten haben, was jedoch gem. \280 Abs. 1 Satz 2 BGB widerleglich vermutet wird. Sofern der Verkäufer nicht beweisen kann, dass er den Mangel nicht zu vertreten hat, könnte die Universität den Ersatz des durch den Datenverlust entstandenen Schaden von dem Verkäufer verlangen. Dieser Schadensersatzanspruch würde neben eventuellen Nacherfüllungsansprüchen der Universität nach den $\$ \int 437 \mathrm{ff}$. BGB stehen und nach der regelmäßigen Frist des \195 BGB innerhalb von drei Jahren ab Ablauf des Jahres verjähren, in dem der Anspruch entstanden ist und die Universität Kenntnis davon erlangt hat. Zu beachten ist, dass auch hier ein Mitverschulden der Universität gem. \254 Abs. 1 BGB vom Schadensersatzanspruch abzuziehen wäre. Dabei wäre ein Verschuldensbeitrag eines Mitarbeiters dieser wiederum gem. \254 Abs. 2 Satz 2 i.V.m. \ 278 BGB zuzurechnen. Ein Mitverschulden könnte dabei insbesondere darin bestehen, dass der Mangel nicht rechtzeitig dem Verkäufer angezeigt wurde. Ferner könnte auch in diesen Fällen eine Pflicht zur Erstellung von Sicherungskopien als Sorgfaltspflicht bestehen.

Sofern die Universität die Hardware gemietet hätte, käme ein Schadensersatzanspruch gem. $\int 536$ a Abs. 1 BGB in Betracht. Sofern der Mangel bereits bei Überlassung der Hardware vorgelegen hätte, müsste der Vermieter unabhängig vom Vertretenmüssen des Mangels für den entstandenen Schaden haften. Sofern der Schaden erst nach Überlassung der Hardware entstanden wäre, müsste er jedoch nur dann haften, wenn er den Umstand, auf dem der Mangel beruht, zu vertreten hat oder er mit der Beseitigung des Mangels in Verzug kommt.

\section{i. Haftungsbegrenzung durch AGB}

Dabei stellt sich auch hier wieder die Frage, ob der Vertragspartner die Haftung im Rahmen seiner AGB begrenzen kann. Dabei ist danach zu unterscheiden, ob zwischen der Universität und dem Dritten ein Kaufvertrag oder ein Mietvertrag vorliegt. 


\section{(1) Haftungsbegrenzung im Rabmen eines Kaufvertrages}

$\mathrm{Zu}$ beachten ist, dass das Verhalten des Verkäufers im konkreten Fall zunächst auch als fahrlässig einzustufen sein müsste. Dies ergibt sich jedoch nicht allein daraus, dass er eine mangelhafte Sache geliefert hat. Insbesondere trifft den Verkäufer nicht von vornherein eine Pflicht, die Kaufsache zu überprüfen. ${ }^{707}$ Etwas anderes kann jedoch ausnahmsweise gelten, wenn es sich um besonders anfällige oder hochwertige Produkte handelt oder der Verkäufer eine besondere Sachkunde aufweist. ${ }^{708} \mathrm{Im}$ Einzelfall ist also zu prüfen, ob es sich bei der mangelhaften Hardwarekomponente um ein besonders fehleranfälliges oder hochwertiges Produkt handelt oder ob der Verkäufer eine besondere Sachkunde aufweist, was in den hier besprochenen Fällen durchaus zutreffen kann, da die Universität die Hardware im Regelfall bei spezialisierten Großhändlern erwerben wird, bei denen eine solche Sachkunde wohl im Normalfall vorausgesetzt werden kann. Ein eventuelles Verschulden des Herstellers wird dem Verkäufer allerdings nicht gem. \$ 278 BGB zugerechnet, da es sich bei ihm nicht um den Erfüllungsgehilfen des Verkäufers handelt. ${ }^{709}$ Hat der Verkäufer dadurch, dass er die Kaufsache zuvor nicht geprüft hat, ausnahmsweise also doch die im Verkehr erforderliche Sorgfalt außer Acht gelassen, könnte seine Haftung dennoch aufgrund der AGB auf vorsätzliches oder grob fahrlässiges Verhalten beschränkt sein. Auch hier muss zunächst geprüft werden, ob die Universität eine solche Haftungsfreizeichnung gegen sich gelten lassen müsste.

(a) Freizeichnung von der Haftung bei grob fahrlässigem Verhalten

Der Verkäufer kann seine Haftung aufgrund der Indizwirkung des $\int 309$ Nr. 7 b) BGB nicht für grob fahrlässiges Handeln ausschließen. Diesbezüglich kann auf die obigen Ausführungen verwiesen werden. ${ }^{710}$

(b) Begrenzung für einfache Fahrlässigkeit

Die Wirksamkeit einer Klausel, die die Haftung des Verkäufers auf grobe Fahrlässigkeit und Vorsatz beschränkt, würde davon abhängen, ob die Universität durch diese gem. \ 307 Abs. 1 BGB unangemessen benachteiligen würde. Zu beachten ist dabei, dass der Verkäufer gem. \ 433 Abs. 1 Satz 2 BGB dazu verpflichtet ist, dem Käufer die Sache frei von Sach- und Rechtsmängeln zu verschaffen. Dies würde damit eine Freizeichnung für die Haftung der Verletzung einer wesentlichen Pflicht darstellen, was nach gefestigter höchstrichterlicher Rechtsprechung

\footnotetext{
707 BGH NJW 1968, 2238, 2239; 1981, 1269, 1270; Grüneberg in Palandt, \280 Rn. 19; Schmidt-Kessel in Prütting/Wegen/Weinreich, \280 Rn. 70.

708 Grüneberg in Palandt, $\ 280$ Rn. 19. H.P. Westermann in Münchener Kommentar BGB, Bd. 2, 5. Aufl. 2008, \$280 Rn. 70.

709 OLG Köln NJW-RR 2006, 677; Weidenkaff in Palandt, \437 Rn. 37; S. Loren₹, ZGS 2004, 408, 410.

710 Siehe oben Seite 204.
} 
jedoch nicht zulässig wäre. ${ }^{711}$ Damit könnte der Verkäufer sich nicht von der Haftung für einen fahrlässig verursachten Mangelfolgeschaden befreien. Sofern den Verkäufer also durch die Lieferung einer mangelhaften Sache ausnahmsweise fahrlässig gehandelt hat, scheidet der Anspruch auf Ersatz des Mangelfolgeschadens also auch nicht aufgrund einer Haftungsfreizeichnung für leichte Fahrlässigkeit aus.

\section{(2) Begrenzung der Haftung im Rabmen eines Mietvertrages}

Denkbar ist ferner, dass die Universität die defekte Hardware gemietet hatte. Auch hier könnte der Vermieter versuchen, durch entsprechende Klauseln im Vertrag seine Haftung weitestgehend auszuschließen.

(a) Ausschluss der verschuldensunabhängigen Haftung

Da der Vermieter gem. \536a Abs.1 Alt.1 BGB für Mängel, die bei Überlassung der Mietsache bereits vorlagen, verschuldensunabhängig haftet, könnte der Vermieter versuchen, diese Garantiehaftung durch seine AGB auf eine verschuldensabhängige Haftung zu beschränken. Diese Klausel wäre jedoch gem. \307 Abs. 1, Abs. 2 Nr. 1 BGB unwirksam, sofern sie mit wesentlichen Grundgedanken der Regelung, von der abgewichen wird, nicht vereinbar ist. Die für Schadensersatzansprüche untypische Garantiehaftung des $\ 536$ Abs. 1 Alt. 1 BGB stellt jedoch gerade kein gesetzliches Leitbild im Sinne des $₫ 307$ Abs. 2 Nr. 1 BGB dar, sondern eine Ausnahme. ${ }^{712}$ Somit wäre ein Ausschluss der Garantiehaftung für anfängliche Mängel an der Mietsache zulässig. ${ }^{713}$

(b) Ausschluss der Haftung für grobe Fahrlässigkeit

Da der Ausschluss der Haftung für grobes Verschulden generell unzulässig ist, wäre eine derartige Klausel auch im Rahmen eines Mietvertrages unzulässig. ${ }^{714}$

(c) Ausschluss der Haftung für einfache Fahrlässigkeit

Schließlich stellt sich die Frage, ob eine Beschränkung der Haftung auf grobe Fahrlässigkeit zulässig ist oder ob der Vermieter auch haften muss, wenn er die Umstände, auf denen der Mangel beruht, leicht oder einfach fahrlässig zu vertreten hat. Eine solche Klausel wäre unwirksam, wenn diese eine Freizeichnung von der Haftung für den Verstoß gegen wesentliche Pflichten darstellte. ${ }^{715}$

\footnotetext{
711 Siehe oben Seite 205Fn. 687.

712 BGHNJW-RR 1993, 519, 520; Häublein in Münchener Kommentar BGB, Bd. 3, 6. Aufl. 2012, $\int 536 a$ Rn. 21; Feldhabn in Prütting/Wegen/Weinreich, \536a Rn.3.

713 BGH NJW-RR 1993, 519, 520; 2002, 3232; Weidenkaff in Palandt, \ 536a Rn. 7; Christensen in Ulmer/Brandner/Hensen, \ 309 Nr. 7 Rn. 41; Feldhahn in Prütting/Wegen/Weinreich, \536a Rn. 3.

714 Zur Unwirksamkeit der Freizeichnung für grobe Fahrlässigkeit siehe schon oben S. 204.

715 Siehe dazu schon oben Seite 205 f. sowie die Rechtsprechungsnachweise in Fn. 687.
} 
Bei der sich aus $\int 535$ BGB ergebenden Pflicht des Vermieters, die Mietsache in einem zum vertragsgemäßen Gebrauch geeigneten Zustand zu erhalten, handelt es sich aber um eine wesentliche Vertragspflicht im Sinne von \307 Abs. 2 Nr. 2 BGB. ${ }^{716}$ Dürfte sich der Vermieter für fahrlässig verursachte Schäden freizeichnen, würde dies seine Instandhaltungspflicht nicht unerheblich zu Lasten des Mieters einschränken. Der Vermieter müsste die Mietsache zwar immer noch instandhalten, müsste jedoch nicht mit Sanktionen rechnen, wenn er diese Pflicht verletzen würde. ${ }^{717}$ Zwar würde sich der zu entrichtende Mietzins ipso iure gem. $\int 536$ Abs. 1 Satz 2 BGB mindern; dies stellt aber keine hinreichende Kompensation der Verschuldenshaftung dar.

Die einschlägige Rechtsprechung zu diesem Problem umfasst zwar überwiegend Sachverhalte aus dem Bereich der Wohnraummiete. Die angeführten Argumente lassen sich jedoch auf die vorliegenden Fälle der Miete von Speicherplatz übertragen. Auch in diesen Fällen wäre der Vertragszweck bei einer Freizeichnung des Vermieters für fahrlässiges Verhalten gefährdet, zumal der Mieter, hier also die Universität Göttingen bzw. der LZA-Verbund, sich nicht zumutbar gegen den aufgrund eines Mangels an den Speichermedien drohenden Datenverlust schützen könnte. Für die Unwirksamkeit einer solchen Klausel spricht darüber hinaus auch ein Urteil des OLG Koblenz, in dem eine Haftungsfreizeichnung für fahrlässiges Verhalten im Rahmen der Gewerberaummiete für zulässig erachtet wurde. ${ }^{718}$ Das Gericht begründete seine Entscheidung nämlich damit, dass ein Großteil der Instandhaltungs- und Instandsetzungspflichten in üblicher und zulässiger Weise dem Mieter auferlegt worden waren. ${ }^{719}$ Damit bestand in diesem Fall jedoch gerade nicht die Gefahr, dass der Vermieter seine Instandhaltungspflicht sanktionslos verletzt, da die Instandhaltung größtenteils sowieso Sache des Mieters war. Dies wird in den Fällen der Anmietung von Speicherplatz aber wohl grundsätzlich nicht der Fall sein. Darüber hinaus ist zu beachten, dass das Urteil des OLG Koblenz ${ }^{720}$ noch vor dem oben zitierten Urteil des $\mathrm{BGH}^{721}$ ergangen ist. Damit kann davon ausgegangen werden, dass auch die Freizeichnung für einfache Fahrlässigkeit unwirksam wäre.

\section{ii. Zwischenergebnis zu b.}

Sofern ein Datenverlust aufgrund mangelhafter Hardware entsteht, kommen grundsätzlich Ansprüche gegen den Verkäufer bzw. gegen den Vermieter der Hardware in Betracht. Ein Schadensersatzanspruch wird jedoch häufig daran scheitern, dass der Verkäufer die Mangelhaftigkeit nicht zu vertreten hat bzw. der

\footnotetext{
716 BGH NJW 2002, 673, 675.

717 BGH NJW 2002, 673, 675.

718 OLG Koblenz NZM 2000, 622.

719 OLG Koblenz NZM 2000, 622.

720 Siehe Fn. 718.

721 Siehe Fn. 716.
} 
Vermieter sich von der gesetzlich vorgesehenen Garantiehaftung für anfängliche Mängel freizeichnen kann. Eine Freizeichnung des Verkäufers und Vermieters von einfach fahrlässig verursachten Mängeln durch AGB würde einer gerichtlichen AGB-Kontrolle demnach grundsätzlich nicht standhalten. Das Gleiche gilt erst Recht für die Freizeichnung von grob fahrlässigem Verhalten oder gar Vorsatz.

II. Ergebnis

Sofern im Rahmen der einzelnen Archivierungsschritte Daten gelöscht oder beschädigt werden, kommt grundsätzlich ein Schadensersatzanspruch gem. \280 I BGB in Betracht. Sofern der Datenverlust durch einen Fehler eines Fremdunternehmens entstanden ist, an das die Archivierung ausgegliedert wurde, richtet sich der Schadensersatzanspruch gegen diesen Vertragspartner. Dabei ist dem Unternehmen ein eventuelles Vertretenmüssen seiner Mitarbeiter zuzurechnen. Sofern der Datenfehler hingegen durch einen Mitarbeiter der Universität bzw. des LZAVerbundes selbst verursacht wird, würde sich der Schadensersatzanspruch gegen diesen richten. Dabei wird der Arbeitnehmer aber aufgrund des im Arbeitsrecht geltenden sog. „innerbetrieblichen Schadensausgleichs“ den Schaden nur dann komplett alleine tragen müssen, wenn er grob fahrlässig oder gar vorsätzlich gehandelt hat. Sofern die Schadenshöhe außer Verhältnis zum Einkommen des Arbeitnehmers steht, würde der Schaden sogar bei grob fahrlässigem Verhalten von Arbeitnehmer und LZA-Verbund bzw. Universität anteilig getragen werden.

Beruht der Datenverlust hingegen nicht auf menschlichem Versagen, sondern auf mangelhafter Hardware, so kommen unter Umständen Schadensersatzansprüche gegen den Verkäufer bzw. den Vermieter der Hardware in Betracht. Diese müssen den Mangel jedoch zu vertreten haben, wobei eine Freizeichnung im Rahmen von AGB nur in Ausnahmefällen möglich ist. 


\section{Kapitel 4 - Fazit}

Abschließend lässt sich feststellen, dass im Rahmen der elektronischen Langzeitarchivierung wissenschaftlicher Primärdaten vor allem urheberrechtliche, datenschutzrechtliche und haftungsrechtliche Probleme auftreten können. Dabei birgt die Archivierung von personenbezogenen Daten für die archivierende Einrichtung die größten rechtlichen Probleme und aufgrund der drohenden Haftung auch Risiken.

Rechtliche Einschränkungen können sich diesbezüglich sowohl aus dem Bundesdatenschutzgesetz, den einzelnen Landesdatenschutzgesetzen sowie dem Sozialgesetzbuch X ergeben. Aus diesem sollte die archivierende Einrichtung vor der Archivierung personenbezogener Daten eine formgerechte Einwilligung des jeweiligen Betroffenen einholen. Sollte dies nicht möglich sein, könnte eine Archivierung der Daten aufgrund der Erlaubnisnorm des $\ 10$ NDSG oder einer entsprechenden Vorschrift der übrigen Landesdatenschutzgesetze zulässig sein. Dies gilt jedoch nur dann, wenn die betreffenden Daten weder zu einem anderen Zweck als dem der wissenschaftlichen Forschung erhoben worden sind noch im Rahmen eines anderen Forschungsvorhabens gewonnen wurden.

Sofern eine wirksame datenschutzrechtliche Einwilligung des Probanden vorliegt, ist darin außerdem gleichzeitig eine (zumindest konkludent erteilte) Entbindung des behandelnden Arztes von seiner ärztlichen Schweigepflicht zu sehen, was vor allem dann relevant ist, wenn medizinische Untersuchungsdaten archiviert 
werden sollen. Die Entbindung von der Schweigepflicht entspricht dabei konsequenterweise in ihrer Reichweite dem Umfang, in welchem der Proband auch der datenschutzrechtlich relevanten Nutzung seiner Daten eingewilligt hat.

Der Betroffene kann seine Einwilligung allerdings jederzeit widerrufen, womit diese mit Wirkung für die Zukunft entfällt. Des Weiteren ist aus datenschutzrechtlicher Sicht zu beachten, dass die Daten sowohl nach den Vorschriften des BDSG als auch nach den Landesdatenschutzgesetzen zu anonymisieren sind, sobald der Forschungszweck dies zulässt. Sofern dies nicht möglich sein sollte, hat die archivierende Einrichtung die Merkmale, mit denen ein Personenbezug hergestellt werden kann, zumindest gesondert zu speichern. Bevor personenbezogene Daten archiviert werden ist also stets zu prüfen, ob die Merkmale, mit denen ein Personenbezug hergestellt werden kann, wirklich noch erforderlich sind. Einer solchen Prüfung sind darüber hinaus regelmäßig die bereits archivierten Daten zu unterziehen. Da die elektronische Archivierung personenbezogener Daten regelmäßig eine automatisierte Datenverarbeitung darstellt, hat die archivierende Einrichtung die jeweiligen datenschutzrechtlichen Vorschriften hinsichtlich der zu ergreifenden technischen und organisatorischen Maßnahmen zu beachten, namentlich des \9 S. 1 i.V.m. der Anlage zu \9 S. 1 BDSG sowie des $₫ 7$ Abs. 2 NDSG beziehungsweise der entsprechenden Vorschriften der übrigen Landesdatenschutzgesetze zu.

Sofern die archivierende Einrichtung gegen die datenschutzrechtlichen Vorgaben verstößt, kann dies zum einen eine Straftat oder Ordnungswidrigkeit darstellen. Darüber hinaus stehen dem Betroffenen unter Umständen auch Schadensersatzansprüche zu, wobei sowohl das BDSG als auch die Landesdatenschutzgesetze in den Fällen einer automatisierten Datenverarbeitung eine verschuldensunabhängige Haftung vorsehen. In den Fällen einer nicht automatisierten Datenverarbeitung kann sich die archivierende Einrichtung zwar exkulpieren, trägt allerdings die Beweislast für das fehlende Verschulden. Besondere Bedeutung erlangt die verschuldensunabhängige Haftung im Rahmen der Auftragsdatenverarbeitung, da der Anspruchsgegner in diesen Fällen der Auftraggeber, im Rahmen der Langzeitarchivierung also die archivierende Einrichtung bleibt. Diese sollte aus diesem Grund in die Verträge mit der beauftragten Einrichtung für den Fall eines VerstoBes gegen datenschutzrechtliche Vorschriften und eines daraus entstehenden Schadensersatzanspruches des Betroffenen, eine Freistellungsklausel in den Vertrag aufnehmen.

Neben den gerade genannten datenschutzrechtlichen Problemen können sich außerdem urheberrechtliche Probleme ergeben, was jedoch vergleichsweise selten der Fall sein wird, da wissenschaftliche Primärdaten grundsätzlich nicht dem Schutz des Urheberrechtsgesetzes unterliegen. Dies gilt in der Regel auch dann, wenn die Daten in Tabellen oder auf andere Art zusammengefasst werden. Eine solche Zusammenstellung könnte zwar ein Datenbankwerk nach \4 Abs. 2 UrhG oder eine Datenbank gem. §87a UrhG darstellen. Zum einen wird es dafür jedoch 
regelmäßig an der erforderlichen geistigen Schöpfungshöhe fehlen, die bei Datenbankwerken nach $\int 4$ Abs.2 UrhG in der individuellen Auswahl oder Anordnung der Daten bestehen muss. Eine solche Individualität wird in den vorliegend relevanten Fällen grundsätzlich nicht vorliegen, da die Anordnung nach logischen Gesichtspunkten erfolgen wird. Zum anderen wird der sui-generis-Schutz der Datensammlung nach $\int 87$ a UrhG in den meisten Fällen daran scheitern, dass für die vorliegend in Betracht kommenden Datensammlungen in der Regel keine wesentliche Investition im Sinne der Vorschrift erforderlich ist. Investitionen werden vielmehr bei der Datenerhebung getätigt, deren Kosten jedoch im Rahmen des $\int 87$ a UrhG gerade nicht zu berücksichtigen sind.

Sofern für eine Datensammlung im Einzelfall doch eine wesentliche Investition erforderlich ist, müsste die archivierende Einrichtung zunächst prüfen, ob sie Datenbankherstellerin und damit Inhaberin der erforderlichen Nutzungsrechte ist, da bezüglich der Archivierungsmaßnahmen und einer eventuellen öffentlichen Zugänglichmachung der archivierten Daten grundsätzlich keine urheberrechtlichen Schranken zugunsten der archivierenden Einrichtung greifen. Im Rahmen der elektronischen Archivierung werden dabei vor allem auf das Recht der Vervielfältigung und das Recht der öffentlichen Zugänglichmachung im Internet tangiert. Insbesondere in den Fällen von Drittmittelprojekten sowie den Fällen der Auftragsforschung, in denen ein Dritter das Investitionsrisiko trägt, ist daher zu prüfen, ob die Rechte des Datenbankherstellers nicht bei der finanzierenden Einrichtung liegen.

Sofern die Nutzungsrechte ursprünglich bei einem Angestellten der archivierenden Einrichtung entstanden sind, könnte diese sie bereits aufgrund des Arbeitsverhältnisses erlangt haben. Zu beachten ist aber, dass dieser Grundsatz aufgrund der durch Art. 5 Abs. 3 GG garantierten Wissenschaftsfreiheit nicht für Hochschulprofessoren gilt, so dass hier nur dann von einem Übergang der Rechte ausgegangen werden kann, wenn der Arbeitsvertrag eine ausdrückliche Rechteübertragungsklausel enthält.

Bei anderen Forschungseinrichtungen kann sich hingegen auch eine stillschweigende Pflicht zur Rechteübertragung aus dem Arbeitsvertrag ergeben. Vor dem Hintergrund, dass die Nutzungsart der öffentlichen Zugänglichmachung im Internet erst seit1995 als bekannt gilt, kann ein Übergang dieses Rechts bei Arbeitsverhältnissen, die vor diesem Zeitpunkt geschlossen wurden, an der Vorschrift des $\int 31$ Abs. 4 a.F.UrhG scheitern.

Dies gilt jedoch nicht für Arbeitsverträge öffentlich-rechtlicher Forschungseinrichtungen, da deren Angestellte in der Regel wirtschaftlich ausreichend abgesichert sind und daher des Schutzes der besagten Vorschrift nicht bedürfen. Bei privaten Forschungseinrichtungen bleibt es hingegen bei der Anwendung von $\int$ 31Abs. 4 UrhG. Bei diesen besteht jedoch die Möglichkeit, das Recht der öffentlichen Zugänglichmachung im Internet aufgrund der Rechtsübertragungsfiktion des \ 1371 UrhG erlangt zu haben, sofern ihr vom Rechteinhaber alle zum Zeitpunkt 
des Vertragsschlusses relevanten Nutzungsrechte an dem betreffenden Datensatz eingeräumt wurden. Dies werden zum einen das Recht der Vervielfältigung sowie das Recht der Verbreitung des Werkes sein, die in der Regel jedoch bereits aufgrund des Arbeitsvertrags auf die archivierende Einrichtung übergegangen sein werden. Daneben kommt jedoch gerade im wissenschaftlichen Bereich das Recht der Nutzung des Werkes als CD-ROM in Betracht, sofern der Arbeitsvertrag nach Bekanntwerden dieser Nutzungsart geschlossen wurde, was frühestens Ende der 1980er Jahre ist.

Da die Verwertung von Datenbanken, die wissenschaftliche Primärdaten zum Inhalt haben, jedoch regelmäßig auch ohne diese Nutzungsart vollumfänglich verwertet werden kann, ist in den vorliegenden Fällen das Recht der CD-ROMNutzung grundsätzlich nicht als wesentliches Recht i.S.d. \137 1 UrhG anzusehen.

Demzufolge werden auch Forschungseinrichtungen auf deren Arbeitsverträge die Vorschrift des $₫ 31$ Abs. 4 a.F. UrhG Anwendung findet, das Recht der öffentlichen Zugänglichmachung grundsätzlich aufgrund der Rechteübertragungsfiktion des $\ 1371$ UrhG erlangt haben, sofern der Rechteinhaber nicht rechtzeitig seinen Widerspruch erklärt und das besagte Recht nicht vor dem 01.01.2008 auf einen Dritten übertragen hat.

Handelt es sich bei dem Rechteinhaber hingegen nicht um einen (ehemaligen) Arbeitnehmer der Forschungseinrichtung, bleibt der Forschungseinrichtung nur die Möglichkeit, sich die benötigten Nutzungsrechte von diesem rechtsgeschäftlich übertragen zu lassen, sofern nicht $\ 1371$ UrhG greifen sollte. Relevant ist dies vor allem bei Forschungsprojekten, bei denen auch ehrenamtliche Beiträger mitgewirkt haben. Aus dem oben Gesagten folgt, dass Forschungseinrichtungen besonderes Augenmerkauf die Ausgestaltung ihrer Arbeitsverträge legen sollten. Insbesondere sollten sie detaillierte Rechteübertragungsklauseln in ihre Arbeitsverträge einfügen, um spätere Rechtsunsicherheiten hinsichtlich der Inhaberschaft von Nutzungsrechten von vornherein zu vermeiden.

Ein weiteres urheberrechtliches Problem ergibt sich, sofern die archivierende Einrichtung mit Fremdforschern und anderen Forschungseinrichtungen Lizenzverträge über die Bereitstellung bestimmter archivierter Daten schließt und diese Verträge die Vervielfältigung und Weitergabe der Daten durch eine Verschwiegenheitsklausel untersagen, da dem Lizenznehmer hierdurch auch urheberrechtliche Schranken abbedungen würden. Dies ist zwar grundsätzlich zulässig, jedoch nur insoweit, wie dadurch nicht grundrechtlich geschützte Interessen des Lizenznehmers verletzt werden, da die Klausel andernfalls nach $₫ 307$ Abs. 1 BGB beziehungsweise, im Falle einer individualvertraglichen Vereinbarung, nach \138 Abs. 1 BGB nichtig wäre. Im Mittelpunkt steht dabei vor allem die in Art. 5 Abs. 3 GG geschützte Wissenschaftsfreiheit.

Aus diesem Grund muss die Klausel ausdrücklich klarstellen, dass der Lizenznehmer die Daten für den eigenen wissenschaftlichen Gebrauch vervielfältigen 
darf. Dies ist insbesondere dann von Bedeutung, wenn es sich bei dem Lizenznehmer nicht um eine natürliche Person, sondern eine andere Forschungseinrichtung handelt, da diese die empfangenen Daten regelmäßig für ihre angestellten Wissenschaftler vervielfältigen muss. Aufgrund des Verbotes einer geltungserhaltenden Reduktion im Rahmen von AGB wäre darüber hinaus eine verfassungskonforme Auslegung einer Klausel nicht möglich, die allgemein die Vervielfältigung und Weitergabe der Datensätze untersagt. Auch die Vereinbarung einer Vertragsstrafe für den Fall, dass der Lizenznehmer gegen die Verschwiegenheitsklausel verstößt, ist grundsätzlich zulässig, sofern die Klausel optisch ausreichend hervorgehoben wird und die AGB einen entsprechenden Hinweis auf die Vertragsstrafe enthalten. Andernfalls läge eine überraschende Klausel nach $\ 305 c$ $\mathrm{BGB}$ vor.

Aus haftungsrechtlicher Sicht lässt sich zunächst feststellen, dass keine generelle Pflicht zu einer elektronischen Archivierung existiert. Allerdings bestehen hinsichtlich medizinischer Daten unter Umständen Dokumentationspflichten aus $\mathbb{S}$ 28 Abs. 4RöntgV, \42 StrlSchV sowie einer von Rechtsprechung und Literatur als Nebenpflichtaus dem Behandlungsvertrag anerkannten Dokumentationspflicht. Darüber hinaus folgt aus $₫ 1$ GenTAufzV eine Aufzeichnungspflicht für den Fall, dass gentechnische Arbeiten oder Freisetzungen durchgeführt werden. Dabei ist festzuhalten, dass es sich bei diesen Dokumentationspflichten nicht speziell um Pflichten zu einer digitalen Langzeitarchivierung handelt, die Dokumentation aufgrund der technischen Gegebenheiten und des technischen Standards aber regelmäßig elektronisch erfolgen werden.

Weitere haftungsrechtliche Probleme entstehen, sofern im Rahmen der Archivierung Dateien gelöscht oder beschädigt werden und dadurch Daten verloren gehen. In diesen ist zunächst danach zu differenzieren, ob die Archivierung von der Einrichtung selbst oder einem beauftragten Unternehmen durchgeführt wurde. Im letztgenannten Fall haftet das Unternehmen der archivierenden Einrichtung nach $\$ 280$ Abs. 1 S. 1BGB, wobei ein eventuelles Verschulden der Mitarbeiter dem beauftragten Unternehmen nach $\ 278$ BGB zugerechnet wird. Eine Haftungsfreizeichnung im Rahmen von AGB ist dabei nur für grobe Fahrlässigkeit und vorsätzliches Handeln einfacher Mitarbeiter zulässig. Dabei muss die Freizeichnungsklausel klarstellen, dass sie nur dann greift, sofern der Mitarbeiter eine nicht hauptleistungsbezogene Pflicht verletzt. Darüber hinaus darf von der Freizeichnung die Haftung für die Verletzung des Lebens, des Körpers oder der Gesundheit ausdrücklich nicht erfasst werden. Da es sich bei der Löschung von Daten durch einen Mitarbeiter des beauftragten Unternehmens allerdings um die Verletzung einer hauptleistungsbezogenen Pflicht handeln wird, haftet der Auftragnehmer der archivierenden Einrichtung auch bei einem wirksamen Haftungsausschluss auf Schadensersatz, sofern die archivierende Einrichtung einen ihr kausal entstandenen Schaden nachweisen kann. 
Da dies gerade bei gelöschten Daten mitunter schwierig sein kann, sollte im Vorfeld ein pauschalisierter Schadensersatz vereinbart werden. Ein Mitverschulden der archivierenden Einrichtung wegen nicht vorgenommener Sicherungskopien der Daten ist zumindest in den Fällen abzulehnen, in denen das Fremdunternehmen gerade mit der Speicherung der Daten beauftragt worden wäre, weil die archivierende Einrichtung gerade nicht über genügend Speicherplatz verfügt. Sofern der Datenverlust hingegen durch einen Mitarbeiter der archivierenden Einrichtung selbst verursacht wird, haftet dieser ihr grundsätzlich ebenfalls auf Schadensersatz gem. $\ 280$ Abs. 1 S. 1 BGB aufgrund einer Pflichtverletzung aus dem Arbeitsverhältnis. Zu beachten sind in diesem Zusammenhang jedoch zum einen die Grundsätze des innerbetrieblichen Schadensausgleich, wonach der Arbeitnehmer bei leichter Fahrlässigkeit gar nicht und bei einfacher Fahrlässigkeit nur anteilig für den entstandenen Schaden haftet. Zum anderen ist auch die Beweislastumkehr des $\ 619 \mathrm{aBGB}$ zu berücksichtigen, nach der die archivierende Einrichtung ihrem Arbeitnehmer das Vertretenmüssen nachzuweisen hätte. Beruht der Datenverlust hingegen auf einem Materialfehler wie etwa defekter Hardware oder fehlerhafter Software, so kommen kauf- oder mietrechtliche Gewährleistungsansprüche der archivierenden Einrichtung in Betracht. Für den Fall, dass ein mit der Archivierung beauftragtes Unternehmen die fehlerhafte Hard- oder Softwareverwendet hätte, könnte auch dieses schadensersatzpflichtig sein, sofern es pflichtwidrig unterlassen hat, eine ordnungsgemäße Wartung vorzunehmen. Aus haftungsrechtlicher Sicht sollten die archivierenden Einrichtungen, sofern sie einen IT-Dienstleister mit der Archivierung beauftragen zum einen Freistellungsklauseln für den Fall einer Verletzung von datenschutzrechtlichen Vorschriften vereinbaren.

Zum anderen sollte für den Fall einer Löschung von Daten ein pauschalisierter Schadensersatz vereinbart werden. Aus den genannten Ergebnissen dieser Arbeit folgt also, dass der rechtliche Problemschwerpunkt der elektronischen Langzeitarchivierung wissenschaftlicher Primärdaten nicht, wie man zunächst annehmen könnte, urheberrechtlicher sondern datenschutzrechtlicher Natur ist. Diese Probleme lassen sich aus Sicht der archivierenden Einrichtung insoweit relativ einfach vermeiden, indem nur Daten ohne Personenbezug archiviert werden. Den urheberrechtlichen und haftungsrechtlichen Problemen kann hingegen, wie gezeigt, größtenteils durch eine durchdachte Gestaltung von Arbeits-und IT-Dienstleistungsverträgen vorgebeugt werden. 
D as Zeitalter der immer weiter fortschreitenden Digitalisierung und die stetige Verbesserung der technischen Rahmenbedingungen bieten Forschungseinrichtung neue Mittel und Wege, ihre gewonnenen Daten zu archivieren und öffentlich zugänglich zu machen. Nicht selten gehen damit rechtliche Problematiken einher. Angefangen bei der Frage, wem denn diese Daten "gehören" bis hin zu der Problematik, ob bestimmte Daten überhaupt archiviert werden dürfen und wer für einen eventuellen Datenverlust haften muss. Das vorliegende Werk legt dabei besonderes Augenmerk auf die urheberrechtlichen, datenschutzrechtlichen sowie haftungsrechtlichen Problematiken, welche eine digitale Archivierung mit sich bringen kann. Des Weiteren wird beleuchtet, welche Überlegungen im Vorfeld einer Archivierung angestellt werden müssen und welche Maßnahmen getroffen werden sollten, um rechtlichen Schwierigkeiten vorzubeugen. Die Arbeit soll daher auch als rechtlicher Leitfaden für Archivierungsprojekte dienen, weshalb unter anderem auch Vorschläge für die Formulierung entsprechender Vertragsklauseln enthalten sind. Aus diesem Grund wird außerdem ausführlich auf die rechtlichen Konsequenzen von Verstößen gegen urheber- und datenschutzrechtliche Vorschriften eingegangen. 